\title{
The Impact of the Second Coordination Sphere in \\ Phosphatase Model Complexes
}

\section{INAUGURAL-DISSERTATION}

\author{
zur \\ Erlangung der Doktorwürde \\ der \\ Naturwissenschaftlich-Mathematischen Gesamtfakultät \\ der \\ Ruprecht-Karls-Universität \\ Heidelberg \\ vorgelegt von \\ Diplom-Chemikerin Simone Bosch \\ aus Nördlingen
}






\title{
INAUGURAL-DISSERTATION
}

\author{
zur \\ Erlangung der Doktorwürde \\ der \\ Naturwissenschaftlich-Mathematischen Gesamtfakultät \\ der \\ Ruprecht-Karls-Universität \\ Heidelberg
}

vorgelegt von

Diplom-Chemikerin Simone Bosch

aus Nördlingen

Tag der mündlichen Prüfung: 24. April 2015 



\section{The Impact of the Second Coordination Sphere in Phosphatase Model Complexes}



Die vorliegende Arbeit wurde in der Zeit von März 2011 bis Januar 2015 unter der Betreuung von Prof. Dr. Peter Comba am Anorganisch-Chemischen Institut der Ruprecht-Karls-Universität Heidelberg angefertigt.

Auszüge dieser Arbeit wurden bereits und werden sinngemäß sowie abschnittsweise wörtlich in den folgenden wissenschaftlichen Journalen veröffentlicht:

1. "Dinuclear Zinc(II) Complexes with Hydrogen Bond Donors as Structural and Functional Phosphatase Models"; Simone Bosch, Peter Comba, Lawrence R. Gahan, Gerhard Schenk, Inorg. Chem. 2014, 53, 9036-9051.

2. "Selective Coordination of Gallium(III), Zinc(II) and Copper(II) by an Asymmetric Dinucleating Ligand: A Model for Metallophosphatases"; Simone Bosch, Peter Comba, Lawrence R. Gahan, Graeme R. Hanson, Christopher J. Noble, Gerhard Schenk, Hubert Wadepohl; manuscript in preparation.

3. "An Approach to More Accurate Model Systems for Purple Acid Phosphatases (PAPs)"; Paul V. Bernhardt, Simone Bosch, Peter Comba, Lawrence R. Gahan, Graeme R. Hanson, Valeriu Mereacre, Christopher J. Noble, Annie K. Powell, Gerhard Schenk, Hubert Wadepohl; manuscript in preparation.

4. "Asymmetric Mono- and Dinuclear Gall' and $\mathrm{Zn}$ " Complexes as Models for Purple Acid Phosphatases (PAPs)"; Simone Bosch, Peter Comba, Lawrence R. Gahan and Gerhard Schenk; manuscript in preparation. 

Meiner Familie. 

„Man sollte alles so einfach wie möglich sehen - aber auch nicht einfacher."

Albert Einstein 



\section{ACKNOWLEDGEMENTS}

First and foremost I thank my advisor, Peter Comba, for the fascinating and challenging topic of my PhD thesis. Throughout the four years, I enjoyed the scientific freedom and I appreciated your all-time 'open' office door for discussions and chats. I am also very thankful for enabling me to cooperate with the University of Queensland. I owe my deepest gratitude to you!

Many thanks also to my associate advisors Lawrence Gahan, Graeme Hanson and Gary Schenk from the University of Queensland, who welcomed and included me in their working groups. I always appreciated you being so encouraging and enthusiastic to talk about my data. It was a great benefit for me!

Thanks to all the people that helped me at the UQ, including Tri Le for NMR assistance - especially for all the 2D experiments and a lot of nice chats, George Blazak for microanalysis measurements, Graham MacFarlane and Brett Hamilton for mass analytic measurements. A big ,thank you' to Paul Bernhardt for X-ray data collection and teaching me all about structure refinement. Thanks to Chris Noble for his assistance with the EPR measurements and advice in the simulation of the spectra. Special thanks to Sandrine Kingston-Ducrot for her help with all the formularities related to this thesis.

Also a big ,thank you' to Marlies Schilli and Karin Stelzer for all your help with administrative issues. I am also greatly indepted to the staff of the chemistry department in Heidelberg, including Markus Enders and Beate Termin for the NMR experiments, Hubert Wadepohl and Heidrun Haungs for the X-ray structures, Jürgen Gross and his team for the mass analytic measurements and the staff of the microanalytic laboratory. Thanks also to Valeriu Mereacre and Annie Powell from the KIT for the Mössbauer experiments.

For the financial support I thank the Graduate College 850 for the scholarship as well as the HGS MathComp and DAAD for travel funds.

A huge 'thank you' to Lena Daumann for introducing me to everyone as well as showing me all the facilities and equipment during my stay at the University of Queensland. I really enjoyed working with you! Thanks for all your advices and scientific and private support. I am also greatly indepted to the others of the UQ group who have assisted me in countless ways; thanks to Maram Basha, Manfredi Miraula, Marcelo Pedroso, Emer Phelan and Asha Roberts.

Special thanks to my 'Forschis', Marie-Louise Morkos, Christiane Antoni, Julia Ricken, Esther Kimmle and Clarissa Benzin. I am also very thankful to Philipp Butzug and Maik Jakob for their consistent technical support and to Marion Kerscher for the great discussions and chats not only during our great after-work beer sessions. I also would like to express my appreciation to Bodo 
Martin for the computational support. A huge 'thank you' to my great lab partners, Tine Graef, Nina Mehrkens, Johannes Straub and Michael Westphal. Thank you very much for 'endless' scientific discussions, answering 'stupid' computer questions, singing, dancing and whizzling, ... the great time in Lab 105! Also a heartfelt thanks to all the past and present colleagues and friends of the Comba and Linti research groups for the encouragement and support, the amusing coffee and beer breaks, the skiing seminars and the memorable time in INF 503. A special 'thanks' to Michael Morgen for reading and correcting this thesis.

I would like to also express my appreciation to the nice people I met in Australia: Alicia, George, Liz, Matthias, Mathilde, Paul, Ryan, Shad, Tanja and Tobsn, making my social life downunder so comfortable and exciting. 'Thanks' also to James Namnoum for practicing my English skills during our great afterwork beer sessions. A huge 'thanks' also to my personal 'food supplier' for the delicious snacks and for the psychological support during writing this thesis.

Finally, I thank my parents, Hilde and Franz, as well as my siblings and their 'plus ones', Vroni \& Marcus, Harry \& Sabine, Marina and Franz \& Nadine, for their encouragement and support when I needed it most. Last, but no least, I thank from the bottom of my heart my wonderful housemate, Sandra Schmitt, for her endless support in various inventive ways ;-). I would have not done it without you! 



\section{TABLE OF CONTENTS}

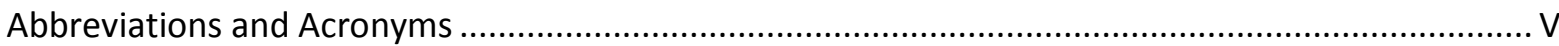

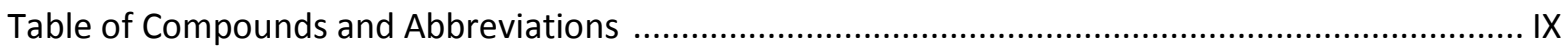

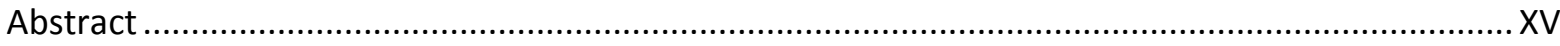

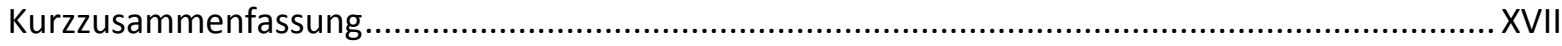

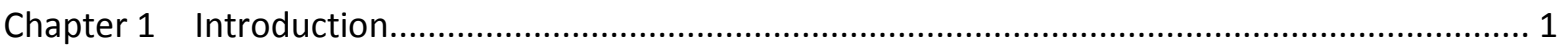

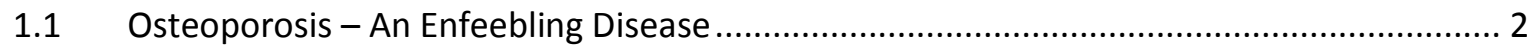

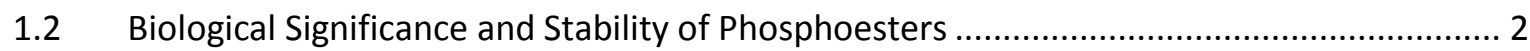

1.3 Phosphatases - Phosphoester Cleaving Enzymes ................................................................ 3

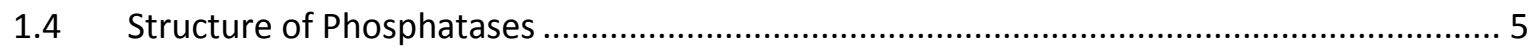

1.5 Significance of the Second Coordination Sphere in Phosphatases ..................................... 8

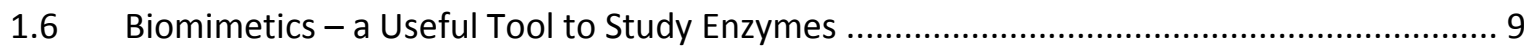

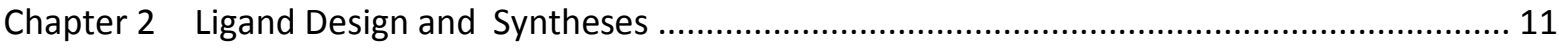

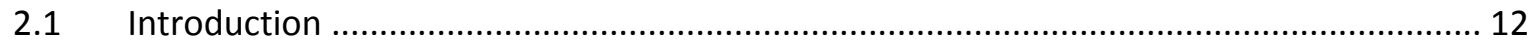

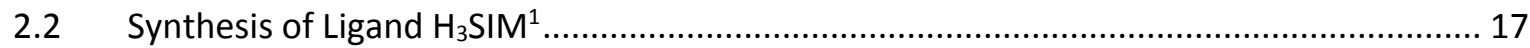

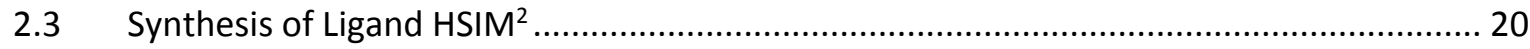

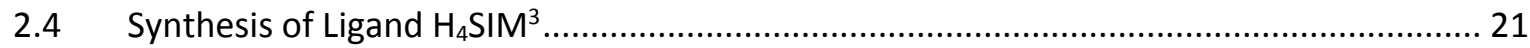

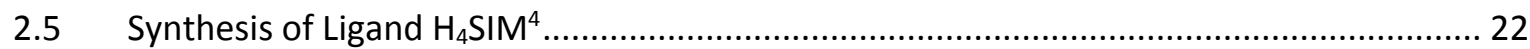

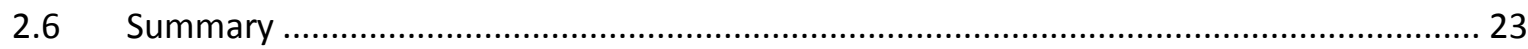

Chapter 3 Coordination Behavior of Asymmetric Ligands ........................................................... 25

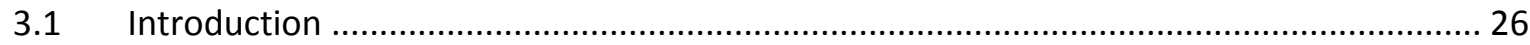

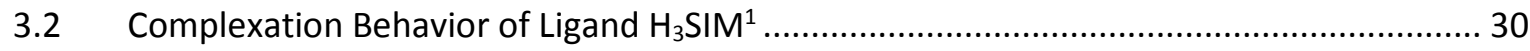

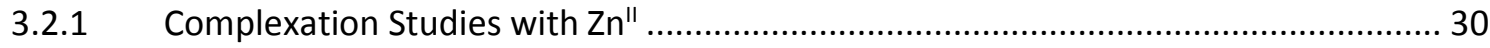

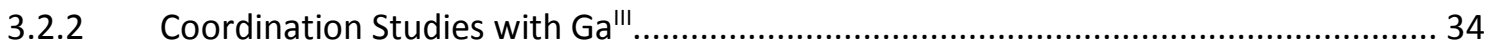

3.2.3 Formation of a Heterodinuclear Ga"'Zn" Complex .................................................... 38

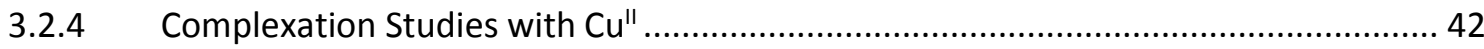

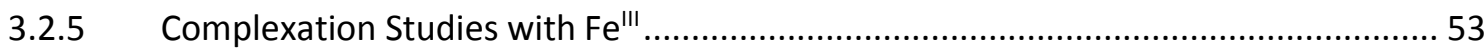

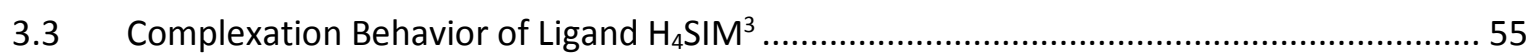

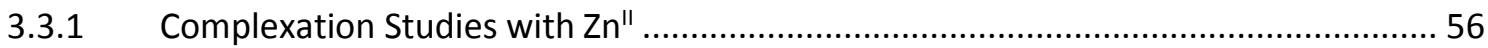

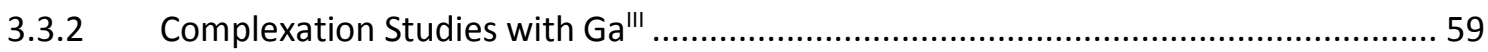

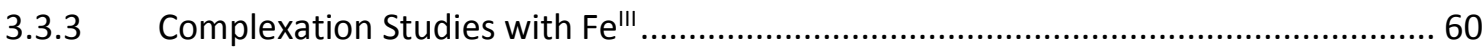

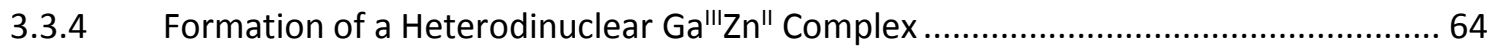

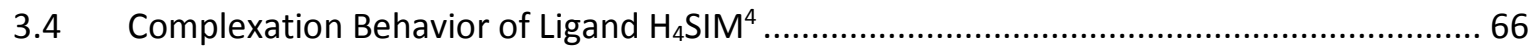

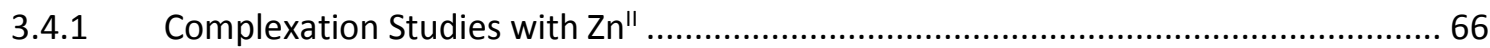




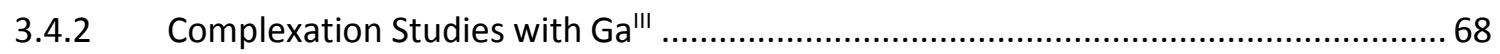

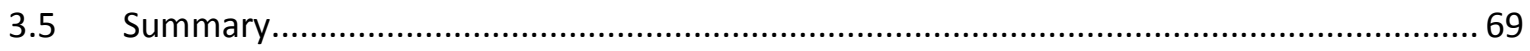

Chapter 4 Dizinc Model Complexes ............................................................................. 71

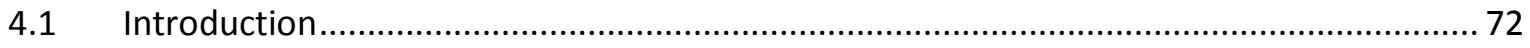

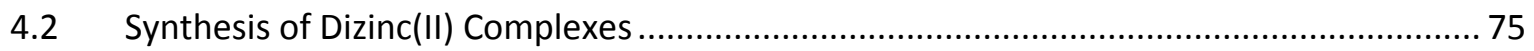

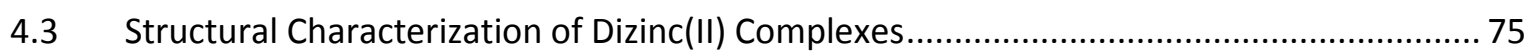

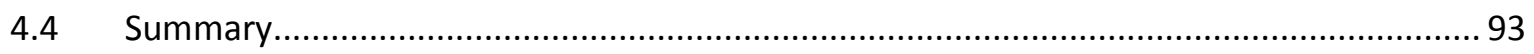

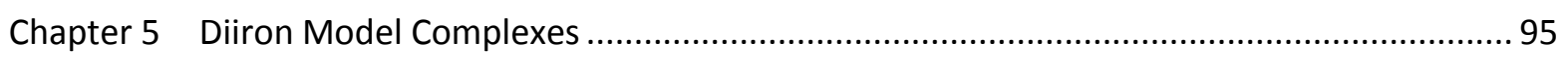

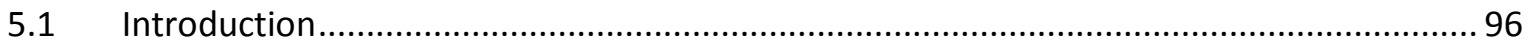

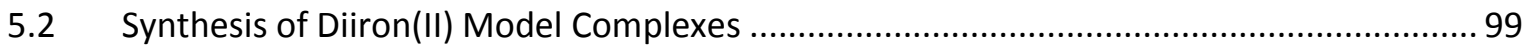

5.3 Structural Characterization of Diiron(II) Complexes ............................................. 100

5.4 Studies toward the Formation of a Heterovalent Fe ${ }^{I I I} \mathrm{Fe}^{\| \prime}$ Complex............................... 125

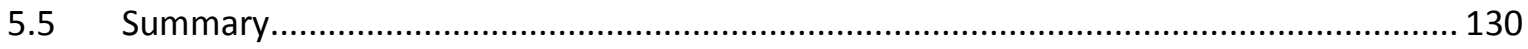

Chapter 6 Studies of the Phosphatase Mechanism .......................................................... 131

6.1 Enzymatic Mechanisms of Phosphoester Cleavage ................................................... 132

6.2 Phosphoester Substrates Employed in Cleavage Studies............................................ 137

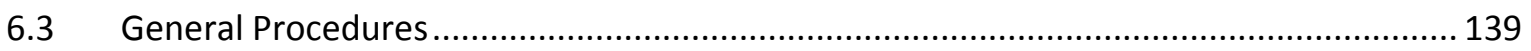

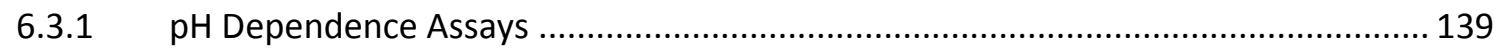

6.3.2 Substrate Concentration Dependence Assays ............................................ 140

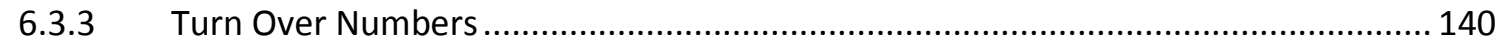

Chapter 7 Phosphatase Activity of Dizinc Complexes ..................................................... 141

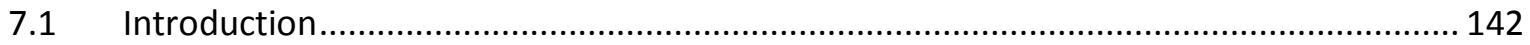

7.2 Phosphoester Hydrolysis Studies of Dizinc(II) Complexes........................................ 148

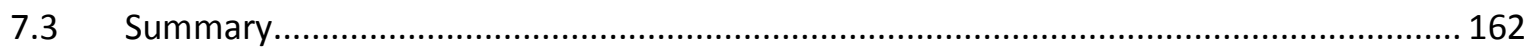

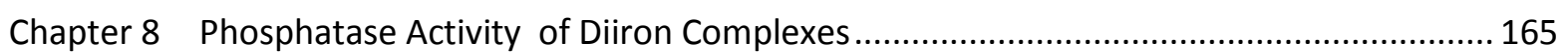

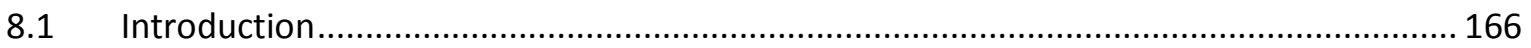

8.2 Phosphoester Hydrolysis Studies of Diiron Complexes ............................................ 168

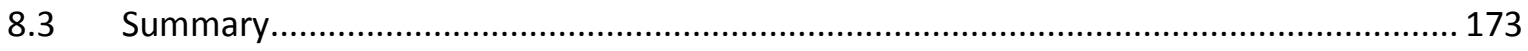

Chapter 9 Phosphatase Activity of Heterovalent Heterodinuclear Complexes ......................... 175

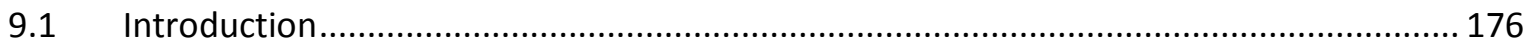

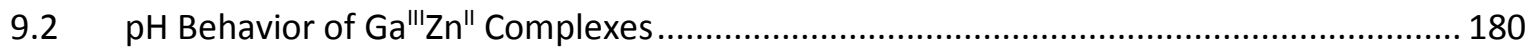

9.3 Phosphoester Hydrolysis Studies with Ga"I"Zn" Complexes ...................................... 181

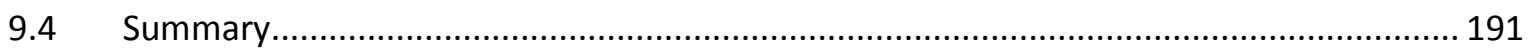

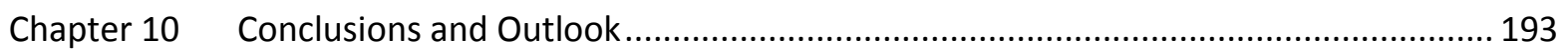

Chapter 11 Experimental Section........................................................................... 199

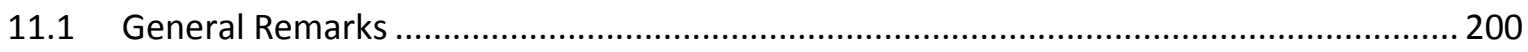




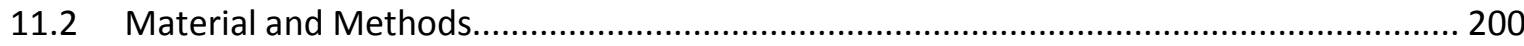

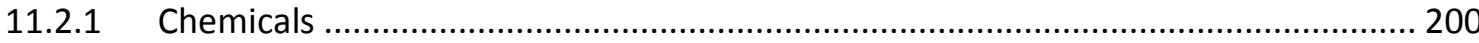

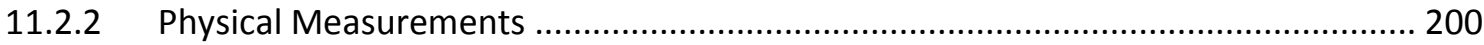

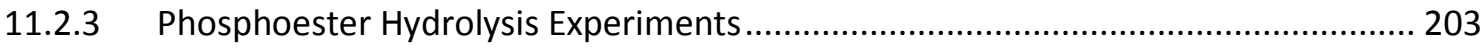

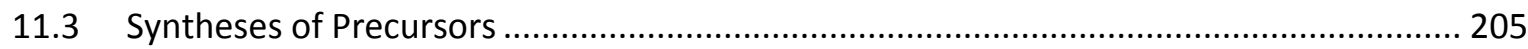

11.3.1 2,6-Bis(chloromethyl)-4-methylphenol (22) ............................................................. 205

11.3.2 3-(Chloromethyl)-2-hydroxy-5-methylbenzaldehyde (5) .......................................... 206

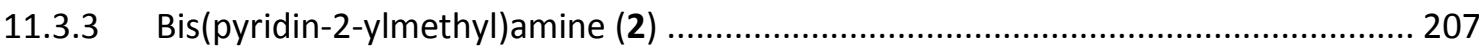

11.3.4 2-(((Pyridin-2-ylmethyl)amino)methyl)phenol (16) ................................................ 208

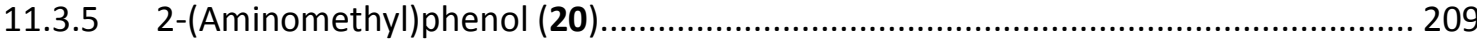

11.3.6 N-(6-pivaloylamido-2-pyridylmethyl)- $N$-(2-pyridylmethyl)amin (18) ....................... 210

11.3.7 N,N-bis((2-pivaloylamidopyridin-6-yl)methyl)amin (3) .......................................... 213

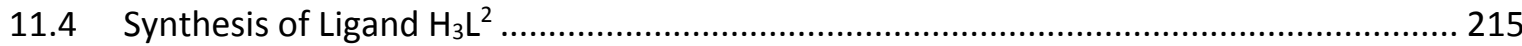

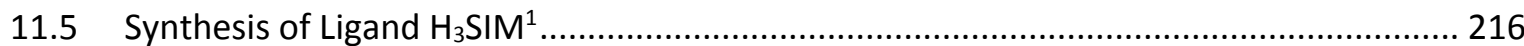

11.5.1 3-((Bis(pyridin-2-ylmethyl)amino)methyl)-2-hydroxy-5-methylbenzaldehyde (12) . 216

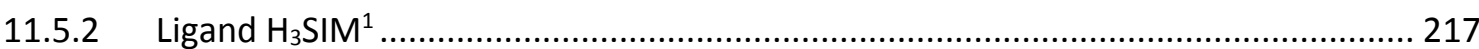

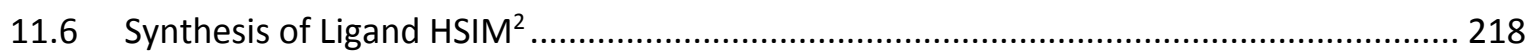

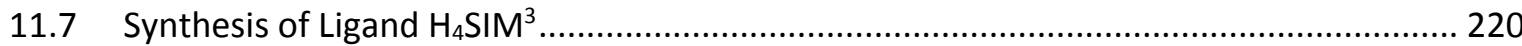

11.7.1 2-Hydroxy-3-(((2-hydroxybenzyl)(pyridin-2-ylmethyl)amino)methyl)-5-methyl-

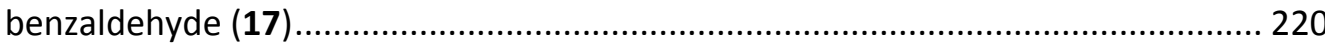

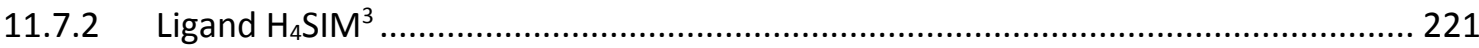

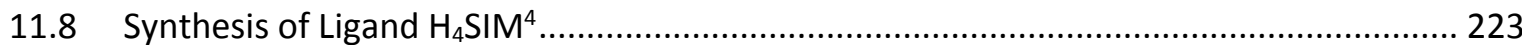

11.8.1 N-(6-(((3-formyl-2-hydroxy-5-methylbenzyl)(pyridin-2-ylmethyl)amino)methyl)pyridin-2-yl)pivalamide (19)

11.8.2 N-(6-(((2-hydroxy-3-(((2-hydroxybenzyl)amino)methyl)-5-methylbenzyl)(pyridin2-ylmethyl)amino)methyl)pyridin-2-yl)pivalamide (21)....................................... 224

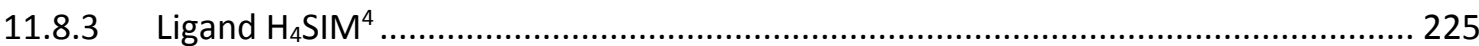

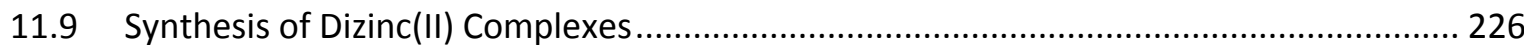

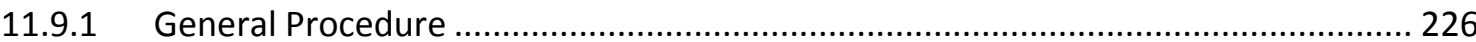

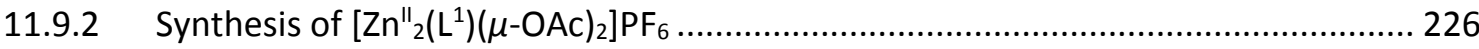

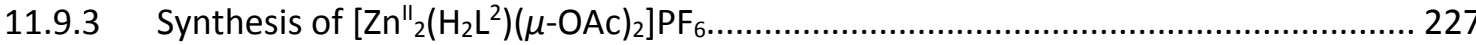

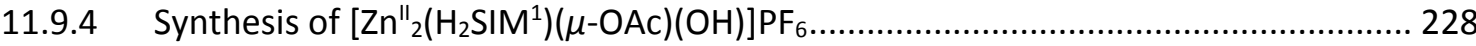

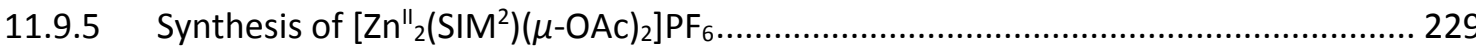

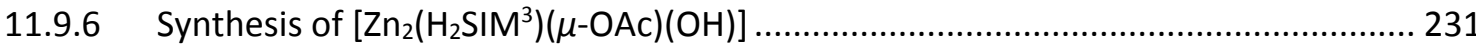

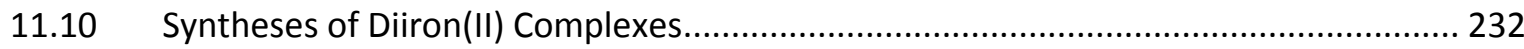

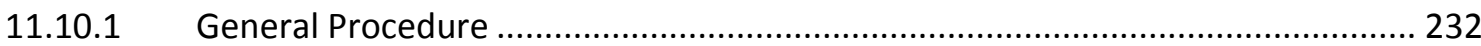

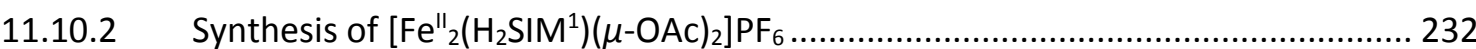




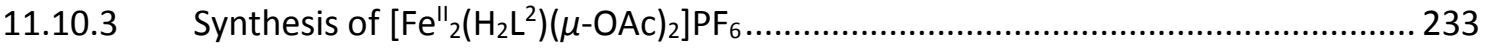

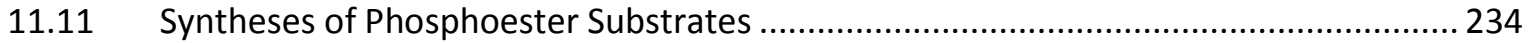

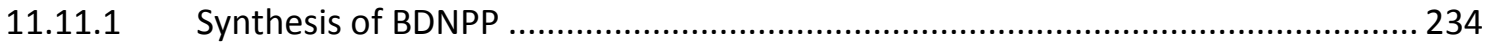

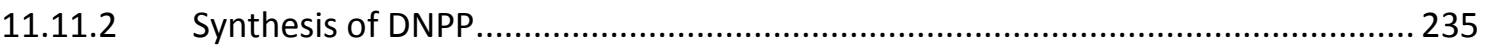

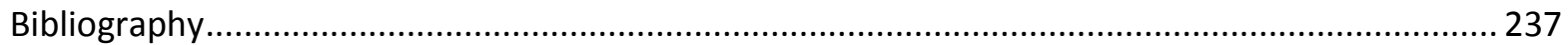

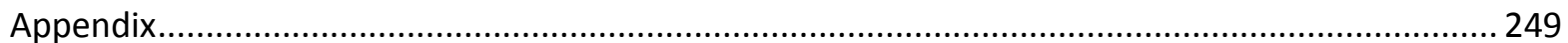




\section{ABBREVIATIONS AND ACRONYMS}

\begin{tabular}{|c|c|}
\hline$\AA$ & Ångström $\left(10^{-10} \mathrm{~m}\right)$ \\
\hline Abs & absorbance \\
\hline AIBN & 2,2'-azobis(2-methylpropionitrile) \\
\hline Ala & alanine \\
\hline Alox & aluminium oxide \\
\hline AP & Alkaline Phosphatase \\
\hline Arg & arginine \\
\hline Asn & asparagine \\
\hline Asp & aspartate \\
\hline ATP & adenosine triphosphate \\
\hline $\mathrm{ax}$ & axial \\
\hline B & magnetic field \\
\hline BDNPP & 2,4-bis(dinitrophenyl)phosphate \\
\hline B.M. & Bohr magneton \\
\hline BPNPP & bis(4-nitrophenyl)phosphate \\
\hline bpy & 2,2'-bipyridine \\
\hline bsPAP & bovine spleen PAP \\
\hline $\mathrm{Bu}$ & butyl \\
\hline calcd. & calculated \\
\hline CAPS & 3-(cyclohexylamino)-1-propanesulfonic acid \\
\hline CCDC & Cambridge Crystallographic Data Centre \\
\hline CEPT & concerted proton-electron transfer \\
\hline CHES & 2-(cyclohexylamino)ethanesulfonic acid \\
\hline conc. & concentrated \\
\hline COSY & correlation spectroscopy \\
\hline $\mathrm{CT}$ & charge transfer \\
\hline CV & cyclovoltammetry \\
\hline Cys & cysteine \\
\hline$d$ & $\operatorname{day}(s)$ \\
\hline $\mathrm{D}$ & axial zero field splitting parameter \\
\hline DEE & diethylether \\
\hline DFT & density functional theory \\
\hline
\end{tabular}




\begin{tabular}{|c|c|}
\hline DMF & $\mathrm{N}, \mathrm{N}$-dimethylformamide \\
\hline DNA & deoxyribonucleic acid \\
\hline DNPP & 2,4-dinitrophenylphosphate \\
\hline DPP & diphenylphosphate \\
\hline E & rhombic zero field splitting parameter \\
\hline$E_{1 / 2}$ & half wave potential \\
\hline E.coli & Escherichia coli \\
\hline EFG & electron field gradient \\
\hline EPR & electron paramagnetic resonance \\
\hline eq. & equivalents / equatorial \\
\hline ESI & electrospray ionization \\
\hline Et & ethyl \\
\hline et al. & et alii \\
\hline EtOAc & ethyl acetate \\
\hline FA & fatty acid \\
\hline $\mathrm{Fc}\left(\mathrm{Fc}^{+}\right)$ & ferrocen (ferrocenium) \\
\hline FNOR & Flavodiiron Nitric Oxide Reductase \\
\hline FTIR & Fourier transform infrared spectroscopy \\
\hline g & Landé g factor \\
\hline Glu & glutamine \\
\hline $\mathrm{h}$ & hour(s) / Boltzmann constant \\
\hline HEPES & 4-(2-hydroxyethyl)piperazine-1-ethanesulfonic acid \\
\hline His & histidine \\
\hline HMBC & heteronuclear multiple bond correlation \\
\hline HPNPP & 2-hydroxypropyl-4-nitrophenylphosphate \\
\hline HRMS & high resolution mass spectrometry \\
\hline hs & high spin \\
\hline HSQC & heteronuclear single quantum correlation \\
\hline IAP & intestinal AP \\
\hline imp & impurity \\
\hline IR & infrared \\
\hline kbPAP & kidney bean PAP \\
\hline $\mathrm{k}_{\text {cat }}$ & first order rate constant \\
\hline Kcom & comproportionation constant \\
\hline $\mathrm{K}_{\mathrm{M}}$ & Michaelis constant \\
\hline
\end{tabular}




\begin{tabular}{|c|c|}
\hline LPS & lipopolysaccharide \\
\hline Is & low spin \\
\hline lut & 2,6-lutidine \\
\hline $\mathrm{m}$ & meta \\
\hline M & molar \\
\hline $\mathrm{m} / \mathrm{z}$ & mass-to-charge ratio \\
\hline MALDI & matrix-assisted laser desorption/ionization \\
\hline Me & methyl \\
\hline MES & 2-(N-morpholino)ethanesulfonic acid \\
\hline $\min$ & minute(s) \\
\hline MMO & Methane Monooxygenase \\
\hline MS & mass spectrometry \\
\hline NBS & $\mathrm{N}$-bromosuccinimide \\
\hline NIR & near infra red \\
\hline NMR & nuclear magnetic resonance \\
\hline NOESY & nuclear Overhauser effect spectroscopy \\
\hline Ns & 4-nitrobenzenesulfonyl \\
\hline 0 & ortho \\
\hline OAc & acetate \\
\hline $\mathrm{OBz}$ & benzoate \\
\hline $\mathrm{p}$ & para \\
\hline p. a. & pro analysi \\
\hline PAP & Purple Acid Phosphatase \\
\hline PCC & pyridinium chlorochromate \\
\hline $\mathrm{Ph}$ & phenyl \\
\hline Piv & pivaloyl \\
\hline PNPP & 4-nitrophenylphosphate \\
\hline ppa & 3-phenylpropionate \\
\hline $\mathrm{PP}_{\mathrm{i}}$ & pyrophosphate \\
\hline ppm & parts per million \\
\hline PTFE & polytetrafluoroethylene \\
\hline Py & pyridine / pyridinyl \\
\hline r & radius \\
\hline $\mathrm{R}_{\mathrm{f}}$ & retention factor \\
\hline RNA & ribonucleic acid \\
\hline
\end{tabular}




\begin{tabular}{|c|c|}
\hline RNR & Ribonucleotide Reductase \\
\hline ROS & reactive oxygen species \\
\hline $\mathrm{rt}$ & room temperature $\left(25^{\circ} \mathrm{C}\right)$ \\
\hline$S$ & spin quantum number \\
\hline Ser & serine \\
\hline SOD & Superoxide Dismutase \\
\hline SQUID & superconducting quantum interference device \\
\hline sub & substitution \\
\hline$t$ & tert \\
\hline THF & tetrahydrofuran \\
\hline TLC & thin layer chromatography \\
\hline TNAP & tissue-nonspecific AP \\
\hline TON & turn over number \\
\hline TRAP & Tartrate-resistant Acid Phosphatase \\
\hline TRAcP & Tartrate-resistant Acid Phosphatase \\
\hline Ts & 4-toluenesulfonyl \\
\hline Tyr & tyrosine \\
\hline ufPAP & uteroferrin PAP \\
\hline UV & ultra violet \\
\hline $\mathrm{V}_{0}$ & initial velocity/rate \\
\hline Vis & visible \\
\hline$V_{\max }$ & maximum velocity/rate \\
\hline vol\% & volume percent \\
\hline vs. & versus \\
\hline w\% & weight percent \\
\hline Z & charge \\
\hline ZFS & zero field splitting \\
\hline$\beta_{e}$ & Bohr magneton \\
\hline$\delta$ & NMR chemical shift \\
\hline$\varepsilon$ & extinction coefficient \\
\hline$\lambda$ & wave length \\
\hline$v$ & frequency \\
\hline
\end{tabular}




\section{TABLE OF COMPOUNDS AND ABBREVIATIONS ${ }^{a}$}

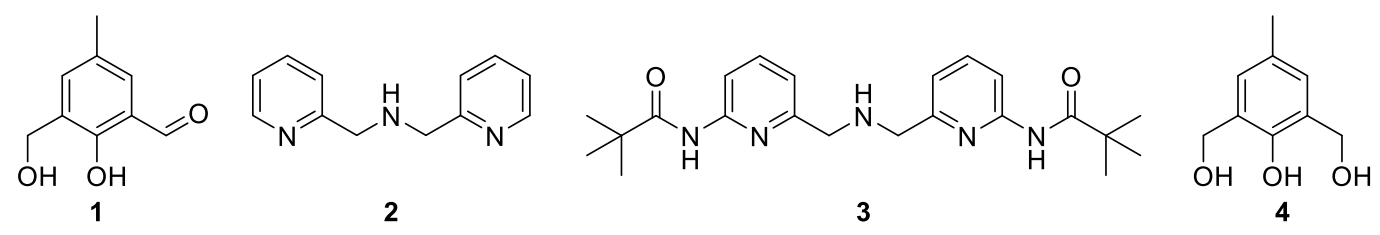<smiles>Cc1cc(C=O)c(O)c(CCl)c1</smiles><smiles>Cc1cc(CO)c2c(c1)COC(C)(C)O2</smiles><smiles>Cc1cc(C=O)c2c(c1)COC(C)(C)O2</smiles><smiles>Cc1cccc(N)n1</smiles><smiles>Cc1cccc(NC(=O)C(C)(C)C)n1</smiles><smiles>CC(C)(C)C(=O)Nc1cccc(CBr)n1</smiles><smiles>CCN(Cc1cccc(NC(=O)C(C)(C)C)n1)S(=O)(=O)c1ccccc1NC(=O)Nc1cccc([N+](=O)[O-])c1</smiles>

8<smiles>Cc1cc(C=O)c(O)c(CN(Cc2ccccn2)Cc2ccccn2)c1</smiles>

10<smiles>Cc1cc(CO)c(O)c(CN(Cc2ccccn2)Cc2ccccn2)c1</smiles><smiles>Oc1ccccc1CNCc1ccccn1</smiles><smiles>Cc1cc(C=O)c(O)c(CN(Cc2ccccn2)Cc2ccccc2O)c1</smiles><smiles>CC(C)(C)C(=O)Nc1cccc(CNCc2ccccn2)n1</smiles><smiles>Cc1cc(C=O)c(O)c(CN(Cc2ccccn2)Cc2cccc(NC(=O)C(C)(C)C)n2)c1</smiles><smiles>NCc1ccccc1O</smiles>

20<smiles>CCCCN(Cc1cccc(CNCc2ccccc2O)n1)Cc1cc([125I])cc(NC(=O)C(C)(C)C)c1O</smiles><smiles>Cc1cc(CCl)c(O)c(CCl)c1</smiles>

22<smiles>Cc1cc(C=O)c(O)c(C=O)c1</smiles>

23<smiles>O=Cc1ccccn1</smiles>

24<smiles>NCc1ccccn1</smiles>

25

26<smiles>NC(=O)c1ccccc1O</smiles>

27 $\overbrace{}^{2}$

HPNPP<smiles>O=[N+]([O-])c1ccc(OP(=O)([O-])Oc2ccc(OP(=O)([O-])Oc3ccc([N+](=O)[O-])cc3[N+](=O)[O-])cc2)cc1</smiles>

a listed are all compounds that are mentioned in this thesis. 

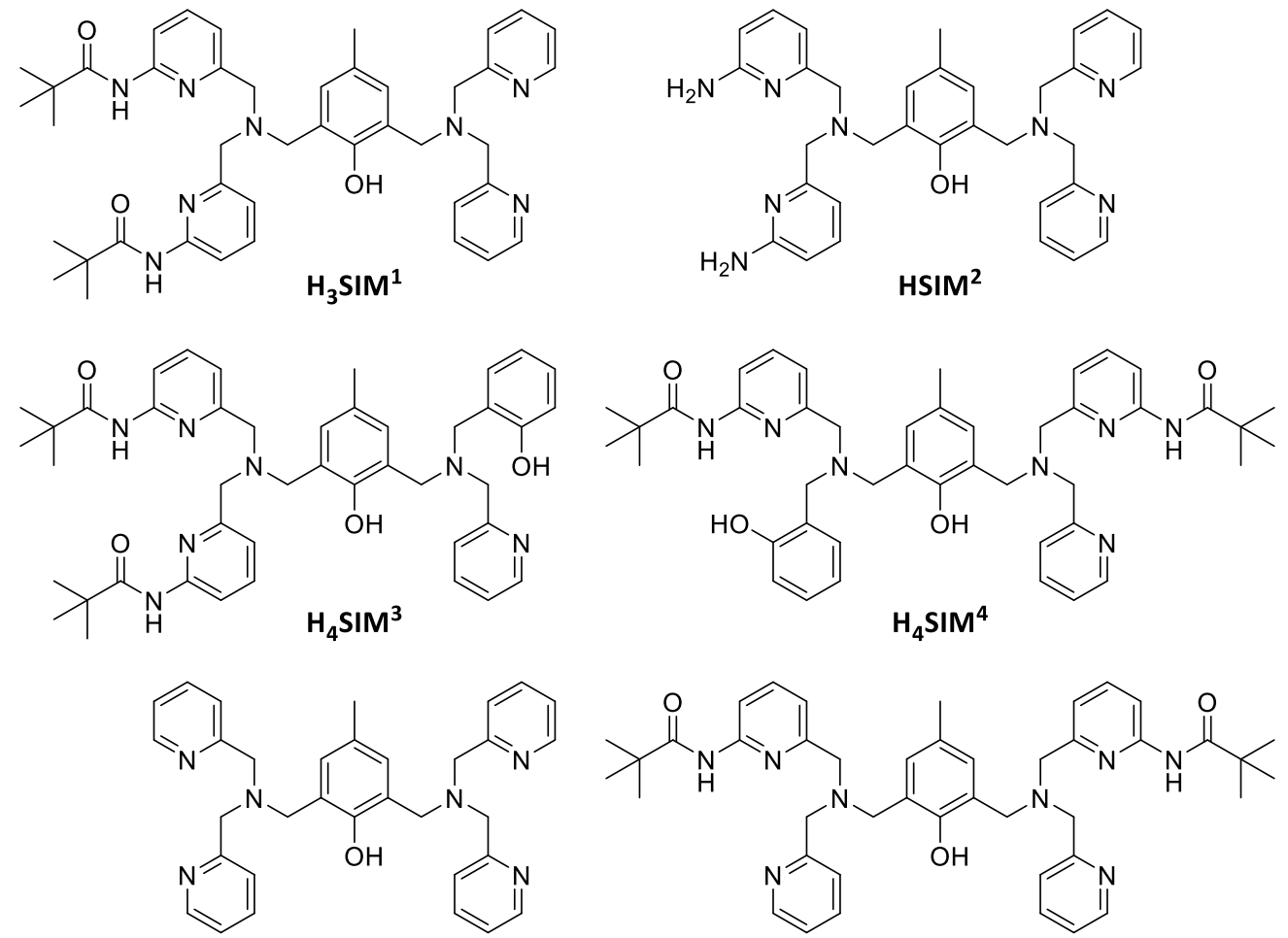

$\mathrm{HL}^{1}$ (HBPMP)<smiles>Cc1cc(CN(Cc2ccccn2)Cc2cccc(NC(=O)C(C)(C)C)n2)c(O)c(CN(Cc2ccccn2)Cc2cccc(NC(=O)C(C)(C)C)n2)c1</smiles>

$\mathrm{H}_{3} \mathrm{~L}^{2}$

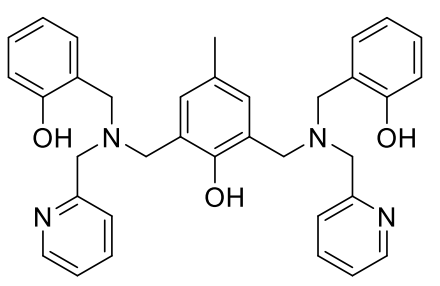

$\mathrm{H}_{3} \mathrm{~L}^{3}\left(\mathrm{H}_{3}\right.$ BBPMP)

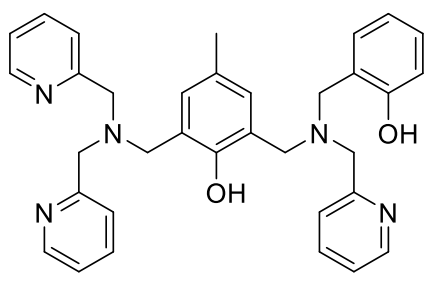

$\mathrm{H}_{2} \mathrm{~L}^{4}\left(\mathrm{H}_{3}\right.$ BPBPMP)

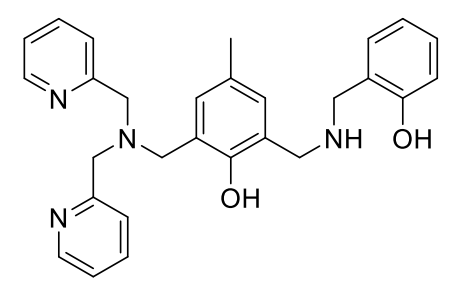

$\mathrm{H}_{2} \mathrm{~L}^{5}$<smiles>[CH2]CCN(CCNC(c1ccccn1)c1ccccn1)CCN1CCCN(C)CCN(CCNC(c2ccccn2)c2ccccn2)CC1</smiles><smiles>Nc1cccc(CN(Cc2cccc(N)n2)CC(O)CN(Cc2cccc(N)n2)Cc2cccc(N)n2)n1</smiles><smiles>Cc1cc(CN(Cc2ccccn2)Cc2cccc(N)n2)c(O)c(CN(Cc2ccccn2)Cc2cccc(N)n2)c1</smiles>

$H L^{8}$<smiles>CC(C)(C)c1cc(CN(Cc2ccccn2)Cc2ccccn2)c(O)c(CN(Cc2ccccn2)Cc2ccccn2)c1</smiles>

$\mathrm{HL}^{9}$ (HBPBP)

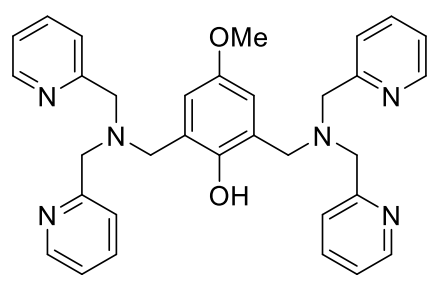

$\mathrm{HL}^{10}$ (HBPMOP)<smiles>Cc1cc(CN(Cc2ccccn2)Cc2ccccn2)c(O)c(CN(Cc2ccccn2)Cc2cccc(C)n2)c1</smiles>

$\mathrm{HL}^{11}$ (HBPLMP)<smiles>Cc1cc(CN(Cc2ccccn2)Cc2ccccn2)c(O)c(CN(Cc2ccccc2O)Cc2ccccc2O)c1</smiles>

$\mathrm{H}_{3} \mathrm{~L}^{12}$<smiles>Cc1cc(C=O)c(O)c(CN2CCN(C(C)C)CCN(C(C)C)CC2)c1</smiles>

$\mathrm{HL}^{13}$ 


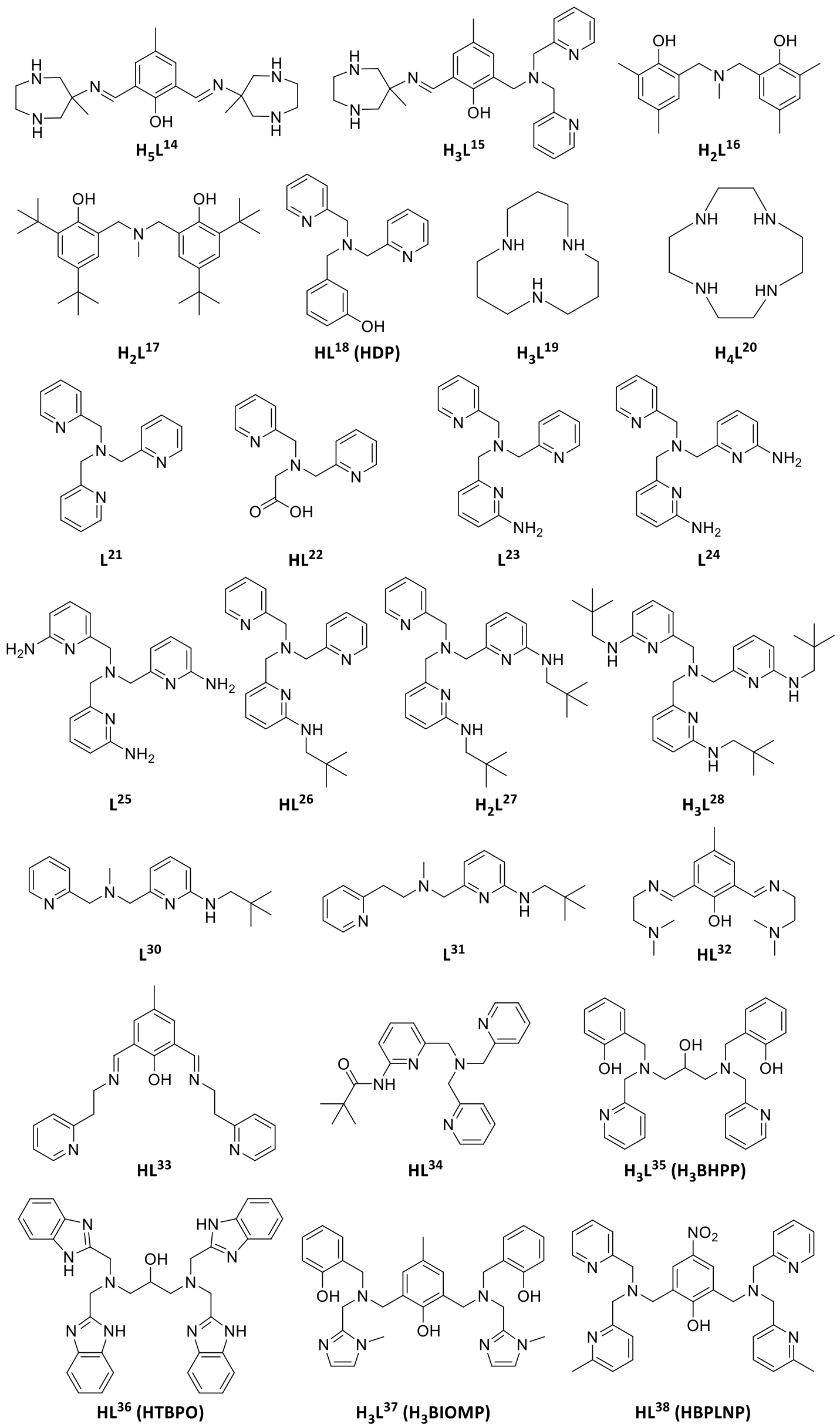


<smiles>Cc1cc(CN(Cc2cccc(NC(=O)C(C)(C)C)n2)Cc2cccc(NC(=O)C(C)(C)C)n2)c(O)c(CN(Cc2cccc(NC(=O)C(C)(C)C)n2)Cc2cccc(NC(=O)C(C)(C)C)n2)c1</smiles>

$$
\mathrm{H}_{5} \mathrm{~L}^{39}
$$<smiles>Cc1cccc(CN(Cc2cccc(C)n2)Cc2cccc(C)n2)n1</smiles>

$\mathrm{L}^{45}$<smiles>Oc1ccccc1CN(Cc1ccccn1)CC(O)CCl</smiles>

$\mathrm{H}_{2} \mathrm{~L}^{46}$<smiles>COC1=CC=CC2=NC=CN=C1C2c1nccn1C</smiles>

$\mathrm{L}^{40}$ (BIPhMe)<smiles>CN(Cc1nc2ccccc2n1C)CC(O)CN(Cc1nc2ccccc2n1C)Cc1nc2ccccc2n1C</smiles>

$\mathrm{HL}^{41}$ (HBMDP)<smiles>CCn1c(CN(Cc2nc3ccccc3n2CC)CC(O)CN(Cc2nc3ccccc3n2CC)Cc2nc3ccccc3n2CC)nc2ccccc21</smiles>

$\mathrm{HL}^{44}$<smiles>c1ccc(-c2ccccn2)nc1</smiles><smiles>c1cnc2c(c1)ccc1cccnc12</smiles><smiles>CC(C)(C)C(=O)Nc1cccc(CN(Cc2ccccn2)Cc2cccc(NC(=O)C(C)(C)C)n2)n1</smiles>

$\mathrm{H}_{2} \mathbf{4}^{49}$<smiles>C1CCCC1</smiles>

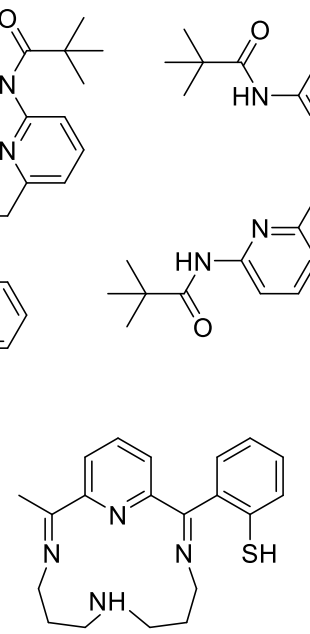

$\mathrm{L}^{47}$ (bpy)

$L^{48}$ (phen)<smiles>CC1=NCCNCCN=C(C)c2cccc1n2</smiles>

$\mathrm{HL}^{52}$
$\mathrm{HL}^{53}$<smiles>Cc1cc(CN(Cc2nc3ccccc3[nH]2)Cc2nc3ccccc3[nH]2)c(O)c(CN(Cc2nc3ccccc3[nH]2)Cc2nc3ccccc3[nH]2)c1</smiles>

$\mathrm{H}_{3} \mathrm{~L}^{50}$<smiles>CN(C)CC#Cc1cccc(-c2cccc(C#CCN(C)C)n2)n1</smiles><smiles>c1ccc(CN(Cc2cccc(CN(Cc3ccccn3)Cc3ccccn3)c2)Cc2ccccn2)nc1</smiles>

$L^{59}$<smiles>N=C(N)NCc1ccc(-c2ccc(CNC(N)N)cn2)nc1</smiles>

$L^{57}$<smiles></smiles>

$\mathrm{HL}^{54}$<smiles>CCCC#Cc1cccc(-c2cccc(C#CCCC)n2)n1</smiles><smiles>N=C(N)NCc1cccc(-c2cccc(CNC(=N)N)n2)n1</smiles>

$\mathrm{L}^{58}$<smiles>Cc1cccc(CN(Cc2cccc(CN(Cc3cccc(C)n3)Cc3cccc(C)n3)c2)Cc2cccc(C)n2)n1</smiles> 

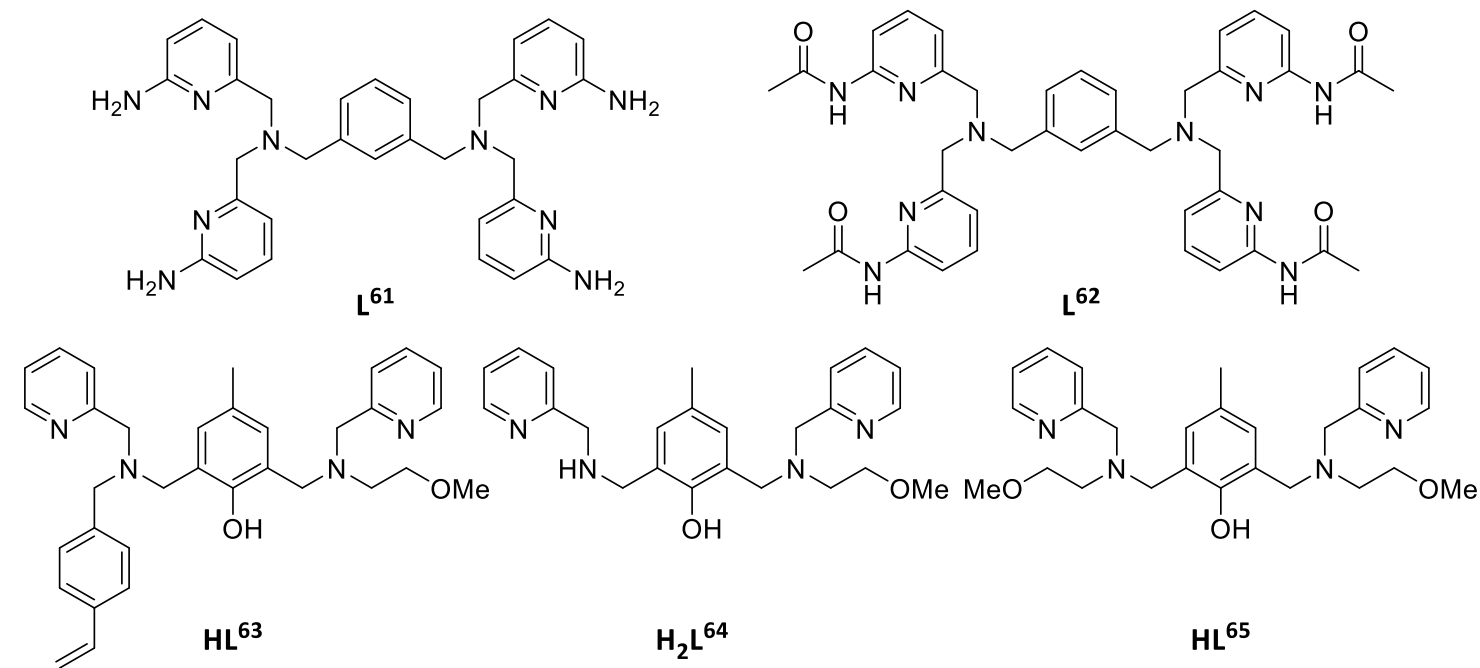<smiles>Cc1cccc(CN(Cc2cccc(N)n2)Cc2cccc(CN(Cc3cccc(C)n3)Cc3c(O)c(C)cc(CN(Cc4ccccn4)Cc4ccccn4)c3O)n2)n1</smiles><smiles>COc1ccc(O)c(CN(CC(=O)O)CC(=O)O)c1</smiles><smiles>[14CH3]</smiles><smiles>Cc1cc(CN(Cc2cccc(CN(Cc3ccccn3)Cc3cccc(N)n3)n2)Cc2cccc(CN(Cc3cccc(C)n3)Cc3cccc(N)n3)n2)c(O)c(CN(Cc2cccc(NCC(C)(C)C)n2)Cc2cccc(NCC(C)(C)C)c2O)c1</smiles><smiles>Cc1cc(CN(Cc2ccccn2)Cc2cccc(N)n2)c(O)c(CN(Cc2ccccc2)Cc2ccccc2O)c1</smiles> 


\section{ABSTRACT}

Osteoporosis is a disease caused by excessive bone resorption resulting in a skeletal disorder and thus leads to higher risk of fractures. More than 75 million people in the United States, Europe and Japan are effected by osteoporosis. Thus, there is a urgent need to develop new and effective therapeutic agents for this enfeebling disease. The enzyme Purple Acid Phosphatase (PAP) is an important target for the development of new anti-osteoporotic drug leads. The major goal of this thesis is to better emulate the synergy that occurs between the primary and secondary coordination sphere within the active site of phosphatase enzymes, such as PAP. This was perceived by development of synthetic methods for new biomimetics, i.e. low-molecular weight metal complexes. Therefore, asymmetric dinucleating ligands which mimic the peptide backbone of the active site of PAP including the ability to form hydrogen bonds with a phosphoester substrate or a nucleophile were designed and synthesized (Chart 1).
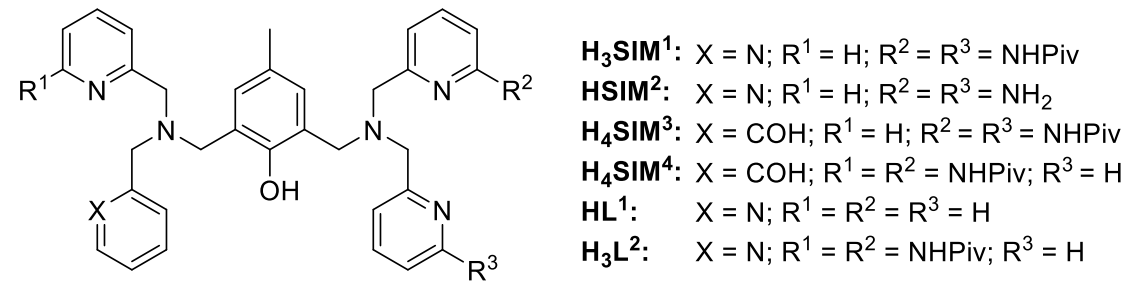

Chart 1: Ligands $\mathrm{H}_{3} \mathrm{SIM}^{1}, \mathrm{HSIM}^{2}, \mathrm{H}_{4} \mathrm{SIM}^{3}$, and $\mathrm{H}_{4} \mathrm{SIM}^{4}$ developed and ligands $\mathrm{HL}^{1}$ and $\mathrm{H}_{3} \mathrm{~L}^{2}$ additionally employed in the course of this thesis.

Using these ligands, more accurate model systems for the enzyme active site were achievable as they combine the two essential structural features known to influence the catalytic activity towards the hydrolysis of phosphoesters, i.e. the asymmetry of the dinuclear active site and the capacity for hydrogen bond formation. The latter was verified by the observation of hydrogen bonds in the X-ray structures of dizinc(II) and diiron(II) complexes. Moreover, two dizinc(II) complexes revealed strikingly different structures compared to the common structure known for phenoxido-bridged phosphatase models. In fact, the two ligands bearing two pivaloyl-amide moieties in an asymmetric manner formed dizinc(II) complexes with two different $\mathrm{Zn}$ " sites and an unusual hydroxido co-ligand, representing two important features in the active site of PAP, the difference of the metal sites and the nucleophile needed for the phosphoester hydrolysis. In addition, hydrogen bond formation detected in these X-ray structures was accounted for the stabilization of the hydroxido co-ligand.

In this work, a new synthetic approach towards more sophisticated model systems for the active form of mammalian PAP was developed. Chemical oxidation of the diiron(II) complex of $\mathrm{H}_{3} \mathrm{SIM}^{1}$ results in the generation of the Fe ${ }^{\prime \prime \prime} \mathrm{Fe} e^{\prime \prime}$ complex that has been specifically designed to both satisfy 
the requirement of a heterovalent diiron core and to mimic the second coordination sphere of the active site of PAP. Similarly, more accurate model systems for the active site of plant PAPs have been generated in the form of heterovalent heterodinuclear complexes of ligand $\mathrm{H}_{3} \mathrm{SIM}^{1}$ and $\mathrm{H}_{4} \mathrm{SIM}^{4}$ bearing, adjacent to the Ga"' $\mathrm{Zn}$ " core, functionalities capable of forming hydrogen bonds. Analysis of the complexation behavior of the respective ligands revealed the selective formation of the Ga"l' $\mathrm{Zn}$ " complexes in solution.

The model complexes described above imitate successfully the extensive hydrogen bond network that is formed by the second coordination sphere within the active site of PAP as well as in a structural and functional similar phosphatase, Alkaline Phosphatase. Thus, those complexes allow to study the impact of hydrogen bonds on the reaction mechanism. The main impact of the secondary interactions in the dizinc(II) complexes was found to be the increased substrate affinity. This catalytic parameter was shown to be dependent on both the hydrogen bonding sites and the type of the hydrogen bonding groups. Although the substrate affinity of the Ga"l'Zn" complex was revealed to be lower compared to the dizinc(II) complex, a 50-fold faster hydrolysis rate and a 6-fold increased efficiency was detected for the heterodinuclear complex. Moreover, the mechanism previously proposed, in which the phosphoester is activated by the

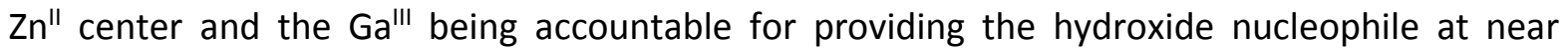
physiological condition, was supported in this study and an accelerating effect by the interplay of the two metal ions was detected. However, the inhibition was found to be favored in the Ga"l' $\mathrm{Zn}$ " complex compared to the respective monogallium(III) complex, most likely due to bridging coordination of the hydrolysis product, additionally stabilized by coordination of the adjacent pivaloyl-amide residue. The proposed arrangement in the catalyst-hydrolysis product adduct derives from a structure of a stable phosphoester-bridged dizinc(II) complex bearing the same ligand backbone. However, the Ga"l' $\mathrm{Zn}$ " complex of $\mathrm{H}_{3} \mathrm{SIM}^{1}$ is the first heterodinuclear model complex that mimics the essential function of PAPs, the ability to cleave phosphomonoesters and therefore supports the crucial impact of the second coordination sphere in the active site of PAP. 


\section{KURZZUSAMMENFASSUNG}

Osteoporose ist eine Krankheit, die aufgrund exzessiver Knochenresorption zu Fehlordnung im Skelett und infolgedessen zu einem erhöhten Frakturrisiko führt. Mehr als 75 Millionen Menschen in den USA, Europa und Japan leiden an Osteoporose. Aus diesem Grund ergibt sich eine akute Notwendigkeit neue und effektive Wirkstoffe für diese Krankheit zu entwickeln. Als Ausgangspunkt für die Entwicklung neuer antiosteoporotischer Wirkstoff-Leitstrukturen ist das Haupttarget das Enzym „violette saure Phosphatase" (Purple Acid Phosphatase, PAP). Das wesentliche Ziel dieser Arbeit ist die Synergie, die zwischen der primären und sekundären Koordinationssphäre innerhalb der aktiven Tasche von Phosphatase-Enzymen wie PAP auftritt, besser nachzubilden. Dies wurde durch die Entwicklung synthetischer Methoden für neuartige Biomimetika (Metallkomplexe mit geringem Molekulargewicht) ermöglicht. Hierfür wurden asymmetrische Liganden mit zwei Koordinationsstellen konzipiert und entwickelt, die das PeptidRückgrat der aktiven Tasche von PAP imitieren, einschließlich der Fähigkeit Wasserstoffbrücken zu Phosphoester-Substraten oder einem Nukleophil zu bilden (Abbildung 1).

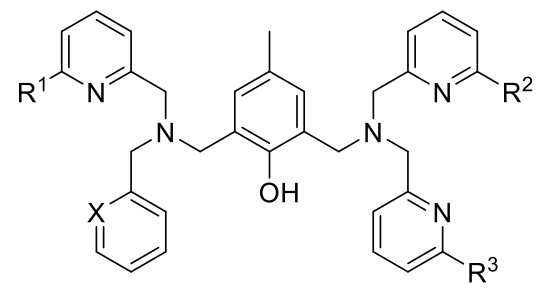

$$
\begin{aligned}
& \mathrm{H}_{3} \text { SIM }^{1}: X=\mathrm{N} ; \mathrm{R}^{1}=\mathrm{H} ; \mathrm{R}^{2}=\mathrm{R}^{3}=\text { NHPiv } \\
& \text { HSIM }{ }^{2}: X=N ; R^{1}=H ; R^{2}=R^{3}=N_{2} \\
& \mathrm{H}_{4} \text { SIM }^{3}: X=\mathrm{COH} ; \mathrm{R}^{1}=\mathrm{H} ; \mathrm{R}^{2}=\mathrm{R}^{3}=\mathrm{NHPiV} \\
& H_{4} \text { SIM }^{4}: X=\mathrm{COH} ; \mathrm{R}^{1}=\mathrm{R}^{2}=\text { NHPiv; } \mathrm{R}^{3}=\mathrm{H} \\
& H^{1}: \quad X=N ; R^{1}=R^{2}=R^{3}=H \\
& H_{3} L^{2}: \quad X=N ; R^{1}=R^{2}=\text { NHPiv; } R^{3}=H
\end{aligned}
$$

Abbildung 1: Im Laufe dieser Arbeit entwickelte Liganden $\mathrm{H}_{3} \mathrm{SIM}^{1}, \mathrm{HSIM}^{2}, \mathrm{H}_{4} \mathrm{SIM}^{3}$ und $\mathrm{H}_{4} \mathrm{SIM}^{4}$ sowie zusätzlich verwendete Liganden $\mathrm{HL}^{1}$ und $\mathrm{H}_{3} \mathrm{~L}^{2}$.

Mit Verwendung dieser Liganden konnten verbesserte Modellsysteme für die aktive Tasche dargestellt werden. Dies wurde durch die Kombination der zwei essentiellen strukturellen Eigenschaften erzielt, welche bekanntlich die katalytische Aktivität bezüglich der PhosphoesterHydrolyse beeinflussen; die Asymmetrie der dinuklearen aktiven Tasche und die Kapazität zur Wasserstoffbrückenbildung. Letztere wurde durch die in den Kristallstrukturen sichtbaren Wasserstoffbrücken der dinuklearen Zink(II)- und Eisen(II)-Komplexe bestätigt. Darüber hinaus zeigten zwei dinukleare Zink(II)-Komplexe auffallend abweichende Strukturen verglichen mit bekannten Strukturen von Phenoxido-verbrückten Phosphatase-Modellen. Unter Verwendung der zwei Liganden, die zwei Pivaloylamid-Substituenten in einer asymmetrischen Anordnung tragen, bildeten sich zweikernige Zink(II)-Komplexe mit unterschiedlichen Zn" Zentren und einem ungewöhnlichen Hydroxido-co-Liganden. Somit imitieren diese Komplexe zwei wichtige Eigenschaften der aktiven Tasche von PAP, die Verschiedenheit der Metallkoordinationssphäre und das für die Phosphoesterhydrolyse benötigte Nukleophil. Die Stabilisierung des Hydroxido- 
co-Liganden wurde den Wasserstoffbrückenbindungen, die in den Kristallstrukturen detektiert wurden, zugeschrieben.

In dieser Arbeit wurde eine neue Synthesestrategie für ausgereiftere Modellsysteme der aktiven

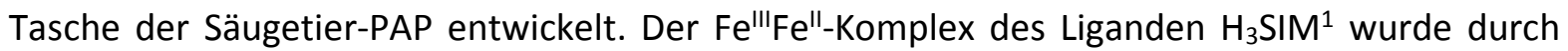
chemische Oxidation des entsprechenden Di-Eisen(II)-Komplexes in Lösung dargestellt. Dieser heterovalente Di-Eisen-Komplex wurde spezifisch entworfen, um den Anforderungen eines heterovalenten Di-Eisen-Kerns zu entsprechen und die zweite Koordinationssphäre der aktiven Tasche der PAP zu imitieren. Gleichermaßen wurden exaktere Modellsysteme für die aktive Tasche von Pflanzen-PAP in Form von heterovalenten heterodinuklearen Komplexen der Liganden $\mathrm{H}_{3} \mathrm{SIM}^{1}$ und $\mathrm{H}_{4} \mathrm{SIM}^{4}$ dargestellt. Diese Komplexe enthalten Funktionalitäten in der näheren Umgebung des Ga"'In" Zentrums, die zur Wasserstoffbrückenbindung befähigt sind. Die Untersuchung des Komplexierungsverhaltens der entsprechenden Liganden zeigten die selektive Bildung der Ga"l'Zn"-Komplexe in Lösung.

In den verwendeten Modellkomplexen wird erfolgreich das ausgedehnte Wasserstoffbrückennetzwerk, das in der zweiten Koordinationssphäre innerhalb der aktiven Tasche von PAP genauso wie in der strukturell und funktionell ähnlichen alkalischen Phosphatase auftritt, imitiert. Diese ermöglichten die Rolle der Wasserstoffbrückenbindungen im Reaktionsmechanismus zu untersuchen. Der größte Einfluss der sekundären Wechselwirkungen in den Di-Zink(II)Komplexen wurde in der erhöhten Substrataffinität gefunden. Es wurde nachgewiesen, dass dieser katalytische Parameter, sowohl von der Anordnung als auch von der Art der Wasserstoffbrückendonoren abhängt. Obwohl die Substrataffinität des Ga"'In"-Komplexes niedriger ist als die des homodinuklearen Zink(II)-Komplexes, wurde eine 50-fach schnellere Hydrolysegeschwindigkeit und eine sechsfach erhöhte Effizienz für den heterodinuklearen Komplex beobachtet. Darüber hinaus konnte in dieser Studie der bisherige vorgeschlagene Mechanismus, in dem der Phosphoester durch das Zn"-Zentrum aktiviert wird und das Gall'-Zentrum das HydroxidNukleophil unter annähernd physiologischen Bedingen bereitstellt, bestätigt und ein Beschleunigungseffekt durch das Zusammenspiel der beiden Metallionen gefunden werden. Ebenso konnte gezeigt werden, dass die Inhibierung des Ga"l'Zn"-Komplexes im Vergleich zum Monogallium(III)-Komplex begünstigt ist. Dies lässt sich auf eine verbrückende Koordination des Hydrolyseproduktes und dessen Stabilisierung durch die Koordination des benachbarten Pivaloylamid-Substituenten zurückführen. Diese postulierte Anordnung im Katalysator-Hydrolyseprodukt-Addukt wurde von einer Struktur eines stabilen Phosphoester-verbrückten DiZink(II)-Komplexes desselben Ligandenrückgrats abgeleitet. Der Ga"'Zn"-Komplex des Liganden $\mathrm{H}_{3} \mathrm{SIM}^{1}$ ist der erste heterodinukleare Modellkomplex, der Phosphomonoester spalten kann, was 
die essentielle Funktion von PAPs darstellt. Dies verdeutlicht die wesentliche Relevanz der zweiten Koordinationssphäre in der aktiven Tasche von PAP. 
Chapter 1

Introduction 


\subsection{OSteoporosis - An ENFEebling DISEASE}

Osteoporosis is a disease resulting in a skeletal disorder caused by excessive bone resorption. ${ }^{1}$ At greatest risk are the elderly and in particular post-menopausal women. ${ }^{2}$ Current treatments for osteoporosis include vitamin and calcium dietary supplements. Bisphosphonates are the most common drugs administered to lower bone resorption rates, but these have significant sideeffects and/or compliance issues. ${ }^{3}$ Thus, osteoporosis is a major health problem and there is an urgent need to develop new and effective therapeutic agents for this enfeebling disease.

Several lines of evidence validate the enzyme Purple Acid Phosphatase (PAP) as an important target for the discovery and development of new anti-osteoporotic drug leads, such as (i) elevated concentrations of PAP in the human serum are associated with an increase in bone turnover, ${ }^{4}$ (ii) elevated serum concentrations of human PAP are observed in patients with osteoporosis, ${ }^{5}$ (iii) mice lacking the gene encoding PAP suffer from deformed bone structure (osteopetrosis), ${ }^{6}$ and (iv) mice over-expressing PAP are osteoporotic. ${ }^{7,8}$ For those reasons PAP is already used as a main biochemical marker for the diagnosis of osteoporosis. ${ }^{9}$ Moreover, detailed understanding of the catalytic mechanism of PAP encourages the rational design of antiosteoporotic drugs. ${ }^{10}$

\subsection{Biological Significance ANd StABILITY OF PHOSPHOESTERS}

Phosphate esters, also called phosphoesters, are very important in the chemistry of life as they fulfill a variety of key roles. ${ }^{11}$ The P-O bonds are extremely stable due to the exceedingly slow uncatalyzed hydrolysis although it is favored thermodynamically. ${ }^{11}$ Therefore, phosphoester linkages are particularly well-suited in biology and therefore ubiquitous in nature as they link for example the nucleosides in deoxyribonucleic acid (DNA) and ribonucleic acid (RNA). For example, the strength of the phosphoester P-O bond is illustrated dramatically in the fact that DNA has a half-life of $30,000,000$ years. ${ }^{12}$ However, phosphoesters are also found in phospholipids, the major component of cell membranes and in many small molecules that play a key role in metabolism including several organic cofactors and secondary messengers. ${ }^{11}$ Reversible phosphorylation of proteins is an extremely important regulatory mechanism. ${ }^{13,}{ }^{14}$ The transformation of serine, threonine or tyrosine residues into phosphomonoesters induces conformational changes in enzymes and receptors leading to activation or inactivation. ${ }^{11}$ In this 
way, phosphorylation and phosphoester cleavage is an effective 'molecular switch' to control protein function. ${ }^{11}$

\subsection{Phosphatases - Phosphoester Cleaving ENZYMES}

Whilst the stability of phosphoester bonds is critical to life, so is the capacity to efficiently manipulate these bonds. Nature has managed to achieve this remarkable feat through the evolution of a diverse range of enzymes that are able to drastically reduce the activation energies of specific P-O hydrolysis reactions and thereby to accelerate those by factors of $>10^{15}$ relative to the uncatalyzed cleavage. ${ }^{11,15}$ This task is adopted by phosphatases which are amongst the most efficient enzymes known and are involved in signal transduction in bacteria, ${ }^{16,17}$ phospholipid degradation, ${ }^{18-20}$ as well as regulation of phosphate levels in plants, fungi and mammals. ${ }^{11,}$ 21-28 Purple Acid Phosphatases (PAPs) and Alkaline Phosphatases (APs) are two of the best characterized hydrolytic enzymes and are predominantly discussed in this thesis. ${ }^{11}$ As the names imply the former are most active at low $\mathrm{pH}$, and the latter under alkaline conditions. ${ }^{11}$ Although it has been thought for a long time that APs and PAPs are strictly phosphomonoesterases it was found that both are also able to operate as phosphodiesterases. ${ }^{29,} 30$

\section{Alkaline Phosphatase}

APs are unique in the class of phosphomonoesterases in the sense that they appear to be nonspecific. ${ }^{30}$ Furthermore, APs hydrolyze not only phosphomonoesters, but also a variety of $O$ and S-phosphothioates, phosphoamidates, thiophosphates and phosphate. ${ }^{30}$ Moreover, APs do not only catalyze hydrolysis reactions but also transphosphorylations in the presence of high concentrations of phosphate acceptors. ${ }^{31}$ Mammalian APs occur in different isozymes with very different in vivo functions. ${ }^{31}$ In general, APs are commonly named after the tissue where they are predominantly expressed..$^{31}$ The major functions of APs established to date is bone mineralization by tissue-nonspecific AP (TNAP), abundant in skeletal tissues, and intestinal absorption by intestinal AP (IAP), abundant in the gut. ${ }^{31}$

Recent studies on TNAP-deficient mice showed that hydrolyzing the mineralization inhibitor pyrophosphate $\left(\mathrm{PP}_{\mathrm{i}}\right)$ was the major function of TNAP in bone tissue, thus facilitating mineral precipitation and growth. ${ }^{32} \mathrm{PP}_{\mathrm{i}}$ prevents initial crystal formation and crystal growth by physically coating the nascent hydroxyapatite crystals. $^{32}$ Therefore, TNAP functions to maintain an appropriate concentration of this inhibitor to ensure normal bone mineralization. ${ }^{32}$ Another 
proposal for the function of TNAP in bone matrix has been the generation of the inorganic phosphate $\left(\mathrm{P}_{\mathrm{i}}\right)$ needed for hydroxyapatite crystallization. ${ }^{32}$

The biological functions of IAP are diverse, including (i) lipid intestinal absorption, ${ }^{33}, 34$ (ii) regulation of bicarbonate secretion, ${ }^{35,36}$ (iii) detoxification of bacterial lipopolysaccharide (LPS), ${ }^{37,38}$ and (iv) protection against LPS-induced intestinal inflammation. ${ }^{39,} 40$ In more detail, pancreatic lipase hydrolyze triglycerides into glycerol and free fatty acids (FAs). For the subsequent FA absorption, the involvement of IAP has been proposed through regulation of the FA carrier protein FA transporter/CD36 (FAT/CD36). ${ }^{40}$ The possibility exists that IAP modulates the FA absorption by controlling the level of phosphorylation of FAT/CD36 and/or by locally hydrolyzing the extracellular ATP, since the CD36-mediated FA uptake (i) decreases with increasing phosphorylation of FAT/CD36, (ii) is inhibited by extracellular ATP, and (iii) is restored after the addition of AP. ${ }^{40}$ The latter modulation mechanism of IAP is also utilized for the regulation of the bicarbonate secretion which works in a loop involving IAP, extracellular ATP and G-coupled purinergic receptor $\mathrm{P} 2 \mathrm{Y}_{1} .{ }^{40}$ ATP stimulates the $\mathrm{P} 2 \mathrm{Y}_{1}$ receptors which, in turn, stimulate the secretion of bicarbonate by enterocytes. ${ }^{40}$ Therefore, the ATPase activity of IAP regulates the local ATP concentration and subsequently the bicarbonate secretion. In contrast, the detoxification of LPS is encompassed directly by the dephosphorylation of its toxic diphosphoryllipid A moiety. ${ }^{40}$ In this way the LPS-induced intestinal inflammation induced by the Toll-like receptor 4 (TLR4) is suppressed, since the dephosphorylated LPS is no longer able to trigger TLR4. ${ }^{40}$

\section{Purple Acid Phosphatase}

PAPs are ubiquitous enzymes found in animals, plants, fungi and bacteria and are associated with a variety of biological functions. PAPs are resistant to inhibition by L-tartrate and thus are frequently referred to as tartrate-resistant acid phosphatase (TRACPs or TRAPs). ${ }^{29}$

Mammalian PAPs, abundantly expressed in osteoclasts, activated macrophages and dendritic cells, ${ }^{41-43}$ have been implicated with (i) iron transport in pregnant pigs, ${ }^{44}$ (ii) the generation of reactive oxygen species (ROS), ${ }^{45}$ and (iii) increased bone resorption. ${ }^{9,}{ }^{46}$ Specifically, it has been suggested that PAPs play an important role in the immune defense system producing hydroxyl and superoxide radicals based on the Haber-Weiss-Fenton-type reactions (Scheme 1). ${ }^{29,47}$

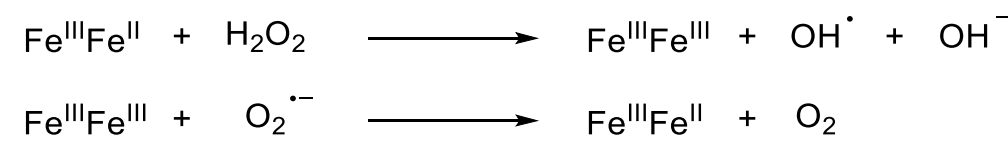

Scheme 1: Generation of ROS by PAPs by Haber-Weiss-Fenton type reactions. ${ }^{29}$ 
As mentioned previously, PAPs were also shown to play a crucial role in bone metabolism since transgenic mice, in which osteoclasts overexpress and secrete PAP, display increased levels of bone resorption and consequently symptoms of mild osteoporosis. ${ }^{7}$ In contrast, knock-out transgenic mice completely lacking PAP reveal the opposite phenotype, osteopetrosis. ${ }^{6}$ It is believed that PAP dephosphorylates bone matrix proteins such as osteopontin which are involved in the adhesion of osteoclasts to the bone surface and are, upon dephosphorylation, no longer capable of fulfilling this function. ${ }^{46}$ However, it has also been speculated that PAP is involved in the ATP-dependent regulation of bone calcium homeostasis due to the fact that PAP is also an efficient ATPase. ${ }^{48}$

Contrary to mammalian PAP, assignment of specific functions has been more difficult for plant PAP due to its appearance in multiple isoforms with diverse metal ion compositions. ${ }^{49-58}$ However, it has been speculated that plant PAPs play a major function in phosphate metabolism mobilizing inorganic phosphate from organophosphates in the soil. ${ }^{50-52,57,59}$ It has also been proposed that plant PAPs are involved in the storage of phosphorus in plant seeds via hydrolysis of phytic acid, the major compound accountable for the phosphorus storage. ${ }^{60}$ Interestingly, it has been found that the extracellular ROS concentration in plants increases in response to a pathogen attack or biotic and abiotic stress, similarly as observed for mammals. ${ }^{61}$ Therefore, plant PAPs seem to be bifunctional, similar to mammalian PAPs, catalyzing both hydrolytic reactions and peroxidations. ${ }^{45,47,62,63}$ Although not yet clear, the purpose of this peroxidation activity in plants, microbe may be killing during pathogen attack. ${ }^{29,61}$

\subsection{Structure of Phosphatases}

APs and PAPs belong to the enzyme family of dinuclear metallohydrolases which require two dinuclear centers to perform a hydrolytic reaction. The presence of two closely spaced metal ions in the active site can have advantages over just one metal ion, such as (i) a lowered thermodynamic driving force for redox reactions due to charge-delocalization, (ii) a lowered activation barrier for solvent and enzyme reorganization, (iii) preorientation and electrostatic activation of substrates, (iv) easier formation of hydrolysis-initiating nucleophiles, and (v) stabilization of transition states generated throughout the hydrolysis reaction. ${ }^{28}$ The binding of the two metals in the active site is facilitated by precisely positioned ligating amino acid side chains of the peptide backbone which are defined as the primary coordination sphere. 


\section{Alkaline Phosphatase}

AP has been structurally characterized as an enzyme-phosphate complex isolated from $E$. coli (Chart 2, left). ${ }^{64}$ Here the phosphate is non-covalently bound at the active center and the structure therefore likely represents an authentic intermediate of the phosphoester hydrolysis reaction. ${ }^{30} \mathrm{AP}$ is a homodimeric protein with 449 amino acids building up the subunits and two $\mathrm{Zn}$ " centers and a single $\mathrm{Mg}^{2+}$ center coordinated in each active site. ${ }^{11}$ Whilst one $\mathrm{Zn}^{11}$ ion is coordinated by two histidine and one aspartate residue of the peptide backbone, the second $\mathrm{Zn}$ " center is ligated by one histidine and two aspartate residues. ${ }^{64}$ In the resting state the proximal serine side chain coordinates the latter $\mathrm{Zn}$ "ion, but is exchanged by a phosphate oxygen atom in the enzyme-phosphate complex. ${ }^{30}$ The $\mathrm{Mg}^{2+}$, ion which appears to play only an ancillary, structural role, is linked to the dizinc(II) site by a Mg-Zn bridging aspartate and is additionally coordinated by a glutamate, a threonine and three water molecules. ${ }^{64}$ The three metal ions in AP form a triangle in which the metal-metal distances are $3.94 \AA$, $4.88 \AA$, and $7.09 \AA .{ }^{30}$ Therefore, the $\mathrm{Mg}^{2+}$ appears not to be close enough to participate directly in the hydrolysis mechanism, but could contribute to the shape of the electrostatic potential around the active center or provide an additional metal bound water in the vicinity of Ser102 functioning as a general acid/base for regulating the protonation state of Ser102. ${ }^{30,65} \mathrm{~A}$ comparison of the amino acid sequence of the phosphorylated peptides isolated from the enzyme with the complete amino acid sequence showed that the phosphorylated residue is Ser102 suggesting that this amino acid residue functions as nucleophile in the catalytic mechanism. ${ }^{30}$
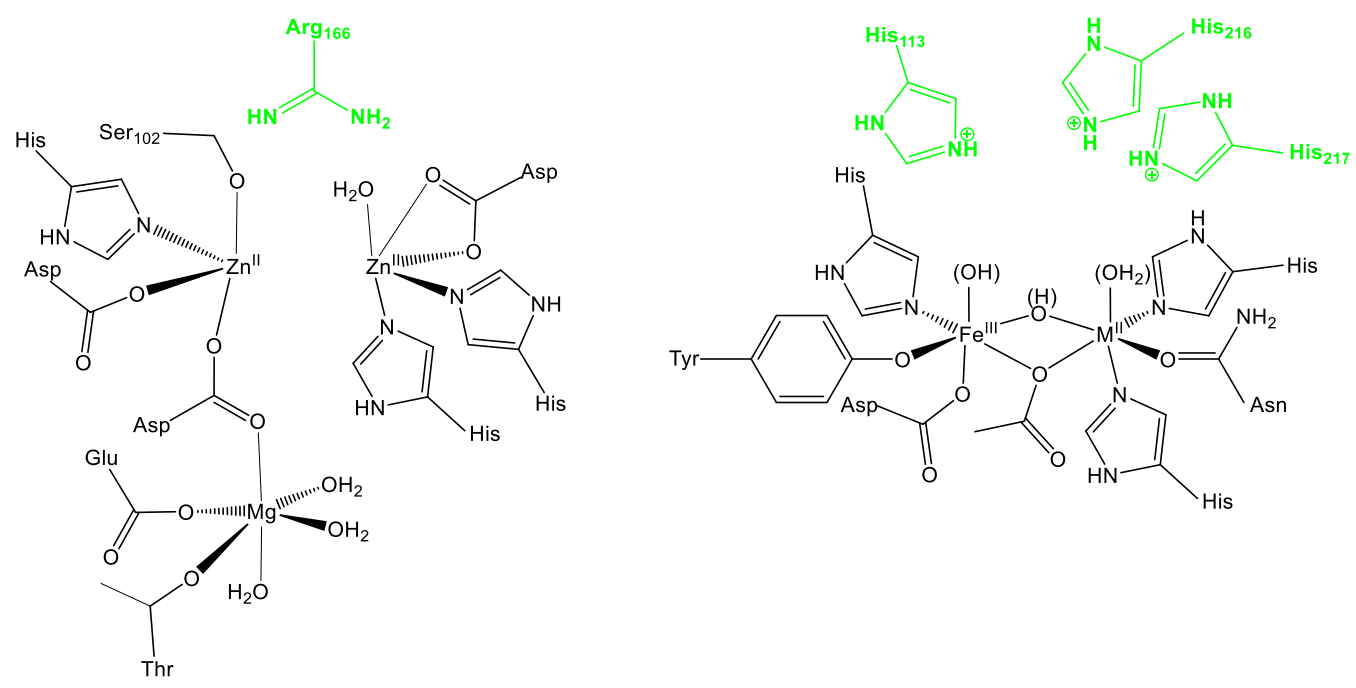

Chart 2: Schematic depiction of the active site of AP (left $)^{30,64,65}$ and PAP (right), ${ }^{45}$ including essential amino acids in the second coordination sphere (depicted in green). 


\section{Purple Acid Phosphatase}

Several crystal structures of PAPs, isolated from various plant, animal, and fungal sources, have been published. ${ }^{29}$ Mammalian PAPs isolated from pig (ufPAP), cow, human, mouse and rat are approximately $35 \mathrm{kDa}$ monomeric proteins with a $\mathrm{Fe}^{\prime \prime \prime} \mathrm{Fe} \mathrm{e}^{\text {II }}$ center and share at least $80 \%$ amino acid sequence identity. ${ }^{21,29,49,66}$ Plant PAPs extracted from red kidney bean (kbPAP), sweet potato, soybean, duckweed, tomato, potato, and thale cress revealed $55-60 \mathrm{kDa}$ homodimeric enzymes and amino acid sequence identity of at least $65 \% .{ }^{21,29,49,66}$ The metal composition in the active sites of plant PAPs is either Fe"'Zn" or Fe"'Inn". ${ }^{\prime 2,24,67,68}$ Although the homology between the mammalian and plant PAP amino acid sequences is less than $20 \%$, their catalytic sites display a remarkable similarity. ${ }^{29}$ The two metal ions were found to be coordinated by seven invariant amino acid residues (Chart 2, right). ${ }^{45}$ The Fe ${ }^{\text {III }}$ ion is coordinated by a histidine, an aspartate and a tyrosine amino acid side chain. This tyrosine ligand in the 'chromophoric' site is accountable for the characteristic purple color of the enzyme due to its ligand to metal chargetransfer (LMCT) transition. ${ }^{69}$ The $\mathrm{M}^{\prime \prime}$ ion ( $\mathrm{M}^{\prime \prime}=\mathrm{Fe}^{\prime \prime}, \mathrm{Zn}^{\prime \prime}$ or $\mathrm{Mn}$ ) in the 'redox-active' site is surrounded by an asparagine and two histidines residues. The coordination spheres of the $\mathrm{Fe}^{\text {III }}$ and $\mathrm{M}^{\prime \prime}$ ions were completed by a bridging aspartate residue, a modeled bridging hydroxide ligand and a modeled terminal water ligand for the resting state for ufPAP, but the number of terminal and bridging aqua and hydroxide ligands is ambiguous. ${ }^{70}$ Although PAPs are rather specific in their metal ion selection in vivo, it has been shown with in vitro studies that on the one hand the $\mathrm{Fe}^{\prime \prime}$ ion of the diiron ufPAP can be exchanged by $\mathrm{Zn}^{\prime \prime}, \mathrm{Ni}^{\prime \prime}, \mathrm{Cu}^{\prime \prime}$, and $\mathrm{Mn}^{\prime \prime}$ and on the other hand the Fe ${ }^{\text {III }}$ ion can be replaced by Gall' and $\mathrm{Al}^{\text {III }}$ without loss of catalytic activity. ${ }^{29}$ Similarly, catalytic activity could be reconstituted by replacement of $\mathrm{Zn}^{\prime \prime}$ by Fe" in kbPAP. ${ }^{29}$ However, PAPs are the only dinuclear metallohydrolases where the necessity for a heterovalent active site for catalysis has been clearly established. ${ }^{21}$

In general, the primary coordination sphere in the active site of dinuclear enzymes is supposed to define (i) the coordination modes, (ii) the separation, and (iii) the electron density of the two metal ions coordinated. Interestingly, in both crystal structures discussed here the two metal centers reside in chemically different environments. The differing coordination environments of dinuclear metallohydrolases are often defined as the $\alpha$ - and $\beta$-site. ${ }^{28}$ In fact, the peptide backbones of the active sites of AP and PAP form an asymmetric dinucleating core with a harder and a softer metal site suggesting that the metal ions have distinct functions in the catalytic mechanism. However, mutation studies (discussed below) of some non-coordinating amino acids of both, AP and PAP, revealed that those are also essential for the activity. ${ }^{13,65,71-74}$ 


\subsection{Significance OF THE SECONd CoORdination SPHERE IN PHOSPHATASES}

Amino acids that are not directly coordinated to the metal ions in the active site but are indespensable to the activity are defined as the second coordination sphere. ${ }^{75}$ Those amino acids alter the physical and chemical properties of the active site, needed for the catalytic transformation by non-covalent interactions, hydrophobic effects and van der Waals forces. ${ }^{75}$

\section{Alkaline Phosphatase}

In the crystal structure of the enzyme-phosphate complex of AP an extensive hydrogen bonding network was determined. More precisely, the two phosphate oxygens, which are not coordinated to the two $\mathrm{Zn}$ " centers, form hydrogen bonds to an adjacent guanidinium group of an arginine residue ( $\mathrm{Arg} 166)$ and one of them also to the amide group of the serine residue in the primary coordination sphere (Ser102). ${ }^{30}$ Furthermore, Arg166 is additionally involved in hydrogen bonding to other amino acid side chains. ${ }^{30}$ The influence of the proximal Arg166 residue was investigated with site-directed mutagenesis studies in which this amino acid was exchanged by lysine, glutamine, serine or alanine. ${ }^{71,72}$ All mutants revealed decreased phosphate affinity but retained catalytic activity. In a similar study the possibility that Arg166 was responsible for the preference of AP for phosphomonoesters was demonstrated. ${ }^{65,73}$ Moreover, investigations of linear free-energy relationships have been suggested that electrostatic interactions of the phosphate with both $\mathrm{Zn}$ " ions and Arg166 are critical stabilizing factors in the trigonal bipyramidal transition structure of the phosphoester hydrolysis reaction. ${ }^{65}$

\section{Purple Acid Phosphatase}

Previous studies have shown that not only the amino acids in the first coordination sphere are crucial for catalytic activity of PAPs but also the three histidine residues nearby the metal centers (Chart 2, right). ${ }^{45,74}$ With the aid of site-directed mutagenesis with rat bone PAP the influence of these residues regarding the phosphatase activity and the generation of reactive oxygen species (ROS) of PAP was analyzed. These experiments led to the conclusion that the replacement of the histidine residues 216 and 113 with alanine or glutamine seriously impaired the phosphatase activity compared to the wild-type enzyme, while the mutants retained a high capacity to form ROS. ${ }^{45,74}$ From this it appears that the acid phosphatase activity and the ROS-generating capacity are independent of each other. More importantly, these results point out that the hydrogen bonding network built up by these histidine residues plays an essential role in the mechanism of 
the phosphoester hydrolysis. ${ }^{26,76}$ Interestingly, the mutant in which His113 was exchanged by a glutamine residue, technically capable to form hydrogen bonds, exhibited also decreased phosphoester hydrolysis activity. This initial contradiction to the former hypothesis was clarified by exchanging His113 with an asparagine residue, which led to an even higher phosphoester hydrolysis activity than the native enzyme. Since asparagine has the same functionalities than glutamine, but with a shorter side chain, the optimum geometry for hydrogen bonding and following phosphoester hydrolysis appears to be achieved in the asparagine mutant.

Although the importance of the second coordination sphere of AP and PAP has been clearly demonstrated, the precise role of the amino acid side chains proximal to the dinuclear active site is still not completely understood. This is primarily due to challenges in examination of functions of specific amino acids in complicated protein matrices, but also caused by difficulties in the characterization of non-covalent bonds. ${ }^{75}$ In this regard the development of simplified model systems, which incorporate the key features of the native metalloenzymes, represents a feasible experimental approach.

\subsection{BIOMIMETICS - A USEFUL TOOL TO STUdY ENZYMES}

Due to the fact that PAP activity is directly linked to bone resorption this enzyme has become a major target for the development of antiosteoporotic chemotherapeutics. ${ }^{29}$ Efforts for a rational design of such compounds is greatly helped by the detailed knowledge about the functional domains of PAPs. In fact, the wealth of information gained in recent years about the structure and the catalytic mechanism of mammalian AP has aided in the development of pharmacological products able to modulate AP activity and in the understanding of AP correlated diseases such as hypophosphatasia, a rare and sometimes fatal metabolic bone disease, caused by the deficiency in TNAP isozyme. ${ }^{32}$

In complement with X-ray structural, spectroscopic, mutagenesis and isotope effect studies of the respective enzyme a useful and important tool to establish the precise mechanism of an enzyme-catalyzed reaction are low-molecular weight synthetic mimics, also called biomimetics. Due to the fact that the active sites of many phosphatases contain metal ions, metal complexes are used as biomimetics to emulate key structural aspects of these enzymes. Model complexes have the advantages over their enzymatic counterparts since (i) they are generally more stable, (ii) they can be more readily crystallized, and (iii) provide easy accessible structural information on metal ion conformation. ${ }^{77}$ In addition, model complexes can be designed to mimic one or more key features of a sophisticated enzyme active site. In this way, the relative importance of 
specific structural or chemical features, or combinations thereof with respect to the overall catalytic efficiency of the enzyme, can be studied separately.

In this thesis the use of dinuclear model complexes as biomimetics of APs and PAPs, in order to gain a deeper understanding of the influence and the precise role of the second coordination sphere with regard to the catalytic phosphoester hydrolysis mechanism, will be presented. 


\title{
Chapter 2
}

\section{Ligand Design and}

\author{
Syntheses ${ }^{b}$
}

${ }^{b}$ Parts of this chapter are published in "Dinuclear Zinc(II) Complexes with Hydrogen Bond Donors as Structural and Functional Phosphatase Models"; Simone Bosch, Peter Comba, Lawrence R. Gahan, Gerhard Schenk, Inorg. Chem. 2014, 53, 9036-9051. and will be published in "Asymmetric Mono- and Dinuclear Ga"l and Zn" Complexes as Models for Purple Acid Phosphatases (PAPs)"; Simone Bosch, Lawrence R. Gahan and Gerhard Schenk; manuscript in preparation. 


\subsection{INTRODUCTION}

In nature transition metal ions bound in the active site of enzymes are used extensively to encompass the catalysis of a broad range of reactions. While in some metalloenzymes the transformation of a substrate molecule takes place at a single metal center, several classes of metalloenzymes make use of the cooperativity of two metal centers within their active site to achieve full catalytic efficiency. ${ }^{78}$ In the latter case the removal of one metal center often results in a decrease of activity. An important example of enzymes containing dinuclear active sites are metallohydrolases, which hydrolyze substrates such as urea, peptides, phosphoesters and -anhydrides, thioesters and even DNA and RNA. While most of the hydrolases bind two metals of the same kind in close proximity to each other, there are two other members of the hydrolase superfamily which have been shown to bind two different metal ions in their active sites, Purple Acid Phosphatases (PAPs) and Ser/Thr Proteins Phosphatases (PPs). ${ }^{9}, 21,79,80$ Moreover, the two chemically distinct coordination environments around the metal centers have been found to be essential for catalytic activity. ${ }^{21,24,81-85}$ The amino acid backbone of the active site in PAPs generate two different coordination sites, which favor the binding to a specific metal, though their individual roles remain a matter of debate.

Owing to the relatively low resolution of protein crystal structures, model complexes often play an important role in the structural characterization of the active sites in metalloproteins. Low molecular weight mimics of phosphoesterases simplify the study of both the environment of the metal ions and the hydrolysis of phosphoesters, and can provide insight into possible catalytic mechanisms. ${ }^{81}$ Therefore, small metal complexes have been used extensively to model the active site of phosphoesterases. ${ }^{81}$ Moreover, dinuclear complexes have been shown to bind phosphates more strongly and to be significantly more reactive for the cleavage of phosphate esters compared to their mononuclear counterparts. ${ }^{86-89}$ Some of the most active dimetallic systems are those in which the ligand structure places the metal ions in a way that it promotes cooperative interactions with the substrate (either via double Lewis acid activation, or Lewis acid activation coupled with provision of a metal-bound hydroxide nucleophile). ${ }^{80,84}$ Due to the fact that for simple oxido- or hydroxido-bridged mononuclear complexes dissociation is likely, attention has been focused on bridging dinucleating ligand systems. In these the ligand must accommodate two metals with an appropriate metal-metal separation that reproduce the intermetallic distance of the enzyme. This is mostly achieved using a linker moiety between the two coordination sites. Different ligand backbone linkers have been successfully used by this means to model the structural features of hydrolases and examples are illustrated in Chart 3. 


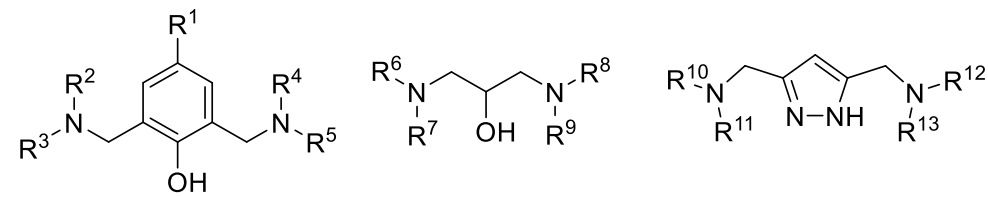

Chart 3: Schematic depiction of ligand types that have been used to model the active site in PAPs: f.I.t.r. phenolate-based, alkoxo-based and imidazolate-based ligands.

Among others, phenolate-, alkoxo-, and imidazolate have been deployed as scaffolds for a range of ligands varying in the aminomethyl-substituents (depicted as $\mathrm{R}^{\mathrm{x}}$ in Chart 3 ) in order to tune the coordination behavior of the ligands towards specific metal ions. ${ }^{81,90-99}$ The phenolate-linker is one of the most commonly used ligand scaffolds, ${ }^{90,92-96,99}$ for example in the symmetric ligands $\mathrm{HL}^{1}$ and $\mathrm{H}_{3} \mathrm{~L}^{3}$ (see Chart 4). ${ }^{100,101}$ (The nomenclature of the ligands denotes the number of protons which can potentially be abstracted from the ligands upon deprotonation; $\mathrm{HL}^{1}$ contains one potentially acidic phenolate residue, accordingly $\mathrm{HL}^{1}$, while $\mathrm{H}_{3} \mathrm{~L}^{3}$ bears three acidic protons and the nomenclature is adjusted accordingly. This nomenclature will be used throughout this thesis.) The phenolate-linker arranges the two coordination sites the way that (i) the two metal ions are separated by about $3-4 \AA$, (ii) the two metal ions are bridged by an oxygen atom as present in the native active site, and (iii) exogenous water and hydroxido ligands are bound in a syn configuration. The latter structural feature was shown to be essential for phosphoester cleavage as a complex with two $\mathrm{H}_{2} \mathrm{O}$ molecules in anti configuration was not active. ${ }^{102}$

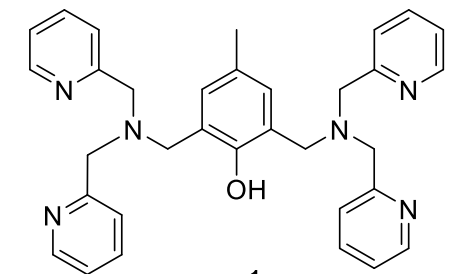

$\mathrm{HL}^{1}$<smiles>Cc1cc(CN(Cc2ccccc2)Cc2ccccn2)c(O)c(CN(Cc2ccccn2)Cc2ccccn2)c1</smiles><smiles>Cc1cc(CN(Cc2ccccn2)Cc2ccccc2O)c(O)c(CN(Cc2ccccn2)Cc2ccccc2O)c1</smiles>

$\mathrm{H}_{3} \mathrm{~L}^{3}$<smiles>Cc1cc(CNCc2ccccc2O)c(O)c(CN(Cc2ccccn2)Cc2ccccn2)c1</smiles>

Chart 4: Symmetric and asymmetric phenolate-based ligands related to the ligands developed in the present thesis, previously used to model the active site peptide backbone of PAP. ${ }^{81,103}$

It should be noted, that an important feature of metalloenzymes, especially those with heterodinuclear active sites, is that the surrounding protein matrix usually provides chemically distinct binding environments for the individual metal centers, commonly referred to as site asymmetry at metallobiosites. ${ }^{91}$ Typically, asymmetric dinucleating ligands are used to mimic this coordination environment of the metals in metallohydrolases, which dictate the steric and 
electronic features of each metal center. ${ }^{81}$ Terminal phenolate residues are often used to mimic the tyrosinate donor found in the active site of PAP. ${ }^{102}$ Numerous asymmetric ligands have been developed so far in order to imitate the peptide backbone of phosphatases with coordination number asymmetry (dissimilar number of donor atoms), i.e. $\mathrm{H}_{2} \mathrm{~L}^{5}$, and/or donor asymmetry (different types of donor atoms), i.e. $\mathrm{H}_{2} \mathrm{~L}^{4}$ and $\mathrm{H}_{2} \mathrm{~L}^{5}$. ${ }^{81,94,103}$

Unfortunately, the accessibility of asymmetric dinucleating ligands is often hampered by various synthetic problems. Several approaches have been proposed to overcome this problem. The most facile route starting from a symmetric, doubly chloro-substituted phenolate-bridging unit was published for the synthesis of $\mathrm{H}_{2} \mathrm{~L}^{4}$ by Neves et al., ${ }^{94}$ whereby successive reaction with two amines generally results in the formation of considerable amounts of symmetric byproducts. The statistical mixture of the desired asymmetric ligand and the two unintended symmetric ligands is usually difficult to separate by conventional chromatography methods utilizing polar silica or aluminia because of the high polarity of the products. An elegant solution for this problem was suggested by Kahn et al. ${ }^{104}$ Starting also from a symmetric dicarbonyl-functionalized phenolate bridging unit, gadolinium(III) ions were used to protect one of the reactive carbonyl functions by coordination, whereby the remaining free carbonyl function leads to an imine by reaction with an amine. Precipitation of $\mathrm{Gd}^{\mathrm{III}}$ in form of gadolinium(III) oxalate yielded the free carbonyl functionalized monoimine, which reacts with another different amine to afford the asymmetric diimine. Due to the fact that this approach only works for small-scale reactions, more efficient routes towards the class of asymmetric ligands were developed starting from already asymmetric bridging units. ${ }^{105}$ In the case of the phenolate-based ligands the stepwise synthetic procedure starts usually with 2-hydroxy-3-(hydroxylmethyl)-5-methylbenzaldehyde (1). Latour et al. (Scheme 2a) and Nordlander et al. (Scheme 2b) reported a procedure generating asymmetric dinucleating ligands using this starting material. ${ }^{106,107}$ Whilst Latour et al. accomplished the incorporation of the two unequal amine side-arms by stepwise generation of a chloro functionality and following nucleophilic substitution, ${ }^{106}$ Nordlander et al. achieved one attachment of an amine side-arm by amination of the carbonyl moiety present in the starting material and the second amine was incorporated by chlorination and subsequent nucleophilic substitution with a second amine following the same procedure as undertaken by Latour et $a l^{107}$ 


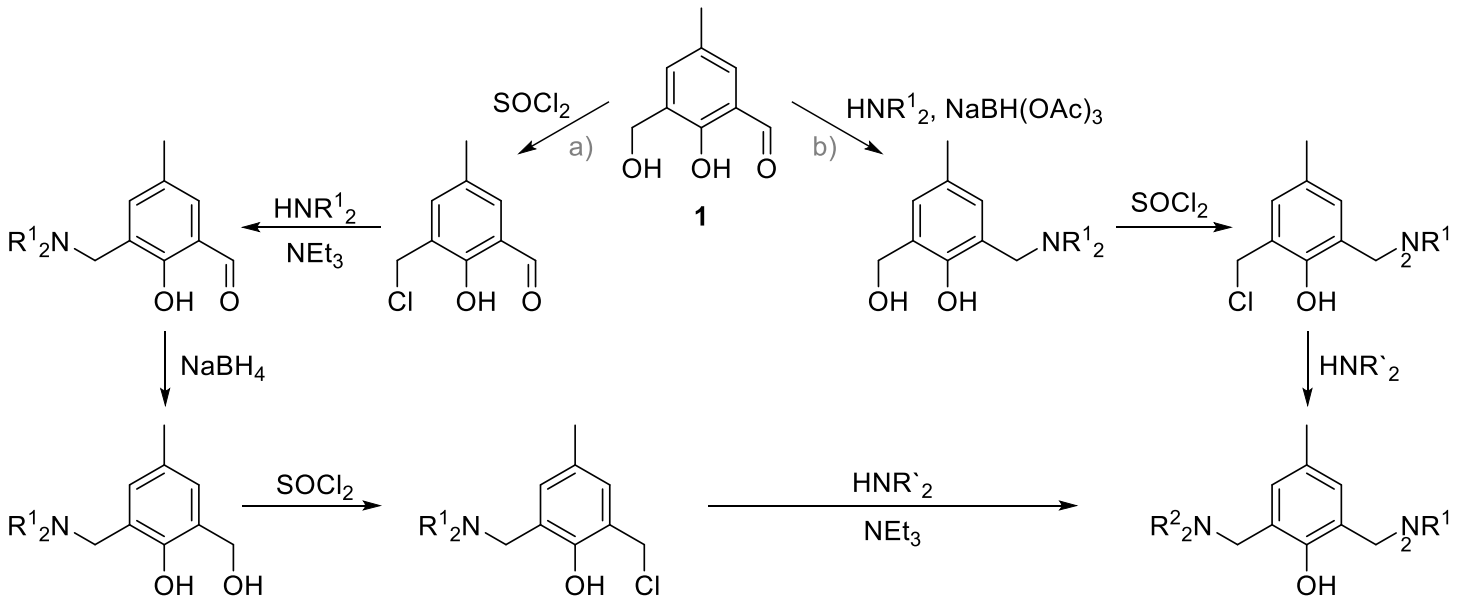

Scheme 2: Schematic representation of procedures applied by Latour et al. and Nordlander et al. to synthesize asymmetric dinucleating ligands. ${ }^{106,107}$

Many metalloenzymes that catalyze the cleavage of phosphate esters also possess a hydrogen bonding microenvironment around the metal centers, which is essential for their activity attributed to transition state stabilization. ${ }^{29,} 108$ In order to study the impact of hydrogen bond donors nearby the metal centers on the hydrolysis activity within the active site of phosphatases, different dinucleating ligands, depicted in Chart 5, have been developed. The hydrogen bonding interaction of PAPs were mimicked either by a cyclam-linker as in $\mathrm{H}_{2} \mathrm{~L}^{6},{ }^{108,109}$ while in the other ligands the hydrogen bonding network was incorporated into the ligands by amino and pivaloylamide residues at the pyridine side-arms. Feng et al. developed a range of alkoxo-based mononucleating ligands and the dinucleating ligand $\mathrm{HL}^{7}$ which also take into account the second coordination sphere. ${ }^{110-113}$ Moreover two $\mathrm{HL}^{1}$ derivatives, $\mathrm{H}_{3} \mathrm{~L}^{2}$ and $\mathrm{HL}^{8}$, which carry hydrogen bond donors proximal to the metal coordinating sites have been developed. ${ }^{109,}{ }^{114}$ In the latter, two hydrogen bond donors, one proximal to the metal center each, are rigidly preorganized to interact with a phosphoester substrate and/or to activate the hydroxido nucleophile coordinated to the dimetal core.<smiles>CN1CCCN(CCNC(c2ccccn2)c2ccccn2)CCCN(CCNC(c2ccccn2)c2ccccn2)CC1</smiles>

$\mathrm{H}_{2} \mathrm{~L}^{6}$<smiles>Cc1cc(CN(Cc2ccccn2)Cc2cccc(NC(=O)C(C)(C)C)n2)c(O)c(CN(Cc2ccccn2)Cc2cccc(NC(=O)C(C)(C)C)n2)c1</smiles>

$\mathrm{H}_{3} \mathrm{~L}^{2}$<smiles>Nc1cccc(CN(Cc2cccc(N)n2)CC(O)CN(Cc2cccc(N)n2)Cc2cccc(N)n2)n1</smiles>

$\mathrm{HL}^{7}$<smiles>Cc1cc(CN(Cc2ccccn2)Cc2cccc(N)n2)c(O)c(CN(Cc2ccccn2)Cc2cccc(N)n2)c1</smiles>

$H^{8}{ }^{8}$

Chart 5: Previously published ligands bearing hydrogen bond donors mimicking the peptide backbone of phosphatases active sites. ${ }^{108,112-114}$ 
The intention of this thesis was to study the role of a hydrogen bond network in the reaction mechanism of phosphatase biomimetics. Thus, dinuclear complexes were designed, which combine the essential structural features of the active site of phosphatases, such as (i) two metal ions accommodated in a well-suited metal -..metal separation, (ii) asymmetry of the two binding sites, and (iii) hydrogen bond donors proximal to the metal centers. Specifically, asymmetric ligands bearing two amide or amine groups as mimics of the second coordination sphere of the enzyme were synthesized and are depicted in Chart 6. Incorporation of two pivaloyl-amide residues in $\mathrm{H}_{3} \mathrm{SIM}^{1}$ in an asymmetrical manner, in contrast to $\mathrm{H}_{3} \mathrm{~L}^{2}$, realized the hydrogen bonding capacity as well as the asymmetry of the two binding sites. Similarly, $\mathrm{HSIM}^{2}$ bears two amine moieties in an asymmetric fashion, contrary to its symmetric counterpart $\mathrm{HL}^{8}$. While in $\mathrm{H}_{3} \mathrm{SIM}^{1}$ and $\mathrm{HSIM}^{2}$ the asymmetry of the metal binding sites is caused by the moieties of the second coordination sphere, $\mathrm{H}_{4} \mathrm{SIM}^{3}$ and $\mathrm{H}_{4} \mathrm{SIM}^{4}$ were designed in the way that the primary coordination sphere of the two binding sites is unlike. Moreover, both asymmetric ligands, $\mathrm{H}_{4} \mathrm{SIM}^{3}$ and $\mathrm{H}_{4} \mathrm{SIM}^{4}$, bear hydrogen bond donors either proximal to one or proximal to both binding sites. The ligands discussed in this thesis leave vacant coordination sites and facilitate the interaction of the metal centers with specific substrates.
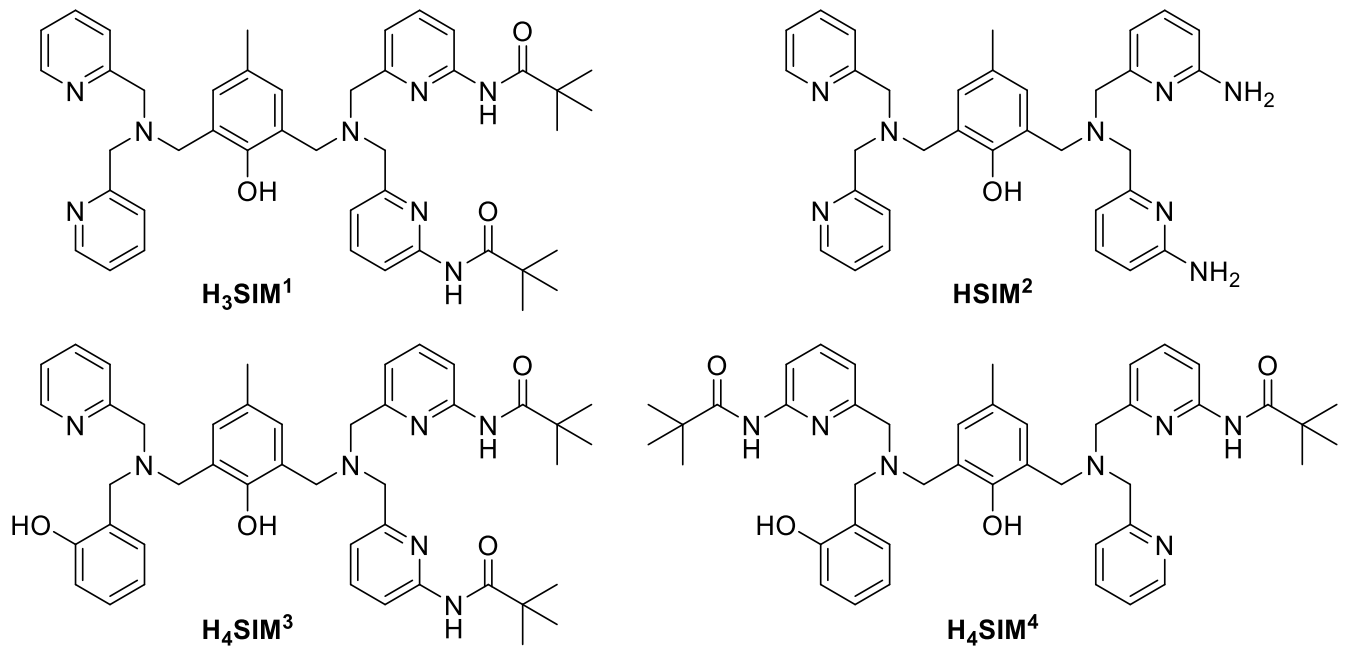

Chart 6: Overview of the ligands synthesized in this work.

The following chapter will give an introduction to the synthetic pathways developed for the preparation of the asymmetric ligands $\mathrm{H}_{3} \mathrm{SIM}^{1}, \mathrm{HSIM}^{2}, \mathrm{H}_{4} \mathrm{SIM}^{3}$ and $\mathrm{H}_{4} \mathrm{SIM}^{4}$ (Chart 6). In addition, detailed experimental procedures as well as analytical results are given in Chapter 11. 


\subsection{SYNTHESIS OF LIGAND $\mathrm{H}_{3} \mathrm{SIM}^{1}$}

In order to gain further insight into the role of the hydrogen bonding network of PAPs in the catalytic mechanism, four asymmetric ligands bearing amide or amine residues with the capacity to form hydrogen bonds were developed. $\mathrm{H}_{3} \mathrm{SIM}^{1}$ was designed in order to investigate especially the impact of the positioning of the hydrogen bond donating groups in the active site of PAPs. The structure of $\mathrm{H}_{3} \mathrm{SIM}^{1}$ is based on the symmetric ligands $\mathrm{HL}^{1}$ and $\mathrm{H}_{3} \mathrm{~L}^{2}$. While $\mathrm{HL}^{1}$ provides no hydrogen bond donors, $\mathrm{H}_{3} \mathrm{~L}^{2}$ bears two pivaloyl-amide moieties, one amide moiety proximal to each coordination site. In contrast, $\mathrm{H}_{3} \mathrm{SIM}^{1}$ was designed in the way that a hydrogen bonding network is only available at one of the two binding sites, while the primary coordination sphere remains unchanged and therefore comparable to $\mathrm{HL}^{1}$ and $\mathrm{H}_{3} \mathrm{~L}^{2}$.

In the process of finding a suitable synthetic pathway for the preparation of $\mathrm{H}_{3} \mathrm{SIM}^{1}$ the approaches illustrated in Scheme 2 were combined and the number of reaction steps minimized. In this way, the retrosynthetic analysis of the ligand $\mathrm{H}_{3} \mathrm{SIM}^{1}$ yield two possible pathways (Scheme 3). Both pathways share the same asymmetric starting material 2-hydroxy-3(hydroxymethyl)-5-methylbenzaldehyde (1). The suggested stepwise reaction with two different secondary amines enables the selective formation of the asymmetric ligand. Therefore, pathway a) suggests an initial amination and the subsequent nucleophilic substitution while pathway b) follows the reverse order.

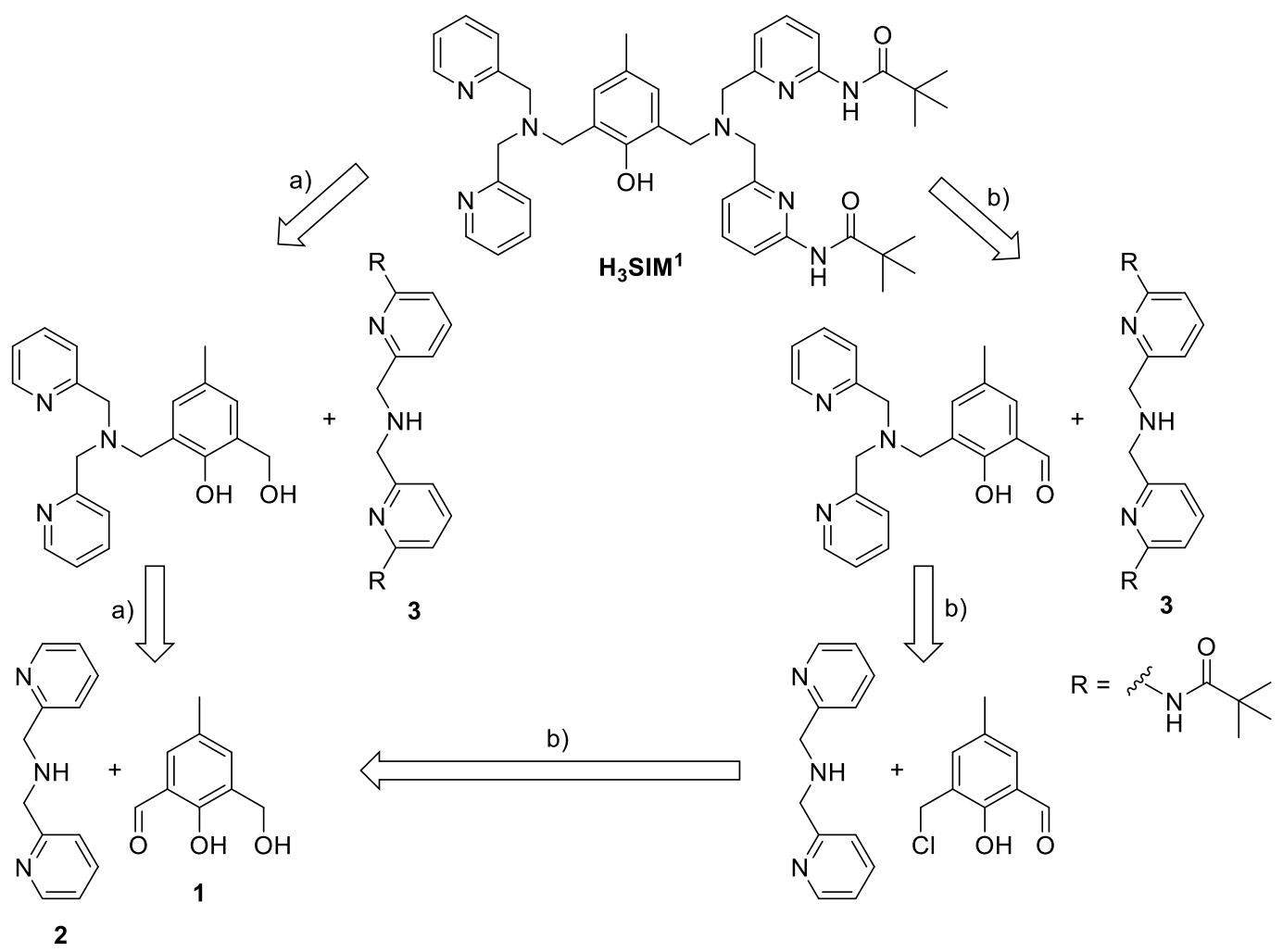

Scheme 3: Retrosynthetic analysis of ligand $\mathrm{H}_{3} \mathrm{SIM}^{1}$. 
The precursors needed for both synthetic routes, i.e. compound $\mathbf{1}, \mathbf{2}$, and $\mathbf{3}$ were synthesized according to known procedures. ${ }^{115-121}$ Starting with 2,6-bis(hydroxylmethyl)-4-methylphenol (4), the aldehyde functionality in compound 1 was formed by oxidation with $\mathrm{MnO}_{2}$ in chloroform (Scheme 4a). ${ }^{120}$ During the reaction both the monoaldehyde 1 and its dialdehyde derivative were formed and separated through column chromatography yielding compound $\mathbf{1}$ in a moderate yield of $46 \%$. A selective procedure for the preparation of compound 1 was also tested. Protection of one alcohol group as the cyclic acetal of 2,2-dimethoxypropane in acetone enables the selective oxidation of the remaining alcohol group in dichloromethane using pyridinium chlorochromate (PCC) as oxidant (Scheme 4b). ${ }^{122}$ The following deprotection with hydrochloric acid in a mixture of acetonitrile and water led exclusively to compound $\mathbf{1}$ with an overall yield of the three reaction steps of $74 \% .{ }^{123}$ However, this alternative route includes two additional synthetic steps and is therefore more time-consuming. Chlorination of the alcohol group in compound $\mathbf{1}$ with concentrated hydrochloric acid in ethanol generated compound 5 in $67 \%$ yield (Scheme 4). ${ }^{121}$ Compound 5, which contains one aldehyde group and one chloromethyl group, was chosen as the building block for the selective synthesis of the asymmetric dinucleating ligands $\mathrm{H}_{3} \mathrm{SIM}^{1}$, $\mathrm{HSIM}^{2}, \mathrm{H}_{4} \mathrm{SIM}^{3}$, and $\mathrm{H}_{4} \mathrm{SIM}^{4}$ as those functionalities make this phenol compound able to react stepwise with two different secondary amines thereby forming the two unequal coordination sites of the ligands.
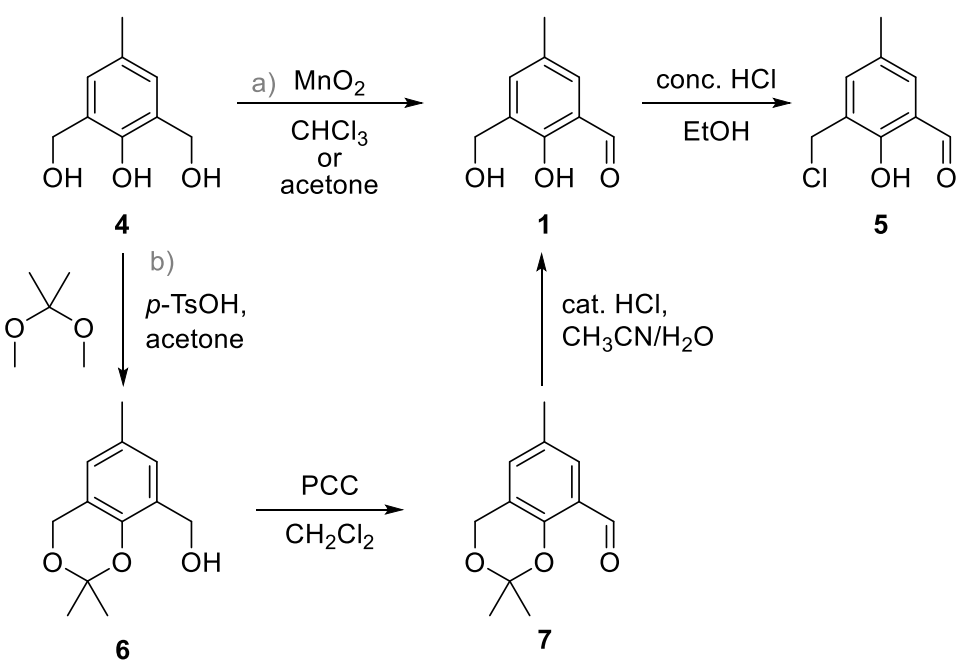

Scheme 4: Comparison of the statistical and selective synthesis of compound $\mathbf{1}$ and the following formation of compound 5.

The two secondary amines, compound $\mathbf{2}$ and compound $\mathbf{3}$, were synthesized adapting published methods. ${ }^{115,} 119$ Compound 2 was generated by amination of picolylaldehyde with 2-(aminomethyl)pyridine in methanol and subsequent hydrogenation with sodium borohydride in a moderate yield of $63 \%$. The preparation of compound $\mathbf{3}$ was more challenging and the synthetic pathway is included in Scheme 5. 


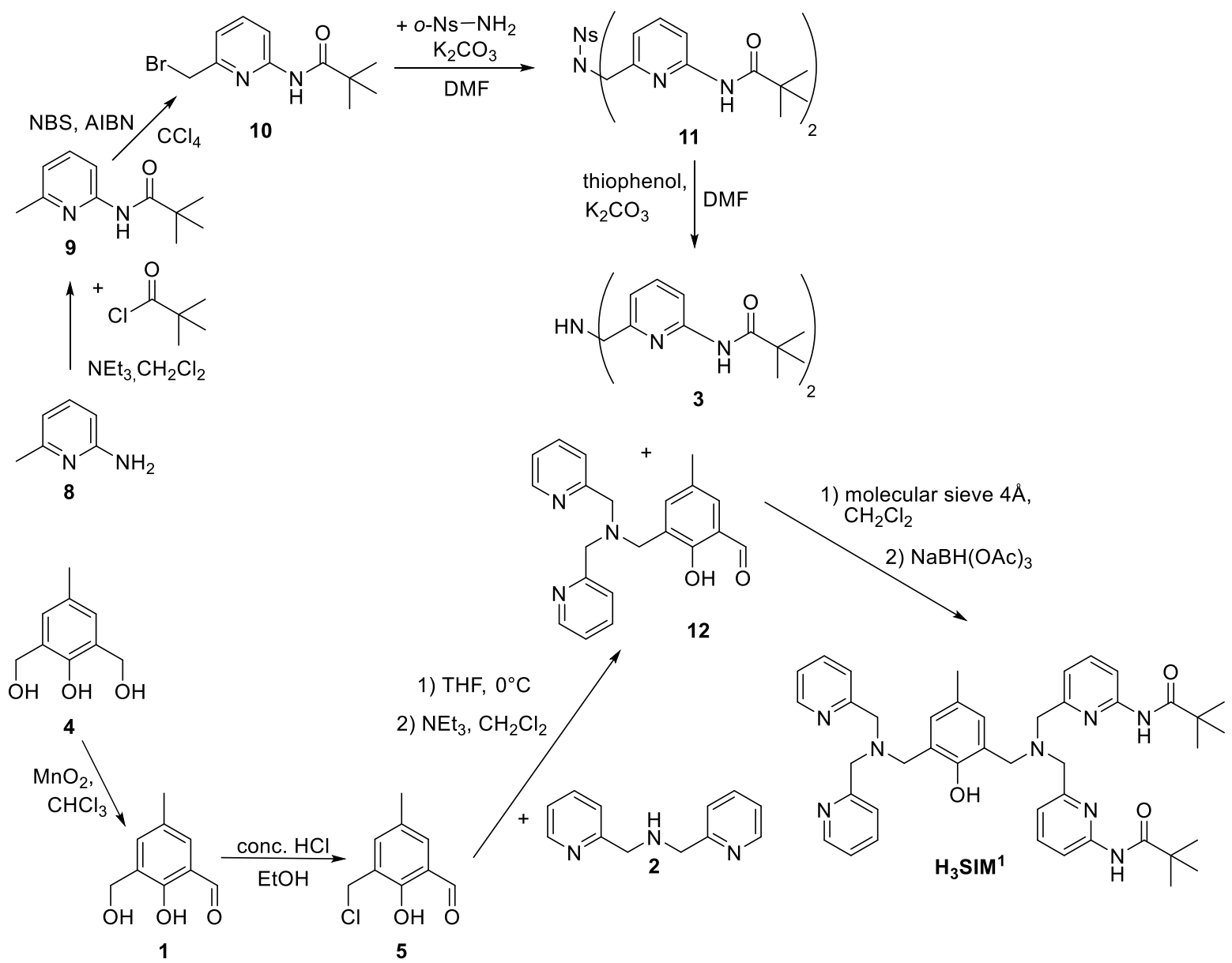

Scheme 5: Synthesis of ligand $\mathrm{H}_{3} \mathrm{SIM}^{1}$.

The amino functionality of 2-amino-6-methylpyridine (8) was protected prior to bromination via generation of the respective pivaloyl-amido derivative ( $95 \%$ yield). During the bromination of $\mathrm{N}$-(6-methylpyridin-2-yl)pivalamide (9) a variety of side products appeared (Scheme 6).

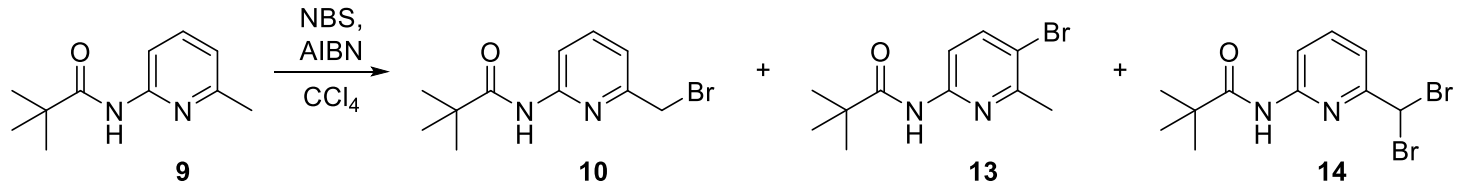

Scheme 6: Bromination of compound 9 yielding the mixture of compound 10, 13, and 14.

Thus, different modifications regarding the type and the concentration of the bromine source, the solvent and the use of UV light were examined in order to minimize the concentration of ringbrominated derivative 13 and dibrominated species 14 at maximal conversion of the starting material (9). In this way, a procedure was developed which leads to an adequate amount of sidechain monobrominated compound $\mathbf{1 0}$. The isolation of the desired compound $\mathbf{1 0}$ was achieved by column chromatography and subsequent crystallization by ultrasonication in a yield of $44 \%$. In the next step two equivalents of compound $\mathbf{1 0}$ were treated with potassium carbonate and one equivalent of 2-nitrobenzoylsulfonamide in dimethylformamide to form compound $\mathbf{1 1}$ in a yield of $98 \%$ (Scheme 5). Subsequent reaction of compound 11 with potassium carbonate and 
thiophenol in anhydrous dimethylformamide realized the formation of compound $\mathbf{3}$ in $81 \%$ yield (Scheme 5).

The attachment of the secondary amines $\mathbf{2}$ and $\mathbf{3}$ to the bridging phenol building block $\mathbf{1}$ was initially tested following pathway a) in Scheme 3, adapting the procedures reported by Nordlander et al. (Scheme 7). ${ }^{107}$ While the amination of compound $\mathbf{1}$ with compound $\mathbf{2}$ yield compound 15 in $85 \%$ yield, the following chlorination with thionylchloride and the nucleophilic substitution with compound $\mathbf{3}$ were found to be an unreliable procedure to obtain $\mathrm{H}_{3} \mathrm{SIM}^{1}$ in a satisfactory yield.
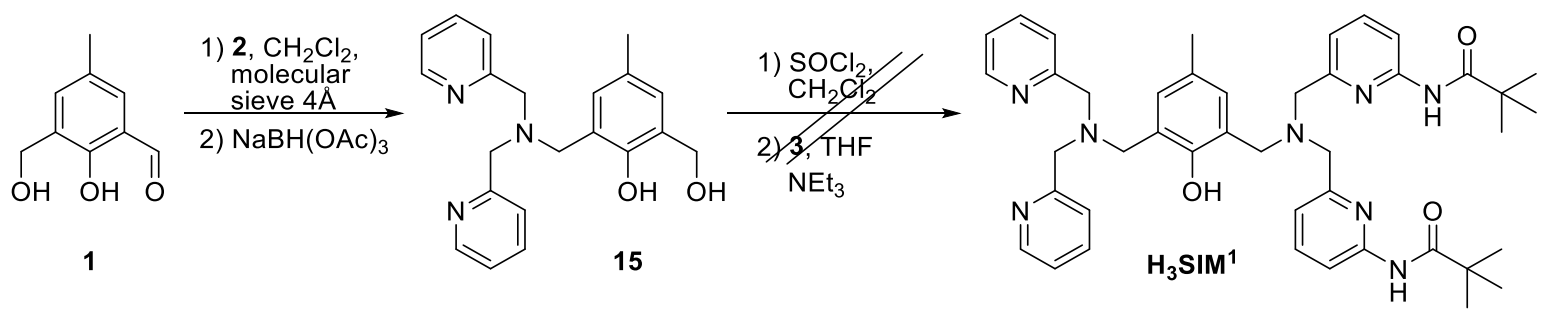

Scheme 7: Failed synthetic route to ligand $\mathrm{H}_{3} \mathrm{SIM}^{1}$.

In order to circumvent the previous drawbacks, the alternative strategy of pathway b) was explored (Scheme 3), and the complete eight-step synthetic route is shown in Scheme 5. Nucleophilic substitution of the chlorine atom of compound $\mathbf{5}$ with amine $\mathbf{2}$ yields compound $\mathbf{1 2}$ (61\% yield). The formation of ligand $\mathrm{H}_{3} \mathrm{SIM}^{1}$ was realized by amination of the remaining carbonyl functionality with the secondary amine $\mathbf{3}$ in anhydrous solution and subsequent hydrogenation with sodium triacetoxyborohydride.

Unfortunately, recrystallization from acetonitrile of the crude product, obtained after the workup of the reaction mixture, gave $\mathrm{H}_{3} \mathrm{SIM}^{1}$ in a yield of only $27 \%$. However, a purification procedure via column chromatography was developed to yield $\mathrm{H}_{3} \mathrm{SIM}^{1}$ in slightly higher yield of $30 \%$. A similar method was also used for the purification of the symmetric ligand $\mathrm{H}_{3} \mathrm{~L}^{2}$, prepared for comparison according to published procedures. ${ }^{109,114}$

\subsection{SYNTHESIS OF LIGAND HSIM²}

Ligand $\mathrm{HSIM}^{2}$ was designed with two amino functionalities adjacent to one binding site, in order to study the influence of the steric hindrance contrived by the bulky pivaloyl-amide residues in $\mathrm{H}_{3} \mathrm{SIM}^{1}$ with unchanged position of the hydrogen bonding donors. Moreover, comparison to the published ligand $\mathrm{HL}^{1}$, lacking hydrogen bond donors, and to $\mathrm{HL}^{8}$, bearing two amine functionalities in a symmetric manner, enables the investigation of the effect of the two asymmetrically incorporated amino substituents. 
Ligand $\mathrm{HSIM}^{2}$ is accessible by deprotection of the two pivaloyl-amide moieties of ligand $\mathrm{H}_{3} \mathrm{SIM}^{1}$. Therefore, the cleavage of the protecting amide bonds in ligand $\mathrm{H}_{3} \mathrm{SIM}^{1}$ was studied under various conditions. It was determined that deprotection of $\mathrm{H}_{3} \mathrm{SIM}^{1}$ under acidic conditions results in decomposition, similar to the chemistry previously detected during treatment of $\mathrm{H}_{3} \mathrm{~L}^{2}$ with hot hydrochloric acid. ${ }^{109}$ However, heating of $\mathrm{H}_{3} \mathrm{SIM}^{1}$ in ethanol solution under basic conditions at $70^{\circ}$ for three days was efficient (Scheme 8). Microwaves radiation under basic conditions also promotes the deprotection of $\mathrm{H}_{3} \mathrm{SIM}^{1}$, but this could not been undertaken on a larger scale due to instrumental restrictions.

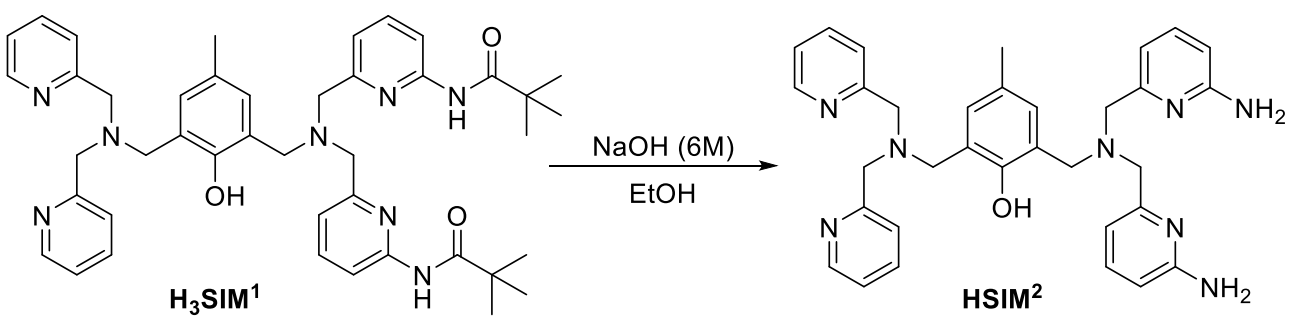

Scheme 8: Synthesis of ligand $\mathrm{HSIM}^{2}$.

Purification of the crude product of $\mathrm{HSIM}^{2}$ obtained after workup of the reaction mixture was undertaken via column chromatography with alumina.

\subsection{SYNTHESIS OF LIGAND $\mathrm{H}_{4} \mathrm{SIM}^{3}$}

The paradigm for the construction of PAP biomimetics, both structural and functional, is that the ligands permit discrimination between trivalent and divalent metal ions and therefore bear the capacity to stabilize a heterodinuclear site. Furthermore, the pivaloyl-amide residues were incorporated into the asymmetric ligand $\mathrm{H}_{2} \mathrm{~L}^{4}$, leading to $\mathrm{H}_{4} \mathrm{SIM}^{3}$ and $\mathrm{H}_{4} \mathrm{SIM}^{4}$, in order to provide both the capability of coordination asymmetry and the ability for hydrogen bonding of the substrate and/or nucleophile. Both ligands provide two different coordination pockets: a softer nitrogen-rich $\mathrm{N}_{3} \mathrm{O}$ compartment for the divalent metal ion and a harder $\mathrm{N}_{2} \mathrm{O}_{2}$ donor set in the other binding site for the trivalent metal ion.

Ligand $\mathrm{H}_{4} \mathrm{SIM}^{3}$ was accessible applying the optimized procedure for the synthesis of ligand $\mathrm{H}_{3} \mathrm{SIM}^{1}$ using compound $\mathbf{1 6}$ as secondary amine in the first step (Scheme 9). Compound 16 was synthesized after a published method by reaction of salicylaldehyde with 2-(aminomethyl)pyridine and subsequent treatment with sodium borohydride ( $99 \%$ yield). ${ }^{124,125}$ 

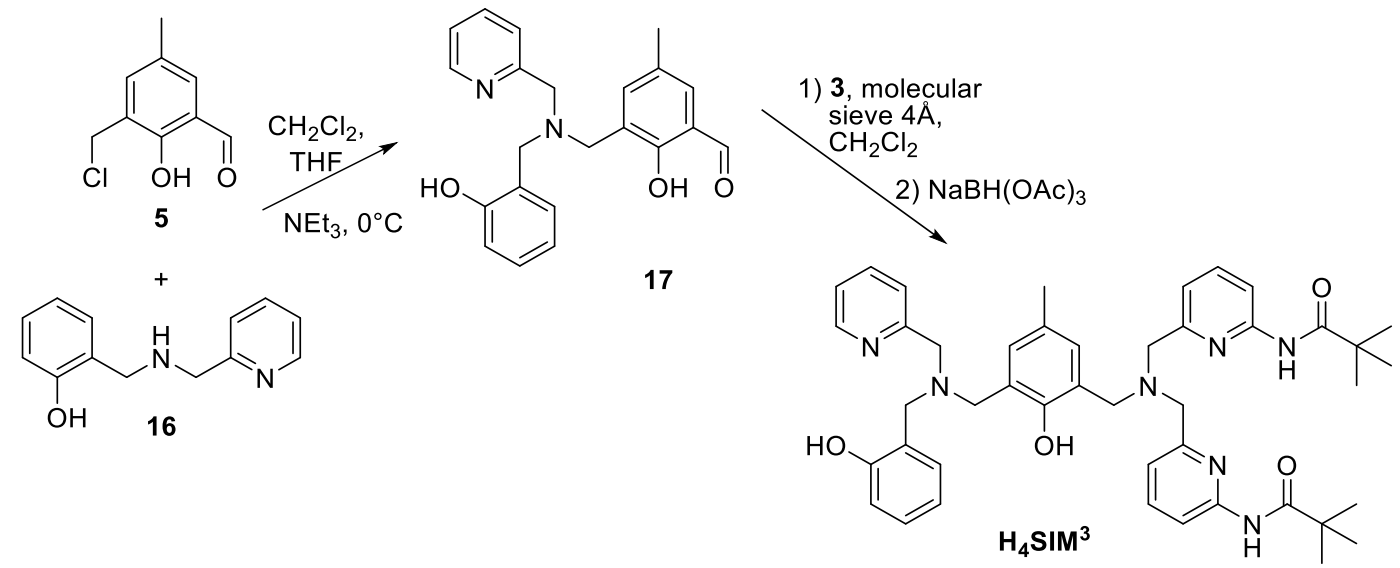

Scheme 9. Synthesis of ligand $\mathrm{H}_{4} \mathrm{SIM}^{3}$.

This secondary amine $\mathbf{1 6}$ was used as nucleophile after treatment with triethylamine in the nucleophilic substitution with compound $\mathbf{5}$ generating compound $\mathbf{1 7}$ in a moderate yield of 55\%. The second amine side-arm was introduced by treatment of compound $\mathbf{1 7}$ with compound $\mathbf{3}$ under anhydrous conditions and following hydrogenation with sodium triacetoxyborohydride. Purification of the crude product of $\mathrm{H}_{4} \mathrm{SIM}^{3}$ obtained after workup of the reaction mixture was undertaken with column chromatography with silica.

\subsection{SYNTHESIS OF LIGAND $\mathrm{H}_{4} \mathrm{SIM}^{4}$}

In $\mathrm{H}_{4} \mathrm{SIM}^{3}$ the hydrogen bond donating pivaloyl-amide residues are incorporated in an asymmetric manner as both are proximal to one binding site. Furthermore, the symmetric counterpart of $\mathrm{H}_{4} \mathrm{SIM}^{3}, \mathrm{H}_{4} \mathrm{SIM}^{4}$, was designed bearing one pivaloyl-amide residue proximal to both binding sites and thus combining an asymmetric primary coordination sphere with a symmetric primary coordination sphere regarding the two metal centers.

For the synthesis of $\mathrm{H}_{4} \mathrm{SIM}^{4}$ the synthetic strategy applied for the preparation of $\mathrm{H}_{3} \mathrm{SIM}^{1}$ and $\mathrm{H}_{4} \mathrm{SIM}^{3}$ was changed slightly. Instead of attaching two secondary amines to the asymmetric phenol building block, the second coordination site was built up in two steps due to the better accessibility of the reactants (Scheme 10). In the first step the secondary amine $\mathbf{1 8}$ was used as nucleophile for substitution of the chloro functionality of compound 5. In this way, compound 19 was formed in $96 \%$ yield. Compound 18 containing only one amidated pyridine was synthesized as published previously. ${ }^{109,114}$ Thereby, the dibrominated byproduct 14 obtained by bromination of compound 9 (Scheme 6) was treated with silver nitrate in acetone to generate a formyl functionality, i.e. $\mathrm{N}$-(6-formylpyridine-2-yl)pivalamide. Subsequent amination with 2-(aminomethyl)pyridine and hydrogenation with sodium borohydride yield compound $\mathbf{1 8}$ in an overall yield of $95 \%$ for the two reaction steps. 
Amination of the remaining aldehyde functionality of compound 19 with 2-(aminomethyl)phenol (20) an subsequent hydrogenation with sodium borohydride resulted in compound 21. 2-(aminomethyl)phenol (20) was prepared according to a literature known procedure by reduction of salicylamide with borane/tetrahydrofuran complex. ${ }^{126}$ Compound $\mathbf{2 1}$ was converted to ligand $\mathrm{H}_{4} \mathrm{SIM}^{4}$ by nucleophilic substitution using compound 10 as reactant.

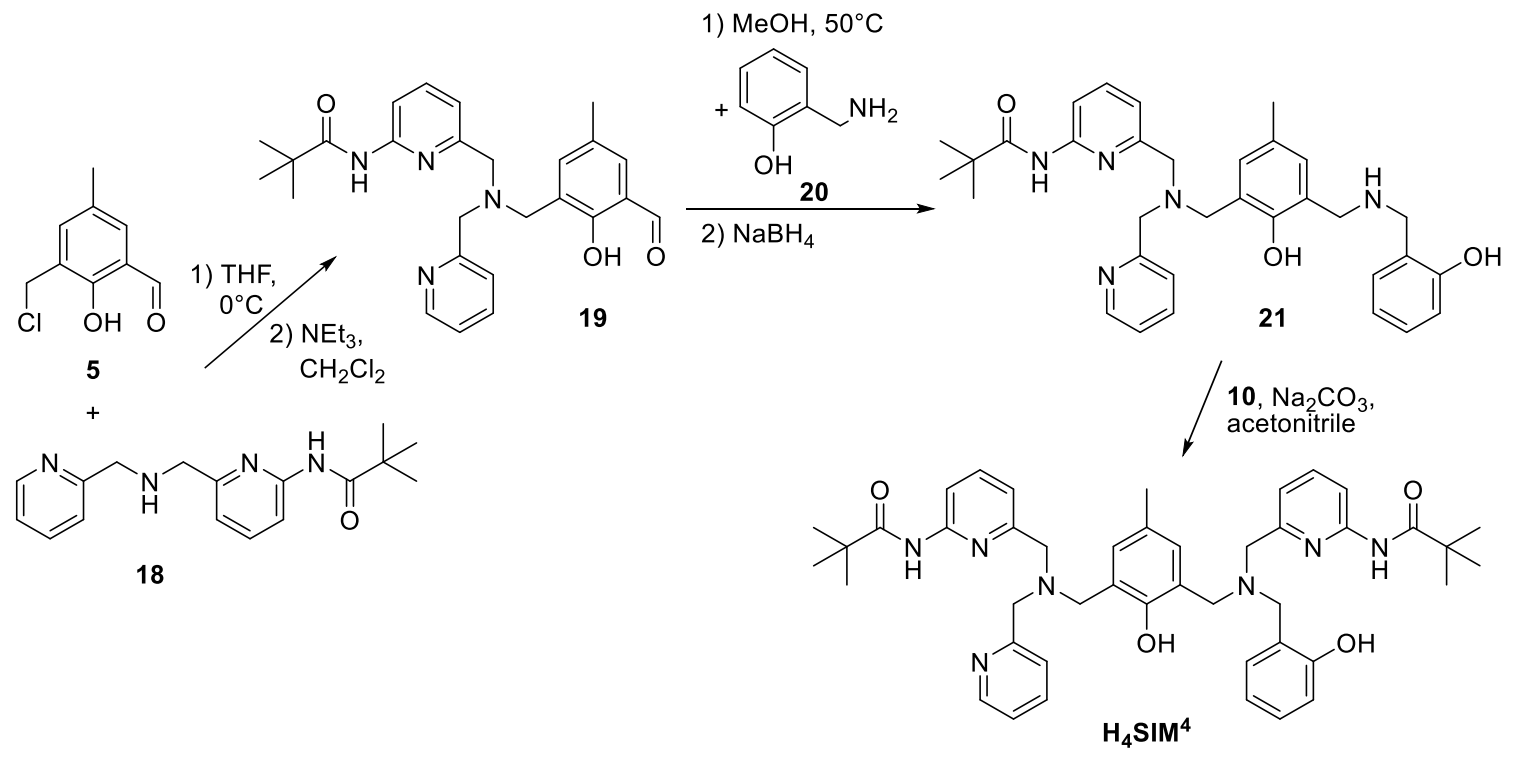

Scheme 10: Synthesis of ligand $\mathrm{H}_{4} \mathrm{SIM}^{4}$.

Similar to the synthetic procedures of $\mathrm{H}_{3} \mathrm{SIM}^{1}$ and $\mathrm{H}_{4} \mathrm{SIM}^{3}$, the crude product of $\mathrm{H}_{4} \mathrm{SIM}^{4}$ was purified via column chromatography.

\subsection{SUMMARY}

Four asymmetric, dinucleating ligands were designed and prepared, which provide two different coordination sites, mimicking the peptide backbone surrounding the dimetallic core in the active site of phosphatases. Ligand $\mathrm{H}_{3} \mathrm{SIM}^{1}$ and $\mathrm{HSIM}^{2}$ are derivatives of $\mathrm{HL}^{1}$, but with the capacity to form a hydrogen bonding network to the substrate/nucleophile during the catalytic hydrolysis of phosphoesters as present in the active site of the native enzymes. In $\mathrm{HSIM}^{2}$ two amino substituents function as hydrogen bond donors, the groups capable to form hydrogen bonds in $\mathrm{H}_{3} \mathrm{SIM}^{1}$ are two sterically demanding pivaloyl-amide residues. In both $\mathrm{HSIM}^{2}$ and $\mathrm{H}_{3} \mathrm{SIM}^{1}$ the hydrogen bond donors are positioned in an asymmetric manner regarding the two coordination sites, while in the previously published ligand $\mathrm{H}_{3} \mathrm{~L}^{2}$ two pivaloyl-amide residues have been incorporated into $\mathrm{HL}^{1}$ in a symmetrical fashion. Similarly, two derivatives of $\mathrm{H}_{2} \mathrm{~L}^{4}$ have been developed bearing two pivaloyl-amide residues either in a symmetric $\left(\mathrm{H}_{4} \mathrm{SIM}^{4}\right)$ or an asymmetric fashion $\left(\mathrm{H}_{4} \mathrm{SIM}^{3}\right)$. Thus, these two ligands $\mathrm{H}_{4} \mathrm{SIM}^{3}$ and $\mathrm{H}_{4} \mathrm{SIM}^{4}$ provide two distinct coordination 
sites, a softer $\mathrm{N}_{3} \mathrm{O}$ and a harder $\mathrm{N}_{2} \mathrm{O}_{2}$, and are therefore able to discriminate the coordination of tri- and divalent metal ions. For the synthesis of $\mathrm{H}_{3} \mathrm{SIM}^{1}$ and $\mathrm{H}_{4} \mathrm{SIM}^{3}$ a three step approach starting with 2-hydroxy-3-(hydroxylmethyl)-5-methylbenzaldehyde was evaluated similar to Nordlander et al., ${ }^{107}$ but avoiding the delicate chlorination with thionylchloride. $\mathrm{H}_{4} \mathrm{SIM}^{4}$ was prepared in a similar procedure except of the stepwise generation of the second coordination site. The synthesis of $\mathrm{HSIM}^{2}$ was realized by deprotection of ligand $\mathrm{H}_{3} \mathrm{SIM}^{1}$ under basic conditions. The detailed synthetic procedures and analytic results are given in Chapter 11. 


\section{Chapter 3}

\section{Coordination Behavior of}

Asymmetric Ligands ${ }^{c}$

\footnotetext{
'Main parts of this chapter will be published in "Selective Coordination of Gallium(III), Zinc(II) and Copper(II) by an Asymmetric Dinucleating Ligand: A Model for Metallophosphatases"; Simone Bosch, Peter Comba, Lawrence R. Gahan, Graeme R. Hanson, Christopher J. Noble, Gerhard Schenk, Hubert Wadepohl; manuscript in preparation.
} 


\subsection{INTRODUCTION}

As certain hydrolases are of particular interest in the development of biologically active compounds against many diseases, there is special interest in understanding the detailed mechanism of the reaction which takes place within their active sites. ${ }^{127}$ In addition to enzymatic studies, modeling the structure and reactivity of the corresponding metalloenzymes using model complexes has been shown to be very instructive in the study of the structural and functional properties of the enzymes themselves.

Several classes of metalloenzymes have a dinuclear active site in which two metal ions cooperate in order to perform the transformation of various substrates. Representative examples of structurally characterized homo- and heterodinuclear active sites relevant for hydrolysis include those in Alkaline Phosphatase [Zn"Zn"], ${ }^{64}$ Purple Acid Phosphatase ([Fe"'Zn"], ${ }^{83,128}\left[\mathrm{Fe}^{\prime \prime \prime} \mathrm{Fe}^{\prime \prime}\right],{ }^{27,}{ }^{129}$ $\left.\left[\mathrm{Fe}^{\prime \prime \prime} \mathrm{Mn}^{\prime \prime}\right]^{67,85}\right)$, human Protein Phosphatase $1\left[\mathrm{Fe}^{\mathrm{III}} \mathrm{Mn}^{\prime \prime}\right]^{130}$ and bovine erythrocyte Superoxide Dismutase [Cu"Zn"]. ${ }^{131-133}$ In the homo- and heterodinuclear active sites of these metalloenzymes the surrounding protein matrix usually provides chemically distinct binding environments for the individual metal centers. Although it is known that the lack of symmetry of the active center is an essential structural feature and decisive factor for the catalytic mechanism and efficiency, ${ }^{107}$, 134,135 there are only a few well-characterized heterodinuclear complexes as model systems for hydrolases. ${ }^{29,81,136}$ The paucity of examples is possibly attributable to the relatively late discovery of heterodinuclear metalloenzymes, but is more likely due to the difficulties in the selective binding of two metal ions at specific sites in the complexes. Nature has had millions of years to perfect site selectivity, unlike coordination chemists.

A challenge in the preparation of PAP biomimetics is the requirement for the stabilization of a heterovalent dinuclear core ${ }^{81}$ because the coordination of two different metal ions by a dinucleating ligand may result in the formation of both discrete homo- and heterodinuclear complexes, and metal site isomers in which the sites are crystallographically disordered occupied. These complications have been circumvented somewhat by designing ligands that either bind metal ions selectively in their pockets or strongly enough to prevent disproportionation and metal mixing. ${ }^{137}$

Some heterovalent and heterometallic systems have been prepared by using symmetrical ligands such as $\mathrm{HL}^{1}, \mathrm{HL}^{9}, \mathrm{HL}^{10}, \mathrm{HL}^{11}$ etc. (see Table of Compounds), which do not exhibit metal site selectivity, but offer the opportunity to prepare heterometallic or heterovalent complexes by careful control of the synthetic conditions. Examples include the biomimetics for the 
heterodivalent reduced form of PAP, $\left[\mathrm{Fe}^{\prime \prime \prime} \mathrm{Fe}^{\prime \prime}\left(\mathrm{L}^{11}\right)(\mathrm{OAC})_{2}\right](\mathrm{BPh})_{2}$, and the mixed-valent $\mathrm{Fe}^{\mathrm{III}} \mathrm{Fe}$ "l and $\mathrm{Fe}^{\prime \prime \prime} \mathrm{Zn} \mathrm{Zn}^{\prime \prime}$ complexes of $\mathrm{HL}^{1}, \quad\left[\mathrm{Fe}^{\prime \prime \prime \prime} \mathrm{Fe}^{\prime \prime}\left(\mathrm{L}^{1}\right)(\mathrm{OPr})_{2}\right]\left(\mathrm{BPh}_{4}\right)_{2}$ and $\left[\mathrm{Fe}^{\prime \prime \prime} \mathrm{Zn}^{\prime \prime}\left(\mathrm{L}^{1}\right)(\mathrm{OAc})_{2}\right]\left(\mathrm{BPh}_{4}\right)_{2}$; crystallographic analysis of the latter suggests the presence of a small degree of disorder in the metal occupancies. ${ }^{138,139}$ However, in many cases the issue of disorder and non-site-selectivity has not been addressed, perhaps not even considered.

Another strategy to circumvent the difficulties in selective binding of two different metal centers has been the use of asymmetric ligands bearing two binding pockets which differ either in the number and/or the type of coordinating donors. One of the most common ligands used for this purpose is $\mathrm{H}_{2} \mathrm{~L}^{4}$ (Chart 7) which contains a harder $\mathrm{N}_{2} \mathrm{O}_{2}$ metal ion binding site $(A)$ and a softer $\mathrm{N}_{3} \mathrm{O}$ metal ion binding site (B). Using $\mathrm{H}_{2} \mathrm{~L}^{4}$ a variety of heterodinuclear model complexes, which mimic the active site structure of PAP more adequately, has been developed and characterized;

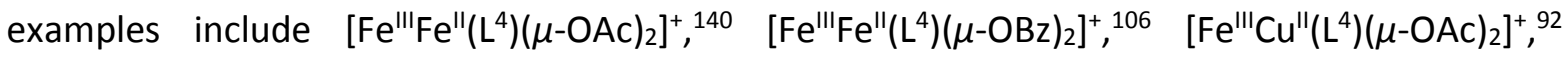
$\left[\mathrm{Fe}^{\prime \prime \prime \prime} \mathrm{Mn} n^{\prime \prime}\left(\mathrm{L}^{4}\right)(\mu-\mathrm{OAc})_{2}\right]^{+}, 96$

$$
\left[\mathrm{Fe}^{\prime \prime \prime} \mathrm{Ni}^{\prime \prime}\left(\mathrm{L}^{4}\right)(\mu-\mathrm{OAC})_{2}\right]^{+},{ }^{141}
$$

$\left[\mathrm{Fe}^{\prime \prime \prime} \mathrm{Zn} \mathrm{n}^{\prime \prime}\left(\mathrm{L}^{4}\right)(\mu-\mathrm{OAC})_{2}\right]^{+}, 143$

$\left[\left[\mathrm{Fe}^{\prime \prime \prime \prime} \mathrm{Hg}^{\prime \prime}\left(\mathrm{L}^{4}\right)(\mu-\mathrm{OH})\right]_{2}\right]^{2+,}, 145$

$$
\left[\mathrm{Fe}^{\prime \prime \prime \prime} \mathrm{Zn}^{\prime \prime}\left(\mathrm{L}^{4}\right)\left(\mathrm{OH}_{2}\right)(\mu-\mathrm{OH})\right]^{2+},{ }^{90} \text {, }
$$

$\left[\mathrm{Fe}^{\prime \prime \prime} \mathrm{Co}^{\prime \prime}\left(\mathrm{L}^{4}\right)(\mu-\mathrm{OAC})_{2}\right]^{+}, 146$

$$
\left[\mathrm{Fe}^{\prime \prime \prime} \mathrm{Ni}^{\prime \prime}\left(\mathrm{L}^{4}\right)(\mu-\mathrm{OAC})\left(\mathrm{OH}_{2}\right)_{2}\right]^{2+},{ }^{142}
$$

144

$$
\left[\mathrm{Fe}^{\mathrm{III}} \mathrm{Cd}^{\prime \prime}\left(\mathrm{L}^{4}\right)(\mu-\mathrm{OAC})_{2}\right]^{+},{ }^{145}
$$

[Ga"'Co" $\left.\left(\mathrm{L}^{4}\right)(\mu-\mathrm{OAC})_{2}\right]^{+}, 146$

$\left[\mathrm{Ga}^{\prime \prime \prime} \mathrm{Zn} \mathrm{n}^{\prime \prime}\left(\mathrm{L}^{4}\right)(\mu-\mathrm{OAc})_{2}\right]^{+66}$ and $\left[\mathrm{Mn}^{\prime \prime \prime} \mathrm{Mn} n^{\prime \prime}\left(\mathrm{L}^{4}\right)(\mu-\mathrm{OAc})_{2}\right]^{+}{ }^{147,}, 148$ The preparation procedure for these complexes commonly involves the simultaneous or stepwise addition of two different metal salts to the ligand solution, where the selective formation of heterodinuclear complexes is theoretically possible. However, investigations of the species distribution were not always undertaken. In at least one case, selective binding has been investigated. Belle et al. illustrated a very elegant way to study the selective binding of ligand $\mathrm{H}_{3} \mathrm{~L}^{12}$ of $\operatorname{zinc}(\mathrm{II})$ ions by NMR spectroscopy via stepwise addition of zinc(II) salt, thereby demonstrating that $\mathrm{H}_{3} \mathrm{~L}^{12}$ selectively forms a monozinc(II) complex, in which the zinc(II) is coordinated to the $\mathrm{N}_{3} \mathrm{O}$ binding compartment. ${ }^{149}$ This finding was confirmed by the crystal structure of $\left[\mathrm{Fe}^{\mathrm{II}} \mathrm{Zn} \mathrm{n}^{\prime \prime}\left(\mathrm{L}^{12}\right)\left(\mu-\mathrm{O}_{2} \mathrm{P}(\mathrm{OPh})_{2}\right)_{2}\right]$ which was obtained after further addition of triethylamine and iron(III) perchlorate to the monozinc(II) synthon. ${ }^{149}$ Similarly, the respective Fe ${ }^{\text {III }} \mathrm{Cu}^{\text {II }}$ complex [Fe $\left.{ }^{\prime \prime \prime} \mathrm{Cu}^{\prime \prime}\left(\mathrm{L}^{12}\right)(\mu-\mathrm{OEt})\right] \mathrm{ClO}_{4}$ was prepared by the stepwise complexation of $\mathrm{Cu}^{\prime \prime}$ in the softer binding compartment and Fe ${ }^{I I I}$ in the harder coordination site after addition of triethylamine. ${ }^{150}$ The sitedirected complexation was confirmed by the crystal structure of the mononuclear complex $\left[\mathrm{Cu}^{\prime \prime}\left(\mathrm{H}_{3} \mathrm{~L}^{12}\right)(\mathrm{OAC})\right] \mathrm{BPh}_{4} ;{ }^{150}$ this study represents one of the few cases when the mononuclear synthon during the synthesis of heterovalent dinuclear complexes was isolated.

Heterovalent dinuclear model complexes have successfully modeled the physiochemical properties of metalloproteins and even the catalytic activity, but with a much lower efficiency. This discrepancy between the catalytic efficiencies of model systems and native enzymes is suggested, to a large extent, to be due to the lack of many important intermolecular interactions. 
In many cases, the amino acid residues close to the active site are known to be necessary for efficient enzymatic activity because mutation of these residues can cause deactivation or even inactivation of the catalyst. ${ }^{74}{ }^{151}$ For example, in PAP, histidine residues proximal to the metal centers in the active site have been shown to play an essential role in substrate binding and activation; mutations of a histidine to an alanine residue in the second coordination sphere of kbPAP resulted in a sharp decrease in activity. ${ }^{74}$ Moreover, theoretical studies have recently reinforced the importance of the proximal histidine residues within the active site of PAP to properly describe coordination of the substrate and stabilization of the transition state. ${ }^{151,152}$ Furthermore, Comba et al. developed a Fe"ll $\mathrm{Fe}^{\text {II }}$ model complex with hydrogen bond donors in close distance to the catalytic site, which presented the highest catalytic efficiency ever reported for a PAP model in relation to phosphodiester hydrolysis. ${ }^{108}$ Similarly, the extension of a Fe"'In" PAP model complex by (3-aminopropyl)silica and a $\mathrm{Fe}^{\text {III }} \mathrm{Cu}^{\prime \prime}$ PAP model complex by a small polyethyleneimine chain led to an increase in phosphoester hydrolysis activity. This finding was attributed to the interaction of the polar side chains with the substrate, mimicking in this way the microenvironment around the active site of the native enzymes. ${ }^{151,153}$

As part of the effort to probe the properties of biologically important, mixed valent, dinuclear centers, and to consider the hydrogen bond network of the second coordination sphere of the native metalloproteins, as well as the influences of asymmetric binding sites, three ligands have been synthesized (Chart 7). In this chapter coordination behavior studies of these asymmetric ligands towards $\mathrm{Zn}^{\prime \prime}, \mathrm{Cu}^{\prime \prime}, \mathrm{Ga}$ III and Fe ${ }^{\text {III }}$ ions will be discussed, in addition to their ability to selectively form a mononuclear synthon, which can then potentially be used to synthesize authentic hetereodinuclear model complexes for PAP.

The choice of the metal ions in this study ( $\mathrm{Zn}^{\prime \prime}, \mathrm{Cu}^{\prime \prime}, \mathrm{Ga}$ "I', and Fe"'I) is driven by a number of factors. The use of $\mathrm{Zn}$ " ions is inspired by the fact that many (> 300) metalloenzymes incorporate $\mathrm{Zn}^{\prime \prime}$ within their active sites. ${ }^{30,154-156}$ Although $\mathrm{Cu}^{\text {Il }}$ has not been found in native phosphatases, it was chosen to be studied within this thesis as there are some $\mathrm{Cu}^{\prime \prime}$ complexes showing catalytic activity towards phosphoester hydrolysis acceleration; Kovari et al. developed monocopper(II) complexes bearing ammonium groups proximal to the metal center which revealed a higher bis(4-nitrophenyl)phosphate (BNPP) hydrolysis rate than its $\mathrm{Zn}^{\prime \prime}$ counterpart; ${ }^{157-159}$ Neves et al. showed asymmetric dicopper(II) complexes accelerated phosphoester hydrolysis, especially via the introduction of a sterically demanding group in close distance to the metal centers; ${ }^{160,161}$ Chin et al. investigated a 6000-fold acceleration of bis(2,4-dinitro-phenyl)phosphate (BDNPP) hydrolysis by the introduction of amino groups in a $\mathrm{Cu}^{\prime \prime}$-o-phenanthroline complex; ${ }^{162}$ and recently Comba et al. reported the phosphatase activity of a dicopper(II) complex of a cyclic pseudopeptide. ${ }^{163}$ 
$\mathrm{Cu}^{\prime \prime}$ has, similar to $\mathrm{Zn}^{\prime \prime}$, substitutional flexibility and a relatively high ionization potential/Lewis acidity (see Table 1), which makes it both promising in regards to polarize $\mathrm{P}-\mathrm{O}$ bonds in phosphate ester substrates and to activate the phosphorus center towards nucleophilic attack.

Table 1: Selected physico-chemical properties of metal ions relevant to this work.

\begin{tabular}{llllll} 
ion & $\begin{array}{l}\text { ionic radius } \\
(\AA)^{\mathrm{a} 164}\end{array}$ & $\begin{array}{l}\text { charge density } \\
(\mathrm{Z} / \mathrm{r})\left(\AA^{-1}\right)^{\mathrm{a} 164}\end{array}$ & $\begin{array}{l}\text { ionization } \\
\text { potential }(\mathrm{eV})^{164}\end{array}$ & $\begin{array}{l}\mathrm{pK}_{\mathrm{a}} \text { of } \\
\text { bound } \mathrm{H}_{2} \mathrm{O}^{165}\end{array}$ & $\mathrm{k}_{\text {sub }}\left(\mathrm{s}^{-1}\right)^{\mathrm{b} 166}$ \\
\hline $\mathrm{Zn}^{\prime \prime}$ & 0.74 & 2.7 & 17.96 & 9.0 & $\sim 6 \cdot 10^{8}$ \\
$\mathrm{Cu}^{\prime \prime}$ & 0.73 & 2.8 & 20.29 & 8.0 & $4.4 \cdot 10^{9}$ \\
$\mathrm{Fe}^{\prime \prime}$ & 0.61 & 3.3 & 16.19 & 9.5 & $4.4 \cdot 10^{6}$ \\
$\mathrm{Ga}^{\prime \prime \prime \prime}$ & 0.62 & 4.8 & 30.73 & 2.6 & $4.0 \cdot 10^{2}$ \\
Fe & 0.55 & 5.5 & 30.65 & 2.2 & $1.6 \cdot 10^{2}$ \\
\hline
\end{tabular}

a ionic radii in crystals for a coordination number of 6 .

${ }^{\mathrm{b}}$ water exchange rate of the hexaaqua metal complexes in water at $25^{\circ} \mathrm{C}$.

Additionally, the $\mathrm{pK}_{\mathrm{a}}$ values of water molecules ligated to $\mathrm{Cu}^{\prime \prime}$ and $\mathrm{Zn}^{\prime \prime}$ ions often fall in the range of 7-9, enabling complexes of these ions to promote hydrolytic reactions under physiological or near physiological conditions. ${ }^{11}$ Moreover, the study of their coordination chemistry is feasible spectroscopically with NMR spectroscopy in the case of $\mathrm{Zn}$ " and EPR spectroscopy in the case of $\mathrm{Cu}^{\prime \prime}$, besides mass spectrometry, UV-vis-NIR spectroscopy and X-ray diffraction.

The investigation of Gall' coordination by the asymmetric ligands was chosen as it is an excellent mimic of Fe $\mathrm{F}^{\text {III }}$ with a similar ionic radius and Lewis acidity (Table 1). Furthermore, the Fe ${ }^{\text {III }}$ center in pig PAP and bovine spleen PAP has been replaced by Ga'"', resulting in Ga"'IF" and Ga"' $\mathrm{Zn}$ "I derivatives, which exhibit catalytic activities similar to those of the native forms. ${ }^{66,142,167}$ These findings give evidence that diamagnetic Gall' can act as a functional analogue of the paramagnetic $\mathrm{Fe}^{\text {IIII }}$ site in PAP model systems. Therefore, it is optimally suited to mimic Fe $\mathrm{e}^{\text {III }}$ ions when NMR techniques are used. Gall' is characterized by a well-defined coordination chemistry and absence of redox chemistry, which allows detailed reactivity studies without interference due to competing oxidation states.

In the present chapter the complexation studies of the asymmetric ligands $\mathrm{H}_{3} \mathrm{SIM}^{1}, \mathrm{H}_{4} \mathrm{SIM}^{3}$ and $\mathrm{H}_{4} \mathrm{SIM}^{4}$ (Chart 7) with Zn", Cu", Ga"I, and Fe"ll ions are reported. Although the primary coordination sphere of the two coordination sites are equivalent in $\mathrm{H}_{3} \mathrm{SIM}^{1}$, the secondary coordination sphere differs due to two pivaloyl-amide moieties adjacent to one binding site (binding site $\mathrm{C}$ ). $\mathrm{H}_{4} \mathrm{SIM}^{3}$ and $\mathrm{H}_{4} \mathrm{SIM}^{4}$ provide two distinctly different binding compartments: a $\mathrm{N}_{3} \mathrm{O}$ donor set in the softer compartment (binding site $\mathrm{C}$ and binding site $\mathrm{E}$ ) versus a rather harder $\mathrm{N}_{2} \mathrm{O}_{2}$ binding pocket (binding site $A$ and binding site D) (Chart 7). While $\mathrm{H}_{4} \mathrm{SIM}^{3}$ and $\mathrm{H}_{4} \mathrm{SIM}^{4}$ bear equal primary coordination spheres, the secondary coordination spheres differ in regard to the position of the two pivaloyl-amide moieties proximal to the metal centers. 

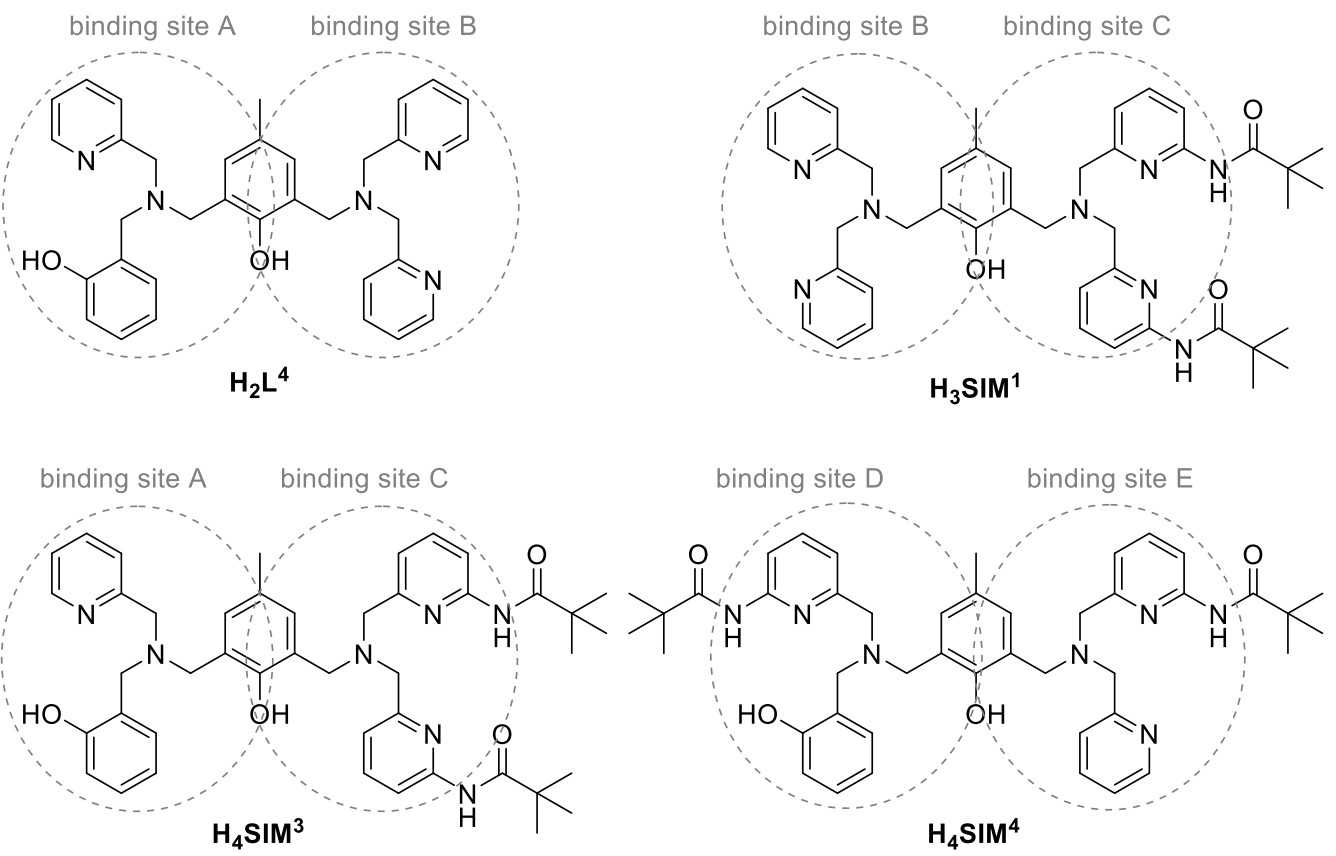

Chart 7: Ligands discussed in this chapter and the nomenclature of their binding compartments.

\subsection{CompleXATION BEhAVIOR OF LIGAND $\mathrm{H}_{3} \mathrm{SIM}^{1}$}

Although ligand $\mathrm{H}_{3} \mathrm{SIM}^{1}$ (Chart 8) provides two coordination pockets with identical primary coordination spheres, built up by a bridging phenoxido group, a tertiary amine and two pyridine moieties, the two coordination sites differ in their surroundings.

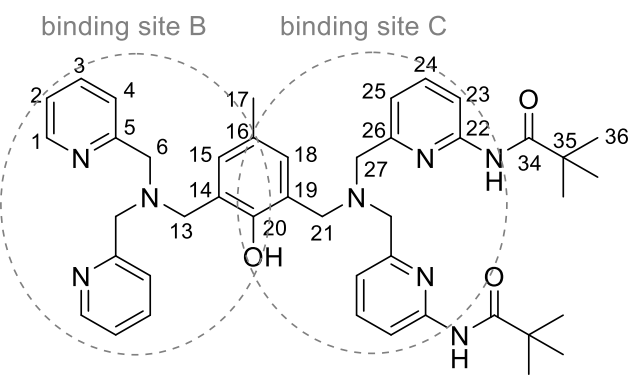

Chart 8: Assignment of the binding sites provided by ligand $\mathrm{H}_{3} \mathrm{SIM}^{1}$ and the numbering of its atoms.

While binding site B (see Chart 8 for the nomenclature) offers an unhindered access to the ligating atoms, binding site $\mathrm{C}$ is sterically hindered by the demanding pivaloyl-amide substituents of the pyridine side-arms. Therefore, the requirement of two inequivalent binding sites as present in native phosphatases is given in this ligand.

\subsubsection{COMPLEXATION STUDIES WITH ZN"}

The coordination features of ligand $\mathrm{H}_{3} \mathrm{SIM}^{1}$ towards $\mathrm{Zn}$ " ions were analyzed using NMR spectroscopic techniques. Aliquots of an acetonitrile solution of zinc(II) perchlorate $(0.518 \mathrm{M})$ 
were added stepwise to the ligand dissolved in acetonitrile $(33.6 \mathrm{mM})$. After each addition, the mixture was heated for five minutes at $50^{\circ} \mathrm{C}$ and the ${ }^{1} \mathrm{H}$ NMR spectrum was then monitored to follow the changes in the proton environments. ${ }^{13} \mathrm{C}$ NMR spectra were recorded and twodimensional NMR experiments (NOESY, COSY, HSQC, and HMBC) were undertaken after addition of one and after addition of two equivalents of metal salt. In addition, mass spectra were recorded of the samples employed in the NMR experiments.

\section{NMR Studies}

Stepwise addition of zinc(II) perchlorate to $\mathrm{H}_{3} \mathrm{SIM}^{1}$ in deuterated acetonitrile resulted in a slight shift of the resonances and the initial rise of a second set of resonances, while the resonances assigned to the free ligand decreased (Figure 1). In the spectrum of an 1:1 mixture of zinc(II) perchlorate and $\mathrm{H}_{3} \mathrm{SIM}^{1}$ the resonances assigned to the ligand disappeared and only a set of nine resonances assigned to the monozinc(II) complex remained (Figure 1d). Further addition of $\mathrm{Zn}^{\prime \prime}$ ions led to the decrease of the resonances assigned to the monozinc(II) complex and the appearance of a set of resonances assigned to the dizinc(II) complex. After the addition of two equivalents of $\mathrm{Zn}^{\|}$ions to $\mathrm{H}_{3} \mathrm{SIM}^{1}$ only the dizinc(II) complex is present in the solution, according to the ${ }^{1} \mathrm{H}$ NMR spectrum (Figure $1 \mathrm{~g}$ ).

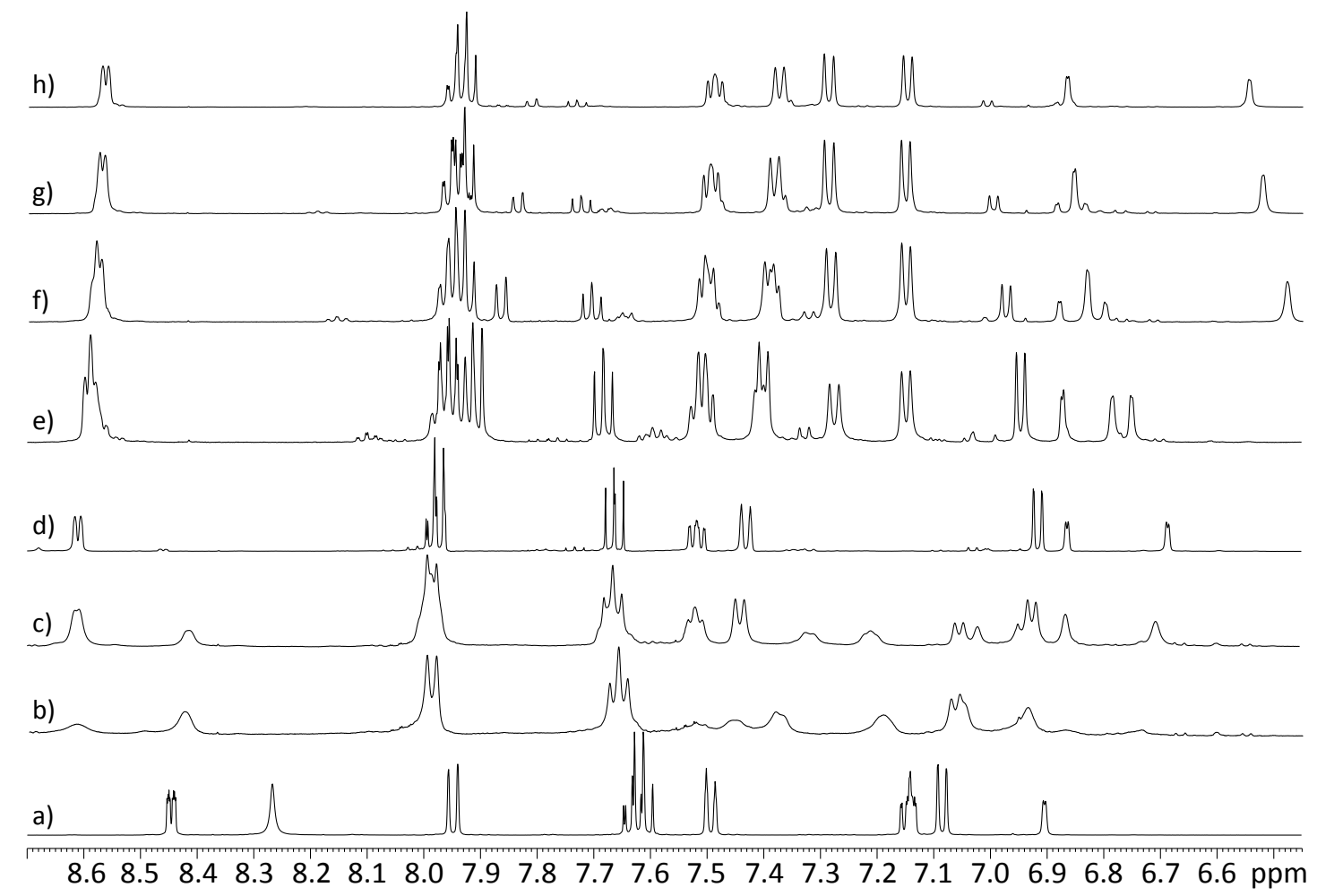

Figure 1: Comparison of ${ }^{1} \mathrm{H}$ NMR spectra of a) $\mathrm{H}_{3} \mathrm{SIM}^{1}$ with b) 0.50 eq, c) 0.75 eq, d) 1.00 eq, e) 1.50 eq , f) 1.75 eq, g) 2.00 eq, and h) 2.50 eq $\mathrm{Zn}^{\prime \prime}\left(\mathrm{ClO}_{4}\right)_{2}$ in $\mathrm{CD}_{3} \mathrm{CN} / \mathrm{D}_{2} \mathrm{O}$.

There is a possibility that the equilibrium between monozinc(II) complexes, in which the $\mathrm{Zn}$ "ion is coordinated in either of the two coordination sites, is more rapid than the NMR time scale. 
Therefore, in order to investigate this possibility the variable temperature ${ }^{1} \mathrm{H}$ NMR spectra of the monozinc(II) complex and the dizinc(II) complex were recorded in the temperature range from $282 \mathrm{~K}$ to $245 \mathrm{~K}$. No change in the spectra were detected, suggesting that the $\mathrm{Zn}$ " ion is selectively bound to one coordination site only.

In order to investigate the exact position of the $\mathrm{Zn}$ " ion within the monozinc(II) complex all resonances in the spectra of the monozinc(II) as well as the dizinc(II) complex species were assigned to the corresponding $\mathrm{H}$-atoms (Figure 2). Two dimensional NMR methods were employed: Firstly, the COSY NMR spectra were used to assign the resonances of the pyridyl $\mathrm{H}$-atoms. $\mathrm{H} 1$ (d) is the most characteristic $\mathrm{H}$-atom, the resonance of which is shifted downfield due to its ortho position to the pyridine $\mathrm{N}$-atom (see Chart 8 for the atom numbering). Commencing with the identified resonance of $H 1(\mathrm{~d})$, the resonances of $H 2(\mathrm{~d}), H 3(\mathrm{t})$ and $H 4(\mathrm{~d})$ were assigned by their cross peaks in the COSY NMR spectrum and their characteristic splitting (given in brackets). The remaining resonance in the region between $6.5 \mathrm{ppm}$ and $8.7 \mathrm{ppm}$ of the ${ }^{1} \mathrm{H}$ NMR spectra, which is split in a triplet, was allocated to $H 24(\mathrm{t})$ due to its position between two similar pyridine $\mathrm{H}$-atoms. The two resonances connected by a cross peak to the $H 24$ resonance and split in doublets are assigned to $H 23$ (d) and $H 25$ (d) (the specific assignments were determined by cross peaks in the HMBC NMR spectra between C26 and H25). Similarly, the remaining two singlets in the aromatic region of the ${ }^{1} \mathrm{H}$ NMR spectra were assigned to $H 15$ (s) and $H 18$ (s) (the specific correlation was assigned by cross peaks to the respective methylene C-atom, C13 and C21, in the HMBC spectra). Subsequently, HSQC and HMBC NMR spectra were used to assign the ${ }^{13} \mathrm{C}$ NMR resonances. The resonances of the methylene $\mathrm{H}$-atoms were allocated by interpretation of the cross peaks in the HMBC NMR spectra.

The comparison of the interpreted ${ }^{1} \mathrm{H}$ NMR spectra of the free ligand, the monozinc(II) and the dizinc(II) species, depicted in Figure 2, revealed that in the case of the monozinc(II) complex, particularly the resonances of the pyridine $\mathrm{H}$-atoms in binding site $\mathrm{B}$, underwent shifts compared to the analogous resonances in the spectrum of the free ligand, while the resonances corresponding to the $\mathrm{H}$-atoms of binding site $\mathrm{C}$ were almost unaffected. However, addition of a second equivalent of the zinc(II) salt resulted in a shift of the amidated pyridine $\mathrm{H}$-atom resonances, while the resonances corresponding to $\mathrm{H}$-atoms in binding site $\mathrm{B}$ remained almost at the same positions as in the spectrum recorded of the monozinc(II) complex. Moreover, the resonance of $H 6$, which was present in form of a singlet in the spectrum of the free ligand, split to a doublet of doublets after addition of one equivalent of zinc(II) perchlorate. All other methylene resonances remained as singlets. The addition of a second equivalent of zinc(II) perchlorate resulted in a splitting of the resonance of $H 27$. It is suggested that the splitting of the resonances arises from the constrained flexibility of the methylene groups by coordination, 
leading to two $\mathrm{H}$-atoms bound to the same $\mathrm{C}$-atom but in different electronic environments. Furthermore, the characteristic ${ }^{13} \mathrm{C}$ resonance of the amide carbonyl C-atom experienced only a slight shift from $177.9 \mathrm{ppm}$ to $178.8 \mathrm{ppm}$ by the formation of a monozinc(II) complex, but after coordination of the second $\mathrm{Zn}$ " ion this resonance is found at $184.2 \mathrm{ppm}$.

These findings are interpreted as the $\mathrm{Zn}^{\prime \prime}$ ions being bound regioselectively by the amide-free coordination site up to the addition of one equivalent of zinc(II) perchlorate, leading to the sole formation of a monozinc(II) complex. Only when a higher than equimolar amount of $\mathrm{Zn}$ " ions is present in solution the dizinc(II) complex of ligand $\mathrm{H}_{3} \mathrm{SIM}^{1}$ was generated. It is worth mentioning that the same results were obtained when the experiment was conducted in deuterated methanol.

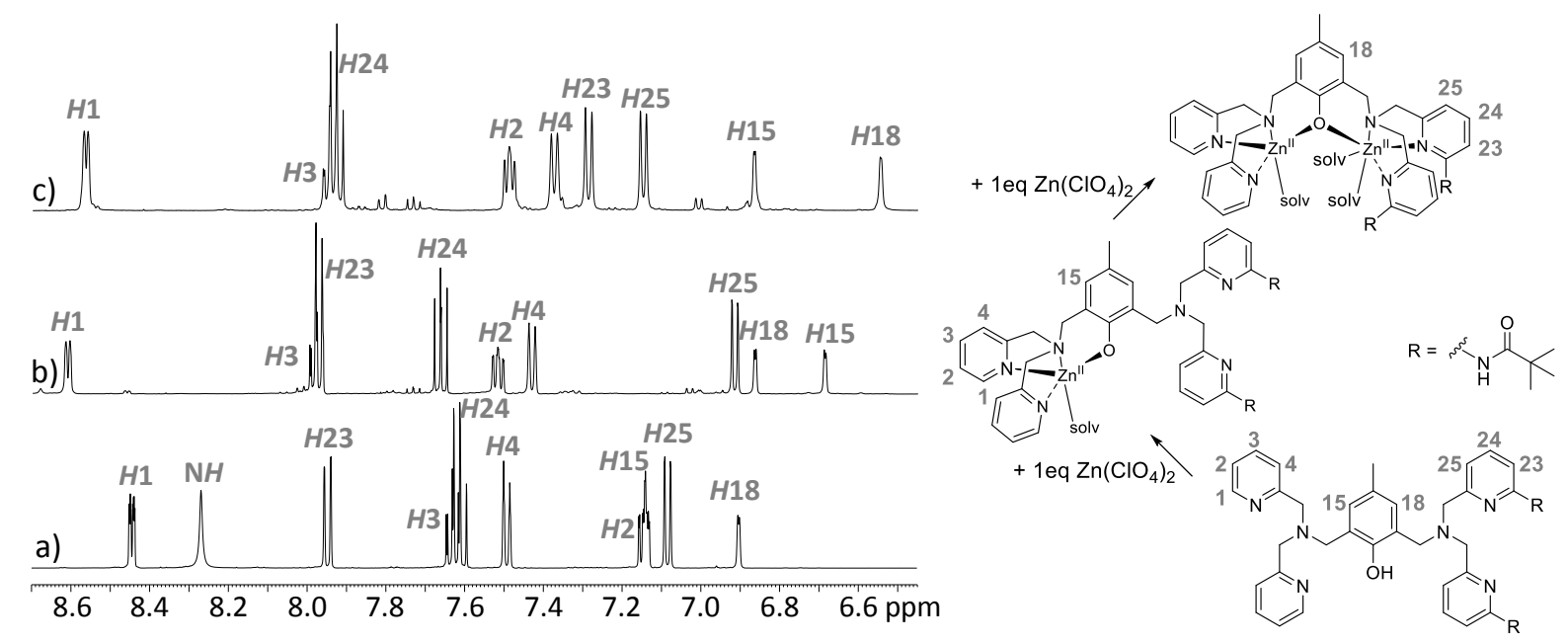

Figure 2: Comparison of ${ }^{1} \mathrm{H}$ NMR spectra of a) $\mathrm{H}_{3} \mathrm{SIM}^{1}$ in $\mathrm{CD}_{3} \mathrm{CN}$, b) with 1 eq, and c) 2 eq $\mathrm{Zn}^{\prime \prime}\left(\mathrm{ClO}_{4}\right)_{2}$.

The distinct coordination of zinc(II) ions to the two unequal binding sites in ligand $\mathrm{H}_{3} \mathrm{SIM}^{1}$ is also observed in the X-ray structure of $\left[\mathrm{Zn}_{2}{ }_{2}\left(\mathrm{H}_{2} \mathrm{SIM}^{1}\right)(\mu-\mathrm{OAc})(\mathrm{OH})\right] \mathrm{PF}_{6}$, which will be discussed in Chapter 4.3 in more detail. In that structure the $\mathrm{Zn}^{\text {"I }}$ ion bound to coordination site $\mathrm{C}$ is six coordinated, while the $\mathrm{Zn}^{\prime \prime}$ ion in binding site $\mathrm{B}$ forms a trigonal bipyramidal coordination geometry.

\section{Mass Spectrometry}

During the NMR titration experiments samples were taken and $\mathrm{ESI}^{+}$mass spectra recorded. The mass spectrum of the equimolar solution of $\mathrm{H}_{3} \mathrm{SIM}^{1}$ and zinc(II) perchlorate showed, in addition to the peak assigned to free ligand, peaks with the isotopic pattern characteristic for a monozinc(II) species (Figure 3a). The main species at $\mathrm{m} / \mathrm{z} 791.3$ could be assigned to $\left[\mathrm{Zn}^{\prime \prime}\left(\mathrm{H}_{2} \mathrm{SIM}^{1}\right)\right]^{+}$. In the mass spectra conducted with the sample taken after addition of two equivalents of zinc(II) perchlorate to $\mathrm{H}_{3} \mathrm{SIM}^{1}$ the peaks show the characteristic isotopic pattern for dizinc(II) species (Figure 3b). In this case the main peak at $\mathrm{m} / \mathrm{z} 951.3$ was assigned to the 
species $\left[\mathrm{Zn}_{2}{ }_{2}\left(\mathrm{D}_{2} \mathrm{SIM}^{1}\right)\left(\mathrm{CH}_{3} \mathrm{OH}\right)_{2}\left(\mathrm{CH}_{3} \mathrm{O}\right)\right]^{+}$. Therefore, the results obtained from mass spectrometry support the assumption of a stepwise coordination of $\mathrm{Zn}$ " ions by the asymmetric ligand $\mathrm{H}_{3} \mathrm{SIM}^{1}$.

a)

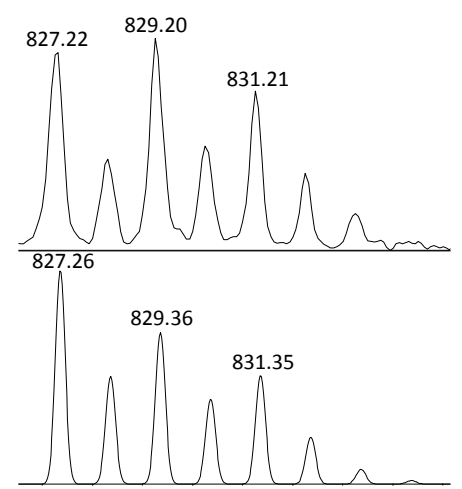

d)

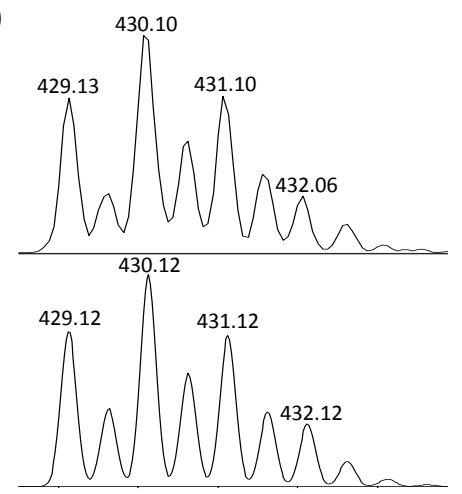

b)

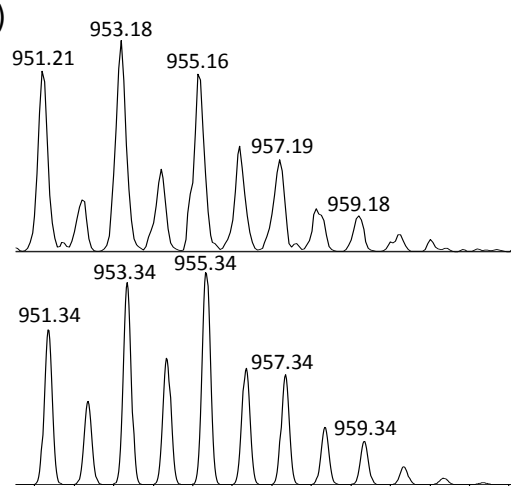

c)

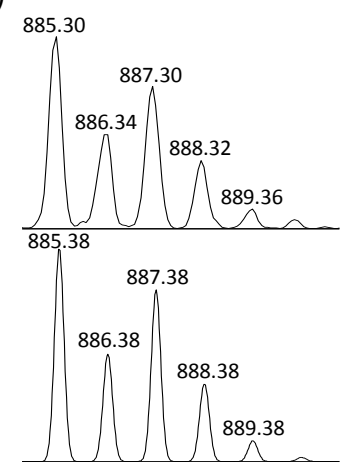

e) 790.34

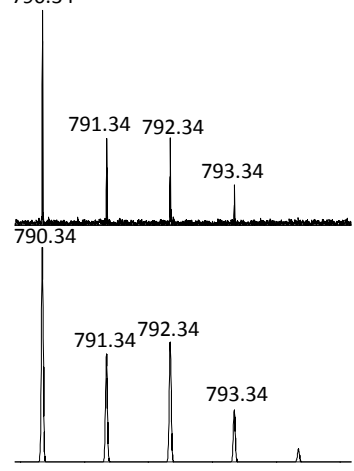

f)

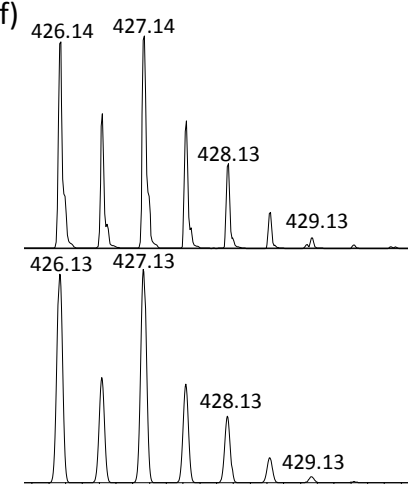

Figure 3: Characteristic isotopic pattern found in $\mathrm{ESI}^{+}$mass spectrometric results (experimental (top) and calculated (bottom)) of $\mathrm{H}_{3} \mathrm{SIM}^{1}$ with a) 1 eq of $\mathrm{Zn}^{\prime \prime}\left(\mathrm{ClO}_{4}\right)_{2}\left(\left[\mathrm{Zn}^{\prime \prime}\left(\mathrm{H}_{2} \mathrm{SIM}^{1}\right)\left(\mathrm{H}_{2} \mathrm{O}\right)_{2}\right]^{+}\right)$, b) 2 eq of $\mathrm{Zn}^{\prime \prime}\left(\mathrm{ClO}_{4}\right)_{2}$ $\left(\left[\mathrm{Zn}_{2}{ }_{2}\left(\mathrm{H}_{2} \mathrm{SIM}^{1}\right)\left(\mathrm{CH}_{3} \mathrm{O}\right)_{2}\left(\mathrm{CD}_{3} \mathrm{O}\right)\right]^{+}\right)$, c) 2 eq of Ga"'( $\left(\mathrm{NO}_{3}\right)_{3}\left(\left[\mathrm{Ga}^{\prime \prime \prime}\left(\mathrm{H}_{2} \mathrm{SIM}^{1}\right)\left(\mathrm{H}_{2} \mathrm{O}\right)_{4}(\mathrm{OH})\right]^{+}\right)$, d) 1 eq of $\mathrm{Zn}^{\prime \prime}\left(\mathrm{ClO}_{4}\right)_{2}$ and 1 eq of Ga"'( $\left(\mathrm{NO}_{3}\right)_{3}\left(\left[\mathrm{Ga}^{\prime \prime \prime} \mathrm{Zn} \text { "' }\left(\mathrm{SIM}^{1}\right)\right]^{2+}\right)$ e) 1 eq of Cu' $(\mathrm{OTf})_{2}\left(\left[\mathrm{Cu}^{\prime \prime}\left(\mathrm{H}_{2} \mathrm{SIM}^{1}\right)\right]^{+}\right)$and f) 2 eq of $\mathrm{Cu}^{\prime \prime}\left(\mathrm{OTf}_{2}\left(\left[\mathrm{Cu}_{2}{ }_{2}\left(\mathrm{HSIM}^{1}\right)\right]^{+}\right)\right.$in methanol.

\subsubsection{COORDINATION STUDIES WITH GA ${ }^{\prime \prime \prime}$}

Analogous to the $\mathrm{Zn}$ " coordination behavior of ligand $\mathrm{H}_{3} \mathrm{SIM}^{1}$, the coordination chemistry of $\mathrm{H}_{3} \mathrm{SIM}^{1}$ with $\mathrm{Ga}$ III ions was investigated employing the NMR titration method, using gallium(III) nitrate or gallium(III) perchlorate as metal source. In these experiments the reaction mixtures were heated for 45 minutes at $50^{\circ} \mathrm{C}$ after every second addition, to ensure complete complexation.

\section{NMR Studies}

Figure 4 shows the coordination process monitored by ${ }^{1} \mathrm{H}$ NMR spectroscopy while gallium(III) nitrate dissolved in $\mathrm{D}_{2} \mathrm{O}(0.518 \mathrm{M})$ was added stepwise to $\mathrm{H}_{3} \mathrm{SIM}^{1}$ in $\mathrm{CD}_{3} \mathrm{CN}$ (33.6 mM). Initially, the increase of the Gall ion concentration resulted in the formation of a new species. In the equimolar mixture of $\mathrm{H}_{3} \mathrm{SIM}^{1}$ with gallium(III) nitrate only one species was present and its ${ }^{1} \mathrm{H} N M R$ 
spectrum remained unchanged during addition of further gallium(III) salt. These results indicate that $\mathrm{H}_{3} \mathrm{SIM}^{1}$ forms selectively a monogallium(III) complex.
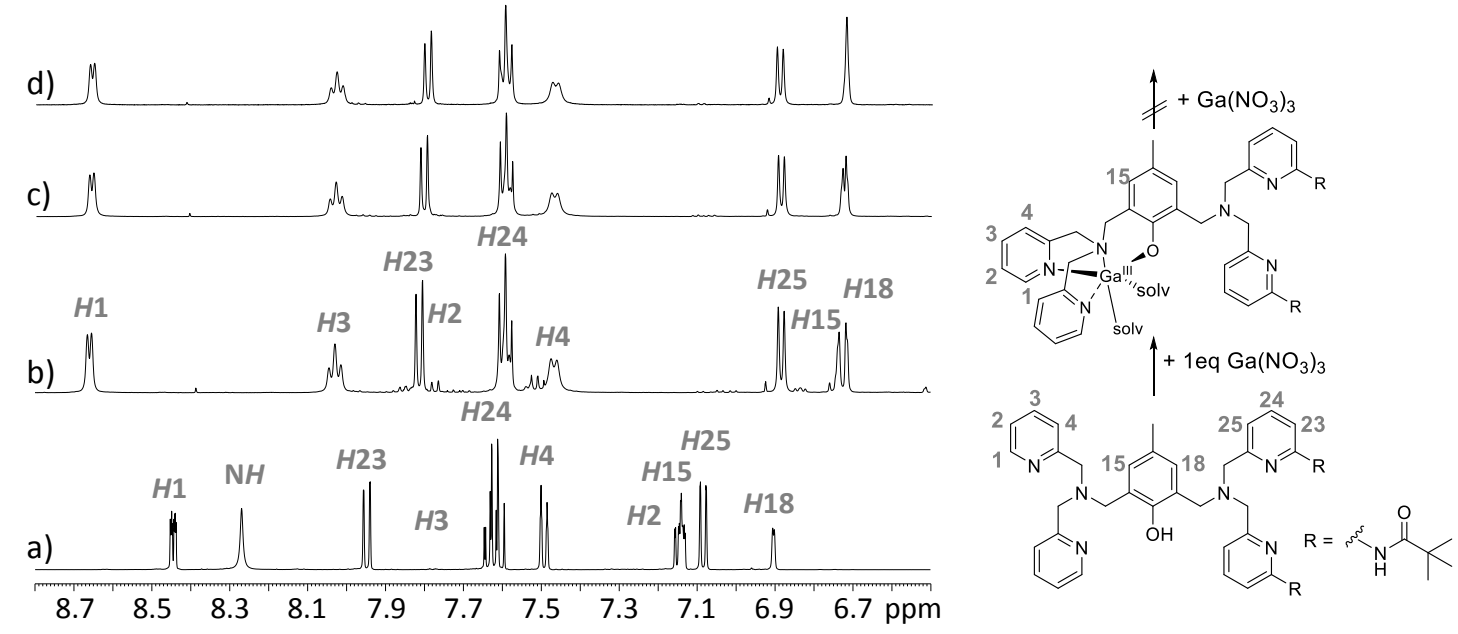

Figure 4: Comparison of ${ }^{1} \mathrm{H}$ NMR spectra of a) $\mathrm{H}_{3} \mathrm{SIM}^{1}$ in $\mathrm{CD}_{3} \mathrm{CN}$, with b) 1.00 eq, c) 1.50 eq and d) 2.00 eq Ga"'"( $\left(\mathrm{NO}_{3}\right)_{3}$ in $\mathrm{D}_{2} \mathrm{O}$.

The assignment of the resonances clarified the exact position of the Gall ion in the monogallium(III) complex. While the resonances associated to binding site $C, H 18, H 23, H 25$, were shifted upfield by about $0.2 \mathrm{ppm}$, or were not effected as in the case of $H 24$, when one equivalent of gallium(III) nitrate is present in solution, the impact on the resonances of binding site $\mathrm{B}$ is more pronounced. The resonances of $H 1, H 2$ and $H 3$ exhibit a downfield shift of up to $0.4 \mathrm{ppm}$, and $H 15$ revealed an upfield shift by $0.4 \mathrm{ppm}$. Furthermore, the carbonyl ${ }^{13} \mathrm{C}$ NMR resonance was found at $179.4 \mathrm{ppm}$, which is similar to the resonance observed in the spectrum of the monozinc(II) complex in which the $\mathrm{Zn}^{\prime \prime}$ ion is bound in the amide-free binding site. In the case of the methylene resonances, only the resonance of $\mathrm{H} 6$ split into two doublets upon complexation with Gall', suggesting the positon of the $\mathrm{Ga}^{\mathrm{III}}$ ion in binding site $\mathrm{B}$, as depicted in Figure 4.

\section{Mass Spectrometry}

The mass spectrometric measurement of a sample taken from the NMR titration experiment after addition of two equivalents of gallium(III) nitrate resulted in a spectrum with the main peak found at $\mathrm{m} / \mathrm{z}$ 885.3. This peak shows the characteristic pattern for a species containing a single Gall' ion (Figure 3c) and was assigned to the monogallium(III) complex [Ga'I'( $\left.\left.\mathrm{H}_{2} \mathrm{SIM}^{1}\right)(\mathrm{OH})\left(\mathrm{OH}_{2}\right)_{4}\right]^{+}$. 


\section{X-ray Diffraction}

During heating a solution of $\mathrm{H}_{3} \mathrm{SIM}^{1}(40.3 \mathrm{mM})$ in deuterated methanol with one equivalent of gallium(III) perchlorate at $50^{\circ} \mathrm{C}$, a white solid precipitated after about 15 minutes. Recrystallization of this solid from acetonitrile with diffusion of diethyl ether yielded white crystals suitable for X-ray data collection. Figure 5 shows a plot of the molecular structure, and Table 2 gives selected structural parameters (Table 2 also includes structural parameters of [Ga"' $\left.\mathrm{Zn}^{\prime \prime}\left(\mathrm{H}_{2} \mathrm{SIM}^{1}\right)(\mathrm{OAc})_{2}(\mu-\mathrm{OH})\right]^{+}$which will be introduced at a later point). The structure consists of a [Ga'l' $\left.\left(\mathrm{H}_{2} \mathrm{SIM}^{1}\right)(\mu-\mathrm{OH})\right]^{+}$dimer, in which the two Gall' ions are coordinated in an octahedral geometry and bridged by two hydroxido ions (Figure 5a). The remaining coordination sites of the Ga'l' ion in the monomer subunit (Figure $5 b$ ) are occupied by two pyridines, the tertiary amine and the phenolate donor groups provided by the ligand. Moreover, hydrogen bonding between the pivaloyl amide moieties of the metal-free binding site $C$ and perchlorate oxygen atoms (3.154 $\AA$ and $3.120 \AA$ ) is visualized in the plot of Figure 5b.

Table 2: Selected bond lengths ( $(\AA)$ and angles $\left({ }^{\circ}\right)$ for $\left[\mathrm{Ga}^{\prime \prime \prime}\left(\mathrm{H}_{2} \mathrm{SIM}^{1}\right)(\mu-\mathrm{OH})\right]^{+}$and $\left[\mathrm{Ga}^{\prime \prime \prime} \mathrm{Zn}^{\prime \prime}\left(\mathrm{H}_{2} \mathrm{SIM}^{1}\right)(\mathrm{OAC})_{2}(\mu-\mathrm{OH})\right]^{+}$.

\begin{tabular}{l|l|l} 
& {$\left[\mathrm{Ga}^{\prime \prime \prime}\left(\mathrm{H}_{2} \mathrm{SIM}^{1}\right)(\mu-\mathrm{OH})\right]^{+}$} & {$\left[\mathrm{Ga}^{\prime \prime \prime} \mathrm{Zn}^{\prime \prime}\left(\mathrm{H}_{2} \mathrm{SIM}^{1}\right)(\mathrm{OAc})_{2}(\mu-\mathrm{OH})\right]^{+}$} \\
\hline $\mathrm{Ga}(1) \ldots \mathrm{Zn}(1)$ & - & $6.1122(9)$ \\
$\mathrm{Ga}(1) \ldots \mathrm{Ga}(2)$ & 3.083 & $3.0183(12)$ \\
$\mathrm{Ga}(1)-\mathrm{O}(1)$ & $1.911(3)$ & $1.871(4)$ \\
$\mathrm{Ga}(1)-\mathrm{O}(6)$ & $1.894(4)$ & $1.976(4)$ \\
$\mathrm{Ga}(1)-\mathrm{O}(6)^{\prime}$ & $2.016(3)$ & $1.880(3)$ \\
$\mathrm{Ga}(1)-\mathrm{N}(1)$ & $2.063(4)$ & $2.063(5)$ \\
$\mathrm{Ga}(1)-\mathrm{N}(2)$ & $2.128(4)$ & $2.097(4)$ \\
$\mathrm{Ga}(1)-\mathrm{N}(3)$ & $2.066(4)$ & $2.066(5)$ \\
$\mathrm{Zn}(1)-\mathrm{N}(4)$ & - & $2.143(5)$ \\
$\mathrm{Zn}(1)-\mathrm{N}(5)$ & - & $2.085(5)$ \\
$\mathrm{Zn}(1)-\mathrm{N}(6)$ & - & $2.199(5)$ \\
$\mathrm{Zn}(1)-\mathrm{O}(2 \mathrm{~A})$ & - & $1.969(4)$ \\
$\mathrm{Zn}(1)-\mathrm{O}(2 \mathrm{~B})$ & - & $2.002(4)$ \\
$\mathrm{Zn}(1)-\mathrm{O}(3 \mathrm{~B})$ & - & $2.386(5)$ \\
$\mathrm{N}(2)-\mathrm{Ga}(1)-\mathrm{N}(1)$ & $77.92(16)$ & $77.5(2)$ \\
$\mathrm{N}(2)-\mathrm{Ga}(1)-\mathrm{N}(3)$ & $80.65(16)$ & $79.82(19)$ \\
$\mathrm{N}(2)-\mathrm{Ga}(1)-\mathrm{O}(1)$ & $94.13(15)$ & $94.15(17)$ \\
$\mathrm{N}(2)-\mathrm{Ga}(1)-\mathrm{O}(6)$ & $169.14(15)$ & $93.98(17)$ \\
$\mathrm{N}(2)-\mathrm{Ga}(1)-\mathrm{O}(6)$ & $93.33(15)$ & $170.86(17)$ \\
$\mathrm{N}(5)-\mathrm{Zn}(1)-\mathrm{N}(4)$ & - & $80.6(2)$ \\
$\mathrm{N}(5)-\mathrm{Zn}(1)-\mathrm{N}(6)$ & - & $77.71(19)$ \\
$\mathrm{N}(5)-\mathrm{Zn}(1)-\mathrm{O}(2 \mathrm{~A})$ & - & $114.59(19)$ \\
$\mathrm{N}(5)-\mathrm{Zn}(1)-\mathrm{O}(2 \mathrm{~B})$ & - & $148.9(2)$ \\
$\mathrm{N}(5)-\mathrm{Zn}(1)-\mathrm{O}(3 \mathrm{~B})$ & - & $90.77(18)$ \\
\hline
\end{tabular}


a)

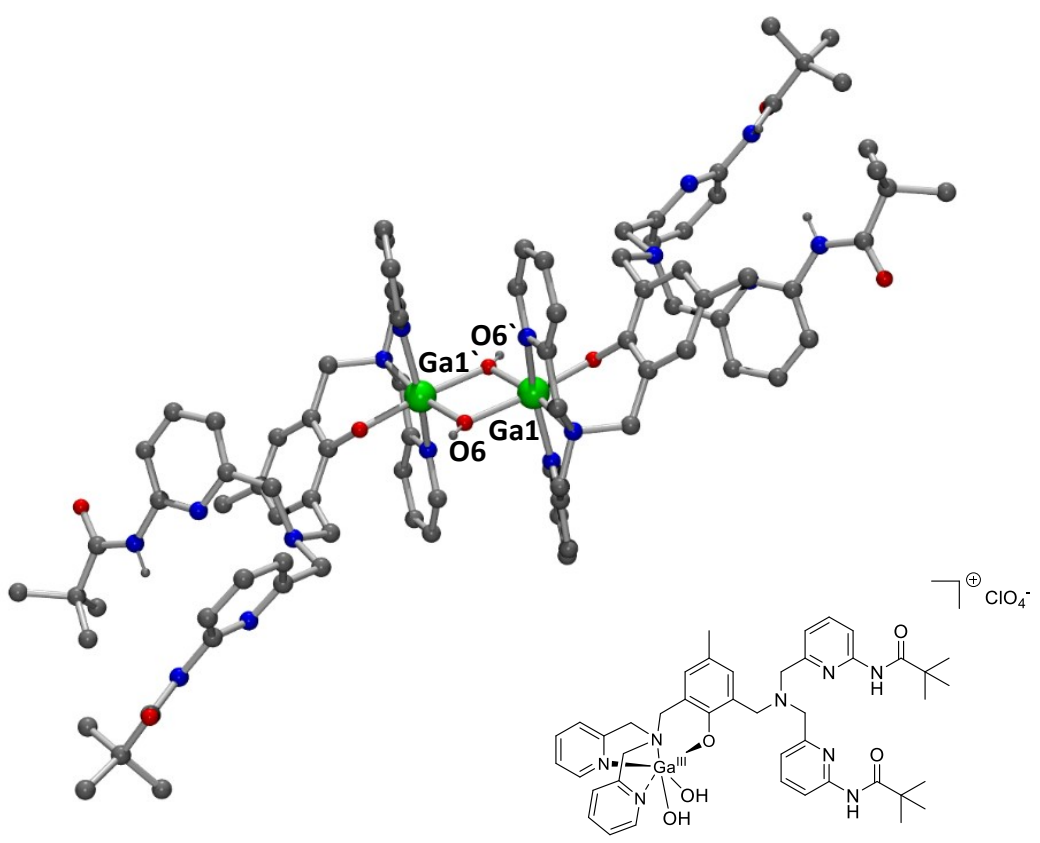

b)

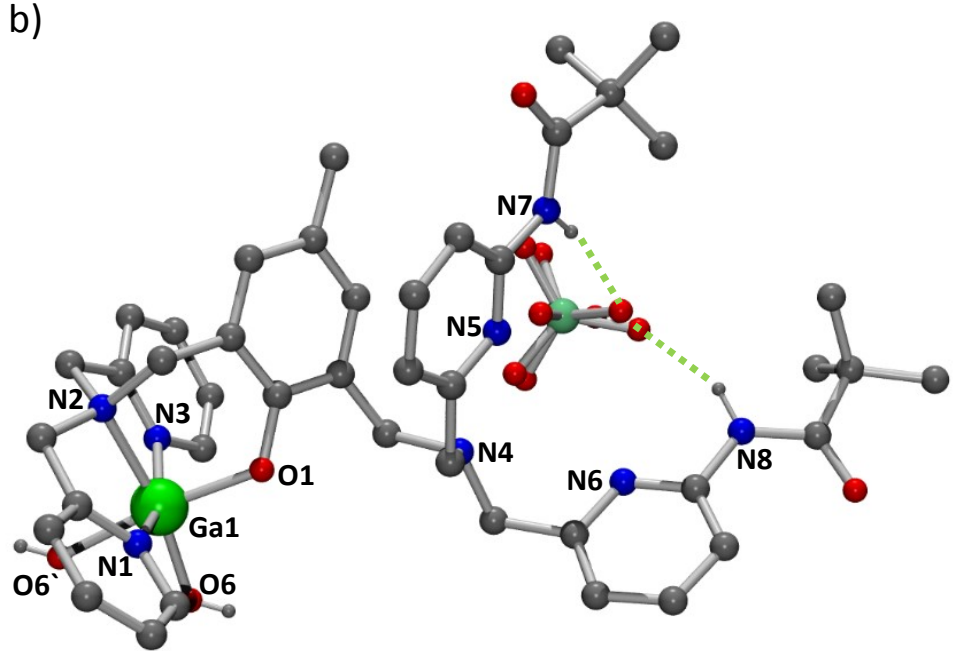

Figure 5: Structures of [Ga"'( $\left.\left(\mathrm{H}_{2} \mathrm{SIM}^{1}\right)(\mu-\mathrm{OH})\right]_{2}\left(\mathrm{ClO}_{4}\right)_{2}$ : a) full dimer and b) monomeric subunit, also showing hydrogen bonding (green dotted lines; counter ions, non-coordinated solvent molecules, and hydrogen atoms, not involved in hydrogen bonding, have been omitted for clarity; crystallographic data and ORTEP plots with $50 \%$ probability level of thermal ellipsoids appear in the Appendix, Table 30 and Figure 70 ).

In contrast, the ligand $\mathrm{HL}^{9}$ with four unsubstituded pyridine arms crystallized as digallium(III) complex of the form [ $\left.\mathrm{Ga}^{\prime \prime \prime}{ }_{2}\left(\mathrm{~L}^{9}\right)(\mathrm{OH})_{2}\left(\mathrm{OH}_{2}\right)_{2}\right]\left(\mathrm{ClO}_{4}\right)_{3}$ when treated with gallium(III) nitrate and tetraethylammonium perchlorate. Whilst the phenolate moiety of the ligand $\mathrm{HL}^{9}$ bridges the two Gall' ions of this dinuclear complex $\left[\mathrm{Ga}^{\prime \prime \prime}{ }_{2}\left(\mathrm{~L}^{9}\right)(\mathrm{OH})_{2}\left(\mathrm{OH}_{2}\right)_{2}\right]^{3+}$, the phenolate of $\mathrm{H}_{3} \mathrm{SIM}^{1}$ is coordinated terminally to the Gall' center in $\left[\mathrm{Ga}^{\prime \prime \prime}\left(\mathrm{H}_{2} \mathrm{SIM}^{1}\right)(\mu-\mathrm{OH})\right]^{+}$. In agreement with this observation is the elongated average Ga"l'- $\mathrm{O}_{\mathrm{Ph}}$ distance in $\left[\mathrm{Ga}^{\prime \prime \prime}{ }_{2}\left(\mathrm{~L}^{9}\right)(\mathrm{OH})_{2}\left(\mathrm{OH}_{2}\right)_{2}\right]^{3+}(2.043 \AA)$, compared to the corresponding value found in $\left[\mathrm{Ga}^{\prime \prime \prime}\left(\mathrm{H}_{2} \mathrm{SIM}^{1}\right)(\mu-\mathrm{OH})\right]^{+}(1.911 \AA)$, while the Ga'l'-Namine (2.122 ̊̊ vs. $2.128 \AA$ ) and Ga'II-N $\mathrm{N}_{\mathrm{Py}}(2.078 \AA$ vs. $2.065 \AA$ ) distances are similar in both complexes. 
The X-ray crystal structural analysis confirms the assignment of the NMR spectra (see above), i.e. that the Gall' ion is bound to binding site $A$ of ligand $\mathrm{H}_{3} \mathrm{SIM}^{1}$ and indicates that the sterically demanding pivaloyl-amide residues hinder the coordination of Ga"l' ions in binding site B of ligand $\mathrm{H}_{3} \mathrm{SIM}^{1}$.

\subsubsection{Formation OF A HETEROdINUCLEAR GA ${ }^{\text {IIIN" }}$ "I COMPLEX}

The results obtained from the investigation of the coordination behavior of $\mathrm{H}_{3} \mathrm{SIM}^{1}$ with $\mathrm{Zn}$ " and Gall' ions showed that $\mathrm{Zn}$ " is coordinated stepwise, firstly in the less hindered binding site B and subsequently, only after complete formation of the monozinc(II) complex, the $\mathrm{Zn}$ " coordination takes place in binding site $\mathrm{C}$. The presence of $\mathrm{Ga}^{\mathrm{III}}$ ions in solution also resulted in the formation of the monogallium(III) complex containing the Gall' ion in binding site $\mathrm{B}$, but the subsequent generation of a digallium(III) complex, could not be achieved. The possibility of the synthesis of a heterodinuclear complex, containing Gall' selectively in binding site $\mathrm{B}$ and $\mathrm{Zn}$ " exclusively in binding site $\mathrm{C}$, was tested using NMR spectroscopic and mass spectrometric techniques.

\section{NMR Studies}

To study the coordination chemistry of $\mathrm{H}_{3} \mathrm{SIM}^{1}$ towards a mixture of Gall and $\mathrm{Zn}$ " different strategies were followed, including (i) addition of zinc(II) perchlorate to the monogallium(III) complex of $\mathrm{H}_{3} \mathrm{SIM}^{1}$, (ii) addition of gallium(III) nitrate to the monozinc(II) complex of $\mathrm{H}_{3} \mathrm{SIM}^{1}$, and (iii) addition of gallium(III) nitrate to the dizinc(II) complex of $\mathrm{H}_{3} \mathrm{SIM}^{1}$. The latter experiment was

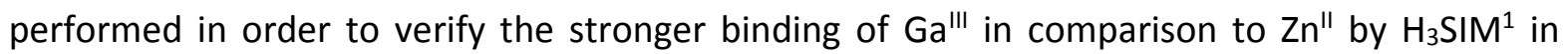
binding site B. All three experiments resulted in a similar spectrum; the resonances assigned to protons in binding site B were broadened and two sets of signals assigned to protons in binding site $C$ were present (Figure 6), suggesting a mixture of two different species, which differ specifically in the coordination mode of the metal bound in binding site $\mathrm{C}$. It was shown that the ratio of the two species is dependent on temperature, time, and donor properties of the solvent used. Moreover, after the NMR experiment samples were left to react at room temperature for seven days the presence of one main species could be detected. 


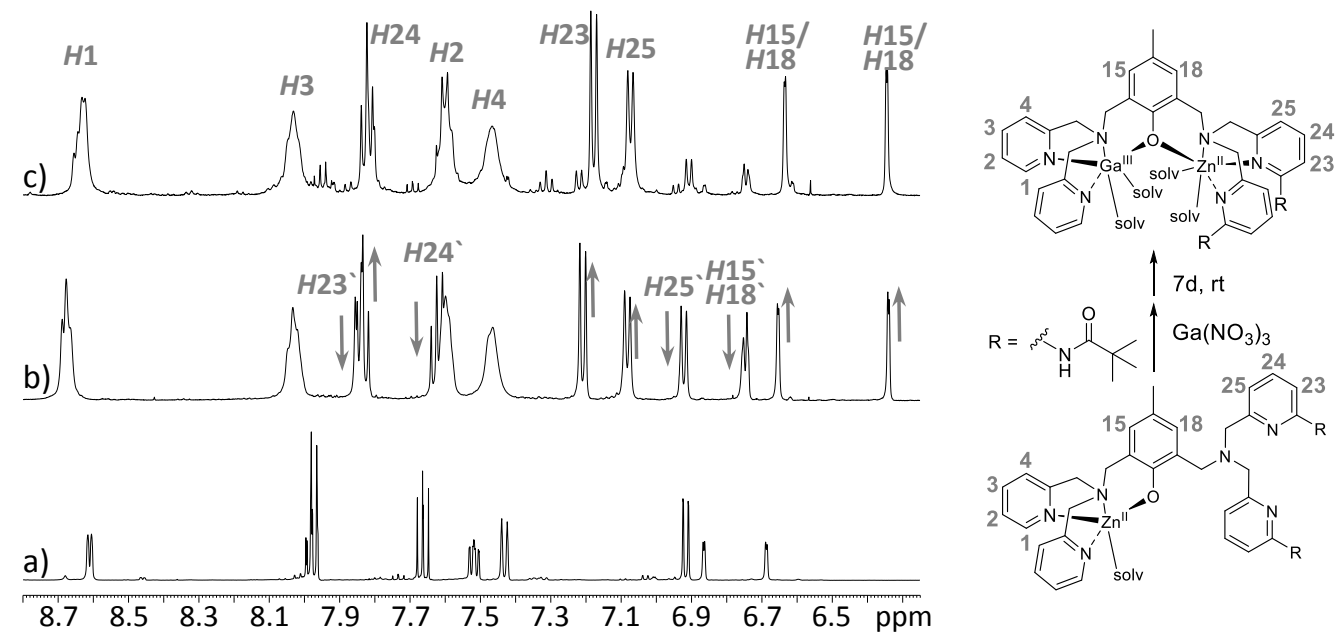

Figure 6: Comparison of ${ }^{1} \mathrm{H}$ NMR spectra of a) $\left[\mathrm{Zn}^{\prime \prime}\left(\mathrm{H}_{2} \mathrm{SIM}^{1}\right)(\text { solv })_{x}\right]$ in $\mathrm{CD}_{3} \mathrm{CN} / \mathrm{D}_{2} \mathrm{O}$ with b) 1.0 eq Ga"'( $\left(\mathrm{NO}_{3}\right)_{3}$ in $\mathrm{D}_{2} \mathrm{O}$ heated for 15 minutes at $50^{\circ} \mathrm{C}$, and $\mathrm{c}$ ) the mixture after 7 days at room temperature.

The chemical shifts of the resonances of both species, detected in the samples treated with Gall' and $\mathrm{Zn}$ ", vary when compared with the resonances found in the monozinc(II) complex, dizinc(II) complex, and monogallium(III) complex of $\mathrm{H}_{3} \mathrm{SIM}^{1}$, discussed above, and therefore exclude the possibility of a mixture of complexes with different metal composition. The amide groups adjacent the metal center in binding site $\mathrm{C}$ are likely to employ multiple coordination modes leading to a mixture of two different species, in which the environment of protons in binding site $C$ varied and therefore the chemical shifts are unequal. The impact of the coordination modes of the amide groups on the protons in binding site $B$ is limited and therefore only a broadening was detected.

The monozinc(II) complex of $\mathrm{H}_{3} \mathrm{SIM}^{1}$ contains the $\mathrm{Zn}^{11}$ ion in the less hindered coordination site $\mathrm{B}$ and Gall ions were not coordinated by the amide containing binding site $\mathrm{C}$, as explained above. Thus, a metal exchange is proposed to proceed during the formation of the heterodinuclear Ga"'Zn" complex. This suggestion is supported by the finding that the addition of gallium(III) nitrate to the dizinc(II) complex of $\mathrm{H}_{3} \mathrm{SIM}^{1}$, as well as the addition of zinc(II) perchlorate to the monogallium(III) complex of $\mathrm{H}_{3} \mathrm{SIM}^{1}$, resulted in the same ${ }^{1} \mathrm{H}$ NMR spectrum as did the addition of gallium(III) nitrate to the monozinc(II) complex.

\section{Mass Spectrometry}

The sole formation of the heterodinuclear complex was also supported by $\mathrm{ESI}^{+}$mass spectrometry, because the main signals exhibit the pattern characteristic for GaZn species and the main peak at $\mathrm{m} / \mathrm{z} 430.1$ could be assigned to the $\left[\mathrm{Ga}^{\prime \prime \prime} \mathrm{Zn} \mathrm{n}^{\prime \prime}\left(\mathrm{SIM}^{1}\right)\right]^{2+}$ complex (Figure 3d). 


\section{X-ray Diffraction}

The heterodinuclear complex was prepared by heating an equimolar mixture of ligand $\mathrm{H}_{3} \mathrm{SIM}^{1}$ and zinc(II) perchlorate in methanol $(40 \mathrm{mM})$ for 15 minutes and subsequent addition of gallium(III) nitrate, sodium acetate and sodium hexafluorophosphate. Diffusion of diethylether in the methanolic complex solution $(22 \mathrm{mM}$ ) gave colorless crystals suitable for data collection. Figure 7 shows the structure obtained; Table 2 shows selected distances and valence angles. The structure was solved and includes a $\left[\mathrm{Ga}^{\prime \prime \prime} \mathrm{Zn}^{\prime \prime}\left(\mathrm{H}_{2} \mathrm{SIM}^{1}\right)(\mathrm{OAc})_{2}(\mu-\mathrm{OH})\right]^{+}$complex cation, a hexafluorophosphate counterion and a diethylether solvent molecule in the elemental cell. Crystallographic data obtained for the structure are displayed in Table 30 (see Appendix). In contrast to complexes derived with similar ligands, included the dizinc(II) complex of $\mathrm{H}_{3} \mathrm{SIM}^{1}$ (discussed in detail in Chapter 4.3), the two metal ions are not bridged by the phenolate backbone. Moreover, the model system forms dimers, bridged by two hydroxide ions between the Gall' ions (Figure 7b) comparable to the monogallium(III) complex of $\mathrm{H}_{3} \mathrm{SIM}^{1}$, $\left[\mathrm{Ga}^{\prime \prime \prime}\left(\mathrm{H}_{2} \mathrm{SIM}^{1}\right)(\mu-\mathrm{OH})\right]^{+}$(Figure 5). The two Gall' ions and the two hydroxido bridges form an asymmetric arrangement with the Gall'-OH distances differing with the values of 1.880 (3) $\AA$ and 1.976(4) A. This arrangement is comparably found in $\left[\mathrm{Ga}^{\prime \prime \prime}\left(\mathrm{H}_{2} \mathrm{SIM}^{1}\right)(\mu-\mathrm{OH})\right]^{+}$, but with a higher difference between the Gal'I-OH distances (1.894(4) $\AA$ and 2.016(3) $\AA$ ); the Ga...Ga separation is slightly elongated in [Ga"' $\left.\left(\mathrm{H}_{2} \mathrm{SIM}^{1}\right)(\mu-\mathrm{OH})\right]^{+}(3.083 \AA)$ compared to [Ga"' $\left.\mathrm{Zn}^{\prime \prime}\left(\mathrm{H}_{2} \mathrm{SIM}^{1}\right)(\mathrm{OAc})_{2}(\mu-\mathrm{OH})\right]^{+}$ (3.0183(12) A). In addition to the two bridging hydroxido co-ligands, the Gall' ions are coordinated by a phenolate, two pyridines, and a tertiary amine group provided by the ligand backbone. While the Gal'I- $\mathrm{N}_{\mathrm{Py}}$ distances between the Gall' center and the coordinated pyridine residues in $\left[\mathrm{Ga}^{\prime \prime \prime} \mathrm{Zn} \mathrm{n}^{\prime \prime}\left(\mathrm{H}_{2} \mathrm{SIM}^{1}\right)(\mathrm{OAc})_{2}(\mu-\mathrm{OH})\right]^{+}$are equal to the ones found in $\left[\mathrm{Ga}^{\prime \prime \prime}\left(\mathrm{H}_{2} \mathrm{SIM}^{1}\right)(\mu-\mathrm{OH})\right]^{+}$, the Ga'"I-Namine and Ga'I-OPh distances between the Gall' ion and the tertiary amine and the phenolate moiety, respectively, are slightly shortened by $0.03 \AA$ and $0.04 \AA$.

In addition to the Gall! ion coordinated in binding site $\mathrm{B}$ a $\mathrm{Zn}$ " ion is coordinated in the more hindered binding site $\mathrm{C}$ of ligand $\mathrm{H}_{3} \mathrm{SIM}^{1}$. The donors around the $\mathrm{Zn}$ " ions form a distorted octahedral coordination geometry with the two coordinating pyridines in the axial positions. The square plane of the $\mathrm{Zn}$ "ions is occupied by the tertiary amine of the ligand backbone and two acetate co-ligands, one in a chelating, the other in a terminal binding mode. The $\mathrm{Zn}$ "-O distances to the chelating acetate co-ligand differ by $0.38 \AA$ (2.002(4) $\AA$ and $2.386(5) \AA ̊$ ) and in accordance with that finding the coordination mode of the acetate is asymmetric. Comparison of the structure of $\left[\mathrm{Ga}^{\prime \prime \prime} \mathrm{Zn}^{\prime \prime}\left(\mathrm{H}_{2} \mathrm{SIM}^{1}\right)(\mathrm{OAc})_{2}(\mu-\mathrm{OH})\right]^{+}$with the structure of $\left[\mathrm{Zn}_{2}{ }_{2}\left(\mathrm{H}_{2} \mathrm{SIM}^{1}\right)(\mathrm{OAc})(\mu-\mathrm{OH})\right]^{+}$, which will be discussed in detail in Chapter 4.3, showed that the coordination arrangements differ as in the latter the pyridine residues are part of the trigonal plane while the tertiary amine occupies the axial position. The $\mathrm{Zn}(1)-\mathrm{N}_{\mathrm{Py}}$ distances in $\left[\mathrm{Ga}^{\prime \prime \prime} \mathrm{Zn}^{\prime \prime}\left(\mathrm{H}_{2} \mathrm{SIM}^{1}\right)(\mathrm{OAc})_{2}(\mu-\mathrm{OH})\right]^{+}$are 
elongated (2.143(5) $\AA$ and 2.199(5) $\AA$ vs. 2.057(3) $\AA$ and 2.102(3) $\AA$ ) and the $\mathrm{Zn}(1)-\mathrm{N}_{\text {amine }}$ distance is shortened $(2.085(5) \AA$ vs. $2.233(2) \AA$ ) compared to the respective distances found in the trigonal bipyramidal coordinated $\mathrm{Zn}^{\prime \prime}$ center of the dizinc(II) complex (discussed in detail in Chapter 4.3). In the octahedral coordination sphere around the Ga"l ion in $\left[\mathrm{Ga}^{\prime \prime \prime} \mathrm{Zn} \mathrm{n}^{\prime \prime}\left(\mathrm{H}_{2} \mathrm{SIM}^{1}\right)(\mathrm{OAc})_{2}(\mu-\mathrm{OH})\right]^{+}$the Gal'-N distances are all shorter than found in $\left[\mathrm{Zn}_{2}{ }_{2}\left(\mathrm{H}_{2} \mathrm{SIM}^{1}\right)(\mathrm{OAc})(\mu-\mathrm{OH})\right]^{+}$, as expected due to the higher charge density of Gall compared to $\mathrm{Zn}$.

Based on the short distances between the amide nitrogen atoms and the nearby acetate oxygen atoms, a hydrogen bond network is proposed in [Ga"'"Zn" $\left.\left(\mathrm{H}_{2} \mathrm{SIM}^{1}\right)(\mathrm{OAc})_{2}(\mu-\mathrm{OH})\right]^{+}$. $\mathrm{N}(7)$ forms a hydrogen bond to the chelating acetate co-ligand ( $N(7)-O(2 B) 2.871 \AA$ ) and $N(8)$, respectively, to the terminal acetate co-ligand $(\mathrm{N}(8)-\mathrm{O}(2 \mathrm{~A}) 3.004 \AA$ ). Moreover, the terminal acetate co-ligand itself stabilizes the hydroxido-bridges between the Gall ions $(\mathrm{O}(3 \mathrm{~A})-\mathrm{O}(6) 2.808 \AA$ ) by hydrogen bonds. The previously observed effect that hydrogen bonding enlarges the angle between the pyridine planes and the plane containing the amide group (NCO) was also found in the structure of $\left[\mathrm{Ga}^{\prime \prime \prime} \mathrm{Zn} \text { " }\left(\mathrm{H}_{2} \mathrm{SIM}^{1}\right)(\mathrm{OAC})_{2}(\mu-\mathrm{OH})\right]^{+}$. $^{168}$ This angle was found to be $18.85^{\circ}$ in case of the $\mathrm{N}(7)$ amide residue, while a value of $11.05^{\circ}$ was measured in the case of the $N(8)$ amide moiety, supposing a preserved $\mathrm{C}-\mathrm{H} \cdots \mathrm{O}=\mathrm{C}$ interaction between the carbonyl oxygen and the nearby hydrogen atom in 5 -position of the pyridine ring in the latter case.

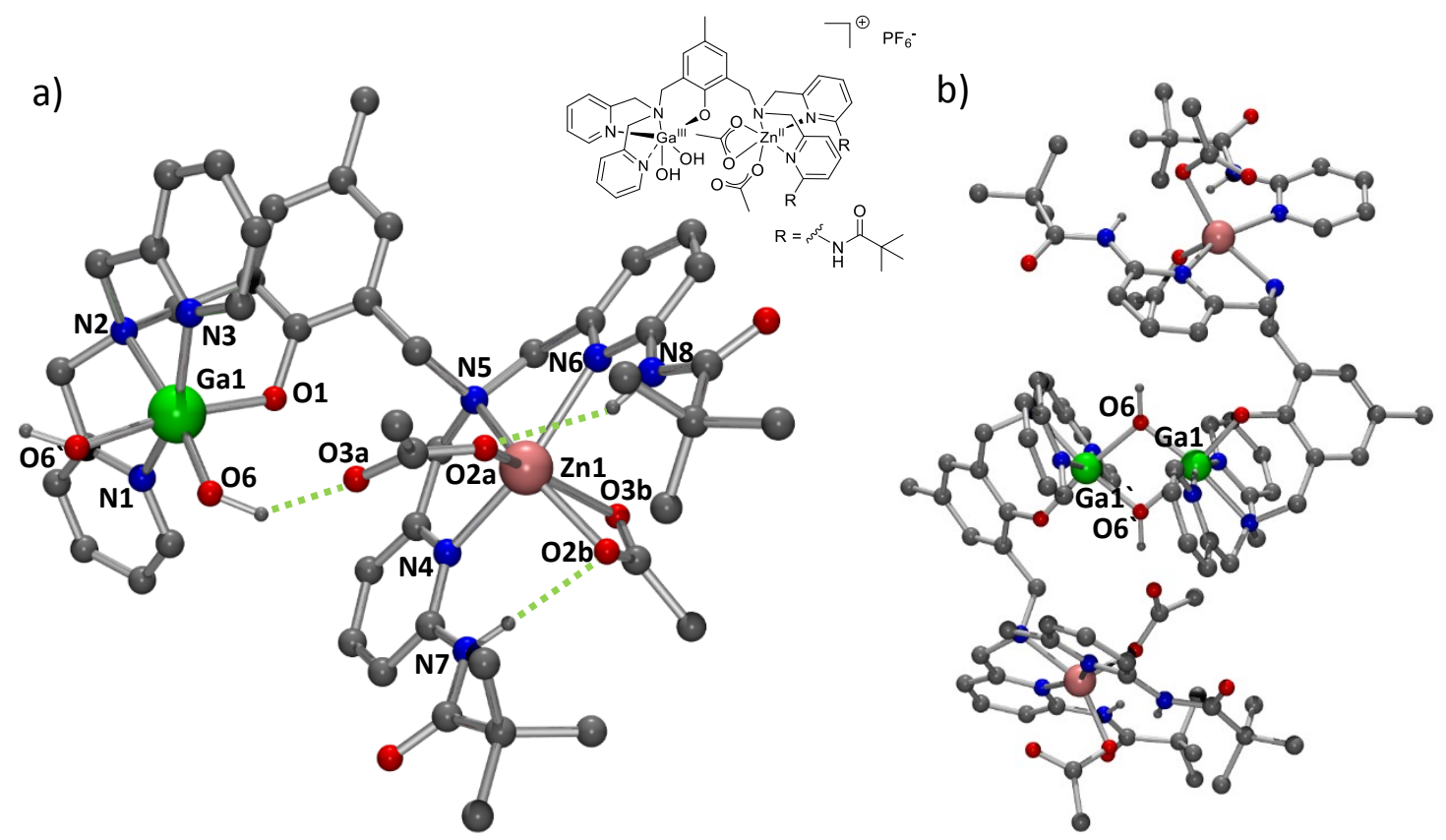

Figure 7: Structures of a) $\left[\mathrm{Ga}^{\prime \prime \prime} \mathrm{Zn} \mathrm{n}^{\prime \prime}\left(\mathrm{H}_{2} \mathrm{SIM}^{1}\right)(\mathrm{OAc})_{2}(\mu-\mathrm{OH})\right]^{+}$and b) the respective dimer [Ga"' $\left.\mathrm{Zn}^{\prime \prime}\left(\mathrm{H}_{2} \mathrm{SIM}^{1}\right)(\mathrm{OAC})_{2}(\mu-\mathrm{OH})\right]_{2}{ }^{2+}$ showing hydrogen bonding (green dotted lines; counter ions, noncoordinated solvent molecules, and hydrogen atoms, not involved in hydrogen bonding, have been omitted for clarity; crystallographic data and ORTEP plots with 50\% probability level of thermal ellipsoids appear in the Appendix, Table 30 and Figure 71). 
The X-ray crystallographic data highlight the selectivity of the two different binding sites in ligand $\mathrm{H}_{3} \mathrm{SIM}^{1}$ towards Gall' and $\mathrm{Zn}^{\prime \prime}$ ions. This enables $\mathrm{H}_{3} \mathrm{SIM}^{1}$ to form a more accurate PAP model complex that takes main structural features of the native enzymes into account, including the presence of two different metal ions with different oxidation states, and which are accompanied by hydrogen bond donors in close distance.

\subsubsection{COMPLEXATION STUDIES WITH CUII}

Due to the diamagnetism of $\mathrm{Zn}^{\prime \prime}$ and Gall' ions their coordination chemistry can be optimally monitored by NMR spectroscopy. In contrast, $\mathrm{Cu}^{\prime \prime}$ ions have an incomplete $\mathrm{d}$ shell configuration $(\operatorname{Ar}) 3 \mathrm{~d}^{9}$ and are paramagnetic. The $\mathrm{d}^{9}$ system, on the other hand, plays a crucial role in several other characterization methods, such as electronic spectroscopy and electron paramagnetic resonance (EPR). Consequently, the coordination behavior of $\mathrm{H}_{3} \mathrm{SIM}^{1}$ with $\mathrm{Cu}$ "l ions was studied using these methods.

\section{UV-vis-NIR Spectroscopy}

The stepwise addition of copper(II) triflate to $\mathrm{H}_{3} \mathrm{SIM}^{1}$ in methanol was followed by UV-vis-NIR spectroscopy (Figure 8). Besides the intense absorptions below $300 \mathrm{~nm}\left(\pi-\pi^{*}\right.$ transitions of the ligand backbone), the spectra show broad transitions at $459 \mathrm{~nm}$ and $654 \mathrm{~nm}$ with intensity ratios of around 3.3:1. Table 3 lists the observed transitions and the corresponding $\varepsilon$ values.

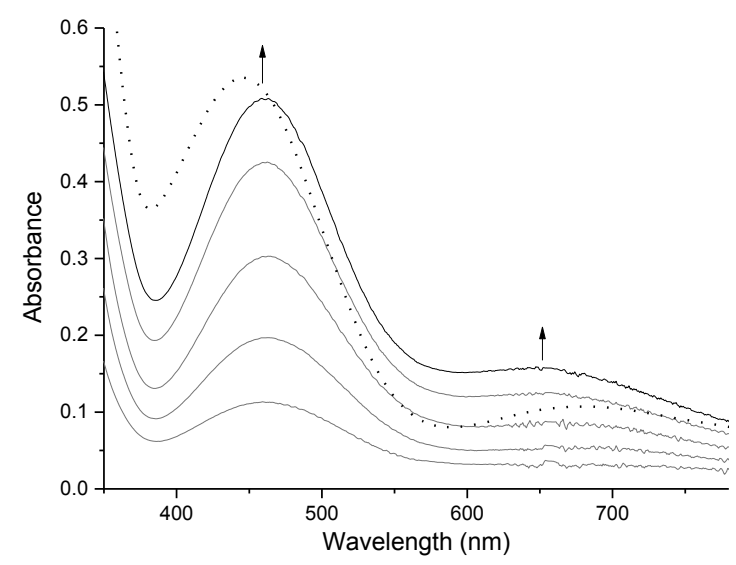

Figure 8: UV-vis-NIR spectra of a methanolic solution of $\mathrm{H}_{3} \mathrm{SIM}^{1}$ treated with $0.6 \mathrm{eq}, 1.0 \mathrm{eq}, 1.4 \mathrm{eq}, 1.8 \mathrm{eq}$ and 2.0 eq (bottom to top) of copper(II) triflate for 12 hours at rt (solid lines) and with 2.0 eq of copper(II) acetate (dotted line).

Treatment of a methanolic solution of $\mathrm{H}_{3} \mathrm{SIM}^{1}$ with two equivalents of copper(II) acetate resulted in a similar spectrum with broad transitions at $447 \mathrm{~nm}$ and $681 \mathrm{~nm}$ with an intensity ratio of 5.0:1. The spectral data are similar to that reported for the complex $\left[\mathrm{Cu}_{2}{ }_{2}\left(\mathrm{~L}^{1}\right)(\mathrm{OAc})_{2}\right]^{+}$, bearing a similar ligand backbone as $\mathrm{H}_{3} \mathrm{SIM}^{1}$, but lacking the two pivaloyl-amide residues. ${ }^{169}$ 
Table 3: UV-vis-NIR spectroscopic properties of the $\mathrm{Cu}^{\text {Il }}$ complexes of $\mathrm{H}_{3} \mathrm{SIM}^{1}$.

\begin{tabular}{cccc}
$\begin{array}{c}\text { mixture } \\
\text { molar ratio }\end{array}$ & $\begin{array}{c}\text { wavelength } \\
{[\mathrm{nm}]}\end{array}$ & $\begin{array}{c}\varepsilon \\
{\left[\mathrm{mol}^{-1} \mathrm{~cm}^{-1}\right]}\end{array}$ & transition \\
\hline $\mathrm{H}_{3} \mathrm{SIM}^{1}: \mathrm{Cu}(\mathrm{OTf})_{2}$ & 459 & 393 & $\mathrm{~d}-\mathrm{d}$ \\
$1: 1$ & 654 & 114 & LMCT \\
\hline $\mathrm{H}_{3} \mathrm{SIM}^{1}: \mathrm{Cu}(\mathrm{OTf})_{2}$ & 459 & 1016 & $\mathrm{~d}-\mathrm{d}$ \\
$1: 2$ & 654 & 314 & LMCT \\
\hline $\mathrm{H}_{3} \mathrm{SIM}^{1}: \mathrm{Cu}(\mathrm{OAc})_{2}$ & 447 & 1068 & $\mathrm{~d}-\mathrm{d}$ \\
$1: 2$ & 681 & 215 & LMCT \\
\hline
\end{tabular}

Furthermore, the presence of the $d-d$ bands in the region between $400 \mathrm{~nm}$ and $500 \mathrm{~nm}$ is typical for axial $\mathrm{Cu}^{\text {Il }}$ sites. The fact that the $\mathrm{d}$ - $\mathrm{d}$ bands of the two $\mathrm{Cu}^{\prime \prime}$ centers are not resolved suggests a rather similar coordination environment, as expected because of the identical primary coordination spheres of the $\mathrm{Cu}^{\prime \prime}$ centers. The continuous increase in intensity of both bands throughout the addition of copper(II) triflate to the methanolic ligand solution is in agreement with both a single mononuclear site and mono- and dinuclear sites of similar coordination geometry. However, the relatively featureless spectra do not allow unambiguous support for a detailed interpretation.

\section{EPR Spectroscopy}

EPR measurements allow the difference between the magnetic energy levels to be determined. Magnetic energy levels are degenerate in absence of a magnetic field, but when a magnetic field is applied the energy levels split up, known as the Zeeman phenomenon (Figure 9). Magnetic energy levels are described by the magnetic quantum number $m_{s}$ and two $m_{s}$ states with an energy difference of $h v=g \beta_{e} B$ are generated by a magnetic field $B$ in the case of a $S=1 / 2$ system (h: Boltzmann constant, v: frequency, g: magnetic tensor, $\beta_{\mathrm{e}}$ : Bohr magneton). The symmetry of the magnetic tensor $\mathrm{g}$, which reflects the symmetry of the coordination environment around the $\mathrm{Cu}^{\prime \prime}$ ions, encompass the detection of one, two or three signals in the spectrum and therefore is used to investigate the coordination geometry of copper(II) complexes.
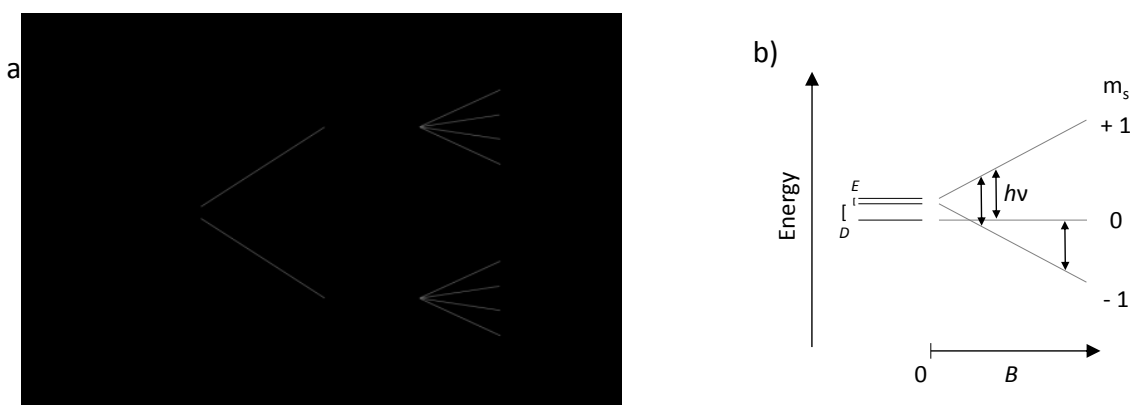

Figure 9: The Zeeman effect in the EPR experiment for $a$ ) $\operatorname{spin} S=1 / 2$ and $b$ ) $\operatorname{spin} S=1$. 
In order to investigate the coordination chemistry of $\mathrm{H}_{3} \mathrm{SIM}^{1}$ with $\mathrm{Cu}$ II ions, $\mathrm{X}$-band EPR spectra of frozen methanolic solutions of $\mathrm{H}_{3} \mathrm{SIM}^{1}$ with copper(II) triflate were recorded. Figure 10 illustrates the spectra obtained and compares them to the spectrum of copper(II) triflate in methanol and Table 4 summarizes the parameters derived from simulating the experimental EPR spectra. Here, $\chi, \sigma$ and $\tau$ present the Euler angles which describe the orientation of the coordination polyhedron of the $\mathrm{Cu}^{\prime \prime}$ centers and are illustrated in Figure 13.

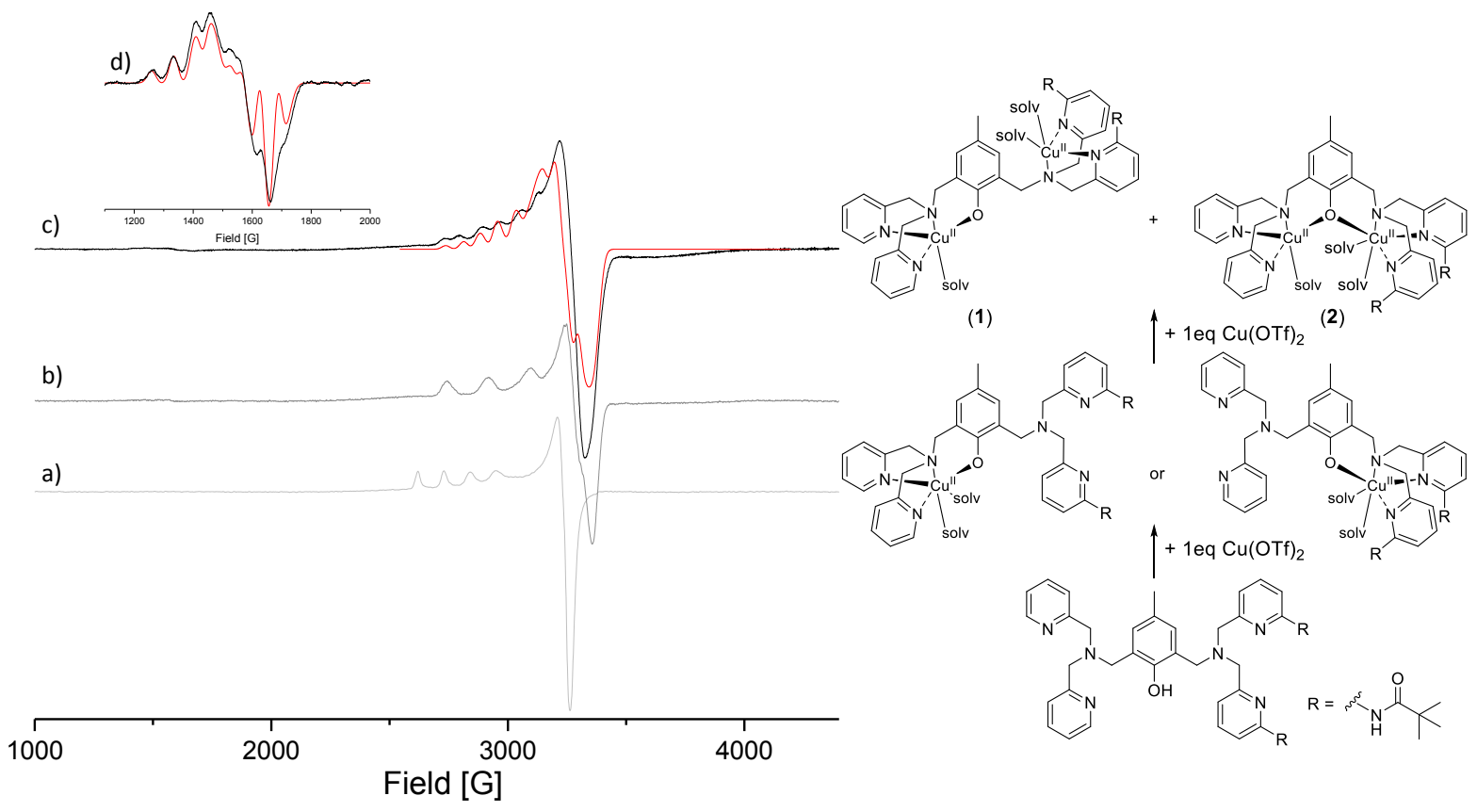

Figure 10: Experimental EPR spectra (black, v $=9.4 \mathrm{GHz}, \mathrm{T}=140 \mathrm{~K}$ ) and computer simulated spectra (red) of a) $\mathrm{Cu}^{\prime \prime}(\mathrm{OTf})_{2}$ in methanol, b) $\mathrm{H}_{3} \mathrm{SIM}^{1}$ and Cu'(OTf) $)_{2}(1: 0.8)$, c) $\mathrm{H}_{3} \mathrm{SIM}^{1}$ and $\mathrm{Cu}^{\prime \prime}(\mathrm{OTf})_{2}(1: 2.0)$, and d) $\Delta \mathrm{m}_{\mathrm{s}}= \pm 2$ transition.

The first and second derivative spectra (Figure 10b and 11) of a mixture of $\mathrm{H}_{3} \mathrm{SIM}^{1}$ and copper(II) trifluoromethanesulfonate in the ratio of 1:0.8 and 1:0.95 in methanol show a single species. Numerical differentiation and Fourier filtering (Hamming function) were undertaken to increase the spectral resolution. Importantly, the cutoff of the Hamming function was adjusted so that the high frequency noise was mininimized without distorting the spectrum. The second derivative spectrum (Figure 11) reveals ${ }^{14} \mathrm{~N}$ superhyperfine splitting on the parallel $\mathrm{Cu}$ "l hyperfine resonances. Computer simulation of the second derivative spectrum with a rhombic spin Hamiltonian yields the spectrum shown in Figure 11, which is in excellent agreement with that of the experimental spectrum. The anisotropic spectrum of the mononuclear complex is typical for a single axially distorted Cull site with a $\mathrm{d}_{\mathrm{x2}-\mathrm{y2}}$ ground state, leading to spin allowed $\Delta \mathrm{m}_{\mathrm{s}}= \pm 1$ transitions above $g=2$. Importantly, the simulation assumed two equivalent nitrogen nuclei, indicating that the equatorial plane contains two nitrogen nuclei. It appears that $\mathrm{Cu}^{\prime \prime}$ selectively occupies a single site and, by analogy to the observations with $\mathrm{Zn}^{\prime \prime}$ (and Ga"I'), this is probably site A, though site B cannot be ruled out. 


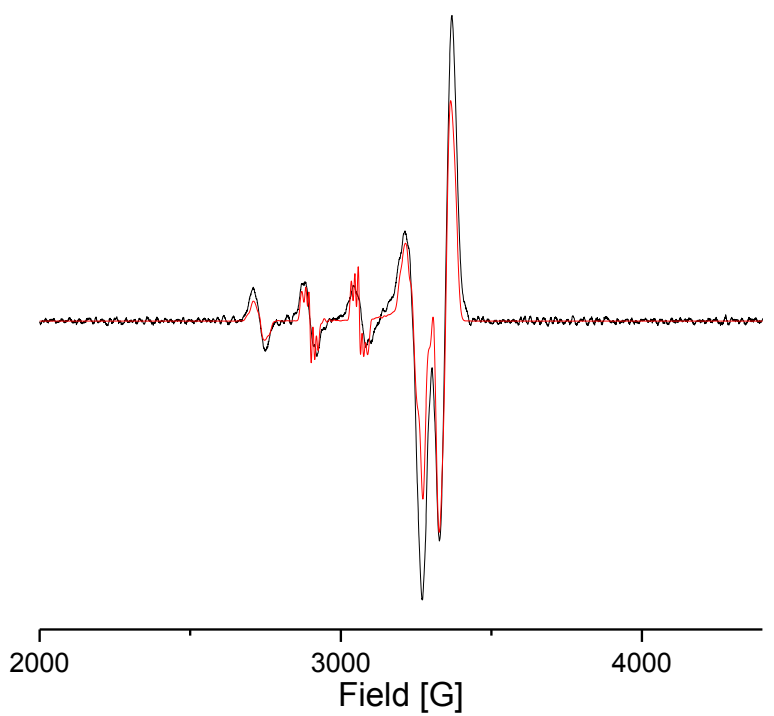

Figure 11: Second derivative of experimental EPR spectrum (black, $v=9.4 \mathrm{GHz}, \mathrm{T}=140 \mathrm{~K}$ ) and computer simulated spectrum (red) of $\mathrm{H}_{3} \mathrm{SIM}^{1}$ and Cu'(OTf) $)_{2}(1: 0.95)$ in methanol. $\left({ }^{63} \mathrm{Cu}\right.$ hyperfine interaction $\mathrm{A}_{x, y, z}\left({ }^{14} \mathrm{~N}\right)=$ $12.0 \cdot 10^{-4}, 12.0 \cdot 10^{-4}, 12.0 \cdot 10^{-4} \mathrm{~cm}^{-1}$ (two magnetically equivalent nitrogen nuclei). Linewidth (g-and A-strain linewidth model), $\sigma \mathrm{R}_{x, y, z}=10.0 \cdot 10^{-4}, 10.0 \cdot 10^{-4}, 5.0 \cdot 10^{-4} \mathrm{~cm}^{-1} ;(\sigma \mathrm{g} / \mathrm{g})_{x, y, z}=1 \cdot 10^{-4}, 1 \cdot 10^{-4},-1 \cdot 10^{-5} ; \sigma \mathrm{A}_{x, y, z}=0.1 \cdot 10^{-4}$, $0.1 \cdot 10^{-4}, 4.7 \cdot 10^{-4} \mathrm{~cm}^{-1}$. The ${ }^{63} \mathrm{Cu}$ and ${ }^{14} \mathrm{~N}$ perpendicular hyperfine resonances are not resolved in the spectra and thus the linewidth in the simulated spectrum results from a combination of $A_{x, y}\left({ }^{63} \mathrm{Cu},{ }^{14} \mathrm{~N}\right)$ and corresponding linewidth parameters).

With increasing $\mathrm{Cu}^{\prime \prime}$ concentration ( $\mathrm{Cu}^{\prime \prime}: \mathrm{H}_{3} \mathrm{SIM}^{1}$ ratio of $2: 1$ ), a new spectrum develops with features typical for dinuclear Cu" systems with two non-identical Cu" centers (Figure 10c). Besides the resonances centered around $\mathrm{g}=2$, arising from allowed $\Delta \mathrm{m}_{\mathrm{s}}= \pm 1$ transitions, weak resonances are also observed around $g_{\text {eff }}=4.3$, arising from the formally forbidden $\Delta \mathrm{m}_{\mathrm{s}}= \pm 2$ transitions (Figure 10d). Interestingly, the EPR spectrum obtained from a mixture of $\mathrm{H}_{3} \mathrm{SIM}^{1}$ with $\mathrm{Cu}^{\prime \prime}$ acetate $(1: 2)$ shows the same general features but with more intense $\Delta \mathrm{m}_{\mathrm{s}}= \pm 2$ resonances (Figure 12). However, the observed spectrum in the spin-allowed region is quite different, with resonances around $320 \mathrm{mT}$, attributable to solvated $\mathrm{Cu}^{\prime \prime}$ ions (depicted in grey in Figure 12) and broad resonances around $260 \mathrm{mT}$ and $360 \mathrm{mT}$, associated with the $\Delta \mathrm{m}_{\mathrm{s}}= \pm 1$ transitions of a dinuclear complex. The latter spectrum is characteristic for a dinuclear $\mathrm{Cu}^{\prime \prime}$ complex, in which the two $\mathrm{Cu}^{\prime \prime}$ ions are dipole-dipole coupled. The strong exchange interaction in the former spectrum could derive from bridging through the phenolate ligand backbone, in a structure supported by a bridging acetate co-ligand.

The features of the strongly interacting dinuclear $\mathrm{Cu}^{\text {Il }}$ system, the $\Delta \mathrm{m}_{\mathrm{s}}= \pm 2$ transition and the two broad bands at $260 \mathrm{mT}$ and $360 \mathrm{mT}$, are also visible in the spectrum obtained with the 1:2 mixture of $\mathrm{H}_{3} \mathrm{SIM}^{1}$ with copper(II) trifluoromethanosulfonate (Figure $10 \mathrm{c}$ ), suggesting that a small amount of the dicopper(II) complex in solution has a similar structure (species (2) in the structures shown in Figure 10). Computer simulation of the spectra are based on two $\mathrm{Cu}^{\prime \prime}$ centers, coordinated to the ligand with a Cu"...Cu" distance of $3.415 \AA$ (Figure 10d; the relative orientations of the two 
$\mathrm{Cu}^{\prime \prime}$ centers are given in Table 4, which also lists the g- and A-matrices used in the simulations of the various spectra). Importantly, this solution structural analysis is in good agreement with the crystal structural analysis discussed below.

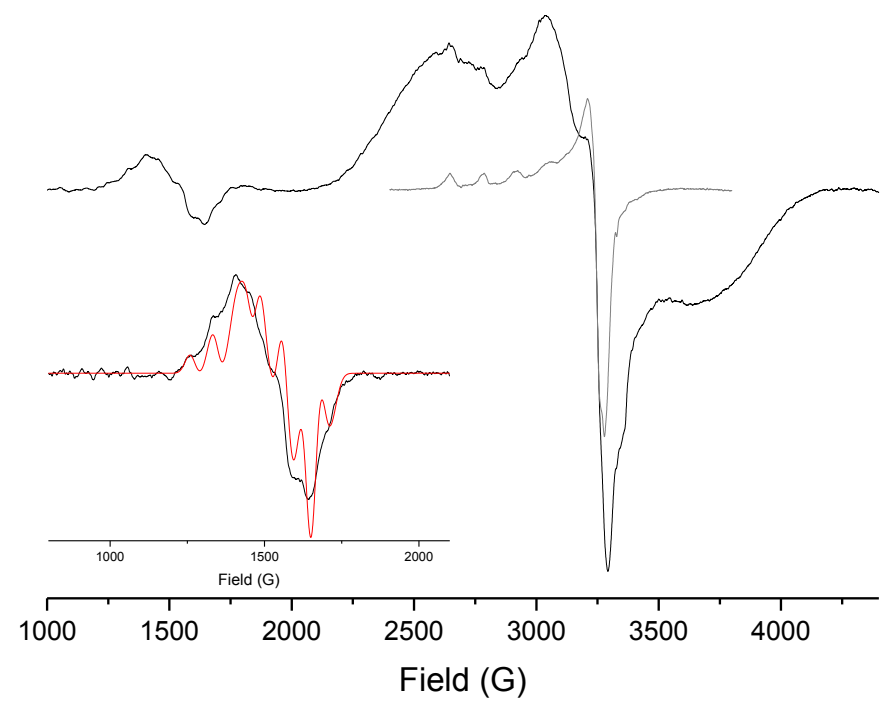

Figure 12: Experimental EPR spectra of a mixture of $\mathrm{H}_{3} \mathrm{SIM}^{1}$ with $\mathrm{Cu}^{\prime \prime}(\mathrm{OAc})_{2}(1: 2)$ (black, v $=9.4 \mathrm{GHz}, \mathrm{T}=140 \mathrm{~K}$ ), computer simulated spectra (red), and experimental EPR spectrum of free Cu"(OAc) 2 (grey) in methanol.

From the EPR simulation of the intense signal in the spectrum in Figure 10d, it emerges that it originates from a dinuclear $\mathrm{Cu}$ "l species with a $\mathrm{Cu}$... Cu distance of $7.2 \AA$ and is proposed to arise from a complex in which the two $\mathrm{Cu}^{\prime \prime}$ centers are not bridged by the phenolate ligand backbone (species (1) in the structures given in Figure 8). Here, the two pyridine donors in each coordination site are on opposite side of the phenolate linker.

Table 4: EPR data for copper(II) complexes of $\mathrm{H}_{3} \mathrm{SIM}^{1}$.

\begin{tabular}{|c|c|c|c|c|c|c|c|}
\hline \multirow[t]{2}{*}{$\begin{array}{l}\frac{\tilde{u}}{\tilde{u}} \\
\frac{\tilde{u}}{\tilde{u}} \\
\frac{\widehat{U}}{0} \\
\frac{\varepsilon}{0}\end{array}$} & \multicolumn{3}{|c|}{ 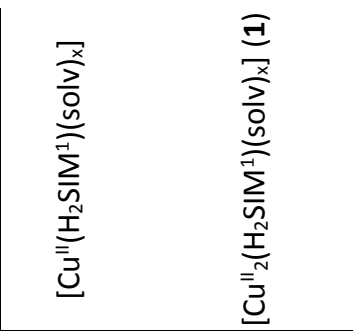 } & \multicolumn{2}{|c|}{ 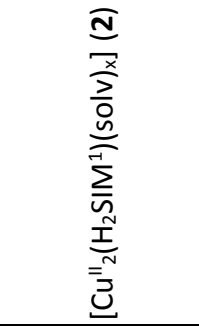 } & \multicolumn{2}{|c|}{ 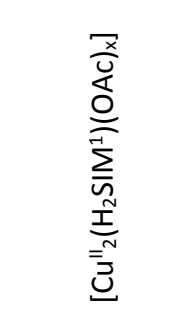 } \\
\hline & & $\mathrm{Cu}(1)$ & $\mathrm{Cu}(2)$ & $\mathrm{Cu}(1)$ & $\mathrm{Cu}(2)$ & $\mathrm{Cu}(1)$ & $\mathrm{Cu}(2)$ \\
\hline$g_{x}$ & 2.044 & 2.054 & 2.055 & 2.054 & 2.054 & 2.054 & 2.060 \\
\hline$g_{y}$ & 2.062 & 2.055 & 2.065 & 2.055 & 2.055 & 2.090 & 2.090 \\
\hline$g_{z}$ & 2.262 & 2.242 & 2.270 & 2.245 & 2.245 & 2.245 & 2.245 \\
\hline$A_{x}\left[10^{-4} \mathrm{~cm}^{-1}\right]$ & $10.6^{a}$ & 15 & 15 & 15 & 15 & 15 & 15 \\
\hline$A_{y}\left[10^{-4} \mathrm{~cm}^{-1}\right]$ & $12.56^{\mathrm{a}}$ & 12 & 12 & 12 & 12 & 12 & 12 \\
\hline$A_{z}\left[10^{-4} \mathrm{~cm}^{-1}\right]$ & $172.02^{\mathrm{a}}$ & 166 & 155 & 166 & 166 & 166 & 166 \\
\hline $\mathrm{R}[\AA ̊]$ & - & & & & 21 & & 6 \\
\hline$\chi\left[{ }^{\circ}\right]$ & - & & & & & & 5 \\
\hline$\rho\left[0^{\circ}\right]$ & - & & & & & & 0 \\
\hline$\tau\left[{ }^{\circ}\right]$ & - & & & & & & \\
\hline
\end{tabular}




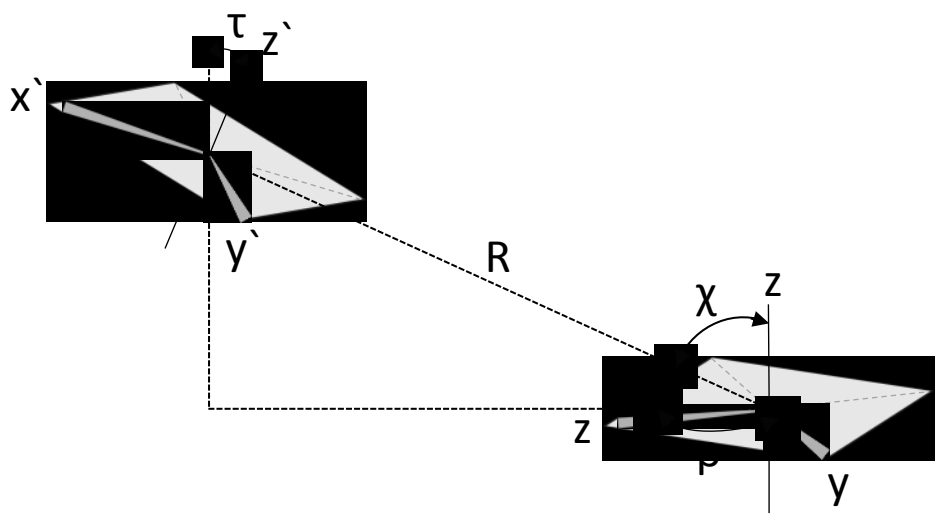

Figure 13: Definition of the Euler angles $\chi, \tau$ and $\rho$ used for EPR simulation using the program Xsophe. ${ }^{170}$

\section{Mass Spectrometry}

The ESI ${ }^{+}$mass spectrometric measurements of a mixture of $\mathrm{H}_{3} \mathrm{SIM}^{1}$ and copper(II) triflate in a 1:2 ratio in methanol resulted in a spectrum with the main peaks at $\mathrm{m} / \mathrm{z} 1003.2$ and $\mathrm{m} / \mathrm{z} 427.1$, which match the mass of the species $\left[\mathrm{Cu}_{2}{ }_{2}\left(\mathrm{HSIM}^{1}\right)\left(\mathrm{F}_{3} \mathrm{CSO}_{3}\right)\right]^{+}$and $\left[\mathrm{Cu}_{2}{ }_{2}\left(\mathrm{HSIM}^{1}\right)\right]^{2+}$ and revealed isotopic pattern typical for species containing two $\mathrm{Cu}$ atoms (Figure 3f). These signals were not detected in the spectrum recorded with the mixture in a ratio of 1:0.8. In contrast, this mixture revealed signals of the metal-free ligand and signals with isotopic pattern characteristic for a mononuclear $\mathrm{Cu}^{\prime \prime}$ complex (Figure 3e), i.e. a signal at $\mathrm{m} / \mathrm{z} 790.3$ which was assigned to $\left[\mathrm{Cu}^{\prime \prime}\left(\mathrm{H}_{2} \mathrm{SIM}^{1}\right)\right]^{+}$. The spectrum recorded with the mixture of $\mathrm{H}_{3} \mathrm{SIM}^{1}$ and two equivalents of copper(II) acetate shows signals with the isotopic pattern typical for dinuclear $\mathrm{Cu}^{\prime \prime}$ complexes. The main signals at $\mathrm{m} / \mathrm{z} \quad 471.2$ and $\mathrm{m} / \mathrm{z} 911.2$ were assigned to $\left[\mathrm{Cu}_{2}{ }_{2}\left(\mathrm{H}_{2} \mathrm{SIM}^{1}\right)(\mathrm{OAc})\left(\mathrm{CH}_{3} \mathrm{OH}\right)\right]^{2+}$ and $\left[\mathrm{Cu}_{2}{ }_{2}\left(\mathrm{HSIM}^{1}\right)(\mathrm{OAC})\right]^{+}$.

\section{X-ray Diffraction}

Treatment of a methanolic solution of $\mathrm{H}_{3} \mathrm{SIM}^{1}(27 \mathrm{mM})$ with Cull acetate (2.0 eq), heating for 15 minutes at $50^{\circ} \mathrm{C}$, followed by addition of sodium hexafluorophosphate (1.5 eq), filtration and diffusion of diethylether, resulted in brown crystals, suitable for X-ray data collection. Figure 14 shows a plot of the molecular structure and Table 5 gives selected structural parameters.

The molecular structure consists of a $\left[\mathrm{Cu}_{2}{ }_{2}\left(\mathrm{HSIM}^{1}\right)(\mu-\mathrm{OAc})\right]^{+}$complex cation and a hexafluorophosphate counter anion. The two $\mathrm{Cu}^{\prime \prime}$ centers have different coordination geometries and are doubly bridged by the phenolate of the ligand backbone and an acetate co-ligand. $\mathrm{Cu}(1)$ is also coordinated to two pyridine moieties and a tertiary amine donor, resulting in a distorted squarepyramidal geometry $(\tau=0.46),{ }^{171}$ while $\mathrm{Cu}(2)$ exhibits a distorted octahedral geometry, emerging from the two bridging ligands, two pyridine residues, a tertiary amine nitrogen, and an oxygen atom of one of the proximal amide groups. The coordination of the pivaloyl-amide moiety is 
stabilized by a hydrogen bond formed with the second pivaloyl-amide group visible by the short $\mathrm{N}(7)-\mathrm{O}(5)$ distance with a value of $3.461 \AA$, illustrated as green dotted lines in Figure 14.

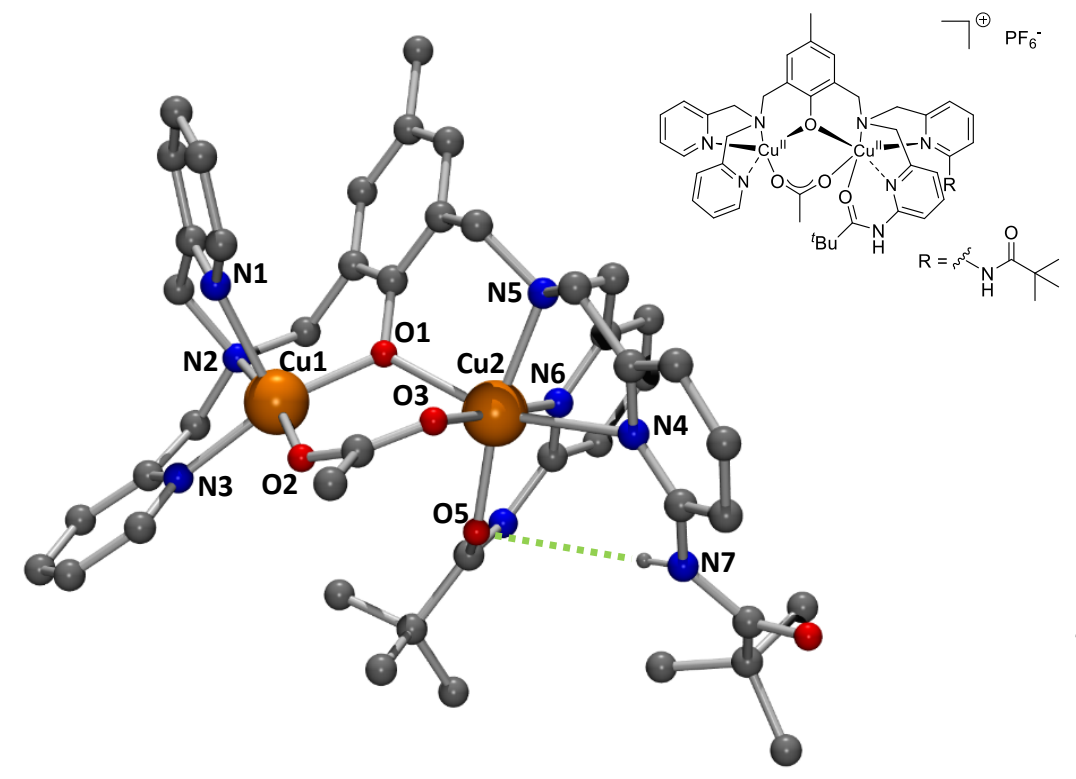

Figure 14: Structure of $\left[\mathrm{Cu}_{2}{ }_{2}\left(\mathrm{HSIM}^{1}\right)(\mu-\mathrm{OAc})\right]^{+}$, also showing intramolecular hydrogen bonding (green dotted lines; counter ions and hydrogen atoms, not involved in hydrogen bonding, have been omitted for clarity; crystallographic data and ORTEP plots with $50 \%$ probability level of thermal ellipsoids appear in the Appendix, Table 30 and Figure 72).

Table 5: Selected bond lengths $(\AA)$ and angles $\left({ }^{\circ}\right)$ for $\left[\mathrm{Cu}_{2}{ }_{2}\left(\mathrm{HSIM}^{1}\right)(\mu-\mathrm{OAC})\right] \mathrm{PF} 6$

\begin{tabular}{l|l} 
& {$\left[\mathrm{Cu}_{2}{ }_{2}\left(\mathrm{HSIM}^{1}\right)(\mu-\mathrm{OAc})\right]^{+}$} \\
\hline $\mathrm{Cu}(1) \ldots \mathrm{Cu}(2)$ & 3.577 \\
$\mathrm{Cu}(1)-\mathrm{O}(1)$ & $1.9061(16)$ \\
$\mathrm{Cu}(1)-\mathrm{O}(2)$ & $1.9525(16)$ \\
$\mathrm{Cu}(1)-\mathrm{N}(1)$ & $2.1981(19)$ \\
$\mathrm{Cu}(1)-\mathrm{N}(2)$ & $2.0710(18)$ \\
$\mathrm{Cu}(1)-\mathrm{N}(3)$ & $2.0075(18)$ \\
$\mathrm{Cu}(2)-\mathrm{O}(1)$ & $2.2567(15)$ \\
$\mathrm{Cu}(2)-\mathrm{O}(3)$ & $1.9378(16)$ \\
$\mathrm{Cu}(2)-\mathrm{O}(5)$ & $1.9316(17)$ \\
$\mathrm{Cu}(2)-\mathrm{N}(4)$ & $2.581(2)$ \\
$\mathrm{Cu}(2)-\mathrm{N}(5)$ & $2.0665(18)$ \\
$\mathrm{Cu}(2)-\mathrm{N}(6)$ & $1.9354(17)$ \\
$\mathrm{Cu}(1)-\mathrm{O}(1)-\mathrm{Cu}(2)$ & $118.24(7)$ \\
$\mathrm{O}(1)-\mathrm{Cu}(1)-\mathrm{N}(1)$ & $95.56(7)$ \\
$\mathrm{O}(1)-\mathrm{Cu}(1)-\mathrm{N}(2)$ & $93.05(6)$ \\
$\mathrm{O}(1)-\mathrm{Cu}(1)-\mathrm{N}(3)$ & $161.08(6)$ \\
$\mathrm{O}(1)-\mathrm{Cu}(1)-\mathrm{O}(2)$ & $95.09(6)$ \\
$\mathrm{O}(1)-\mathrm{Cu}(2)-\mathrm{N}(4)$ & 161.42 \\
$\mathrm{O}(1)-\mathrm{Cu}(2)-\mathrm{N}(5)$ & $90.42(7)$ \\
$\mathrm{O}(1)-\mathrm{Cu}(2)-\mathrm{N}(6)$ & $91.13(7)$ \\
$\mathrm{O}(1)-\mathrm{Cu}(2)-\mathrm{O}(3)$ & $91.21(6)$ \\
$\mathrm{O}(1)-\mathrm{Cu}(2)-\mathrm{O}(5)$ & $101.64(7)$ \\
\hline
\end{tabular}


The structure of $\left[\mathrm{Cu}_{2}{ }_{2}\left(\mathrm{HSIM}^{1}\right)(\mu-\mathrm{OAc})\right]^{+}$contrasts with the previously published structure of the dicopper(II) complex of the symmetric ligand $\mathrm{HL}^{1},\left[\mathrm{Cu}_{2}{ }_{2}\left(\mathrm{~L}^{1}\right)(\mathrm{OAC})_{2}\right] \mathrm{ClO}_{4}$, which bears two square pyramidal coordinated $\mathrm{Cu}^{\prime \prime}$ centers. ${ }^{169}$ Consequently, the asymmetrically substituted ligand $\mathrm{H}_{3} \mathrm{SIM}^{1}$ provides two different coordination sites for the $\mathrm{Cu}^{\prime \prime}$ ions. This asymmetry is additionally visible in the bond length between the $\mathrm{Cu}$ "l centers and the phenolate oxygen atom; while this value measures $2.173 \AA$ for both $\mathrm{Cu}^{\prime \prime}$ centers in $\left[\mathrm{Cu}_{2}{ }_{2}\left(\mathrm{~L}^{1}\right)(\mathrm{OAc})_{2}\right]^{+}, 169$ in $\left[\mathrm{Cu}_{2}{ }_{2}\left(\mathrm{HSIM}^{1}\right)(\mu-\mathrm{OAc})\right]^{+}$this value is shortened in the case of the square pyramidal $\mathrm{Cu}(1)(1.9061(16) \AA)$ and elongated in the case of the octahedral $\mathrm{Cu}(2)(2.2567(15) \AA)$. Interestingly, the $\mathrm{Cu} \cdots \mathrm{Cu}$ separation in $\left[\mathrm{Cu}_{2}{ }_{2}\left(\mathrm{HSIM}^{1}\right)(\mu-\mathrm{OAC})\right]^{+}(3.577 \AA)$ is much shorter than in $\left[\mathrm{Cu}_{2}{ }_{2}\left(\mathrm{~L}^{1}\right)(\mathrm{OAC})_{2}\right]^{+}(3.916 \AA),{ }^{169}$ probably due to the bridging acetate co-ligand, as $\left[\mathrm{Cu}_{2}{ }_{2}\left(\mathrm{~L}^{1}\right)(\mathrm{OAC})_{2}\right]^{+}$comprises two terminally coordinated acetate co-ligands.

\section{Magnetic Susceptibility Measurement}

The molar magnetic susceptibtibility $\left(\chi_{M}\right)$ describes the degree of the magnetization of a material and characterizes the way in which an applied magnetic field $\mathrm{H}$ interacts with the angular momenta associated with the thermally populated states of a molecule. ${ }^{172,173}$ When a sample is perturbed by an external magnetic field $\mathrm{H}$, its magnetization is related to its energy variation by Equation 1 which defines the microscopic magnetizations $\mu_{n}$ for each energy level $E_{n}(n=1,2, \ldots)$.

$$
\text { Equation } 1 \quad \mu_{\mathrm{n}}=-\frac{\delta \mathrm{E}_{\mathrm{n}}}{\delta \mathrm{H}}
$$

The macroscopic molar magnetization $M$ is then obtained by summing the microscopic magnetizations weighted according to the Boltzmann distribution (Equation 2). The molar magnetic susceptibility is deduced from the molar magnetization by Equation 3.

$$
\begin{array}{ll}
\text { Equation 2 } & \mathrm{M}=\frac{\mathrm{N} \sum_{\mathrm{n}}\left(-\frac{\delta \mathrm{E}_{\mathrm{n}}}{\delta \mathrm{H}}\right) \mathrm{e}^{-\frac{E_{\mathrm{n}}}{\mathrm{kT}}}}{\sum_{\mathrm{n}} \mathrm{e}^{-\frac{E_{\mathrm{n}}}{\mathrm{kT}}}} \\
\text { Equation 3 } & \chi=\frac{\delta \mathrm{M}}{\delta \mathrm{H}}
\end{array}
$$

The function of a magnetometer is to measure the induced or remnant magnetic moment in a sample, usually as a function of applied field and temperature. ${ }^{174} \mathrm{~A}$ graph of $\chi_{M} T$ vs. temperature (T) is commonly used for the analysis of the magnetic behavior of a substance, whereby a constant $\chi_{M} T$ value is characteristic for a paramagnetic species. Antiferromagnetism leads to the deviation of $\chi_{M} T$ to lower values by decreasing $T(J<0)$, while the deviation to higher $\chi_{M} T$ values by decreasing $T$ reveals ferromagnetism $(J>0)$. The effective magnetic moment may be calculated from the susceptibility measurements with Equation 4.

$$
\text { Equation } 4 \quad \mu_{\text {eff }}=2.828 \sqrt{\chi_{M}^{T}}
$$


The experimentally obtained value for $\mu_{\text {eff }}$ can be compared with the expected spin-only value for a dinuclear system calculated with Equation 5 (for two strongly interacting spins with the resulting total spin quantum number $S_{\text {tot }}$ ) or Equation 6 (for two non-interacting spins $S_{1}$ and $S_{2}$ ). Equation 6 simplifies for two non-interacting spins of the same value $\left(S_{1}=S_{2}=S\right)$ resulting in Equation 7.

$$
\begin{array}{ll}
\text { Equation 5 } & \mu_{\mathrm{eff}}=2 \sqrt{\mathrm{S}_{\text {tot }}\left(\mathrm{S}_{\text {tot }}+1\right)} \\
\text { Equation 6 } & \mu_{\mathrm{eff}}=2 \sqrt{\mathrm{S}_{1}\left(\mathrm{~S}_{1}+1\right)+\mathrm{S}_{2}\left(\mathrm{~S}_{2}+1\right)} \\
\text { Equation 7 } & \mu_{\mathrm{eff}}=2 \sqrt{\mathrm{S}(\mathrm{S}+1)} * \sqrt{2}
\end{array}
$$

In order to understand the magnetic property variable temperature dc magnetic susceptibility studies in solid state were performed with ground crystals of complex $\left[\mathrm{Cu}_{2}{ }_{2}\left(\mathrm{H}_{2} \mathrm{SIM}^{1}\right)(\mu-\mathrm{OAc})_{2}\right] \mathrm{PF}_{6}$ in the temperature range from 2 to $300 \mathrm{~K}$. The data for $\chi_{M} T$ vs. T are shown in Figure 15. The strong peak between $50 \mathrm{~K}$ and $100 \mathrm{~K}$ was attributed to oxygen present in the instrument.

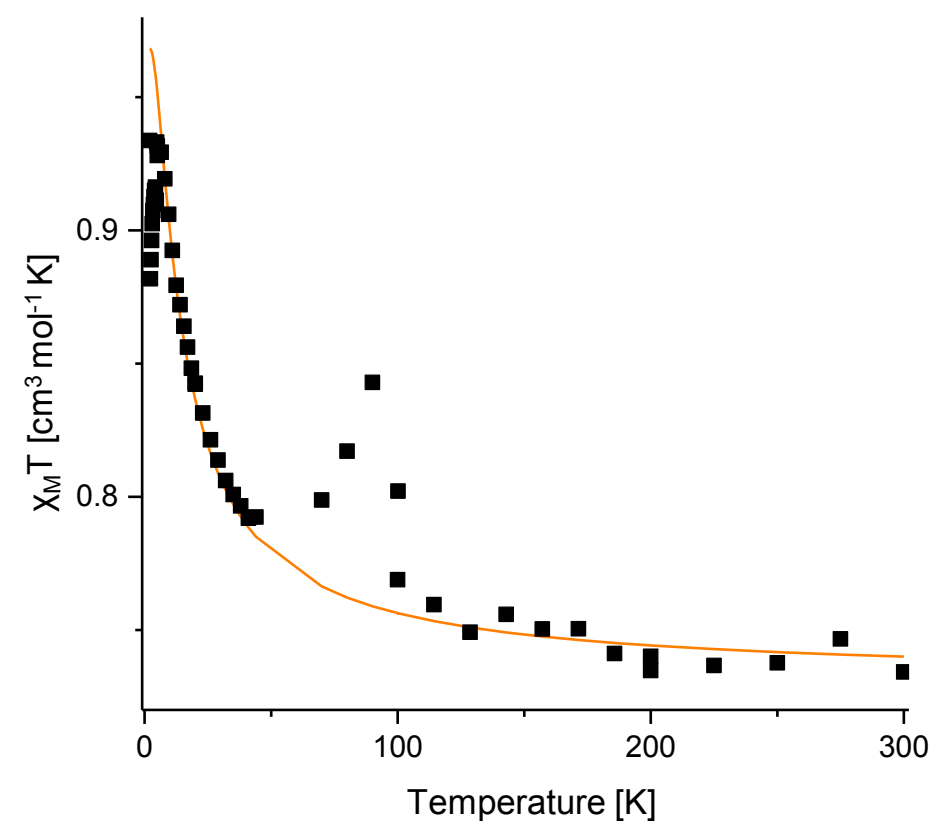

Figure 15: Temperature dependence of $\chi_{M} T$ for $\left[\mathrm{Cu}_{2}{ }_{2}\left(\mathrm{HSIM}^{1}\right)(\mu-\mathrm{OAc})\right] \mathrm{PF} \mathrm{F}_{6}$ recorded in a magnetic field of $1000 \mathrm{Oe}$ and the respective fit curve (orange line).

The $\chi_{M} T$ vs. T plot shows the typical process of a ferromagnetically coupled system. The value of $\chi_{M} T$ increases from a value of $0.8818 \mathrm{~cm}^{3} \mathrm{~mol}^{-1} \mathrm{~K}$ at $2.25 \mathrm{~K}$ to $0.9331 \mathrm{~cm}^{3} \mathrm{~mol}^{-1} \mathrm{~K}$ at $4.75 \mathrm{~K}$, and then decreases at higher temperatures. The $\chi_{M} T$ value tends to $1 \mathrm{~cm}^{3} \mathrm{~mol}^{-1} \mathrm{~K}$ at low temperature which is the expected value for two interacting $S_{1,2}=1 / 2$ metal centers $\left(S_{\text {tot }}=1\right)$. The value of $0.7406 \mathrm{~cm}^{3} \mathrm{~mol}^{-1} \mathrm{~K}$ at $300 \mathrm{~K}$ is close to the expected value for two non-coupled electrons $\left(0.75 \mathrm{~cm}^{3} \mathrm{~mol}^{-1} \mathrm{~K}\right)$.

The data were fitted by the following spin Hamiltonian (Equation 8), where $J$ is the isotropic exchange coupling constant, $S_{i}$ denotes the spin states $(S=1 / 2)$, and $D_{i}$ and $E_{i}$ are the axial and rhombic zero-field splitting parameters. 
Equation 8

$$
\mathrm{H}=-2 \mathrm{~S}_{1} \mathrm{~S}_{2}+\sum_{\mathrm{i}=1}^{2}\left[\mathrm{D}_{\mathrm{i}}\left(\mathrm{S}_{\mathrm{zi}}{ }^{2}-\frac{1}{3} \mathrm{~S}_{\mathrm{i}}\left(\mathrm{S}_{\mathrm{i}}+1\right)\right)+\mathrm{E}_{\mathrm{i}}\left(\mathrm{S}_{\mathrm{xi}}{ }^{2}-\mathrm{S}_{\mathrm{yi}}{ }^{2}\right)+\beta \mathrm{S}_{\mathrm{i}} \mathrm{g}_{\mathrm{i}} \mathrm{H}\right]
$$

This model reproduced reasonably well the experimental data when the data points between $50 \mathrm{~K}$ and $100 \mathrm{~K}$ were excluded. The final parameter set of the best fit were found to be $\mathrm{J}=5.0 \mathrm{~cm}^{-1}$, $\mathrm{g}_{1}=\mathrm{g}_{2}=2.0$, and the D- and E-values to be 0 , considering $5 \%$ of a paramagnetic impurity derived from the monocopper(II) complex.

The majority of dicopper(II) compounds have an antiferromagnetically coupled ground state with $\mathrm{S}=0 .{ }^{173}$ Therefore, $\left[\mathrm{Cu}^{1{ }^{\prime \prime}}\left(\mathrm{HSIM}^{1}\right)(\mu-\mathrm{OAC})\right] \mathrm{PF}_{6}$ is a rare example for a dicopper(II) system with weak ferromagnetic coupling and an $S=1$ ground state. This observation contrasts to previously published phenolate-bridged dicopper(II) complexes, which usually show antiferromagnetic coupling, i.e. the symmetric complex $\left[\mathrm{Cu}_{2}{ }_{2}\left(\mathrm{~L}^{1}\right)(\mathrm{OAc})_{2}\right] \mathrm{ClO}_{4}\left(\mathrm{~J}=-0.6 \mathrm{~cm}^{-1} ; \Varangle(\mathrm{Cu}-\mathrm{O}-\mathrm{Cu})=128.65^{\circ}\right)^{169}$ and the asymmetric complexes $\left[\mathrm{Cu}_{2}{ }_{2}\left(\mathrm{~L}^{13}\right) \mathrm{Cl}_{3}\right]\left(\mathrm{J}=-168 \mathrm{~cm}^{-1} ; \Varangle(\mathrm{Cu}-\mathrm{O}-\mathrm{Cu})=111.52^{\circ}\right),{ }^{175}$ $\left[\mathrm{Cu}_{2}{ }_{2}\left(\mathrm{H}_{4} \mathrm{~L}^{14}\right)(\mu-\mathrm{OH})\right]\left(\mathrm{ClO}_{4}\right)_{2} \quad\left(\mathrm{~J}=-238.1 \quad \mathrm{~cm}^{-1} ; \quad \Varangle(\mathrm{Cu}-\mathrm{O}-\mathrm{Cu})=95.3^{\circ}\right),{ }^{176}$ and $\left[\mathrm{Cu}_{2}{ }_{2}\left(\mathrm{H}_{2} \mathrm{~L}^{15}\right)(\mu-\mathrm{OH})\left(\mathrm{ClO}_{4}\right)\right] \mathrm{ClO}_{4}\left(\mathrm{~J}=-100.5 \mathrm{~cm}^{-1} ; \Varangle(\mathrm{Cu}-\mathrm{O}-\mathrm{Cu})=91.9^{\circ}\right) .{ }^{176} \mathrm{It}$ must be pointed out here that the determination of $\mathrm{J}$ from the magnetic data is much more accurate for $\mathrm{J}<0$ than for $\mathrm{J}>0 .{ }^{173} \mathrm{~A}$ comparison of key structural parameters and exchange integrals for bis( $\mu$-phenoxido)dicopper(II) complexes reveals the dependence of $\mathrm{J}$ on (i) the $\mathrm{Cu}-\mathrm{OPh}_{\mathrm{ph}} \mathrm{Cu}$ angle, (ii) the $\mathrm{Cu}$ ‥Cu distance, (iii) the pyramidal geometry around the phenolate oxygen atom, and (iv) the deviation of the copper(II) coordination geometry from square pyramidal. ${ }^{177}$ Similarly, the structural requirements for a strongly coupled hydroxido-bridged dicopper(II) system was investigated by Ruiz et al. theoretically, and it was found that (i) small Cu-O-Cu angles, (ii) short Cu-O distances, (iii) large out of plane shifts of the hydrogen atoms on the bridge, and (iv) hingedistorted $\mathrm{Cu}_{2} \mathrm{O}_{2}$ rings lead to strong exchange coupling. ${ }^{178}$ The major factor controlling the exchange coupling is the bridging $\mathrm{Cu}-\mathrm{O}-\mathrm{Cu}$ angle, and a linear variation of $2 \mathrm{~J}$ with this parameter was observed for hydroxido-bridged and alkoxido-bridged dicopper(II) complexes. ${ }^{178,}{ }^{179}$ For hydroxido-bridged dicopper(II) complexes the crossover point, below which the magnetic behavior changes from antiferromagnetic to ferromagnetic coupling, is characterized by a $\mathrm{Cu}-\mathrm{O}(\mathrm{H})-\mathrm{Cu}$ angle of $97.5^{\circ} .177$ In the case of bis( $\mu$-phenoxido)dicopper(II) complexes only five compounds revealing weak ferromagnetic coupling were observed, in which the $\mathrm{Cu}-\mathrm{O}_{\mathrm{Ph}}-\mathrm{Cu}$ angle lies in the range of $85.9-96.1^{\circ} .^{177}$ The $\mathrm{Cu}(1)-\mathrm{O}(1)-\mathrm{Cu}(2)$ angle of $\left[\mathrm{Cu}_{2}{ }_{2}\left(\mathrm{HSIM}^{1}\right)(\mu-\mathrm{OAc})\right] \mathrm{PF}_{6}$ is comparably large with a value of $118.24(7) \AA ̊$ when compared to other ferromagnetically coupled diphenoxido-bridged complexes, and in the medium range of antiferromagnetically coupled 
phenoxido-bridged dicopper(II) complexes $\left[\mathrm{Cu}_{2}\left(\mathrm{~L}^{1}\right)(\mathrm{OAC})_{2}\right] \mathrm{ClO}_{4},{ }^{169} \quad\left[\mathrm{Cu}_{2}{ }_{2}\left(\mathrm{~L}^{13}\right) \mathrm{Cl}_{3}\right],{ }^{175}$ $\left[\mathrm{Cu}_{2}{ }_{2}\left(\mathrm{H}_{4} \mathrm{~L}^{14}\right)(\mu-\mathrm{OH})\right]\left(\mathrm{ClO}_{4}\right)_{2},{ }^{176}$ and $\left[\mathrm{Cu}_{2}{ }_{2}\left(\mathrm{H}_{2} \mathrm{~L}^{15}\right)(\mu-\mathrm{OH})\left(\mathrm{ClO}_{4}\right)\right] \mathrm{ClO}_{4} \cdot{ }^{176}$

Interestingly, Chaudhuri et al. developed two bis( $\mu$-phenoxido)dicopper(II) complexes, which contain the same ONO donor atoms but reveal opposite exchange interactions, $\left[\mathrm{Cu}_{2}{ }_{2}\left(\mathrm{~L}^{16}\right)_{2}\right]$ the usual antiferromagnetic exchange coupling and $\left[\mathrm{Cu}_{2}{ }_{2}\left(\mathrm{~L}^{17}\right)_{2}\right]$ ferromagnetic exchange coupling (Chart 9). ${ }^{177}$ Several structural parameters were discussed to contribute to this contrasting magnetic behavior, the smaller $\mathrm{Cu}-\mathrm{O}-\mathrm{Cu}$ angle of $\left[\mathrm{Cu}_{2}{ }_{2}\left(\mathrm{~L}^{17}\right)_{2}\right]\left(86.34^{\circ}\right.$ and $85.92^{\circ}$ vs. $97.0^{\circ}$ and $99.4^{\circ}$ in $\left.\left[\mathrm{Cu}_{2}{ }_{2}\left(\mathrm{~L}^{16}\right)_{2}\right]\right)$, the shorter $\mathrm{Cu} \cdots \mathrm{Cu}$ separation of $\left[\mathrm{Cu}_{2}{ }_{2}\left(\mathrm{~L}^{17}\right)_{2}\right](2.697 \AA$ vs. $2.967 \AA$ in $\left.\left[\mathrm{Cu}_{2}{ }_{2}\left(\mathrm{~L}^{16}\right)_{2}\right]\right)$, and the more severe deviation from square planarity of the $\mathrm{Cu}^{\prime \prime}$ centers in $\left[\mathrm{Cu}_{2}{ }_{2}\left(\mathrm{~L}^{17}\right)_{2}\right]$. However, the parallel spin coupling of $\left[\mathrm{Cu}_{2}{ }_{2}\left(\mathrm{~L}^{17}\right)_{2}\right]$ was mainly ascribed to the folded $\mathrm{Cu}_{2}\left(\mathrm{~L}^{17}\right)_{2}$ structure, in which the two copper(II) planes make an angle of $75.4^{\circ}$.

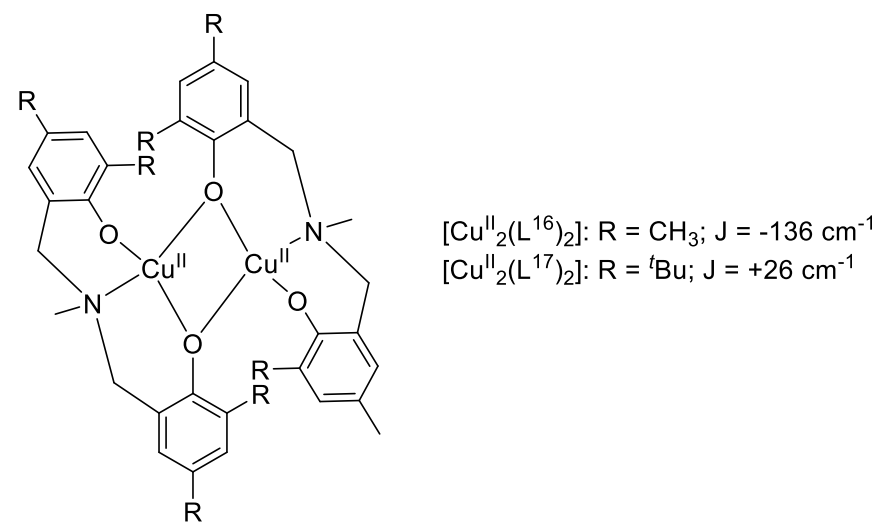

Chart 9: Bis(phenolate)-bridged dicopper(II) complexes with antiferromagnetic interaction $\left(\left[\mathrm{Cu}_{2}{ }_{2}\left(\mathrm{~L}^{16}\right)_{2}\right]\right)$ and ferromagnetic interaction $\left(\left[\mathrm{Cu}_{2}{ }_{2}\left(\mathrm{~L}^{17}\right)_{2}\right]\right) .{ }^{177}$

Similarly, in $\left[\mathrm{Cu}_{2}{ }_{2}\left(\mathrm{HSIM}^{1}\right)(\mu-\mathrm{OAc})\right] \mathrm{PF}_{6}$ the $\mathrm{Cu}(1)-\mathrm{O}(1)-\mathrm{O}(2)-\mathrm{N} 2-\mathrm{N} 4$ plane forms an angle of $42.09^{\circ}$ with the $\mathrm{Cu}(2)-\mathrm{O}(1)-\mathrm{O}(3)-\mathrm{N}(4)-\mathrm{N}(6)$ plane, which is rather small compared to the antiferromangetically coupled complex $\left[\mathrm{Cu}_{2}{ }_{2}\left(\mathrm{~L}^{1}\right)(\mathrm{OAc})_{2}\right] \mathrm{ClO}_{4}$ with an angle of $57.65^{\circ}$. Thus, the stronger folding in $\left[\mathrm{Cu}_{2}{ }_{2}\left(\mathrm{~L}^{1}\right)(\mathrm{OAC})_{2}\right] \mathrm{ClO}_{4}$ lowers the magnitude of the antiferromagnetic interaction, which dominates exchange coupling between two $\mathrm{Cu}^{\prime \prime}$ centers due to loss of orbital overlap. Similar diminution of antiferromagnetic coupling due to a folded $\mathrm{Cu}_{2} \mathrm{O}_{2}$ and a $\mathrm{Cr}_{2} \mathrm{O}_{2}$ core has been observed previously. ${ }^{177,} 180$ Moreover, the distortion of the $\mathrm{O}_{\mathrm{Ph}}-\mathrm{C}_{\mathrm{Ph}}$ bond with respect to the $\mathrm{Cu}-\mathrm{O}_{\mathrm{ph}}-\mathrm{Cu}$ plane is also known to affect the magnetic behavior. ${ }^{181} \mathrm{In}$ fact, the $\mathrm{O}(1)-\mathrm{C}_{\mathrm{ph}}$ bond in $\left[\mathrm{Cu}_{2}{ }_{2}\left(\mathrm{HSIM}^{1}\right)(\mu-\mathrm{OAc})\right] \mathrm{PF}_{6}$ is placed out of the $\mathrm{Cu}-\mathrm{O}(1)-\mathrm{Cu}$ plane which is not the case in $\left[\mathrm{Cu}_{2}{ }_{2}\left(\mathrm{~L}^{1}\right)(\mathrm{OAC})_{2}\right] \mathrm{ClO}_{4} \cdot{ }^{169}$ Therefore, it is apparent that the major magnetic exchange interaction pathway between the two $\mathrm{Cu}^{\prime \prime}$ centers is the oxygen atom of the bridging phenolate ligand. However, Horn et al. detected an impact of bridging acetate co-ligands on the coupling of a diiron(III) complex suggesting it to be a secondary pathway for the mediation of interaction. ${ }^{102}$ 


\subsubsection{COMPLEXATION STUDIES WITH FE ${ }^{I I I}$}

In addition to the investigations with Ga'l', used to mimic the Fe ${ }^{\text {III }}$ center in native PAPs, the coordination behavior of $\mathrm{H}_{3} \mathrm{SIM}^{1}$ towards Fe ${ }^{\text {III }}$ was examined. Similar to $\mathrm{Cu}^{\text {"l, }} \mathrm{Fe}^{\text {III }}$ is paramagnetic with a $(\mathrm{Ar}) 3 \mathrm{~d}^{5}$ configuration. Therefore, the coordination chemistry towards Fe $\mathrm{e}^{\text {III }}$ was studied by electronic and NMR spectroscopic techniques, suitable for paramagnetic substances.

\section{UV-vis-NIR Titration}

Recording a UV-vis-NIR spectroscopic time scan with mixtures of $\mathrm{H}_{3} \mathrm{SIM}^{1}$ and iron(III) perchlorate in methanol both with and without base for about ten hours, the change in the number and intensity of absorption bands was monitored during the first 1.5 hours; afterwards no change in the spectra was detected. Therefore, all samples were allowed to react at room temperature for 14 hours before the UV-vis-NIR spectroscopic analysis was undertaken in order to assure that the reactions were completed. The spectrum of a mixture of $\mathrm{H}_{3} \mathrm{SIM}^{1}$ and iron(III) perchlorate in a ratio of 1:1 showed two bands at $348 \mathrm{~nm}$ and $552 \mathrm{~nm}$. The relatively broad band at $552 \mathrm{~nm}$ $\left(\varepsilon=1238 \mathrm{M}^{-1} \mathrm{~cm}^{-1}\right)$ originates from the charge transfer (CT) transition from filled $p_{\pi}$ orbitals of the phenolate oxygen to the empty $d_{\pi^{*}}$ orbitals of the Fe ${ }^{\text {III }}$ metal center. All phenoxido-Fe ${ }^{\text {III }}$ complexes display this characteristic phenolate-to-iron(III) CT transition, ${ }^{81}$ for example the complex $\left[\mathrm{Fe}{ }^{\prime \prime \prime} \mathrm{Mn}\left(\mathrm{L}^{1}\right)(\mu-\mathrm{OAC})_{2}\right]^{+}$in acetone at $596 \mathrm{~nm} \quad\left(\varepsilon=980 \mathrm{M}^{-1} \mathrm{~cm}^{-1}\right)^{182}$ and the complex $\left[\mathrm{Fe}^{\mathrm{III}}{ }_{2}\left(\mathrm{~L}^{18}\right)_{2}(\mu-\mathrm{O})(\mu-\mathrm{OBz})\right]^{+}$in acetonitrile at $522 \mathrm{~nm}\left(\varepsilon=2700 \mathrm{M}^{-1} \mathrm{~cm}^{-1}\right)^{183}$. The additional band at $348 \mathrm{~nm}\left(\varepsilon=3838 \mathrm{M}^{-1} \mathrm{~cm}^{-1}\right)$ arises from $\mathrm{p}_{\pi^{-}}-\mathrm{d}_{\sigma^{*}}$ type transitions.

Comparison of the UV-vis-NIR spectra, recorded with methanolic solutions of $\mathrm{H}_{3} \mathrm{SIM}^{1}$ and iron(III) perchlorate up to a ratio of 1:2 (Figure 16a), illustrates the coordination of one equivalent Fe $\mathrm{III}$ ions by ligand $\mathrm{H}_{3} \mathrm{SIM}^{1}$ resulting in a green solution. Complexation of a second Fe"ll ion is unlikely due to the detection of a plateau of the absorbance at $552 \mathrm{~nm}$ vs. Fe ${ }^{\text {III }}$ content plot (insert in Figure 16a) for mixtures with excess Fe ${ }^{\prime \prime \prime}$. The increase of the absorbance at $348 \mathrm{~nm}$ at Fe contents higher than one equivalent with respect to the ligand possibly arises from noncoordinated free Fe $\mathrm{F}^{\mathrm{III}}$ in solution and the resulting hexaaqua complex.

Addition of one equivalent of triethylamine as a base led to a shift of the CT band from $552 \mathrm{~nm}$ to $488 \mathrm{~nm}\left(\varepsilon=1718 \mathrm{M}^{-1} \mathrm{~cm}^{-1}\right)$, directly visible in the change of the solution color to blue and possibly due to deprotonation of the phenol moiety. A further increase of the base concentration did not result in additional changes. The $\mathrm{p}_{\pi^{-}}-\mathrm{d}_{\sigma^{*}}$ band at $342 \mathrm{~nm}\left(\varepsilon=5132 \mathrm{M}^{-1} \mathrm{~cm}^{-1}\right)$ experienced almost no change during the addition of base. Similarly to the experiments undertaken without addition of base, the absorbance vs. equivalence of Fe $\mathrm{F}^{\text {III }}$ plot for the mixtures of $\mathrm{H}_{3} \mathrm{SIM}^{1}$, treated with one equivalent of triethylamine and iron(III) perchlorate, shows the increase in intensity of 
the bands at $342 \mathrm{~nm}$ and $488 \mathrm{~nm}$ up to a 1:1.25 mixture (Figure 16b). At higher Fe'II concentrations the curve (inset) shows a plateau, suggesting that $\mathrm{H}_{3} \mathrm{SIM}^{1}$ is able to bind only one $\mathrm{Fe}^{\mathrm{III}}$ ion, independently of the presence of base.
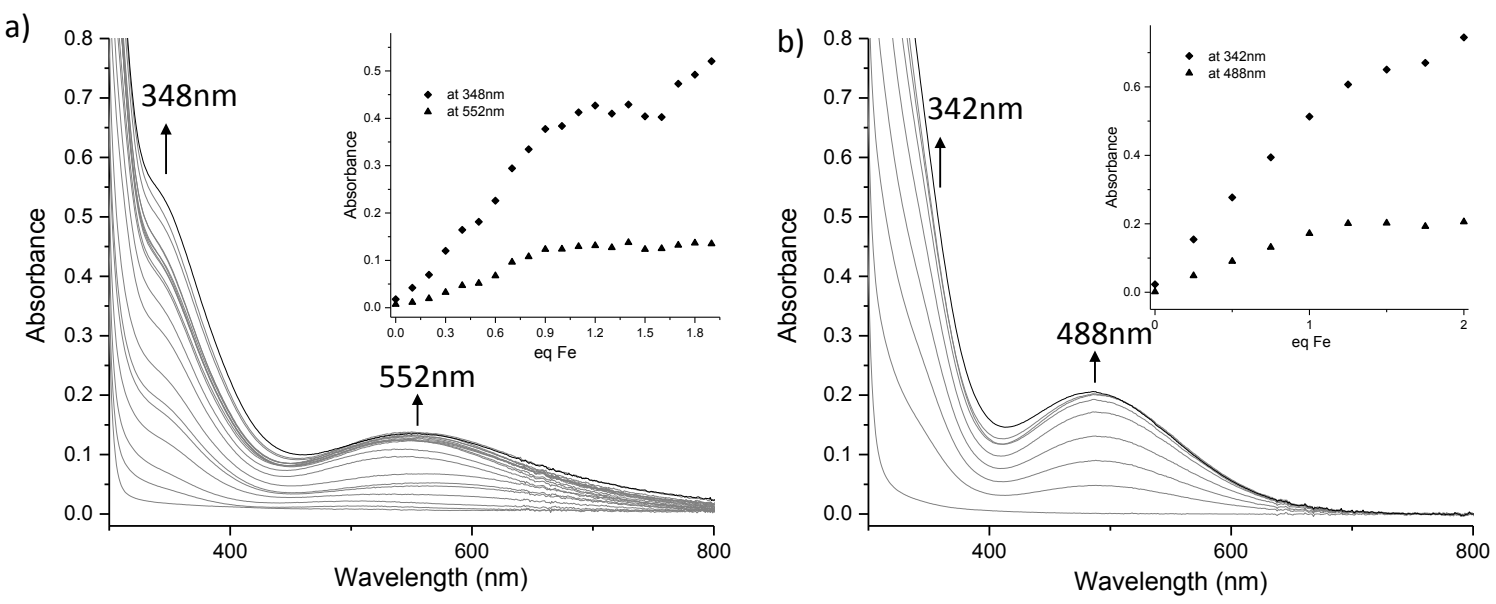

Figure 16: UV-vis-NIR spectroscopic titration of $\mathrm{H}_{3} \mathrm{SIM}^{1}(0.4 \mathrm{mM})$ with $\mathrm{Fe}^{\prime \prime \prime}\left(\mathrm{ClO}_{4}\right)_{3}$ in methanol a) without $\mathrm{NEt}_{3}$ and b) with 1 eq of $\mathrm{NEt}_{3}$.

These results are in contrast to the observation gained during a similar UV-vis-NIR spectroscopic titration of ligand $\mathrm{H}_{3} \mathrm{~L}^{2}$ with $\left[\mathrm{FeCl}_{4}\right]\left(\mathrm{NEt}_{4}\right)$ in methanol, because a progressive shift of the $\mathrm{CT}$ transitions from $533 \mathrm{~nm}$ to $629 \mathrm{~nm}$ was monitored by addition up to one equivalent of Fe ${ }^{\text {IIII }}$ and a constant increase in intensity of the band with further addition of $\mathrm{Fe}^{\mathrm{III}}$. These findings were explained by the simultaneous formation of mono- and dinuclear iron(III) complexes at low Fe $\mathrm{III}$ content in solution and the shift of the equilibrium in favor of the diiron(III) complex with raising Fe ${ }^{\text {III }}$ concentrations. ${ }^{109}$ Considering these observations, the positioning of two bulky pivaloylamide residues proximal to one binding site seems to hinder the coordination of Fe ${ }^{\text {III }}$ in coordination site $\mathrm{C}$, which was still possible when only one pivaloyl-amide moiety was adjacent to the coordination sites as in $\mathrm{H}_{3} \mathrm{~L}^{2}$.

\section{NMR Studies}

A mixture of $\mathrm{H}_{3} \mathrm{SIM}^{1}$ with 0.5 equivalents of iron(II) tetrafluoroborate in deuterated acetonitrile was treated with $4 \AA$ molecular sieves and left to react under air for 12 hours. The recorded ${ }^{1} \mathrm{H}$ NMR spectrum (-60 ppm to $260 \mathrm{ppm}$ ) of the blue solution shows broad resonances in the aromatic region associated with the uncomplexed ligand. However, resonances with chemical shifts higher than $10 \mathrm{ppm}$ were not detected in contrast to the spectrum of $\left[\mathrm{Fe}_{2}{ }_{2}\left(\mathrm{H}_{2} \mathrm{SIM}^{1}\right)(\mu-\mathrm{OAc})_{2}\right]^{+}$, which will be discussed in Chapter 5.3, supporting the proposal of the full oxidation of $\mathrm{Fe}^{\prime \prime}$ to Fe $\mathrm{F}^{\mathrm{II}}$ during the reaction under aerobic conditions. Unfortunately, differences in the integrals assigned to protons of binding site $B$ and binding site $C$ could not be detected due to broadening of the signals. 
The measurement of the magnetic susceptibility of the mixture by the Evans method resulted in a value of $\mu_{\text {eff }}=4.79$ B.M. Based on a comparison with the calculated spin-only values for a lowspin Fe ${ }^{\text {III }}$ complex $(S=1 / 2)$ of 1.73 B.M. and for a high-spin Fe ${ }^{\text {III }}$ complex $(S=5 / 2)$ of 5.92 B.M., $\mathrm{H}_{3} \mathrm{SIM}^{1}$ is proposed to form a mononuclear high-spin Fe ${ }^{\text {III }}$ complex.

It is apparent from the results of the NMR and UV-vis-NIR spectroscopic examinations of mixtures with $\mathrm{H}_{3} \mathrm{SIM}^{1}$ and iron salts that $\mathrm{H}_{3} \mathrm{SIM}^{1}$ is capable of coordinating one $\mathrm{Fe}^{\mathrm{III}}$ ion, generating a highspin Fe"II complex. The coordination of the Fe ${ }^{\text {III }}$ ion most likely takes place in the more accessible binding site $B$. This proposal is made on the basis of the observations from the ${ }^{1} \mathrm{H}$ NMR titration

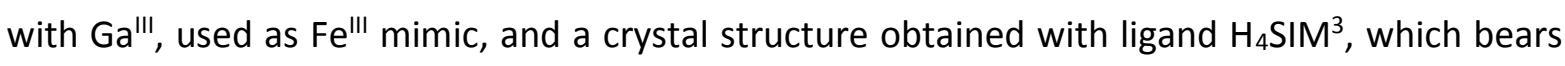
also binding site $\mathrm{B}$, and iron(III) perchlorate. In this way, the second coordination site $\mathrm{C}$ of $\mathrm{H}_{3} \mathrm{SIM}^{1}$ stays vacant for other, preferably divalent, metal ions to form heterodinuclear complexes and thereby form a more accurate model for PAPs.

\subsection{Complexation Behavior OF LIGAND $\mathrm{H}_{4} \mathrm{SIM}^{3}$}

In addition to the asymmetry in the second coordination sphere $\mathrm{H}_{4} \mathrm{SIM}^{3}$ provides also asymmetry in the donor sets, in contrast to $\mathrm{H}_{3} \mathrm{SIM}^{1}$. The two compartments in $\mathrm{H}_{4} \mathrm{SIM}^{3}$ are built up by different donors, forming a $\mathrm{N}_{3} \mathrm{O}$ coordination site (binding site $\mathrm{C}$, Chart 10) and a harder $\mathrm{N}_{2} \mathrm{O}_{2}$ coordination site, containing a terminal phenolate moiety (binding site $A$, Chart 10).

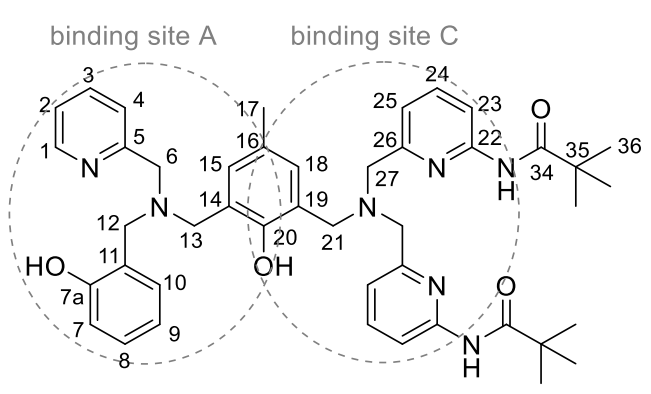

Chart 10: Assignment of the binding sites provided by ligand $\mathrm{H}_{4} \mathrm{SIM}^{3}$ and the numbering of its atoms.

However, the positions of the two pivaloyl-amide residues, mimicking the hydrogen bond donors in the active site of PAP, are comparable to $\mathrm{H}_{3} \mathrm{SIM}^{1}$. Therefore, the comparison of the coordination behavior of $\mathrm{H}_{3} \mathrm{SIM}^{1}$ and $\mathrm{H}_{4} \mathrm{SIM}^{3}$ provides the opportunity to investigate the impact of the exchange of one pyridine by a phenolate residue at preserved positions of the hydrogen bond donors. 


\subsubsection{COMPLEXATION STUDIES WITH ZN"}

Similar to the investigation of the $\mathrm{Zn}^{\|}$complexation behavior of $\mathrm{H}_{3} \mathrm{SIM}^{1}$, the coordination chemistry of $\mathrm{H}_{4} \mathrm{SIM}^{3}$ with $\mathrm{Zn}^{\|}$ions was studied applying the above introduced NMR titration technique (Chapter 3.2.1).

\section{NMR Studies}

Comparable to the analogous experiments undertaken with $\mathrm{H}_{3} \mathrm{SIM}^{1}$, the generation of a monozinc(II) complex could be followed during addition of zinc(II) perchlorate by ${ }^{1} \mathrm{H}$ NMR spectra, measured after each addition step of 0.25 eq to $\mathrm{H}_{4} \mathrm{SIM}^{3}$, dissolved in acetonitrile. However, after addition of one equivalent, further increase of the $\mathrm{Zn}$ " concentration did not result in a second set of resonances, suggesting that the generation of a dizinc(II) complex of $\mathrm{H}_{4} \mathrm{SIM}^{3}$ does not occur. In the monozinc(II) complex spectrum the resonances assigned to binding site $\mathrm{C}$ are shifted compared to those in the spectrum of the free ligand. This observation was duplicated during the complexation of $\mathrm{Zn}^{\prime \prime}$ in binding site $\mathrm{C}$ of ligand $\mathrm{H}_{3} \mathrm{SIM}^{1}$, suggesting the $\mathrm{Zn}$ " ion to be bound by the two amidated pyridine residues in binding site $\mathrm{C}$ of $\mathrm{H}_{4} \mathrm{SIM}^{3}$ (see Figures 2 and Figure 17).

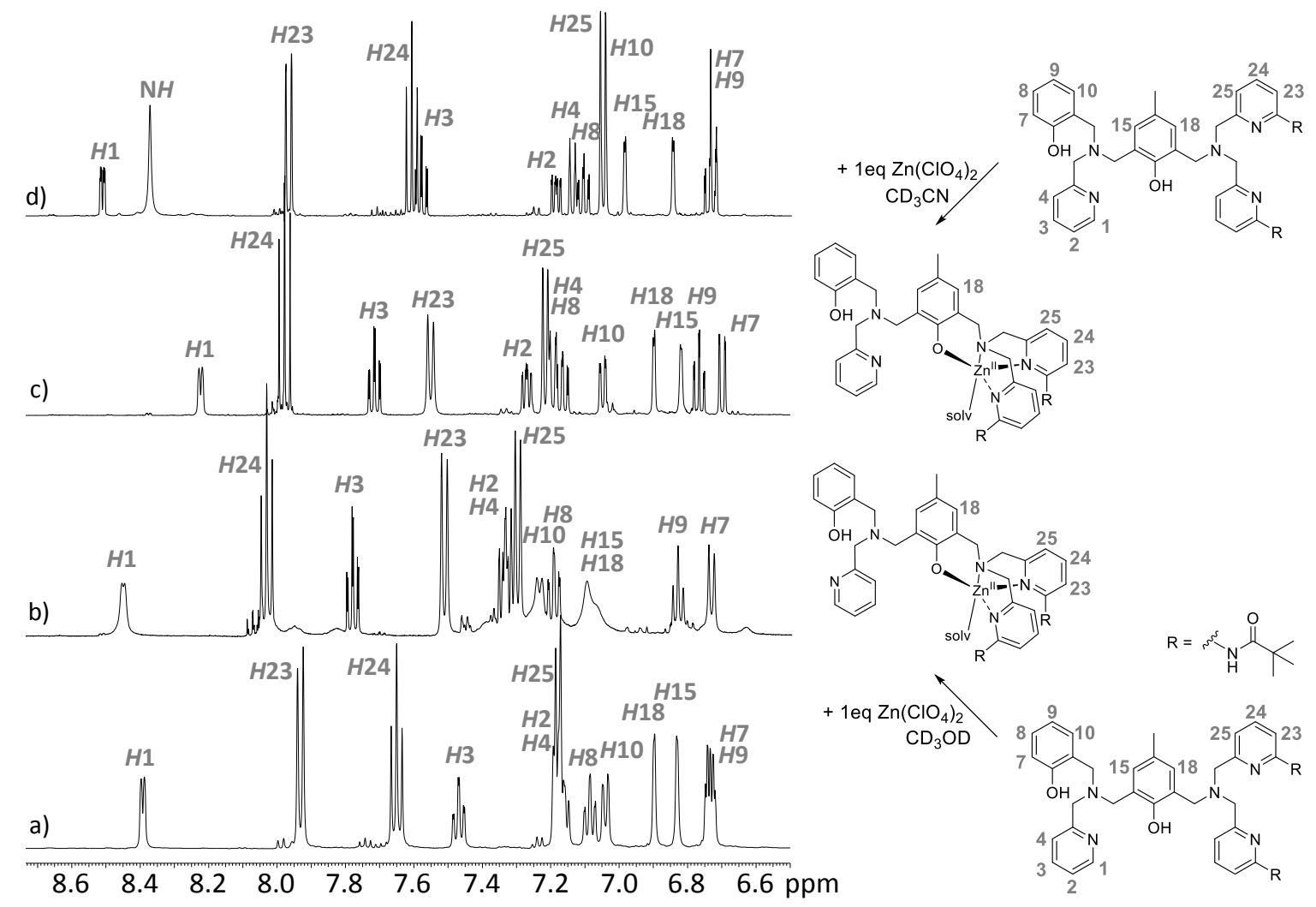

Figure 17: Comparison of the aromatic regions in the ${ }^{1} \mathrm{H}$ NMR of a) $\mathrm{H}_{4} \mathrm{SIM}^{3}$ in $\mathrm{CD}_{3} \mathrm{OD}$ with b) $\mathrm{H}_{4} \mathrm{SIM}^{3}$ with 1.0 eq $\mathrm{Zn}^{\prime \prime}\left(\mathrm{ClO}_{4}\right)_{2}$ in $\left.\mathrm{CD}_{3} \mathrm{OD}, \mathrm{c}\right) \mathrm{H}_{4} \mathrm{SIM}^{3}$ with 1.0 eq $\mathrm{Zn}^{\prime \prime}\left(\mathrm{ClO}_{4}\right)_{2}$ in $\mathrm{CD}_{3} \mathrm{CN} / \mathrm{D}_{2} \mathrm{O}$, and d) $\mathrm{H}_{4} \mathrm{SIM}^{3}$ in $\mathrm{CD}_{3} \mathrm{CN}$. 
Moreover, the methylene resonance assigned to $H 27$ was split into two doublets upon addition of zinc(II) perchlorate, confirming the coordination of $\mathrm{Zn}$ " to binding site C. A similar behavior was found when the experiment was performed in methanol. However, the chemical shifts of the resonances assigned to the pyridine residue in binding site $A$ differ in the two different solvents used (Figure 17).

When the experiment was undertaken in methanol the resonance of $H 1$ was only slightly affected (shifting by $0.05 \mathrm{ppm}$ ), but when acetonitrile was used as solvent and zinc(II) perchlorate was dissolved in deuterium oxide, this resonance was shifted by $0.3 \mathrm{ppm}$ upon formation of a monozinc(II) complex (which is not completely an effect of the change in polarity of the solvent as (i) further addition of zinc(II) perchlorate in deuterium oxide did not influence the chemical shift of this resonance, and (ii) the $H 1$ resonance assigned to the monozinc(II) complex was also detected at the same position after addition of only 0.5 eq of zinc(II) perchlorate and therefore less deuterium oxide). For the resonance assigned to $H 3$ the opposite behavior was observed; a larger shift in acetonitrile/deuterium oxide than in methanol. The resonances allocated to the terminal phenol residue in binding site $A$ did not change to this extent in both solvents used. Based on these findings the proposal of two differing complexes in the different solvents used with variation orientation of the pyridine residue in binding site A was made. Possibly, the orientation of the pyridine nitrogen atom in the direction of the $\mathrm{Zn}$ " center in acetonitrile/deuterium oxide are assisted by hydrogen bonding via deuterium oxide solvent molecules.

Interestingly, addition of a methanolic solution of zinc(II) acetate to ligand $\mathrm{H}_{4} \mathrm{SIM}^{3}$ in methanol led directly to a dizinc(II) complex. This was proposed due to the fact that the concentration of the new formed species only increased by half of the factor the concentration of $\mathrm{Zn}$ " was raised. The addition of 0.25 eq of $\mathrm{Zn}$ " resulted in an 0.125:0.875 mixture of new species and remaining free ligand. Although the resonances broadened during addition of two equivalents of $\mathrm{Zn}$ ", it is recognizable that the resonances of the terminal phenolate are shifted by the coordination of $\mathrm{Zn}$ "Figure 18). The ability of ligand $\mathrm{H}_{4} \mathrm{SIM}^{3}$ to form a dizinc(II) complex by reaction with zinc(II) acetate is additionally supported by the obtained crystal structure, which will be discussed in Chapter 4.3. 


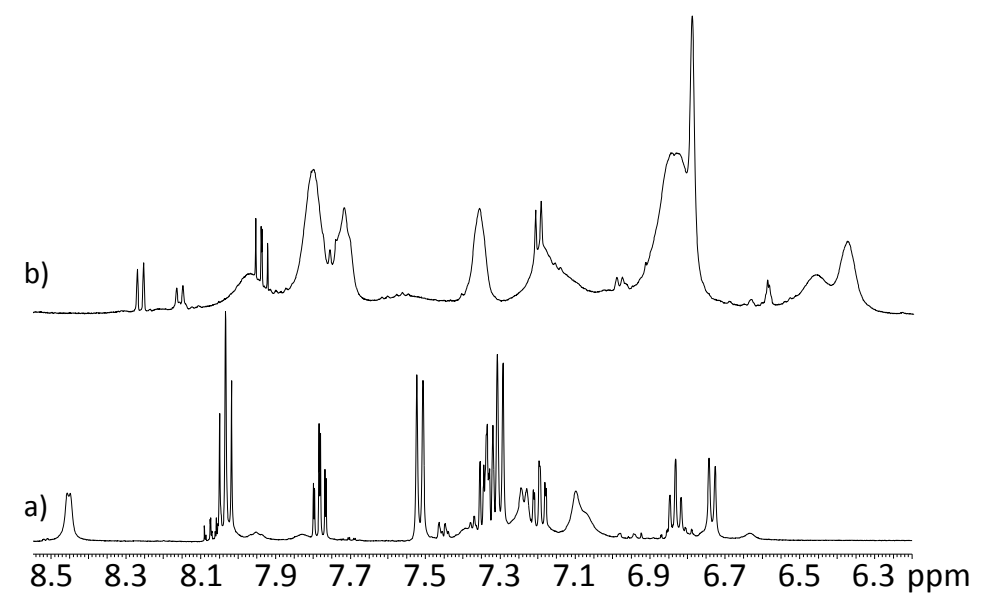

Figure 18: Comparison of the aromatic regions in the ${ }^{1} \mathrm{H} \mathrm{NMR}$ of $\mathrm{H}_{4} \mathrm{SIM}^{3}$ in $\mathrm{CD}_{3} \mathrm{OD}$ with a) 2.0 eq $\mathrm{Zn}^{\prime \prime}\left(\mathrm{ClO}_{4}\right)_{2}$ and b) 2.0 eq $\mathrm{Zn} "(\mathrm{OAc}) 2$.

\section{Mass Spectrometry}

In order to further investigate the composition of the species formed during the addition of zinc(II) salts to $\mathrm{H}_{4} \mathrm{SIM}^{3}$ containing solution samples were taken during the NMR titration at several addition steps and $\mathrm{ESI}^{+}$mass spectra were recorded. The main peaks detected in the spectra of mixtures up to a 1:1 ratio of $\mathrm{H}_{4} \mathrm{SIM}^{3}$ and zinc(II) perchlorate were found at $\mathrm{m} / \mathrm{z} 744.4$, which match the mass of metal-free ligand $\mathrm{H}_{4} \mathrm{SIM}^{3}$, and at $\mathrm{m} / \mathrm{z} 806.2$ assigned to the $\left[\mathrm{Zn}^{\prime \prime}\left(\mathrm{H}_{3} \mathrm{SIM}^{3}\right)\right]^{+}$ complex. Further addition of zinc(II) perchlorate led to an additional signal at $\mathrm{m} / \mathrm{z} 868.1$ assigned to the dizinc(II) complex $\left[\mathrm{Zn}_{2}{ }_{2}\left(\mathrm{HSIM}^{3}\right)\right]^{+}$, as well as to the signals at $\mathrm{m} / \mathrm{z} 403.7\left(\left[\mathrm{Zn}^{\prime \prime}\left(\mathrm{H}_{3} \mathrm{SIM}^{3}\right)\right]^{2+}\right)$ and $\mathrm{m} / \mathrm{z} 434.7\left(\left[\mathrm{Zn}_{2}{ }_{2}\left(\mathrm{HSIM}^{3}\right)\right]^{2+}\right)$. For the latter two signals the mass to charge ratio between the peaks in the isotopic pattern was decreased two fold, in accord with the formation of doubly charged species.

a)

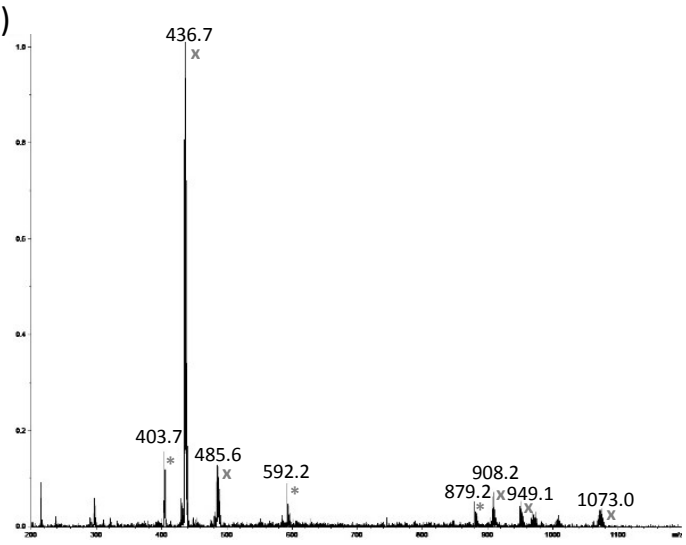

b)

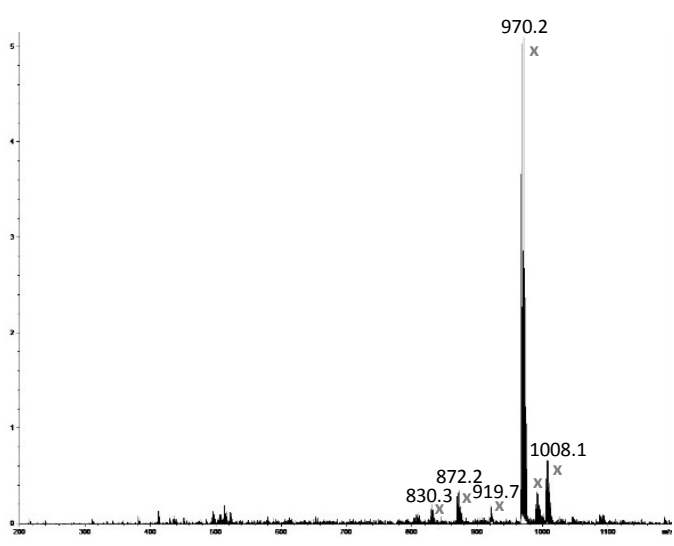

Figure 19: $\mathrm{ESI}^{+}$mass spectra of $\mathrm{H}_{4} \mathrm{SIM}^{3}$ with a) 2.0 eq $\mathrm{Zn}^{\prime \prime}\left(\mathrm{ClO}_{4}\right)_{2}$ and b) 2.0 eq $\mathrm{Zn}^{\prime \prime}(\mathrm{OAc})_{2}$ (asterisks mark signals with monozinc isotopic pattern, crosses mark signals with dizinc isotopic pattern).

The contribution of the species accountable for the signals detected was identified by their isotopic patterns, which were characteristic either for monozinc(II) or for dizinc(II) species, as 
explained in Chapter 3.2.1 (Figure 3a and 3b). During mass spectrometric investigations there is often the debate about the presence of dizinc(II) complexes already present in the sample solution, since the generation is also possible during the mass spectrometric measurement from the monozinc(II) complex in presence of $\mathrm{Zn}^{\prime \prime}$ ions. However, the constant detection of monozinc(II) complexes in mixtures with 2.0 eq (Figure 19a), and even 2.15 eq of zinc(II) perchlorate imply that the dizinc(II) complexes are generated during the mass spectrometric measurement. Based on this consideration, and taking into account the result from the ${ }^{1} \mathrm{H} N M R$ titration, the sole formation of a monozinc(II) complex without presence of a dizinc(II) complex by the addition of zinc(II) perchlorate to $\mathrm{H}_{4} \mathrm{SIM}^{3}$ is proposed.

In contrast, after addition of two equivalents of zinc(II) acetate to $\mathrm{H}_{4} \mathrm{SIM}^{3}$ an $\mathrm{ESI}^{+}$mass spectrum (Figure 19b) was recorded with one main peak at $\mathrm{m} / \mathrm{z} 966.2$ with an isotopic pattern characteristic for a dizinc(II) complex, assigned to $\left[\mathrm{Zn}_{2}{ }_{2}\left(\mathrm{H}_{2} \mathrm{SIM}^{3}\right)(\mathrm{OAc})\left(\mathrm{H}_{2} \mathrm{O}\right)\left(\mathrm{D}_{2} \mathrm{O}\right)\right]^{+}$, in the absence of signals corresponding to a monozinc(II) species. Therfore, it is apparent that the presence of acetate anions in solution favors the complexation of two $\mathrm{Zn}$ " ions by ligand $\mathrm{H}_{4} \mathrm{SIM}^{3}$. This observation is in agreement with the preparation of $\left[\mathrm{Zn}_{2}{ }_{2}\left(\mathrm{H}_{2} \mathrm{SIM}^{3}\right)(\mu-\mathrm{OAc})(\mathrm{OH})\right]$ in high yield which will be discussed in Chapter 4.2.

\subsubsection{COMPLEXATION STUDIES WITH GA ${ }^{\prime \prime \prime}$}

Comparable to the Gall' coordination studies of $\mathrm{H}_{3} \mathrm{SIM}^{1}$, the Gall' complexation by $\mathrm{H}_{4} \mathrm{SIM}^{3}$ was examined by NMR titration as well as mass spectrometric measurements.

\section{NMR Studies}

Gallium(III) perchlorate and gallium(III) nitrate were used as Gall! source for the ${ }^{1} \mathrm{H}$ NMR titration experiment. The coordination of Gall' ions by $\mathrm{H}_{4} \mathrm{SIM}^{3}$ was found to be less kinetically favored than $\mathrm{Zn}$ " coordination, as the mixtures of $\mathrm{H}_{4} \mathrm{SIM}^{3}$ and Gall needed to be heated for an hour at $50^{\circ} \mathrm{C}$ before the reaction was completed. Addition of one equivalent of gallium(III) perchlorate to $\mathrm{H}_{4} \mathrm{SIM}^{3}$ in methanol led to a new species, in which especially the resonances corresponding to binding site $A$ are shifted (Figure 20b) compared to the metal-free ligand. 
b)

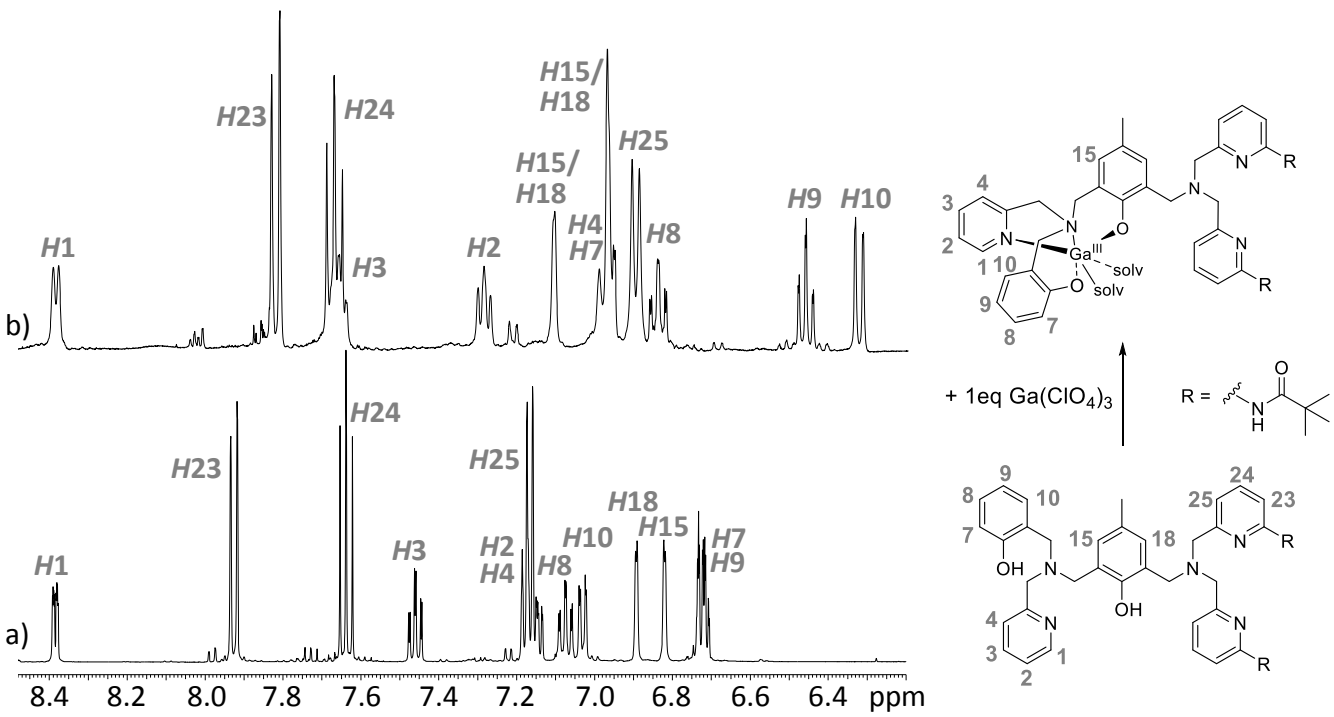

Figure 20: Comparison of the aromatic regions in the ${ }^{1} \mathrm{H} N M R$ of a) $\mathrm{H}_{4} \mathrm{SIM}^{3}$ in $\mathrm{CD}_{3} \mathrm{OD}$ with b) 2.0 eq $\mathrm{Ga}^{\text {III }}\left(\mathrm{ClO}_{4}\right)_{3}$.

Addition of two equivalents of gallium(III) nitrate in deuterium oxide to $\mathrm{H}_{4} \mathrm{SIM}^{3}$ in acetonitrile resulted in a very similar spectrum, suggesting that the formation of a digallium(III) complex of $\mathrm{H}_{4} \mathrm{SIM}^{3}$ is not possible and that the Gall ions are selectively bound to binding site $A$, independent of the gallium(III) salt and solvent used. This is in accordence with the results derived from the reaction of Ga"l' with $\mathrm{H}_{3} \mathrm{SIM}^{1}$, providing the identical binding site $\mathrm{C}$ (Chapter 3.2.2), suggesting that in this binding site Gall' ions are unlikely to be bound.

\section{Mass Spectrometry}

The inability of $\mathrm{H}_{4} \mathrm{SIM}^{3}$ to form a digallium(III) complex was also confirmed in the mass spectrum obtained with a 1:2 mixture of $\mathrm{H}_{4} \mathrm{SIM}^{3}$ with gallium(III) nitrate. The only major peak was detected at $\mathrm{m} / \mathrm{z} 810.2$ with a characteristic monogallium isotopic pattern corresponding to the $\left[\mathrm{Ga}^{\prime \prime \prime}\left(\mathrm{H}_{2} \mathrm{SIM}^{3}\right)\right]^{+}$complex.

\subsubsection{COMPLEXATION StUdies WITH FE}

The investigations with Gall' as Fe ${ }^{\text {III }}$ mimic showed that Gall' ions are only bound in binding site $\mathrm{A}$ of ligand $\mathrm{H}_{4} \mathrm{SIM}^{3}$, leaving binding site $\mathrm{C}$ vacant, as already observed with $\mathrm{H}_{3} \mathrm{SIM}^{1}$. In order to verify this proposal, the coordination of $\mathrm{Fe}^{\mathrm{III}}$ ions by ligand $\mathrm{H}_{4} \mathrm{SIM}^{3}$ was examined by $\mathrm{X}$-ray diffraction and mass spectrometric analysis. 


\section{X-ray Diffraction}

Treatment of $\mathrm{H}_{4} \mathrm{SIM}^{3}$ in methanol $(27 \mathrm{mM}$ ) with iron(III) perchlorate (2.0 eq) and heating for $15 \mathrm{~min}$ at $50^{\circ} \mathrm{C}$ resulted in a purple solution. Following addition of sodium tetrafluoroborate (4.0 eq) and diethylether diffusion yielded small black crystals, suitable for X-ray data collection. Figure 21 shows a plot of the molecular structure and Table 6 gives selected structural parameters.
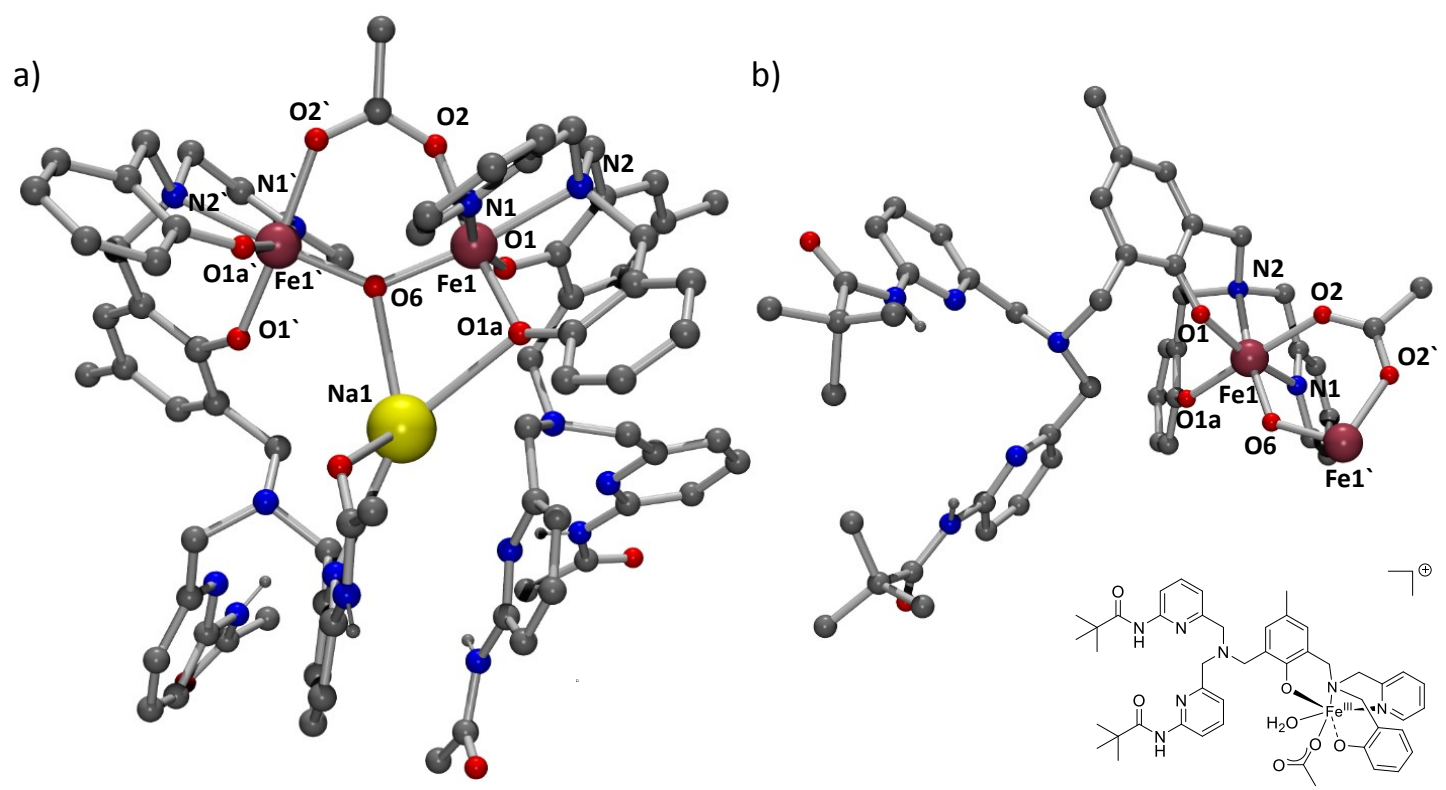

Figure 21: Structure of a) $\left\{\mathrm{Na}\left[\mathrm{Fe}{ }^{\prime \prime \prime}\left(\mathrm{H}_{2} \mathrm{SIM}^{3}\right)\right]_{2}(\mu-\mathrm{OAc})\left(\mu-\mathrm{OH}_{2}\right)\right\}^{+}$and b) its monomeric section (counter ions, noncoordinated solvent molecules, and hydrogen atoms, not involved in hydrogen bonding, have been omitted for clarity; crystallographic data and ORTEP plots with 50\% probability level of thermal ellipsoids appear in the Appendix, Table 31 and Figure 73).

Table 6: Selected bond lengths $(\AA)$ and angles $\left(^{\circ}\right)$ for $\left\{\mathrm{Na}\left[\mathrm{Fe}^{\prime \prime \prime}\left(\mathrm{H}_{2} \mathrm{SIM}^{3}\right)\right]_{2}(\mu-\mathrm{OAc})\left(\mu-\mathrm{OH}_{2}\right)\right\}\left(\mathrm{BF}_{4}\right)_{2}$.

\begin{tabular}{|c|c|c|}
\hline & $\multicolumn{2}{|c|}{\mathrm{Na}\left[\mathrm{Fe}^{\mathrm{e}}{ }^{\prime \prime \prime}\left(\mathrm{H}_{2} \mathrm{SIM}^{3}\right)\right]_{2}(\mu-\mathrm{OAc})\left(\mu-\mathrm{OH}_{2}\right)}^{2+}$ \\
\hline & $\mathrm{Fe}(1)$ & $\mathrm{Fe}\left(1^{\prime}\right)^{\mathrm{a}}$ \\
\hline $\mathrm{Fe}(1) \cdots \mathrm{Fe}\left(1^{\prime}\right)$ & \multicolumn{2}{|c|}{3.567} \\
\hline $\mathrm{Fe}(1)-\mathrm{O}(1)$ & $1.918(6)$ & $1.936(5)$ \\
\hline $\mathrm{Fe}(1)-\mathrm{O}(1 \mathrm{~A})$ & $1.971(5)$ & $1.870(6)$ \\
\hline $\mathrm{Fe}(1)-\mathrm{O}(2)$ & $2.019(5)$ & $2.063(6)$ \\
\hline $\mathrm{Fe}(1)-\mathrm{N}(1)$ & $2.152(7)$ & $2.137(7)$ \\
\hline $\mathrm{Fe}(1)-\mathrm{N}(2)$ & $2.201(7)$ & $2.206(6)$ \\
\hline $\mathrm{Fe}(1)-\mathrm{O}(6)$ & $1.943(6)$ & $1.933(5)$ \\
\hline $\mathrm{Fe}(1)-\mathrm{O}(6)-\mathrm{Fe}\left(1^{\prime}\right)$ & \multicolumn{2}{|c|}{$134.0(3)$} \\
\hline $\mathrm{O}(1)-\mathrm{Fe}(1)-\mathrm{N}(1)$ & $163.6(3)$ & $86.4(2)$ \\
\hline $\mathrm{O}(1)-\mathrm{Fe}(1)-\mathrm{N}(2)$ & $86.1(3)$ & $88.7(2)$ \\
\hline$O(1)-F e(1)-O(1 A)$ & $95.3(2)$ & $95.5(2)$ \\
\hline $\mathrm{O}(1)-\mathrm{Fe}(1)-\mathrm{O}(2)$ & $94.4(2)$ & $170.8(2)$ \\
\hline $\mathrm{O}(1)-\mathrm{Fe}(2)-\mathrm{O}(6)$ & $100.5(2)$ & $91.9(2)$ \\
\hline
\end{tabular}

The structure reveals a diiron(III) core bridged by an aqua and an acetate co-ligand. Both Fe ${ }^{\text {III }}$ centers are additionally coordinated by a pyridine and two phenolate moieties provided by two deprotonated $\mathrm{H}_{4} \mathrm{SIM}^{3}$ ligand molecules. Furthermore, the crystal cell of 
$\left\{\mathrm{Na}\left[\mathrm{Fe}^{\prime \prime \prime}\left(\mathrm{H}_{2} \mathrm{SIM}^{3}\right)\right]_{2}(\mu-\mathrm{OAc})\left(\mu-\mathrm{OH}_{2}\right)\right\}\left(\mathrm{BF}_{4}\right)_{2}$ includes a sodium ion and a tetrafluoroborate counterion. A similar dimeric structure of an Fe"ll complex was published by Que et al., obtained with the mononucleating ligand $\mathrm{N}$-(o-hydroxybenzyl)- $\mathrm{N}, \mathrm{N}$-bis(2-pyridylmethyl)amine (HDP, $\left.\mathrm{HL}^{18}\right)$. This published structure of $\left[\mathrm{Fe}_{2}{ }_{2}{ }_{2}\left(\mathrm{~L}^{18}\right)_{2}(\mu-\mathrm{O})(\mu-\mathrm{OBz})\right] \mathrm{BPh}_{4}$ is formed by a $\left(\mu\right.$-oxo)( $\mu$-benzoate)diiron(III) core. $^{183}$

The two Fe ${ }^{\text {III }}$ ions in $\left\{\mathrm{Na}\left[\mathrm{Fe}^{\prime \prime \prime}\left(\mathrm{H}_{2} \mathrm{SIM}^{3}\right)\right]_{2}(\mu-\mathrm{OAc})\left(\mu-\mathrm{OH}_{2}\right)\right\}\left(\mathrm{BF}_{4}\right)_{2}$, separated by $3.567 \AA$, are coordinated in the harder binding site $A$ of the dinucleating ligand $\mathrm{H}_{4} \mathrm{SIM}^{3}$ and reveal short Fe-O distances (average $1.957 \AA$ ), comparable to those of published complexes of $\mathrm{H}_{2} \mathrm{~L}^{4}$, in which $\mathrm{Fe} \mathrm{e}^{\text {III }}$ is also coordinated in binding site A. Examples are $\left[\mathrm{Fe}^{\mathrm{III}} \mathrm{Fe}^{\prime \prime}\left(\mathrm{L}^{4}\right)(\mu-\mathrm{OAC})_{2}\right]^{+}(1.967 \AA),{ }^{140}$

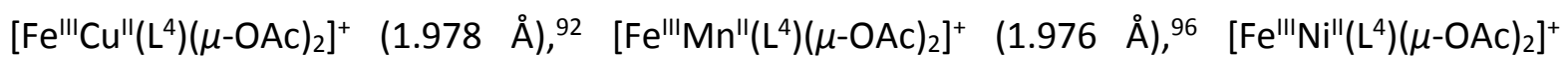

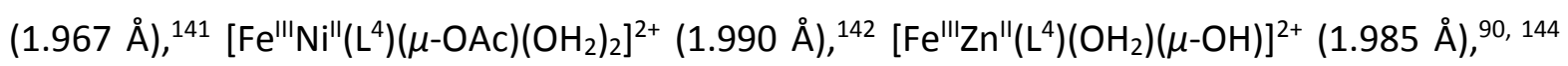
$\left[\mathrm{Fe}^{\prime \prime \prime} \mathrm{Cd}^{\prime \prime}\left(\mathrm{L}^{4}\right)(\mu-\mathrm{OAc})_{2}\right]^{+}(1.978 \AA),{ }^{145}\left[\left[\mathrm{Fe}^{\prime \prime \prime} \mathrm{Hg}^{\prime \prime}\left(\mathrm{L}^{4}\right)(\mu-\mathrm{OH})\right]_{2}\right]^{2+}(1.985 \AA),{ }^{145}$ and $\left[\mathrm{Fe}^{\prime \prime \prime} \mathrm{Co}^{\prime \prime}\left(\mathrm{L}^{4}\right)(\mu-\mathrm{OAc})_{2}\right]^{+}$ $\left(1.971 \AA\right.$ A). ${ }^{146}$ However, it should be mentioned that the metal ion coordinated in the second coordination site may have an effect on the average $\mathrm{Fe}^{\mathrm{III}}$-O distance.

The structure of $\left\{\mathrm{Na}\left[\mathrm{Fe}^{\prime \prime \prime}\left(\mathrm{H}_{2} \mathrm{SIM}^{3}\right)\right]_{2}(\mu-\mathrm{OAc})\left(\mu-\mathrm{OH}_{2}\right)\right\}\left(\mathrm{BF}_{4}\right)_{2}$ demonstrates that the Fe ${ }^{\text {III }}$ ion is preferably coordinated in the harder binding site $A$, which is in agreement with the NMR titration experiments performed with Gall' as an Fe ${ }^{\text {III }}$ mimic. Moreover, although an excess of iron(III) perchlorate was present in solution, binding site $\mathrm{C}$ was left vacant during the crystallization. The structure of $\left[\mathrm{NaFe}^{\prime \prime \prime}\left(\mathrm{H}_{2} \mathrm{SIM}^{1}\right)(\mu-\mathrm{OAc})_{2}\right] \mathrm{PF}_{6}$, which will be discussed in Chapter 5.3 in more detail, also carries the $\mathrm{Fe}^{\mathrm{III}}$ ion only in the non-amidated binding site of $\mathrm{H}_{3} \mathrm{SIM}^{1}$, while binding site $\mathrm{C}$ was occupied by a $\mathrm{Na}^{+}$ion. Therefore, the coordination of $\mathrm{Fe}^{\text {III }}$ in binding site $\mathrm{C}$ seems to be unfavored.

\section{Mass Spectrometry}

A methanolic solution of $\mathrm{H}_{4} \mathrm{SIM}^{3}(6.86 \mathrm{mM})$ was treated with two equivalents of sodium hydroxide and one equivalent of iron(II) tetrafluoroborate and the mixture was stirred for 14 hours under aerobic conditions (investigations with UV-vis-NIR spectroscopy and NMR spectroscopy revealed the completed oxidation of $\mathrm{Fe}^{\mathrm{Il}}$ to $\mathrm{Fe}^{\mathrm{III}}$ after this period of time). The recorded $\mathrm{ESI}^{+}$mass spectrum of this mixture (Figure 22a) shows one main signal at $\mathrm{m} / \mathrm{z} 797.3$, which matches the mass of the $\left[\mathrm{Fe}^{\text {III }}\left(\mathrm{H}_{2} \mathrm{SIM}^{3}\right)\right]^{+}$complex and shows the characteristic pattern for a monoiron complex (Figure 23a). The detection of a monoiron complex supports the finding obtained from the ${ }^{1} \mathrm{H}$ NMR titration with Gal' as an Fe ${ }^{\text {III }}$ mimic as well as from the X-ray diffraction analysis, that Ga"l' ions can only be bound in one coordination site, namely in binding site A. This proposal is supported by the UV-vis-NIR spectroscopic titration of $\mathrm{H}_{3} \mathrm{SIM}^{1}$, which contains also binding site $\mathrm{C}$, with iron(III) perchlorate as well as the ${ }^{1} \mathrm{H}$ NMR titration and the $\mathrm{X}$-ray structural 
analysis of $\mathrm{H}_{3} \mathrm{SIM}^{1}$ with Ga"II as an Fe"II mimic, which revealed that $\mathrm{Fe}^{\text {III }}$ and Gal' cannot be bound in the hindered binding site $\mathrm{C}$.
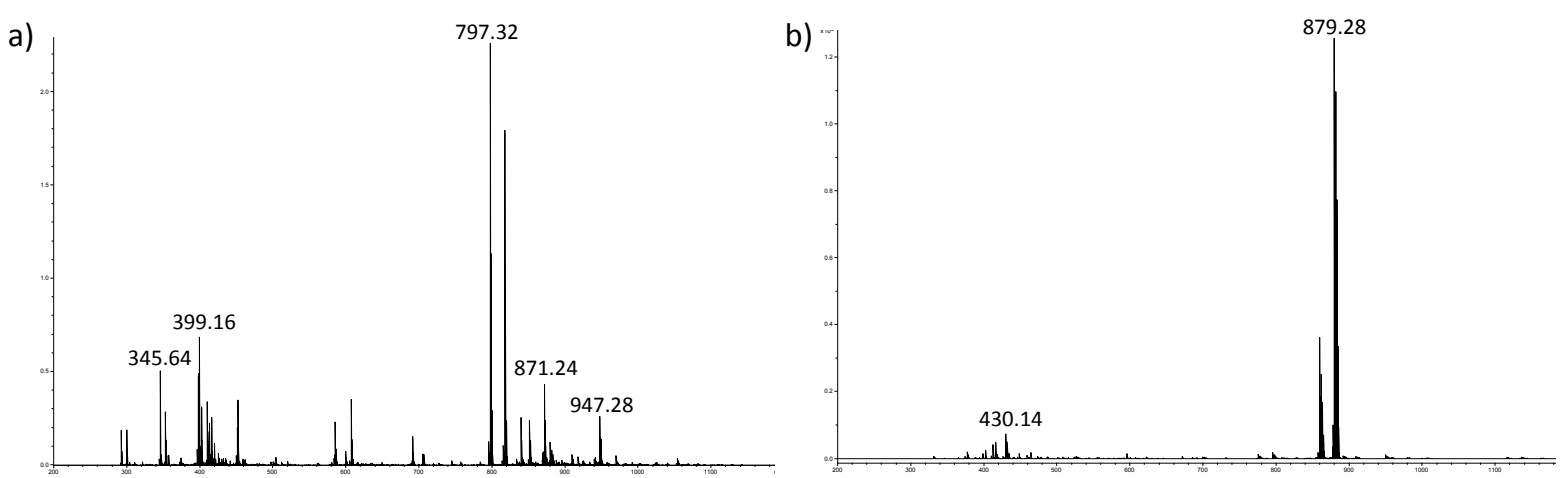

Figure 22: $\mathrm{ESI}^{+}$mass spectra of $\mathrm{H}_{4} \mathrm{SIM}^{3}$ with a) 1.0 eq Fe" $\left(\mathrm{BF}_{4}\right)_{2}$ after 14 hours exposure to air and b) 1.0 eq $\mathrm{Fe}^{\prime \prime}\left(\mathrm{BF}_{4}\right)_{2}$ after 14 hours of exposure to air and addition of $1.0 \mathrm{eq} \mathrm{Zn}$ "(OMe $)_{2}$ in methanol.
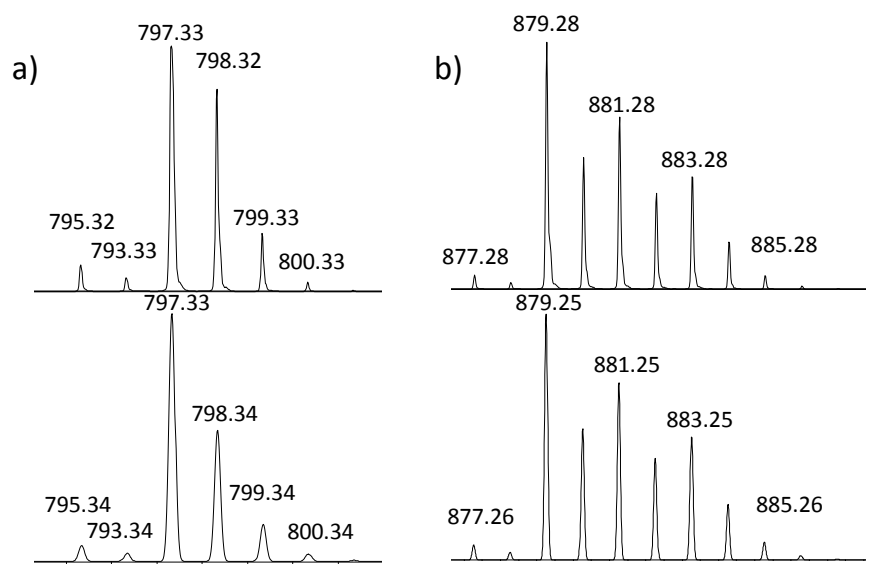

Figure 23: Characteristic isotopic pattern found in $\mathrm{ESI}^{+}$mass spectra (experimental (top) and calculated (bottom)) of $\mathrm{H}_{4} \mathrm{SIM}^{3}$ with a) 1 eq of Fe" $\left(\mathrm{BF}_{4}\right)_{2}$ (after 14 hours of exposure to air) $\left(\left[\mathrm{Fe}^{\prime \prime \prime}\left(\mathrm{H}_{2} \mathrm{SIM}^{3}\right)^{+}\right)\right.$and b) 1 eq of $\mathrm{Fe}^{\prime \prime}\left(\mathrm{BF}_{4}\right)_{2}$ (after 14 hours of exposure to air) and 1 eq of $\mathrm{Zn}^{\prime \prime}(\mathrm{OMe})_{2}\left(\left[\mathrm{Fe}^{\prime \prime \prime} \mathrm{Zn} \mathrm{n}^{\prime \prime}\left(\mathrm{HSIM}^{3}\right) \mathrm{F}\right]^{+}\right)$in methanol.

Moreover, one equivalent of zinc(II) methoxide was added after 14 hours to the mixture of $\mathrm{H}_{4} \mathrm{SIM}^{3}$ and one equivalent of iron(II) tetrafluoroborate under aerobic conditions. The resulting $\mathrm{ESI}^{+}$mass spectrum (Figure 21b) contains signals with a characteristic FeZn isotopic pattern (Figure 22b), besides the small signal at $\mathrm{m} / \mathrm{z} 797.3$ stemming from residues of the monoiron complex. The FeZn signals at $\mathrm{m} / \mathrm{z} 430.1, \mathrm{~m} / \mathrm{z} 859.2$ and $\mathrm{m} / \mathrm{z} 879.3$ were assigned to $\left[\mathrm{Fe}^{\prime \prime \prime} \mathrm{Zn}^{\prime \prime}\left(\mathrm{HSIM}^{3}\right)\right]^{2+}, \quad\left[\mathrm{Fe}^{\prime \prime \prime} \mathrm{Zn}{ }^{\prime \prime}\left(\mathrm{SIM}^{3}\right)\right]^{+}$and $\left[\mathrm{Fe}^{\prime \prime \prime} \mathrm{Zn} n^{\prime \prime}\left(\mathrm{HSIM}^{3}\right) \mathrm{F}\right]^{+}$. This indicates that $\mathrm{Zn}^{\prime \prime}$ ions are selectively coordinated in the softer vacant binding site $C$, while the harder binding site $A$ is occupied by the harder Fe ${ }^{\text {III }}$ ion, generating an accurate model for the FeZn active site of PAPs. 


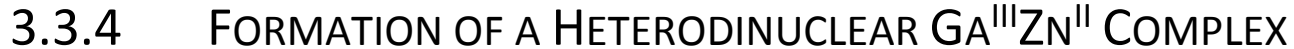

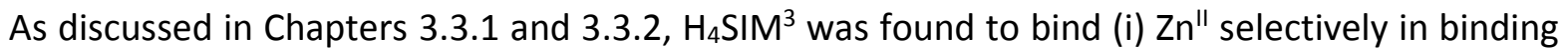
site $\mathrm{C}$, and (ii) Gall' in the harder binding site A. These findings are in agreement with the observed crystal structure of $\left[\mathrm{Ga}^{\prime \prime \prime} \mathrm{Zn} \mathrm{n}^{\prime \prime}\left(\mathrm{L}^{4}\right)(\mu-\mathrm{OAc})_{2}\right]^{+}{ }^{66}$ This ligand lacks the pivaloyl-amide residues at the 6-position of the pyridine residues compared to $\mathrm{H}_{4} \mathrm{SIM}^{3}$, but provides a similar primary coordination sphere. In [Ga"' $\left.\mathrm{Zn}^{\prime \prime}\left(\mathrm{L}^{4}\right)(\mu-\mathrm{OAc})_{2}\right]^{+}, \mathrm{Ga}^{\prime \prime \prime}$ is bound in the harder binding $\mathrm{N}_{2} \mathrm{O}_{4}$-binding site and $\mathrm{Zn}$ " is coordinated in the softer $\mathrm{N}_{3} \mathrm{O}_{3}$-binding site. ${ }^{66}$ The possibility to generate a Ga"' $\mathrm{Zn}$ " complex with $\mathrm{H}_{4} \mathrm{SIM}^{3}$, in which the two different metal ions are bound in distinct binding sites, was investigated via NMR spectroscopy and mass spectrometry.

\section{NMR Studies}

Ligand $\mathrm{H}_{4} \mathrm{SIM}^{3}$, dissolved in acetonitrile, was treated with one equivalent of zinc(II) perchlorate dissolved in deuterium oxide and the formation of the monozinc(II) complex was confirmed by the measurement of a ${ }^{1} \mathrm{H}$ NMR spectrum (Figure $24 \mathrm{~b}$ ). Afterwards, gallium(III) nitrate, dissolved in deuterium oxide, was added stepwise and the changes were followed using NMR spectroscopy. After addition of one equivalent of gallium(III) nitrate to the monozinc(II) complex of $\mathrm{H}_{4} \mathrm{SIM}^{3}$ one new main species is visible in the ${ }^{1} \mathrm{H}$ NMR spectrum, in addition to at least three other new species (Figure 24c).

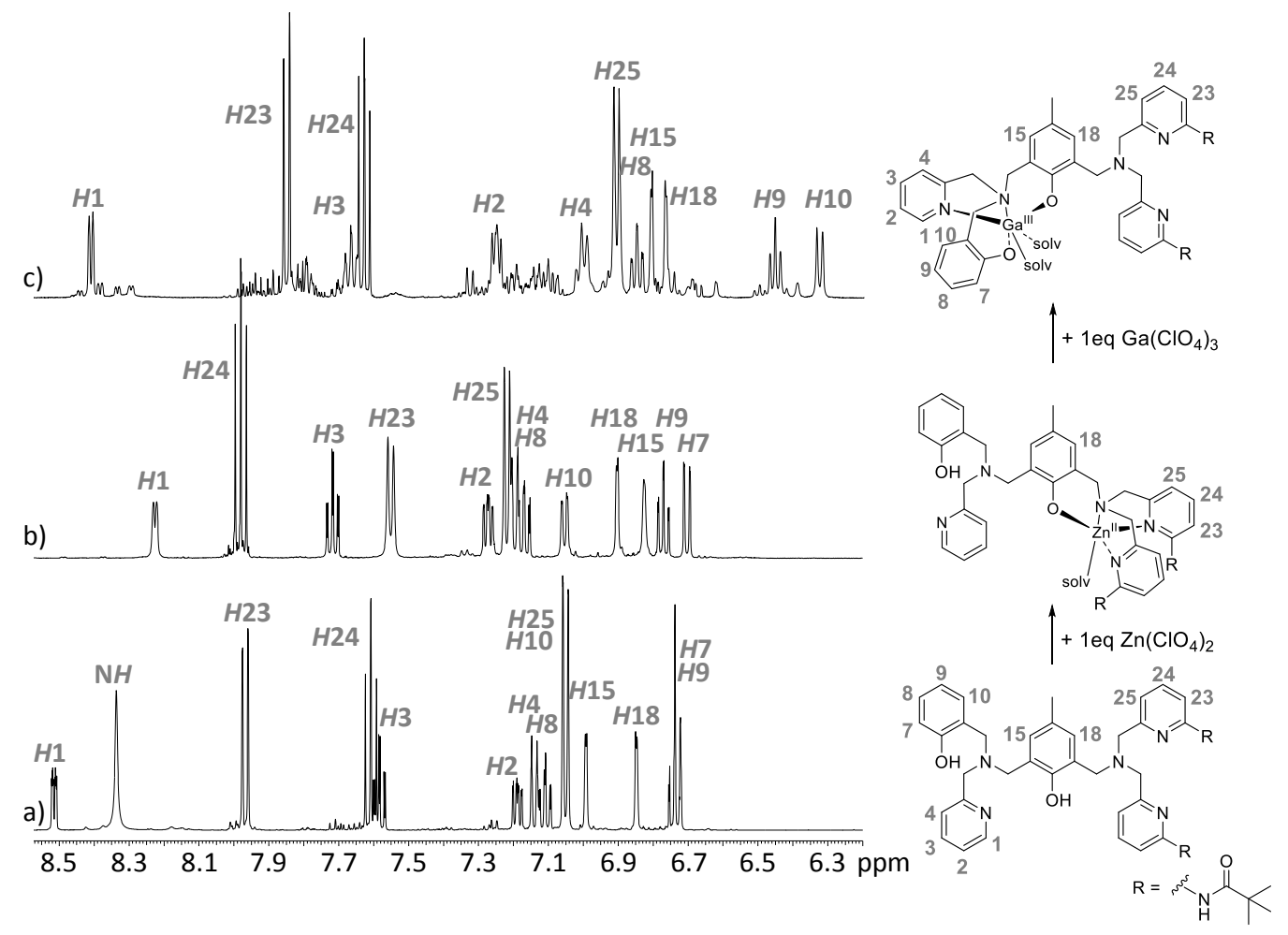

Figure 24 Comparison of the aromatic regions in the ${ }^{1} \mathrm{H} N M R$ of a) $\mathrm{H}_{4} \mathrm{SIM}^{3}$ in $\mathrm{CD}_{3} \mathrm{CN}$, b) $\mathrm{H}_{4} \mathrm{SIM}^{3}$ with 1.0 eq of $\mathrm{Zn}^{\prime \prime}\left(\mathrm{ClO}_{4}\right)_{2}$ in $\mathrm{CD}_{3} \mathrm{CN} / \mathrm{D}_{2} \mathrm{O}$, and c) $\mathrm{H}_{4} \mathrm{SIM}^{3}$ with 1.0 eq of $\mathrm{Zn}^{\prime \prime}\left(\mathrm{ClO}_{4}\right)_{2}$ and 1.0 eq of $\mathrm{Ga}{ }^{\prime \prime \prime}\left(\mathrm{ClO}_{4}\right)_{3}$ in $\mathrm{CD}_{3} \mathrm{CN}_{2} / \mathrm{D}_{2} \mathrm{O}$. 
The resonances of $H 9$ and $H 10$ are shifted upfield similar as found in the monogallium(III) complex spectrum. Furthermore, the resonances assigned to $H 23, H 24$ and $H 25$ have also a similar chemical shift in the monogallium(III) complex. Therefore, the formation of the monogallium(III) complex, lacking the $\mathrm{Zn}$ "ion in the second coordination site, is proposed during this experiment.

Interestingly, the analogous experiment undertaken in methanol gave a different result. Although the addition of gallium(III) perchlorate to the monozinc(II) complex also resulted in the formation of three species the chemical shifts of the resonances assigned to $H 23, H 24$ and $H 25$ did not result in the identical chemical shifts of those measured in the monogallium(III) complex. Moreover, when the experimental procedure was changed, i.e. when zinc(II) perchlorate was added to the monogallium(III) complex, which had been generated in methanol prior to the addition of the zinc(II) ion (Figure 25a) only one new species was detected (Figure 25c, d). In order to preserve complete conversion to the Ga"' $\mathrm{Zn}^{\text {" }}$ complex treatment with one equivalent of base ( $\mathrm{NaOD}$ in $\mathrm{D}_{2} \mathrm{O}$ ) was necessary (Figure $25 \mathrm{~d}$ ). To confirm that the base was responsible for the complexation in the latter experiment, the addition of $\mathrm{D}_{2} \mathrm{O}$ was tested in a separate experiment. Addition of $\mathrm{D}_{2} \mathrm{O}$ was found to have no influence on the species distribution.

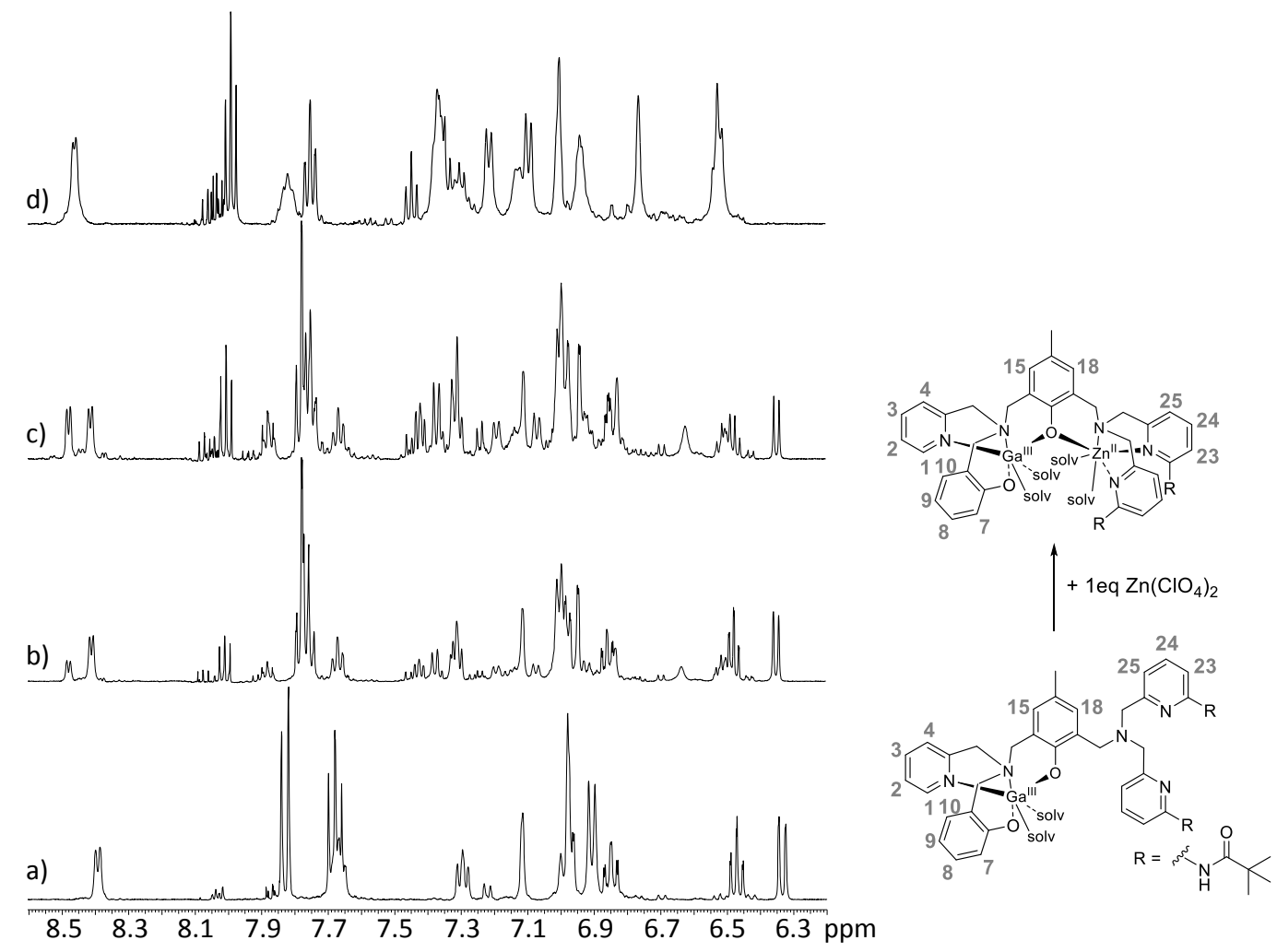

Figure 25: Comparison of the aromatic regions in the ${ }^{1} \mathrm{H}$ NMR of a) $\mathrm{H}_{4} \mathrm{SIM}^{3}$ with 1.1 eq of $\mathrm{Ga}$ '"' $\left(\mathrm{ClO}_{4}\right)_{3}$ in $\mathrm{CD}_{3} \mathrm{OD}$, b) $\mathrm{H}_{4} \mathrm{SIM}^{3}$ with 1.1 eq of $\mathrm{Ga}^{\prime \prime \prime}\left(\mathrm{ClO}_{4}\right)_{3}$ and 0.5 eq of $\mathrm{Zn}^{\prime \prime}\left(\mathrm{ClO}_{4}\right)_{2}$ in $\mathrm{CD}_{3} \mathrm{OD}$, c) $\mathrm{H}_{4} \mathrm{SIM}^{3}$ with 1.1 eq of $\mathrm{Ga}^{\prime \prime \prime}\left(\mathrm{ClO}_{4}\right)_{3}$ and 1.0 eq of $\mathrm{Zn}^{\prime \prime}\left(\mathrm{ClO}_{4}\right)_{2}$ heated to $60^{\circ} \mathrm{C}$ for $8 \mathrm{~h}$, and d) $\mathrm{H}_{4} \mathrm{SIM}^{3}$ with 1.1 eq of $\mathrm{Ga}^{\prime \prime \prime}\left(\mathrm{ClO}_{4}\right)_{3}, 1.0$ eq of $\mathrm{Zn}^{\prime \prime}\left(\mathrm{ClO}_{4}\right)_{2}$ and 1.0 eq of $\mathrm{NaOD}$ in $\mathrm{CD}_{3} \mathrm{OD} / \mathrm{D}_{2} \mathrm{O}$. 


\section{Mass Spectrometry}

The mass spectrometric investigation of $\mathrm{H}_{4} \mathrm{SIM}^{3}$ treated with zinc(II) perchlorate and gallium(III) nitrate showed three main peaks in the spectrum at $\mathrm{m} / \mathrm{z} 437.6, \mathrm{~m} / \mathrm{z} 446.6$ and $\mathrm{m} / \mathrm{z}$ 992.1, all three displaying a GaZn isotopic pattern. The peaks were assigned to the complexes $\left[\mathrm{Ga}^{\prime \prime \prime} \mathrm{Zn} "\left(\mathrm{HSIM}^{3}\right)\right]^{2+},\left[\mathrm{Ga}^{\prime \prime \prime} \mathrm{Zn} "\left(\mathrm{HSIM}^{3}\right)\left(\mathrm{H}_{2} \mathrm{O}\right)\right]^{2+}$, and $\left[\mathrm{Ga}^{\prime \prime \prime} \mathrm{Zn}^{\prime \prime}\left(\mathrm{H}_{2} \mathrm{SIM}^{3}\right)\left(\mathrm{CH}_{3} \mathrm{CN}\right)(\mathrm{OH})_{2}\right]^{+}$.

\subsection{COMPLEXATION BehaVIOR OF LIGAND $\mathrm{H}_{4} \mathrm{SIM}^{4}$}

$\mathrm{H}_{4} \mathrm{SIM}^{4}$ bears two chemically distinct coordination sites and the primary coordination sphere is comparable to $\mathrm{H}_{4} \mathrm{SIM}^{3}$. However, the second coordination sphere in $\mathrm{H}_{4} \mathrm{SIM}^{4}$ provides hydrogen bonding proximal both binding sites, while $\mathrm{H}_{4} \mathrm{SIM}^{4}$ only introduced hydrogen bonding adjacent to the softer coordination site.

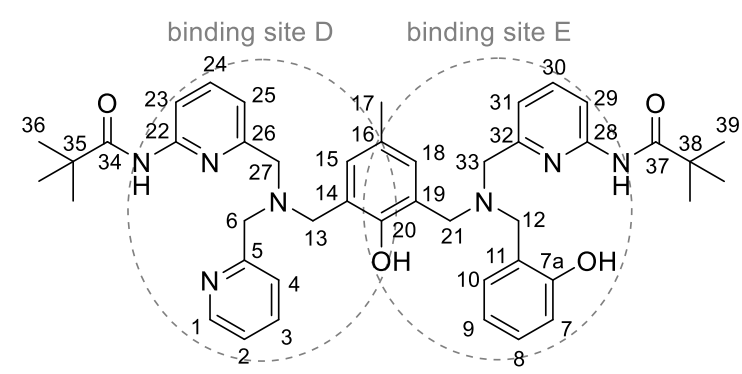

Chart 11: Assignment of the binding sites provided by ligand $\mathrm{H}_{4} \mathrm{SIM}^{4}$ and the numbering of its atoms.

In fact, the two sterically demanding pivaloyl-amide moieties are attached to both side-arms in $\mathrm{H}_{4} \mathrm{SIM}^{4}$ leading to two rather equally accessible binding sites, contrary to $\mathrm{H}_{4} \mathrm{SIM}^{3}$.

\subsubsection{COMPLEXATION STUDIES WITH ZN"}

The binding behavior of ligand $\mathrm{H}_{4} \mathrm{SIM}^{4}$ with $\mathrm{Zn}$ " ions was investigated by NMR titration and mass spectrometric measurements, as with $\mathrm{H}_{3} \mathrm{SIM}^{1}$ and $\mathrm{H}_{4} \mathrm{SIM}^{3}$ (Chapter 3.2.1 and 3.3.1).

\section{NMR Studies}

The formation of a monozinc(II) complex was monitored during the addition of zinc(II) perchlorate to a solution of $\mathrm{H}_{4} \mathrm{SIM}^{4}$ in methanol. Next to the resonances assigned to the metalfree ligand, resonances of a new species appeared in mixtures with low $\mathrm{Zn}$ " ion concentration. Finally, in the ${ }^{1} \mathrm{H}$ NMR spectrum obtained with the 1:1 mixture of zinc(II) perchlorate and $\mathrm{H}_{4} \mathrm{SIM}^{4}$ the resonances corresponding to the monozinc(II) complex of $\mathrm{H}_{4} \mathrm{SIM}^{4}$ were the only resonances left, while the resonances of the metal-free ligand disappeared. The resonances assigned to the non-amidated pyridine residue and one of the two amidated-pyridine residues revealed shifts in 
the spectrum of the monozinc(II) complex of $\mathrm{H}_{4} \mathrm{SIM}^{4}$ compared to the metal-free ligand, while the resonances of the other two side-arm moieties did not show pronounced changes (Figure 26). This suggests the coordination of $\mathrm{Zn}^{\|}$ions in binding site $\mathrm{D}$ of $\mathrm{H}_{4} \mathrm{SIM}^{4}$. Increase of the $\mathrm{Zn}$ "I concentration to a ratio higher than 1:1 did not lead to changes in the recorded ${ }^{1} \mathrm{H}$ NMR spectrum indicating the selective formation of the monozinc(II) complex. However, when the NMR titration experiment was conducted in acetonitrile as solvent the formation of the monozinc(II) complex could also be detected, but addition of further zinc(II) perchlorate to the monozinc(II) complex resulted in a mixture with a second species, most likely the dizinc(II) complex, but complete conversion could not be detected.

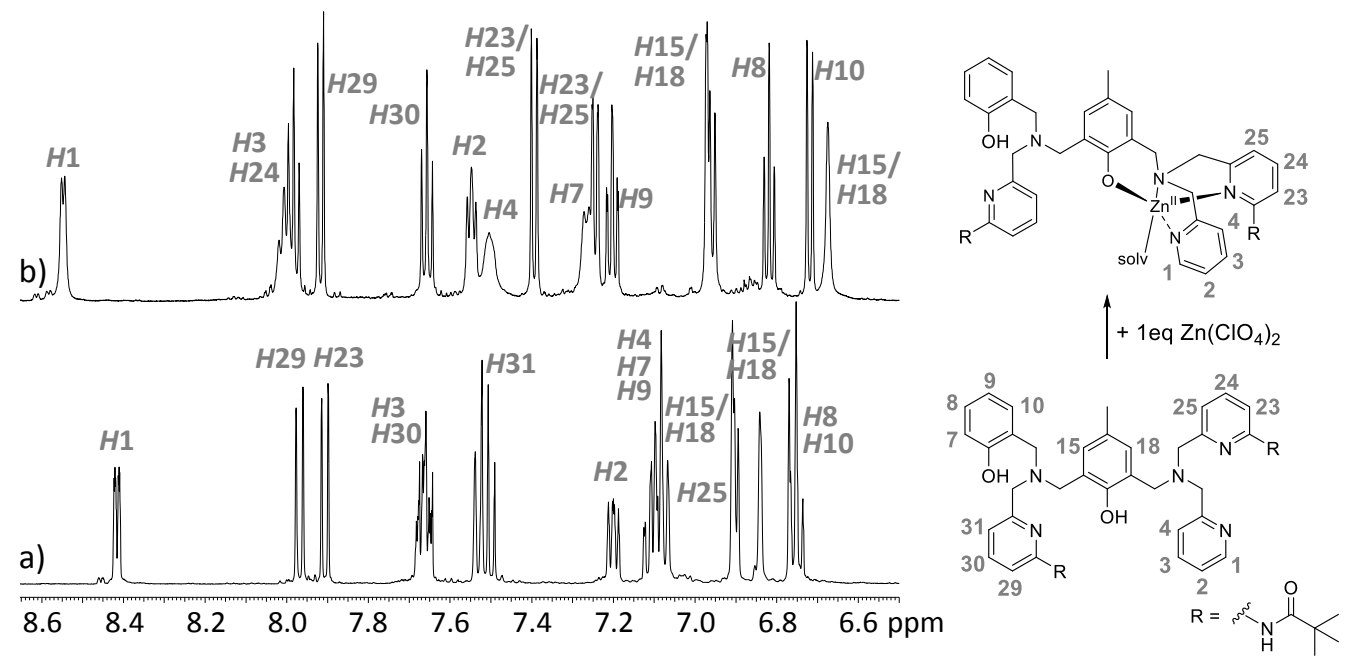

Figure 26: Comparison of the aromatic regions in the ${ }^{1} \mathrm{H} N M R$ of a) $\mathrm{H}_{4} \mathrm{SIM}^{4}$ in $\mathrm{CD}_{3} \mathrm{OD}$ with b) 1.0 eq of $\mathrm{Zn}^{\prime \prime}\left(\mathrm{ClO}_{4}\right)_{2}$.

\section{Mass Spectrometry}

$\mathrm{ESI}^{+}$mass spectrometric measurements were conducted in parallel to the ${ }^{1} \mathrm{H}$ NMR spectroscopic investigations regarding the $\mathrm{Zn}^{\prime \prime}$ coordination behavior of $\mathrm{H}_{4} \mathrm{SIM}^{4}$ by taking samples throughout the experiments. The spectrum recorded after addition of one equivalent of $\mathrm{Zn}^{\prime \prime}$ ions in acetonitrile showed a main peak at $\mathrm{m} / \mathrm{z} 806.4$ associated with the monozinc(II) complex $\left[\mathrm{Zn}^{\prime \prime}\left(\mathrm{H}_{3} \mathrm{SIM}^{4}\right)\right]^{+}$with the characteristic isotopic pattern for monozinc species. Further addition of zinc(II) perchlorate led to a spectrum with a main peak at $\mathrm{m} / \mathrm{z} 436.7$ exhibiting the isotopic pattern characteristic for a dizinc species and corresponding to a complex containing two zinc(II) centers, $\left[\mathrm{Zn}_{2}{ }_{2}\left(\mathrm{H}_{2} \mathrm{SIM}^{4}\right)\right]^{2+}$. These findings support the observation of the selective formation of a monozinc(II) complex by addition of one equivalent of $\mathrm{Zn}^{\|}$to $\mathrm{H}_{4} \mathrm{SIM}^{4}$ in agreement with the results acquired from the NMR spectroscopic measurements. 


\subsubsection{COMPLEXATION STUDIES WITH GA}

Similar to the complexation of $\mathrm{H}_{4} \mathrm{SIM}^{4}$ with $\mathrm{Zn}$ ", the capacity of $\mathrm{H}_{4} \mathrm{SIM}^{4}$ to form gallium(III) complexes was examined by NMR titration and mass spectrometric measurements.

\section{NMR Studies}

The ${ }^{1} \mathrm{H}$ NMR spectrum recorded with a mixture of one equivalent of gallium(III) perchlorate with ligand $\mathrm{H}_{4} \mathrm{SIM}^{4}$ in acetonitrile after heating for 12 hours shows mainly 16 resonances in the aromatic region. These resonances are accountable for the 16 inequivalent protons of a selectively formed species. The shift of the resonances assigned to the protons of the terminal phenol residue indicates the coordination of the Gall' ion in binding site $E$ in the resulting monogallium(III) complex of $\mathrm{H}_{4} \mathrm{SIM}^{4}$.

b)
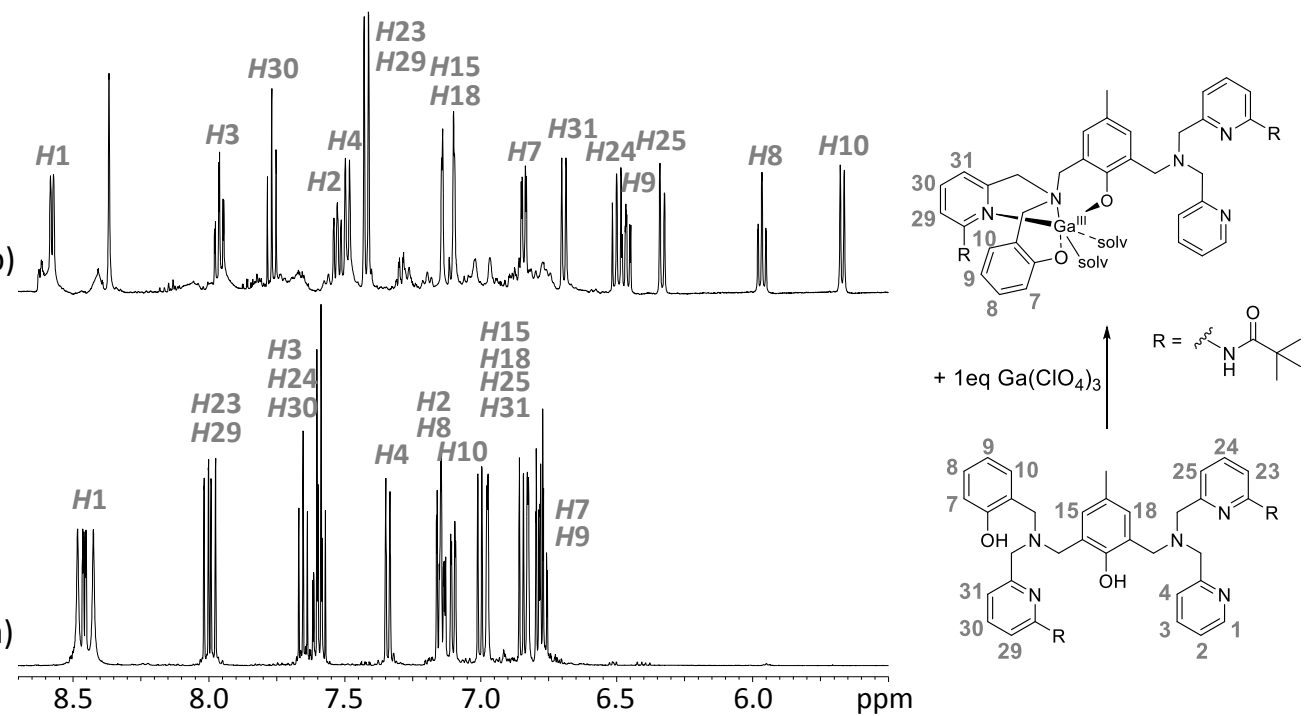

Figure 27: Comparison of the aromatic regions in the ${ }^{1} \mathrm{H} N M R$ of a) $\mathrm{H}_{4} \mathrm{SIM}^{4}$ in $\mathrm{CD}_{3} \mathrm{CN}$ with b) 1.0 eq of $\mathrm{Ga}^{\text {III }}\left(\mathrm{ClO}_{4}\right)_{3}$ heated at $60^{\circ} \mathrm{C}$ for $12 \mathrm{~h}$.

\section{Mass Spectrometry}

The $\mathrm{ESI}^{+}$mass spectrum recorded with a solution of $\mathrm{H}_{4} \mathrm{SIM}^{4}$ treated with one equivalent of gallium(III) perchlorate revealed main signals with the pattern characteristic for monogallium compounds. The signals were found at $\mathrm{m} / \mathrm{z} 405.7, \mathrm{~m} / \mathrm{z} 810.3$, and $\mathrm{m} / \mathrm{z} 912.3$ corresponding to the species $\left[\mathrm{Ga}^{\prime \prime \prime}\left(\mathrm{H}_{3} \mathrm{SIM}^{4}\right)\right]^{2+},\left[\mathrm{Ga}^{\prime \prime \prime}\left(\mathrm{H}_{2} \mathrm{SIM}^{4}\right)\right]^{+}$, and $\left[\mathrm{Ga}^{\prime \prime \prime}\left(\mathrm{H}_{3} \mathrm{SIM}^{4}\right)\left(\mathrm{ClO}_{4}\right)\right]^{+}$, respectively. The mass spectrometric investigation of a solution of $\mathrm{H}_{4} \mathrm{SIM}^{4}$ with one equivalent of zinc(II) perchlorate and one equivalent of gallium(III) perchlorate heated for 12 hours at $60^{\circ} \mathrm{C}$ resulted in a spectrum with signals associated with GaZn species due to their characteristic pattern (besides the signals assigned to remnants of the monogallium(III) complex). The GaZn species found had the mass to 
charge ratio of $\mathrm{m} / \mathrm{z} 436.62$ and $\mathrm{m} / \mathrm{z} 912.28$ and match the mass of [Ga"'Zn" $\left.\left(\mathrm{HSIM}^{4}\right)\right]^{2+}$ and $\left.\left[\mathrm{Ga}^{\mathrm{II}} \mathrm{Zn} \mathrm{n}(\mathrm{SIM})^{4}\right)\right]^{+}$.

\subsection{SUMMARY}

The coordination behavior towards $\mathrm{Zn}^{\prime \prime}, \mathrm{Cu}^{\prime \prime}, \mathrm{Ga}{ }^{\prime \prime \prime}$, and Fe ${ }^{\prime \prime \prime}$ of the asymmetric ligands $\mathrm{H}_{3} \mathrm{SIM}^{1}$, $\mathrm{H}_{4} \mathrm{SIM}^{3}$, and $\mathrm{H}_{4} \mathrm{SIM}^{4}$ was examined with various techniques, including NMR spectroscopy, $\mathrm{X}$-ray diffraction, mass spectrometry, UV-vis-NIR and EPR spectroscopy. In this way, the selective formation of specific complexes due to the asymmetry of the ligands was established.

Ligand $\mathrm{H}_{3} \mathrm{SIM}^{1}$ was shown to coordinate $\mathrm{Zn}$ " stepwise, i.e. $\mathrm{H}_{3} \mathrm{SIM}^{1}$ generates selectively the monozinc(II) complex in presence of $\mathrm{Zn}^{\prime \prime}$ with the $\mathrm{Zn}$ " ion bound in binding site $\mathrm{B}$, prior to coordination of a second $\mathrm{Zn}$ " ion in binding site $\mathrm{C}$. A similar behavior of $\mathrm{H}_{3} \mathrm{SIM}^{1}$ with $\mathrm{Cu}^{\prime \prime}$ ions was detected since the selective formation of a monocopper(II) complex was revealed after addition of one equivalent of $\mathrm{Cu}^{\prime \prime}$. In presence of two equivalents of $\mathrm{Cu}^{\prime \prime}, \mathrm{H}_{3} \mathrm{SIM}^{1}$ generates a dicopper(II) complex, which could be structurally characterized as $\left[\mathrm{Cu}_{2}{ }_{2}\left(\mathrm{HSIM}^{1}\right)(\mu-\mathrm{OAc})\right]^{+}$. In this structure, the asymmetry of the complex is visible due to the presence of two unequal $\mathrm{Cu}^{\prime \prime}$ centers, one in a trigonal bipyramidal and one in a distorted octahedral coordination geometry. Interestingly, the additional coordination site in the octahedral $\mathrm{Cu}^{\prime \prime}$ center is occupied by the pivaloyl-amide oxygen atom of coordination site $C$, stabilized by hydrogen bond formation by the second adjacent pivaloyl-amide residue. In contrast to the observations gained with $\mathrm{Zn}^{\prime \prime}$ and $\mathrm{Cu}^{\prime \prime}$ salts, $\mathrm{H}_{3} \mathrm{SIM}^{1}$ achieved only the coordination of one trivalent metal ion when Ga"l or Fe"ll salts were used as metal source. The coordination of the trivalent metal ion takes place in the less hindered binding site $\mathrm{B}$, as shown in the $\mathrm{X}$-ray structure of $\left[\mathrm{Ga}^{\prime \prime \prime}\left(\mathrm{H}_{2} \mathrm{SIM}^{1}\right)(\mu-\mathrm{OH})\right]_{2}\left(\mathrm{ClO}_{4}\right)_{2}$. The selectivity of the two different binding sites of the asymmetric ligand $\mathrm{H}_{3} \mathrm{SIM}^{1}$ was particularly demonstrated in the generation of a heterodinuclear Ga"' $\mathrm{Zn}$ " complex, independent of the order of addition of the two metal salts or an excess of one of the two metal salts. The X-ray structure of $\left[\mathrm{Ga}^{\prime \prime \prime} \mathrm{Zn} n^{\prime \prime}\left(\mathrm{H}_{2} \mathrm{SIM}^{1}\right)(\mathrm{OAc})_{2}(\mu-\mathrm{OH})\right]_{2}\left(\mathrm{PF}_{6}\right)_{2}$ verifies the coordination of $\mathrm{Ga}^{\prime \prime \prime}$ in the less hindered binding site $B$ and of $\mathrm{Zn}$ in the amidated binding site $C$.

Contrary to $\mathrm{H}_{3} \mathrm{SIM}^{1}, \mathrm{H}_{4} \mathrm{SIM}^{3}$, and $\mathrm{H}_{4} \mathrm{SIM}^{4}$ provide two chemically different binding sites with regards to the primary coordination sphere, i.e. a softer nitrogen-rich binding site and a harder binding site with a terminal phenolate residue. By means of NMR titration and mass spectrometric experiments it could be shown that the softer $\mathrm{Zn}$ " ion favors the softer binding site, while the harder trivalent Gall' ion selectively coordinates to the harder binding site. In case 
of $\mathrm{H}_{4} \mathrm{SIM}^{3}$ the coordination of $\mathrm{Fe}^{\mathrm{III}}$ in the harder binding site was also verified by X-ray diffraction of the dimeric iron(III) complex $\left\{\mathrm{Na}\left[\mathrm{Fe}^{\mathrm{III}}\left(\mathrm{H}_{2} \mathrm{SIM}^{3}\right)\right]_{2}(\mu-\mathrm{OAc})\left(\mu-\mathrm{OH}_{2}\right)\right\}\left(\mathrm{BF}_{4}\right)_{2}$. 


\section{Chapter 4}

\section{Dizinc Model Complexes ${ }^{d}$}

d Main parts of this chapter are published in "Dinuclear Zinc(II) Complexes with Hydrogen Bond Donors as Structural and Functional Phosphatase Models"; Simone Bosch, Peter Comba, Lawrence R. Gahan, Gerhard Schenk, Inorg. Chem. 2014, 53, 9036-9051. 


\subsection{INTRODUCTION}

Due to its geometric flexibility and high ionization potential/Lewis acidity the zinc(II) ion is one of the most frequently incorporated metal ions in hydrolases. These features facilitate zinc(II) containing enzymes to polarize $\mathrm{P}-\mathrm{O}$ bonds in phosphate ester substrates and simultaneously to activate the phosphorus center towards nucleophilic attack. ${ }^{11} \mathrm{~A}$ water molecule coordinated to $\mathrm{Zn}^{\prime \prime}$ is highly activated by polarization and ionization. The generation of a hydroxide ion at neutral $\mathrm{pH}$ can then act as a nucleophile. ${ }^{184} \mathrm{~A}$ key property, therefore, in the function of the catalytic sites is the acidity ( $\mathrm{pK}_{\mathrm{a}}$ value) of a metal-bound water molecule. ${ }^{185} \mathrm{It}$ is known that the number and the nature of the zinc(II) coordinating ligands play a critical role in the polarization capacity. ${ }^{186}$ Studying the zinc(II) complexes $\left[\mathrm{Zn}^{\prime \prime}\left(\mathrm{H}_{3} \mathrm{~L}^{19}\right)\left(\mathrm{OH}_{2}\right)\right]^{2+}$ and $\left[\mathrm{Zn}^{\prime \prime}\left(\mathrm{H}_{4} \mathrm{~L}^{20}\right)\left(\mathrm{OH}_{2}\right)\right]^{2+}($ Chart 12) it was found that, as the coordination number decreases from five to four, the $\mathrm{pK}_{\mathrm{a}}$ value of the $\mathrm{Zn}$ "-bound water molecule decreases from 8.0 to $7.3 .{ }^{187-189}$ In general, as the coordination number increases the charge on the $\mathrm{Zn}^{\|}$center is reduced and, consequently, the capacity to polarize the $\mathrm{Zn}$ "-bound water molecule is decreased. ${ }^{184}$

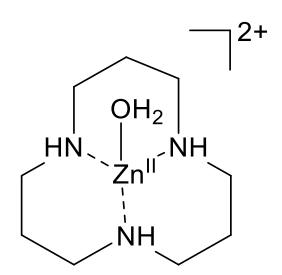

$$
\left[\mathrm{Zn}^{\prime \prime}\left(\mathrm{H}_{3} \mathrm{~L}^{19}\right)\left(\mathrm{OH}_{2}\right)\right]^{2+}
$$

$\mathrm{pK}_{\mathrm{a}}=7.3$

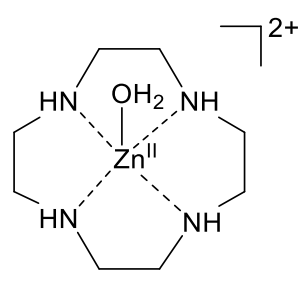

$$
\left[\mathrm{Zn}^{\prime \prime}\left(\mathrm{H}_{4} \mathrm{~L}^{20}\right)\left(\mathrm{OH}_{2}\right)\right]^{2+}
$$$$
\mathrm{pK}_{\mathrm{a}}=8.0
$$

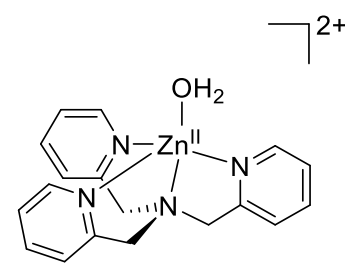

$$
\begin{gathered}
{\left[\mathrm{Zn}^{\prime \prime}\left(\mathrm{L}^{21}\right)\left(\mathrm{OH}_{2}\right)\right]^{2+}} \\
\mathrm{pK}_{\mathrm{a}}=8.0
\end{gathered}
$$

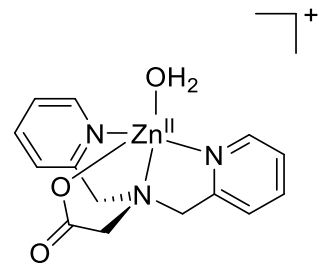

$$
\begin{gathered}
{\left[\mathrm{Zn}^{\prime \prime}\left(\mathrm{L}^{22}\right)\left(\mathrm{OH}_{2}\right)\right]^{+}} \\
\mathrm{pK}_{\mathrm{a}}=9.1
\end{gathered}
$$

Chart 12: Zinc(II) complexes used to study the impact of the primary coordination sphere of the $\mathrm{Zn}^{\text {"I center }}$ towards the $\mathrm{pK}$ a of $\mathrm{Zn}^{\text {"I}}$-bound water. ${ }^{190}$

A similar effect was found by changing coordinating ligands in the primary coordination sphere of the $\mathrm{Zn}$ " center. The change of one neutral pyridine ligand in $\left[\mathrm{Zn}^{\prime \prime}\left(\mathrm{L}^{21}\right)\left(\mathrm{OH}_{2}\right)\right]^{2+}($ Chart 12) by one anionic carboxylate results in a decrease of the Lewis acidity of the $\mathrm{Zn}$ " ion and consequently in a lowered capacity to polarize the bound water molecule $\left(\mathrm{pK}_{\mathrm{a}} 8.0\right.$ for $\left[\mathrm{Zn}^{\prime \prime}\left(\mathrm{L}^{21}\right)\left(\mathrm{OH}_{2}\right)\right]^{2+}$ and $\mathrm{pK}$ 9.1 for $\left.\left[\mathrm{Zn}^{\prime \prime}\left(\mathrm{L}^{22}\right)\left(\mathrm{OH}_{2}\right)\right]^{+}\right) .{ }^{191}$ This was confirmed by ab initio calculations and site-directed mutagenesis studies with carbonic anhydrase, and demonstrates the importance of the first coordination sphere on the $\mathrm{Zn}^{\text {"-water acidity. }}{ }^{186,192}$ However, the microenvironment of the active site in phosphatases is also known to influence the function of metalloproteins by non-covalent interactions such as hydrogen bonding. ${ }^{25,82,83}$ 
Mareque-Rivas et al. investigated the impact of hydrogen bond donors adjacent to the $\mathrm{Zn}$ " center towards the $\mathrm{pK}_{\mathrm{a}}$ value of the $\mathrm{Zn}$-bound water using derivatives of $\left[\mathrm{Zn}^{\prime \prime}\left(\mathrm{L}^{21}\right)\left(\mathrm{OH}_{2}\right)\right]^{2+}($ Chart 12$)$ the model complexes (Chart 13). ${ }^{190}$

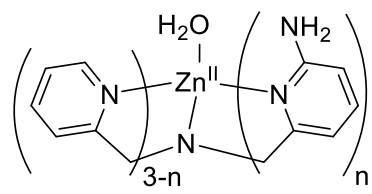

$\left[\mathrm{Zn}^{\prime \prime}\left(\mathrm{L}^{23}\right)\left(\mathrm{OH}_{2}\right)\right]: \mathrm{n}=1 ; \mathrm{pK}_{\mathrm{a}}=7.6$ $\left[\mathrm{Zn}^{\prime \prime}\left(\mathrm{L}^{24}\right)\left(\mathrm{OH}_{2}\right)\right]: \mathrm{n}=2 ; \mathrm{pK}_{\mathrm{a}}=6.7$ $\left[\mathrm{Zn}^{\prime \prime}\left(\mathrm{L}^{25}\right)\left(\mathrm{OH}_{2}\right)\right]: \mathrm{n}=3 ; \mathrm{pK}_{\mathrm{a}}=6.0$

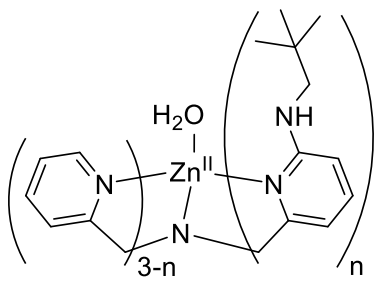

$\left[\mathrm{Zn} "\left(\mathrm{HL}^{26}\right)\left(\mathrm{OH}_{2}\right)\right]: \mathrm{n}=1 ; \mathrm{pK}_{\mathrm{a}}=7.7$ $\left[\mathrm{Zn}^{\prime \prime}\left(\mathrm{H}_{2} \mathrm{~L}^{27}\right)\left(\mathrm{OH}_{2}\right)\right]: \mathrm{n}=2 ; \mathrm{pK}_{\mathrm{a}}=6.4$ $\left[\mathrm{Zn}^{\prime \prime}\left(\mathrm{H}_{3} \mathrm{~L}^{28}\right)\left(\mathrm{OH}_{2}\right)\right]: \mathrm{n}=3 ; \mathrm{pK}_{\mathrm{a}}=7.2$

Chart 13: Zinc(II) complexes used to study the impact of hydrogen bond donors and hydrophobic groups in proximity to the ZnII center towards the pKa of ZnII-bound water. ${ }^{190}$

Since amino groups are electron donating, the $\mathrm{pK}_{\mathrm{a}}$ values of the zinc(II)-bound water molecule is expected to increase when more zinc(II) coordinating pyridyl groups are exchanged by aminopyridyl residues. The fact that the inverse was observed can be rationalized as being due to intramolecular hydrogen bonding as the $\mathrm{N}-\mathrm{H}$ hydrogen bond donors, when close to the $\mathrm{Zn}$ " center, can polarize the water $\mathrm{O}-\mathrm{H}$ bond. Moreover, the better hydrogen bond accepting properties of the anionic zinc(II)-bound hydroxide relative to water leads to a greater stabilization of the zinc(II)-hydroxide by the amino hydrogen bonding groups. In comparison to $\left[\mathrm{Zn}^{11}\left(\mathrm{~L}^{24}\right)\left(\mathrm{OH}_{2}\right)\right]^{2+}$, introduction of two neopentylamino group as in $\left[\mathrm{Zn}^{\prime \prime}\left(\mathrm{H}_{2} \mathrm{~L}^{27}\right)\left(\mathrm{OH}_{2}\right)\right]^{2+}$ (Chart 13), which simultaneously create a hydrophobic and hydrogen bonding microenvironment in proximity to the metal site, resulted in an even lower $\mathrm{pK}_{\mathrm{a}}$ value of the $\mathrm{Zn}^{\prime \prime}$-bound water $\left(\mathrm{pK}_{\mathrm{a}}\right.$ is 6.7 for $\left[\mathrm{Zn}^{\prime \prime}\left(\mathrm{L}^{24}\right)\left(\mathrm{OH}_{2}\right)\right]^{2+}$ and 6.4 for $\left.\left[\mathrm{Zn}^{\prime \prime}\left(\mathrm{H}_{2} \mathrm{~L}^{27}\right)\left(\mathrm{OH}_{2}\right)\right]^{2+}\right)$. Remarkably, these observations suggest that the second coordination sphere, which cooperates with the Lewis acidic $\mathrm{Zn}$ " center, can be of similar importance as the first coordination sphere in enhancing the $\mathrm{Zn}$ "-bound water acidity. ${ }^{184}$

In general, model systems that contain two metal centers were found to cleave various substrates at faster rates than the corresponding mononuclear analogues. ${ }^{11}$ When the two metal centers are brought in close distance to each other cooperation is facilitated either by double Lewis acid activation of the substrate or Lewis acid activation of the substrate, coupled with the provision of a metal bound hydroxide nucleophile. However, recently it was shown that the introduction of non-coordinated pyridyl residues in a mononuclear zinc(II) complex is as effective as the introduction of a second $\mathrm{Zn}$ " ion for enhancing its acidity towards the transesterification of phosphodiesters. This was accounted for hydrogen bonding of the vacant coordination site (Chart 14). ${ }^{111}$ 


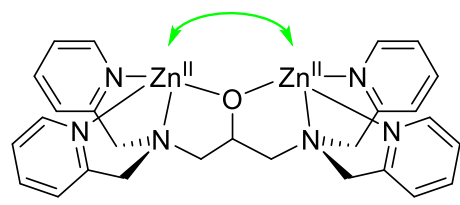

$\left[\mathrm{Zn}_{2}{ }_{2}\left(\mathrm{~L}^{29}\right)\right]$

metal-metal cooperation

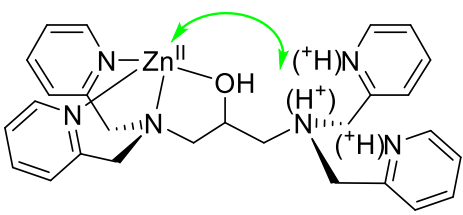

$\left[\mathrm{Zn}^{\mathrm{II}}\left(\mathrm{HL}^{29}\right)\right]$

metal-second sphere cooperation

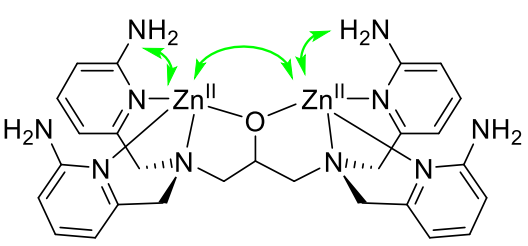

$\left[\mathrm{Zn}_{2}{ }_{2}\left(\mathrm{~L}^{7}\right)\right]$

metal-metal cooperation \& metal-second sphere cooperation

Chart 14: Introduction of metal-metal cooperation and metal-second sphere cooperation in zinc(II) model complexes for hydrolases. ${ }^{193}$

Combining both strategies, i.e. metal-metal cooperation, and metal-hydrogen bonding cooperation, accomplished in complex $\left[\mathrm{Zn}_{2}\left(\mathrm{~L}^{7}\right)\right]$ (Chart 14), resulted in tighter binding of phosphate derivatives such as pyrophosphate, RNA fragments and dimethyl phosphate, than with dizinc(II) complexes lacking second coordination sphere models or monozinc(II) complexes with hydrogen bond donors. ${ }^{112,113,194}$

Therefore, a variety of ligands were developed in this work, which differ in the type and geometric position of hydrogen bond donors and acceptors, more precisely pivaloyl-amide and amine moieties. The ligands were synthesized as described in Chapter 2. Using these ligands the corresponding dizinc(II) complexes, shown in Chart 15, were prepared and investigated. Experimental procedures including ligand and complex syntheses are given in detail in Chapter 11.

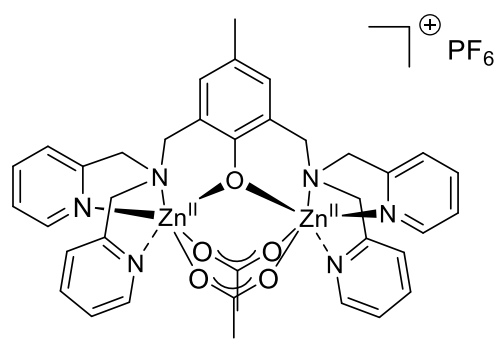

$\left[\mathrm{Zn}_{2}{ }_{2}\left(\mathrm{~L}^{1}\right)(\mu-\mathrm{OAc})_{2}\right] \mathrm{PF}_{6}$

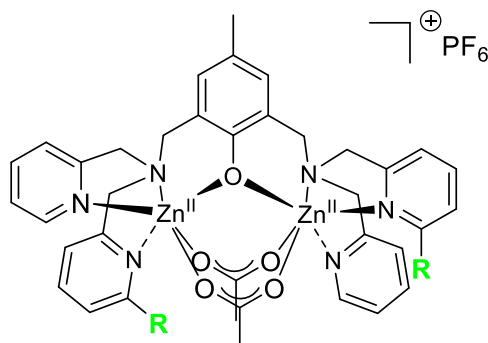

$\left[\mathrm{Zn}_{2}{ }_{2}\left(\mathrm{H}_{2} \mathrm{~L}^{2}\right)(\mu-\mathrm{OAc})_{2}\right] \mathrm{PF}{ }_{6}$

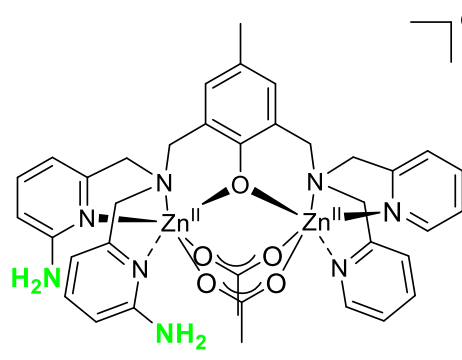

$\left[\mathrm{Zn}_{2}{ }_{2}\left(\mathrm{SIM}^{2}\right)(\mu-\mathrm{OAc})_{2}\right] \mathrm{PF}{ }_{6}$

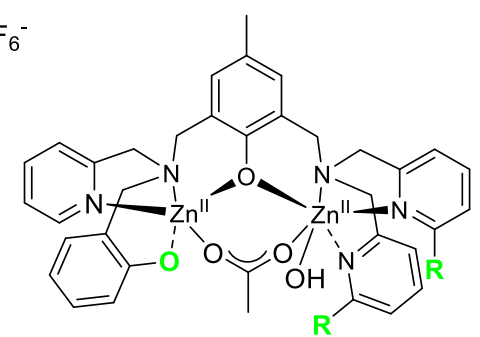

$\left[\mathrm{Zn}_{2}{ }_{2}\left(\mathrm{H}_{2} \mathrm{SIM}^{3}\right)(\mu-\mathrm{OAc})(\mathrm{OH})\right]$

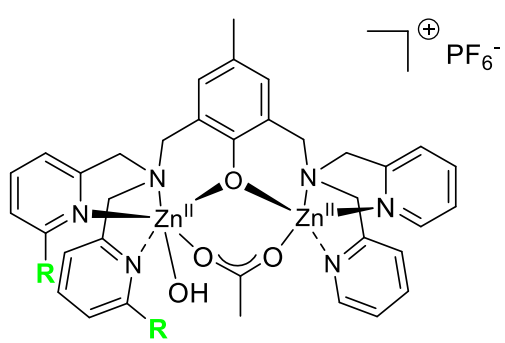

$\left[\mathrm{Zn}_{2}{ }_{2}\left(\mathrm{H}_{2} \mathrm{SIM}^{1}\right)(\mu-\mathrm{OAc})(\mathrm{OH})\right] \mathrm{PF}{ }_{6}$

Chart 15: Dizinc(II) complexes employed to mimic the active site of phosphoesterases.

These dizinc(II) model complexes were structurally characterized in the solid state and also in solution, using X-ray crystallography, NMR spectroscopy, mass spectrometry and UV-vis spectroscopy. Moreover, kinetic assays were employed in order to investigate the effect of the 
introduced moieties into the reference complex $\left[\mathrm{Zn}_{2}{ }_{2}\left(\mathrm{~L}^{1}\right)(\mu-\mathrm{OAC})_{2}\right] \mathrm{PF}_{6}$ (Chart 15). These introduced functionalities are able to form hydrogen bonds, responsible for the positioning of the substrate and release of the product molecules.

\subsection{SYNTHESIS OF DIZINC(II) COMPLEXES}

The dinuclear zinc(II) complexes of $\mathrm{HL}^{1}, \mathrm{H}_{3} \mathrm{~L}^{2}, \mathrm{H}_{3} \mathrm{SIM}^{1}, \mathrm{HSIM}^{2}$ and $\mathrm{H}_{4} \mathrm{SIM}^{3}$ were prepared by reaction with two equivalents of zinc(II) acetate dihydrate in methanol at $50^{\circ} \mathrm{C}$ for ten minutes. After addition of sodium hexafluorophosphate to the hot solution X-ray quality crystals were obtained upon standing. In this way the complexes $\left[\mathrm{Zn}_{2}{ }_{2}\left(\mathrm{~L}^{1}\right)(\mu-\mathrm{OAc})_{2}\right] \mathrm{PF}_{6}$, $\left[\mathrm{Zn}_{2}{ }_{2}\left(\mathrm{H}_{2} \mathrm{~L}^{2}\right)(\mu-\mathrm{OAc})_{2}\right] \mathrm{PF}_{6}, \quad\left[\mathrm{Zn}_{2}{ }_{2}\left(\mathrm{H}_{2} \mathrm{SIM}^{1}\right)(\mu-\mathrm{OAc})(\mathrm{OH})\right] \mathrm{PF}{ }_{6}$, and $\left[\mathrm{Zn}_{2}{ }_{2}\left(\mathrm{SIM}^{2}\right)(\mu-\mathrm{OAc})_{2}\right] \mathrm{PF}_{6}$ were isolated as white crystals. In the case of $\mathrm{H}_{4} \mathrm{SIM}^{3}$, the ligand containing solution was treated with aqueous sodium hydroxide before addition of zinc(II) acetate dihydrate, in order to ensure deprotonation of both phenol residues. Slow evaporation of the solvent led to the complex $\left[\mathrm{Zn}_{2}{ }_{2}\left(\mathrm{H}_{2} \mathrm{SIM}^{3}\right)(\mu-\mathrm{OAc})(\mathrm{OH})\right]$ in the form of crystals, suitable for X-ray diffraction, irrespective of the presence of sodium hexafluorophosphate. All five dizinc(II) complexes were characterized and studied in the solid state and in solution (acetonitrile was chosen as solvent since it is the common organic solvent used for hydrolysis activity studies with this type of model complexes). Bearing amino and amide residues adjacent the $\mathrm{Zn}$ " centers, these complexes offer the potential for the investigation of the effect of hydrogen bonding on the phosphoester hydrolytic activity.

\subsection{Structural Characterization of Dizinc(II) COMPLEXES}

\section{Solid State Structures}

The colorless single crystals of $\left[\mathrm{Zn}_{2}{ }_{2}\left(\mathrm{~L}^{1}\right)(\mu-\mathrm{OAc})_{2}\right] \mathrm{PF} \mathrm{F}_{6},\left[\mathrm{Zn}_{2}{ }_{2}\left(\mathrm{H}_{2} \mathrm{~L}^{2}\right)(\mu-\mathrm{OAc})_{2}\right] \mathrm{PF} \mathrm{F}_{6}$, $\left[\mathrm{Zn}_{2}{ }_{2}\left(\mathrm{H}_{2} \mathrm{SIM}^{1}\right)(\mu-\mathrm{OAc})(\mathrm{OH})\right] \mathrm{PF}_{6},\left[\mathrm{Zn}_{2}{ }_{2}\left(\mathrm{SIM}^{2}\right)(\mu-\mathrm{OAc})_{2}\right] \mathrm{PF}_{6}$ and $\left[\mathrm{Zn}_{2}{ }_{2}\left(\mathrm{H}_{2} \mathrm{SIM}^{3}\right)(\mu-\mathrm{OAc})(\mathrm{OH})\right]$ were used for X-ray diffraction data collection. Crystallographic data obtained for the structures are displayed in Table 33 (see Appendix).

The structures obtained show that the dizinc(II) complexes formed with the ligands $\mathrm{HL}^{1}, \mathrm{H}_{3} \mathrm{~L}^{2}$ and $\mathrm{HSIM}^{2}$ have the common formation for this type of phenolate-based complexes, composed of the corresponding ligand monoanion, resulting from the deprotonation of the phenol backbone, two $\mathrm{Zn}$ " ions, two bridging acetato anions, with a hexafluorophosphate 
counter ion. ${ }^{100,195,196}$ In accordance with the structure of $\left[\mathrm{Zn}_{2}{ }_{2}\left(\mathrm{~L}^{1}\right)(\mu-\mathrm{OAc})_{2}\right] \mathrm{BF}$, published by Adams et al., ${ }^{196}$ both $\mathrm{Zn}^{\prime \prime}$ ions in $\left[\mathrm{Zn}_{2}{ }_{2}\left(\mathrm{~L}^{1}\right)(\mu-\mathrm{OAc})_{2}\right] \mathrm{PF}_{6}, \quad\left[\mathrm{Zn}_{2}{ }_{2}\left(\mathrm{H}_{2} \mathrm{~L}^{2}\right)(\mu-\mathrm{OAc})_{2}\right] \mathrm{PF}_{6}$ and $\left[\mathrm{Zn}_{2}{ }_{2}\left(\mathrm{SIM}^{2}\right)(\mu-\mathrm{OAC})_{2}\right] \mathrm{PF}_{6}$ are coordinated in a six coordinate arrangement and are bridged by the ligand backbone phenoxido group and two acetate co-ligands. The remaining coordination sites are occupied by the nitrogen donors of two pyridine ligands and the tertiary amines. The structures are shown in Figure 28 and selected distances and valence angles are summarized in Table 7. Nordlander et al. recently reported a structure for the complex $\left[\mathrm{Zn}_{2}{ }_{2}\left(\mathrm{~L}^{1}\right)(\mu-\mathrm{OAc})\left(\mathrm{CH}_{3} \mathrm{OH}\right)\right] \mathrm{ClO}_{4}$, obtained when $\mathrm{HL}^{1}$ was reacted with zinc(II) acetate, but using sodium perchlorate, instead of sodium hexafluorophosphate as source for the counterion. ${ }^{197}$ The crude product was initially treated with water before the complex was crystallized by slow diffusion of diethyl ether. In $\left[\mathrm{Zn}_{2}{ }_{2}\left(\mathrm{~L}^{1}\right)(\mu-\mathrm{OAc})\left(\mathrm{CH}_{3} \mathrm{OH}\right)\right] \mathrm{ClO}_{4}$ one $\mathrm{Zn}^{\prime \prime}$ ion is coordinated in a slightly distorted octahedral geometry, while the second $\mathrm{Zn}$ " ion is the center of an distorted square pyramidal coordination geometry. ${ }^{197}$

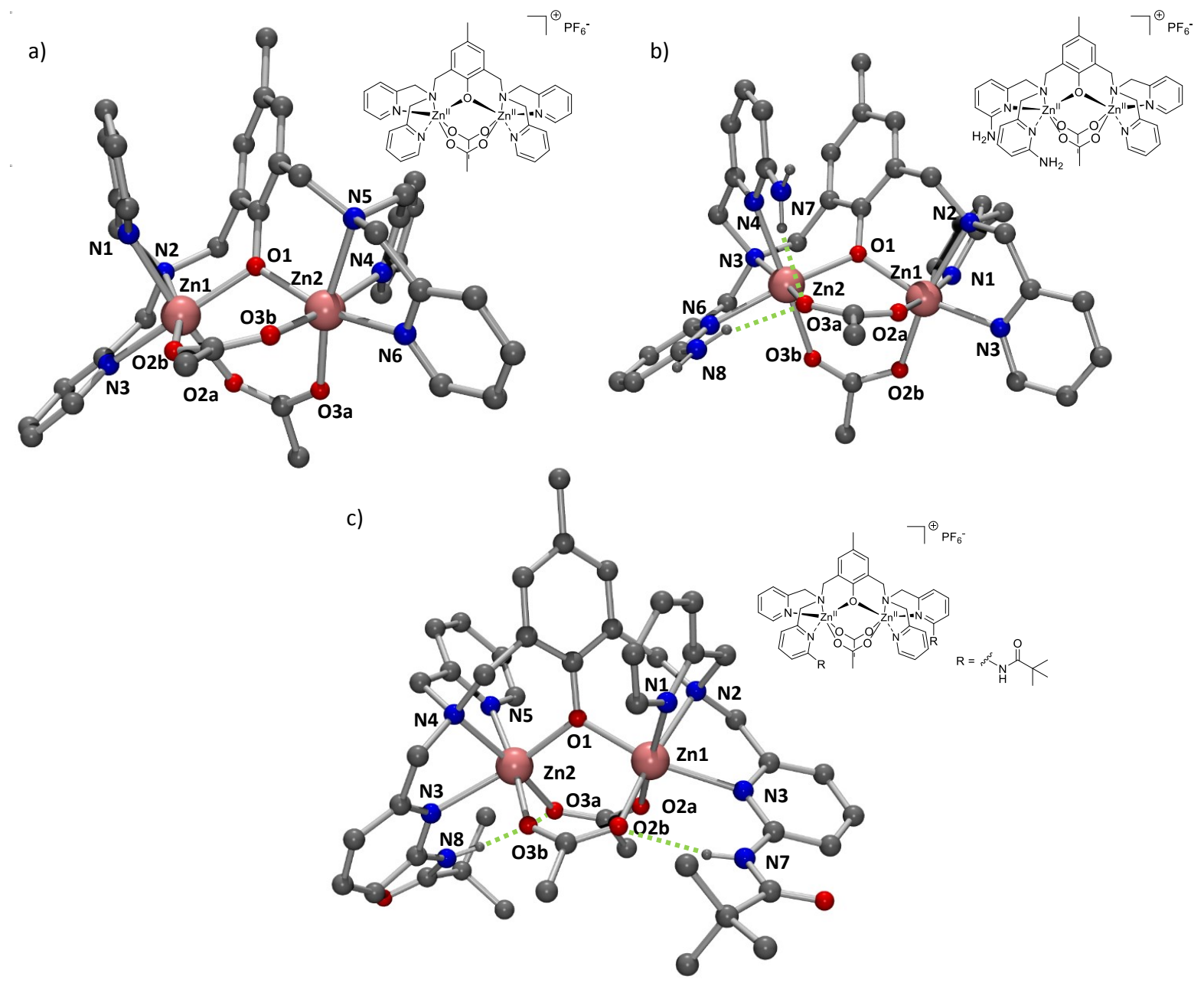

Figure 28: Structures of a) $\left[\mathrm{Zn}_{2}{ }_{2}\left(\mathrm{~L}^{1}\right)(\mu-\mathrm{OAc})_{2}\right] \mathrm{PF} 6_{6}$, b) $\left[\mathrm{Zn}_{2}{ }_{2}\left(\mathrm{SIM}^{2}\right)(\mu-\mathrm{OAc})_{2}\right] \mathrm{PF} 6_{6}$, and c) $\left[\mathrm{Zn}_{2}{ }_{2}\left(\mathrm{H}_{2} \mathrm{~L}^{2}\right)(\mu-\mathrm{OAc})_{2}\right] \mathrm{PF}_{6}$ showing hydrogen bonding (green dotted lines; counter ions, non-coordinated solvent molecules, and hydrogen atoms, not involved in hydrogen bonding, have been omitted for clarity; crystallographic data and ORTEP plots with 50\% probability level of thermal ellipsoids appear in the Appendix, Table 33 and Figure 74). 
Table 7: Selected bond lengths $(\AA)$ and angles $\left({ }^{\circ}\right)$ for $\left[\mathrm{Zn}_{2}{ }_{2}\left(\mathrm{~L}^{1}\right)(\mu-\mathrm{OAc})_{2}\right] \mathrm{PF}_{6},\left[\mathrm{Zn}_{2}{ }_{2}\left(\mathrm{H}_{2} \mathrm{SIM}^{1}\right)(\mu-\mathrm{OAc})(\mathrm{OH})\right] \mathrm{PF} 6_{6}$, $\left[\mathrm{Zn}_{2}{ }_{2}\left(\mathrm{H}_{2} \mathrm{~L}^{2}\right)(\mu-\mathrm{OAC})_{2}\right] \mathrm{PF}_{6},\left[\mathrm{Zn}_{2}\left(\mathrm{SIM}^{2}\right)(\mu-\mathrm{OAC})_{2}\right] \mathrm{PF}_{6}$ and $\left[\mathrm{Zn}_{2}{ }_{2}\left(\mathrm{H}_{2} \mathrm{SIM}^{3}\right)(\mu-\mathrm{OAC})(\mathrm{OH})\right]$.

\begin{tabular}{|c|c|c|c|c|c|}
\hline & 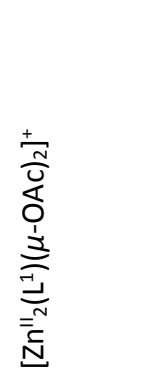 & 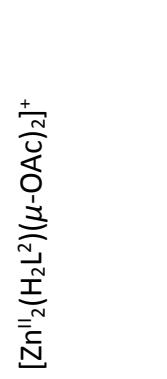 & 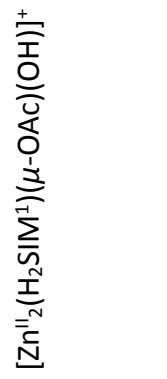 & 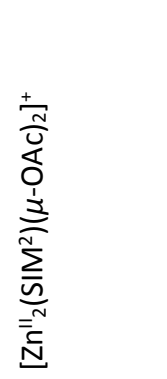 & 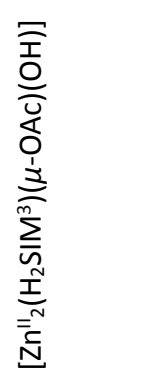 \\
\hline $\mathrm{Zn}(1) \cdots \mathrm{Zn}(2)$ & $3.3714(4)$ & $3.3992(6)$ & $3.5422(7)$ & $3.3948(10)$ & $3.5892(6)$ \\
\hline $\mathrm{Zn}(1)-\mathrm{O}(1)$ & $2.0135(14)$ & $2.025(3)$ & $1.954(3)$ & $1.994(4)$ & 1.9904(19) \\
\hline $\mathrm{Zn}(1)-\mathrm{O}(2 \mathrm{~A})$ & $2.1825(16)$ & $2.092(3)$ & $2.003(3)$ & $2.112(4)$ & $2.065(2)$ \\
\hline $\mathrm{Zn}(1)-\mathrm{O}(2 \mathrm{~B})$ & $2.0088(15)$ & $2.056(3)$ & - & $2.008(4)$ & - \\
\hline $\mathrm{Zn}(1)-\mathrm{N}(1)$ & $2.2346(19)$ & $2.169(3)$ & $2.057(3)$ & $2.216(5)$ & $2.096(2)$ \\
\hline $\mathrm{Zn}(1)-\mathrm{N}(2)$ & $2.2306(19)$ & $2.214(3)$ & $2.233(2)$ & $2.206(5)$ & $2.213(2)$ \\
\hline $\mathrm{Zn}(1)-\mathrm{N}(3)^{*}$ & $2.1443(19)$ & $2.284(3)$ & $2.102(3)$ & $2.132(5)$ & $1.934(2)$ \\
\hline $\mathrm{Zn}(2)-\mathrm{O}(1)$ & $2.0342(14)$ & $2.000(3)$ & $2.120(2)$ & $1.995(4)$ & $2.1380(19)$ \\
\hline $\mathrm{Zn}(2)-\mathrm{O}(3 \mathrm{~A})^{* *}$ & $2.0198(17)$ & $2.039(3)$ & $2.133(3)$ & $2.008(4)$ & $2.102(2)$ \\
\hline $\mathrm{Zn}(2)-\mathrm{O}(3 \mathrm{~B})$ & $2.0849(16)$ & $2.077(3)$ & - & $2.114(4)$ & - \\
\hline $\mathrm{Zn}(2)-\mathrm{N}(4)$ & $2.223(2)$ & $2.155(3)$ & $2.322(3)$ & $2.239(5)$ & $2.295(2)$ \\
\hline $\mathrm{Zn}(2)-\mathrm{N}(5)$ & $2.247(2)$ & $2.199(3)$ & $2.136(3)$ & $2.165(5)$ & $2.148(2)$ \\
\hline $\mathrm{Zn}(2)-\mathrm{N}(6)$ & $2.1764(19)$ & $2.289(3)$ & $2.336(3)$ & $2.123(5)$ & $2.473(2)$ \\
\hline $\mathrm{Zn}(2)-\mathrm{O}(6)$ & - & - & $1.945(2)$ & - & $1.9571(19)$ \\
\hline$Z n(1)-O(1)-Z n(2)$ & $112.80(7)$ & $115.23(13)$ & $120.75(12)$ & $116.65(18)$ & 120.73(9) \\
\hline$O(1)-Z n(1)-N(1)$ & $87.65(6)$ & $89.95(12)$ & $118.71(12)$ & $88.63(18)$ & 130.04(9) \\
\hline$O(1)-Z n(1)-N(2)$ & $88.88(7)$ & $87.20(11)$ & $91.48(11)$ & $88.12(17)$ & $91.80(8)$ \\
\hline $\mathrm{O}(1)-\mathrm{Zn}(1)-\mathrm{N}(3)^{*}$ & $163.19(7)$ & $160.92(12)$ & $124.82(11)$ & $161.07(18)$ & 109.92(9) \\
\hline$O(1)-Z n(1)-O(2 A) * *$ & $87.91(6)$ & $89.64(11)$ & $99.48(12)$ & $89.65(16)$ & $90.21(8)$ \\
\hline $\mathrm{O}(1)-\mathrm{Zn}(1)-\mathrm{O}(2 \mathrm{~B})$ & $100.20(6)$ & $100.55(11)$ & - & $101.16(17)$ & - \\
\hline$O(1)-Z n(2)-N(4)$ & $86.73(7)$ & $93.20(12)$ & $88.66(10)$ & $91.46(16)$ & $86.80(8)$ \\
\hline$O(1)-Z n(2)-N(5)$ & $87.53(6)$ & $88.29(12)$ & $90.65(11)$ & $89.20(17)$ & $91.44(9)$ \\
\hline$O(1)-Z n(2)-N(6)$ & $163.04(7)$ & $159.77(12)$ & $161.27(10)$ & $162.28(16)$ & $161.12(8)$ \\
\hline$O(1)-Z n(2)-O(3 A)^{* *}$ & $100.51(6)$ & $101.25(11)$ & $87.91(11)$ & $98.04(17)$ & $91.27(8)$ \\
\hline $\mathrm{O}(1)-\mathrm{Zn}(2)-\mathrm{O}(3 \mathrm{~B})$ & $92.43(6)$ & $90.00(11)$ & - & $88.12(15)$ & - \\
\hline$O(1)-Z n(2)-O(6)$ & - & - & $102.84(10)$ & - & $107.30(8)$ \\
\hline
\end{tabular}

$*$ in the case of $\left[\mathrm{Zn}_{2}{ }_{2}\left(\mathrm{H}_{2} \mathrm{SIM}^{3}\right)(\mu-\mathrm{OAc})(\mathrm{OH})\right] \mathrm{N}(3)$ is $\mathrm{O}(1 \mathrm{~A})$.

** in the case of $\left[\mathrm{Zn}_{2}{ }_{2}\left(\mathrm{H}_{2} \mathrm{SIM}^{1}\right)(\mu-\mathrm{OAC})(\mathrm{OH})\right]^{+}$and $\left[\mathrm{Zn}_{2}{ }_{2}\left(\mathrm{H}_{2} \mathrm{SIM}^{1}\right)\left(\mu-\mathrm{O}_{3} \mathrm{POC}_{6} \mathrm{H}_{4} \mathrm{NO}_{2}\right)\right]^{+} \mathrm{O}(2 \mathrm{~A})$ is $\mathrm{O}(2)$ and $\mathrm{O}(3 \mathrm{~A})$ is $\mathrm{O}(3)$.

In $\left[\mathrm{Zn}_{2}{ }_{2}\left(\mathrm{H}_{2} \mathrm{~L}^{2}\right)(\mu-\mathrm{OAc})_{2}\right]^{+}$the ligands in the trans position to the bridging phenolate are the pivaloyl-substituted pyridine residues at both $\mathrm{Zn}$ " centers and its $\mathrm{Zn}-\mathrm{N}_{\mathrm{ax}}$ distances (2.289(3) $\AA$ and 2.284(3) $\AA$ ) are elongated compared to the $\mathrm{Zn}-\mathrm{N}_{\text {eq }}$ distances $(2.155(3) \AA$ and $2.169(3) \AA$ ) of the non-substituted pyridine residues. This contrasts with distances measured in $\left[\mathrm{Zn}_{2}{ }_{2}\left(\mathrm{~L}^{1}\right)(\mu-\mathrm{OAc})_{2}\right]^{+}$, in which the $\mathrm{Zn}-\mathrm{N}_{\mathrm{ax}}$ distances $(2.1443(19) \AA$ and $2.1764(19) \AA$ ) are shorter than the $\mathrm{Zn}-\mathrm{N}_{\text {eq }}$ distances $(2.2346(19) \AA$ and $2.223(2) \AA ̊$ ), presumably due to the electron withdrawing effect of the pivaloyl-amide residues. In agreement with the electron donating effect of the amino substituent, the $\mathrm{Zn}-\mathrm{N}_{\mathrm{ax}}$ distance to the amino substituted pyridine moiety 
$(2.123(5) \AA)$ is slightly shorter than to the unsubstituted pyridine ligand $(2.132(5) \AA)$ in $\left[\mathrm{Zn}^{\prime \prime}\left(\mathrm{SIM}^{2}\right)(\mu-\mathrm{OAc})_{2}\right]^{+}$.

Reaction of $\mathrm{H}_{3} \mathrm{SIM}^{1}$ and $\mathrm{H}_{4} \mathrm{SIM}^{3}$ with zinc(II) acetate dihydrate resulted in the complexes $\left[\mathrm{Zn}_{2}{ }_{2}\left(\mathrm{H}_{2} \mathrm{SIM}^{1}\right)(\mu-\mathrm{OAc})(\mathrm{OH})\right] \mathrm{PF}_{6}$ and $\left[\mathrm{Zn}_{2}{ }_{2}\left(\mathrm{H}_{2} \mathrm{SIM}^{3}\right)(\mathrm{OAc})(\mu-\mathrm{OH})\right]$ (Figure 29), in which the two

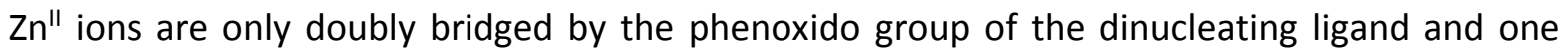
acetato co-ligand. $\mathrm{Zn}(1)$, the $\mathrm{Zn}$ " ion in the amide-free binding site, adopts a five-coordinate geometry. Applying the method of Addison et al., ${ }^{171}$ which evaluates the degree of trigonality by means of the structural parameter $\tau(\tau=(\beta-\alpha) / 60$, where $\alpha$ and $\beta$ are the two largest angles around the central atom with $\beta>\alpha$ ) and assigns a value of $\tau=0$ to a regular square pyramidal geometry and $\tau=1$ to a trigonal bipyramidal geometry, the coordination environment of $\mathrm{Zn}(1)$ in $\left[\mathrm{Zn}_{2}{ }_{2}\left(\mathrm{H}_{2} \mathrm{SIM}^{1}\right)(\mu-\mathrm{OAc})(\mathrm{OH})\right] \mathrm{PF}_{6}$ with $\tau=0.94$ is a trigonal bipyramidal with the trigonal plane formed by two pyridines and the backbone phenolate.

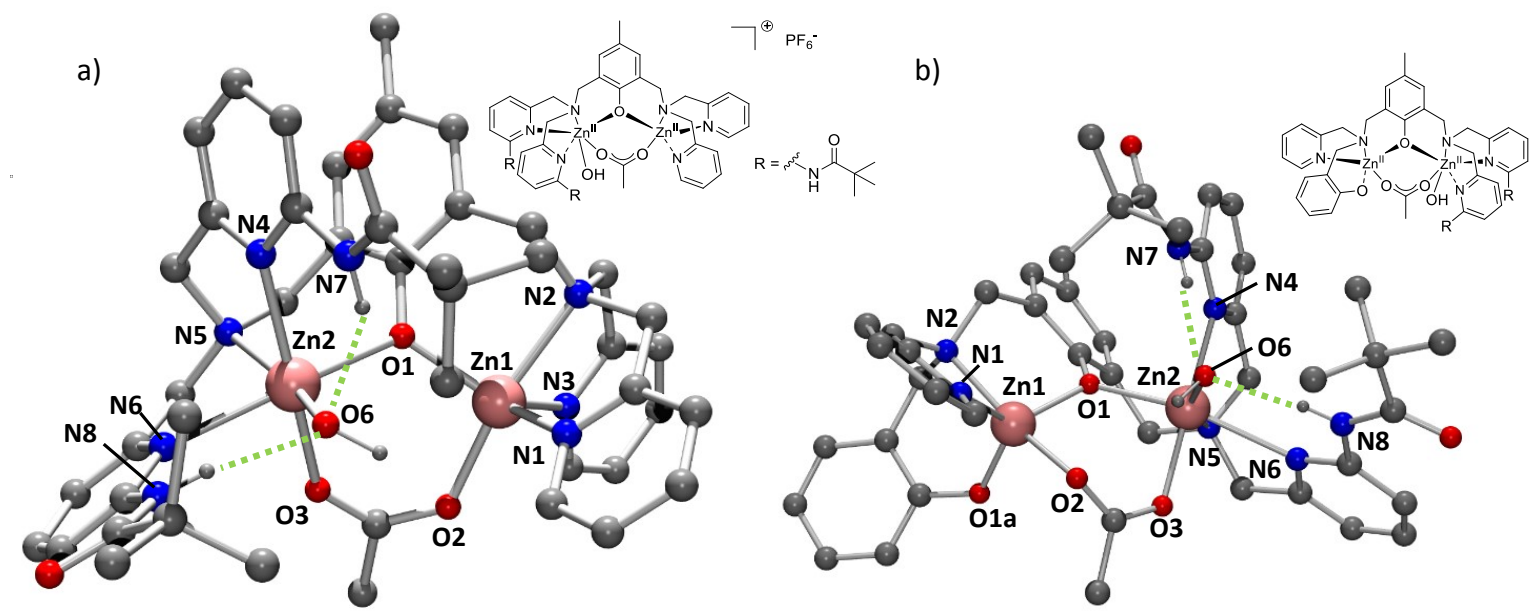

Figure 29: Structures of $\left[\mathrm{Zn}_{2}{ }_{2}\left(\mathrm{H}_{2} \mathrm{SIM}^{1}\right)(\mu-\mathrm{OAc})(\mathrm{OH})\right] \mathrm{PF} 6$ (left) and $\left[\mathrm{Zn}_{2}{ }_{2}\left(\mathrm{H}_{2} \mathrm{SIM}^{3}\right)(\mu-\mathrm{OAc})(\mathrm{OH})\right]$ (right) showing hydrogen bonding (green dotted lines; counter ions and hydrogen atoms, not involved in hydrogen bonding, have been omitted for clarity; crystallographic data and ORTEP plots with $50 \%$ probability level of thermal ellipsoids appear in the Appendix, Table 34 and Figure 74).

The five donors around $\mathrm{Zn}(1)$ in $\left[\mathrm{Zn}_{2}{ }_{2}\left(\mathrm{H}_{2} \mathrm{SIM}^{3}\right)(\mu-\mathrm{OAc})(\mathrm{OH})\right]$ are arranged in a coordination geometry with a $\tau$-value of 0.58 , in between trigonal bipyramidal and square pyramidal. Assuming trigonal bipyramidal, the trigonal plane is defined by one pyridine and two phenolate residues. The axial positions are occupied by the tertiary amine and an acetato oxygen atom in both structures, $\left[\mathrm{Zn}_{2}{ }_{2}\left(\mathrm{H}_{2} \mathrm{SIM}^{1}\right)(\mu-\mathrm{OAc})(\mathrm{OH})\right] \mathrm{PF}_{6}$ and $\left[\mathrm{Zn}_{2}{ }_{2}\left(\mathrm{H}_{2} \mathrm{SIM}^{3}\right)(\mu-\mathrm{OAc})(\mathrm{OH})\right]$. The variation of the coordination geometry in these two complexes is proposed to be due to the larger phenolate moiety in $\mathrm{H}_{4} \mathrm{SIM}^{3}$ compared to the pyridine residue in $\mathrm{H}_{3} \mathrm{SIM}^{1}$. For $\left[\mathrm{Zn}_{2}{ }_{2}\left(\mathrm{H}_{2} \mathrm{SIM}^{3}\right)(\mu-\mathrm{OAc})(\mathrm{OH})\right]$, the angle $(O(1)-Z n(1)-O(1 a))$ is reduced, with respect to the angle $(O(1)-Z n(1)-N(3))$ in $\left[\mathrm{Zn}_{2}{ }_{2}\left(\mathrm{H}_{2} \mathrm{SIM}^{1}\right)(\mu-\mathrm{OAc})(\mathrm{OH})\right] \mathrm{PF}_{6}$, and the bridging acetate ligand is twisted relative to the $\mathrm{Zn}(1)-\mathrm{O}(1)-\mathrm{Zn}(2)$ plane (torsion angles $\mathrm{O}(1)-\mathrm{Zn}(1)-\mathrm{O}(2)-\mathrm{C}(1 \mathrm{a})$ and $\mathrm{O}(1)-\mathrm{Zn}(2)-\mathrm{O}(3)-\mathrm{C}(1 \mathrm{a})$ are $6.75^{\circ}$ 
and $29.46^{\circ}$ in $\left[\mathrm{Zn}_{2}{ }_{2}\left(\mathrm{H}_{2} \mathrm{SIM}^{1}\right)(\mu-\mathrm{OAC})(\mathrm{OH})\right] \mathrm{PF}_{6}$ as well as $50.57^{\circ}$ and $64.53^{\circ}$ in $\left.\left[\mathrm{Zn}_{2}{ }_{2}\left(\mathrm{H}_{2} \mathrm{SIM}^{3}\right)(\mu-\mathrm{OAc})(\mathrm{OH})\right]\right)$.

Similar to the zinc(II) sites in $\left[\mathrm{Zn}_{2}{ }_{2}\left(\mathrm{~L}^{1}\right)(\mu-\mathrm{OAc})_{2}\right] \mathrm{PF}_{6}, \quad\left[\mathrm{Zn}_{2}{ }_{2}\left(\mathrm{H}_{2} \mathrm{~L}^{2}\right)(\mu-\mathrm{OAc})_{2}\right] \mathrm{PF} 6$ and $\left[\mathrm{Zn}_{2}{ }_{2}\left(\mathrm{SIM}^{2}\right)(\mu-\mathrm{OAC})_{2}\right] \mathrm{PF}_{6}$, the second $\mathrm{Zn}$ " ion in $\left[\mathrm{Zn}_{2}{ }_{2}\left(\mathrm{H}_{2} \mathrm{SIM}^{1}\right)(\mu-\mathrm{OAc})(\mathrm{OH})\right] \mathrm{PF} 6$ and $\left[\mathrm{Zn}_{2}{ }_{2}\left(\mathrm{H}_{2} \mathrm{SIM}^{3}\right)(\mu-\mathrm{OAc})(\mathrm{OH})\right]$, bound to the amide containing binding site $(\mathrm{Zn}(2))$, is six-coordinate. The coordination environment of $\mathrm{Zn}(2)$ is formed by two pyridine donors, a tertiary amine, the bridging phenolate group and the bridging acetate co-ligand and completed by a hydroxido group.

In comparison to $\left[\mathrm{Zn}_{2}{ }_{2}\left(\mathrm{~L}^{1}\right)(\mu-\mathrm{OAc})_{2}\right] \mathrm{PF}{ }_{6},\left[\mathrm{Zn}_{2}{ }_{2}\left(\mathrm{H}_{2} \mathrm{~L}^{2}\right)(\mu-\mathrm{OAc})_{2}\right] \mathrm{PF}_{6}$ and $\left[\mathrm{Zn}_{2}{ }_{2}\left(\mathrm{SIM}^{2}\right)(\mu-\mathrm{OAc})_{2}\right] \mathrm{PF}_{6}$, the distances between $\mathrm{Zn}(1)$, the $\mathrm{Zn}$ " atom coordinated in a trigonal bipyramidal geometry, and the coordinating ligands of the primary coordination sphere are shorter. Furthermore, the length of the bonds between $\mathrm{Zn}(2)$ and its six donors are elongated compared to the complexes $\left[\mathrm{Zn}_{2}{ }_{2}\left(\mathrm{~L}^{1}\right)(\mu-\mathrm{OAc})_{2}\right] \mathrm{PF}_{6},\left[\mathrm{Zn}_{2}{ }_{2}\left(\mathrm{H}_{2} \mathrm{~L}^{2}\right)(\mu-\mathrm{OAc})_{2}\right] \mathrm{PF}_{6}$, and $\left[\mathrm{Zn}_{2}{ }_{2}\left(\mathrm{SIM}^{2}\right)(\mu-\mathrm{OAc})_{2}\right] \mathrm{PF} \mathrm{F}_{6}$, presumably due to the electron-rich hydroxido co-ligand.

The presence of the unusual hydroxido co-ligand was confirmed by comparison of the $\mathrm{Zn}-\mathrm{O}$ distance with the respective value in structures of previously published zinc(II) complexes with aqua, $\mu$-hydroxido and terminal hydroxido donor groups. For example, for the dizinc(II) complex of $\mathrm{HL}^{1},\left[\mathrm{Zn}_{2}{ }_{2}\left(\mathrm{~L}^{1}\right)\left(\mathrm{OH}_{2}\right)_{2}\right]\left(\mathrm{ClO}_{4}\right)_{3}, \mathrm{Zn}-\mathrm{OH}_{2}$ distances are of the order of $2.04 \AA$ and the distances to bridging hydroxido ligands are reduced to $1.99 \AA$, as in $\left[\mathrm{Zn}_{2}{ }_{2}\left(\mathrm{~L}^{1}\right)(\mu-\mathrm{OH})\right]\left(\mathrm{ClO}_{4}\right)_{2}$ (see Figure 30). ${ }^{198}$

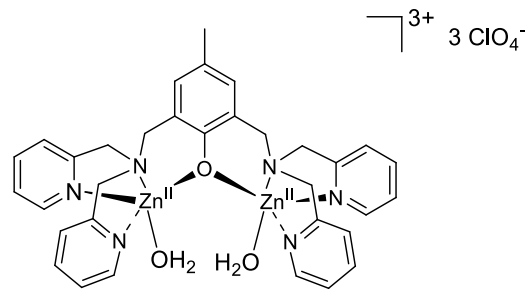

$\mathrm{Zn}-\mathrm{OH}_{(2)}$ distance

$2.043(5)$

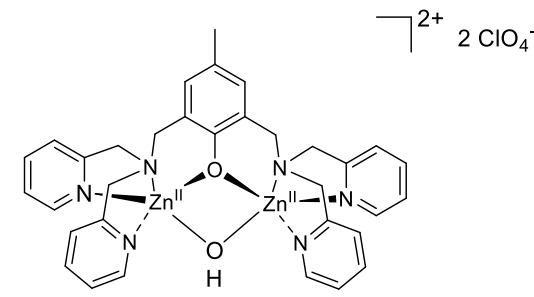

$1.987(3)$

Figure 30: $\left[\mathrm{Zn}_{2}{ }_{2}\left(\mathrm{~L}^{1}\right)\left(\mathrm{OH}_{2}\right)_{2}\right]\left(\mathrm{ClO}_{4}\right)_{3}$ and $\left[\mathrm{Zn}_{2}{ }_{2}\left(\mathrm{~L}^{1}\right)(\mu-\mathrm{OH})\right]\left(\mathrm{ClO}_{4}\right)_{2}$ and the respective zinc(II)-aqua and zinc(II)-( $\mu$-hydroxido) distances. ${ }^{198}$

Similar $\mathrm{Zn}-(\mu-\mathrm{OH})$ distances have been found for a variety of dizinc(II) complexes: $\left[\left[\mathrm{Zn}^{\prime \prime}\left(\mathrm{L}^{30}\right)\right]_{2}(\mu-\mathrm{OH})_{2}\right]\left(\mathrm{ClO}_{4}\right)_{2} \quad(1.983(3) \AA ̊ \AA) ;\left[\left[\mathrm{Zn}^{\prime \prime}\left(\mathrm{L}^{31}\right)\right]_{2}(\mu-\mathrm{OH})_{2}\right]\left(\mathrm{ClO}_{4}\right)_{2} \cdot 0.5 \mathrm{CH}_{3} \mathrm{CN}$ (2.070(3) $\AA$ ); $\left[\mathrm{Zn}_{2}{ }_{2}\left(\mathrm{~L}^{32}\right)(\mu-\mathrm{OH})(\mathrm{py})_{2}\right]\left(\mathrm{ClO}_{4}\right)_{2} \quad(1.946(3) \quad \AA) ; \quad\left[\mathrm{Zn}_{2}\left(\mathrm{~L}^{33}\right)(\mu-\mathrm{OH})(\mathrm{py})_{3}\right]\left(\mathrm{ClO}_{4}\right)_{2} \quad(1.951(4) \quad \AA) \quad$ and $\left[\mathrm{Zn}_{2}\left(\mathrm{~L}^{1}\right)(\mu-\mathrm{OH})\right]\left(\mathrm{ClO}_{4}\right)_{2}(1.943(3) \AA)$ (structures of the respective ligands are given in the Table of Molecules). ${ }^{198-200}$ These metrics compare with the $\mathrm{Zn}-\mathrm{O}$ distances in $\left[\mathrm{Zn}_{2}{ }_{2}\left(\mathrm{H}_{2} \mathrm{SIM}^{3}\right)(\mu-\mathrm{OAc})(\mathrm{OH})\right]$ $(1.9575(19) \AA)$ and $\left[\mathrm{Zn}_{2}{ }_{2}\left(\mathrm{H}_{2} \mathrm{SIM}^{1}\right)(\mu-\mathrm{OAc})(\mathrm{OH}) \mathrm{PF}_{6}(1.943(3) \AA)\right.$, which are significantly shorter than in complexes reported with a terminal aqua ligand and in the range of complexes containing bridging hydroxido ligands. This supports the notion of the presence of a terminal hydroxido co- 
ligand. Moreover, the long $\mathrm{Zn}(1) \cdots \mathrm{OH}$ distances of $3.936 \AA$ in $\left[\mathrm{Zn}_{2}{ }_{2}\left(\mathrm{H}_{2} \mathrm{SIM}^{1}\right)(\mu-\mathrm{OAc})(\mathrm{OH})\right] \mathrm{PF} 6$ and $4.199 \AA$ in $\left[\mathrm{Zn}_{2}{ }_{2}\left(\mathrm{H}_{2} \mathrm{SIM}^{3}\right)(\mu-\mathrm{OAc})(\mathrm{OH})\right]$ support the assignment of the $\mathrm{OH}^{-}$group in question as a terminal hydroxido co-ligand.

The fact that a terminal hydroxido co-ligand has not been observed previously in dinuclear zinc(II) complexes with similar phenolate-based ligands, but lacking the hydrogen-bond donors, is consistent with the proposition that the hydrogen-bond donors, i.e. the two adjacent amide residues at the binding site of one $\mathrm{Zn}$ " center in $\left[\mathrm{Zn}_{2}{ }_{2}\left(\mathrm{H}_{2} \mathrm{SIM}^{1}\right)(\mu-\mathrm{OAc})(\mathrm{OH})\right] \mathrm{PF}_{6}$ and $\left[\mathrm{Zn}_{2}{ }_{2}\left(\mathrm{H}_{2} \mathrm{SIM}^{3}\right)(\mu-\mathrm{OAc})(\mathrm{OH})\right]$, stabilize this unusual hydroxido co-ligand. Moreover, the hydrogen bond formation within the structure of $\left[\mathrm{Zn}_{2}{ }_{2}\left(\mathrm{H}_{2} \mathrm{SIM}^{1}\right)(\mu-\mathrm{OAc})(\mathrm{OH})\right] \mathrm{PF}_{6}$ is confirmed by the observed arrangement of the amide hydrogen atoms with respect to the free electron pairs of the hydroxido oxygen atom (hydrogen bonds are depicted as green dotted lines in Figure 28 and Figure 29). This is in accordance with the previous observation that the incorporation of two $\mathrm{H}$-bond donors, i.e. neopentylamino groups, in tripodal $\mathrm{N}_{4}$ ligands lead to the generation of a stable $\mathrm{Zn}^{\prime \prime}-\mathrm{OH}$ complex $\left[\mathrm{Zn}^{11}\left(\mathrm{H}_{2} \mathrm{~L}^{27}\right)(\mathrm{OH})\right] \mathrm{ClO}_{4}$ due to $\mathrm{H}$-bonding (Figure 31). ${ }^{190}$

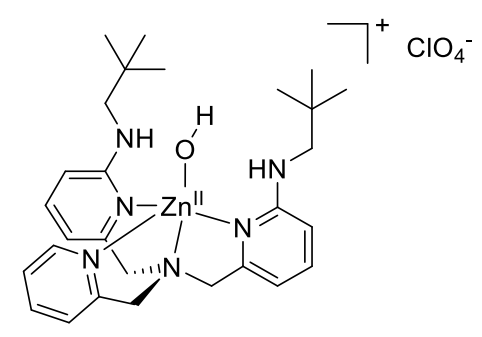

Figure 31: Previously published $\mathrm{Zn}^{\prime \prime}$-hydroxido complex $\left[\mathrm{Zn}^{\prime \prime}\left(\mathrm{H}_{2} \mathrm{~L}^{27}\right)(\mathrm{OH})\right] \mathrm{ClO}$, stabilized by hydrogen bond formation. ${ }^{190}$

Furthermore, hydrogen bond formation was detected in $\left[\mathrm{Zn}^{\prime \prime}\left(\mathrm{H}_{2} \mathrm{~L}^{27}\right)(\mathrm{OH})\right] \mathrm{ClO}_{4}$ proposed from the short distances between the amino nitrogen and hydroxido oxygen atom $(2.741 \AA$ and $2.727 \AA)^{190}$; the corresponding distances for $\left[\mathrm{Zn}_{2}{ }_{2}\left(\mathrm{H}_{2} \mathrm{SIM}^{1}\right)(\mu-\mathrm{OAc})(\mathrm{OH})\right] \mathrm{PF}_{6}$ and $\left[\mathrm{Zn}_{2}{ }_{2}\left(\mathrm{H}_{2} \mathrm{SIM}^{3}\right)(\mu-\mathrm{OAc})(\mathrm{OH})\right]$ are listed in Table 8. This table also contains the corresponding distances for $\left[\mathrm{Zn}_{2}{ }_{2}\left(\mathrm{H}_{2} \mathrm{~L}^{2}\right)(\mu-\mathrm{OAc})_{2}\right] \mathrm{PF}_{6}$ and $\left[\mathrm{Zn}_{2}\left(\mathrm{SIM}^{2}\right)(\mu-\mathrm{OAc})_{2}\right] \mathrm{PF}_{6}$, and those between the amine or amide nitrogen atom and the nearby acetate oxygen atoms are in the range 2.750(8) $\AA$ and 2.982(4) $\AA$. These short distances demonstrate that the desired hydrogen bond formation is also present in these complexes. Importantly, the protons of the amine or amide moieties also point towards oxygen atoms of the bridging acetate groups in $\left[\mathrm{Zn}_{2}\left(\mathrm{H}_{2} \mathrm{~L}^{2}\right)(\mu-\mathrm{OAC})_{2}\right] \mathrm{PF}_{6}$ and $\left[\mathrm{Zn}_{2}{ }_{2}\left(\mathrm{SIM}^{2}\right)(\mu-\mathrm{OAC})_{2}\right] \mathrm{PF}_{6}$. 
Table 8: Selected distances (Å) for $\left[\mathrm{Zn}_{2}{ }_{2}\left(\mathrm{H}_{2} \mathrm{~L}^{2}\right)(\mu-\mathrm{OAc})_{2}\right] \mathrm{PF} F_{6}, \quad\left[\mathrm{Zn}_{2}{ }_{2}\left(\mathrm{H}_{2} \mathrm{SIM}^{1}\right)(\mu-\mathrm{OAc})(\mathrm{OH})\right] \mathrm{PF} 6_{6}$, $\left[\mathrm{Zn}_{2}{ }_{2}\left(\mathrm{SIM}^{2}\right)(\mu-\mathrm{OAc})_{2}\right] \mathrm{PF}_{6}$, and $\left[\mathrm{Zn}_{2}{ }_{2}\left(\mathrm{H}_{2} \mathrm{SIM}^{3}\right)(\mu-\mathrm{OAc})(\mathrm{OH})\right]$ corresponding to $\mathrm{H}$-bond formation.

\begin{tabular}{|c|c|c|c|c|}
\hline & 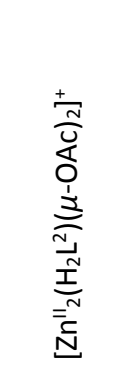 & 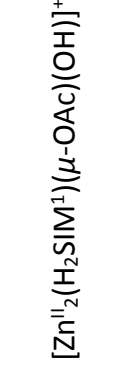 & 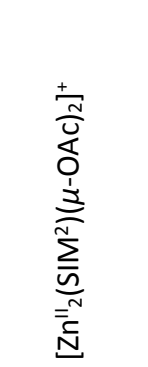 & 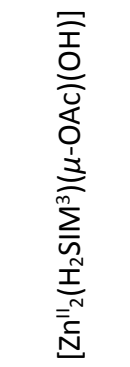 \\
\hline $\mathrm{N}(7)-\mathrm{O}(2 \mathrm{~B})$ & $2.982(4)$ & - & - & \\
\hline$N(7)-O(3 A)$ & - & - & $2.802(9)$ & \\
\hline$N(7)-O(6)$ & - & $2.809(4)$ & - & $2.764(3)$ \\
\hline$N(8)-O(3 A)$ & $2.976(4)$ & - & $2.750(8)$ & \\
\hline $\mathrm{N}(8)-\mathrm{O}(6)$ & - & $2.874(4)$ & - & $2.870(3)$ \\
\hline
\end{tabular}

Moreover, the hydrogen bonding appears to direct the position of the pivaloyl-amide groups resulting in an enlarged angle between the pyridine planes and the plane containing the amide group $(\mathrm{N}(7) \mathrm{C}(34) \mathrm{O}(4)$ and $\mathrm{N}(8) \mathrm{C}(37) \mathrm{O}(5))\left(28.3^{\circ}\right.$ and $17.8^{\circ}$ for $\left[\mathrm{Zn}_{2}{ }_{2}\left(\mathrm{H}_{2} \mathrm{~L}^{2}\right)(\mu-\mathrm{OAC})_{2}\right]^{+}$; $12.0^{\circ}$ and $19.2^{\circ}$ for $\left[\mathrm{Zn}_{2}{ }_{2}\left(\mathrm{H}_{2} \mathrm{SIM}^{1}\right)(\mu-\mathrm{OAc})(\mathrm{OH})\right]^{+} ; \quad 9.2^{\circ}$ and $19.1^{\circ}$ for $\left.\left[\mathrm{Zn}_{2}{ }_{2}\left(\mathrm{H}_{2} \mathrm{SIM}^{3}\right)(\mu-\mathrm{OAc})(\mathrm{OH})\right]\right)$. This angle was found to be small in the structure of the 6-(pivaloylamido)pyridine containing ligand $\mathrm{HL}^{34}$ with a value of $11.4^{\circ}$ due to a $\mathrm{C}-\mathrm{H} \cdots \mathrm{O}=\mathrm{C}$ interaction between the carbonyl oxygen and the nearby hydrogen atom in 5-position of the pyridine ring. ${ }^{168}$ Interestingly, in the asymmetric complexes $\left[\mathrm{Zn}_{2}{ }_{2}\left(\mathrm{H}_{2} \mathrm{SIM}^{1}\right)(\mu-\mathrm{OAc})(\mathrm{OH})\right]^{+}$and $\left[\mathrm{Zn}_{2}{ }_{2}\left(\mathrm{H}_{2} \mathrm{SIM}^{3}\right)(\mu-\mathrm{OAc})(\mathrm{OH})\right]$ only the angle at the 6-(pivaloylamido)pyridine moiety, which is coordinated opposite of the bridging acetate group, is expanded, presumably due to steric hindrance within the cavity.

In all five complexes discussed in this chapter, the $\mathrm{Zn}$ " ions are coordinated in the anticonfiguration with respect to the phenoxide ring. The resulting $\mathrm{Zn}^{\| \prime . . Z \mathrm{Zn}}$ " distances (see Table 7) are at the short end of the range reported for similar dizinc(II) complexes. The variation of ligand symmetry and substituents in the range of five ligands discussed here only leads to a relatively small variation of the $\mathrm{Zn}^{\prime \prime} \cdots \mathrm{Zn}^{\prime \prime}$ distance (3.37 $\AA$ to $3.59 \AA$ ). Importantly, the $\mathrm{Zn}^{\prime \prime} \cdots \mathrm{Zn}$ " distances are slightly elongated in the asymmetric complexes $\left[\mathrm{Zn}_{2}{ }_{2}\left(\mathrm{H}_{2} \mathrm{SIM}^{1}\right)(\mu-\mathrm{OAc})(\mathrm{OH})\right] \mathrm{PF}_{6}$ and $\left[\mathrm{Zn}_{2}{ }_{2}\left(\mathrm{H}_{2} \mathrm{SIM}^{3}\right)(\mu-\mathrm{OAc})(\mathrm{OH})\right]$ to $3.54 \AA$ and $3.59 \AA$. Consequently, the $\mathrm{Zn}$ "-O- $\mathrm{Zn}$ " angle is increased in those two complexes to about $120^{\circ}$, close to a highly symmetrical trigonal angle with respect to the phenoxide bridge, and this compares with $\mathrm{Zn}^{\prime \prime}-\mathrm{O}-\mathrm{Zn}$ " angles in the range $112.8^{\circ}$ to $116.1^{\circ}$ in the more symmetric derivatives $\left[\mathrm{Zn}_{2}{ }_{2}\left(\mathrm{~L}^{1}\right)(\mu-\mathrm{OAc})_{2}\right] \mathrm{PF}{ }_{6},\left[\mathrm{Zn}_{2}{ }_{2}\left(\mathrm{H}_{2} \mathrm{~L}^{2}\right)(\mu-\mathrm{OAc})_{2}\right] \mathrm{PF}_{6}$, and $\left[\mathrm{Zn}_{2}{ }_{2}\left(\mathrm{SIM}^{2}\right)(\mu-\mathrm{OAc})_{2}\right] \mathrm{PF}{ }_{6}$. 
The X-ray crystallographic results for the acetate-bridged dizinc(II) complexes give rise to the conclusions that (i) $\mathrm{Zn}$ " ions bound in phenoxido-based ligands with pyridine donors (e.g. the less hindered ligands $\mathrm{HL}^{1}, \mathrm{H}_{3} \mathrm{~L}^{2}$ and $\mathrm{HSIM}^{2}$ ) favor a six-coordinate geometry (by binding to two bridging acetate ligands) over the trigonal bipyramidal geometry with only one bridging acetate co-ligand, which was only observed in the case of $\mathrm{H}_{3} \mathrm{SIM}^{1}$ and $\mathrm{H}_{4} \mathrm{SIM}^{3}$ due to the sterically demanding tert-butyl groups, and (ii) the two bulky pivaloyl-amide residues of $\mathrm{H}_{3} \mathrm{~L}^{2}$ are positioned on opposite sides of the phenolate backbone upon coordination of two zinc(II) ions. Moreover, the asymmetric ligands $\mathrm{H}_{3} \mathrm{SIM}^{1}$ and $\mathrm{H}_{4} \mathrm{SIM}^{3}$ enforce a cavity that leads to the complexation of two $\mathrm{Zn}$ " ions in different coordination geometries: one zinc(II) site is octahedral, the other site is trigonal bipyramidal, the coordination geometry found for both $\mathrm{Zn}$ " ions in the active site of Phospholipase C ${ }^{201}$ and for one $Z n^{\prime \prime}$ ion in Ser/Thr Phosphatase-1, ${ }^{202}$ which is accompanied by a zinc(II) in a square-pyramidal geometry. ${ }^{202}$ The biomimetic systems $\left[\mathrm{Zn}_{2}{ }_{2}\left(\mathrm{H}_{2} \mathrm{SIM}^{1}\right)(\mu-\mathrm{OAc})(\mathrm{OH})\right] \mathrm{PF}_{6}, \quad\left[\mathrm{Zn}_{2}{ }_{2}\left(\mathrm{H}_{2} \mathrm{~L}^{2}\right)(\mu-\mathrm{OAc})_{2}\right] \mathrm{PF}_{6}, \quad\left[\mathrm{Zn}_{2}\left(\mathrm{SIM}^{2}\right)(\mu-\mathrm{OAc})_{2}\right] \mathrm{PF}_{6}, \quad$ and $\left[\mathrm{Zn}_{2}{ }_{2}\left(\mathrm{H}_{2} \mathrm{SIM}^{3}\right)(\mu-\mathrm{OAC})(\mathrm{OH})\right]$ demonstrate the formation of a hydrogen-bond network similar to that found in the active site of the native enzymes.

\section{Infrared Spectroscopy}

Examination of solid state FT-IR spectra of the dizinc(II) complexes and comparison among each other as well as to the free ligands provide a good method for assigning the different infrared vibrations and investigating the symmetry of the complexes and the strength of the hydrogen bonds formed (Figure 32). ${ }^{203}$
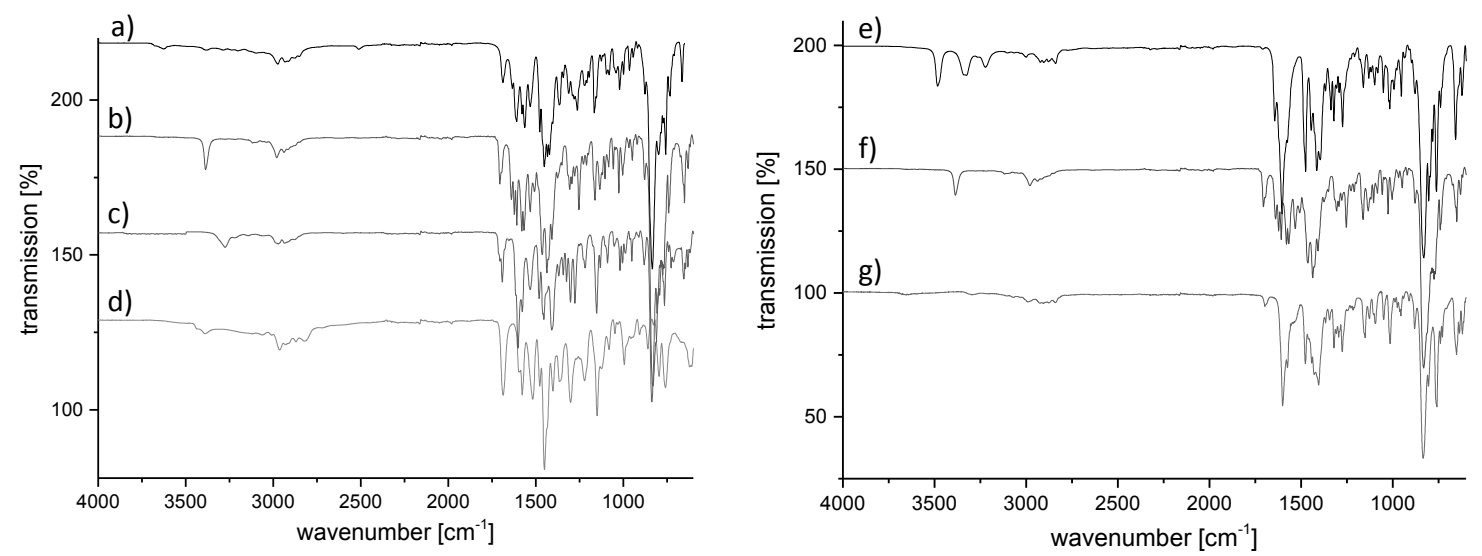

Figure 32: Comparison of IR spectra of a) $\left[\mathrm{Zn}_{2}{ }_{2}\left(\mathrm{H}_{2} \mathrm{SIM}^{3}\right)(\mu-\mathrm{OAc})(\mathrm{OH})\right]$, b) $\left.\left[\mathrm{Zn}_{2}{ }_{2}\left(\mathrm{H}_{2} \mathrm{SIM}^{1}\right)(\mu-\mathrm{OAc})(\mathrm{OH})\right] \mathrm{PF} F_{6}, \mathrm{c}\right)$ $\left[\mathrm{Zn}_{2}\left(\mathrm{H}_{2} \mathrm{~L}^{2}\right)(\mu-\mathrm{OAC})_{2}\right] \mathrm{PF}_{6}$, d) $\mathrm{H}_{2} \mathrm{SIM}^{1}$, e) $\left[\mathrm{Zn}_{2}{ }_{2}\left(\mathrm{SIM}^{2}\right)(\mu-\mathrm{OAC})_{2}\right] \mathrm{PF} F_{6}$, f) $\left[\mathrm{Zn}_{2}\left(\mathrm{H}_{2} \mathrm{SIM}^{1}\right)(\mu-\mathrm{OAc})(\mathrm{OH})\right] \mathrm{PF}_{6}$ and $\left.\mathrm{g}\right)$ $\left[\mathrm{Zn}_{2}\left(\mathrm{~L}^{1}\right)(\mu-\mathrm{OAC})_{2}\right] \mathrm{PF} 6$.

The very similar IR spectra of $\mathrm{H}_{3} \mathrm{SIM}^{1}$ and $\mathrm{H}_{3} \mathrm{~L}^{2}$ show the $\mathrm{N}-\mathrm{H}$ vibration band at around $3440 \mathrm{~cm}^{-1}$ (Figure 32d); this is lowered by $145 \mathrm{~cm}^{-1}\left(1.73 \mathrm{~kJ} \cdot \mathrm{mol}^{-1}\right)$ in the spectrum of 
$\left[\mathrm{Zn}_{2}{ }_{2}\left(\mathrm{H}_{2} \mathrm{~L}^{2}\right)(\mu-\mathrm{OAc})_{2}\right] \mathrm{PF}_{6}$ (Figure 32c). This can be explained by hydrogen bonding, which results in $\mathrm{N}-\mathrm{H}$ bond elongation and therefore in a lowering of the vibrational frequency and a broadening of the corresponding bands. Thus, the detection of only a very broad $\mathrm{N}-\mathrm{H}$ vibration band for $\left[\mathrm{Zn}_{2}{ }_{2}\left(\mathrm{H}_{2} \mathrm{SIM}^{1}\right)(\mu-\mathrm{OAc})(\mathrm{OH})\right] \mathrm{PF}_{6}$ around $3120 \mathrm{~cm}^{-1}\left(\Delta \mathrm{v}=220 \mathrm{~cm}^{-1}, 2.63 \mathrm{~kJ} \cdot \mathrm{mol}^{-1}\right)$ suggests that the hydrogen bonds are even stronger in this complex (Figure 32b). ${ }^{204}{ }^{205}$ Moreover, the terminal hydroxido-ligand in $\left[\mathrm{Zn}_{2}{ }_{2}\left(\mathrm{H}_{2} \mathrm{SIM}^{1}\right)(\mu-\mathrm{OAc})(\mathrm{OH})\right] \mathrm{PF}_{6}$ results in a sharp O-H stretch with a frequency of $3385 \mathrm{~cm}^{-1}$. The small energy value of $3385 \mathrm{~cm}^{-1}\left(40.5 \mathrm{~kJ} \cdot \mathrm{mol}^{-1}\right)$ for the excitation of the $\mathrm{O}-\mathrm{H}$ stretch additionally reflects the strength of the hydrogen bonding within this complex. Unfortunately, for $\left[\mathrm{Zn}_{2}{ }_{2}\left(\mathrm{H}_{2} \mathrm{SIM}^{3}\right)(\mu-\mathrm{OAc})(\mathrm{OH})\right]$ both bands, associated with hydrogen bonding, are very broad (Figure 32a).

The spectrum of the reference complex $\left[\mathrm{Zn}_{2}{ }_{2}\left(\mathrm{~L}^{1}\right)(\mu-\mathrm{OAc})_{2}\right] \mathrm{PF}_{6}$ lacks bands in the region between $3100 \mathrm{~cm}^{-1}$ to $3600 \mathrm{~cm}^{-1}$ (Figure 32g), whereas its amino derivative, $\left[\mathrm{Zn}_{2}{ }_{2}\left(\mathrm{SIM}^{2}\right)(\mu-\mathrm{OAC})_{2}\right] \mathrm{PF}_{6}$, has three transitions in this region, assigned to $\mathrm{N}-\mathrm{H}$ stretching vibrations (Figure 32e). Additionally, the separation of the asymmetric and symmetric acetate stretches at around $1400 \mathrm{~cm}^{-1}$ and $1550 \mathrm{~cm}^{-1}$ confirms bidentate binding in the solid state of all dizinc(II) complexes, discussed herein. ${ }^{205,206}$ Interestingly, for the asymmetric complexes $\left[\mathrm{Zn}_{2}{ }_{2}\left(\mathrm{H}_{2} \mathrm{SIM}^{1}\right)(\mu-\mathrm{OAc})(\mathrm{OH})\right] \mathrm{PF}_{6}$ and $\left[\mathrm{Zn}_{2}{ }_{2}\left(\mathrm{H}_{2} \mathrm{SIM}^{3}\right)(\mu-\mathrm{OAc})(\mathrm{OH})\right]$ the intensity of the band assigned to the asymmetric acetate stretching vibration is lower $\left(\sim 1580 \mathrm{~cm}^{-1}\right)$ than for the symmetric counterpart, in contrast to the symmetric complexes $\left[\mathrm{Zn}_{2}{ }_{2}\left(\mathrm{~L}^{1}\right)(\mu-\mathrm{OAc})_{2}\right] \mathrm{PF}_{6}$, $\left[\mathrm{Zn}_{2}{ }_{2}\left(\mathrm{H}_{2} \mathrm{~L}^{2}\right)(\mu-\mathrm{OAc})_{2}\right] \mathrm{PF}_{6}$ and $\left[\mathrm{Zn}_{2}{ }_{2}\left(\mathrm{SIM}^{2}\right)(\mu-\mathrm{OAc})_{2}\right] \mathrm{PF}_{6}\left(\sim 1600 \mathrm{~cm}^{-1}\right)$, suggesting a slightly different coordination geometry. In the cases in which hexafluorophosphate was present as the counter ion stretching and deformation bands were detected around $830 \mathrm{~cm}^{-1}$ and $555 \mathrm{~cm}^{-1}$.

\section{Mass Spectrometry}

In order to investigate the complex structure of the dizinc(II) complexes in solution, mass spectrometric data were obtained in dichloromethane as well as in acetonitrile; Table 9 summarizes the highest mass peaks detected. All highest mass peaks showed the distinctive isotopic pattern of dizinc(II) complexes (Figure 33). As expected, the separation of the peaks for the doubly charged species was $0.5 \mathrm{~m} / \mathrm{z}$ units.

The mass spectrometric measurement showed solvent dependency in the case of the doubly acetate-bridged complexes $\left[\mathrm{Zn}_{2}{ }_{2}\left(\mathrm{~L}^{1}\right)(\mu-\mathrm{OAc})_{2}\right] \mathrm{PF}_{6}, \quad\left[\mathrm{Zn}_{2}{ }_{2}\left(\mathrm{H}_{2} \mathrm{~L}^{2}\right)(\mu-\mathrm{OAc})_{2}\right] \mathrm{PF}_{6}, \quad$ and $\left[\mathrm{Zn}_{2}{ }_{2}\left(\mathrm{SIM}^{2}\right)(\mu-\mathrm{OAC})_{2}\right] \mathrm{PF}_{6}$. Thus, the highest mass peaks obtained by $\mathrm{ESI}^{+}$mass spectrometric measurements in dichloromethane were assigned to the intact cation, but similar measurements 
in acetonitrile resulted in highest mass peaks that imply ligand exchange within the complex cationic structure. In contrast, the complexes $\left[\mathrm{Zn}_{2}{ }_{2}\left(\mathrm{H}_{2} \mathrm{SIM}^{1}\right)(\mu-\mathrm{OAc})(\mathrm{OH})\right] \mathrm{PF}_{6}$ and $\left[\mathrm{Zn}_{2}{ }_{2}\left(\mathrm{H}_{2} \mathrm{SIM}^{3}\right)(\mu-\mathrm{OAC})(\mathrm{OH})\right]$, containing only one bridging acetate co-ligand and an additional hydroxido co-ligand led in both solvents to highest mass peaks that could be assigned to species missing the acetate bridge. These findings imply ligand exchange by solvent or water molecules in acetonitrile (rather than dichloromethane) for all complexes discussed in this chapter.
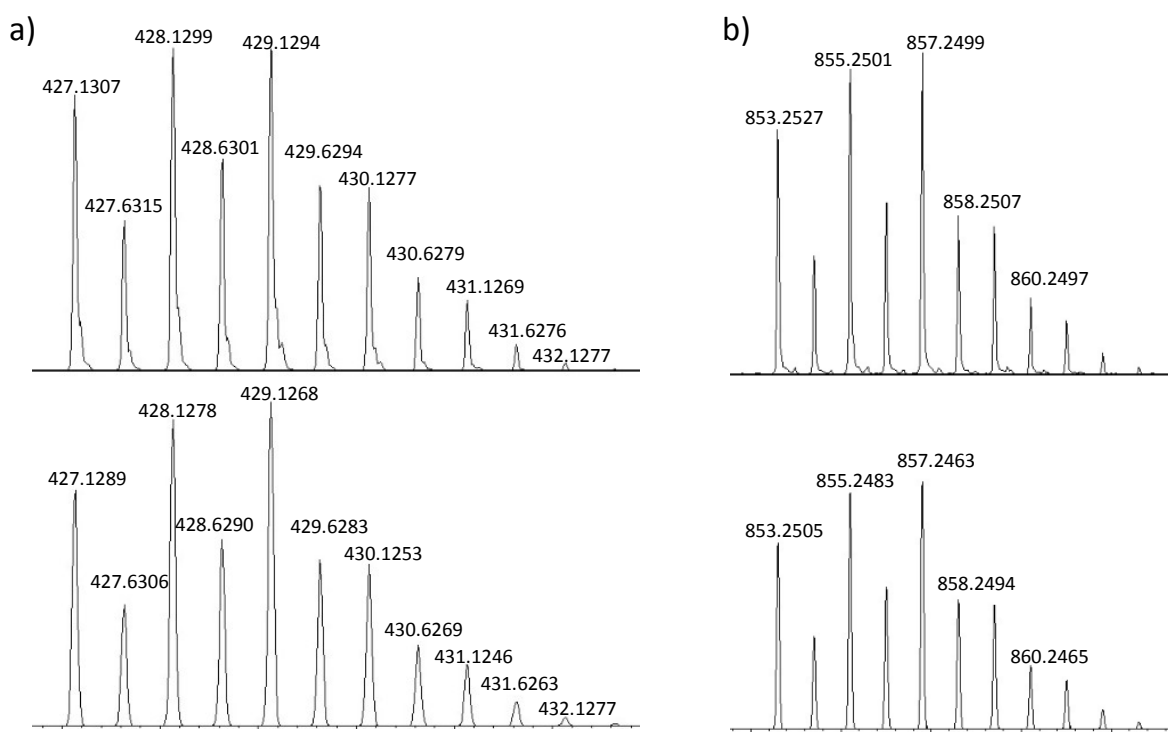

Figure 33: Recorded (top) and calculated (bottom) isotopic pattern for a) $\left[\mathrm{Zn}_{2}{ }_{2}\left(\mathrm{H}_{2} \mathrm{~L}^{2}\right)(\mathrm{OH})\left(\mathrm{H}_{2} \mathrm{O}\right)\right]^{2+}$ and b) $\left[\mathrm{Zn}_{2}\left(\mathrm{~L}^{2}\right)\right]^{2+}$ detected in the mass spectrum of $\left[\mathrm{Zn}_{2}{ }_{2}\left(\mathrm{H}_{2} \mathrm{~L}^{2}\right)(\mu-\mathrm{OAc})_{2}\right] \mathrm{PF} 6$ in acetonitrile.

Table 9: Highest mass peaks in $\mathrm{ESI}^{+}$mass spectrometric measurements of $\left[\mathrm{Zn}_{2}{ }_{2}\left(\mathrm{~L}^{1}\right)(\mu-\mathrm{OAc})_{2}\right] \mathrm{PF}_{6}$, $\left[\mathrm{Zn}_{2}{ }_{2}\left(\mathrm{H}_{2} \mathrm{~L}^{2}\right)(\mu-\mathrm{OAc})_{2}\right] \mathrm{PF}{ }_{6},\left[\mathrm{Zn}_{2}{ }_{2}\left(\mathrm{H}_{2} \mathrm{SIM}^{1}\right)(\mu-\mathrm{OAc})(\mathrm{OH})\right] \mathrm{PF} 6_{6},\left[\mathrm{Zn}_{2}{ }_{2}\left(\mathrm{SIM}^{2}\right)(\mu-\mathrm{OAc})_{2}\right] \mathrm{PF}_{6}$, and $\left[\mathrm{Zn}_{2}{ }_{2}\left(\mathrm{H}_{2} \mathrm{SIM}^{3}\right)(\mu-\mathrm{OAc})(\mathrm{OH})\right]$ in dichloromethane and acetonitrile.

\begin{tabular}{|c|c|c|c|c|}
\hline \multirow{3}{*}{ complex } & \multicolumn{4}{|c|}{ highest $\mathrm{ESI}^{+}$mass peaks } \\
\hline & \multicolumn{2}{|c|}{ in dichloromethane } & \multicolumn{2}{|c|}{ in acetonitrile } \\
\hline & $\begin{array}{l}\text { peak found } \\
(\mathrm{m} / \mathrm{z})\end{array}$ & $\begin{array}{l}\text { assigned to } \\
\text { (calcd m/z) }\end{array}$ & $\begin{array}{l}\text { peak found } \\
(\mathrm{m} / \mathrm{z})\end{array}$ & $\begin{array}{l}\text { assigned to } \\
\text { (calcd m/z) }\end{array}$ \\
\hline \multirow[t]{4}{*}[\mathrm{Zn}_{2}{}_{2}(\mathrm{L}^{1})(\mu-\mathrm{OAc})_{2}]{$\mathrm{PF}_{6}$} & 779.1537 & {$\left[\mathrm{Zn}_{2}{ }_{2}\left(\mathrm{~L}^{1}\right)(\mathrm{OAc})_{2}\right]^{+}$} & 360.0704 & {$\left[\mathrm{Zn}_{2}{ }_{2}\left(\mathrm{~L}^{1}\right)(\mathrm{OAc})\right]^{2+}$} \\
\hline & & 779.1517 & & 360.0689 \\
\hline & & & 779.1507 & {$\left[\mathrm{Zn}_{2}\left(\mathrm{~L}^{1}\right)(\mathrm{OAc})_{2}\right]^{+}$} \\
\hline & & & & 779.1517 \\
\hline \multirow[t]{4}{*}[\mathrm{Zn}_{2}{}_{2}(\mathrm{H}_{2}\mathrm{SIM}^{1})(\mu-\mathrm{OAc})(\mathrm{OH})]{$\mathrm{PF}_{6}$} & 921.2715 & {$\left[\mathrm{Zn}_{2}\left(\mathrm{H}_{2} \mathrm{SIM}^{1}\right)(\mathrm{OH})_{2}\left(\mathrm{CH}_{2} \mathrm{O}\right)\right]^{+}$} & 429.1298 & {$\left[\mathrm{Zn}_{2}{ }_{2}\left(\mathrm{HSIM}^{1}\right)\right]^{2+}$} \\
\hline & & 921.2791* & & 429.1268 \\
\hline & & & 917.2757 & {$\left[\mathrm{Zn}_{2}{ }_{2}\left(\mathrm{HSIM}^{1}\right)(\mathrm{OAc})\right]^{+}$} \\
\hline & & & & 917.2674 \\
\hline \multirow[t]{2}{*}[\mathrm{Zn}_{2}{}_{2}(\mathrm{H}_{2}\mathrm{L}^{2})(\mu-\mathrm{OAc})_{2}]{$\mathrm{PF}_{6}$} & 977.2952 & {$\left[\mathrm{Zn}_{2}{ }_{2}\left(\mathrm{H}_{2} \mathrm{~L}^{2}\right)(\mathrm{OAc})_{2}\right]^{+}$} & 429.1283 & {$\left[\mathrm{Zn}_{2}{ }_{2}\left(\mathrm{HL}^{2}\right)\right]^{2+}$} \\
\hline & & 977.2886 & & 429.1268 \\
\hline \multirow[t]{2}{*}[\mathrm{Zn}_{2}{}_{2}(\mathrm{SIM}^{2})(\mu-\mathrm{OAC})_{2}]{$\mathrm{PF}_{6}$} & 809.1766 & {$\left[\mathrm{Zn}_{2}{ }_{2}\left(\mathrm{SIM}^{2}\right)(\mathrm{OAC})_{2}\right]^{+}$} & 344.0730 & {$\left[\mathrm{Zn}_{2}\left(\mathrm{SIM}^{2}\right)\right]^{2+}$} \\
\hline & & 809.1735 & & 344.0701 \\
\hline \multirow[t]{4}{*}[\mathrm{Zn}_{2}{}_{2}(\mathrm{H}_{2}\mathrm{SIM}^{3})(\mu-\mathrm{OAc})(\mathrm{OH})]{} & 872.2469 & {$\left[\mathrm{Zn}_{2}\left(\mathrm{HSIM}^{3}\right)\right]^{+}$} & 435.6 & {$\left[\mathrm{Zn}_{2}\left(\mathrm{SIM}^{3}\right)\right]^{2+}$} \\
\hline & & 872.2459 & & 435.6272 \\
\hline & & & 908.3 & {$\left[\mathrm{Zn}_{2}{ }_{2}\left(\mathrm{HSIM}^{3}\right)\left(\mathrm{H}_{2} \mathrm{O}\right)_{2}\right]^{+}$} \\
\hline & & & & 908.2682 \\
\hline
\end{tabular}

* Best fit found for this pattern. 


\section{NMR Studies}

In order to probe the symmetry of the complexes in solution, ${ }^{1} \mathrm{H}$ and ${ }^{13} \mathrm{C}$ NMR measurements of the dizinc(II) complexes were carried out in deuterated acetonitrile. Two-dimensional methods (NOESY, COSY, HSQC and HMBC) were used to assign the signals to the explicit hydrogen or carbon atoms in the manner described in Chapter 3.2.1. The number, chemical shifts and splitting of the resonances detected illustrate the symmetry of the coordination compounds in solution.

\section{$\left[\mathrm{Zn}_{2}{ }_{2}\left(\mathrm{~L}^{1}\right)(\mu-\mathrm{OAC})_{2}\right] \mathrm{PF}_{6}$ and $\left[\mathrm{Zn}_{2}{ }_{2}\left(\mathrm{H}_{2} \mathrm{~L}^{2}\right)(\mu-\mathrm{OAC})_{2}\right] \mathrm{PF}_{6}$}

The equivalence of the two binding sites within $\left[\mathrm{Zn}_{2}{ }_{2}\left(\mathrm{H}_{2} \mathrm{~L}^{2}\right)(\mu-\mathrm{OAc})_{2}\right] \mathrm{PF} \mathrm{F}_{6}$, found by $\mathrm{X}$-ray diffractometric investigation, was confirmed as the 16 aromatic protons resulted in eight resonances in the aromatic region of the ${ }^{1} \mathrm{H}$ NMR spectrum, with each of the multiplet resonances integrating for two protons (Figure 34, top). Comparison with the ${ }^{1} \mathrm{H} N M R$ spectrum of the free ligand $\mathrm{H}_{3} \mathrm{~L}^{2}$ (Figure 34 , bottom) shows a downfield shift of protons of the amidated pyridine residues and an upfield shift of protons of the unsubstituted pyridines upon the binding of $\mathrm{Zn}$ "ions. As expected, $\left[\mathrm{Zn}_{2}{ }_{2}\left(\mathrm{~L}^{1}\right)(\mu-\mathrm{OAc})_{2}\right] \mathrm{PF}_{6}$ showed the same behavior, leading to a ${ }^{1} \mathrm{H}$ NMR spectrum corresponding to a pair of two equal pyridine groups within the complex. Moreover, due to the same surroundings the two bridging acetate groups in $\left[\mathrm{Zn}_{2}{ }_{2}\left(\mathrm{~L}^{1}\right)(\mu-\mathrm{OAc})_{2}\right] \mathrm{PF}_{6}$ and $\left[\mathrm{Zn}_{2}\left(\mathrm{H}_{2} \mathrm{~L}^{2}\right)(\mu-\mathrm{OAC})_{2}\right] \mathrm{PF}_{6}$, and the tert-butyl residues in $\left[\mathrm{Zn}_{2}{ }_{2}\left(\mathrm{H}_{2} \mathrm{~L}^{2}\right)(\mu-\mathrm{OAc})_{2}\right] \mathrm{PF}_{6}$ lead to a single resonance. This supports the symmetry appearance of $\left[\mathrm{Zn}_{2}{ }_{2}\left(\mathrm{~L}^{1}\right)(\mu-\mathrm{OAc})_{2}\right] \mathrm{PF} 6$ and $\left[\mathrm{Zn}_{2}{ }_{2}\left(\mathrm{H}_{2} \mathrm{~L}^{2}\right)(\mu-\mathrm{OAc})_{2}\right] \mathrm{PF}_{6}$, also when dissolved in acetonitrile.

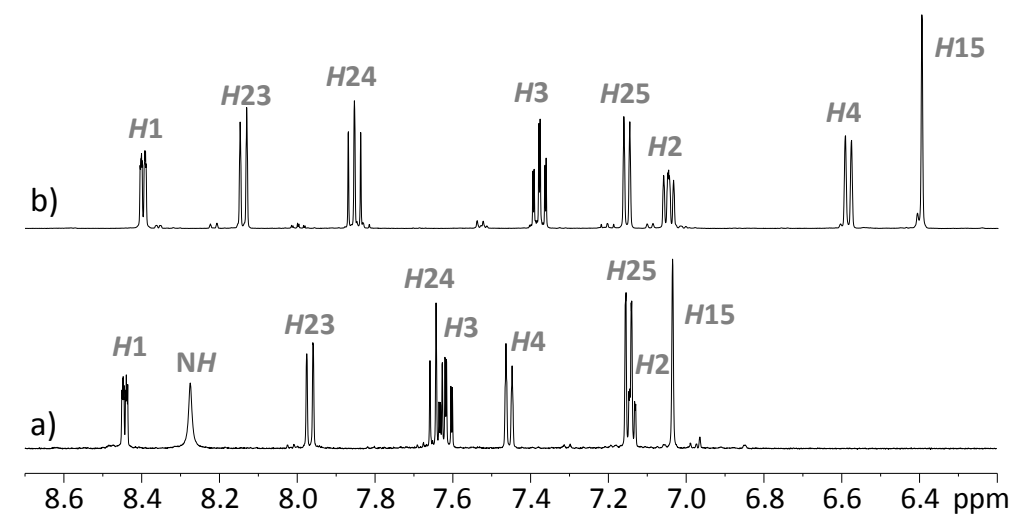

Figure 34: Aromatic region of the ${ }^{1} \mathrm{H}-\mathrm{NMR}$ spectra of a) ligand $\mathrm{H}_{3} \mathrm{~L}^{2}$ and b) complex $\left[\mathrm{Zn}{ }_{2}\left(\mathrm{H}_{2} \mathrm{~L}^{2}\right)(\mu-\mathrm{OAc})_{2}\right] \mathrm{PF} \mathrm{F}_{6}$ in $\mathrm{CD}_{3} \mathrm{CN}$.

Additional experiments were undertaken to study the stability of complex $\left[\mathrm{Zn}_{2}{ }_{2}\left(\mathrm{H}_{2} \mathrm{~L}^{2}\right)(\mu-\mathrm{OAc})_{2}\right] \mathrm{PF}_{6}$ in water/acetonitrile mixtures, as well as under basic conditions (conditions comparable to the activity assays which will be discussed in Chapter 7). Therefore, $\left[\mathrm{Zn}_{2}{ }_{2}\left(\mathrm{H}_{2} \mathrm{~L}^{2}\right)(\mu-\mathrm{OAc})_{2}\right] \mathrm{PF}_{6}$ was dissolved in $\mathrm{CD}_{3} \mathrm{CN}$, and $\mathrm{D}_{2} \mathrm{O}$ was added stepwise. Figure 35 shows the spectra of $\left[\mathrm{Zn}_{2}{ }_{2}\left(\mathrm{H}_{2} \mathrm{~L}^{2}\right)(\mu-\mathrm{OAc})_{2}\right] \mathrm{PF}$ in pure $\mathrm{CD}_{3} \mathrm{CN}$ and $\mathrm{CD}_{3} \mathrm{CN} / \mathrm{D}_{2} \mathrm{O}$ 
mixtures $(8: 1,2: 1,1: 1)$. The addition of $D_{2} \mathrm{O}$ led to no variation in the chemical shifts but induced a broadening of the detected resonances. Further addition of $\mathrm{NaOD}$ in $\mathrm{D}_{2} \mathrm{O}$ resulted in no change in the ${ }^{1} \mathrm{H}$ NMR spectrum as shown in Figure $35 \mathrm{e}$. This implies that no decoordination of $\mathrm{Zn}$ " takes place under basic conditions.

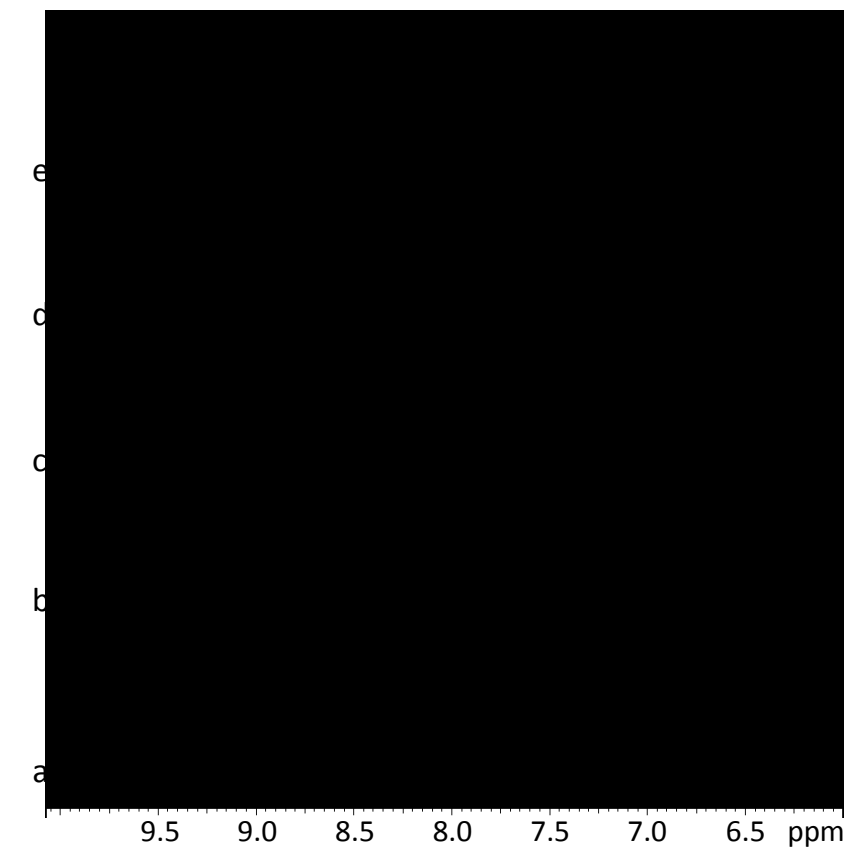

Figure 35: Aromatic region of the ${ }^{1} \mathrm{H}$ NMR spectra of a) complex $\left[\mathrm{Zn}_{2}{ }_{2}\left(\mathrm{H}_{2} \mathrm{~L}^{2}\right)(\mu-\mathrm{OAC})_{2}\right] \mathrm{PF}_{6}$ in $\mathrm{CD}_{3} \mathrm{CN}$ and in $\mathrm{CD}_{3} \mathrm{CN} / \mathrm{D}_{2} \mathrm{O}$ mixtures (b) 8:1 , c) 2:1, d) 1:1) as well as e) in $\mathrm{CD}_{3} \mathrm{CNN}_{2} \mathrm{D}_{2} \mathrm{O}$ mixture with $\mathrm{pH} 10.4$.

\section{$\left[\mathrm{Zn}_{2}{ }_{2}\left(\mathrm{H}_{2} \mathrm{SIM}^{1}\right)(\mu-\mathrm{OAC})(\mathrm{OH})\right] P F_{6}$}

The asymmetry of $\left[\mathrm{Zn}_{2}{ }_{2}\left(\mathrm{H}_{2} \mathrm{SIM}^{1}\right)(\mu-\mathrm{OAc})(\mathrm{OH})\right] \mathrm{PF}_{6}$, observed in the solid state by $\mathrm{X}$-ray diffraction, also emerges from the ${ }^{1} \mathrm{H}$ NMR spectrum, where the aromatic region exhibits 16 resonances (Figure 36, top). In this complex, every hydrogen atom has a different electronic environment leading to the difference in the spectrum, when compared to the metal-free ligand (Figure 36, bottom). The resonances from the protons proximal to the $\mathrm{Zn}^{\prime \prime}$ centers show a downfield shift while protons, that are distant from the metal centers, especially those pointing to the opposite site of the phenolate backbone, show an upfield shift. Similarly, the proton signals of each methylene group are separated, which gives rise to a set of 12 doublets in the region between 3.3 and $4.5 \mathrm{ppm}$ (Figure 37, top). This implies that the electronic environment of protons bound to the same methylene carbon are not identical, in contrast to the spectrum of the free ligand (Figure 37, bottom), and this again is consistent with the observed X-ray structure. The coupling to the other hydrogen atom at the same carbon atom leads to the appearance of doublets. As a result of the asymmetry in this structure, the two tert-butyl groups in $\left[\mathrm{Zn}_{2}{ }_{2}\left(\mathrm{H}_{2} \mathrm{SIM}^{1}\right)(\mu-\mathrm{OAc})(\mathrm{OH})\right]^{+}$give rise to two resonances in the ${ }^{1} \mathrm{H}$ NMR spectrum. 
b)

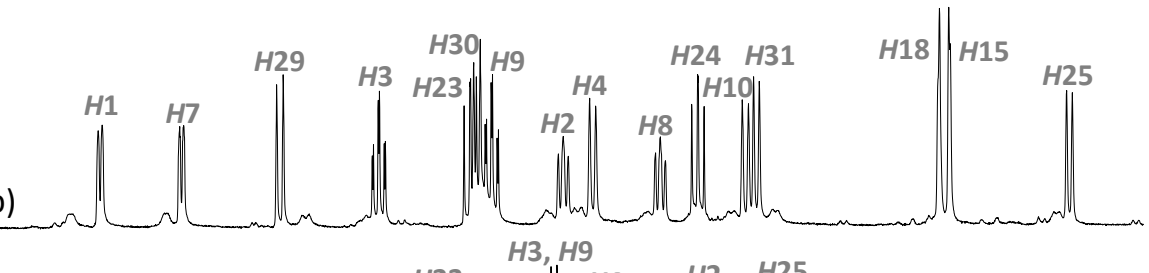

a)

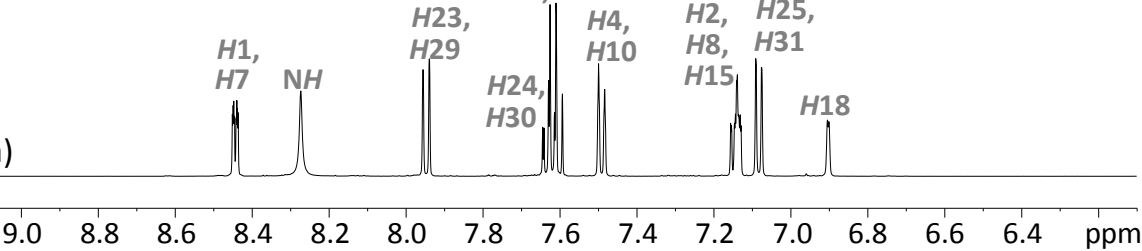

Figure 36: Aromatic region of ${ }^{1} \mathrm{H}$ NMR spectra of a) ligand $\mathrm{H}_{3} \mathrm{SIM}^{1}$ and b) complex $\left[\mathrm{Zn}^{\prime \prime}{ }_{2}\left(\mathrm{H}_{2} \mathrm{SIM}^{1}\right)(\mu-\mathrm{OAc})(\mathrm{OH})\right] \mathrm{PF} 6$ in $\mathrm{CD}_{3} \mathrm{CN}$.

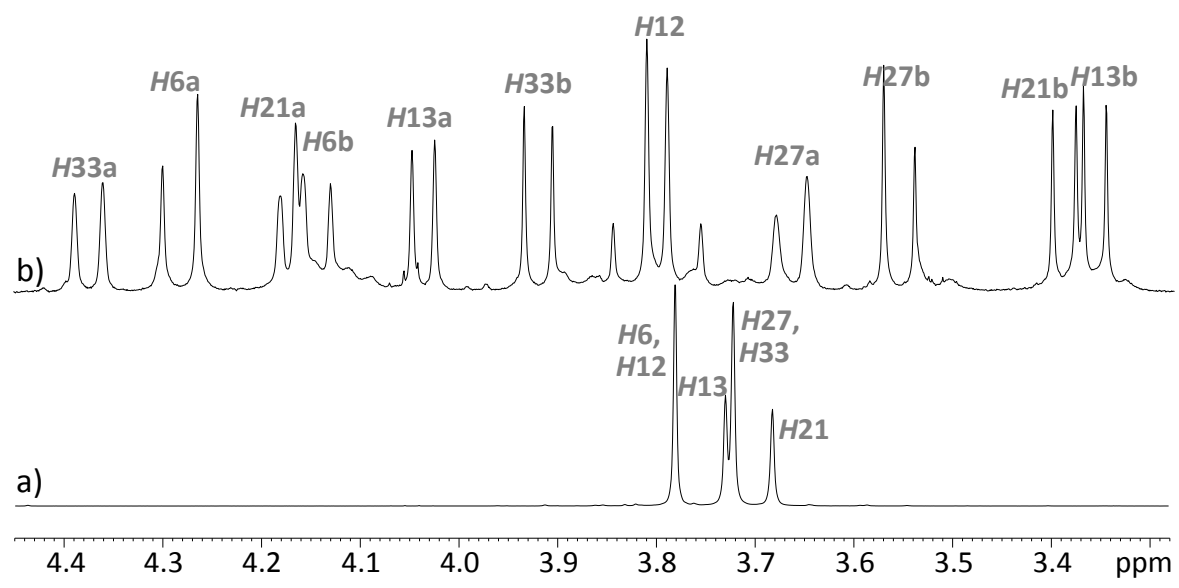

Figure 37: Methylene resonances in the ${ }^{1} \mathrm{H}$ NMR spectra of a) ligand $\mathrm{H}_{3} \mathrm{SIM}^{1}$ and b) complex $\left[\mathrm{Zn}_{2}{ }_{2}\left(\mathrm{H}_{2} \mathrm{SIM}^{1}\right)(\mu-\mathrm{OAC})(\mathrm{OH})\right] \mathrm{PF}_{6}$ in $\mathrm{CD}_{3} \mathrm{CN}$.

\section{$\left[\mathrm{Zn}_{2}{ }_{2}\left(\mathrm{SIM}^{2}\right)(\mu-\mathrm{OAC})_{2}\right] \mathrm{PF}_{6}$}

Although the crystal structure of $\left[\mathrm{Zn}_{2}{ }_{2}\left(\mathrm{SIM}^{2}\right)(\mu-\mathrm{OAc})_{2}\right] \mathrm{PF}_{6}$ suggests that, in the solid state, the complex is symmetrical, the ${ }^{1} \mathrm{H}$ NMR spectrum exhibits 16 resonances in the aromatic region (Figure 38, top). Similar to the spectrum of $\left[\mathrm{Zn}_{2}{ }_{2}\left(\mathrm{H}_{2} \mathrm{SIM}^{1}\right)(\mu-\mathrm{OAc})(\mathrm{OH})\right]^{+}, 12$ resonances in the form of doublets are detected for the methylene protons in $\left[\mathrm{Zn}_{2}\left(\mathrm{SIM}^{2}\right)(\mu-\mathrm{OAc})_{2}\right]^{+}$. In contrast to $\left[\mathrm{Zn}_{2}{ }_{2}\left(\mathrm{~L}^{1}\right)(\mu-\mathrm{OAC})_{2}\right]^{+}$and $\left[\mathrm{Zn}_{2}{ }_{2}\left(\mathrm{H}_{2} \mathrm{~L}^{2}\right)(\mu-\mathrm{OAC})_{2}\right]^{+}$, the two bridging acetate co-ligands in $\left[\mathrm{Zn}_{2}{ }_{2}\left(\mathrm{SIM}^{2}\right)(\mu-\mathrm{OAC})_{2}\right]^{+}$produce two resonances in the ${ }^{1} \mathrm{H}$ NMR spectrum. Therefore, the substitution of two pyridine groups in one of the binding sites of $\mathrm{HL}^{1}$ creates two different coordination environments in $\mathrm{HSIM}^{2}$, which induce the formation of an asymmetric dizinc(II) complex in solution, in contrast to the symmetric structure observed in the solid state by X-ray crystallography. 
b)

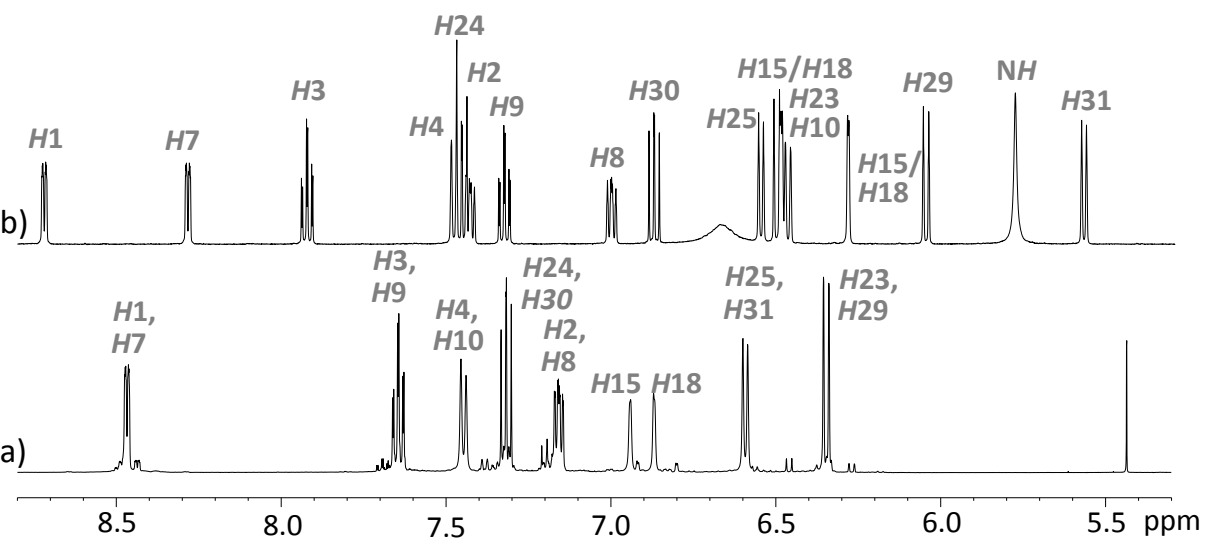

Figure 38: Aromatic region of the ${ }^{1} \mathrm{H}$ NMR spectra of a) ligand $\mathrm{HSIM}^{2}$ and $\left.\mathrm{b}\right)$ complex $\left[\mathrm{Zn}_{2}{ }_{2}\left(\mathrm{SIM}^{2}\right)(\mu-\mathrm{OAc})_{2}\right] \mathrm{PF} 6$ in $\mathrm{CD}_{3} \mathrm{CN}$.

\section{$\left[\mathrm{Zn}_{2}{ }_{2}\left(\mathrm{H}_{2} \mathrm{SIM}^{3}\right)(\mu-\mathrm{OAC})(\mathrm{OH})\right]$}

Unfortunately, the poor solubility of $\left[\mathrm{Zn}_{2}{ }_{2}\left(\mathrm{H}_{2} \mathrm{SIM}^{3}\right)(\mu-\mathrm{OAc})(\mathrm{OH})\right]$ made it impossible to conduct a comparable set of ${ }^{1} \mathrm{H}$ NMR measurements for this complex, because addition of perchloric acid was necessary to achieve solubility. Figure 39 illustrates the ${ }^{1} \mathrm{H}$ NMR spectra obtained for the ligand $\mathrm{H}_{4} \mathrm{SIM}^{3}$ and $\left[\mathrm{Zn}_{2}{ }_{2}\left(\mathrm{H}_{2} \mathrm{SIM}^{3}\right)(\mu-\mathrm{OAc})(\mathrm{OH})\right]$ in deuterated acetonitrile after addition of four volume percent perchloric acid.

Comparison of the spectra exhibits two essential findings. Firstly, resonances for protons of the two amidated pyridine residues in binding site $A$ (defined in Chart 7, Chapter 3 ) as well as resonances for protons of the unsubstituted pyridine moiety in binding site B are shifted in the complex spectrum compared to the free ligand. Secondly, the resonances of only one methylene proton pair is split into two doublets. Therefore, the addition of perchloric acid appears to lead to loss of the phenolate side-arm from the metal ion, since the proton resonances exhibit the same chemical shifts as those for this phenolate in the free ligand. The shift of the resonances assigned to the amidated pyridine residues in addition to the splitting of the methylene group assigned to the same side-arms implies sustained binding of a $\mathrm{Zn}^{\prime \prime}$ ion in binding site $\mathrm{A}$. Whether the pyridine residue of binding site $\mathrm{B}$ is still bound to a $\mathrm{Zn}$ " center remains unclear, since on the one side the aromatic resonances of this side-arm are shifted, but on the other side the methylene group transformed in a singlet. The $\mathrm{SSI}^{+}$mass spectrometric measurement of $\left[\mathrm{Zn}_{2}{ }_{2}\left(\mathrm{H}_{2} \mathrm{SIM}^{3}\right)(\mu-\mathrm{OAc})(\mathrm{OH})\right]$ in deuterated acetonitrile after addition of hydrochloric acid resulted in a spectrum showing the main peak with a pattern typical for a dizinc(II) species $\left(\mathrm{m} / \mathrm{z} 870.2\right.$ assigned to $\left.\left[\mathrm{Zn}_{2}\left(\mathrm{HSIM}^{3}\right)\right]^{+}\right)$, but also a peak with a typical monozinc(II) pattern with a similar intensity was detected $\left(\mathrm{m} / \mathrm{z} 806.2\right.$ assigned to $\left.\left[\mathrm{Zn}\left(\mathrm{H}_{3} \mathrm{SIM}^{3}\right)\right]^{+}\right)$. 


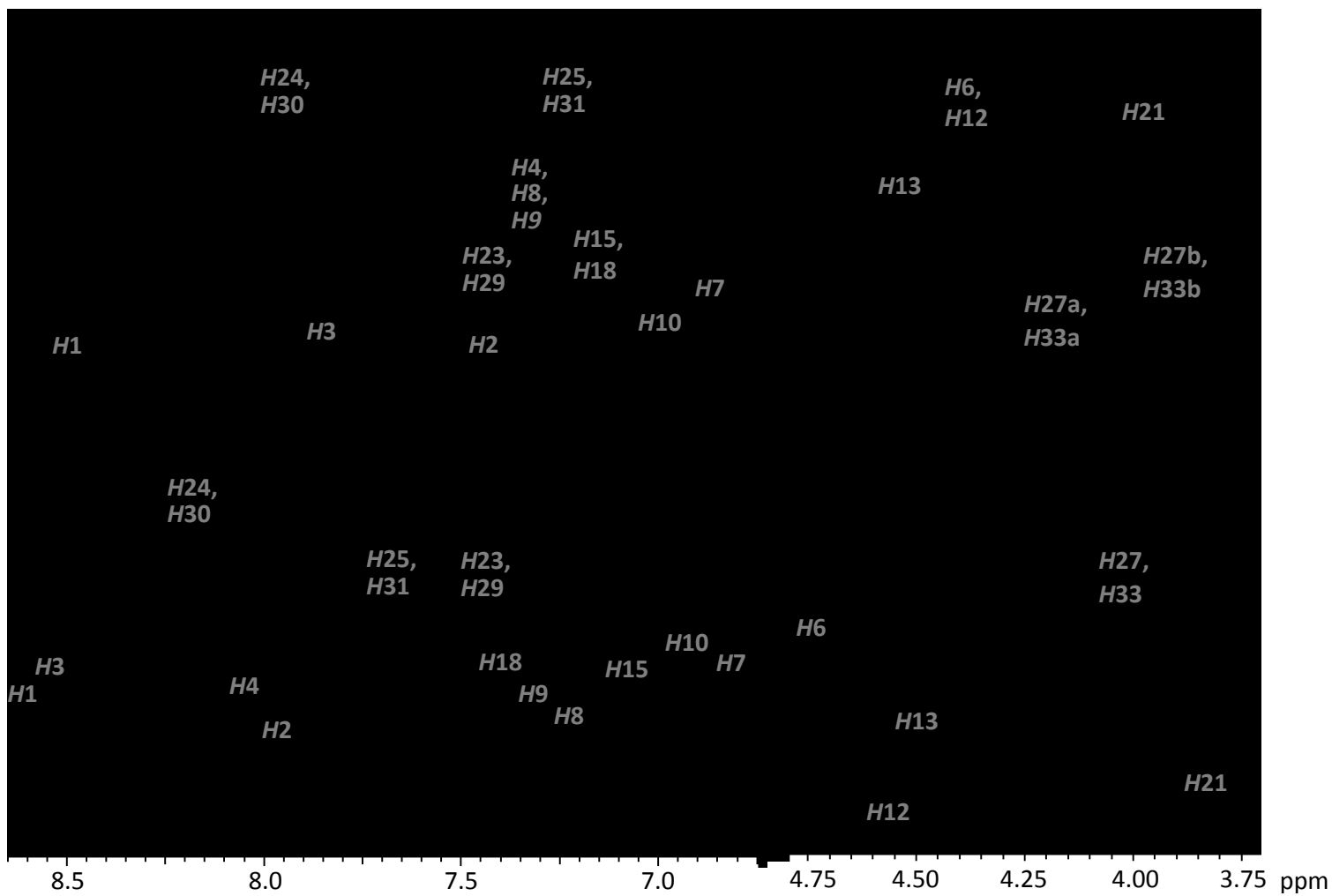

Figure 39: Aromatic region (a) and methylen proton resonances (b) in the ${ }^{1} \mathrm{H}$ NMR spectra of $\mathrm{H}_{4} \mathrm{SIM}^{3}$ (bottom) and $\left[\mathrm{Zn}_{2}{ }_{2}\left(\mathrm{H}_{2} \mathrm{SIM}^{3}\right)(\mu-\mathrm{OAC})(\mathrm{OH})\right]\left(\right.$ top) in $\mathrm{CD}_{3} \mathrm{CN}$ with 4 vol\% $\mathrm{HClO}_{4}$.

The results obtained by NMR spectroscopic techniques demonstrate that only the two complexes $\left[\mathrm{Zn}_{2}{ }_{2}\left(\mathrm{~L}^{1}\right)(\mu-\mathrm{OAc})_{2}\right]^{+}$and $\left[\mathrm{Zn}_{2}{ }_{2}\left(\mathrm{H}_{2} \mathrm{~L}^{2}\right)(\mu-\mathrm{OAc})_{2}\right]^{+}$contain two metal centers in identical geometry, when dissolved in acetonitrile. $\left[\mathrm{Zn}_{2}{ }_{2}\left(\mathrm{H}_{2} \mathrm{SIM}^{1}\right)(\mu-\mathrm{OAc})(\mathrm{OH})\right]^{+}$and $\left[\mathrm{Zn}_{2}{ }_{2}\left(\mathrm{SIM}^{2}\right)(\mu-\mathrm{OAc})_{2}\right]^{+}$, the complexes formed with the asymmetrically substituted ligands $\mathrm{H}_{3} \mathrm{SIM}^{1}$ and $\mathrm{HSIM}^{2}$, contain two $\mathrm{Zn}$ " ions in different surroundings. This suggests that the asymmetry of the amide-based complex $\left[\mathrm{Zn}_{2}{ }_{2}\left(\mathrm{H}_{2} \mathrm{SIM}^{1}\right)(\mu-\mathrm{OAc})(\mathrm{OH})\right] \mathrm{PF}_{6}$ is not only the result of the two different coordination geometries of the two $\mathrm{Zn}$ " centers but is also due to the hydrogen bond donating groups in proximity to one of the coordination sites.

\section{UV-vis Spectroscopic Studies}

Electronic spectra of the complexes $\left[\mathrm{Zn}_{2}{ }_{2}\left(\mathrm{~L}^{1}\right)(\mu-\mathrm{OAc})_{2}\right] \mathrm{PF}_{6}, \quad\left[\mathrm{Zn}_{2}{ }_{2}\left(\mathrm{H}_{2} \mathrm{~L}^{2}\right)(\mu-\mathrm{OAc})_{2}\right] \mathrm{PF} \mathrm{F}_{6}$, $\left[\mathrm{Zn}_{2}{ }_{2}\left(\mathrm{H}_{2} \mathrm{SIM}^{1}\right)(\mu-\mathrm{OAc})(\mathrm{OH})\right] \mathrm{PF}_{6}$, and $\left[\mathrm{Zn}_{2}{ }_{2}\left(\mathrm{SIM}^{2}\right)(\mu-\mathrm{OAc})_{2}\right] \mathrm{PF}_{6}$ were recorded in acetonitrile as well as in acetonitrile/aqueous buffer mixtures (1:1). The aqueous buffer solutions were $50 \mathrm{mM}$ in HEPES, $50 \mathrm{mM}$ in MES, $50 \mathrm{mM}$ in CHES, $50 \mathrm{mM}$ in CAPS and $250 \mathrm{mM}$ in lithium perchlorate and were adjusted to a specific $\mathrm{pH}$ value by addition of aqueous sodium hydroxide solution. These buffer solutions were also used for the hydrolytic activity assays discussed in Chapter 6.3. 
All four dizinc(II) complexes show two bands in the regions at $230-260 \mathrm{~nm}$ and $280-320 \mathrm{~nm}$ attributed to $p_{\pi} \rightarrow p_{\pi^{*}}$ ligand internal transitions (see Figure 40 and Table 10).

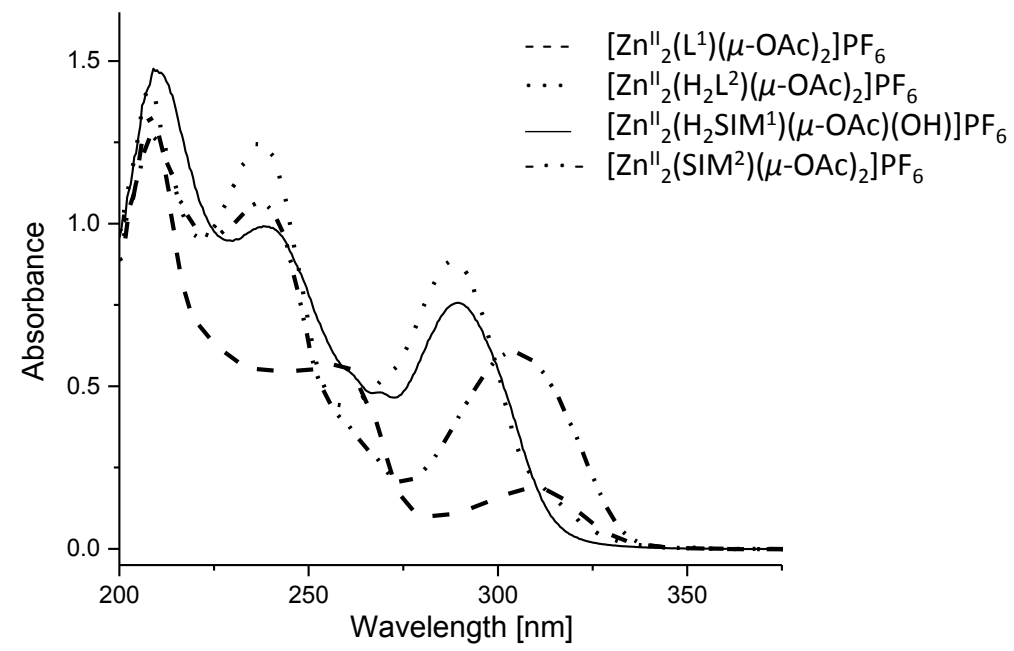

Figure 40: UV-vis absorption spectra of dizinc(II) complexes ( $5 \mu \mathrm{M}$ solution in $\mathrm{CH}_{3} \mathrm{CN}$ ).

Table 10: UV-vis spectral properties of the dizinc(II) complexes (5 $\mu \mathrm{M}$ in $\left.\mathrm{CH}_{3} \mathrm{CN}\right)$.

\begin{tabular}{llll}
\multicolumn{1}{c}{ complex } & $\begin{array}{c}\text { UV-vis } \\
{[\mathrm{nm}]}\end{array}$ & \multicolumn{1}{c}{$\varepsilon$} & transitions \\
{$\left[\mathrm{M}^{-1} \mathrm{~cm}^{-1}\right]$} & \\
\hline$\left[\mathrm{Zn}_{2}{ }_{2}\left(\mathrm{~L}^{1}\right)(\mu-\mathrm{OAC})_{2}\right] \mathrm{PF}_{6}$ & 260 & 113168 & $\mathrm{p}_{\pi} \rightarrow \mathrm{p}_{\pi^{*}}$ \\
& 311 & 37161 & $\mathrm{p}_{\pi} \rightarrow \mathrm{p}_{\pi^{*}}$ \\
\hline$\left[\mathrm{Zn}_{2}{ }_{2}\left(\mathrm{H}_{2} \mathrm{~L}^{2}\right)(\mu-\mathrm{OAC})_{2}\right] \mathrm{PF}_{6}$ & 238 & 249438 & $\mathrm{p}_{\pi} \rightarrow \mathrm{p}_{\pi^{*}}$ \\
& 289 & 177075 & $\mathrm{p}_{\pi} \rightarrow \mathrm{p}_{\pi^{*}}$ \\
\hline$\left[\mathrm{Zn}_{2}{ }_{2}\left(\mathrm{H}_{2} \mathrm{SIM}^{1}\right)(\mu-\mathrm{OAC})(\mathrm{OH})\right] \mathrm{PF}_{6}$ & 239 & 198843 & $\mathrm{p}_{\pi} \rightarrow \mathrm{p}_{\pi^{*}}$ \\
& 290 & 151351 & $\mathrm{p}_{\pi} \rightarrow \mathrm{p}_{\pi^{*}}$ \\
\hline$\left[\mathrm{Zn}_{2}{ }_{2}\left(\mathrm{SIM}^{2}\right)(\mu-\mathrm{OAC})_{2}\right] \mathrm{PF}_{6}$ & 237 & 213347 & $\mathrm{p}_{\pi} \rightarrow \mathrm{p}_{\pi^{*}}$ \\
& 305 & 120667 & $\mathrm{p}_{\pi} \rightarrow \mathrm{p}_{\pi^{*}}$ \\
\hline
\end{tabular}

The substitution with a pivaloyl-amide group in position six of two pyridine residues in $\left[\mathrm{Zn}_{2}{ }_{2}\left(\mathrm{H}_{2} \mathrm{SIM}^{1}\right)(\mu-\mathrm{OAc})(\mathrm{OH})\right]^{+}$and $\left[\mathrm{Zn}_{2}{ }_{2}\left(\mathrm{H}_{2} \mathrm{~L}^{2}\right)(\mu-\mathrm{OAc})_{2}\right]^{+}$leads to a hypsochromic shift in the UVvis spectroscopic measurements, compared with the spectra of the unsubstituted compound $\left[\mathrm{Zn}_{2}{ }_{2}\left(\mathrm{~L}^{1}\right)(\mu-\mathrm{OAc})_{2}\right]^{+}$. The substitution by amino moieties, as in $\left[\mathrm{Zn}_{2}{ }_{2}\left(\mathrm{SIM}^{2}\right)(\mu-\mathrm{OAc})_{2}\right]^{+}$, has a similar but less pronounced effect.

\section{pH Behavior}

In order to examine the behavior of the complexes $\left[\mathrm{Zn}_{2}{ }_{2}\left(\mathrm{~L}^{1}\right)(\mu-\mathrm{OAc})_{2}\right]^{+},\left[\mathrm{Zn}_{2}{ }_{2}\left(\mathrm{H}_{2} \mathrm{~L}^{2}\right)(\mu-\mathrm{OAc})_{2}\right]^{+}$, $\left[\mathrm{Zn}_{2}{ }_{2}\left(\mathrm{H}_{2} \mathrm{SIM}^{1}\right)(\mu-\mathrm{OAc})(\mathrm{OH})\right]^{+}$and $\left[\mathrm{Zn}_{2}\left(\mathrm{SIM}^{2}\right)(\mu-\mathrm{OAc})_{2}\right]^{+}$under basic conditions, UV-vis spectroscopic measurements were also conducted in acetonitrile-aqueous buffer mixtures (1:1); the $\mathrm{pH}$ values recorded refer to the aqueous component. With increasing basicity, the band in the absorbance spectrum of $\left[\mathrm{Zn}_{2}\left(\mathrm{H}_{2} \mathrm{~L}^{2}\right)(\mu-\mathrm{OAc})_{2}\right]^{+}$at $287 \mathrm{~nm}$ experiences a shift to $294 \mathrm{~nm}$. Also, the band at $233 \mathrm{~nm}$ decreases in intensity, while new bands at $259 \mathrm{~nm}$ and 
$326 \mathrm{~nm}$ arise (Figure 41a). Figure 41b shows the change of absorbance at different wavelengths as a function of rising $\mathrm{pH}$ value. The overlay of the spectra at different $\mathrm{pH}$ values indicates the presence of three isosbestic points at $246 \mathrm{~nm}, 270 \mathrm{~nm}$ and $297 \mathrm{~nm}$. The observation of isosbestic points indicates that the dizinc(II) complex is stable in the investigated $\mathrm{pH}$ range and undergoes a single deprotonation step. Therefore, the curves can be analyzed by the Henderson-Hasselbalch Equation 9, where $[\mathrm{HA}]$ and $\left[\mathrm{A}^{-}\right]$are the concentrations of the protonated and deprotonated complex species, respectively, in the equilibrium ( $\mathrm{K}_{\mathrm{a}}$ : equilibrium constant).

$$
\text { Equation } 9 \quad \mathrm{pH}=\mathrm{pK}_{\mathrm{a}}+\log \frac{\left[\mathrm{A}^{-}\right]}{[\mathrm{HA}]}
$$

$[\mathrm{HA}]$ and $\left[\mathrm{A}^{-}\right]$follow the correlation given in Equation 10 with the initial complex concentration $[\mathrm{HA}]_{0}$.

$$
\text { Equation } 10 \quad\left[\mathrm{~A}^{-}\right]=[\mathrm{HA}]_{0}-[\mathrm{HA}]
$$

Therefore, the concentration of protonated species in solution can be described as given in Equation 11.

$$
\text { Equation } 11 \quad[\mathrm{HA}]=\frac{[\mathrm{HA}]_{0}}{10^{\mathrm{pH}-\mathrm{pK}_{\mathrm{s}}+1}}
$$

The Beer-Lambert Law assuming two species in solution is given in Equation 12, where $\varepsilon_{1}$ and $\varepsilon_{2}$ are the extinction coefficients of the protonated $(\mathrm{HA})$ and deprotonated complex species $\left(A^{-}\right)$and $d$ is the path length.

$$
\text { Equation } 12 \quad \mathrm{Abs}=\varepsilon_{1} * \mathrm{~d} *[\mathrm{HA}]+\varepsilon_{2} * \mathrm{~d} *\left[\mathrm{~A}^{-}\right]
$$

Consideration of Equation 10, leads to Equation 13.

$$
\text { Equation } 13 \mathrm{Abs}=\left(\varepsilon_{1}-\varepsilon_{2}\right) * \mathrm{~d} *[\mathrm{HA}]+\varepsilon_{2} * \mathrm{~d} *[\mathrm{HA}]_{0}
$$

Substituting [HA] in Equation 13, as defined in Equation 11, provides Equation 14.

$$
\text { Equation } 14 \quad \mathrm{Abs}=\left(\varepsilon_{1}-\varepsilon_{2}\right) * \frac{[\mathrm{HA}]_{0} * \mathrm{~d}}{10^{\mathrm{pH}-\mathrm{pK} \mathrm{a}+1}}+\varepsilon_{2} *[\mathrm{HA}]_{0} * \mathrm{~d}
$$

Here, the absorbance depends on the extinction coefficients, $\varepsilon_{1}$ and $\varepsilon_{2}$, and the $\mathrm{pK}_{a}$ value. Fitting of the $\mathrm{pH}$-dependent spectroscopic data to Equation 14 results in an estimate of the $\mathrm{pK}_{\mathrm{a}}$ value of $\left[\mathrm{Zn}_{2}{ }_{2}\left(\mathrm{H}_{2} \mathrm{~L}^{2}\right)(\mu-\mathrm{OAc})_{2}\right]^{+}$of $8.14 \pm 0.25$. $\mathrm{pH}$-dependent UV-vis spectroscopic measurements of $\left[\mathrm{Zn}_{2}{ }_{2}\left(\mathrm{H}_{2} \mathrm{SIM}^{1}\right)(\mu-\mathrm{OAc})(\mathrm{OH})\right]^{+}$show similar effects (Figure $41 \mathrm{c}, \mathrm{d}$ ), and from fitting of these data to Equation 14, the $\mathrm{pK}_{\mathrm{a}}$ value of $\left[\mathrm{Zn}_{2}{ }_{2}\left(\mathrm{H}_{2} \mathrm{SIM}^{1}\right)(\mu-\mathrm{OAc})(\mathrm{OH})\right]^{+}$is: $\mathrm{pK}_{\mathrm{a}}=7.27 \pm 0.65$. The variation of the $\mathrm{pH}$ value from 5 to 11 did not result in any change of the UV-vis spectra of $\left[\mathrm{Zn}_{2}{ }_{2}\left(\mathrm{~L}^{1}\right)(\mu-\mathrm{OAc})_{2}\right] \mathrm{PF}_{6}$ and $\left[\mathrm{Zn}_{2}{ }_{2}\left(\mathrm{SIM}^{2}\right)(\mu-\mathrm{OAC})_{2}\right] \mathrm{PF} 6$. 

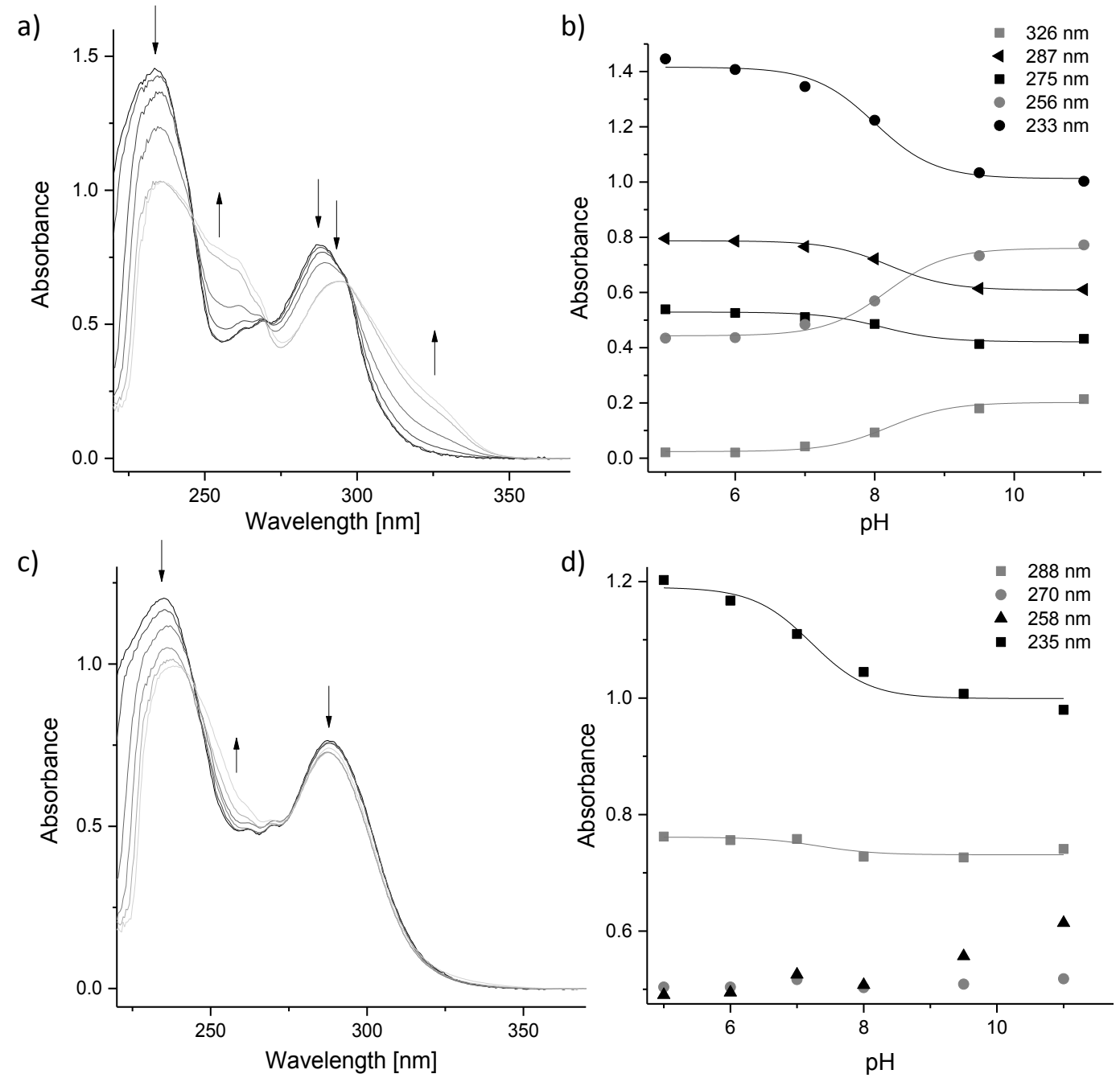

Figure 41: UV-vis spectroscopic titration spectra (5 $\mu \mathrm{M}$ in acetonitrile/buffer between pH 5 and 11) for a) $\left[\mathrm{Zn}_{2}{ }_{2}\left(\mathrm{H}_{2} \mathrm{~L}^{2}\right)(\mu-\mathrm{OAc})_{2}\right] \mathrm{PF}_{6}$ and c) $\left[\mathrm{Zn}_{2}{ }_{2}\left(\mathrm{H}_{2} \mathrm{SIM}^{1}\right)(\mu-\mathrm{OAc})(\mathrm{OH})\right] \mathrm{PF} 6$ as well as absorbance vs. pH plots for b) $\left[\mathrm{Zn}_{2}\left(\mathrm{H}_{2} \mathrm{~L}^{2}\right)(\mu-\mathrm{OAC})_{2}\right] \mathrm{PF}_{6}$ and d) $\left[\mathrm{Zn}_{2}\left(\mathrm{H}_{2} \mathrm{SIM}^{1}\right)(\mu-\mathrm{OAc})(\mathrm{OH})\right] \mathrm{PF}_{6}$.

The spectrophotometrically determined $\mathrm{pK}_{a}$ values of $\left[\mathrm{Zn}_{2}{ }_{2}\left(\mathrm{H}_{2} \mathrm{SIM}^{1}\right)(\mu-\mathrm{OAc})(\mathrm{OH})\right]^{+}$and $\left[\mathrm{Zn}_{2}{ }_{2}\left(\mathrm{H}_{2} \mathrm{~L}^{2}\right)(\mu-\mathrm{OAc})_{2}\right]^{+}$can be compared to the corresponding data obtained from the analysis of the kinetic data of the phosphoester hydrolysis, and this supports the mechanistic interpretation (see Chapter 7). Importantly, the data described here also support the solution structural analysis, i.e. the observation of isosbestic points in the $\mathrm{pH}$ range of 5 to 11 indicates that the dizinc(II) complexes are stable (and the only species of relevance) in these solutions, and this emerges also from the NMR studies, specifically in mixed $C_{3} C N / D_{2} \mathrm{O}$ solutions at varying $\mathrm{pH}$ values (see above). 


\subsection{SUMMARY}

The five ligands $\mathrm{HL}^{1}$, with no substituents, $\mathrm{H}_{3} \mathrm{SIM}^{1}, \mathrm{H}_{4} \mathrm{SIM}^{3}$, with two asymmetrically disposed amide residues, $\mathrm{H}_{3} \mathrm{~L}^{2}$, with two symmetrically disposed amide residues, and $\mathrm{HSIM}^{2}$, with two asymmetrically disposed amine anchor groups, lead to strikingly different structures of the dizinc(II) complexes $\left[\mathrm{Zn}_{2}{ }_{2}\left(\mathrm{~L}^{1}\right)(\mu-\mathrm{OAc})_{2}\right]^{+},\left[\mathrm{Zn}_{2}{ }_{2}\left(\mathrm{H}_{2} \mathrm{~L}^{2}\right)(\mu-\mathrm{OAc})_{2}\right]^{+},\left[\mathrm{Zn}_{2}{ }_{2}\left(\mathrm{H}_{2} \mathrm{SIM}^{1}\right)(\mu-\mathrm{OAc})(\mathrm{OH})\right]^{+}$, $\left[\mathrm{Zn}_{2}{ }_{2}\left(\mathrm{SIM}^{2}\right)(\mu-\mathrm{OAC})_{2}\right]^{+}$, and $\left[\mathrm{Zn}_{2}{ }_{2}\left(\mathrm{H}_{2} \mathrm{SIM}^{3}\right)(\mu-\mathrm{OAc})(\mathrm{OH})\right]$. While the three complexes $\left[\mathrm{Zn}_{2}{ }_{2}\left(\mathrm{~L}^{1}\right)(\mu-\mathrm{OAC})_{2}\right]^{+}, \quad\left[\mathrm{Zn}_{2}{ }_{2}\left(\mathrm{H}_{2} \mathrm{~L}^{2}\right)(\mu-\mathrm{OAc})_{2}\right]^{+}$, and $\left[\mathrm{Zn}_{2}{ }_{2}\left(\mathrm{SIM}^{2}\right)(\mu-\mathrm{OAc})_{2}\right]^{+}$show the common structure of phenoxido-bridged phosphatase models with two bridging acetate co-ligands, $\left[\mathrm{Zn}_{2}{ }_{2}\left(\mathrm{H}_{2} \mathrm{SIM}^{1}\right)(\mu-\mathrm{OAc})(\mathrm{OH})\right]^{+}$and $\left[\mathrm{Zn}_{2}{ }_{2}\left(\mathrm{H}_{2} \mathrm{SIM}^{3}\right)(\mu-\mathrm{OAc})(\mathrm{OH})\right]$ possess two strikingly different $\mathrm{Zn}$ " sites, one being five- and one six-coordinate, with rare example of a $\mathrm{Zn}$ "-OH bond. The appearance of a terminal hydroxido co-ligand is ascribed to the steric hindrance caused by the asymmetric pivaloyl-substituted ligand backbone and the emerging incapacity of the resulting dizinc(II) complexes to bind two acetate co-ligands. Stabilization of this hydroxido co-ligand by hydrogen bond formation is supported by X-ray structures and the IR spectra of $\left[\mathrm{Zn}_{2}{ }_{2}\left(\mathrm{H}_{2} \mathrm{SIM}^{1}\right)(\mu-\mathrm{OAc})(\mathrm{OH})\right]^{+}$and $\left[\mathrm{Zn}_{2}{ }_{2}\left(\mathrm{H}_{2} \mathrm{SIM}^{3}\right)(\mu-\mathrm{OAc})(\mathrm{OH})\right]$. Hydrogen bond formation was also seen in the structure of the complexes $\left[\mathrm{Zn}_{2}{ }_{2}\left(\mathrm{H}_{2} \mathrm{~L}^{2}\right)(\mu-\mathrm{OAc})_{2}\right]^{+}$and $\left[\mathrm{Zn}_{2}\left(\mathrm{SIM}^{2}\right)(\mu-\mathrm{OAc})_{2}\right]^{+}$ to the acetate co-ligands.

The structures of the dizinc(II) complexes in solution were studied via NMR spectroscopy. The asymmetry of $\left[\mathrm{Zn}_{2}{ }_{2}\left(\mathrm{H}_{2} \mathrm{SIM}^{1}\right)(\mu-\mathrm{OAc})(\mathrm{OH})\right]^{+}$in solution was verified. Furthermore, the solution structure of $\left[\mathrm{Zn}_{2}{ }_{2}\left(\mathrm{SIM}^{2}\right)(\mu-\mathrm{OAc})_{2}\right]^{+}$, which is also built up by an asymmetric ligand backbone, was found to contain two unequal $\mathrm{Zn}^{\text {" }}$ centers due to the two amino residues adjacent one of the two $\mathrm{Zn}^{\prime \prime}$ centers. In conclusion these three asymmetric complexes $\left[\mathrm{Zn}_{2}{ }_{2}\left(\mathrm{H}_{2} \mathrm{SIM}^{1}\right)(\mu-\mathrm{OAc})(\mathrm{OH})\right]^{+}, \quad\left[\mathrm{Zn}_{2}{ }_{2}\left(\mathrm{SIM}^{2}\right)(\mu-\mathrm{OAc})_{2}\right]^{+}$and $\left[\mathrm{Zn}_{2}{ }_{2}\left(\mathrm{H}_{2} \mathrm{SIM}^{3}\right)(\mu-\mathrm{OAc})(\mathrm{OH})\right]$ can be considered as accurate model complexes for phosphoesters type metalloenzymes as they bear the two $\mathrm{Zn}$ " centers in different coordination environments and have the capacity to form a hydrogen bond network as exhibited for the native enzymes. 


\section{Chapter 5}

\section{Diiron Model Complexes ${ }^{\mathrm{e}}$}

e Main parts of this chapter will be published in "An Approach to More Accurate Model Systems for Purple Acid Phosphatases (PAPs)"; Paul V. Bernhardt, Simone Bosch, Peter Comba, Lawrence R. Gahan, Graeme R. Hanson, Valeriu Mereacre, Christopher J. Noble, Gerhard Schenk, Hubert Wadepohl; manuscript in preparation. 


\subsection{INTRODUCTION}

Iron is one of the most versatile of the biochemically active metals and, therefore, the role of iron in its various oxidation states in the active sites of metalloenzymes has been widely studied. ${ }^{207-211}$ Even considering only non-haem diiron proteins, the diiron core takes part in a multitude of functions including oxygen transport (Hemerythrin), methane oxidation (Methane Monooxygenase, $\mathrm{MMO}$ ), conversion of ribonucleotides to deoxyribonucleotides (Ribonucleotide Reductase, RNR), detoxification of NO (Flavodiiron Nitric Oxide Reductase, FNOR) and hydrolysis of phosphate esters (Purple Acid Phosphatase, PAP). ${ }^{210-218}$ In these enzymes the diiron sites, in all of their accessible oxidation states, are typically bridged by at least one carboxylate moiety. ${ }^{183}$ Most of the iron-containing non-haem diiron proteins function through interaction of their diiron(II) form with dioxgen. In contrast, PAPs perform a hydrolytic function, at least in vitro, via their heterovalent diiron(III/II) form and in this way are involved in the regulation of phosphate and phosphorylated metabolite levels in a wide range of organisms. ${ }^{102}$ More precisely, PAPs catalyze the hydrolysis of phosphomonoesters at acidic to neutral $\mathrm{pH}$. The active sites of the mammalian PAPs isolated from bovine spleen (bsPAP) and porcine uterus (ufPAP) were determined to contain a dinuclear iron center in two accessible oxidation states: the $\mathrm{Fe}^{\mathrm{III}}{ }_{2}$ form, which is characterized by typical visible absorption maxima between $550 \mathrm{~nm}$ and $570 \mathrm{~nm}$ $\left(\varepsilon \sim 4000 \mathrm{M}^{-1} \mathrm{~cm}^{-1}\right)$, and the reduced $\mathrm{Fe}^{\prime \prime \prime} \mathrm{Fe}^{\prime \prime}$ form, which exhibits blue shifted absorption maxima between $505 \mathrm{~nm}$ and $510 \mathrm{~nm}\left(\varepsilon \sim 4000 \mathrm{M}^{-1} \mathrm{~cm}^{-1}\right) \cdot{ }^{69,138,217,219,220}$ Interestingly, the Fe ${ }_{2}{ }_{2}$ form of ufPAP was found to have an upper limit of less than $1 \%$ of the activity of the heterovalent Fe ${ }^{\text {III }} \mathrm{Fe}$ "I form. ${ }^{21,218,221}$ The redox potential for the $\mathrm{Fe} \mathrm{e}_{2} / \mathrm{Fe}^{\mathrm{III}} \mathrm{Fe} \mathrm{e}^{\text {II }}$ couple of ufPAP was found to be $0.367 \mathrm{~V}$ at $\mathrm{pH} 5$ and $0.306 \mathrm{~V}$ at pH 6 vs. NHE. Therefore, the enzyme is easily and reversibly oxidized to the Fe ${ }^{\prime \prime \prime}{ }_{2}$ form owing to the low redox potential of the Fe" center. ${ }^{222,}{ }^{223}$ This observation led to the suggestion that PAPs may regulate their catalytic activity in vivo by reversible oxidation/reduction of the active site. ${ }^{222}$

PAPs further differ from other non-heme diiron proteins having a tyrosinate ligand leading to the characteristic absorption bands and consequently to the characteristic purple color. The absorption bands have been assigned by resonance Raman studies to tyrosinate-to-Fe ${ }^{\text {III }}$ CT transitions. ${ }^{219}$ With Mössbauer spectroscopy the iron centers of ufPAP and bsPAP were revealed to have distinct coordination environments, and this was confirmed by X-ray crystallographic studies of ufPAP. ${ }^{219,224,225}$ Magnetic susceptibility studies indicate that the Fe ${ }^{\text {III }}$ and Fe ${ }^{\text {Il }}$ centers are weakly antiferromagnetically coupled with $\mathrm{J}$ values ranging from $-5 \mathrm{~cm}^{-1}$ to $-11 \mathrm{~cm}^{-1}$ $\left(H=-2 J S_{1} S_{2}\right) .{ }^{219}, 226$ Similarly, weak antiferromagnetic coupling was emerged from magnetic 
susceptibility studies for the oxidized Fe ${ }^{\text {III }}{ }_{2}$ form of ufPAP $\left(\mathrm{J}>-15 \mathrm{~cm}^{-1}\right) \cdot{ }^{227}$ Moreover, the reduced form of ufPAP exhibits rhombic EPR signals at $g=1.94,1.73$ and 1.58 , consistent with antiferromagnetically coupled high-spin Fe"l and Fe ${ }^{\text {III }}$ centers. ${ }^{217,} 228$

In recent years, a number of structural models for the active site of PAPs have been developed using phenoxido-containing multidentate ligands. ${ }^{81} \mathrm{Fe}^{\mathrm{II \prime}}{ }_{2}$ complexes, which were reported for symmetrical ligands such as $\mathrm{H}_{3} \mathrm{~L}^{3}, \mathrm{H}_{3} \mathrm{~L}^{35}, \mathrm{HL}^{36}$, and $\mathrm{H}_{3} \mathrm{~L}^{37}$, were used to model the oxidized form of PAPs. ${ }^{101}, 125,229,230$ Moreover, heterovalent diiron complexes were also generated using symmetric ligands such as $\mathrm{HL}^{1}, \mathrm{HL}^{11}, \mathrm{HL}^{38}$, and $\mathrm{HL}^{10}$ and have been examined as structural models for PAPs. ${ }^{99}, 138,139,231,232$ From the separation of their one-electron redox processes assigned to

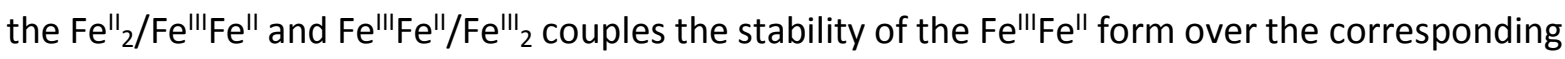
homovalent $\mathrm{Fe}_{2}{ }_{2}$ or $\mathrm{Fe}^{\mathrm{II \prime}}{ }_{2}$ complexes was investigated. The comproportionation constant $\left(\mathrm{K}_{\text {com }}\right)$ for this equilibrium was found to be in the order of $10^{10}-10^{12}$ and therefore confirms the substantial stability of the mixed-valent Fe ${ }^{\text {III }} \mathrm{Fe}^{\text {II }}$ form. ${ }^{81}$

Perhaps the most widely employed Fe"ll Fe"l model complex mimicking the mixed-valent diiron active site of PAP is $\left[\mathrm{Fe}^{\prime \prime \prime} \mathrm{Fe}^{\prime \prime}\left(\mathrm{L}^{4}\right)(\mu-\mathrm{OAc})_{2}\right] \mathrm{ClO}_{4}$. This acetate-bridged $\mathrm{Fe}^{\prime \prime \prime} \mathrm{Fe}$ complex of the asymmetric ligand $\mathrm{H}_{2} \mathrm{~L}^{4}$ revealed significant similarities in the physical properties compared to the native enzyme ufPAP. In particular, $\left[\mathrm{Fe}^{\prime \prime \prime \prime} \mathrm{Fe}^{\prime \prime}\left(\mathrm{L}^{4}\right)(\mu-\mathrm{OAc})_{2}\right] \mathrm{ClO}_{4}$ exhibits (i) a comparable

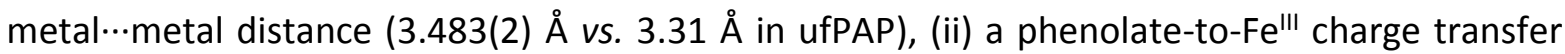
band at $550 \mathrm{~nm}$ (vs. the tyrosinate-to-Fe ${ }^{\prime \prime \prime}$ charge transfer band at $~ 510 \mathrm{~nm}$ in ufPAP), (iii) weakly antiferromagnetically coupled high spin Fe ${ }^{I I I}$ and Fe ions $\left(\mathrm{J}=-7.4 \mathrm{~cm}^{-1}\right.$ vs. -5 to $-11 \mathrm{~cm}^{-1}$ for ufPAP), (iv) localized iron valences in the solid state, and (v) a reversible $\mathrm{Fe}^{\prime \prime \prime} \mathrm{Fe}^{\prime \prime} / \mathrm{Fe}^{\prime \prime \prime}{ }_{2}$ redox couple at a

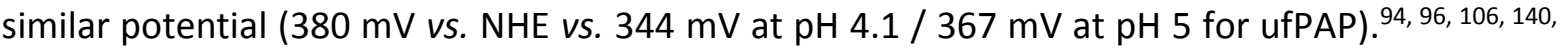
141

Although $\left[\mathrm{Fe}^{\prime \prime \prime} \mathrm{Fe}{ }^{\prime \prime}\left(\mathrm{L}^{4}\right)(\mu-\mathrm{OAC})_{2}\right] \mathrm{ClO}_{4}$ mimics accurately the first coordination sphere in the active site of PAP, the important structural features derived from the second coordination sphere of PAPs have not been addressed. The PAPs active site includes non-coordinated histidine groups proximal to the iron centers, which may help to position the substrate, as proposed from crystallographic data as well as from mechanistic studies. ${ }^{74}$ These amino acid residues have been considered in the diiron(III) complexes, depicted in Chart 16, in form of basic groups proximal to the metal centers, in order to mimic the hydrogen bonding network within the active site of PAPs. ${ }^{108,} 114$ Moreover, the generation of a $\mathrm{Fe}^{\text {IIII } \mathrm{Fe}}{ }^{\text {II }}$ form by bulk electrolysis with $\left[\mathrm{Fe}_{2}{ }_{2}{ }_{2}\left(\mathrm{~L}^{8}\right)(\mu-\mathrm{OH})\left(\mathrm{OH}_{2}\right)_{2}\right]^{2+}$ was achieved. ${ }^{109,114}$ 


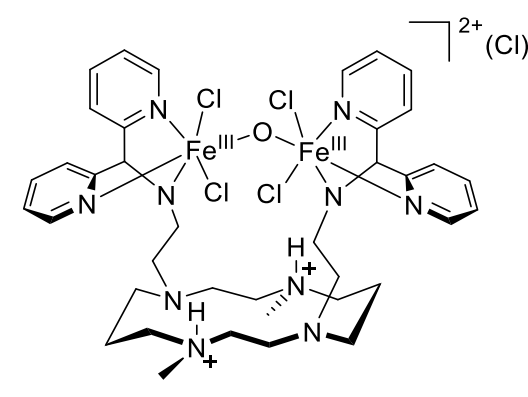

$\left[\mathrm{Fe}_{2}{ }_{2}{ }_{2}\left(\mathrm{~L}^{6}\right)(\mu-\mathrm{O}) \mathrm{Cl}_{4}\right](\mathrm{Cl})_{2}$

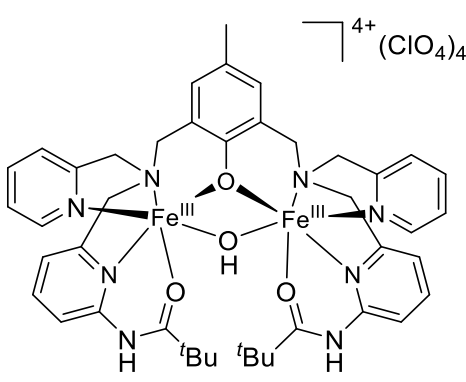

$\left[\mathrm{Fe}_{2}{ }_{2}\left(\mathrm{~L}^{2}\right)(\mu-\mathrm{OH})\right]\left(\mathrm{ClO}_{4}\right)_{4}$

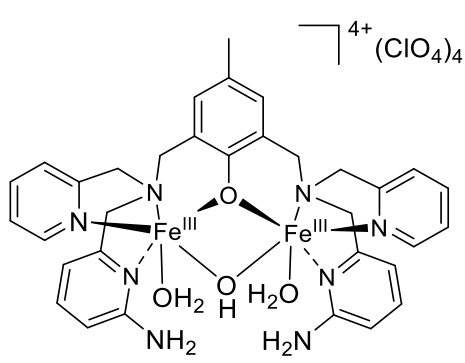

$\left[\mathrm{Fe}_{2}{ }_{2}\left(\mathrm{~L}^{8}\right)(\mu-\mathrm{OH})\left(\mathrm{OH}_{2}\right)_{2}\right]\left(\mathrm{ClO}_{4}\right)_{4}$

Chart 16: Previously reported diiron(III) complexes containing hydrogen bond donors. ${ }^{108,114}$

Examination and comparison of the phosphoester hydrolysis, accomplished with the complexes possesing the capacity for hydrogen bonding, as well as the $\mathrm{Fe}^{\prime \prime \prime}{ }_{2}$ complex of $\mathrm{HL}^{1}$, lacking hydrogen bond donors, gave further insights on the impact of hydrogen bond donors proximal to the diiron core on the catalytic activity. The results emerging from this progress are discussed in more detail in Chapter 8.

In this work, the role of the hydrogen bond donor position is probed, based on the hypothesis that hydrogen bonding in an asymmetrical manner favors monodentate or asymmetrical bridging phosphate binding and therefore helps to release the product formed after hydrolysis. Hence, the ligands $\mathrm{H}_{3} \mathrm{SIM}^{1}$ and $\mathrm{H}_{4} \mathrm{SIM}^{3}$ were designed and synthesized. In this chapter the synthesis and characterization of three $\mathrm{Fe}_{2}{ }_{2}$ complexes, shown in Chart 17, are discussed. All three complexes bear two pivaloyl-amide residues that are able to form hydrogen bonds to substrate molecules. The two hydrogen bond donors in $\left[\mathrm{Fe}_{2}{ }_{2}\left(\mathrm{H}_{2} \mathrm{SIM}^{1}\right)(\mu-\mathrm{OAc})_{2}\right] \mathrm{PF}_{6}$ and $\left[\mathrm{Fe}_{2}{ }_{2}\left(\mathrm{H}_{2} \mathrm{~L}^{2}\right)(\mu-\mathrm{OAc})_{2}\right] \mathrm{PF}_{6}$ are positioned in two different fashions, (i) both pivaloyl-amide moieties adjacent to one binding site, or (ii) one pivaloyl-amide moiety adjacent to each binding site. Similar to $\left[\mathrm{Fe}_{2}{ }_{2}\left(\mathrm{H}_{2} \mathrm{SIM}^{1}\right)(\mu-\mathrm{OAc})_{2}\right] \mathrm{PF}_{6},\left[\mathrm{Fe}_{2}{ }_{2}\left(\mathrm{H}_{2} \mathrm{SIM}^{3}\right)(\mu-\mathrm{OAc})_{2}\right]$ contains both pivaloyl-amide residues in one binding site but the second binding site has one phenolate instead of a pyridine residue. Among other investigations, the stability of the $\mathrm{Fe}^{\mathrm{III}} \mathrm{Fe}{ }^{\prime \prime}$ complex of the asymmetric ligand $\mathrm{H}_{3} \mathrm{SIM}^{1}$ over the corresponding homovalent $\mathrm{Fe}_{2}{ }_{2}$ and $\mathrm{Fe} \mathrm{II}_{2}$ complexes was studied by examination of the comproportionation constant $\mathrm{K}_{\text {com }}$. 


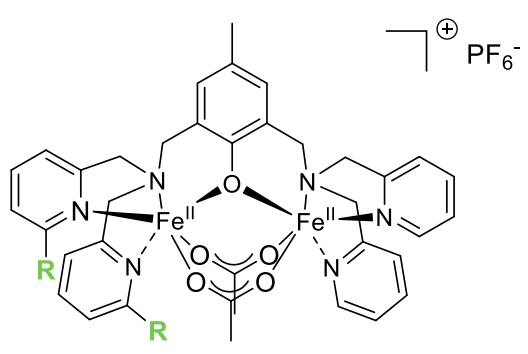

$\left[\mathrm{Fe}_{2}{ }_{2}\left(\mathrm{H}_{2} \mathrm{SIM}^{1}\right)(\mu-\mathrm{OAc})_{2}\right] \mathrm{PF}_{6}$

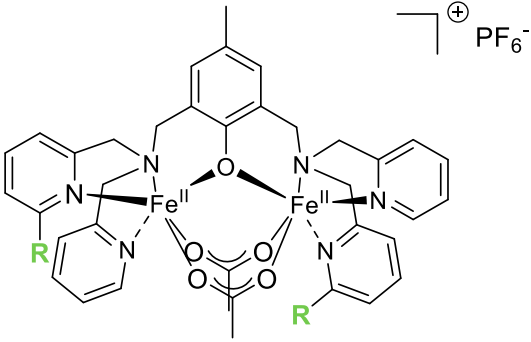

$\left[\mathrm{Fe}_{2}{ }_{2}\left(\mathrm{H}_{2} \mathrm{~L}^{2}\right)(\mu-\mathrm{OAc})_{2}\right] \mathrm{PF}_{6}$
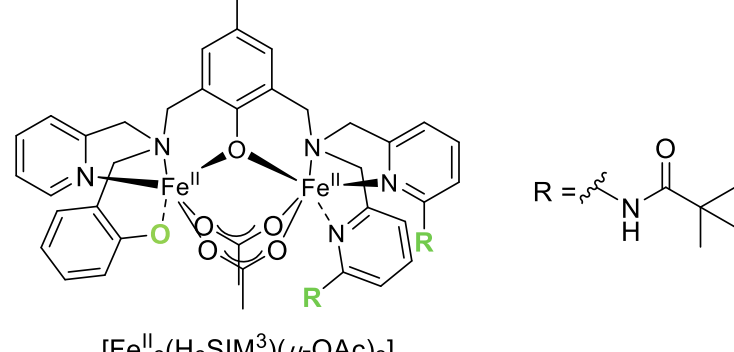

$\left[\mathrm{Fe}_{2}{ }_{2}\left(\mathrm{H}_{2} \mathrm{SIM}^{3}\right)(\mu-\mathrm{OAc})_{2}\right]$

Chart 17: Diiron(II) complexes discussed in this chapter.

As discussed in Chapter 3.2.5 the coordination of two $\mathrm{Fe}^{\text {III }}$ ions in a single $\mathrm{H}_{3} \mathrm{SIM}^{1}$ ligand molecule could not be detected. Therefore, the preparation of a Fe ${ }^{\mathrm{III}} 2$ complex and the subsequent generation of the $\mathrm{Fe}^{\text {IIIIFell }}$ complex by bulk electrolysis as it was done with $\left[\mathrm{Fe}_{2}{ }_{2}\left(\mathrm{~L}^{8}\right)(\mu-\mathrm{OH})\left(\mathrm{OH}_{2}\right)_{2}\right]\left(\mathrm{ClO}_{4}\right)_{4}$ is not feasible and alternative synthetic pathways were tested. For example, Suzuki et al. and Maeda et al. prepared first the Fe" ${ }_{2}$ complex of $\mathrm{HL}^{1}$ and, subsequently,

generated the heterovalent diiron form by selective oxidation in the presence of oxygen. ${ }^{231,233}$ With this knowledge, the stability of $\left[\mathrm{Fe}_{2}{ }_{2}\left(\mathrm{H}_{2} \mathrm{SIM}^{1}\right)(\mu-\mathrm{OAc})_{2}\right] \mathrm{PF}_{6}$ under aerobic conditions was tested. Moreover, the generation of an $\mathrm{Fe}^{\mathrm{III}} \mathrm{Fe}^{\mathrm{Il}}$ complex of $\mathrm{H}_{3} \mathrm{SIM}^{1}$ was studied with electrochemical methods as well as by the use of the oxidant ferrocenium hexafluorophosphate.

\subsection{SYNTHESIS OF DIIRON(II) MODEL COMPLEXES}

The dinuclear iron(II) complexes of $\mathrm{H}_{3} \mathrm{SIM}^{1}$ and $\mathrm{H}_{3} \mathrm{~L}^{2}$ were prepared under an inert atmosphere in the glovebox by reaction with two equivalents of iron(II) acetate in degassed methanol. After the yellow solution was stirred for 12 hours at room temperature, sodium hexafluorophosphate was added. The mixture was filtered through a syringe filter and subsequent diffusion of degassed diethylether into the solution yielded X-ray quality crystals. In this way, the complexes $\left[\mathrm{Fe}_{2}{ }_{2}\left(\mathrm{H}_{2} \mathrm{SIM}^{1}\right)(\mu-\mathrm{OAC})_{2}\right] \mathrm{PF}_{6}$ and $\left[\mathrm{Fe}_{2}{ }_{2}\left(\mathrm{H}_{2} \mathrm{~L}^{2}\right)(\mu-\mathrm{OAc})_{2}\right] \mathrm{PF}_{6}$ were isolated as green crystals. Applying this procedure to $\mathrm{H}_{4} \mathrm{SIM}^{3}$, the ligand containing solution was treated with one equivalent of sodium hydroxide before addition of iron(II) acetate and sodium hexafluorophosphate. Diffusion of degassed diethylether into the claret-colored solution resulted in the formation of only a few crystals of the complex $\left\{\mathrm{Na}\left[\mathrm{Fe}_{2}{ }_{2}\left(\mathrm{H}_{2} \mathrm{SIM}^{3}\right)(\mu-\mathrm{OAc})_{2}\right]_{2}\right\} \mathrm{PF}_{6}$, 
suitable for X-ray diffraction but insufficient for further investigations. $\left[\mathrm{Fe}_{2}{ }_{2}\left(\mathrm{H}_{2} \mathrm{SIM}^{1}\right)(\mu-\mathrm{OAc})_{2}\right] \mathrm{PF}_{6}$ and $\left[\mathrm{Fe}^{\|}{ }_{2}\left(\mathrm{H}_{2} \mathrm{~L}^{2}\right)(\mu-\mathrm{OAc})_{2}\right] \mathrm{PF}_{6}$ were characterized and studied in the solid state and also in solution. Furthermore, the possibility of obtaining an Fe ${ }^{\text {III }} \mathrm{Fe}{ }^{\prime \prime}$ complex with the asymmetric complex $\left[\mathrm{Fe}_{2}{ }_{2}\left(\mathrm{H}_{2} \mathrm{SIM}^{1}\right)(\mu-\mathrm{OAc})_{2}\right] \mathrm{PF}_{6}$ was examined. Bearing two amide residues adjacent to one iron(II) center in $\left[\mathrm{Fe}_{2}{ }_{2}\left(\mathrm{H}_{2} \mathrm{SIM}^{1}\right)(\mu-\mathrm{OAc})_{2}\right] \mathrm{PF}_{6}$ or one amide residue adjacent to each iron(II) center in $\left[\mathrm{Fe}_{2}{ }_{2}\left(\mathrm{H}_{2} \mathrm{~L}^{2}\right)(\mu-\mathrm{OAc})_{2}\right] \mathrm{PF}_{6}$, these complexes offer the potential for the investigation of the positioning effect of hydrogen bonding on the phosphoester hydrolytic activity.

\subsection{Structural Characterization of Dilron(II) COMPLEXES}

The three diiron(II) complexes were structurally characterized in the solid state and $\left[\mathrm{Fe}_{2}{ }_{2}\left(\mathrm{H}_{2} \mathrm{SIM}^{1}\right)(\mu-\mathrm{OAC})_{2}\right] \mathrm{PF}_{6}$ and $\left[\mathrm{Fe}_{2}{ }_{2}\left(\mathrm{H}_{2} \mathrm{~L}^{2}\right)(\mu-\mathrm{OAc})_{2}\right] \mathrm{PF}_{6}$ also in solution. Due to the air sensitivity of the samples, especially in solution, analyses were undertaken strictly anaerobically. The solid state structures were investigated by X-ray diffraction analysis and SQUID experiments. Furthermore, the structure as well as the electronic, magnetic, and electrochemical properties in solution of $\left[\mathrm{Fe}_{2}{ }_{2}\left(\mathrm{H}_{2} \mathrm{SIM}^{1}\right)(\mu-\mathrm{OAc})_{2}\right] \mathrm{PF}_{6}$ and $\left[\mathrm{Fe}_{2}{ }_{2}\left(\mathrm{H}_{2} \mathrm{~L}^{2}\right)(\mu-\mathrm{OAc})_{2}\right] \mathrm{PF}_{6}$ were studied with UVvis-NIR spectroscopy, NMR spectroscopy, and cyclic voltammetry. In addition, the oxidation of the diiron(II) complexes under aerobic conditions was investigated.

\section{Solid State Structure under Anaerobic Conditions}

The yellow crystals of $\left[\mathrm{Fe}_{2}{ }_{2}\left(\mathrm{H}_{2} \mathrm{SIM}^{1}\right)(\mu-\mathrm{OAc})_{2}\right] \mathrm{PF}_{6}$ and $\left[\mathrm{Fe}^{\|}{ }_{2}\left(\mathrm{H}_{2} \mathrm{~L}^{2}\right)(\mu-\mathrm{OAc})_{2}\right] \mathrm{PF}_{6}$ and the black crystals of $\left\{\mathrm{Na}\left[\mathrm{Fe}_{2}{ }_{2}\left(\mathrm{H}_{2} \mathrm{SIM}^{3}\right)(\mu-\mathrm{OAc})_{2}\right]_{2}\right\} \mathrm{PF}_{6}$ were used for X-ray diffraction data collection. Crystallographic data obtained are displayed in Table 35 (see Appendix).

The structures of $\left[\mathrm{Fe}_{2}{ }_{2}\left(\mathrm{H}_{2} \mathrm{SIM}^{1}\right)(\mu-\mathrm{OAC})_{2}\right]^{+}$and $\left[\mathrm{Fe}_{2}{ }_{2}\left(\mathrm{H}_{2} \mathrm{~L}^{2}\right)(\mu-\mathrm{OAc})_{2}\right]^{+}$were solved including a complex cation and a hexafluorophosphate counterion. With $\mathrm{H}_{4} \mathrm{SIM}^{3}$ as ligand in the complex preparation, the $\mathrm{X}$-ray crystal structure revealed a dimeric structure in which the $\left[\mathrm{Fe}_{2}{ }_{2}\left(\mathrm{H}_{2} \mathrm{SIM}^{3}\right)(\mu-\mathrm{OAc})_{2}\right]$ monomers are bridged by a sodium cation between the $\mathrm{Fe}(1)$ ions (separated by $6.119 \AA$ ) coordinated in the amide-free binding sites. The charge is neutralized by the hexafluorophosphate counterion present in the crystal cell. The structures of the complex cations are shown in Figure 42 and selected distances and valence angles are summarized in Table 11. 
In contrast to the corresponding $\mathrm{Zn}_{2}{ }_{2}$ complexes discussed in Chapter 4.3, all three $\mathrm{Fe}_{2}$ complexes, are bridged threefold by two acetate co-ligands and the phenolate-linker of the respective ligand. In addition to the three bridging ligands, the two $\mathrm{Fe}^{\text {ll }}$ ions in $\left[\mathrm{Fe}_{2}{ }_{2}\left(\mathrm{H}_{2} \mathrm{SIM}^{1}\right)(\mu-\mathrm{OAC})_{2}\right]^{+}$and $\left[\mathrm{Fe}_{2}{ }_{2}\left(\mathrm{H}_{2} \mathrm{~L}^{2}\right)(\mu-\mathrm{OAC})_{2}\right]^{+}$are coordinated by two pyridine moieties and a tertiary amine, forming an octahedral coordination environment.

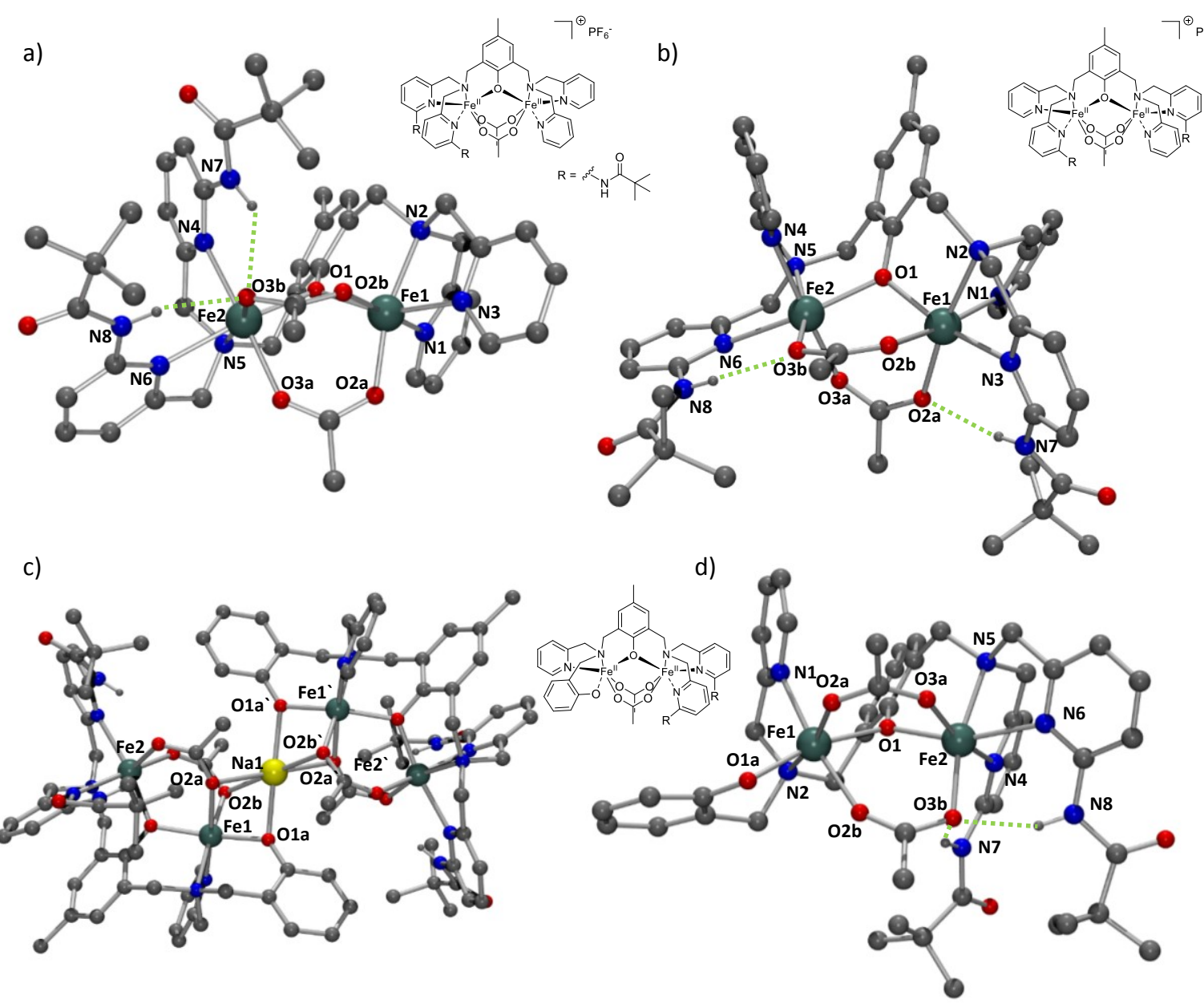

Figure 42: Structures of a) $\left[\mathrm{Fe}_{2}{ }_{2}\left(\mathrm{H}_{2} \mathrm{SIM}^{1}\right)(\mu-\mathrm{OAc})_{2}\right]^{+}$, b) $\left.\left[\mathrm{Fe}_{2}{ }_{2}\left(\mathrm{H}_{2} \mathrm{~L}^{2}\right)(\mu-\mathrm{OAc})_{2}\right]^{+}, \mathrm{c}\right)\left\{\mathrm{Na}\left[\mathrm{Fe}_{2}{ }_{2}\left(\mathrm{H}_{2} \mathrm{SIM}^{3}\right)(\mu-\mathrm{OAc})_{2}\right]_{2}\right\}^{+}$and d) the monomeric section of the latter complex, showing hydrogen bonding (green dotted lines; counter ions, non-coordinated solvent molecules, and hydrogen atoms, not involved in hydrogen bonding, have been omitted for clarity; crystallographic data and ORTEP plots with 50\% probability level of thermal ellipsoids appear in the Appendix, Table 35 and Figure 75).

While the Fe $-\mathrm{N}_{\mathrm{Py}}$ distances for the pyridine residues lacking the amide substituents are in the range of $2.159 \AA$ to $2.178 \AA$, the distance between the Fe"l center and the amidated pyridine moieties are elongated with bond distances from $2.290 \AA$ to $2.364 \AA$ as expected because of the electron withdrawing effect of the pivaloyl-amide residues. This finding accords with the results obtained from a Fe" ${ }_{2}$ complex $\left[\mathrm{Fe}_{2}{ }_{2}\left(\mathrm{H}_{4} \mathrm{~L}^{39}\right)(\mu-\mathrm{OBz})_{2}\right]^{+}$of the fourfold pivaloyl-amide substituted ligand $\mathrm{H}_{5} \mathrm{~L}^{39}$ in which the $\mathrm{Fe}^{\mathrm{II}}-\mathrm{N}_{\mathrm{Py}}$ distances to the amidated pyridines are in the range of $2.250 \AA$ to $2.265 \AA^{234}$ The Fe" ${ }^{\prime \prime} \mathrm{N}_{\text {amine }}$ distances of $\left[\mathrm{Fe}_{2}{ }_{2}\left(\mathrm{H}_{2} \mathrm{SIM}^{1}\right)(\mu-\mathrm{OAc})_{2}\right]^{+}$and $\left[\mathrm{Fe}_{2}{ }_{2}\left(\mathrm{H}_{2} \mathrm{~L}^{2}\right)(\mu-\mathrm{OAc})_{2}\right]^{+}$, ranging from $2.188 \AA$ to $2.270 \AA$, are in the medium range compared with the Fe-N $\mathrm{N}_{\text {py }}$ distances, While this parameter is similar in the two binding sites of $\left[\mathrm{Fe}_{2}{ }_{2}\left(\mathrm{H}_{2} \mathrm{~L}^{2}\right)(\mu-\mathrm{OAc})_{2}\right]^{+}$containing an 
amide-substituted pyridine in each binding site (2.2311(15) $\AA$ and $2.2270(16) \AA$ ), the Fe" $-\mathrm{N}_{\text {amine }}$ distance in the amide-free binding site in $\left[\mathrm{Fe}_{2}\left(\mathrm{H}_{2} \mathrm{~L}^{2}\right)(\mu-\mathrm{OAc})_{2}\right]^{+}$is elongated $(2.270(5) \AA)$ and shortened to 2.188(6) $\AA$ in the amide-containing binding site. An explanation for this finding may be the hydrogen bond formation of the amide residues to the bridging acetate co-ligands, distorting the other coordination bonds. Moreover, comparison to the pivaloyl-amide-free complex $\left[\mathrm{Fe}_{2}{ }_{2}\left(\mathrm{~L}^{1}\right)(\mu-\mathrm{OPr})_{2}\right]^{+}(2.250(2) \AA \text { and } 2.259(2) \AA)^{235}$ both $\mathrm{Fe}^{\prime \prime}-\mathrm{N}_{\text {amine }}$ distances in $\left[\mathrm{Fe}_{2}{ }_{2}\left(\mathrm{H}_{2} \mathrm{~L}^{2}\right)(\mu-\mathrm{OAc})_{2}\right]^{+}$are shortened as in both binding sites hydrogen bond formation takes place. In contrast, in $\left[\mathrm{Fe}_{2}{ }_{2}\left(\mathrm{H}_{2} \mathrm{SIM}^{1}\right)(\mu-\mathrm{OAC})_{2}\right]^{+}$only the amide-containing coordination sites contains a shorter Fe"-Namine distance, while the other Fe" $-\mathrm{N}_{\text {amine }}$ distance in the amide-free binding site is elongated compared to the found values in $\left[\mathrm{Fe}_{2}\left(\mathrm{~L}^{1}\right)(\mu-\mathrm{OPr})_{2}\right]^{+},{ }^{235}$ as the hydrogen bonding network is only created in the amidated binding site.

Table 11: Selected bond lengths $(\AA ̊ \AA)$ and angles ( $\left.{ }^{\circ}\right)$ for $\left[\mathrm{Fe}_{2}{ }_{2}\left(\mathrm{H}_{2} \mathrm{SIM}^{1}\right)(\mu-\mathrm{OAc})_{2}\right] \mathrm{PF}{ }_{6},\left[\mathrm{Fe}^{\prime \prime}{ }_{2}\left(\mathrm{H}_{2} \mathrm{~L}^{2}\right)(\mu-\mathrm{OAc})_{2}\right] \mathrm{PF} 6$ and $\left\{\mathrm{Na}\left[\mathrm{Fe}_{2}{ }_{2}\left(\mathrm{H}_{2} \mathrm{SIM}^{3}\right)(\mu-\mathrm{OAC})_{2}\right]_{2}\right\} \mathrm{PF}_{6}$.

\begin{tabular}{|c|c|c|c|c|}
\hline & {$\left[\mathrm{Fe}_{2}{ }_{2}\left(\mathrm{H}_{2} \mathrm{SIM}^{1}\right)(\mu-\mathrm{OAc})_{2}\right]^{+}$} & {$\left[\mathrm{Fe}_{2}{ }_{2}\left(\mathrm{H}_{2} \mathrm{~L}^{2}\right)(\mu-\mathrm{OAc})_{2}\right]^{+}$} & $\multicolumn{2}{|c|}{\mathrm{Na}\left[\mathrm{Fe}_{2}{ }_{2}\left(\mathrm{H}_{2} \mathrm{SIM}^{3}\right)(\mu-\mathrm{OAC})_{2}\right]_{2}}^{+}$ \\
\hline & & & $\mathrm{Fe}(1)$ & $\mathrm{Fe}\left(1^{\prime}\right)$ \\
\hline $\mathrm{Fe}(1) \cdots \mathrm{Fe}\left(1^{\prime}\right)$ & - & - & \multicolumn{2}{|c|}{6.118} \\
\hline $\mathrm{Fe}(1) \cdots \mathrm{Fe}(2)$ & 3.430 & $3.4457(3)$ & 3.536 & 3.541 \\
\hline $\mathrm{Fe}(1)-\mathrm{O}(1)$ & $2.039(4)$ & $2.0746(13)$ & $2.046(3)$ & $2.053(3)$ \\
\hline $\mathrm{Fe}(1)-\mathrm{O}(1 \mathrm{~A})$ & - & - & $2.005(3)$ & $2.001(3)$ \\
\hline $\mathrm{Fe}(1)-\mathrm{O}(2 \mathrm{~A})$ & $2.024(4)$ & $2.0930(13)$ & $2.131(3)$ & $2.142(3)$ \\
\hline $\mathrm{Fe}(1)-\mathrm{O}(2 \mathrm{~B})$ & $2.185(4)$ & $2.0712(13)$ & $2.287(3)$ & $2.249(3)$ \\
\hline $\mathrm{Fe}(1)-\mathrm{N}(1)$ & $2.167(5)$ & $2.1753(16)$ & $2.178(3)$ & $2.168(3)$ \\
\hline $\mathrm{Fe}(1)-\mathrm{N}(2)$ & $2.270(5)$ & $2.2311(15)$ & $2.256(3)$ & $2.277(3)$ \\
\hline $\mathrm{Fe}(1)-\mathrm{N}(3)$ & $2.159(5)$ & $2.3638(15)$ & - & - \\
\hline $\mathrm{Fe}(2)-\mathrm{O}(1)$ & $2.040(4)$ & $2.0378(13)$ & $2.046(3)$ & $2.040(3)$ \\
\hline $\mathrm{Fe}(2)-\mathrm{O}(3 \mathrm{~A})$ & $2.204(4)$ & $2.1184(14)$ & $2.117(3)$ & $2.114(3)$ \\
\hline $\mathrm{Fe}(2)-\mathrm{O}(3 \mathrm{~B})$ & $2.079(4)$ & $2.1020(14)$ & $2.093(3)$ & $2.105(3)$ \\
\hline $\mathrm{Fe}(2)-\mathrm{N}(4)$ & $2.290(6)$ & $2.1703(16)$ & $2.299(3)$ & $2.299(3)$ \\
\hline $\mathrm{Fe}(2)-\mathrm{N}(5)$ & $2.188(6)$ & $2.2270(16)$ & $2.207(3)$ & $2.212(4)$ \\
\hline $\mathrm{Fe}(2)-\mathrm{N}(6)$ & $2.311(5)$ & $2.2786(15)$ & $2.315(3)$ & $2.325(3)$ \\
\hline $\mathrm{Fe}(1)-\mathrm{O}(1)-\mathrm{Fe}(2)$ & $114.45(18)$ & $113.83(6)$ & $119.60(12)$ & $119.82(12)$ \\
\hline $\mathrm{O}(1)-\mathrm{Fe}(1)-\mathrm{N}(1)$ & $91.16(18)$ & $87.69(6)$ & $90.25(11)$ & $90.55(11)$ \\
\hline $\mathrm{O}(1)-\mathrm{Fe}(1)-\mathrm{N}(2)$ & $86.17(17)$ & $86.53(5)$ & $86.94(10)$ & $86.82(11)$ \\
\hline $\mathrm{O}(1)-\mathrm{Fe}(1)-\mathrm{N}(3)$ & 156.49(19) & $160.48(5)$ & - & - \\
\hline $\mathrm{O}(1)-\mathrm{Fe}(1)-\mathrm{O}(1 \mathrm{~A})$ & - & - & $165.10(11)$ & $165.81(11)$ \\
\hline $\mathrm{O}(1)-\mathrm{Fe}(1)-\mathrm{O}(2 \mathrm{~A})$ & 104.69(17) & $102.08(5)$ & $94.75(10)$ & $94.82(10)$ \\
\hline $\mathrm{O}(1)-\mathrm{Fe}(1)-\mathrm{O}(2 \mathrm{~B})$ & $87.79(17)$ & $90.73(5)$ & $85.79(10)$ & $84.94(10)$ \\
\hline $\mathrm{O}(1)-\mathrm{Fe}(2)-\mathrm{N}(4)$ & $87.09(18)$ & $90.36(5)$ & $87.46(10)$ & $87.72(11)$ \\
\hline $\mathrm{O}(1)-\mathrm{Fe}(2)-\mathrm{N}(5)$ & $87.69(18)$ & $88.88(6)$ & $87.83(11)$ & $87.96(12)$ \\
\hline $\mathrm{O}(1)-\mathrm{Fe}(2)-\mathrm{N}(6)$ & $162.03(18)$ & $160.55(6)$ & $160.81(12)$ & $161.05(12)$ \\
\hline $\mathrm{O}(1)-\mathrm{Fe}(2)-\mathrm{O}(3 \mathrm{~A})$ & $93.65(16)$ & $89.27(6)$ & $89.89(10)$ & $91.04(11)$ \\
\hline $\mathrm{O}(1)-\mathrm{Fe}(2)-\mathrm{O}(3 \mathrm{~B})$ & $103.30(16)$ & $97.90(5)$ & $105.74(10)$ & $104.19(10)$ \\
\hline
\end{tabular}

The $\mathrm{Fe}^{\| \prime \ldots F e "}$ distance is the shortest in $\left[\mathrm{Fe}_{2}{ }_{2}\left(\mathrm{H}_{2} \mathrm{SIM}^{1}\right)(\mu-\mathrm{OAc})_{2}\right]^{+}$formed with the asymmetric pyridine-rich ligand $\mathrm{H}_{3} \mathrm{SIM}^{1}$ but still longer than that found in $\left[\mathrm{Fe}^{\prime \prime}{ }_{2}\left(\mathrm{~L}^{1}\right)(\mu-\mathrm{OPr})_{2}\right]^{+}(3.348(2) \AA) .{ }^{235}$ 
Although the first coordination sphere in $\left[\mathrm{Fe}_{2}{ }_{2}\left(\mathrm{H}_{2} \mathrm{~L}^{2}\right)(\mu-\mathrm{OAc})_{2}\right]^{+}$is comparable to that in $\left[\mathrm{Fe}_{2}{ }_{2}\left(\mathrm{H}_{2} \mathrm{SIM}^{1}\right)(\mu-\mathrm{OAC})_{2}\right]^{+}$the $\mathrm{Fe}^{\| \prime . . . F e}{ }^{\| l}$ separation is $0.015 \AA$ shorter in the latter. From the elongation of the $\mathrm{Fe}^{\| \prime \ldots}$..Fe" separation results a widening of the $\mathrm{Fe}(1)-\mathrm{O}(1)-\mathrm{Fe}(2)$ angle $\left(114.45(18) \AA\right.$ for $\left[\mathrm{Fe}_{2}{ }_{2}\left(\mathrm{H}_{2} \mathrm{SIM}^{1}\right)(\mu-\mathrm{OAc})_{2}\right]^{+}$and $113.83(6) \AA ̊$ for $\left.\left[\mathrm{Fe}_{2}{ }_{2}\left(\mathrm{H}_{2} \mathrm{~L}^{2}\right)(\mu-\mathrm{OAc})_{2}\right]^{+}\right)$, compared to $\left[\mathrm{Fe}_{2}{ }_{2}\left(\mathrm{~L}^{1}\right)(\mu-\mathrm{OPr})_{2}\right]^{+}\left(108.93(6)^{\circ}\right)$. In the respective $\mathrm{Zn}_{2}{ }_{2}$ complexes the behavior is reversed, that is, the $\left[\mathrm{Zn}_{2}{ }_{2}\left(\mathrm{H}_{2} \mathrm{~L}^{2}\right)(\mu-\mathrm{OAc})_{2}\right]^{+}$exhibits a $0.143 \AA$ shorter $\mathrm{Zn}^{\prime \prime} \cdots \mathrm{Zn}$ " distance, possibly due to the doubly acetate-bridged arrangement, which was not found in $\left[\mathrm{Zn}_{2}{ }_{2}\left(\mathrm{H}_{2} \mathrm{SIM}^{1}\right)(\mu-\mathrm{OAc})(\mathrm{OH})\right]^{+}$. Comparison of the $\mathrm{Fe}_{2}$ and $\mathrm{Zn}_{2}{ }_{2}$ complexes of $\mathrm{H}_{3} \mathrm{~L}^{2}$, which are both doubly acetate-bridged, shows a longer

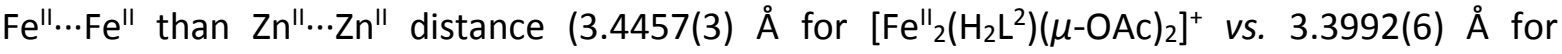
$\left.\left[\mathrm{Zn}_{2}{ }_{2}\left(\mathrm{H}_{2} \mathrm{~L}^{2}\right)(\mu-\mathrm{OAC})_{2}\right]^{+}\right)$. In the case of the complexes derived from ligand $\mathrm{H}_{3} \mathrm{SIM}^{1}$ the observation is opposite, most likely to the lack of the second bridging acetate co-ligand in $\left[\mathrm{Zn}_{2}{ }_{2}\left(\mathrm{H}_{2} \mathrm{SIM}^{1}\right)(\mu-\mathrm{OAc})(\mathrm{OH})\right]^{+}\left(3.5422(7) \AA\right.$ vs. $3.430 \AA$ in $\left.\left[\mathrm{Fe}_{2}{ }_{2}\left(\mathrm{H}_{2} \mathrm{SIM}^{1}\right)(\mu-\mathrm{OAc})_{2}\right]^{+}\right)$. Similar to the $\mathrm{Zn}_{2}{ }_{2}$ complex, the $\mathrm{Fe}^{\|}{ }_{2}$ complex of ligand $\mathrm{H}_{4} \mathrm{SIM}^{3}$ with two phenol moieties in contrast to $\mathrm{H}_{3} \mathrm{SIM}^{1}$ and $\mathrm{H}_{3} \mathrm{~L}^{2}$, the metal $\cdots$ metal distance is the longest by about $0.1 \AA$. In $\left[\mathrm{Fe}_{2}{ }_{2}\left(\mathrm{H}_{2} \mathrm{SIM}^{3}\right)(\mu-\mathrm{OAc})_{2}\right]$ the $\mathrm{Fe}(1)$ ion is surrounded by the bridging phenolate, two bridging acetate co-ligands, a tertiary amine, only one pyridine and one terminal phenolate residue. The distance between the Fe" center and the terminal phenolate, $\mathrm{Fe}(1)-\mathrm{O}(1 \mathrm{~A})(2.007(4) \AA / 2.003(4) \AA)$, is shorter than the $\mathrm{Fe}(1)-\mathrm{N}(3)$ distances found in $\left[\mathrm{Fe}_{2}{ }_{2}\left(\mathrm{H}_{2} \mathrm{SIM}^{1}\right)(\mu-\mathrm{OAC})_{2}\right]^{+}(2.159(5) \AA)$ and $\left[\mathrm{Fe}_{2}{ }_{2}\left(\mathrm{H}_{2} \mathrm{~L}^{2}\right)(\mu-\mathrm{OAC})_{2}\right]^{+}$ (2.3638(15) A), due to the higher electron density of the anionic coordinating ligand. As a result, the $\mathrm{Fe}(1)$ center is forced away from the $\mathrm{Fe}(2)$ center, explaining the elongated $\mathrm{Fe}^{\| l . . . F e} \mathrm{Fe}^{\| \prime}$ separation. In $\left[\mathrm{Fe}_{2}{ }_{2}\left(\mathrm{H}_{2} \mathrm{SIM}^{3}\right)(\mu-\mathrm{OAc})_{2}\right]$ the impact of the sodium ion between the $\mathrm{Fe}(1)$ ions of the monomer subunits should not be neglected. The sodium ion is surrounded by six oxygen atoms deriving from the terminal phenolate and both acetate co-ligands of both monomer subunits. This arrangement results in an asymmetrically chelating acetate co-ligand, while the bridging acetate is coordinated in a more symmetric fashion; more precisely, the $\mathrm{Fe}^{\mathrm{II}} \mathrm{-O}$ distances of the asymmetric acetate differ by $0.204 \AA / 0.143 \AA$ and by $0.022 \AA / 0.023 \AA$ for the symmetric acetate. However, this can also be attributed to the asymmetry of the complex, induced by the asymmetric ligand backbone, as both acetate co-ligands are also coordinated in an asymmetric fashion in $\left[\mathrm{Fe}_{2}{ }_{2}\left(\mathrm{H}_{2} \mathrm{SIM}^{1}\right)(\mu-\mathrm{OAC})_{2}\right]^{+}$. Therefore, each iron center in $\left[\mathrm{Fe}_{2}{ }_{2}\left(\mathrm{H}_{2} \mathrm{SIM}^{1}\right)(\mu-\mathrm{OAC})_{2}\right]^{+}$ develops a short and a long bond to the acetate oxygen atoms, although the differences in the bond lengths are not as pronounced as in $\left[\mathrm{Fe}_{2}{ }_{2}\left(\mathrm{H}_{2} \mathrm{SIM}^{3}\right)(\mu-\mathrm{OAc})_{2}\right]$. In contrast, in $\left[\mathrm{Fe}_{2}{ }_{2}\left(\mathrm{H}_{2} \mathrm{~L}^{2}\right)(\mu-\mathrm{OAC})_{2}\right]^{+}$the two bridging acetate co-ligands are bound in a symmetric manner with differences in the Fe" $-\mathrm{O}$ distances of the same acetate anion of $0.0254 \AA$ and $0.0308 \AA$. 
As mentioned above, a hydrogen bond network was present in the structures of $\left[\mathrm{Fe}_{2}{ }_{2}\left(\mathrm{H}_{2} \mathrm{SIM}^{1}\right)(\mu-\mathrm{OAc})_{2}\right]^{+},\left[\mathrm{Fe}_{2}{ }_{2}\left(\mathrm{H}_{2} \mathrm{~L}^{2}\right)(\mu-\mathrm{OAC})_{2}\right]^{+}$, and $\left[\mathrm{Fe}_{2}{ }_{2}\left(\mathrm{H}_{2} \mathrm{SIM}^{3}\right)(\mu-\mathrm{OAc})_{2}\right]$ and the presence of this network is proposed based on (i) the short distances between the amido nitrogen and acetate oxygen atoms, listed in Table 12, and (ii) the orientation of the pivaloyl-amide substituents with respect to the acetate co-ligands and the pyridine rings.

Table 12: Selected distance $(\AA)$ for $\left[\mathrm{Fe}_{2}{ }_{2}\left(\mathrm{H}_{2} \mathrm{SIM}^{1}\right)(\mu-\mathrm{OAc})_{2}\right]^{+}$, [ $\left.\mathrm{Fe}^{\prime \prime}{ }_{2}\left(\mathrm{H}_{2} \mathrm{~L}^{2}\right)(\mu-\mathrm{OAc})_{2}\right]^{+}$, and $\left[\mathrm{Fe}_{2}{ }_{2}\left(\mathrm{H}_{2} \mathrm{SIM}^{3}\right)(\mu-\mathrm{OAc})_{2}\right]$ corresponding to $\mathrm{H}$-bond formation.

\begin{tabular}{cccc} 
& {$\left[\mathrm{Fe}_{2}{ }_{2}\left(\mathrm{H}_{2} \mathrm{SIM}^{1}\right)(\mu-\mathrm{OAc})_{2}\right]^{+}$} & {$\left[\mathrm{Fe}_{2}{ }_{2}\left(\mathrm{H}_{2} \mathrm{~L}^{2}\right)(\mu-\mathrm{OAc})_{2}\right]^{+}$} & {$\left[\mathrm{Fe}_{2}{ }_{2}\left(\mathrm{H}_{2} \mathrm{SIM}^{3}\right)(\mu-\mathrm{OAc})_{2}\right]$} \\
\hline $\mathrm{N}(7)-\mathrm{O}(2 \mathrm{~A})$ & - & $3.032(2)$ & \\
$\mathrm{N}(7)-\mathrm{O}(3 \mathrm{~B})$ & 3.057 & - & $3.124 / 3.076$ \\
$\mathrm{~N}(8)-\mathrm{O}(3 \mathrm{~B})$ & 2.969 & $3.006(2)$ & $2.936 / 2.997$ \\
\hline
\end{tabular}

The formation of hydrogen bonds with the acetate oxygen atoms appears to direct the position of the pivaloyl-amide groups. This results in enlarged angles between the pyridine planes and the plane containing the amide group ( $\mathrm{N}-\mathrm{C}-\mathrm{O})$ in some cases. This angle was found to be $12.09^{\circ}$ and $30.14^{\circ}$ for $\left[\mathrm{Fe}_{2}{ }_{2}\left(\mathrm{H}_{2} \mathrm{SIM}^{1}\right)(\mu-\mathrm{OAC})_{2}\right]^{+}, 18.51^{\circ}$ and $30.72^{\circ}$ for $\left[\mathrm{Fe}_{2}{ }_{2}\left(\mathrm{H}_{2} \mathrm{~L}^{2}\right)(\mu-\mathrm{OAC})_{2}\right]^{+}$and $30.38^{\circ} / 34.58^{\circ}$ and $38.10^{\circ} / 35.12^{\circ}$ for $\left[\mathrm{Fe}_{2}{ }_{2}\left(\mathrm{H}_{2} \mathrm{SIM}^{1}\right)(\mu-\mathrm{OAc})_{2}\right]$, but differs from $0.86^{\circ}$ to $23.02^{\circ}$ in uncoordinated pivaloyl-amide substituted pyridines in the mononuclear structures discussed in Chapter 3.

\section{Solid State Structure under Aerobic Conditions}

Treatment of $\mathrm{H}_{3} \mathrm{SIM}^{1}$, dissolved in methanol, with two equivalents of iron(II) acetate and one equivalent of sodium hexafluorophosphate under an inert atmosphere for 12 hours resulted in a yellow solution. Following diffusion of diethylether, green crystals of the complex $\left[\mathrm{Fe}_{2}{ }_{2}\left(\mathrm{H}_{2} \mathrm{SIM}^{1}\right)(\mu-\mathrm{OAc})_{2}\right] \mathrm{PF}_{6}$ formed, as discussed above. When the yellow solution was exposed to air the color turned purple immediately. Diffusion of diethylether into this purple solution yielded black crystals of $\left[\mathrm{Fe}^{\prime \prime \prime} \mathrm{Na}\left(\mathrm{H}_{2} \mathrm{SIM}^{1}\right)(\mu-\mathrm{OAc})_{2}\right] \mathrm{PF}_{6}$, suitable for X-ray diffraction data collection. Crystallographic data obtained are displayed in Table 36 (see Appendix).

The structure was solved including the cationic complex $\left[\mathrm{Fe}^{\prime \prime \prime} \mathrm{Na}\left(\mathrm{H}_{2} \mathrm{SIM}^{1}\right)(\mu-\mathrm{OAc})_{2}\right]^{+}$and a hexafluorophosphate counterion in the crystal cell. The structure is depicted in Figure 43 , and selected distances and valence angles are summarized in Table 13. The complex cation contains the deprotonated phenolate backbone, an Fe ${ }^{\text {III }}$ ion in the amide-free binding site, a $\mathrm{Na}^{+}$ion in the amidated binding site and two bridging acetate co-ligands. The two metal ions are bridged threefold, comparable to $\left[\mathrm{Fe}_{2}{ }_{2}\left(\mathrm{H}_{2} \mathrm{SIM}^{1}\right)(\mu-\mathrm{OAc})_{2}\right]^{+}$. Repetition of the experiment showed that the synthesis of $\left[\mathrm{Fe}{ }^{I I I} \mathrm{Na}\left(\mathrm{H}_{2} \mathrm{SIM}^{1}\right)(\mu-\mathrm{OAc})_{2}\right]^{+}$is reproducible and proved the unusual coordination of a $\mathrm{Na}^{+}$ion by the phenolate-based ligand $\mathrm{H}_{3} \mathrm{SIM}^{1}$. 


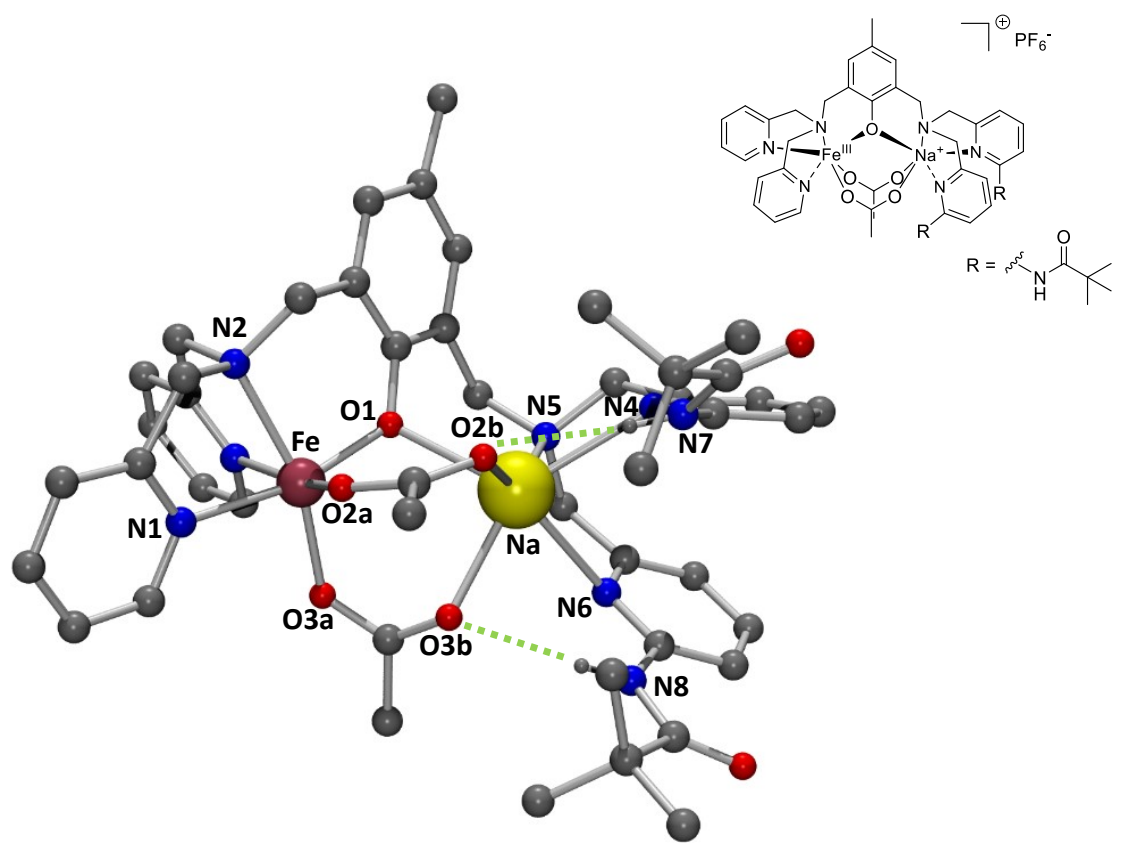

Figure 43: Structure of $\left[\mathrm{Fe}{ }^{\prime \prime \prime} \mathrm{Na}\left(\mathrm{H}_{2} \mathrm{SIM}^{1}\right)(\mu-\mathrm{OAc})_{2}\right]^{+}$showing hydrogen bonding (green dotted lines; counter ions and hydrogen atoms, not involved in hydrogen bonding, have been omitted for clarity; crystallographic data and ORTEP plots with $50 \%$ probability level of thermal ellipsoids appear in the Appendix, Table 36 and Figure 76).

Table 13: Selected bond lengths $(\AA)$ and angles $\left(^{\circ}\right)$ for $\left[\mathrm{Fe}^{\prime \prime \prime} \mathrm{Na}\left(\mathrm{H}_{2} \mathrm{SIM}^{1}\right)(\mu-\mathrm{OAc})_{2}\right] \mathrm{PF}_{6}$ and $\left[\mathrm{Fe}_{2}{ }_{2}\left(\mathrm{H}_{2} \mathrm{SIM}^{1}\right)(\mu-\mathrm{OAc})_{2}\right] \mathrm{PF}_{6}$.

\begin{tabular}{|c|c|c|}
\hline & {$\left[\mathrm{Fe}^{\prime \prime \prime} \mathrm{Na}\left(\mathrm{H}_{2} \mathrm{SIM}^{1}\right)(\mu-\mathrm{OAc})_{2}\right]^{+}$} & {$\left[\mathrm{Fe}_{2}{ }_{2}\left(\mathrm{H}_{2} \mathrm{SIM}^{1}\right)(\mu-\mathrm{OAc})_{2}\right]^{+}$} \\
\hline $\mathrm{Fe} \cdots \mathrm{M}^{\mathrm{a}}$ & $3.4802(6)$ & 3.430 \\
\hline $\mathrm{Fe}-\mathrm{O}(1)^{\mathrm{b}}$ & $1.8906(11)$ & $2.039(4)$ \\
\hline $\mathrm{Fe}-\mathrm{O}(2 \mathrm{~A})^{\mathrm{b}}$ & $1.9620(11)$ & $2.024(4)$ \\
\hline $\mathrm{Fe}-\mathrm{O}(2 \mathrm{~B})^{\mathrm{b}}$ & $1.9556(11)$ & $2.185(4)$ \\
\hline $\mathrm{Fe}-\mathrm{N}(1)^{b}$ & $2.1702(13)$ & $2.167(5)$ \\
\hline $\mathrm{Fe}-\mathrm{N}(2)^{\mathrm{b}}$ & $2.2130(13)$ & $2.270(5)$ \\
\hline $\mathrm{Fe}-\mathrm{N}(3)^{b}$ & $2.1767(13)$ & $2.159(5)$ \\
\hline $\mathrm{M}-\mathrm{O}(1)^{\mathrm{a}}$ & $2.3710(12)$ & $2.040(4)$ \\
\hline $\mathrm{M}-\mathrm{O}(3 \mathrm{~A})^{\mathrm{a}}$ & $2.3578(13)$ & $2.204(4)$ \\
\hline $\mathrm{M}-\mathrm{O}(3 \mathrm{~B})^{\mathrm{a}}$ & $2.3575(13)$ & $2.079(4)$ \\
\hline$M-N(4)^{a}$ & $2.5819(14)$ & $2.290(6)$ \\
\hline$M-N(5)^{a}$ & $2.4845(14)$ & $2.188(6)$ \\
\hline$M-N(6)^{a}$ & $2.5862(15)$ & $2.311(5)$ \\
\hline $\mathrm{Fe}-\mathrm{O}(1)-\mathrm{M}^{\mathrm{a}, \mathrm{b}}$ & $108.98(5)$ & $114.45(18)$ \\
\hline $\mathrm{O}(1)-\mathrm{Fe}-\mathrm{N}(1)^{\mathrm{b}}$ & $87.54(5)$ & $91.16(18)$ \\
\hline $\mathrm{O}(1)-\mathrm{Fe}-\mathrm{N}(2)^{\mathrm{b}}$ & $91.54(5)$ & $86.17(17)$ \\
\hline $\mathrm{O}(1)-\mathrm{Fe}-\mathrm{N}(3)^{\mathrm{b}}$ & $166.43(5)$ & $156.49(19)$ \\
\hline$O(1)-\mathrm{Fe}-\mathrm{O}(2 \mathrm{~A})^{\mathrm{b}}$ & $98.85(5)$ & 104.69(17) \\
\hline $\mathrm{O}(1)-\mathrm{Fe}-\mathrm{O}(2 \mathrm{~B})^{\mathrm{b}}$ & $101.88(5)$ & $87.79(17)$ \\
\hline$O(1)-M-N(4)^{a}$ & $121.84(5)$ & $87.09(18)$ \\
\hline$O(1)-M-N(5)^{a}$ & $79.69(4)$ & $87.69(18)$ \\
\hline $\mathrm{O}(1)-\mathrm{M}-\mathrm{N}(6)^{\mathrm{a}}$ & $133.73(5)$ & $162.03(18)$ \\
\hline$O(1)-M-O(3 A)^{a}$ & $85.18(4)$ & $93.65(16)$ \\
\hline$O(1)-M-O(3 B)^{a}$ & $91.86(4)$ & $103.30(16)$ \\
\hline
\end{tabular}

${ }^{a} \mathrm{M}$ is $\mathrm{Na}$ in $\left[\mathrm{Fe}{ }^{\prime \prime \prime} \mathrm{Na}\left(\mathrm{H}_{2} \mathrm{SIM}^{1}\right)(\mu-\mathrm{OAc})_{2}\right]^{+}$and $\mathrm{Fe}(2)$ in $\left[\mathrm{Fe}^{11}{ }_{2}\left(\mathrm{H}_{2} \mathrm{SIM}^{1}\right)(\mu-\mathrm{OAC})_{2}\right]^{+}$.

${ }^{b} \mathrm{Fe}$ is called $\mathrm{Fe}(1)$ in $\left[\mathrm{Fe}_{2}{ }_{2}\left(\mathrm{H}_{2} \mathrm{SIM}^{1}\right)(\mu-\mathrm{OAC})_{2}\right]^{+}$. 
The Fe ${ }^{\text {III }}$ center in $\left[\mathrm{Fe}^{\text {III }} \mathrm{Na}\left(\mathrm{H}_{2} \mathrm{SIM}^{1}\right)(\mu-\mathrm{OAc})_{2}\right]^{+}$is coordinated in a similar manner to the $\mathrm{Fe}$ "l centers in $\left[\mathrm{Fe}_{2}{ }_{2}\left(\mathrm{H}_{2} \mathrm{SIM}^{1}\right)(\mu-\mathrm{OAc})_{2}\right]^{+}$, forming a distorted octahedral coordination geometry with two pyridines, a tertiary amine, the bridging phenolate linker, and two bridging acetate co-ligands. The distances of the Fe center to the pyridine nitrogen atoms in $\left[\mathrm{Fe}^{\prime \prime \prime} \mathrm{Na}\left(\mathrm{H}_{2} \mathrm{SIM}^{1}\right)(\mu-\mathrm{OAc})_{2}\right]^{+}$are slightly elongated compared to those in $\left[\mathrm{Fe}_{2}{ }_{2}\left(\mathrm{H}_{2} \mathrm{SIM}^{1}\right)(\mu-\mathrm{OAc})_{2}\right]^{+}$, while the coordination bond between the Fe ion and the amine nitrogen is slightly shortened by $0.057 \AA$. The oxidation of the

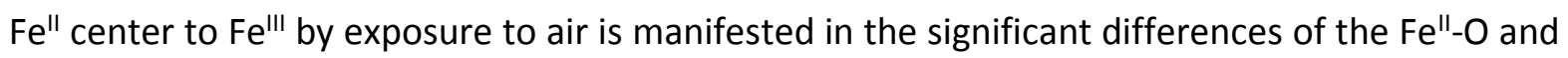

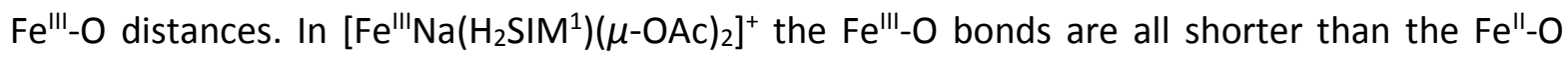
bonds in $\left[\mathrm{Fe}_{2}{ }_{2}\left(\mathrm{H}_{2} \mathrm{SIM}^{1}\right)(\mu-\mathrm{OAc})_{2}\right]^{+}$, as expected due to the higher Lewis acidity of Fe ${ }^{\text {III }}$ compared to $\mathrm{Fe}^{\prime \prime}$. The differences in the Fe-O bonds are comparable to those in the hexaaqua ions $\left[\mathrm{Fe}^{\prime \prime}\left(\mathrm{H}_{2} \mathrm{O}\right)_{6}\right]^{2+}$ and $\left[\mathrm{Fe}^{\prime \prime \prime}\left(\mathrm{H}_{2} \mathrm{O}\right)_{6}\right]^{3+}$, where $\mathrm{Fe}^{\prime \prime}-\mathrm{OH}_{2}$ is $2.13 \AA$ and $\mathrm{Fe}^{\mathrm{III}}-\mathrm{OH}_{2}$ is $2.00 \AA .{ }^{166}$ A similar observation was found for the complexes $\left\{\mathrm{Na}\left[\mathrm{Fe}^{\prime \prime \prime}\left(\mathrm{H}_{2} \mathrm{SIM}^{3}\right)\right]_{2}(\mu-\mathrm{OAc})\left(\mu-\mathrm{OH}_{2}\right)\right\}\left(\mathrm{BF}_{4}\right)_{2} \quad$ and $\left\{\mathrm{Na}\left[\mathrm{Fe}_{2}{ }_{2}\left(\mathrm{H}_{2} \mathrm{SIM}^{3}\right)(\mu-\mathrm{OAc})_{2}\right]_{2}\right\}^{+}$as the Fe ${ }^{\text {III }}$ complex exhibits an average Fe" $-\mathrm{O}_{\mathrm{Ph}}$ distance of $1.957 \AA$ and this value is $2.103 \AA$ for the Fe" complex. Furthermore, the average Fe ${ }^{\text {III-O }}$ bond length of $\left[\mathrm{Fe}{ }^{\prime \prime \prime} \mathrm{Na}\left(\mathrm{H}_{2} \mathrm{SIM}^{1}\right)(\mu-\mathrm{OAc})_{2}\right]^{+}(1.9361 \AA)$ is comparable to that found for the Fe $\mathrm{F}^{\text {III }}$ center in $\left[\mathrm{Fe}^{\prime \prime \prime} \mathrm{Fe} \mathrm{e}^{\prime \prime}\left(\mathrm{L}^{1}\right)(\mu-\mathrm{OPr})_{2}\right]^{+}$with a value of $1.96 \AA$, while the average of the Fe"-O bond lengths was $2.09 \AA$, close to that found for $\left[\mathrm{Fe}_{2}{ }_{2}\left(\mathrm{H}_{2} \mathrm{SIM}^{1}\right)(\mu-\mathrm{OAc})_{2}\right]^{+}(2.083 \AA$ and $2.108 \AA) .{ }^{139}$ However, it should be mentioned that both acetate co-ligands are bound in an asymmetric fashion in $\left[\mathrm{Fe}_{2}{ }_{2}\left(\mathrm{H}_{2} \mathrm{SIM}^{1}\right)(\mu-\mathrm{OAc})_{2}\right]^{+}$and $\left[\mathrm{Fe}{ }^{\prime \prime \prime} \mathrm{Na}\left(\mathrm{H}_{2} \mathrm{SIM}^{1}\right)(\mu-\mathrm{OAc})_{2}\right]^{+}$, but in the $\mathrm{Fe}^{\prime \prime \prime} \mathrm{Na}$ complex both form a short bond to the $\mathrm{Fe}^{\mathrm{III}}$ ion and a longer bond to the $\mathrm{Na}^{+}$ion. This different arrangement of the bridging acetate co-ligands in the complexes also influences the average of the $\mathrm{Fe}^{\mathrm{II}}-\mathrm{O}$ and $\mathrm{Fe}^{\mathrm{III}}-\mathrm{O}$ distances.

The sodium ion in $\left[\mathrm{Fe}^{\prime \prime \prime} \mathrm{Na}\left(\mathrm{H}_{2} \mathrm{SIM}^{1}\right)(\mu-\mathrm{OAc})_{2}\right]^{+}$is surrounded by the same primary coordination sphere as the Fe ${ }^{\text {III }}$ center, but in a trigonal prismatic geometry. The distances of the coordinating atoms to the $\mathrm{Na}^{+}$ion are without exceptions longer compared to the corresponding distances in $\left[\mathrm{Fe}_{2}{ }_{2}\left(\mathrm{H}_{2} \mathrm{SIM}^{1}\right)(\mu-\mathrm{OAc})_{2}\right]^{+}$by at least $0.15 \AA$, due to the decreased charge and the larger ionic radius of the metal center. Consequently, the separation between the two coordination centers in $\left[\mathrm{Fe}{ }^{\prime \prime \prime} \mathrm{Na}\left(\mathrm{H}_{2} \mathrm{SIM}^{1}\right)(\mu-\mathrm{OAc})_{2}\right]^{+}$is increased $\left(3.4802 \AA\right.$ vs. $3.430 \AA$ in $\left.\left[\mathrm{Fe}_{2}{ }_{2}\left(\mathrm{H}_{2} \mathrm{SIM}^{1}\right)(\mu-\mathrm{OAc})_{2}\right]^{+}\right)$.

The two oxygen atoms coordinating the $\mathrm{Na}^{+}$ion in the amidated binding site are part of a hydrogen bond formed by the pivaloyl-amide substituents. Such a hydrogen bonding network is also visible in the previously discussed complexes containing pivaloyl-amide residues. Table 14 compares the corresponding $\mathrm{N}-\mathrm{O}$ distances of the different complexes derived from ligand $\mathrm{H}_{3} \mathrm{SIM}^{1}$. 
Table 14: Selected distances (Å) for $\left[\mathrm{Fe}^{\prime \prime \prime} \mathrm{Na}\left(\mathrm{H}_{2} \mathrm{SIM}^{1}\right)(\mu-\mathrm{OAc})_{2}\right]^{+}, \quad\left[\mathrm{Fe}_{2}{ }_{2}\left(\mathrm{H}_{2} \mathrm{SIM}^{1}\right)(\mu-\mathrm{OAc})_{2}\right]^{+}, \quad$ and $\left[\mathrm{Zn}_{2}{ }_{2}\left(\mathrm{H}_{2} \mathrm{SIM}^{1}\right)(\mu-\mathrm{OAc})_{2}\right]^{+}$, corresponding to H-bond formation.

\begin{tabular}{lccc} 
& {$\left[\mathrm{Fe}^{\prime \prime \prime} \mathrm{Na}\left(\mathrm{H}_{2} \mathrm{SIM}^{1}\right)(\mu-\mathrm{OAc})_{2}\right]^{+}$} & {$\left[\mathrm{Fe}_{2}{ }_{2}\left(\mathrm{H}_{2} \mathrm{SIM}^{1}\right)(\mu-\mathrm{OAc})_{2}\right]^{+}$} & {$\left[\mathrm{Zn}_{2}{ }_{2}\left(\mathrm{H}_{2} \mathrm{SIM}^{1}\right)(\mu-\mathrm{OAc})(\mathrm{OH})\right]^{+}$} \\
\hline $\mathrm{N}(7)-\mathrm{O}(3 \mathrm{~A})$ & 3.153 & - & - \\
$\mathrm{N}(7)-\mathrm{O}(3 \mathrm{~B})$ & - & 3.057 & - \\
$\mathrm{N}(8)-\mathrm{O}(3 \mathrm{~B})$ & 3.087 & 2.969 & - \\
$\mathrm{N}(7)-\mathrm{O}(6)$ & - & - & $2.809(4)$ \\
$\mathrm{N}(8)-\mathrm{O}(6)$ & - & - & $2.874(4)$ \\
\hline
\end{tabular}

However, in contrast to the complexes $\left[\mathrm{Zn}_{2}{ }_{2}\left(\mathrm{H}_{2} \mathrm{SIM}^{1}\right)(\mu-\mathrm{OAc})(\mathrm{OH})\right]^{+}$and $\left[\mathrm{Fe}_{2}{ }_{2}\left(\mathrm{H}_{2} \mathrm{SIM}^{1}\right)(\mu-\mathrm{OAc})_{2}\right]^{+}$, the two pivaloyl-amide nitrogen atoms, $\mathrm{N}(7)$ and $\mathrm{N}(8)$, in $\left[\mathrm{Fe}^{\mathrm{III}} \mathrm{Na}\left(\mathrm{H}_{2} \mathrm{SIM}^{1}\right)(\mu-\mathrm{OAc})_{2}\right]^{+}$do not point in the direction of the same oxygen atom, but to two different oxygen atoms, each part of an acetate co-ligand.

The structure of $\left[\mathrm{Fe}{ }^{\prime \prime \prime} \mathrm{Na}\left(\mathrm{H}_{2} \mathrm{SIM}^{1}\right)(\mu-\mathrm{OAc})_{2}\right] \mathrm{PF}_{6}$ suggests that the crystallization of the Fe ${ }^{\text {III }} \mathrm{Na}$ form of the complex is more likely than of the Fe ${ }_{2}{ }_{2}$ complex of the respective ligand $\mathrm{H}_{3} \mathrm{SIM}^{1}$. It seems that, when the two $\mathrm{Fe}^{\|}$ions of $\left[\mathrm{Fe}_{2}{ }_{2}\left(\mathrm{H}_{2} \mathrm{SIM}^{1}\right)(\mu-\mathrm{OAc})_{2}\right]^{+}$are oxidized by exposure to air, the resulting diiron(III) complex is unstable. Thus, only the iron(III) center in the less sterically demanding binding site remains bound, while in the more sterically hindered binding site a metal exchange from $\mathrm{Fe}^{\text {III }}$ to $\mathrm{Na}^{+}$takes place prior crystallization.

\section{Elemental Analysis}

The unusual presence of a $\mathrm{Na}^{+}$ion in a phenolate-based complex was also confirmed by elemental analysis. Table 15 compares the experimentally obtained values for two separately prepared samples of $\left[\mathrm{Fe}^{\prime \prime \prime} \mathrm{Na}\left(\mathrm{H}_{2} \mathrm{SIM}^{1}\right)(\mu-\mathrm{OAc})_{2}\right] \mathrm{PF}_{6}$ with the expected values.

Table 15: Elemental analytical results obtained with two samples of $\left[\mathrm{Fe}^{\mathrm{III}} \mathrm{Na}\left(\mathrm{H}_{2} \mathrm{SIM}^{1}\right)(\mu-\mathrm{OAc})_{2}\right] \mathrm{PF} 6$ and the corresponding calculated values.

\begin{tabular}{lcccccc} 
& $\mathrm{C}[\%]$ & $\mathrm{H}[\%]$ & $\mathrm{N}[\%]$ & $\mathrm{F}[\%]$ & $\mathrm{Fe}[\%]$ & $\mathrm{Na}[\%]$ \\
\hline sample 1 & 51.11 & 4.62 & 9.96 & 10.17 & 5.84 & 2.27 \\
sample 2 & 51.88 & 5.58 & 10.20 & n.a. & n.a. & n.a. \\
calc. for $\left[\mathrm{Fe}{ }^{\prime \prime \prime} \mathrm{Na}\left(\mathrm{H}_{2} \mathrm{SIM}^{1}\right)(\mu-\mathrm{OAc})_{2}\right] \mathrm{PF}_{6}$ & 52.77 & 5.37 & 10.47 & 10.66 & 5.22 & 2.15 \\
\hline
\end{tabular}

The calculation of the molecular formula derived from the analytical data is $\mathrm{C}_{48} \mathrm{H}_{49} \mathrm{~N}_{8} \mathrm{~F}_{6} \mathrm{FeNa}$, which compares well with the required molecular formula $\mathrm{C}_{47} \mathrm{H}_{57} \mathrm{~N}_{8} \mathrm{O}_{7} \mathrm{~F}_{6} \mathrm{FeNa}$ for $\left[\mathrm{Fe}{ }^{I ! \prime} \mathrm{Na}\left(\mathrm{H}_{2} \mathrm{SIM}^{1}\right)(\mu-\mathrm{OAC})_{2}\right] \mathrm{PF}_{6}$ and proves the ratio of $\mathrm{Fe}: \mathrm{Na}=1: 1$. 


\section{Solid State Susceptibility Measurements}

In order to understand the magnetic properties of $\left[\mathrm{Fe}_{2}{ }_{2}\left(\mathrm{H}_{2} \mathrm{SIM}^{1}\right)(\mu-\mathrm{OAc})_{2}\right] \mathrm{PF}_{6}$ and $\left[\mathrm{Fe}{ }^{I I I} \mathrm{Na}\left(\mathrm{H}_{2} \mathrm{SIM}^{1}\right)(\mu-\mathrm{OAc})_{2}\right] \mathrm{PF}_{6}$, variable temperature dc SQUID magnetic susceptibility studies were performed with ground crystals in the temperature range from $2-300 \mathrm{~K}$.

The $\chi_{M} T$ vs. $T$ curve obtained with the powdered sample of $\left[\mathrm{Fe}_{2}{ }_{2}\left(\mathrm{H}_{2} \mathrm{SIM}^{1}\right)(\mu-\mathrm{OAC})_{2}\right] \mathrm{PF}_{6}$ is reproduced in Figure 44. At $300 \mathrm{~K}$, the $\chi_{M} T$ value of $\left[\mathrm{Fe}_{2}{ }_{2}\left(\mathrm{H}_{2} \mathrm{SIM}^{1}\right)(\mu-\mathrm{OAC})_{2}\right] \mathrm{PF}_{6}$ is $6.47 \mathrm{~cm}^{3} \mathrm{~mol}^{-1} \mathrm{~K}$ ( $\mu_{\text {eff }}=7.19$ B.M.). This value is slightly above $6.00 \mathrm{~cm}^{3} \mathrm{~mol}^{-1} \mathrm{~K}\left(\mu_{\mathrm{eff}}=6.92\right.$ B.M.), the value expected for two non-interacting high-spin Fe"l ions. The continuous decrease of the $\chi_{M} T$ product upon decrease in temperature suggests an antiferromagnetic interaction between the two Fe" centers in $\left[\mathrm{Fe}_{2}{ }_{2}\left(\mathrm{H}_{2} \mathrm{SIM}^{1}\right)(\mu-\mathrm{OAc})_{2}\right] \mathrm{PF}_{6}$. This conclusion is confirmed by the fact that $\chi_{M} T$ reaches $0.35 \mathrm{~cm}^{3} \mathrm{~mol}^{-1} \mathrm{~K}$ ( $\mu_{\text {eff }}=1.35$ B.M.) below $2 \mathrm{~K}$, indicating an $\mathrm{S}=0$ ground state for $\left[\mathrm{Fe}_{2}{ }_{2}\left(\mathrm{H}_{2} \mathrm{SIM}^{1}\right)(\mu-\mathrm{OAC})_{2}\right] \mathrm{PF}_{6}$. An impurity of paramagnetic monoiron(III) complex is suggested accountable for the higher experimental $\chi_{M} T$ values compared to the expected ones.

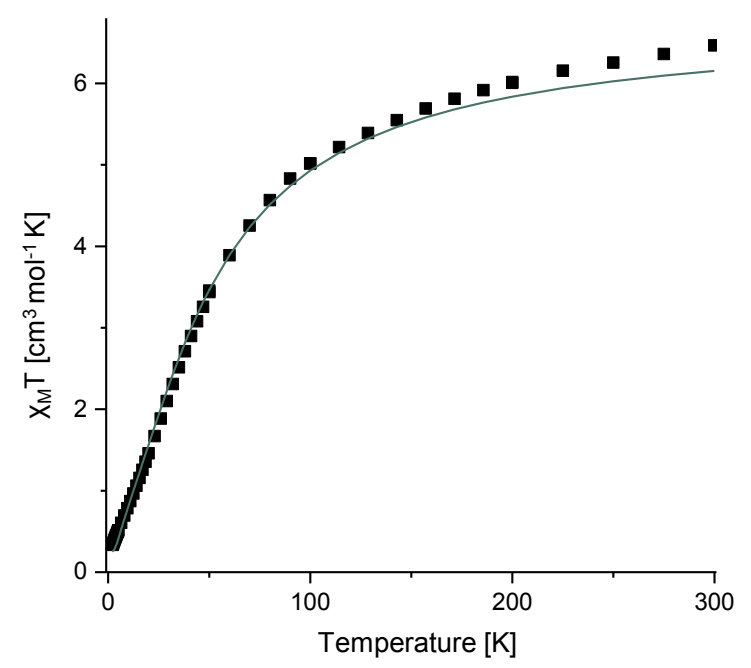

Figure 44: Temperature dependence of $\chi_{M} T$ for $\left[\mathrm{Fe}_{2}{ }_{2}\left(\mathrm{H}_{2} \mathrm{SIM}^{1}\right)(\mu-\mathrm{OAc})_{2}\right] \mathrm{PF} 6$, recorded in a magnetic field of 1000 Oe and the respective fit curve (green line).

The data were fitted according to the simplified van Vleck formula for two interacting $S=2$ spins, including paramagnetic impurities (imp) (Equation 16), derived from the Heisenberg Hamiltonian formulated in Equation 15. ${ }^{236}$

$$
\begin{array}{ll}
\text { Equation 15 } & \mathrm{H}=-2 \mathrm{JS}_{1} \mathrm{~S}_{2} \\
\text { Equation 16 } & \chi_{\mathrm{M}}=\frac{\mathrm{N}_{\mathrm{A}} \mathrm{g}^{2} \mu_{\mathrm{B}}^{2}}{3 \mathrm{kT}} \mathrm{S}(\mathrm{S}+1)+\chi_{\mathrm{imp}}
\end{array}
$$

The best fit was obtained with $\mathrm{J}=-5.05 \mathrm{~cm}^{-1}$ and a fixed $\mathrm{g}$ factor at $\mathrm{g}=2.15$, which is in agreement with the antiferromagnetism. $5.8 \%$ monoiron(III) complex as paramagnetic impurity $(S=5 / 2)$ was determined. The weak antiferromagnetism is similar to the observed magnetism for native PAPs, with $\mathrm{J}$ values between $-5 \mathrm{~cm}^{-1}$ and $-11 \mathrm{~cm}^{-1}$. 
Studying $\mathrm{Fe}_{2}{ }_{2}$ complexes of $\mathrm{HL}^{1}$, Hendrich et al. found that replacing two bridging carboxylate coligands as in $\left[\mathrm{Fe}_{2} \mathrm{~L}\left(\mathrm{~L}^{1}\right)(\mu-\mathrm{OPr})_{2}\right]^{+}$with bridging phosphate co-ligands, as in $\left[\mathrm{Fe}_{2}{ }_{2}\left(\mathrm{~L}^{1}\right)\left(\mu-\mathrm{O}_{2} \mathrm{P}(\mathrm{OPh})_{2}\right)_{2}\right]^{+}$, results in the change of the magnetic behavior from ferromagnetic to antiferromagnetic. ${ }^{237}$ This was attributed to the change in the $\mathrm{Fe}^{\|}-\mathrm{O}_{\mathrm{Ph}}-\mathrm{Fe}^{\|}$angle $\left(108.93(6)^{\circ}\right.$ for $\left[\mathrm{Fe}_{2}{ }_{2}\left(\mathrm{~L}^{1}\right)(\mu-\mathrm{OPr})_{2}\right]^{+}$and $122.7(2)^{\circ}$ for $\left.\left[\mathrm{Fe}_{2}{ }_{2}\left(\mathrm{~L}^{1}\right)\left(\mu-\mathrm{O}_{2} \mathrm{P}(\mathrm{OPh})_{2}\right)_{2}\right]^{+}\right)$and the simultaneous modification of the metal-metal interaction. ${ }^{237}$ The $\mathrm{Fe}^{\mathrm{II}}-\mathrm{O}_{\mathrm{Ph}}-\mathrm{Fe}^{\prime \prime}$ angle in $\left[\mathrm{Fe}_{2}{ }_{2}\left(\mathrm{H}_{2} \mathrm{SIM}^{1}\right)(\mu-\mathrm{OAc})_{2}\right] \mathrm{PF}_{6}$ is $114.45(18)^{\circ}$ and falls between those two values. Moreover, the magnitude of the antiferromagnetic exchange constant for $\left[\mathrm{Fe}_{2}{ }_{2}\left(\mathrm{H}_{2} \mathrm{SIM}^{1}\right)(\mu-\mathrm{OAC})_{2}\right] \mathrm{PF}_{6}$ is in the range of those reported for other oxygen-bridged diiron(II) complexes (Table 16).

Table 16: Selected structural parameters for diiron(II) complexes proposed to correlate with the magnetic exchange constant J.

\begin{tabular}{|c|c|c|c|c|}
\hline complex & $\Varangle\left(\mathrm{Fe}^{\prime \prime}-\mathrm{O}-\mathrm{Fe}^{\prime \prime}\right)\left[^{\circ}\right]$ & $\mathrm{d}\left(\mathrm{Fe} \mathrm{e}^{\prime \prime}-\mu-\mathrm{O}\right)[\AA]$ & $\varnothing\left(\mathrm{d}\left(\mathrm{Fe}^{\prime \prime}-\mu-\mathrm{O}\right)\right)[\AA \AA]$ & $\mathrm{J}\left[\mathrm{cm}^{-1}\right]$ \\
\hline \multirow{2}{*}[\mathrm{Fe}^{\prime\prime}(\mathrm{L}^{40})_{2}(\mathrm{O}_{2}\mathrm{CH})_{3}(\mathrm{O}_{2}\mathrm{CH})]{$^{\mathrm{a} 238}$} & \multirow{2}{*}{$113.0(1)$} & $2.172(2)$ & \multirow{2}{*}{2.143} & \multirow{2}{*}{-0.16} \\
\hline & & $2.113(2)$ & & \\
\hline \multirow[t]{2}{*}[\mathrm{Fe}_{2}{}_{2}(\mathrm{H}_{2}\mathrm{SIM}^{1})(\mu-\mathrm{OAc})_{2}]{$\mathrm{PF}_{6}$} & \multirow{2}{*}{114.5} & $2.039(4)$ & \multirow{2}{*}{2.032} & \multirow{2}{*}{-5.0} \\
\hline & & $2.024(4)$ & & \\
\hline \multirow[t]{2}{*}[\mathrm{Fe}_{2}{}_{2}(\mathrm{L}^{41})(\mu-\mathrm{OBz})]{$\left(\mathrm{BF}_{4}\right)_{2}{ }^{239}$} & \multirow{2}{*}{$125.7(6)$} & $2.028(13)$ & \multirow{2}{*}{1.998} & \multirow{2}{*}{-5.5} \\
\hline & & $1.968(10)$ & & \\
\hline \multirow[t]{2}{*}[\mathrm{Fe}_{2}{}_{2}(\mathrm{L}^{42})(\mu-\mathrm{OBz})(\mathrm{H}_{2}\mathrm{O})]{$\mathrm{BF}_{4}{ }^{240}$} & \multirow{2}{*}{131.2} & $2.060(2)$ & \multirow{2}{*}{2.022} & \multirow{2}{*}{-8.6} \\
\hline & & $1.984(2)$ & & \\
\hline \multirow[t]{2}{*}[\mathrm{Fe}_{2}{}_{2}(\mathrm{L}^{43})(\mu-\mathrm{OBz})]{$\left(\mathrm{BF}_{4}\right)_{2}{ }^{241}$} & \multirow{2}{*}{124.0} & $1.960(6)$ & \multirow{2}{*}{1.967} & \multirow{2}{*}{-10.5} \\
\hline & & $1.973(7)$ & & \\
\hline
\end{tabular}

a the two Fell centers are formaldehyde-bridged.

However, comparing the antiferromagnetic exchange constant $\mathrm{J}$ in relation with the $\mathrm{Fe}$ "I-O-Fe" angle, there is not a direct correlation detectable (Pearson's correlation coefficient: -0.75 ), but the critical value of the $\mathrm{Fe}^{\mathrm{ll}}-\mathrm{O}-\mathrm{Fe}$ ll angle regarding the change from ferromagnetic to antiferromagnetic interaction seems to be between $108.9^{\circ}$ and $113.2^{\circ} .^{237,} 238 \mathrm{~A}$ potential influence of the $\mathrm{Fe}^{\mathrm{I}}-(\mu-\mathrm{O})$ distance was also suggested to be accountable for the magnetic behavior of diiron(II) complexes and it appears that this parameter shows a correlation with the antiferromagnetic exchange interaction (Pearson`s correlation coefficient: 0.90). ${ }^{181,237}$ Thus, the weakened metal-metal interaction manifested by the longer $\mathrm{Fe}^{\prime \prime}-(\mu-0)$ bond leads to the decrease or elimination of antiferromagnetic coupling between the two Fe" centers. However, it should be mentioned that due to the limited number of examples of alkoxido/phenoxido-bridged diiron(II) complexes this proposal should be considered as speculative.

For the monoiron(III) complex $\left[\mathrm{Fe}{ }^{I I I} \mathrm{Na}\left(\mathrm{H}_{2} \mathrm{SIM}^{1}\right)(\mu-\mathrm{OAc})_{2}\right] \mathrm{PF}_{6} \chi_{\mathrm{M}} \mathrm{T}=4.29 \mathrm{~cm}^{3} \mathrm{~mol}^{-1} \mathrm{~K}\left(\mu_{\text {eff }}=5.84 \mathrm{~B} . \mathrm{M}\right.$.) at $300 \mathrm{~K}$ was obtained. This value is close to the expected value for five unpaired electrons of $\chi_{M} T=4.39 \mathrm{~cm}^{3} \mathrm{~mol}^{-1} \mathrm{~K}\left(\mu_{\mathrm{eff}}=5.92\right.$ B.M.). 


\section{NMR Studies}

\section{Susceptibility Measurements with the Evans Method under Anaerobic Conditions}

The magnetic properties of $\left[\mathrm{Fe}_{2}{ }_{2}\left(\mathrm{H}_{2} \mathrm{SIM}^{1}\right)(\mu-\mathrm{OAc})_{2}\right]^{+}$and $\left[\mathrm{Fe}_{2}{ }_{2}\left(\mathrm{H}_{2} \mathrm{~L}^{2}\right)(\mu-\mathrm{OAc})_{2}\right]^{+}$were also investigated in solution, applying the methodology described by Evans. ${ }^{242}$ In this method the frequency shift in the NMR spectrum between two solutions, the pure solvent and a known concentration of the paramagnetic material in the same solvent, is measured. The frequency shift is proportional to the paramagnetic susceptibility of the compound (Equation 17).

$$
\text { Equation } 17 \quad \chi_{\text {para,subst }}=\frac{3}{4 \pi c}\left(\frac{\Delta v}{v}\right)+\chi_{\text {dia,solv }}+\chi_{\text {dia,subst }}
$$

The effective magnetic moment $\mu_{\text {eff }}$ is calculated with Equation 18.

$$
\text { Equation } 18 \mu_{\text {eff }}=2.828 \sqrt{\chi_{\text {para,subst }} \mathrm{T}}
$$

The magnetic moments of the complexes were evaluated with Equations 17 and 18, after taking appropriate corrections for diamagnetism of the solvent and the complex into account. In solution, the complexes exhibit effective magnetic moments of 6.10 B.M. for $\left[\mathrm{Fe}_{2}{ }_{2}\left(\mathrm{H}_{2} \mathrm{SIM}^{1}\right)(\mu-\mathrm{OAC})_{2}\right]^{+}$and 5.35 B.M. for $\left[\mathrm{Fe}_{2}{ }_{2}\left(\mathrm{H}_{2} \mathrm{~L}^{2}\right)(\mu-\mathrm{OAc})_{2}\right]^{+}$. The experimentally derived values for these complexes were lower than the calculated value for two non-interacting highspin Fe" centers $\left(\mu_{\text {eff }}=6.93\right.$ B.M.) indicate the presence of exchange coupling between the two metal centers.

\section{Susceptibility Measurements with the Evans Method under Aerobic Conditions}

The magnetic susceptibility of $\left[\mathrm{Fe}_{2}{ }_{2}\left(\mathrm{H}_{2} \mathrm{SIM}^{1}\right)(\mu-\mathrm{OAc})_{2}\right]^{+}$dissolved in deuterated acetonitrile was found to reduce from $\mu_{\text {eff }}=6.10$ B.M. to $\mu_{\text {eff }}=4.55$ B.M. within the first hour after the solution was exposed to air, and subsequently to $\mu_{\text {eff }}=3.82$ B.M. within 24 hours. The decrease of $\mu_{\text {eff }}$ can be explained by the formation of relatively strongly coupled complexes, but the composition is not entirely clear. It has to be mentioned that $\mu_{\text {eff }}=1.73$ B.M. for a strongly coupled (hs)Fe ${ }^{\text {III-}-(h s) F e " ~ s y s t e m ~ a n d ~} \mu_{\text {eff }}=0$ for a strongly coupled (hs)Fe ${ }^{\text {III-}}$-(hs) Fe ${ }^{\text {III }}$ system is only expected at low temperature. On the basis of published work on similar systems, only moderate coupling through the phenoxide bridge is expected. ${ }^{114,125,230}$ Interestingly, slightly increased $\mu_{\text {eff }}$ values were observed when the solutions were measured after several weeks again ( $\mu_{\text {eff }}=4.85$ B.M.). This might be the result of a slow decomposition of initially formed Fe ${ }^{\prime \prime \prime}{ }_{2}$ species, i.e. formation of a monoiron(III) complex and ligand-free Fe'II. This is in agreement with the observed X-ray structures under aerobic and anaerobic conditions. 


\section{${ }^{1}$ H NMR Spectroscopy under Anaerobic Conditions}

${ }^{1} \mathrm{H}$ NMR spectroscopy was used to investigate the symmetry of the diiron(II) complexes in solution. The paramagnetic ${ }^{1} \mathrm{H} \quad \mathrm{NMR}$ spectra of $\left[\mathrm{Fe}_{2}{ }_{2}\left(\mathrm{H}_{2} \mathrm{SIM}^{1}\right)(\mu-\mathrm{OAc})_{2}\right] \mathrm{PF}_{6}$ and $\left[\mathrm{Fe}_{2}{ }_{2}\left(\mathrm{H}_{2} \mathrm{~L}^{2}\right)(\mu-\mathrm{OAc})_{2}\right] \mathrm{PF}_{6}$, dissolved in deuterated acetonitrile, were recorded (Figure 45$)$. The ${ }^{1} \mathrm{H}$ NMR spectra of the diiron(II) complexes display well-resolved and relatively narrow resonances, consistent with the fast electronic relaxation of the high-spin Fe" centers and their energetically low excited levels. ${ }^{243}$ The chemical shifts of the resonances extend over 230 ppm, from -30 ppm to $200 \mathrm{ppm}$. Overall, the spectrum is highly similar to that of the Fe" $\mathrm{Mn}^{\prime \prime}$ complex of $\mathrm{HL}^{1}$ and $\mathrm{HL}^{44} \cdot 244,245$

b)

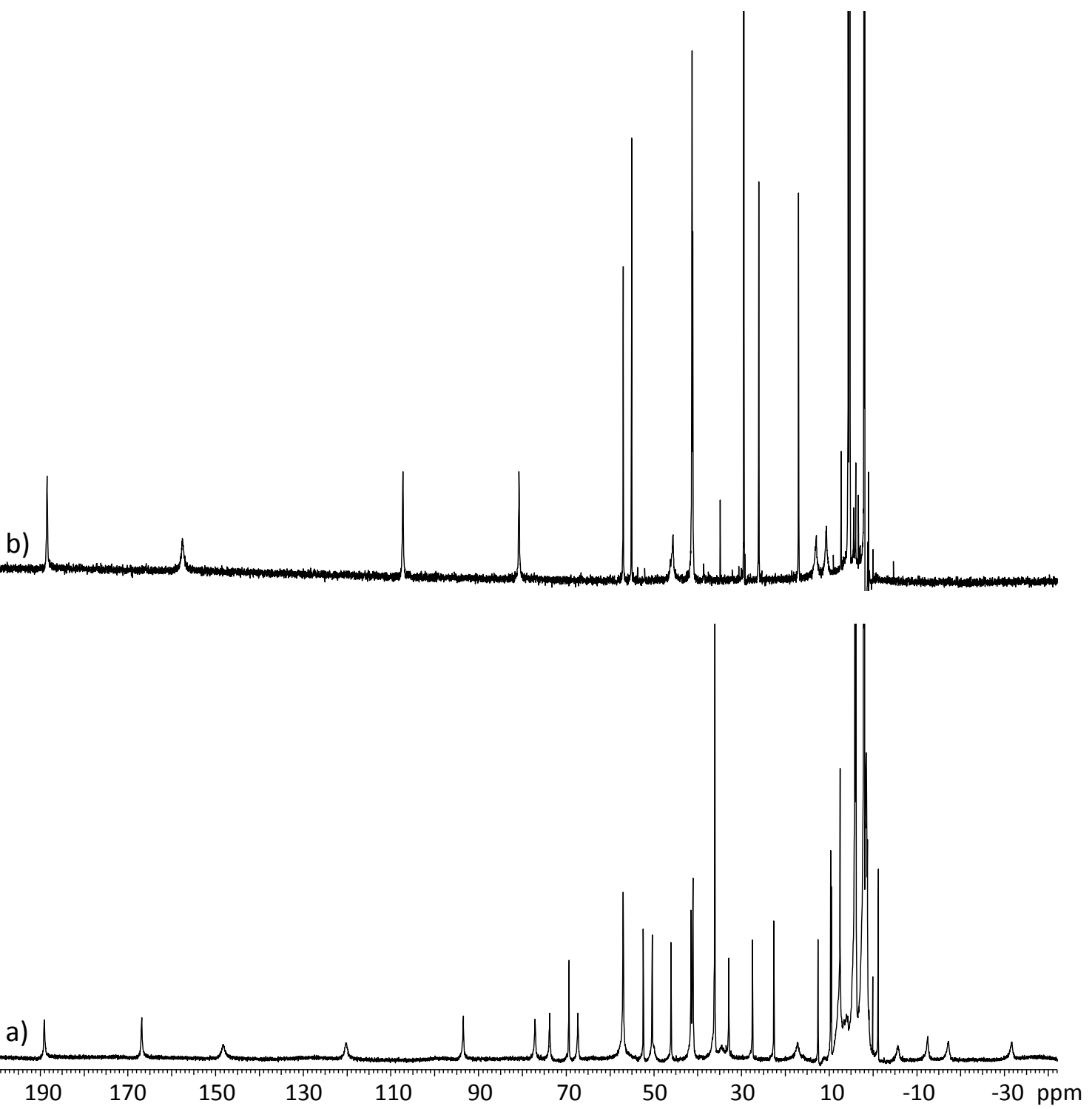

Figure 45: ${ }^{1} \mathrm{H}$ NMR spectrum of a) $\left[\mathrm{Fe}^{{ }^{\prime \prime}}{ }_{2}\left(\mathrm{H}_{2} \mathrm{SIM}^{1}\right)(\mu-\mathrm{OAc})_{2}\right] \mathrm{PF} 6$ and $\left.\mathrm{b}\right)\left[\mathrm{Fe}_{2}{ }_{2}\left(\mathrm{H}_{2} \mathrm{~L}^{2}\right)(\mu-\mathrm{OAc})_{2}\right] \mathrm{PF} 6$ in $\mathrm{CD}_{3} \mathrm{CN}$.

Unfortunately, connectivity in the complexes could not be resolved sufficiently with COSY experiments to assign the protons. However, in the spectrum recorded with $\left[\mathrm{Fe}_{2}{ }_{2}\left(\mathrm{H}_{2} \mathrm{~L}^{2}\right)(\mu-\mathrm{OAc})_{2}\right]^{+}, \quad 13$ resonances were detected, while in the spectrum of 
$\left[\mathrm{Fe}_{2}{ }_{2}\left(\mathrm{H}_{2} \mathrm{SIM}^{1}\right)(\mu-\mathrm{OAC})_{2}\right]^{+}, 26$ resonances were observed, i.e. one resonance for each proton. In the case of $\left[\mathrm{Fe}_{2}{ }_{2}\left(\mathrm{H}_{2} \mathrm{~L}^{2}\right)(\mu-\mathrm{OAC})_{2}\right]^{+}$, due to a rotation axis two protons resonate at similar fields and, therefore, a single resonance for two protons was detected. Moreover, the spectrum of $\left[\mathrm{Fe}_{2}{ }_{2}\left(\mathrm{H}_{2} \mathrm{SIM}^{1}\right)(\mu-\mathrm{OAC})_{2}\right]^{+}$exhibits two equally intense sharp resonances at $3.86 \mathrm{ppm}$ and $4.08 \mathrm{ppm}$, which are assigned to the two tert-butyl groups of the pivaloyl-amide residues due to their integration for nine protons. Interestingly, the spectrum of $\left[\mathrm{Fe}_{2}{ }_{2}\left(\mathrm{H}_{2} \mathrm{~L}^{2}\right)(\mu-\mathrm{OAc})_{2}\right]^{+}$shows a signal integrating for 18 protons at $5.40 \mathrm{ppm}$. The comparison of the spectra obtained of $\left[\mathrm{Fe}_{2}{ }_{2}\left(\mathrm{H}_{2} \mathrm{SIM}^{1}\right)(\mu-\mathrm{OAc})_{2}\right] \mathrm{PF}_{6}$, bearing a symmetric ligand backbone, and $\left[\mathrm{Fe}_{2}{ }_{2}\left(\mathrm{H}_{2} \mathrm{SIM}^{1}\right)(\mu-\mathrm{OAc})_{2}\right] \mathrm{PF}_{6}$, bearing an asymmetric ligand backbone, suggest that the symmetry provided by the ligands is still present in the respective diiron(II) complexes.

\section{${ }^{1} \mathrm{H}$ NMR Spectroscopy under Aerobic Conditions}

The green solution of $\left[\mathrm{Fe}_{2}{ }_{2}\left(\mathrm{H}_{2} \mathrm{SIM}^{1}\right)(\mu-\mathrm{OAc})_{2}\right]^{+}$, dissolved in deuterated acetonitrile, turned rapidly purple when exposed to air. The ${ }^{1} \mathrm{H}$ NMR spectrum obtained with this purple solution exhibited only one sharp downfield shifted resonance in the region between 20 ppm and 200 ppm, as opposed to the original spectrum of the complex measured under anaerobic conditions (Figure 46).

b)
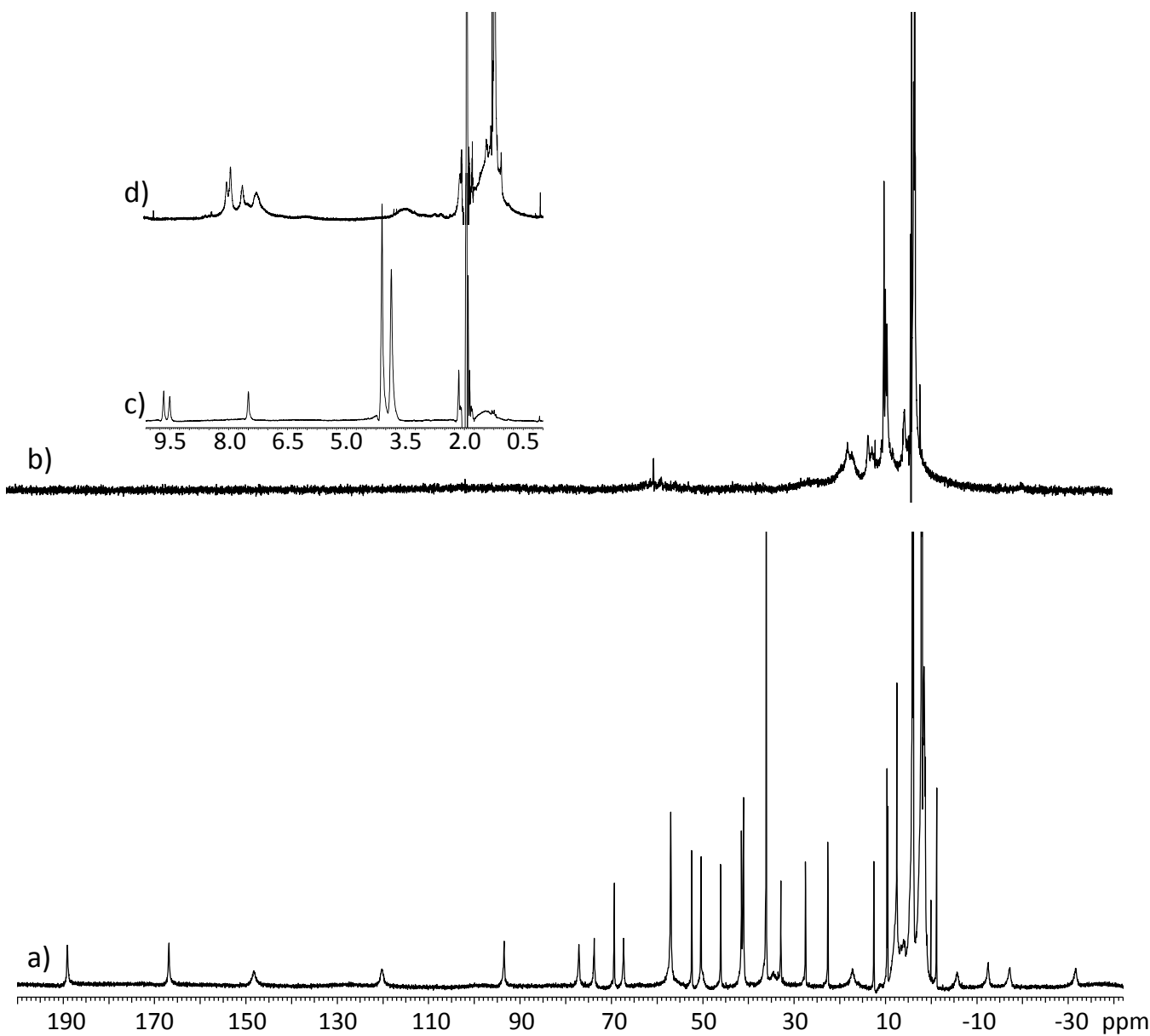

Figure 46: ${ }^{1} \mathrm{H}$ NMR spectra of $\left[\mathrm{Fe}^{\|}{ }_{2}\left(\mathrm{H}_{2} \mathrm{SIM}^{1}\right)(\mu-\mathrm{OAc})_{2}\right] \mathrm{PF}_{6}$ in $\mathrm{CD}_{3} \mathrm{CN}$ under a) and $\left.c\right)$ anaerobic conditions and $\left.\mathrm{b}\right)$ and d) after exposure to air. 
In $\left[\mathrm{Fe}_{2}{ }_{2}\left(\mathrm{H}_{2} \mathrm{SIM}^{1}\right)(\mu-\mathrm{OAC})_{2}\right]^{+}$the short electronic relaxation time of the $\mathrm{Fe}^{\prime \prime}$ ion makes the overall system relax fast enough so that sharp NMR resonances are observed. After exposure to air, the antiferromagnetic interactions are not strong enough to quench the electronic spin at room temperature. Therefore, the Fe ${ }^{I I I}$ complex formed presents only very broad unresolved resonances. Interestingly, reaction with air results in four broad resonances in the region between $6 \mathrm{ppm}$ and $8 \mathrm{ppm}$, while there was only one single resonance visible in the intact $\left[\mathrm{Fe}_{2}{ }_{2}\left(\mathrm{H}_{2} \mathrm{SIM}^{1}\right)(\mu-\mathrm{OAc})_{2}\right]^{+}$complex. This can be due to (i) one binding site being metal-free with non-coordinating pyridine residues, or (ii) some protons in the resulting diiron(III) complex are only slightly affected by the paramagnetic metal ions exhibiting resonances with chemical shifts similar to the metal-free ligand.

\section{Mass Spectrometry}

$\mathrm{ESI}^{+}$mass spectrometric investigations of $\left[\mathrm{Fe}_{2}{ }_{2}\left(\mathrm{H}_{2} \mathrm{SIM}^{1}\right)(\mu-\mathrm{OAc})_{2}\right] \mathrm{PF}_{6}$ in dichloromethane resulted in a spectrum with a main peak at $\mathrm{m} / \mathrm{z} 924.4$ and two smaller peaks at $\mathrm{m} / \mathrm{z} 889.2$ and $\mathrm{m} / \mathrm{z} 957.3$ with isotopic patterns characteristic for diiron complexes (Figure 47). The signals are assigned to $\left[\mathrm{Fe}_{2}{ }_{2} \mathrm{Na}\left(\mathrm{HSIM}^{1}\right)\left(\mathrm{CH}_{3} \mathrm{O}\right)_{2}\right]^{+},\left[\mathrm{Fe}_{2}{ }_{2}\left(\mathrm{HSIM}^{1}\right)\left(\mathrm{CH}_{3} \mathrm{OH}\right) \mathrm{F}\right]^{+}$and the intact complex $\left[\mathrm{Fe}_{2}{ }_{2}\left(\mathrm{H}_{2} \mathrm{SIM}^{1}\right)(\mathrm{OAC})_{2}\right]^{+}$, respectively. When the solution of $\left[\mathrm{Fe}_{2}{ }_{2}\left(\mathrm{H}_{2} \mathrm{SIM}^{1}\right)(\mu-\mathrm{OAc})_{2}\right] \mathrm{PF}_{6}$ in acetonitrile was exposed to air for about ten minutes prior to the measurement, one main signal was detected at $\mathrm{m} / \mathrm{z} 782.3$ consisting to the monoiron(III) complex $\left[\mathrm{Fe}^{\prime \prime \prime}\left(\mathrm{HSIM}^{1}\right)\right]^{+}$.
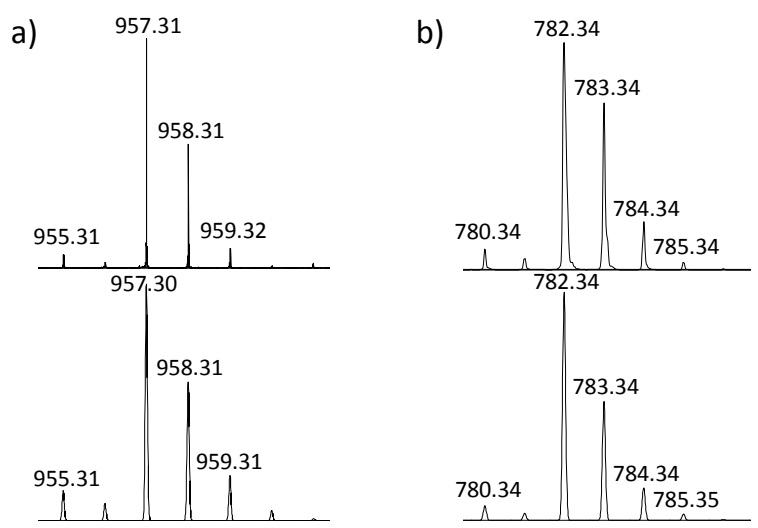

Figure 47: Characteristic isotopic pattern found in $\mathrm{ESI}^{+}$mass spectra (experimental (top) and calculated (bottom)) of $\left[\mathrm{Fe}_{2}{ }_{2}\left(\mathrm{H}_{2} \mathrm{SIM}^{1}\right)(\mu-\mathrm{OAc})_{2}\right] \mathrm{PF}_{6}$ a) measured directly after solvation in dichloromethane $\left(\left[\mathrm{Fe}_{2}{ }_{2}\left(\mathrm{H}_{2} \mathrm{SIM}^{1}\right)(\mathrm{OAC})_{2}\right]^{+}\right)$and $\left.b\right)$ in acetonitrile after exposure to air for $5 \mathrm{~min}\left(\left[\mathrm{Fe}^{\prime \prime \prime}\left(\mathrm{HSIM}^{1}\right)\right]^{+}\right)$.

Unfortunately, the solubility of $\left[\mathrm{Fe}_{2}{ }_{2}\left(\mathrm{H}_{2} \mathrm{~L}^{2}\right)(\mu-\mathrm{OAc})_{2}\right] \mathrm{PF}_{6}$ in dichloromethane was lower compared to $\left[\mathrm{Fe}_{2}{ }_{2}\left(\mathrm{H}_{2} \mathrm{SIM}^{1}\right)(\mu-\mathrm{OAC})_{2}\right] \mathrm{PF}_{6}$ and made the addition of methanol necessary, in order to obtain a spectrum. This resulted in a main signal at $\mathrm{m} / \mathrm{z} 729.4$, matching the protonated ligand $\left[\mathrm{H}_{4} \mathrm{~L}^{2}\right]^{+}$and a rather intense signal at $\mathrm{m} / \mathrm{z} 782.3$, matching the oxidation product $\left[\mathrm{Fe}^{\prime \prime \prime}\left(\mathrm{HL}^{2}\right)\right]^{+}$. Therefore, the mass spectrometric investigation of the $\mathrm{Fe}_{2}{ }_{2}$ complex in solution is hampered due to fast 
oxidation. However, signals with the characteristic diiron isotopic pattern were detected at $\mathrm{m} / \mathrm{z}$ 929.3, $\mathrm{m} / \mathrm{z}$ 943.3, and $\mathrm{m} / \mathrm{z}$ 957.3, and these are assigned to the diiron species $\left[\mathrm{Fe}_{2}{ }_{2}\left(\mathrm{H}_{2} \mathrm{~L}^{2}\right)(\mathrm{OAc})\left(\mathrm{CH}_{3} \mathrm{O}\right)\right]^{+}, \quad\left[\mathrm{Fe}_{2}{ }_{2}\left(\mathrm{H}_{2} \mathrm{~L}^{2}\right)(\mathrm{OAc})\left(\mathrm{CH}_{3} \mathrm{CH}_{2} \mathrm{O}\right)\right]^{+}$, and the intact complex $\left[\mathrm{Fe}_{2}{ }_{2}\left(\mathrm{H}_{2} \mathrm{~L}^{2}\right)(\mathrm{OAC})_{2}\right]^{+}$.

The $\mathrm{ESI}^{+}$mass spectrometric investigations undertaken with the purple solid $\left[\mathrm{Fe}{ }^{\mathrm{III}} \mathrm{Na}\left(\mathrm{H}_{2} \mathrm{SIM}^{1}\right)(\mu-\mathrm{OAc})_{2}\right] \mathrm{PF}_{6}$, dissolved in methanol resulted in a spectrum with a main peak at $\mathrm{m} / \mathrm{z}$ 872.4, assigned to $\left[\mathrm{Fe}^{\prime \prime \prime}\left(\mathrm{H}_{2} \mathrm{SIM}^{1}\right)\left(\mathrm{CH}_{3} \mathrm{OH}\right)(\mathrm{OAc})\right]^{+}$. The softer ionization method conducted with MALDI experiments resulted in a spectrum with two main peaks at $\mathrm{m} / \mathrm{z} 821.3$ and $\mathrm{m} / \mathrm{z} 839.3$, which both show the pattern characteristic for an iron complex similar to the one depicted in Figure $47 \mathrm{~b})$. These signals match the species of $\left[\mathrm{Fe}^{\prime \prime \prime} \mathrm{Na}\left(\mathrm{SIM}^{1}\right)\left(\mathrm{H}_{2} \mathrm{O}\right)\right]^{+}$and $\left[\mathrm{Fe}{ }^{\prime \prime \prime} \mathrm{Na}\left(\mathrm{SIM}^{1}\right)\left(\mathrm{H}_{2} \mathrm{O}\right)_{2}\right]^{+}$.

\section{Mössbauer Spectroscopy}

${ }^{57} \mathrm{Fe}$ Mössbauer spectroscopy is a very sensitive technique, which has been very useful for ascertaining the oxidation and spin states of iron centers. This technique is based on the Mössbauer effect, consisting of recoil-free, resonant absorption and emission of gamma rays. ${ }^{246}$ Mössbauer experiments involve the determination of the energy difference between the ground state and the excited states by exposure of the sample to a beam of gamma radiation. The energy difference between the ground and the excited states depends on the interaction of the nucleus and electric (and magnetic) fields at the nucleus (Scheme 11). ${ }^{247}$ Thus, ${ }^{57} \mathrm{Fe}$ Mössbauer spectra reflect chemical information of the iron centers in the Mössbauer parameters, i.e. isomer shift and quadrupole splitting. The isomer shift results from the Coulomb interaction of the nuclear charge distribution and the s-electron charge cloud, penetrating the nucleus (electric monopole interaction) and depends, therefore, most strongly on the ionization of the iron center. ${ }^{246}$ The quadrupole splitting originates from the interaction between the electric quadrupole moment $\mathrm{eQ}$ of the nucleus itself and the Electron Field Gradient (EFG) at the nucleus (electric quadrupole interaction). ${ }^{246}$ The EFG arises from an aspherical distribution of electrons in the valence orbitals and/or aspherical charge distribution in the ligand sphere. Thus, the quadrupole splitting depends on the symmetry of the environment of the iron center. ${ }^{246}$ 


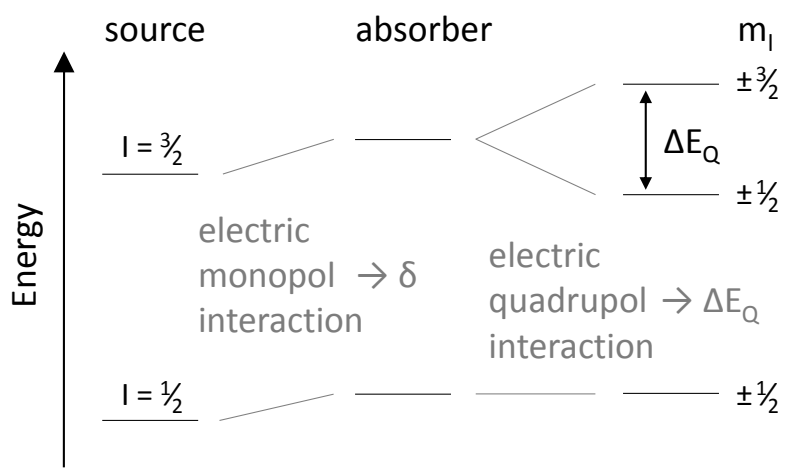

Scheme 11: Origin of the isomer shift $\delta$ (electric monopol ineraction) and the quadrupol splitting $\Delta E_{Q}$ (electric quadrupol interaction) in ${ }^{57} \mathrm{Fe}$ Mössbauer spectra. ${ }^{246,247}$

\section{Mössbauer Spectroscopy under Anaerobic Conditions}

In order to investigate the coordination chemistry of the two $\mathrm{Fe}^{\text {ll }}$ centers in $\left[\mathrm{Fe}_{2}{ }_{2}\left(\mathrm{H}_{2} \mathrm{SIM}^{1}\right)(\mu-\mathrm{OAc})_{2}\right] \mathrm{PF}_{6}$, a ${ }^{57} \mathrm{Fe}$ enriched sample was prepared and a ${ }^{57} \mathrm{Fe}$ Mössbauer spectrum was measured in frozen acetonitrile solution at $170 \mathrm{~K}$ (Figure 48a). The spectrum obtained shows a single quadrupole doublet, indicating similar electronic environments for both Fe" centers. The parameters determined by fitting the spectrum, using the NORMOS Mössbauer Fitting Program ${ }^{248}, \delta=1.13(1) \mathrm{mm} / \mathrm{s}$ and $\Delta \mathrm{E}_{\mathrm{Q}}=2.66(1) \mathrm{mm} / \mathrm{s}(\Gamma=0.45(1) \mathrm{mm} / \mathrm{s})$, are consistent with Fe" in the high-spin configuration and in agreement with values reported for other dinuclear highspin Fe" complexes, i.e. $\left[\mathrm{Fe}_{2}{ }_{2}\left(\mathrm{~L}^{42}\right)\left(\mu-\mathrm{O}_{2} \mathrm{CCF}_{3}\right)\left(\mathrm{H}_{2} \mathrm{O}\right)_{2}\right]\left(\mathrm{BF}_{4}\right)_{2}\left(\delta=1.05 \mathrm{~mm} / \mathrm{s}\right.$ and $\left.\Delta \mathrm{E}_{\mathrm{Q}}=2.69 \mathrm{~mm} / \mathrm{s}\right) .{ }^{240}$ The $\delta$ shift of $\left[\mathrm{Fe}_{2}{ }_{2}\left(\mathrm{H}_{2} \mathrm{SIM}^{1}\right)(\mu-\mathrm{OAc})_{2}\right] \mathrm{PF}_{6}$ to more positive values compared to $\left[\mathrm{Fe}_{2}{ }_{2}\left(\mathrm{~L}^{42}\right)\left(\mu-\mathrm{O}_{2} \mathrm{CCF}_{3}\right)\left(\mathrm{H}_{2} \mathrm{O}\right)_{2}\right]\left(\mathrm{BF}_{4}\right)_{2}$ is attributed to the increased electronegativity of the coordinating ligands in the former complex.

\section{Mössbauer Spectroscopy under Aerobic Conditions}

The Mössbauer spectrum of a sample of $\left[\mathrm{Fe}_{2}{ }_{2}\left(\mathrm{H}_{2} \mathrm{SIM}^{1}\right)(\mu-\mathrm{OAC})_{2}\right] \mathrm{PF}_{6}$, dissolved in acetonitrile and exposed to air for 24 hours, was collected at $170 \mathrm{~K}$ and showed a slightly asymmetric doublet (Figure 48b). The ${ }^{57} \mathrm{Fe}$ Mössbauer spectrum obtained after exposure to air for further 24 hours was essentially the same (Figure 48c). Least-squares fitting determined two doublets of equal intensity with the isomer shifts of $\delta(I)=0.42(1) \mathrm{mm} / \mathrm{s}$ and $\delta(I I)=0.42(1) \mathrm{mm} / \mathrm{s}$ and the quadrupole splitting of $\Delta \mathrm{E}_{\mathrm{Q}}(\mathrm{I})=0.77(2) \mathrm{mm} / \mathrm{s}(\Gamma=0.44(1) \mathrm{mm} / \mathrm{s})$ and $\Delta \mathrm{E}_{\mathrm{Q}}(\mathrm{II})=1.31 \mathrm{~mm} / \mathrm{s}$ $(\Gamma=0.40(1) \mathrm{mm} / \mathrm{s})$, respectively. The Mössbauer parameters are in agreement with two high-spin Fe ${ }^{\text {III }}$ ions in similar electronic environments and are comparable with those previously reported for phenolate-bridged diiron(III) complexes, i.e. $\left[\mathrm{Fe}^{\mathrm{III}}{ }_{2}\left(\mathrm{H}_{2} \mathrm{~L}^{2}\right)(\mu-\mathrm{OH})\right]^{4+}(\delta=0.47 \mathrm{~mm} / \mathrm{s}$, $\left.\Delta \mathrm{E}_{\mathrm{Q}}=0.84 \mathrm{~mm} / \mathrm{s}\right),{ }^{114}\left[\mathrm{Fe}^{\prime \prime \prime \prime}{ }_{2}(\mu-\mathrm{O})\left(\mathrm{L}^{45}\right)_{2}(\mathrm{OH})\left(\mathrm{H}_{2} \mathrm{O}\right)\right] \mathrm{ClO}_{4}\left(\delta=0.45 \mathrm{~mm} / \mathrm{s}, \Delta \mathrm{E}_{\mathrm{Q}}(\mathrm{I})=1.63 \mathrm{~mm} / \mathrm{s}\right.$, $\left.\Delta \mathrm{E}_{\mathrm{Q}}(\mathrm{II})=1.11 \mathrm{~mm} / \mathrm{s}\right),{ }^{249}$ and $\left[\mathrm{Fe}^{\prime \prime \prime \prime}{ }_{2}\left(\mathrm{~L}^{46}\right)_{2}(\mu-\mathrm{OAC})\right] \mathrm{ClO}_{4}\left(\delta=0.39 \mathrm{~mm} / \mathrm{s}, \Delta \mathrm{E}_{\mathrm{Q}}=0.93 \mathrm{~mm} / \mathrm{s}\right) .{ }^{102} \mathrm{The}$ fitting with two quadupole doublets is consistent with two different Fe ${ }^{\text {III }}$ centers and, therefore, 
the formation of the complex $\left[\mathrm{Fe}^{\prime \prime \prime}{ }_{2}\left(\mathrm{H}_{2} \mathrm{SIM}^{1}\right)(\mu-\mathrm{OAc})_{2}\right]^{3+}$. In contrast to $\left[\mathrm{Fe}_{2}{ }_{2}\left(\mathrm{H}_{2} \mathrm{SIM}^{1}\right)(\mu-\mathrm{OAc})_{2}\right] \mathrm{PF}_{6}$, the asymmetry of electron density distribution around the two Fe ${ }^{\text {III }}$ centers in $\left[\mathrm{Fe}^{\mathrm{III}}{ }_{2}\left(\mathrm{H}_{2} \mathrm{SIM}^{1}\right)(\mu-\mathrm{OAC})_{2}\right]^{3+}$ is larger and therefore large enough to afford two distinct quadrupole doublets.
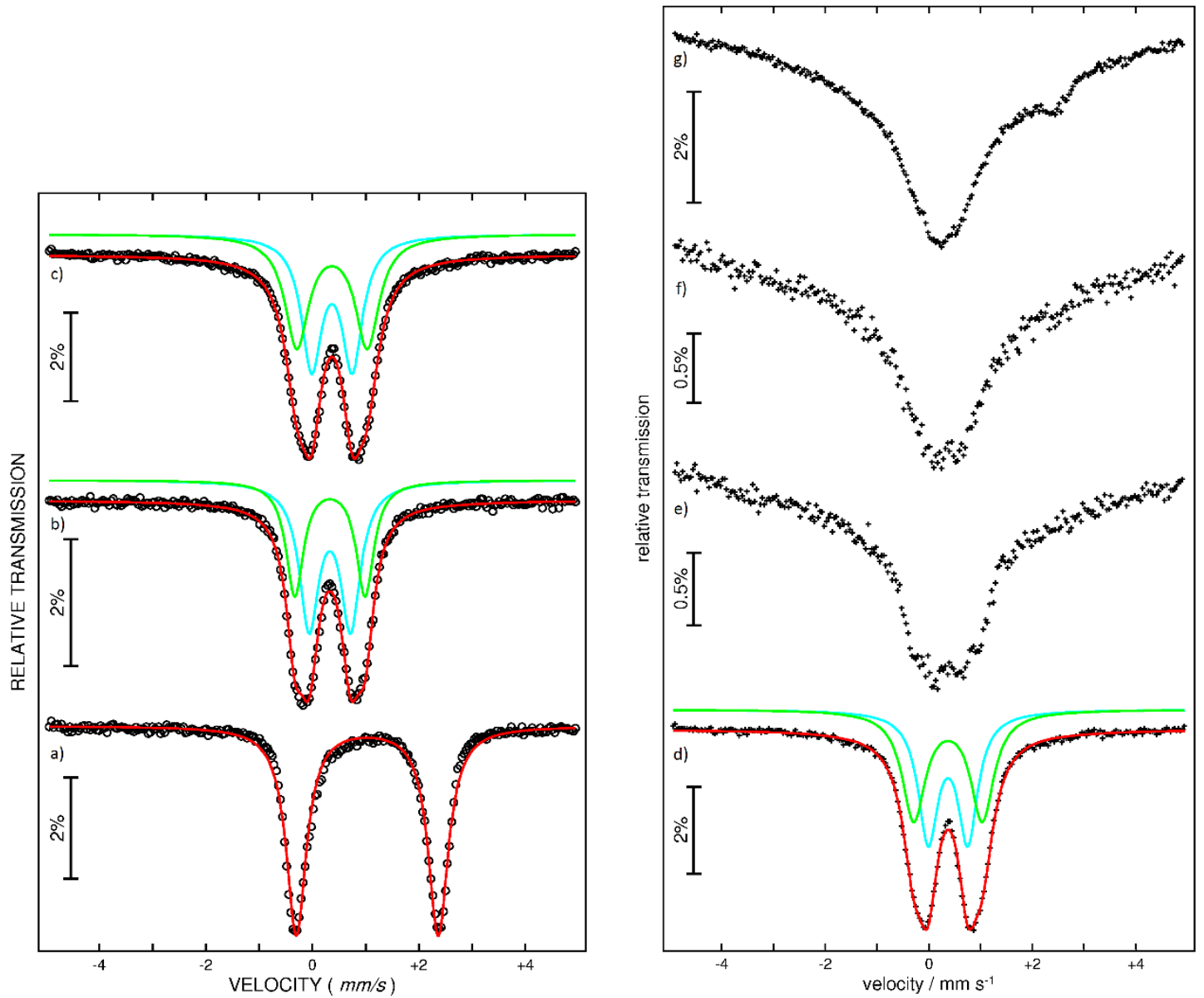

Figure 48: Comparison of the ${ }^{57} \mathrm{Fe}$ Mössbauer spectra obtained from frozen acetonitrile solution (except spectrum g)) of a) $\left[\mathrm{Fe}_{2}{ }_{2}\left(\mathrm{H}_{2} \mathrm{SIM}^{1}\right)(\mu-\mathrm{OAc})_{2}\right] \mathrm{PF}_{6}$, b) $\left[\mathrm{Fe}^{\|_{2}}{ }_{2}\left(\mathrm{H}_{2} \mathrm{SIM}^{1}\right)(\mu-\mathrm{OAc})_{2}\right] P F_{6}$ after exposure to air for $\left.24 \mathrm{~h} \mathrm{c}\right)$ $\left[\mathrm{Fe}_{2}{ }_{2}\left(\mathrm{H}_{2} \mathrm{SIM}^{1}\right)(\mu-\mathrm{OAc})_{2}\right] \mathrm{PF}_{6}$ after exposure to air for $\left.48 \mathrm{~h}, \mathrm{~d}\right)\left[\mathrm{Fe}_{2}{ }_{2}\left(\mathrm{H}_{2} \mathrm{SIM}^{1}\right)(\mu-\mathrm{OAc})_{2}\right] \mathrm{PF} 6$ treated with NaPF $\mathrm{F}_{6}$ after exposure to air for $72 \mathrm{~h}$, e) $\left[\mathrm{Fe}^{\prime \prime \prime} \mathrm{Na}\left(\mathrm{H}_{2} \mathrm{SIM}^{1}\right)(\mu-\mathrm{OAc})_{2}\right] \mathrm{PF}_{6}$ after exposure to air for $\left.24 \mathrm{~h}, \mathrm{f}\right)$ $\left[\mathrm{Fe}^{\prime \prime \prime} \mathrm{Na}\left(\mathrm{H}_{2} \mathrm{SIM}^{1}\right)(\mu-\mathrm{OAc})_{2}\right] \mathrm{PF}_{6}$ after exposure to air for $2 \mathrm{~h}$ and $\left.\mathrm{g}\right)\left[\mathrm{Fe}^{\mathrm{III}} \mathrm{Na}\left(\mathrm{H}_{2} \mathrm{SIM}^{1}\right)(\mu-\mathrm{OAc})_{2}\right] \mathrm{PF} 6$ as a solid.

The purple solid [ $\left.\mathrm{Fe}^{1 \mid \prime \prime} \mathrm{Na}\left(\mathrm{H}_{2} \mathrm{SIM}^{1}\right)(\mu-\mathrm{OAc})_{2}\right] \mathrm{PF}_{6}$ exhibits a broad singlet in the ${ }^{57} \mathrm{Fe}$ Mössbauer spectrum, ascribed to a relaxing high-spin Fe ${ }^{\text {III }}$ mononuclear species (Figure $48 \mathrm{~g}$ ) and a small signal indicating a Fe" species. The spectrum obtained of $\left[\mathrm{Fe}^{\prime \prime \prime} \mathrm{Na}^{\prime \prime}\left(\mathrm{H}_{2} \mathrm{SIM}^{1}\right)(\mu-\mathrm{OAc})_{2}\right] \mathrm{PF}_{6}$, dissolved in acetonitrile (Figure 48f), is essentially the same as the one obtained of the solid, except for an intensity decrease of the Fe"l species. The Fe"l species found in these spectra is assigned to impurities of $\left[\mathrm{Fe}^{\prime \prime \prime} \mathrm{Fe}^{\prime \prime}\left(\mathrm{H}_{2} \mathrm{SIM}^{1}\right)(\mu-\mathrm{OAc})_{2}\right]^{2+}$ and the corresponding fitting curves are shown in Figure 49c. This interpretation derives from comparison with the corresponding ${ }^{57} \mathrm{Fe}$ Mössbauer spectrum of $\left[\mathrm{Fe}^{\prime \prime \prime} \mathrm{Fe}{ }^{\prime \prime}\left(\mathrm{H}_{2} \mathrm{SIM}^{1}\right)(\mu-\mathrm{OAc})_{2}\right]^{2+}$ (Figure $54 \mathrm{~b}$ ), generated by chemical oxidation of $\left[\mathrm{Fe}_{2}{ }_{2}\left(\mathrm{H}_{2} \mathrm{SIM}^{1}\right)(\mu-\mathrm{OAC})_{2}\right]^{+}$and will be discussed later in this chapter. During further exposure to air, the spectrum becomes more narrow (Figure 48e) and exhibits features similar to the spectrum 
of $\left[\mathrm{Fe}^{\mathrm{III}}{ }_{2}\left(\mathrm{H}_{2} \mathrm{SIM}^{1}\right)(\mu-\mathrm{OAC})_{2}\right]^{3+}$ (Figure $\left.48 \mathrm{~d}\right)$, suggesting the formation of the diiron(III) complex in acetonitrile solution under aerobic conditions. Coordination of two $\mathrm{Fe}^{\| l}$ ions in the corresponding ligand $\mathrm{H}_{3} \mathrm{SIM}^{1}$ and subsequent oxidation of the iron centers appears to be the preferred way to form $\left[\mathrm{Fe}^{\prime \prime \prime}{ }_{2}\left(\mathrm{H}_{2} \mathrm{SIM}^{1}\right)(\mu-\mathrm{OAc})_{2}\right]^{3+}$ compared to direct coordination of two $\mathrm{Fe}^{\text {III }}$ ions (discussed in Chapter 3.2.5). Moreover, the crystallization of $\left[\mathrm{Fe}^{\mathrm{III}}{ }_{2}\left(\mathrm{H}_{2} \mathrm{SIM}^{1}\right)(\mu-\mathrm{OAc})_{2}\right]^{3+}$ from methanol solution seems to be less likely than crystallization of $\left[\mathrm{Fe}{ }^{I I I} \mathrm{Na}\left(\mathrm{H}_{2} \mathrm{SIM}^{1}\right)(\mu-\mathrm{OAc})_{2}\right] \mathrm{PF}_{6}$, because only the latter compound was isolated via diethylether diffusion.

Treatment of $\left[\mathrm{Fe}^{\prime \prime \prime}{ }_{2}\left(\mathrm{H}_{2} \mathrm{SIM}^{1}\right)(\mu-\mathrm{OAC})_{2}\right]^{3+}$ with perchloric acid results in decomposition of the complex apparent by its loss of color in solution. Interestingly, a solution of [Fe $\left.{ }^{I I I} \mathrm{Na}\left(\mathrm{H}_{2} \mathrm{SIM}^{1}\right)(\mu-\mathrm{OAc})_{2}\right] \mathrm{PF}_{6}$, which was exposed to air for 72 hours (generating the mixture of $\left[\mathrm{Fe}^{\mathrm{II \prime}}{ }_{2}\left(\mathrm{H}_{2} \mathrm{SIM}^{1}\right)(\mu-\mathrm{OAc})_{2}\right]^{3+}$ and metal-free ligand $\left.\mathrm{H}_{3} \mathrm{SIM}^{1}\right)$, and subsequent treatment with perchloric acid resulted in a ${ }^{57} \mathrm{Fe}$ Mössbauer spectrum with a single quadrupole doublet, characteristic for a high-spin Fe" species (Figure 49b). This indicates the reduction of the Fe ${ }^{\text {III }}$ ion by the phenol-based ligand $\mathrm{H}_{3} \mathrm{SIM}^{1}$ in acidic solution.

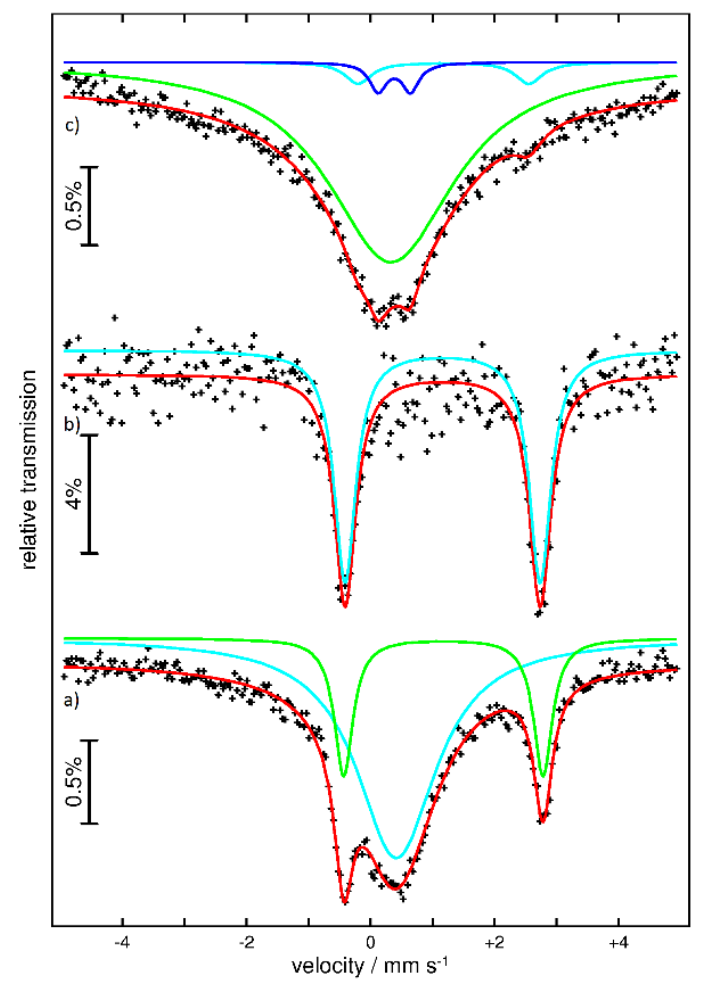

Figure 49: Comparison of the ${ }^{57} \mathrm{Fe}$ Mössbauer spectra obtained from frozen acetonitrile solution of a) $\left[\mathrm{Fe}_{2}{ }_{2}\left(\mathrm{H}_{2} \mathrm{SIM}^{1}\right)(\mu-\mathrm{OAc})_{2}\right] \mathrm{PF}_{6}$ after exposure to air for $72 \mathrm{~h}$ and addition of $\left.\mathrm{HClO}_{4}, \mathrm{~b}\right)\left[\mathrm{Fe} \mathrm{Fi}^{\prime \prime \prime} \mathrm{Na}\left(\mathrm{H}_{2} \mathrm{SIM}^{1}\right)(\mu-\mathrm{OAc})_{2}\right] \mathrm{PF}_{6}$ after exposure to air for $72 \mathrm{~h}$ and addition of $\mathrm{HClO}_{4}$, and c) $\left[\mathrm{Fe}^{\mathrm{III}} \mathrm{Na}\left(\mathrm{H}_{2} \mathrm{SIM}^{1}\right)(\mu-\mathrm{OAc})_{2}\right] \mathrm{PF}_{6}$.

Mayer et al. used phenols with pendant substituted pyridines to study the concerted protonelectron transfer in hydrogen-bonded pyridine-phenols. ${ }^{250,251}$ In this process, the electron is removed by an outer-sphere one-electron oxidant such as $\left[\mathrm{Fe}^{\prime \prime \prime}\left(\mathrm{L}^{47}\right)_{3}\right]^{3+}$ and $\left[\mathrm{Fe}^{\mathrm{III}}\left(\mathrm{L}^{48}\right)_{3}\right]^{3+}$ and derivatives thereof, and the phenolic proton is transferred concomitantly to the pendant basic 
pyridyl residue to give a distonic radical cation (Scheme 12). ${ }^{250,251}$ Another task which the basic sites fulfill is the stabilization of the oxidized product. ${ }^{251}$
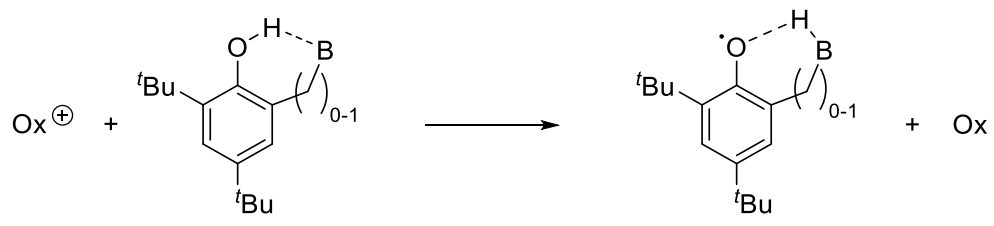

Scheme 12: CPET reactivity of phenols with pendant basic moieties. ${ }^{251}$

The addition of perchloric acid to $\left[\mathrm{Fe}{ }^{\prime \prime \prime} \mathrm{Na}\left(\mathrm{H}_{2} \mathrm{SIM}^{1}\right)(\mu-\mathrm{OAc})_{2}\right] \mathrm{PF}_{6}$ in acetonitrile solution results in the protonation of the bridging phenolate, apparent in the color change to blue, forming most likely $\left[\mathrm{Fe}^{\mathrm{III}}\left(\mathrm{H}_{3} \mathrm{SIM}^{1}\right)(\text { solv })_{2}\right]^{3+}$ (Scheme 13$)$. The unexpected appearance of a Fe" species in the ${ }^{57} \mathrm{Fe}$ Mössbauer spectrum, recorded with the colorless solution obtained after ultrasonication, is proposed to be due to a CPET mechanism similar to the one shown in Scheme 12. The Fe ${ }^{\text {III }}$ complex is proposed to act as a one-electron oxidant generating a phenol radical and the corresponding $\mathrm{Fe}^{l l}$ complex. The proximal pyridine residues may act as pendant basic site (Scheme 13). It is not clear if the oxidant needed for the oxidation of the phenol-based ligand is an $\mathrm{Fe}^{\text {III }}$ ion coordinated to the same ligand or the Fe ${ }^{\text {III }}$ center of another complex molecule.

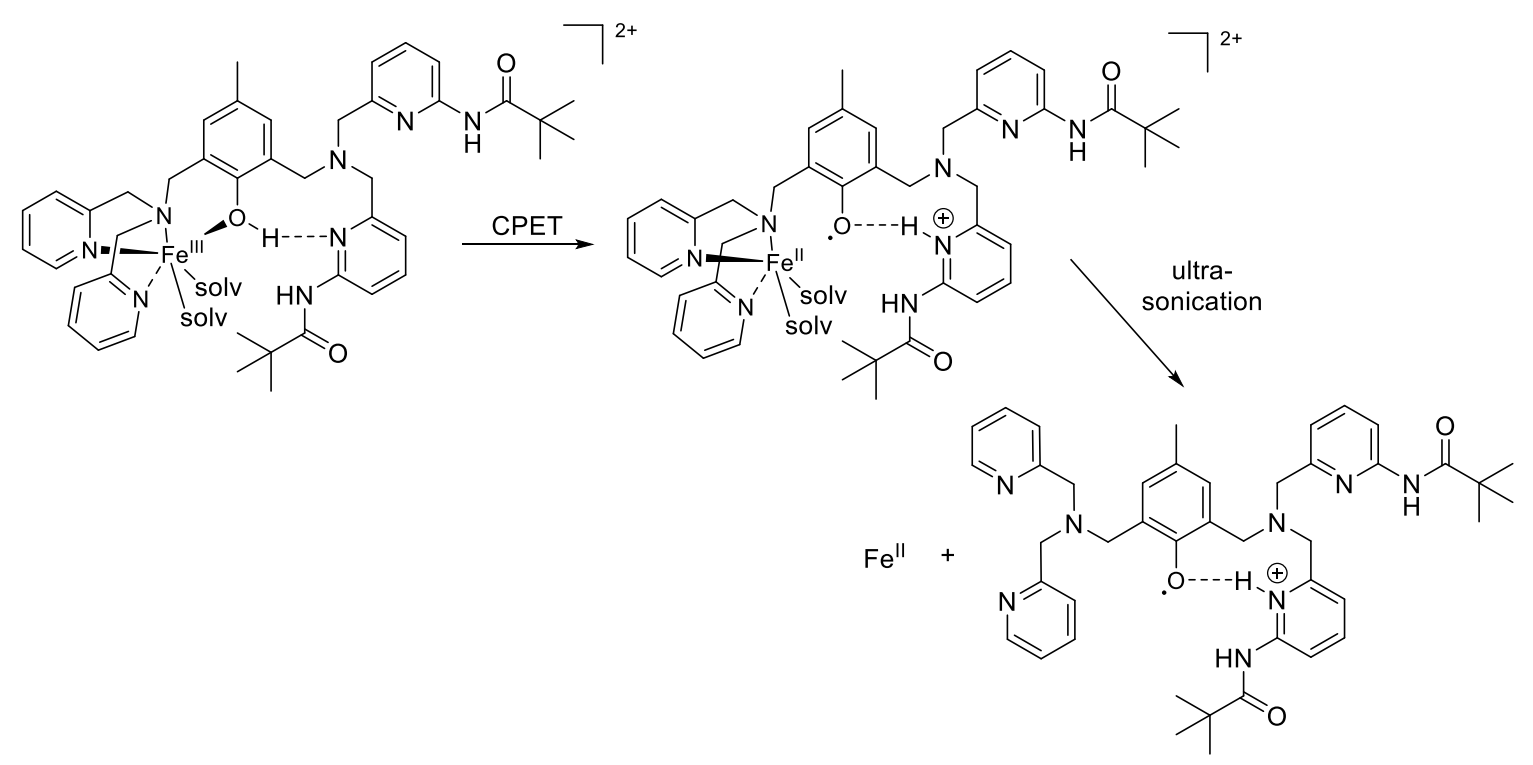

Scheme 13: Proposed mechanism for the CPET in $\left[\mathrm{Fe}^{\text {III }}\left(\mathrm{H}_{3} \mathrm{SIM}^{1}\right)(\text { solv })_{2}\right]^{2+}$.

Similar to the observation obtained from the acidified solution of $\left[\mathrm{Fe}^{I ! \prime} \mathrm{Na}\left(\mathrm{H}_{2} \mathrm{SIM}^{1}\right)(\mu-\mathrm{OAc})_{2}\right] \mathrm{PF}_{6}$, the $\left[\mathrm{Fe}^{\mathrm{III}}{ }_{2}\left(\mathrm{H}_{2} \mathrm{SIM}^{1}\right)(\mu-\mathrm{OAC})_{2}\right]^{3+}$ compound (formed via oxidation under aerobic conditions of a solution of $\left[\mathrm{Fe}_{2}{ }_{2}\left(\mathrm{H}_{2} \mathrm{SIM}^{1}\right)(\mu-\mathrm{OAC})_{2}\right]^{+}$in acetonitrile) showed also an $\mathrm{Fe}^{\prime \prime}$ species in the ${ }^{57} \mathrm{Fe}$ Mössbauer spectrum, measured after treatment with perchloric acid (Figure 49a). Besides the quadrupole doublet, assigned to ligand-free $\mathrm{Fe}^{\| l}$ ions, a broad signal was determined in the spectrum, indicating the presence of a Fe ${ }^{\text {III }}$ species in the mixture. This finding is consistent with 
the 2:1 ratio of $\mathrm{Fe}^{\mathrm{III}}$ ions and phenol-based ligand in the initial mixture, which enables only the reduction of half of the Fe ${ }^{\text {III }}$ ions, as no more phenol-based species are available in solution.

\section{Electrochemical Studies using Cyclic Voltammetry}

The electrochemical behavior of $\left[\mathrm{Fe}_{2}{ }_{2}\left(\mathrm{H}_{2} \mathrm{SIM}^{1}\right)(\mu-\mathrm{OAc})_{2}\right]^{+}$and $\left[\mathrm{Fe}_{2}{ }_{2}\left(\mathrm{H}_{2} \mathrm{~L}^{2}\right)(\mu-\mathrm{OAc})_{2}\right]^{+}$was investigated by cyclic voltammetry (CV). The CV experiments were conducted in degassed acetonitrile solution ( $0.1 \mathrm{M} \mathrm{NBu}_{4} \mathrm{ClO}_{4}, 0.5 \mathrm{mM}$ complex) at room temperature and the results are presented in Figure 50. Ferrocene was used as internal standard. ${ }^{252}$ Multiple CV scans were essentially identical when the experiments were undertaken under anaerobic conditions, indicating that decomposition in solution does not occur.

The $\mathrm{CV}$ of $\left[\mathrm{Fe}_{2}{ }_{2}\left(\mathrm{H}_{2} \mathrm{SIM}^{1}\right)(\mu-\mathrm{OAC})_{2}\right] \mathrm{PF}_{6}$ shows two sequential reductions, a reversible one-electron transfer at $0.16 \mathrm{~V} v s$. SCE $\left(\Delta \mathrm{E}_{\mathrm{p}}=88 \mathrm{mV}\right)$ and a quasi-reversible one-electron transfer at $0.56 \mathrm{~V} v s$. $\mathrm{SCE}\left(\Delta \mathrm{E}_{\mathrm{p}}=153 \mathrm{mV}\right)$ (Figure 50a) which can be assigned to the $\mathrm{Fe}_{2}{ }_{2} / \mathrm{Fe}^{\prime \prime \prime} \mathrm{Fe}{ }^{\prime \prime}$ and $\mathrm{Fe}{ }^{\prime \prime \prime} \mathrm{Fe}{ }^{\prime \prime} / \mathrm{Fe}^{\prime \prime \prime}{ }_{2}$ couples, respectively. Interestingly, the potential at $0.16 \mathrm{~V}$ ( $v s$. SCE) is similar to the redox couple examined for the native ufPAP enzyme at $\sim 0.34 \mathrm{~V}$ vs. NHE (0.10 V vs. SCE). ${ }^{222}$

In the case of $\left[\mathrm{Fe}_{2}{ }_{2}\left(\mathrm{H}_{2} \mathrm{~L}^{2}\right)(\mu-\mathrm{OAc})_{2}\right] \mathrm{PF}_{6}$ a more complex $\mathrm{CV}$ was observed, with two anodic and two cathodic waves (Figure 50b). Thus, several species appear to coexist in both redox states in solution and these complicate the redox behavior. While the $\mathrm{Fe}^{\prime \prime \prime} \mathrm{Fe} \mathrm{e}^{\prime \prime} / \mathrm{Fe}^{\prime \prime \prime}{ }_{2}$ couples are in the same region as in the case of $\left[\mathrm{Fe}_{2}{ }_{2}\left(\mathrm{H}_{2} \mathrm{SIM}^{1}\right)(\mu-\mathrm{OAc})_{2}\right] \mathrm{PF}_{6}$, the $\mathrm{Fe}_{2}{ }_{2} / \mathrm{Fe}^{\prime \prime \prime} \mathrm{Fe}{ }^{\prime \prime}$ couples are shifted to more positive redox potentials around $0.8 \mathrm{~V}$ vs. SCE. The redox potential of the Fe ${ }^{\prime \prime \prime}{ }_{2}$ complex of ligand $\mathrm{H}_{2} \mathrm{~L}^{2}$ was detected at $0.40 \mathrm{~V}$ vs. SCE. ${ }^{114}$

Table 17 compares these values with the redox potentials found for similar complexes but lacking the pivaloyl-amide residues. Interestingly, the redox potentials found for $\left[\mathrm{Fe}_{2}{ }_{2}\left(\mathrm{H}_{2} \mathrm{~L}^{2}\right)(\mu-\mathrm{OAc})_{2}\right] \mathrm{PF}_{6}$ are all shifted to more positive potentials, while in the case of $\left[\mathrm{Fe}_{2}{ }_{2}\left(\mathrm{H}_{2} \mathrm{SIM}^{1}\right)(\mu-\mathrm{OAc})_{2}\right] P F_{6} \mathrm{E}_{1}$ is shifted to more positive and $E_{2}$ to more negative potentials, compared to the unsubstituted complexes derived from $\mathrm{HL}^{1}$. 
a)

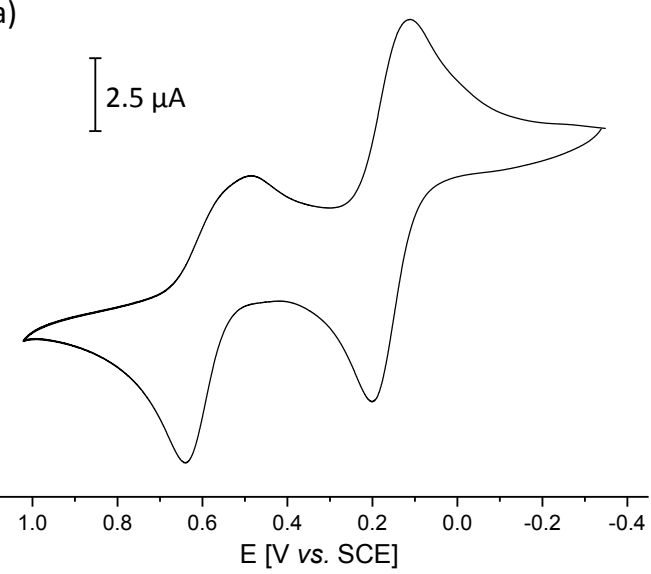

c)

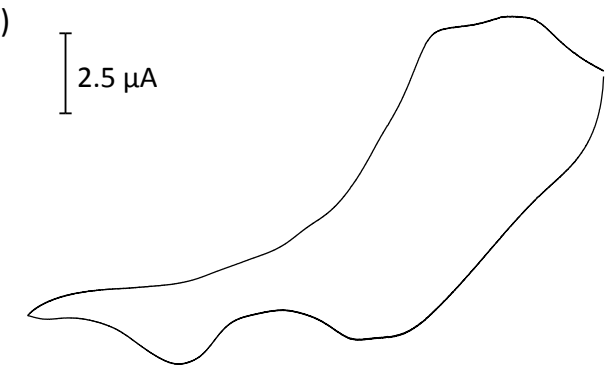

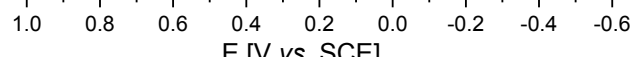

b)
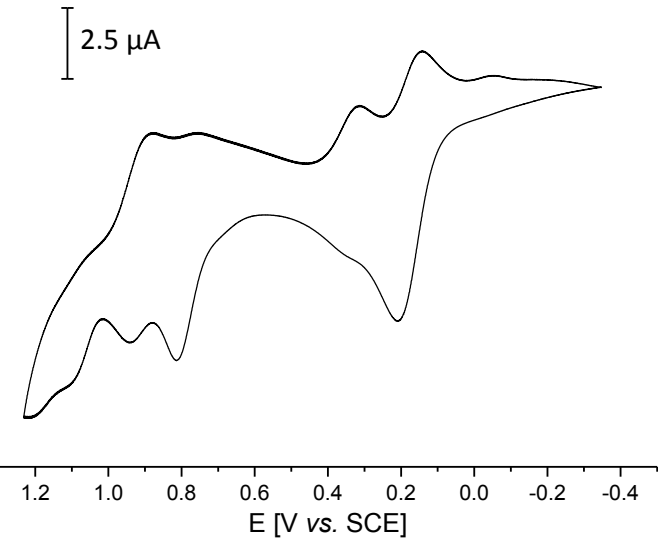

d) $[2.5 \mu \mathrm{A}$

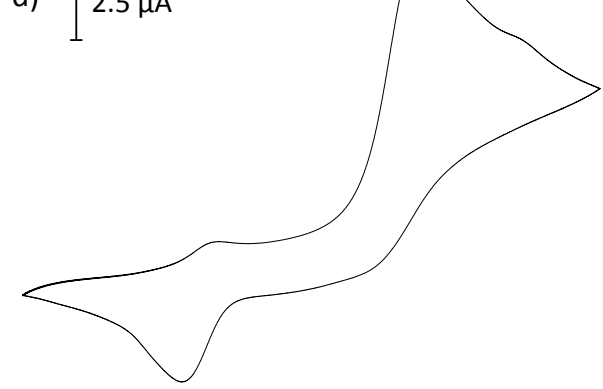

$\begin{array}{lllllllll}1.0 & 0.8 & 0.6 & 0.4 & 0.2 & 0.0 & -0.2 & -0.4 & -0.6\end{array}$

E [V vs. SCE]

Figure 50: Cyclic voltammograms of a) $\left[\mathrm{Fe}_{2}{ }_{2}\left(\mathrm{H}_{2} \mathrm{SIM}^{1}\right)(\mu-\mathrm{OAc})_{2}\right] \mathrm{PF}_{6}, \quad$ b) $\left[\mathrm{Fe}_{2}{ }_{2}\left(\mathrm{H}_{2} \mathrm{~L}^{2}\right)(\mu-\mathrm{OAc})_{2}\right] \mathrm{PF}_{6}, \quad$ c) $\left[\mathrm{Fe}_{2}{ }_{2}\left(\mathrm{H}_{2} \mathrm{SIM}^{1}\right)(\mu-\mathrm{OAc})_{2}\right] \mathrm{PF}_{6}$ after exposure to air and d) $\left[\mathrm{Fe}_{2}{ }_{2}\left(\mathrm{H}_{2} \mathrm{~L}^{2}\right)(\mu-\mathrm{OAc})_{2}\right] \mathrm{PF}_{6}$ after exposure to air, recorded in degassed acetonitrile ( $0.5 \mathrm{mM}$ complex, $0.1 \mathrm{M} \mathrm{NBu}_{4} \mathrm{ClO}_{4}$ ) at rt under Ar (glassy carbon working electrode, platinum wire counter electrode, $\mathrm{Ag} / \mathrm{Ag}^{+}(0.1 \mathrm{mM}$ in $\mathrm{NaCl}$ ) reference electrode).

Table 17: Cyclic voltammetric data of phenoxido-bridged diiron complexes in acetonitrile.

\begin{tabular}{|c|c|c|c|c|}
\hline complex & $\mathrm{E}_{1}[\mathrm{~V}$ vs. SCE $]$ & $\mathrm{E}_{2}[\mathrm{~V}$ vs. SCE] & $\Delta \mathrm{E}[\mathrm{V}]$ & $\mathrm{K}_{\mathrm{com}^{\mathrm{a}}}$ \\
\hline$\left[\mathrm{Fe}_{2}{ }_{2}\left(\mathrm{H}_{2} \mathrm{SIM}^{1}\right)(\mu-\mathrm{OAC})_{2}\right] \mathrm{PF}_{6}{ }^{\mathrm{b}}$ & 0.16 & 0.56 & 0.41 & $8.5 \cdot 10^{6}$ \\
\hline$\left[\mathrm{Fe}_{2}{ }_{2}\left(\mathrm{H}_{2} \mathrm{~L}^{2}\right)(\mu-\mathrm{OAc})_{2}\right] \mathrm{PF}_{6}{ }^{\mathrm{b}}$ & $0.18 / 0.34$ & $0.79 / 0.92$ & $\sim 0.60$ & $\sim 1.4 \cdot 10^{10}$ \\
\hline$\left[\mathrm{Fe}^{\prime \prime \prime} \mathrm{Fe}^{\prime \prime}\left(\mathrm{L}^{1}\right)(\mu-\mathrm{OAc})_{2}\right]\left(\mathrm{BF}_{4}\right)_{2}{ }^{\mathrm{c}} 231$ & -0.03 & 0.68 & 0.71 & $1.6 \cdot 10^{12}$ \\
\hline$\left[\mathrm{Fe}^{\prime \prime \prime} \mathrm{Fe}^{\prime \prime}\left(\mathrm{L}^{1}\right)(\mu-\mathrm{OPr})_{2}\right]\left(\mathrm{BPh}_{4}\right)_{2}$ d 139 & -0.01 & 0.69 & 0.70 & $7.5 \cdot 10^{11}$ \\
\hline$\left[\mathrm{Fe}^{\prime \prime \prime} \mathrm{Fe}^{\prime \prime}\left(\mathrm{L}^{1}\right)(\mu-\mathrm{OBz})_{2}\right]\left(\mathrm{PF}_{6}\right)_{2}$ d 139 & 0.06 & 0.75 & 0.69 & $4.7 \cdot 10^{11}$ \\
\hline$\left[\mathrm{Fe}^{\mathrm{III}} \mathrm{Fe}^{\prime \prime}\left(\mathrm{L}^{1}\right)(\mu-\mathrm{OBz})_{2}\right]\left(\mathrm{BF}_{4}\right)_{2}{ }^{\mathrm{c} 231}$ & 0.05 & 0.73 & 0.68 & $5.0 \cdot 10^{11}$ \\
\hline$\left[\mathrm{Fe}^{\prime \prime \prime} \mathrm{Fe}^{\prime \prime}\left(\mathrm{L}^{1}\right)(\mu-\mathrm{OBz})_{2}\right]\left(\mathrm{BF}_{4}\right)_{2}$ e 253 & -0.02 & 0.67 & 0.69 & $6.8 \cdot 10^{11}$ \\
\hline$\left[\mathrm{Fe}^{\prime \prime \prime} \mathrm{Fe}^{\prime \prime}\left(\mathrm{L}^{11}\right)(\mu-\mathrm{OAc})_{2}\right]\left(\mathrm{BPh}_{4}\right)_{2}{ }^{\mathrm{f}} 232$ & -0.09 & 0.66 & 0.75 & $3.3 \cdot 10^{12}$ \\
\hline
\end{tabular}

${ }^{\text {a }}$ see Equation 19.

${ }^{\mathrm{b}} 0.1 \mathrm{M} \mathrm{NBu}_{4} \mathrm{ClO}_{4}$, glassy carbon working electrode, platinum wire counter electrode, $\mathrm{Ag} / \mathrm{Ag}^{+}(0.1 \mathrm{mM}$ in $\mathrm{NaCl})$ reference electrode.

${ }^{c} \mathrm{Bu}_{4} \mathrm{NClO}_{4}$, glassy carbon working electrode, auxiliary electrode, saturated calomel reference electrode.

${ }^{d} 0.1 \mathrm{M} \mathrm{NBu}_{4} \mathrm{BF}_{4}$, platinum disk working electrode, platinum wire auxiliary electrode, saturated calomel reference electrode.

e $0.1 \mathrm{M} \mathrm{NBu}_{4} \mathrm{ClO}_{4}$, glassy carbon working electrode, platinum counter electrode, saturated $\mathrm{Ag} / \mathrm{AgCl}$ reference electrode.

${ }^{f} 0.1 \mathrm{M} \mathrm{NBu}_{4} \mathrm{ClO}_{4}$, platinum net working electrode, platinum wire auxiliary electrode, $\mathrm{Ag} / \mathrm{Ag}^{+}$or calomel working electrode.

The trend to more positive potentials by an increase of the number of pivaloyl-amide substituents was already observed with pivaloyl-amide substituted Co" and Cu" complexes. ${ }^{254,255}$ 
The hydrogen bond donors implemented in the complexes induce a shift to less negative values for $\mathrm{E}_{\mathrm{P}}$ (Chart 18). More precisely, the complex [Co"I $\left(\mathrm{L}^{21}\right)$ (acetohydroximate) $\mathrm{Cl}$ was found to have a Co"/Co"l' couple at $-0.99 \mathrm{~V}$ vs. SCE, while the 6-pivaloylamide substituted derivative showed this potential at $-0.72 \mathrm{~V}$ vs. SCE. It was proposed that the easier Co"/Co"l' reduction is due to the decreased charge of the hydroximate oxygen because of hydrogen bonding with the pivaloylamide residue. ${ }^{254}$ Similarly, the $\mathrm{Cu}^{\prime \prime}$ complexes of the tripodal ligand $\mathrm{L}^{21}$ show a reduction wave in the CV at more positive redox potentials with an increasing number of 6-pivaloyl-amide substitution of the pyridine moieties. ${ }^{255}$ Therefore, the two pivaloyl-amide residues in $\left[\mathrm{Fe}_{2}{ }_{2}\left(\mathrm{H}_{2} \mathrm{~L}^{2}\right)(\mu-\mathrm{OAC})_{2}\right] \mathrm{PF}_{6}$, one at each iron center, seem to influence the redox potentials of the $\mathrm{Fe}_{2}{ }_{2} / \mathrm{Fe}^{I I I} \mathrm{Fe}^{\prime \prime}$ and $\mathrm{Fe}^{\text {III }} \mathrm{Fe}^{\prime \prime} / \mathrm{Fe}^{\text {III}}{ }_{2}$ couples in the same way, probably due to hydrogen bond formation.

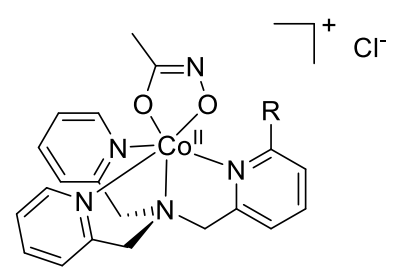

$\left[\mathrm{Co}^{\prime \prime}\left(\mathrm{L}^{21}\right)(\right.$ acetohydroximate $\left.)\right] \mathrm{Cl}$ :

$\mathrm{R}=\mathrm{H} ; \mathrm{E}_{\mathrm{P}}=-0.99 \mathrm{~V}$ vs. SCE

$\left[\mathrm{Co}^{\prime \prime}\left(\mathrm{HL}^{34}\right)(\right.$ acetohydroximate $\left.)\right] \mathrm{Cl}$ :

$\mathrm{R}=\mathrm{NHCO}{ }^{\mathrm{t}} \mathrm{Bu} ; \mathrm{E}_{\mathrm{P}}=-0.72 \mathrm{~V}$ vs. SCE

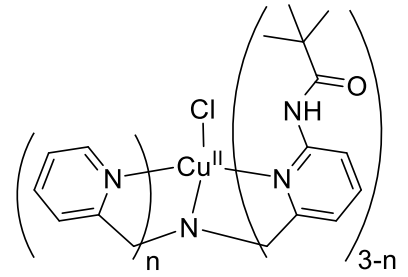

$\left[\mathrm{Cu}^{\prime \prime}\left(\mathrm{L}^{21}\right)\left(\mathrm{OH}_{2}\right)\right]^{+}: \quad \mathrm{n}=3 ; \mathrm{E}_{\mathrm{p}}=-0.72 \vee v s . \mathrm{Fc}^{+} / \mathrm{Fc}$ $\left[\mathrm{Cu}^{\prime \prime}\left(\mathrm{HL}^{34}\right)\left(\mathrm{OH}_{2}\right)\right]^{+}: \mathrm{n}=2 ; \mathrm{E}_{\mathrm{p}}=-0.65 \vee v s . \mathrm{Fc}^{+} / \mathrm{Fc}$ $\left[\mathrm{Cu}^{\prime \prime}\left(\mathrm{H}_{2} \mathrm{~L}^{49}\right)\left(\mathrm{OH}_{2}\right)\right]^{+}: \mathrm{n}=1 ; \mathrm{E}_{\mathrm{p}}=-0.48 \vee v s$. $\mathrm{Fc}^{+} / \mathrm{Fc}$ $\left[\mathrm{Cu}^{\mathrm{Il}}\left(\mathrm{H}_{3} \mathrm{~L}^{50}\right)\left(\mathrm{OH}_{2}\right)\right]^{+}: \mathrm{n}=0 ; \mathrm{E}_{\mathrm{p}}=-0.18 \vee v s$. $\mathrm{Fc}^{+} / \mathrm{Fc}$

Chart 18: Co" and Cu" complexes used to study the impact of pivaloyl-amide substituents in proximity to the metal center on the redox potential. ${ }^{254,255}$

Table 17 also compares the methyl-substituted complex $\left[\mathrm{Fe}^{\prime \prime \prime} \mathrm{Fe}^{\prime \prime}\left(\mathrm{L}^{11}\right)(\mu-\mathrm{OAC})_{2}\right]\left(\mathrm{BPh}_{4}\right)_{2}{ }^{232}$ with the heterovalent diiron complexes of $\mathrm{HL}^{1}$. The more negative potentials of the redox couples for $\left[\mathrm{Fe}^{\mathrm{III}} \mathrm{Fe}^{\prime \prime}\left(\mathrm{L}^{11}\right)(\mathrm{OAC})_{2}\right]\left(\mathrm{BPh}_{4}\right)_{2}{ }^{232}$ compared to $\left[\mathrm{Fe}^{\prime \prime \prime} \mathrm{Fe}^{\prime \prime}\left(\mathrm{L}^{1}\right)(\mu-\mathrm{OAC})_{2}\right]\left(\mathrm{BPh}_{4}\right)_{2},{ }^{231}$ was explained by the effect of the electron-donating ability of methyl substituents of the 6-methylpyridine rings. ${ }^{231,232}$ Therefore, the cathodic shift of the redox potentials of $\left[\mathrm{Fe}_{2}{ }_{2}\left(\mathrm{H}_{2} \mathrm{SIM}^{1}\right)(\mu-\mathrm{OAc})_{2}\right] \mathrm{PF}_{6}$ and $\left[\mathrm{Fe}_{2}{ }_{2}\left(\mathrm{H}_{2} \mathrm{~L}^{2}\right)(\mu-\mathrm{OAC})_{2}\right] \mathrm{PF}_{6}$ compared to $\left[\mathrm{Fe}^{\mathrm{III}} \mathrm{Fe}^{\prime \prime}\left(\mathrm{L}^{1}\right)(\mu-\mathrm{OAC})_{2}\right]\left(\mathrm{BF}_{4}\right)_{2}$ may result from the electron withdrawing effect of the pivaloyl-amide residues.

The contrasting effect of lowering the redox potential by about $500 \mathrm{mV}$ by incorporation of pivaloyl-amide residues into a diiron(II) complex was reported by Arii et al. and proposed to be caused by an increase in the energy level of the HOMO of the iron centers due to coordination of pivaloyl-amide oxygen atoms. ${ }^{256}$ Therefore, the lower redox potential of the $\mathrm{Fe}_{2}{ }_{2} / \mathrm{Fe}{ }^{\text {III }} \mathrm{Fe}{ }^{\prime \prime}$ couple of $\left[\mathrm{Fe}_{2}{ }_{2}\left(\mathrm{H}_{2} \mathrm{SIM}^{1}\right)(\mu-\mathrm{OAC})_{2}\right] \mathrm{PF}_{6}$ by $0.12 \mathrm{~V}$ compared to $\left[\mathrm{Fe}^{\prime \prime \prime} \mathrm{Fe}^{\prime \prime}\left(\mathrm{L}^{1}\right)(\mu-\mathrm{OAC})_{2}\right]\left(\mathrm{BF}_{4}\right)_{2}$ is probably induced by the two pivaloyl-amide residues adjacent to one of the two iron centers. Moreover, a diiron(II) complex of a $\mathrm{HL}^{1}$ derivative with pivaloyl-amide residues at each pyridine moiety 
$\left(\mathrm{H}_{5} \mathrm{~L}^{39}\right)$ resulted in a CV with a strong wave at $0.39 \mathrm{~V}$ vs. SCE and shoulders at $-0.20 \mathrm{~V}$ and $-0.36 \mathrm{~V}$ vs. SCE. ${ }^{234}$

The formation of an Fe ${ }^{I I I} \mathrm{Fe}$ "l complex is important to obtain a more accurate model for the active form of PAP. The thermodynamic stability of the Fe ${ }^{\text {III }} \mathrm{Fe}$ "l complex of the asymmetric ligand $\mathrm{H}_{3} \mathrm{SIM}^{1}$ was estimated by examining the following comproportionation equilibrium.

$$
\left[\mathrm{Fe}_{2}{ }_{2}\left(\mathrm{H}_{2} \mathrm{SIM}^{1}\right)(\mathrm{OAc})_{2}\right]^{+}+\left[\mathrm{Fe}_{2}{ }_{2}\left(\mathrm{H}_{2} \mathrm{SIM}^{1}\right)(\mathrm{OAc})_{2}\right]^{3+} \stackrel{\mathrm{K}_{\mathrm{com}}}{\rightleftharpoons}\left[\mathrm{Fe}^{\prime \prime \prime \prime} \mathrm{Fe}^{\prime \prime}\left(\mathrm{H}_{2} \mathrm{SIM}^{1}\right)(\mathrm{OAc})_{2}\right]^{2+}
$$

From the separation of the redox potentials $\Delta \mathrm{E}$, the comproportionation constant $\mathrm{K}_{\text {com }}$ can be calculated from Equation 19 and is listed in combination with the values reported for pivaloylamide-free diiron complexes in Table 7. ${ }^{139,} 231,232,253$

$$
\text { Equation } 19 \quad \Delta \mathrm{E}=\left(\frac{\mathrm{RT}}{\mathrm{nF}}\right) \ln \mathrm{K}_{\text {com }}
$$

The small value of $\Delta \mathrm{E}$ for $\left[\mathrm{Fe}_{2}{ }_{2}\left(\mathrm{H}_{2} \mathrm{SIM}^{1}\right)(\mu-\mathrm{OAc})_{2}\right] \mathrm{PF}_{6}(0.41 \mathrm{~V})$ yielded $\mathrm{K}_{\text {com }}=8.5 \cdot 10^{6}$, which indicates a substantial stability of the $\mathrm{Fe}^{\mathrm{III}} \mathrm{Fe}^{\mathrm{Il}}$ complex over the corresponding $\mathrm{Fe}_{2}{ }_{2}$ and $\mathrm{Fe}^{\mathrm{II}}{ }_{2}$ complexes. However, the value is rather small compared to those of other phenoxido-bridged diiron complexes (Table 17). However, the corresponding heterovalent diiron complex of ligand $\mathrm{H}_{3} \mathrm{SIM}^{1}$ would take the asymmetry and the hydrogen bonding network present in the active site of the native PAP enzyme into consideration. Therefore, a suitable method for oxidation of $\left[\mathrm{Fe}_{2}{ }_{2}\left(\mathrm{H}_{2} \mathrm{SIM}^{1}\right)(\mu-\mathrm{OAc})_{2}\right] \mathrm{PF}_{6}$ was explored and will be discussed in Chapter 5.4.

The green solutions of $\left[\mathrm{Fe}_{2}{ }_{2}\left(\mathrm{H}_{2} \mathrm{SIM}^{1}\right)(\mu-\mathrm{OAc})_{2}\right] \mathrm{PF}_{6}$ and $\left[\mathrm{Fe}{ }_{2}\left(\mathrm{H}_{2} \mathrm{~L}^{2}\right)(\mu-\mathrm{OAc})_{2}\right] \mathrm{PF}_{6}$ in acetonitrile were exposed to air, subsequently degassed and CVs recorded. These data are also shown in Figure 50. The purple solution derived from $\left[\mathrm{Fe}_{2}{ }_{2}\left(\mathrm{H}_{2} \mathrm{SIM}^{1}\right)(\mu-\mathrm{OAc})_{2}\right] \mathrm{PF}_{6}$ resulted in a $\mathrm{CV}$ with an irreversible redox wave around $0.5 \mathrm{~V} v$ s. SCE and two quasi-reversible redox waves at $0.02 \mathrm{~V}$ and $-0.16 \mathrm{~V} v s$. SCE. The corresponding CV derived from $\left[\mathrm{Fe}_{2}{ }_{2}\left(\mathrm{H}_{2} \mathrm{~L}^{2}\right)(\mu-\mathrm{OAC})_{2}\right] \mathrm{PF}_{6}$ has two irreversible redox couples at $0.54 \mathrm{~V}$ and $0.04 \mathrm{~V} v$ s. SCE. The irreversibility of the redox processes indicates structural changes associated with the oxidation. Moreover, consecutive CV scans were not identical, and this is attributed to (partial) decomposition after oxidation.

\section{UV-vis-NIR Spectroscopy}

\section{Electronic Properties under Anaerobic Conditions}

In addition to the intense absorptions below $300 \mathrm{~nm}$ ( $\pi-\pi^{*}$ transitions of the ligand backbone), the electronic spectra of $\left[\mathrm{Fe}_{2}{ }_{2}\left(\mathrm{H}_{2} \mathrm{SIM}^{1}\right)(\mu-\mathrm{OAC})_{2}\right]^{+}$and $\left[\mathrm{Fe}_{2}{ }_{2}\left(\mathrm{H}_{2} \mathrm{~L}^{2}\right)(\mu-\mathrm{OAC})_{2}\right]^{+}$dissolved in acetonitrile show only one band in the region of $400-450 \mathrm{~nm}$. $\left[\mathrm{Fe}_{2}{ }_{2}\left(\mathrm{H}_{2} \mathrm{SIM}^{1}\right)(\mu-\mathrm{OAc})_{2}\right]^{+}$has a transition at $416 \mathrm{~nm}\left(\varepsilon=1446 \mathrm{M}^{-1} \mathrm{~cm}^{-1}\right)$ and $\left[\mathrm{Fe}_{2}{ }_{2}\left(\mathrm{H}_{2} \mathrm{~L}^{2}\right)(\mu-\mathrm{OAc})_{2}\right]^{+}$has a band at $409 \mathrm{~nm}$ $\left(\varepsilon=1315 \mathrm{M}^{-1} \mathrm{~cm}^{-1}\right)$, ascribed to MLCT transitions of $\mathrm{Fe}^{\| l}$ to the pyridine donors. This is in 
accordance with previously published $\mathrm{Fe}_{2} \mathrm{I}_{2}$ complexes. ${ }^{234,} 235,257,258$ The lack of low-energy absorption bands indicates that $\left[\mathrm{Fe}_{2}{ }_{2}\left(\mathrm{H}_{2} \mathrm{SIM}^{1}\right)(\mu-\mathrm{OAc})_{2}\right]^{+}$and $\left[\mathrm{Fe}_{2}{ }_{2}\left(\mathrm{H}_{2} \mathrm{~L}^{2}\right)(\mu-\mathrm{OAc})_{2}\right]^{+}$are high-spin Fe" complexes. ${ }^{258}$

\section{Electronic Properties under Aerobic Conditions}

The UV-vis-NIR spectrum of $\left[\mathrm{Fe}^{\prime \prime \prime \prime} \mathrm{Na}\left(\mathrm{H}_{2} \mathrm{SIM}^{1}\right)(\mu-\mathrm{OAc})_{2}\right] \mathrm{PF}_{6}$ in acetonitrile shows one broad transition at $568 \mathrm{~nm}\left(\varepsilon=2499 \mathrm{M}^{-1} \mathrm{~cm}^{-1}\right)$ and a shoulder at $335 \mathrm{~nm}\left(\varepsilon=5049 \mathrm{M}^{-1} \mathrm{~cm}^{-1}\right)$, in addition to the intense absorptions below $300 \mathrm{~nm}$. The band at $568 \mathrm{~nm}$ is associated with the phenolateto-iron(III) charge transfer transition from the oxygen $\mathrm{p}_{\pi}$ orbital to the $\mathrm{Fe}^{\text {III }} \mathrm{d}_{\pi^{*}}$ orbitals and is energetically close to the tyrosinate-to-iron(III) charge transfer observed for PAPs. ${ }^{69,} 138,217,219$,

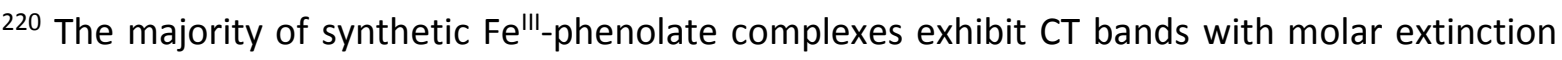
coefficients in the order of $1000-2000 \mathrm{M}^{-1} \mathrm{~cm}^{-1}$ per Fe'll-phenolate bond. ${ }^{259,} 260$ The molar extinction coefficient of $2499 \mathrm{M}^{-1} \mathrm{~cm}^{-1}$ for $\left[\mathrm{Fe}{ }^{I ! \prime} \mathrm{Na}\left(\mathrm{H}_{2} \mathrm{SIM}^{1}\right)(\mu-\mathrm{OAc})_{2}\right] \mathrm{PF}_{6}$ is slightly outside this

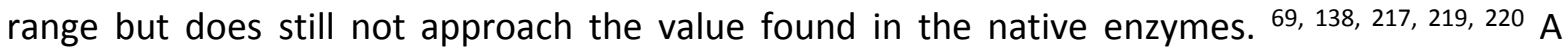
comparison of a variety of similar doubly acetate-bridged Fe ${ }^{\prime \prime \prime} \mathrm{M}^{\prime \prime}$ complexes of $\mathrm{H}_{2} \mathrm{~L}^{4}$ reveals that the molar extinction coefficient strongly depends on the nature of the $M^{\prime \prime}$ ion, i.e. $\left[\mathrm{Fe}^{\prime \prime \prime} \mathrm{Mn}^{\prime \prime}\left(\mathrm{L}^{4}\right)(\mu-\mathrm{OAC})_{2}\right] \mathrm{ClO}_{4} \quad\left(544 \mathrm{~nm}, 2680 \mathrm{M}^{-1} \mathrm{~cm}^{-1}\right),{ }^{96} \quad\left[\mathrm{Fe}^{\prime \prime \prime} \mathrm{Cu}^{\prime \prime}\left(\mathrm{L}^{4}\right)(\mu-\mathrm{OAc})_{2}\right] \mathrm{ClO}_{4} \quad(546 \mathrm{~nm}$, $\left.3400 \mathrm{M}^{-1} \mathrm{~cm}^{-1}\right)^{, 92}\left[\mathrm{Fe}^{\prime \prime \prime \prime} \mathrm{Zn} "\left(\mathrm{~L}^{4}\right)(\mu-\mathrm{OAc})_{2}\right] \mathrm{ClO}_{4}\left(540 \mathrm{~nm}, 3700 \mathrm{M}^{-1} \mathrm{~cm}^{-1}\right),{ }^{95}\left[\mathrm{Fe}^{\prime \prime \prime \prime} \mathrm{Fe}^{\prime \prime}\left(\mathrm{L}^{4}\right)(\mu-\mathrm{OAc})_{2}\right] \mathrm{BF}_{4}$ $\left(556 \mathrm{~nm}, 4560 \mathrm{M}^{-1} \mathrm{~cm}^{-1}\right),{ }^{94}$ and $\left[\mathrm{Fe}^{\prime \prime \prime} \mathrm{Ni}^{\prime \prime}\left(\mathrm{L}^{4}\right)(\mu-\mathrm{OAC})_{2}\right] \mathrm{ClO}_{4}\left(538 \mathrm{~nm}, 4813 \mathrm{M}^{-1} \mathrm{~cm}^{-1}\right) \cdot{ }^{81,141}$

\section{pH Behavior}

In order to examine the behavior of $\left[\mathrm{Fe}_{2}{ }_{2}\left(\mathrm{H}_{2} \mathrm{SIM}^{1}\right)(\mu-\mathrm{OAc})_{2}\right]^{+}$and $\left[\mathrm{Fe}_{2}{ }_{2}\left(\mathrm{H}_{2} \mathrm{~L}^{2}\right)(\mu-\mathrm{OAc})_{2}\right]^{+}$under basic condition UV-vis-NIR spectroscopic measurement were also conducted in acetonitrileaqueous buffer mixtures (1:1). The $\mathrm{pH}$ of the aqueous buffer was varied from $\mathrm{pH} 6$ to $\mathrm{pH} 11$; the $\mathrm{pH}$ recorded refers to the aqueous component. With increasing basicity the absorbance of $\left[\mathrm{Fe}_{2}{ }_{2}\left(\mathrm{H}_{2} \mathrm{~L}^{2}\right)(\mu-\mathrm{OAC})_{2}\right]^{+}$(Figure 51c) shows a decrease in intensity of the two bands at $235 \mathrm{~nm}$ and $286 \mathrm{~nm}$, while two new bands at $261 \mathrm{~nm}$ and $314 \mathrm{~nm}$ arise. Moreover, the band at $414 \mathrm{~nm}$ experiences a shift to $440 \mathrm{~nm}$ with increasing basicity. Figure $51 \mathrm{~d}$ shows the change of absorbance at different wavelengths as a function of $\mathrm{pH}$ value. The overlay of the spectra at different $\mathrm{pH}$ values indicates the presence of three isosbestic points at $245 \mathrm{~nm}, 274 \mathrm{~nm}$, and $395 \mathrm{~nm}$. This implies that the diiron(II) complex is stable in the investigated $\mathrm{pH}$ range and undergoes a single deprotonation step. Therefore, the curves can be analyzed by the Equation 6, as discussed in Chapter 4.3. 

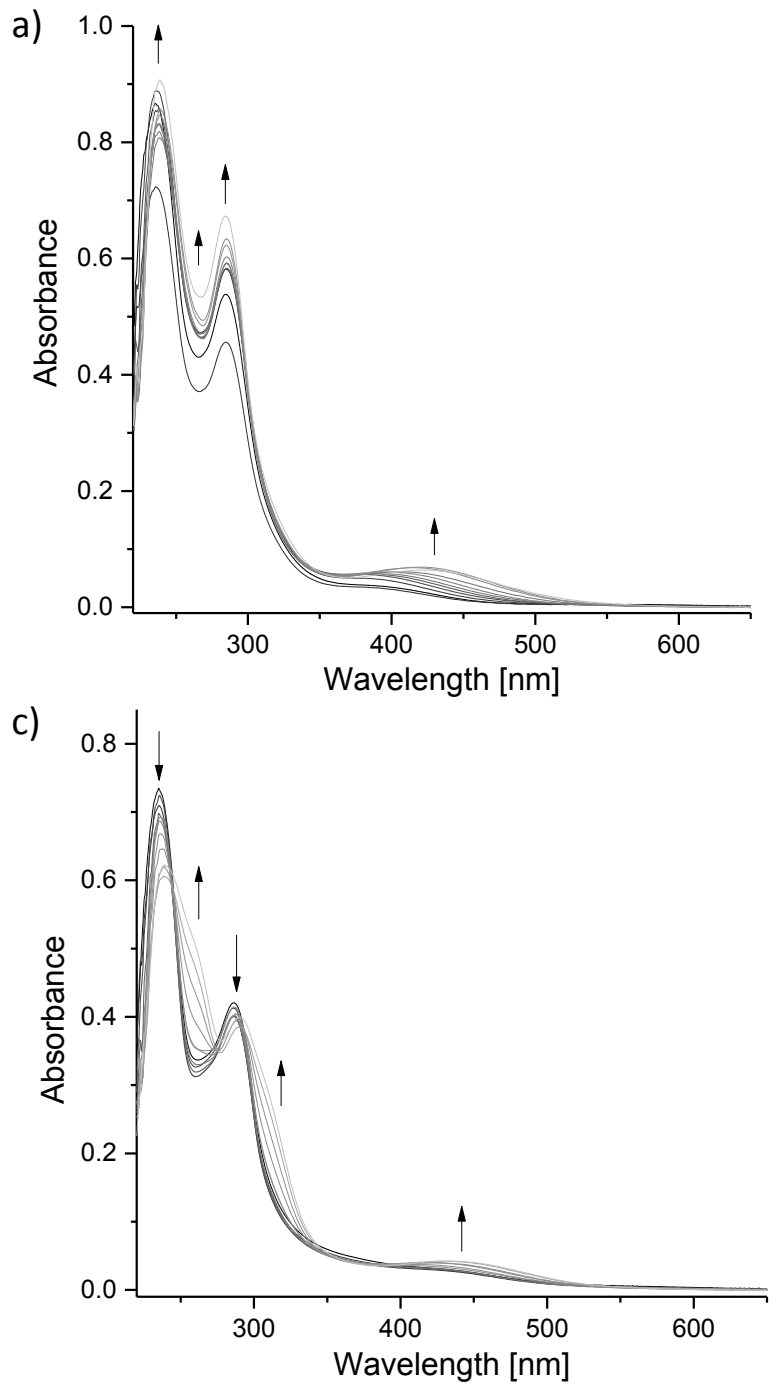
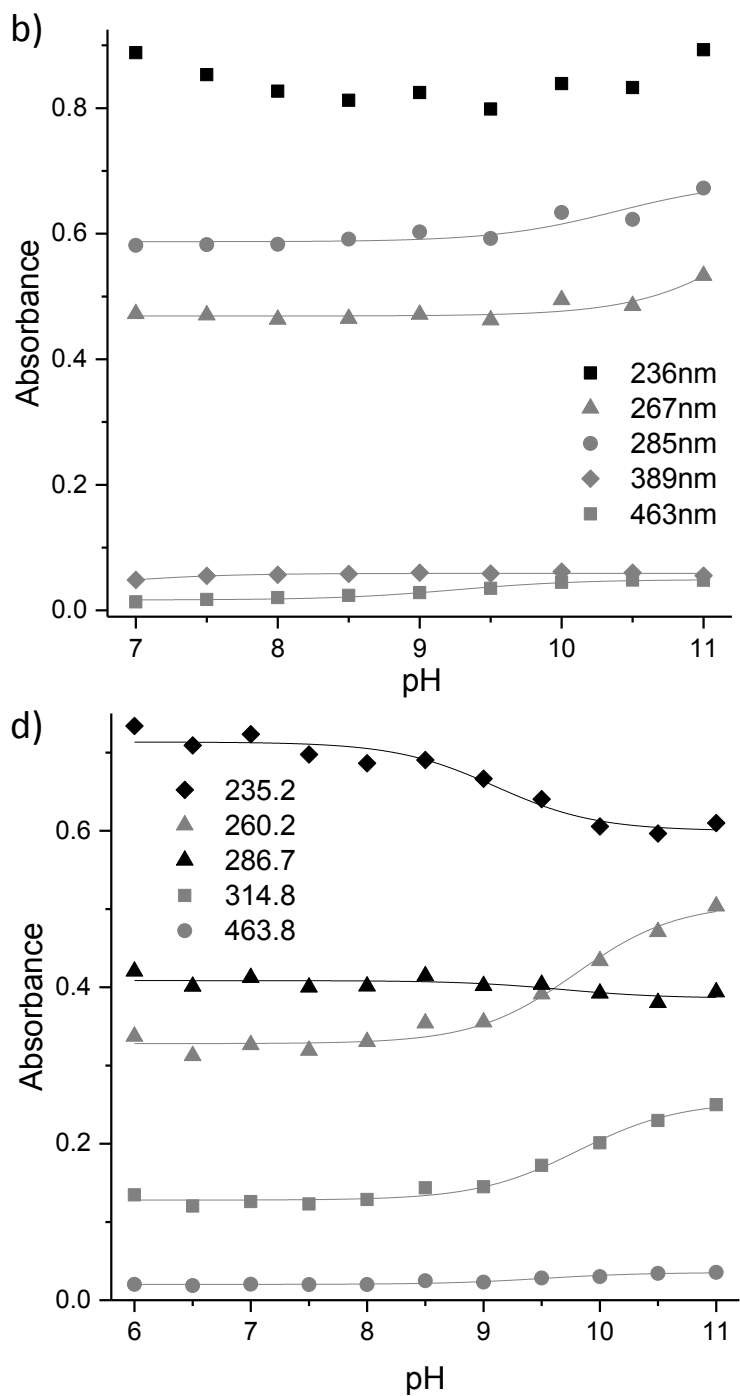

Figure 51: UV-vis spectroscopic titration (50 $\mu \mathrm{M}$ in acetonitrile/buffer between $\mathrm{pH} 6$ and 11) for a) $\left[\mathrm{Fe}_{2}{ }_{2}\left(\mathrm{H}_{2} \mathrm{SIM}^{1}\right)(\mu-\mathrm{OAc})_{2}\right] \mathrm{PF}_{6}$ and c) $\left[\mathrm{Fe}_{2}{ }_{2}\left(\mathrm{H}_{2} \mathrm{~L}^{2}\right)(\mu-\mathrm{OAc})_{2}\right] \mathrm{PF}_{6}$ as well as absorbance vs. pH plots for b) $\left[\mathrm{Fe}_{2}{ }_{2}\left(\mathrm{H}_{2} \mathrm{SIM}^{1}\right)(\mu-\mathrm{OAc})_{2}\right] \mathrm{PF}_{6}$ and d) $\left[\mathrm{Fe}_{2}{ }_{2}\left(\mathrm{H}_{2} \mathrm{~L}^{2}\right)(\mu-\mathrm{OAc})_{2}\right] \mathrm{PF}_{6}$.

Fitting of the $\mathrm{pH}$-dependent spectroscopic data as described Chapter 4.3, leads to the $\mathrm{pK}_{\mathrm{a}}$ value of $9.58 \pm 0.65$ for $\left[\mathrm{Fe}_{2}{ }_{2}\left(\mathrm{H}_{2} \mathrm{~L}^{2}\right)(\mu-\mathrm{OAc})_{2}\right]^{+}$. This is about $1.5 \mathrm{pH}$ units higher than that observed with the corresponding dizinc(II) complex $\left[\mathrm{Zn}_{2}\left(\mathrm{H}_{2} \mathrm{~L}^{2}\right)(\mu-\mathrm{OAc})_{2}\right]^{+}$and follows the same trend as the corresponding metal aqua complexes. ${ }^{165}$

The $\mathrm{pH}$-dependent UV-vis-NIR spectra of $\left[\mathrm{Fe}_{2}{ }_{2}\left(\mathrm{H}_{2} \mathrm{SIM}^{1}\right)(\mu-\mathrm{OAc})_{2}\right]^{+}$show a similar shifting of the band at $399 \mathrm{~nm}$ to $426 \mathrm{~nm}$ (Figure 51a). In contrast to $\left[\mathrm{Fe}_{2}{ }_{2}\left(\mathrm{H}_{2} \mathrm{~L}^{2}\right)(\mu-\mathrm{OAc})_{2}\right]^{+}$, both bands between $200 \mathrm{~nm}$ and $300 \mathrm{~nm}$ in the spectrum of $\left[\mathrm{Fe}_{2}{ }_{2}\left(\mathrm{H}_{2} \mathrm{SIM}^{1}\right)(\mu-\mathrm{OAc})_{2}\right]^{+}$increase in intensity with increasing basicity. The absorbance vs. pH plot (Figure 51b) exhibits changes in the pH regions around 7 and around 10.5, indicating two deprotonation steps with $\mathrm{pK}_{\mathrm{a}}$ values around 7 and 10.5 . 


\subsection{StUdies TOWARD THE FORMATION OF A Heterovalent Fe IIIFE" COMPLEX}

As explained before the presence of a heterovalent diiron core and the hydrogen bonding network that arises from the peptide backbone are essential features of the active site of PAPs. Therefore, the generation of a Fe ${ }^{I I I} \mathrm{Fe}^{\text {II }}$ complex of $\mathrm{H}_{3} \mathrm{SIM}^{1}$ creates an accurate model system for PAP. In order to test the generation of $\left[\mathrm{Fe}^{I I I} \mathrm{Fe}^{\prime \prime}\left(\mathrm{H}_{2} \mathrm{SIM}^{1}\right)(\text { solv })_{\mathrm{x}}\right]^{4+}$, the chemical oxidation was analyzed by UV-vis, EPR, and ${ }^{57} \mathrm{Fe}$ Mössbauer spectroscopy.

\section{UV-vis-NIR Spectroscopy}

Due to the fact that the two redox waves in the CV of $\left[\mathrm{Fe}_{2}{ }_{2}\left(\mathrm{H}_{2} \mathrm{SIM}^{1}\right)(\mu-\mathrm{OAC})_{2}\right]^{+}$are well separated and that the redox potential of ferrocene is in between the two waves, the chemical oxidation of $\left[\mathrm{Fe}_{2}{ }_{2}\left(\mathrm{H}_{2} \mathrm{SIM}^{1}\right)(\mu-\mathrm{OAc})_{2}\right]^{+}$was tested using ferrocenium hexafluorophosphate as oxidant. The solution of $\left[\mathrm{Fe}_{2}{ }_{2}\left(\mathrm{H}_{2} \mathrm{SIM}^{1}\right)(\mu-\mathrm{OAc})_{2}\right]^{+}$in acetonitrile was therefore treated stepwise with a solution of ferrocenium hexafluorophosphate under anaerobic conditions. After addition of one and after the two equivalents of oxidant the mixture was allowed to react for 12 hours.

It is apparent that the oxidant reacts with the diiron(II) complex in a ratio of $\left[\mathrm{Fe}_{2}{ }_{2}\left(\mathrm{H}_{2} \mathrm{SIM}^{1}\right)(\mu-\mathrm{OAC})_{2}\right]^{+}$to ferrocenium of 1:1. UV-vis bands arise at $362 \mathrm{~nm}\left(\varepsilon=6357 \mathrm{M}^{-1} \mathrm{~cm}^{-1}\right)$ and $500 \mathrm{~nm}\left(\varepsilon=1271 \mathrm{M}^{-1} \mathrm{~cm}^{-1}\right)$, while the bands at $283 \mathrm{~nm}$ and $654 \mathrm{~nm}$ decrease (Figure $52 \mathrm{c}$ ). Addition of a second equivalent of ferrocenium hexafluorophosphate does not significantly change the UV-vis spectrum ( $362 \mathrm{~nm}, \varepsilon=7460 \mathrm{M}^{-1} \mathrm{~cm}^{-1}$ and $500 \mathrm{~nm}, \varepsilon=1471 \mathrm{M}^{-1} \mathrm{~cm}^{-1}$ ), but a shoulder at $619 \mathrm{~nm}$ appears. This transition at $619 \mathrm{~nm}$ arises from unreacted ferrocenium hexafluorophosphate and indicates the incapacity of the oxidant to oxidize the second Fe" ion in the initial $\left[\mathrm{Fe}_{2}{ }_{2}\left(\mathrm{H}_{2} \mathrm{SIM}^{1}\right)(\mu-\mathrm{OAc})_{2}\right]^{+}$complex. This indicates a selective formation of the heterovalent complex $\left[\mathrm{Fe}^{\text {IIII }} \mathrm{Fe}^{\prime \prime}\left(\mathrm{H}_{2} \mathrm{SIM}^{1}\right)(\text { solv })_{\mathrm{x}}\right]^{4+}$. 
a)

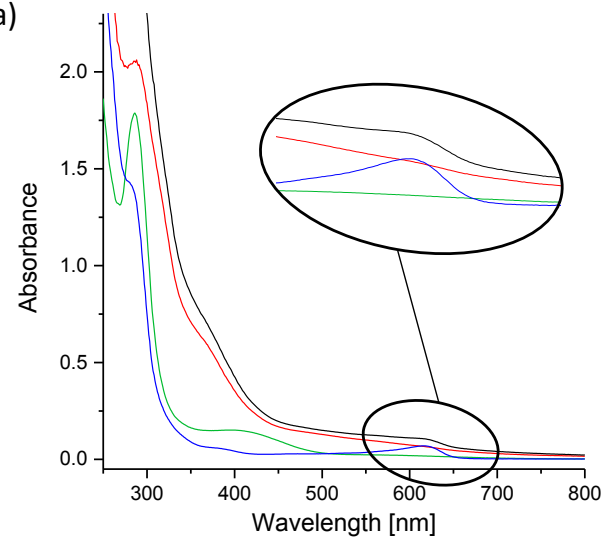

c)

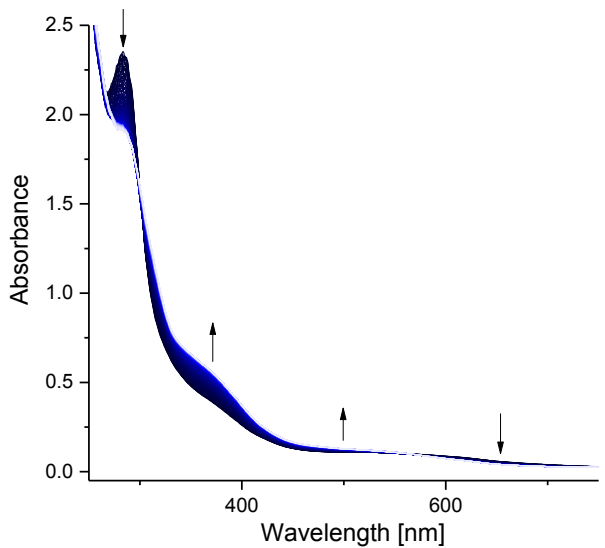

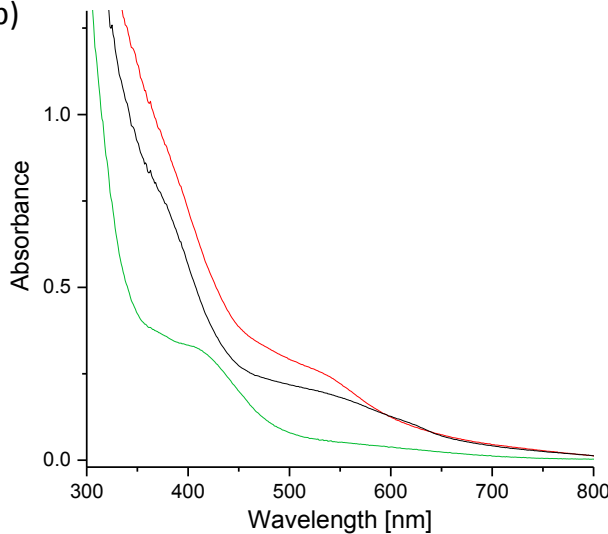

d)

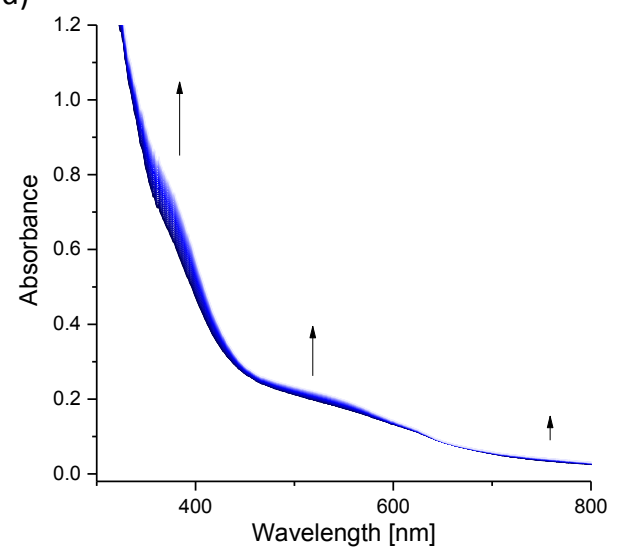

Figure 52: UV-vis-NIR spectra after addition of ferrocenium hexafluorophosphate to a) $\left[\mathrm{Fe}_{2}\left(\mathrm{H}_{2} \mathrm{SIM}^{1}\right)(\mu-\mathrm{OAc})_{2}\right] \mathrm{PF}_{6}$ and b) $\left[\mathrm{Fe}_{2}{ }_{2}\left(\mathrm{H}_{2} \mathrm{~L}^{2}\right)(\mu-\mathrm{OAc})_{2}\right] \mathrm{PF}_{6}$ (green lines: without $\mathrm{FeCp}_{2} \mathrm{PF}_{6}$; red lines: 1 eq $\mathrm{FeCp}_{2} \mathrm{PF}_{6}$; black lines: 2 eq $\mathrm{FeCp}_{2} \mathrm{PF}_{6}$; blue line: $\left.\mathrm{FeCp}_{2} \mathrm{PF}_{6}\right)$ and time-dependent experiments of $\mathrm{c}$ $\left[\mathrm{Fe}_{2}{ }_{2}\left(\mathrm{H}_{2} \mathrm{SIM}^{1}\right)(\mu-\mathrm{OAc})_{2}\right] \mathrm{PF}_{6}$ with 1 eq FeCp $2 \mathrm{PF}_{6}$ and d) $\left[\mathrm{Fe}_{2}{ }_{2}\left(\mathrm{H}_{2} \mathrm{~L}^{2}\right)(\mu-\mathrm{OAc})_{2}\right] \mathrm{PF}_{6}$ with 1 eq FeCp $\mathrm{PF}_{6}$ (a spectrum every $20 \mathrm{~min}$ for a period of $12 \mathrm{~h}$ ).

For the purpose of comparison the oxidation of the symmetric complex $\left[\mathrm{Fe}_{2}{ }_{2}\left(\mathrm{H}_{2} \mathrm{~L}^{2}\right)(\mu-\mathrm{OAc})_{2}\right] \mathrm{PF}_{6}$ with ferrocenium hexafluorophosphate was also examined. The reaction with one equivalent of oxidant lead to transitions at $373 \mathrm{~nm}\left(\varepsilon=7688 \mathrm{M}^{-1} \mathrm{~cm}^{-1}\right)$ and $515 \mathrm{~nm}\left(\varepsilon=2114 \mathrm{M}^{-1} \mathrm{~cm}^{-1}\right)$. In contrast to the experiments with $\left[\mathrm{Fe}_{2}{ }_{2}\left(\mathrm{H}_{2} \mathrm{SIM}^{1}\right)(\mu-\mathrm{OAc})_{2}\right]^{+}$, addition of a second equivalent of ferrocenium hexafluorophosphate lead to further changes in the spectrum, i.e. a shift of the band at $515 \mathrm{~nm}$ to $540 \mathrm{~nm}\left(\varepsilon=2415 \mathrm{M}^{-1} \mathrm{~cm}^{-1}\right)$ (Figure $52 \mathrm{~b}$ and d). Thus, the oxidant seems to be capable to oxidize both $\mathrm{Fe}^{\|}$centers in $\left[\mathrm{Fe}_{2}{ }_{2}\left(\mathrm{H}_{2} \mathrm{~L}^{2}\right)(\mu-\mathrm{OAc})_{2}\right] \mathrm{PF}_{6}$. However, it does not emerge whether the two Fe"l centers are oxidized stepwise or simultaneously. However, the steady rise of the absorbance band at $515 \mathrm{~nm}$ during the stepwise addition of up to one equivalent of ferrocenium hexafluorophosphate and the following shift with further addition of oxidant as well as the $\mathrm{K}_{\text {com }}$ value of $\sim 1.4 \cdot 10^{10}$ (see Table 17 ) indicate a substantial stability of the heterovalent complex $\left[\mathrm{Fe}^{\prime \prime \prime \prime} \mathrm{Fe}^{\prime \prime}\left(\mathrm{H}_{2} \mathrm{~L}^{2}\right)(\text { solv })_{\mathrm{x}}\right]^{4+}$. 


\section{EPR Spectroscopy}

EPR spectroscopy has been shown to be a useful technique for the characterization of the Fe ${ }^{I I I} \mathrm{Fe}$ core in heterovalent diiron model complexes and proteins. ${ }^{261}$ At low temperature Fe ${ }^{\text {III }} \mathrm{Fe}{ }^{\prime \prime}$ sites exhibit a broad EPR signal at $\mathrm{g}_{\mathrm{av}}<2$, indicative for antiferromagnetically coupled high-spin Fe ${ }^{\mathrm{II}}$ $\left(S_{1}=5 / 2,{ }^{6} \mathrm{~A}\right.$ state $)$ and high-spin Fe" $\left(S_{2}=2,{ }^{5} \mathrm{~T}\right.$ state), resulting in a spin ground state of $S_{\text {total }}=1 / 2$. These signals are only observed near liquid helium temperature and disappear above $30 \mathrm{~K}^{262}$ Such a behavior is very similar to that of the heterovalent diiron proteins. ${ }^{262}$

EPR spectra in frozen acetonitrile solution with one or two equivalents of oxidant added were recorded at temperatures below $5 \mathrm{~K}$ to investigate the oxidation state of $\left[\mathrm{Fe}_{2}{ }_{2}\left(\mathrm{H}_{2} \mathrm{SIM}^{1}\right)(\mu-\mathrm{OAc})_{2}\right]^{+}$ after reaction with one and two equivalents of ferrocenium hexafluorophosphate for 12 hours at room temperature. The resulting spectra are presented in Figure 53. Both spectra show a broad intense signal centered near $\mathrm{g}=1.6\left(\mathrm{~g}_{1}=1.43, \mathrm{~g}_{2}=1.67, \mathrm{~g}_{3}=1.82\right.$, derived from fitting the spectrum assuming as an $\mathrm{S}=1 / 2 \mathrm{Fe} \mathrm{e}^{\mathrm{III}}$ core), in addition to a strong signal near $\mathrm{g}=4.3$. These spectra are similar to that obtained from the electrochemically generated heterovalent complex $\left[\mathrm{Fe}^{\text {IIII } \mathrm{Fe}}{ }^{\prime \prime}\left(\mathrm{H}_{2} \mathrm{~L}^{2}\right)(\mu-\mathrm{OH})\right]^{3+} .114$

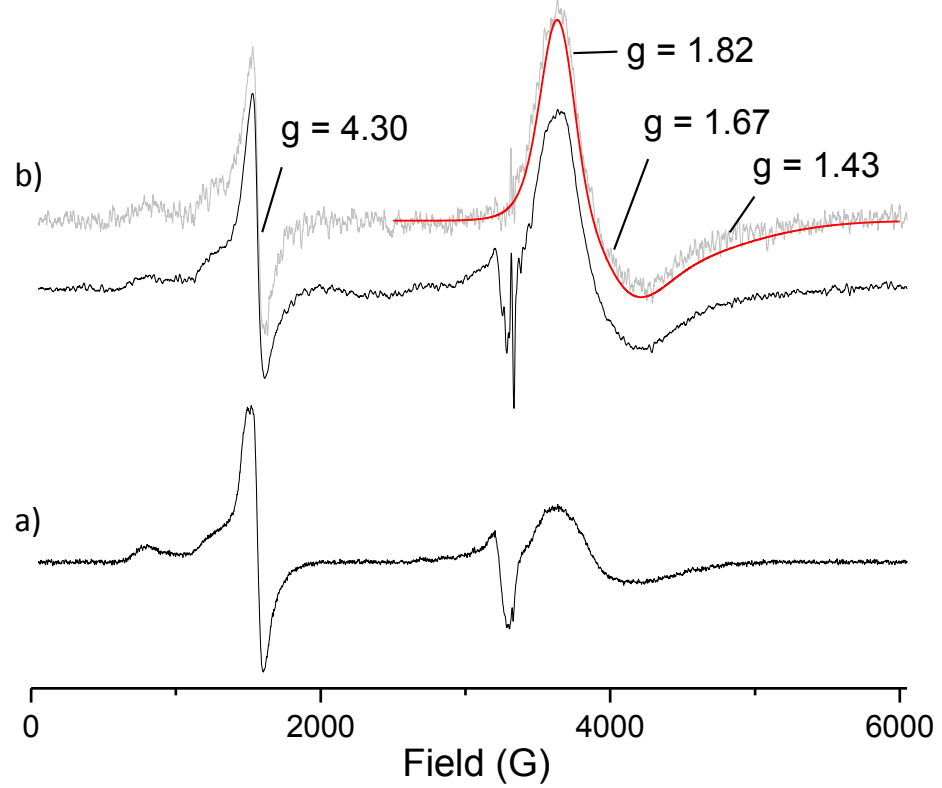

Figure 53: EPR spectra $(9.4 \mathrm{GHz})$ of mixtures of $\left[\mathrm{Fe}_{2}{ }_{2}\left(\mathrm{H}_{2} \mathrm{SIM}^{1}\right)(\mu-\mathrm{OAc})_{2}\right]^{+}$with a) 1 eq $\mathrm{FeCp}_{2} \mathrm{PF}_{6}(\mathrm{~T}=5 \mathrm{~K})$ and b) 2 eq of $\mathrm{FeCp}_{2} \mathrm{PF}_{6}(\mathrm{~T}=1.7 \mathrm{~K}, 1 \mathrm{mM}$ in acetonitrile-toluene solution (1:1), experimental spectra in black, spectrum after subtraction of the copper impurity in grey, simulated spectrum in red. The signals at $\mathrm{g}=2.04$ is an artefact due to a copper(II) impurity in the cavity of the spectrometer).

The signal at $\mathrm{g} \sim 1.6$ is attributed to a strongly antiferromagnetically coupled Fe ${ }^{\text {III }} \mathrm{Fe}{ }^{\prime \prime}$ core. Similar broad signals in this region have previously been reported for $\left[\mathrm{Fe}^{\prime \prime \prime} \mathrm{Fe}^{\prime \prime}\left(\mathrm{L}^{1}\right)(\mu-\mathrm{OPr})_{2}\right]^{+}$, $\left[\mathrm{Fe}^{\prime \prime \prime} \mathrm{Fe}^{\prime \prime}\left(\mathrm{L}^{11}\right)(\mu-\mathrm{OAC})_{2}\right]^{2+}, \quad\left[\mathrm{Fe}^{\prime \prime \prime} \mathrm{Fe} e^{\prime \prime}\left(\mathrm{L}^{51}\right)(\mu-\mathrm{OAC})_{2}\right]^{2+}$, and $\left[\mathrm{Fe}^{\prime \prime \prime \prime} \mathrm{Fe}^{\prime \prime}\left(\mathrm{L}^{4}\right)(\mu-\mathrm{OAC})_{2}\right]^{+} .139, \quad 140,232,235,262$ Similarly, bsPAP exhibits a rhombic spectrum with apparent $g$ values of $1.58,1.73$ and 1.85 at 
$\mathrm{pH}>5 .{ }^{219}$ The fact that both mixtures show similar EPR spectra with features typical for an Fe $\mathrm{III}^{\text {III }} \mathrm{Fe}^{\text {II }}$ species supports the prediction (CV) and observation (UV-vis) that ferrocenium can only oxidize one of the two $\mathrm{Fe}^{\|}$centers of $\left[\mathrm{Fe}_{2}{ }_{2}\left(\mathrm{H}_{2} \mathrm{SIM}^{1}\right)(\mu-\mathrm{OAc})_{2}\right]^{+}$.

Both frozen solution spectra exhibit a sharp signal at $g \sim 4.3\left(g_{1}=4.28, g_{2}=5.57, g_{3}=9.44\right)$. This

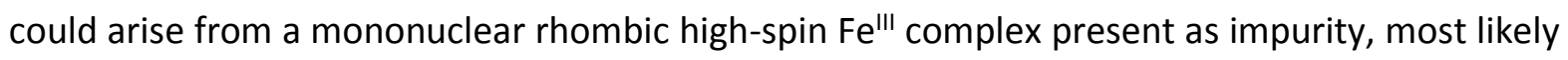
formed by decomposition of $\left[\mathrm{Fe}^{\prime \prime \prime} \mathrm{Fe}^{\prime \prime}\left(\mathrm{H}_{2} \mathrm{SIM}^{1}\right)(\mu-\mathrm{OAc})_{2}\right]^{2+}$ in solution, or a decomposition product of ferrocenium hexafluorophosphate. The detection of a similar signal from a ferrocenium hexafluorophosphate solution which was left under aerobic conditions for two days indicates that the latter is the case. This is also supported by the fact that the spectrum of a powder of $\left[\mathrm{Fe}^{\prime \prime \prime} \mathrm{Fe}^{\prime \prime}\left(\mathrm{L}^{51}\right)(\mathrm{OBz})_{2}(\mathrm{OH})\right] \mathrm{BF}_{4}$ did not exhibit such signal. ${ }^{262}$ The intense signals near $\mathrm{g} \sim 4.3$ originate from the Fe"l' center with almost axial symmetry $(g=8.56,5.45)$ and rhombic symmetry $(g=4.30)$. However, such an impurity was often detected with solution EPR spectra of $\mathrm{Fe}^{\text {III }} \mathrm{Fe}$ "l complexes, but the intensity was not as strong as in the spectra obtained with the mixtures of $\left[\mathrm{Fe}_{2}{ }_{2}\left(\mathrm{H}_{2} \mathrm{SIM}^{1}\right)(\mu-\mathrm{OAc})_{2}\right]^{+}$with ferrocenium hexafluorophosphate. Therefore, it is not clear if the generated $\mathrm{Fe}^{\prime \prime \prime} \mathrm{Fe}$ "l complex is stable enough to exist in solution, since the intense signal at $\mathrm{g} \sim 4.3$ could arise from at least partial decomposition.

It should be noted that in the spectra recorded with heterovalent complexes of ligand $\mathrm{HL}^{51}$ in solution there is, besides the signal at $\mathrm{g} \sim 1.7$, a broad signal at $\mathrm{g} \sim 5-6$ which is absent in the iron proteins and $\mathrm{HL}^{1}$ complexes. This signal shows also temperature dependence which is similar to that of the signal at $g \sim 1.7$. Therefore, the signals at $g \sim 5-6$ may be ascribed to the mononuclear complex in $S=3 / 2$ spin state. ${ }^{262}$ Those features were not detected in the spectra recorded with the mixture of $\left[\mathrm{Fe}_{2}{ }_{2}\left(\mathrm{H}_{2} \mathrm{SIM}^{1}\right)(\mu-\mathrm{OAc})_{2}\right]^{+}$with ferrocenium hexafluorophosphate indicating that the intense signal at $g \sim 1.7$ arises from the decomposition of ferrocenium.

\section{Mössbauer Spectroscopy}

The heterovlalent form of $\left[\mathrm{Fe}^{\prime \prime \prime} \mathrm{Fe}{ }^{\prime \prime}\left(\mathrm{H}_{2} \mathrm{SIM}^{1}\right)(\text { solv })_{\mathrm{x}}\right]^{4+}$, generated by oxidation of $\left[\mathrm{Fe}_{2}{ }_{2}\left(\mathrm{H}_{2} \mathrm{SIM}^{1}\right)(\mu-\mathrm{OAC})_{2}\right]^{+}$with ferrocenium hexafluorophosphate was also studied by ${ }^{57} \mathrm{Fe}$ Mössbauer spectroscopy. A mixture of ${ }^{57} \mathrm{Fe}$ enriched $\left[\mathrm{Fe}_{2}{ }_{2}\left(\mathrm{H}_{2} \mathrm{SIM}^{1}\right)(\mu-\mathrm{OAc})_{2}\right]^{+}$with one equivalent of ferrocenium hexafluorophosphate (as ${ }^{56} \mathrm{Fe}$ ) as oxidant clearly showed after 12 hours at room temperature signals for both Fe ${ }^{I I I}$ and Fe" (Figure 54b). Moreover, the presence of Fe $\mathrm{F}^{\mathrm{III}}$ and Fe $\mathrm{Fe}^{\mathrm{II}}$ equimolar ratio emerges from the fact that the ratio of areas of the two sets of quadrupole doublets is nearly unity. Thus, $\left[\mathrm{Fe}^{\text {III }} \mathrm{Fe}^{\prime \prime}\left(\mathrm{H}_{2} \mathrm{SIM}^{1}\right)(\mathrm{solv})_{\mathrm{x}}\right]^{4+}$ appears to be stable in solution. In addition, the Mössbauer parameters of the characteristic high-spin Fe"ll quadrupole doublet, $\delta(\mathrm{I})=0.41(1) \mathrm{mm} / \mathrm{s}$ and $\Delta \mathrm{E}_{\mathrm{Q}}(\mathrm{I})=0.36(2) \mathrm{mm} / \mathrm{s}(\Gamma(\mathrm{I})=0.38(1) \mathrm{mm} / \mathrm{s})$, and the characteristic highspin Fe" quadrupole doublet, $\delta(I I)=1.17(1) \mathrm{mm} / \mathrm{s}$ and $\Delta \mathrm{E}_{\mathrm{Q}}(\mathrm{II})=2.78(1) \mathrm{mm} / \mathrm{s}$ 
$(\Gamma(I I)=0.53(2) \mathrm{mm} / \mathrm{s})$, are comparable to these of previously reported phenolate-bridged $\mathrm{Fe}^{\mathrm{III}} \mathrm{Fe}{ }^{\mathrm{II}}$ complexes, i.e. $\left[\mathrm{Fe}^{\mathrm{III}} \mathrm{Fe}^{\prime \prime}\left(\mathrm{L}^{1}\right)(\mu-\mathrm{OPr})_{2}\right]\left(\mathrm{BPh}_{4}\right)_{2}\left(\delta(\mathrm{I})=0.48 \mathrm{~mm} / \mathrm{s}\right.$ and $\Delta \mathrm{E}_{\mathrm{Q}}(\mathrm{I})=0.50 \mathrm{~mm} / \mathrm{s}$; $\delta(I I)=1.13 \mathrm{~mm} / \mathrm{s}$ and $\left.\Delta \mathrm{E}_{\mathrm{Q}}(\mathrm{II})=2.69 \mathrm{~mm} / \mathrm{s}\right),{ }^{235}\left[\mathrm{Fe}^{\mathrm{III}} \mathrm{Fe}^{\mathrm{II}}\left(\mathrm{L}^{1}\right)(\mu-\mathrm{ppa})_{2}\right]\left(\mathrm{BF}_{4}\right)_{2}(\delta(\mathrm{I})=0.48 \mathrm{~mm} / \mathrm{s}$ and $\Delta \mathrm{E}_{\mathrm{Q}}(\mathrm{I})=0.56 \mathrm{~mm} / \mathrm{s} ; \delta(\mathrm{II})=1.14 \mathrm{~mm} / \mathrm{s}$ and $\left.\Delta \mathrm{E}_{\mathrm{Q}}(\mathrm{II})=2.75 \mathrm{~mm} / \mathrm{s}\right),{ }^{233}\left[\mathrm{Fe}^{\mathrm{III}} \mathrm{Fe} \mathrm{C}^{I \prime}\left(\mathrm{L}^{4}\right)(\mu-\mathrm{OAc})_{2}\right] \mathrm{ClO}_{4}$ $\left(\delta(\mathrm{I})=0.52 \mathrm{~mm} / \mathrm{s}\right.$ and $\Delta \mathrm{E}_{\mathrm{Q}}(\mathrm{I})=1.10 \mathrm{~mm} / \mathrm{s} ; \delta(\mathrm{II})=1.13 \mathrm{~mm} / \mathrm{s}$ and $\left.\Delta \mathrm{E}_{\mathrm{Q}}(\mathrm{II})=2.54 \mathrm{~mm} / \mathrm{s}\right),{ }^{94}$ and $\left[\mathrm{Fe}^{\mathrm{III}} \mathrm{Fe}^{\mathrm{II}}\left(\mathrm{L}^{51}\right)(\mu-\mathrm{OAC})_{2}\right]\left(\mathrm{BF}_{4}\right)_{2}\left(\delta(\mathrm{I})=0.58 \mathrm{~mm} / \mathrm{s}\right.$ and $\Delta \mathrm{E}_{\mathrm{Q}}(\mathrm{I})=0.40 \mathrm{~mm} / \mathrm{s} ; \delta(\mathrm{II})=1.29 \mathrm{~mm} / \mathrm{s}$ and $\left.\Delta \mathrm{E}_{\mathrm{Q}}(\mathrm{II})=2.81 \mathrm{~mm} / \mathrm{s}\right) \cdot{ }^{262}$ The ${ }^{57} \mathrm{Fe}$ Mössbauer spectrum of the asymmetric complex $\left[\mathrm{Fe}^{\text {III }} \mathrm{Fe}^{\prime \prime}\left(\mathrm{H}_{2} \mathrm{SIM}^{1}\right)(\text { solv })_{\mathrm{x}}\right]^{4+}$ shows distinct, i.e. valence trapped, $\mathrm{Fe}^{\text {III }}$ and $\mathrm{Fe}^{\prime \prime}$ centers, indicating that each of the two metal ions is bound specifically in one of the two binding sites.

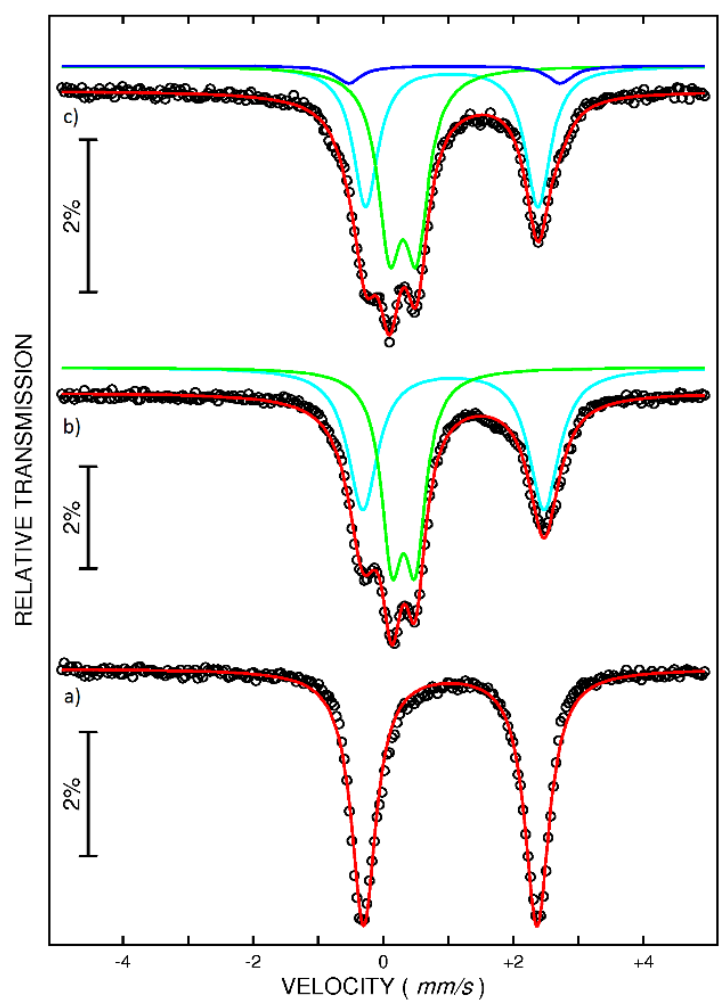

Figure 54: Comparison of ${ }^{57} \mathrm{Fe}$ Mössbauer spectra of $\left[\mathrm{Fe}_{2}{ }_{2}\left(\mathrm{H}_{2} \mathrm{SIM}^{1}\right)(\mu-\mathrm{OAc})_{2}\right] \mathrm{PF}_{6}$ a) in acetonitrile, b) with 1 eq of $\mathrm{FeCp}_{2} \mathrm{PF}_{6}$ and c) with 2 eq of $\mathrm{FeCp}_{2} \mathrm{PF}_{6}$.

Addition of two equivalents of ferrocenium hexafluorophosphate to the solution of ${ }^{57} \mathrm{Fe}$ enriched $\left[\mathrm{Fe}_{2}{ }_{2}\left(\mathrm{H}_{2} \mathrm{SIM}^{1}\right)(\mu-\mathrm{OAC})_{2}\right]^{+}$in acetonitrile resulted in a comparable ${ }^{57} \mathrm{Fe}$ Mössbauer spectrum. The fitted parameters are $\delta(I)=0.40(1) \mathrm{mm} / \mathrm{s}$ and $\Delta E_{Q}(I)=0.41(1) \mathrm{mm} / \mathrm{s}(\Gamma(I)=0.43(1) \mathrm{mm} / \mathrm{s})$ for Fe ${ }^{I I I}$ and $\delta(I I)=1.15(1) \mathrm{mm} / \mathrm{s}$ and $\Delta \mathrm{E}_{\mathrm{Q}}(\mathrm{II})=2.65(1) \mathrm{mm} / \mathrm{s}(\Gamma(\mathrm{II})=0.46(2) \mathrm{mm} / \mathrm{s})$ for Fe" . This supports the proposed incapacity of the oxidant to oxidize both $\mathrm{Fe}^{\| \prime}$ centers of $\left[\mathrm{Fe}_{2}{ }_{2}\left(\mathrm{H}_{2} \mathrm{SIM}^{1}\right)(\mu-\mathrm{OAc})_{2}\right]^{+}$and the selective formation of the heterovalent $\left[\mathrm{Fe}^{\prime \prime \prime} \mathrm{Fe}^{\prime \prime}\left(\mathrm{H}_{2} \mathrm{SIM}^{1}\right)(\text { solv })_{\mathrm{x}}\right]^{4+}$ form. 


\subsection{SUMMARY}

The two ligands $\mathrm{H}_{3} \mathrm{SIM}^{1}$ and $\mathrm{H}_{3} \mathrm{~L}^{2}$ with two asymmetrically and two symmetrically disposed pivaloyl-amide moieties, respectively, lead to $\left[\mathrm{Fe}_{2}{ }_{2}\left(\mathrm{H}_{2} \mathrm{SIM}^{1}\right)(\mu-\mathrm{OAc})_{2}\right] \mathrm{PF}_{6}$ and $\left[\mathrm{Fe}_{2} \mathrm{I}_{2}\left(\mathrm{H}_{2} \mathrm{~L}^{2}\right)(\mu-\mathrm{OAC})_{2}\right] \mathrm{PF}_{6}$, after treatment with iron(II) acetate and hexafluorophosphate under anaerobic conditions. These complexes revealed the common structure for phenoxido-bridged phosphatase models bearing two bridging acetate co-ligands. NMR spectroscopy demonstrates that the symmetry provided by the ligands is still present in the diiron(II) complexes, $\left[\mathrm{Fe}_{2}{ }_{2}\left(\mathrm{H}_{2} \mathrm{SIM}^{1}\right)(\mu-\mathrm{OAC})_{2}\right]^{+}$and $\left[\mathrm{Fe}_{2}{ }_{2}\left(\mathrm{H}_{2} \mathrm{~L}^{2}\right)(\mu-\mathrm{OAc})_{2}\right]^{+}$, in solution. Under aerobic conditions only $\left[\mathrm{Fe}{ }^{I I \prime} \mathrm{Na}\left(\mathrm{H}_{2} \mathrm{SIM}^{1}\right)(\mu-\mathrm{OAc})_{2}\right] \mathrm{PF}_{6}$ could be isolated. However, with ${ }^{57} \mathrm{Fe}$ Mössbauer spectroscopy the formation of the corresponding $\mathrm{Fe}^{\mathrm{III}}{ }_{2}$ complex was monitored in solutions of $\left[\mathrm{Fe}_{2}{ }_{2}\left(\mathrm{H}_{2} \mathrm{SIM}^{1}\right)(\mu-\mathrm{OAc})_{2}\right] \mathrm{PF}_{6}$ and $\left[\mathrm{Fe}^{\prime \prime \prime} \mathrm{Na}\left(\mathrm{H}_{2} \mathrm{SIM}^{1}\right)(\mu-\mathrm{OAc})_{2}\right] \mathrm{PF}_{6}$ under aerobic conditions. This diiron(III) complex is unstable under acidic conditions, reacting possibly via a concerted proton-electron transfer to generate an $\mathrm{Fe}^{\mathrm{Il}}$ species and a ligand-phenol radical. SQUID susceptibility measurements indicate that $\left[\mathrm{Fe}_{2}{ }_{2}\left(\mathrm{H}_{2} \mathrm{SIM}^{1}\right)(\mu-\mathrm{OAC})_{2}\right] \mathrm{PF}_{6}$ consists of two weakly antiferromagnetically coupled high-spin Fell ions. This also emerges from solution magnetic susceptibility measurements, UV-vis and Mössbauer spectroscopy. The CV of $\left[\mathrm{Fe}_{2}{ }_{2}\left(\mathrm{H}_{2} \mathrm{SIM}^{1}\right)(\mu-\mathrm{OAc})_{2}\right]^{+}$exhibits two reasonably well separated redox waves for the redox couples $\mathrm{Fe}{ }_{2} / \mathrm{Fe}^{\prime \prime \prime} \mathrm{Fe}^{\prime \prime}$ and $\mathrm{Fe}^{\prime \prime \prime} \mathrm{Fe}^{\prime \prime} / \mathrm{Fe}^{\prime \prime \prime}$, and the $\mathrm{K}_{\text {com }}$ value indicates an appreciable stability of the mixed-valent Fe ${ }^{I I I} \mathrm{Fe}{ }^{I I}$ species. Therefore, the generation of the heterovalent diiron complex of $\mathrm{H}_{3} \mathrm{SIM}^{1}$ was probed. The successful formation of complex $\left[\mathrm{Fe}^{\prime \prime \prime} \mathrm{Fe}{ }^{\prime \prime}\left(\mathrm{H}_{2} \mathrm{SIM}^{1}\right)(\text { solv })_{x}\right]^{4+}$ by chemical oxidation of $\left[\mathrm{Fe}_{2}{ }_{2}\left(\mathrm{H}_{2} \mathrm{SIM}^{1}\right)(\mu-\mathrm{OAC})_{2}\right]^{+}$with ferrocenium hexafluorophosphate under anaerobic conditions was verified by ${ }^{57} \mathrm{Fe}$ Mössbauer, UV-vis and EPR spectroscopy. As a result of these studies an accurate model complex $\left[\mathrm{Fe}^{\text {III }} \mathrm{Fe}^{\prime \prime}\left(\mathrm{H}_{2} \mathrm{SIM}^{1}\right)(\mathrm{solv})_{\mathrm{x}}\right]^{4+}$ for the active form of mammalian PAPs became available with a heterovalent diiron core in a asymmetric coordination environment with the capacity to form a hydrogen bond network as exhibited for the native enzymes. 


\section{Chapter 6}

Studies of the

Phosphatase Mechanism 


\subsection{EnzyMATIC MeChanisms of PhOSPHOESTER Cleavage}

Phosphatases are amongst the most efficient enzymes known with their ability to drastically reduce the activation energies associated with the hydrolytic cleavage of phosphoester bonds. ${ }^{11}$ Acid Phosphatases, e.g. PAPs, which are most active at low pH, and Alkaline Phosphatases (APs), which exhibit their highest activity under basic conditions, are two particularly important classes. ${ }^{11}$

\section{Hydrolysis Mechanism Proposed for AP}

In the active site of AP the phosphoester substrate is activated by interaction with both $\mathrm{Zn}$ " centers (Scheme 14). ${ }^{11,263,264}$ A proximal arginine residue is proposed to be involved in the substrate binding and the simultaneous activation of the substrate. The nucleophilic attack of the phosphoester substrate is accomplished by an alcohol group of a serine residue adjacent the dizinc(II) active core. The resulting transition state is also stabilized by the arginine residue adjacent to the dizinc(II) core. The release of the alcoholate leaving group is supported by the interaction with one $\mathrm{Zn}^{\prime \prime}$ center. Subsequently, alcohol/water ligand exchange provides the nucleophilic water molecule necessary for the hydrolysis and this nucleophile attacks the bridging phosphate and thereby initiates the dissociation of the phosphate product, and the regeneration of the resting state. ${ }^{11,263,264}$

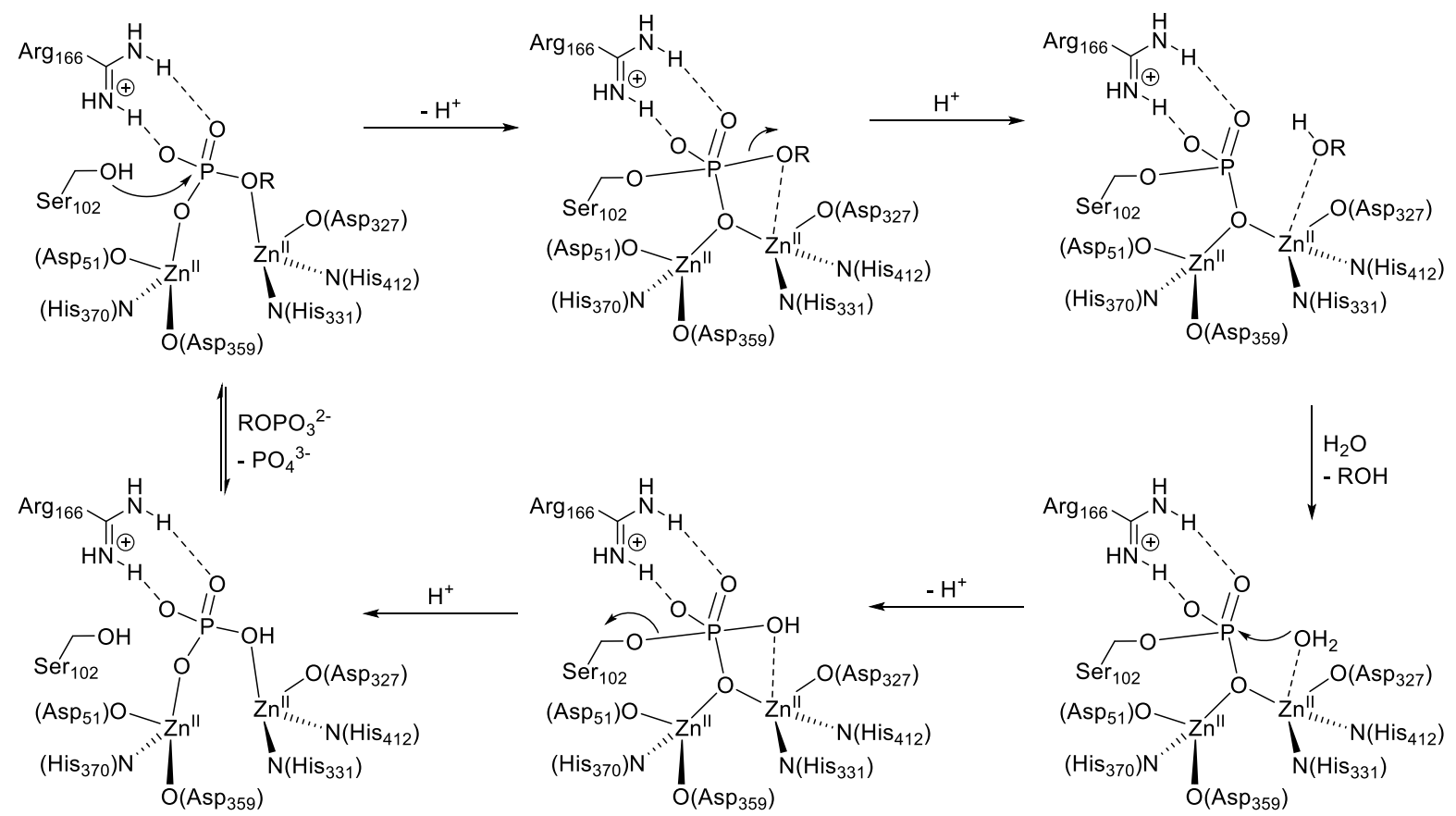

Scheme 14: Proposed catalytic cycle for the AP of E. coli. ${ }^{11,263,264}$ 


\section{Catalytic Mechanisms Proposed for PAP}

Recently, an eight step mechanism for the phosphoester hydrolysis by the heterodinuclear active site of PAPs was proposed (Scheme 15). ${ }^{25,29}$ This catalytic cycle is based on X-ray structures, spectroscopic studies of different enzyme-inhibitor complexes and kinetic data for phosphoester hydrolysis. Prior to nucleophilic attack it was suggested that the phosphoester substrate is precoordinated in the second coordination sphere and stabilized via an extensive hydrogen bonding network, involving the $\mu$-hydroxido ligand, the $\mathrm{Zn}$ " coordinated asparagine residue and adjacent histidine residues. This proposal derives from stopped-flow kinetic measurements and a crystal structure of a kbPAP-sulfate complex in which a sulfate anion is coordinated in this fashion. ${ }^{25,265,266}$ Following this, the preorientation of the phosphoester leads to a monodentate and subsequently bridging coordination mode of the phosphoester substrate. The attacking nucleophile is proposed to be the asymmetrically bound $\mu$-hydroxido ligand, although the nature of the nucleophile is still a matter of debate, and different proposals are discussed later in this chapter. ${ }^{25,} 67,69,82,85,267,268$ After the esterolysis, the phosphate remains bound to the active site either in a tripodal or a $\mu-1,3$-bridging coordination fashion, proposed on basis of crystal structures of different types of PAPs. ${ }^{26,83,85}$ The least understood step of the catalytic cycle is the regeneration of the resting state of the enzyme, which requires the removal of the phosphate group. The authors suggested a plausible sequence depicted in Scheme $15 .^{29}$

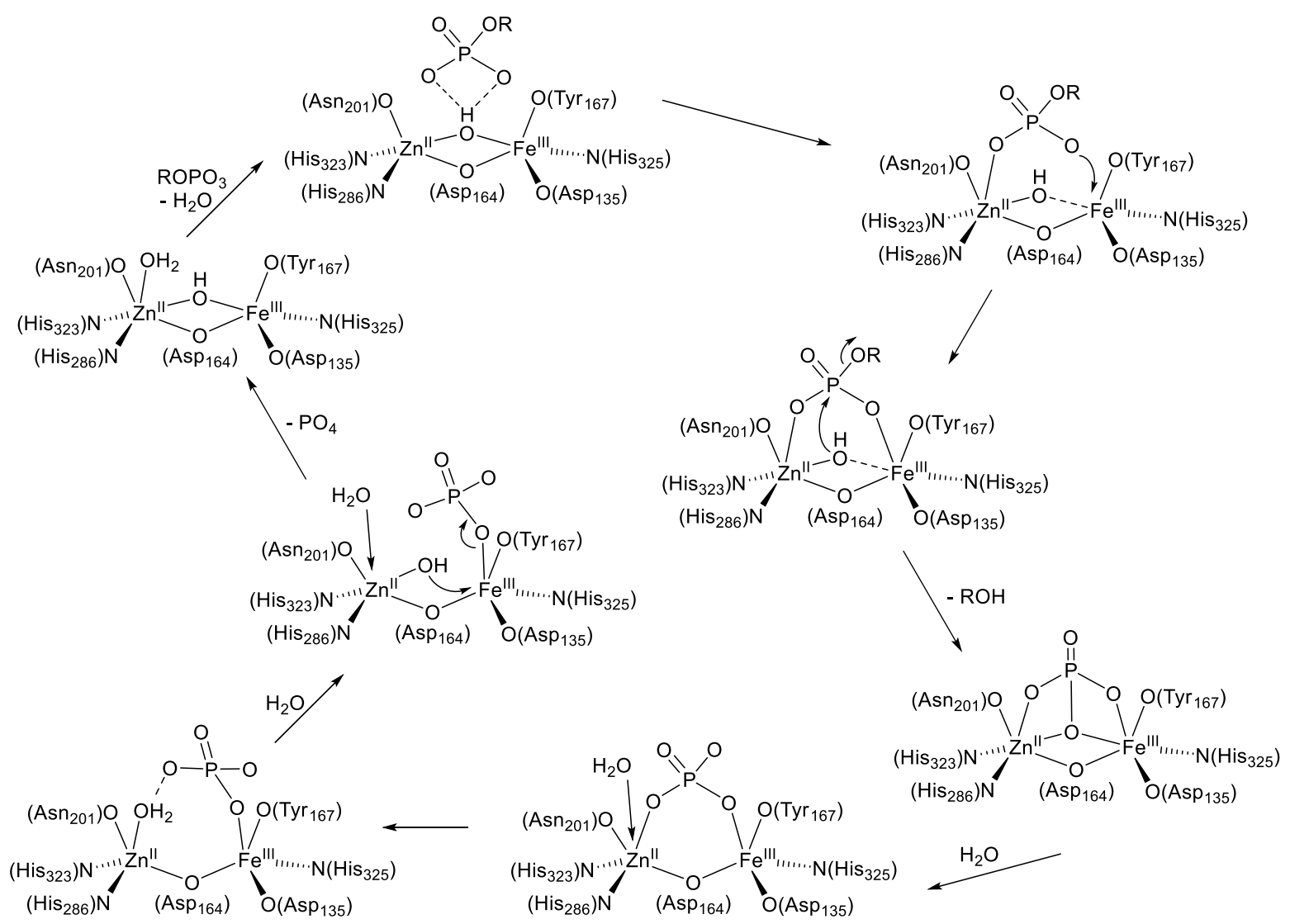

Scheme 15: Proposed reaction mechanism for PAP-catalyzed phosphoester hydrolysis based on X-ray structures. ${ }^{25,} 29$ 
Various other suggestions regarding the catalytic mechanism of dinuclear phosphoesterases have been proposed, and these are summarized in Scheme $16 .{ }^{269}$ Early publications proposed the activation of the phosphoester substrate via monodentate coordination to the metal in binding site $\beta$ and the subsequent attack of a $M^{\alpha}$-bound terminal hydroxide ( $M^{\alpha}=F e^{I I I}$ in PAP), generating a pentacoordinated, associative-type phosphorus intermediate or transition state (Scheme 16, pathway a). ${ }^{83,228}$

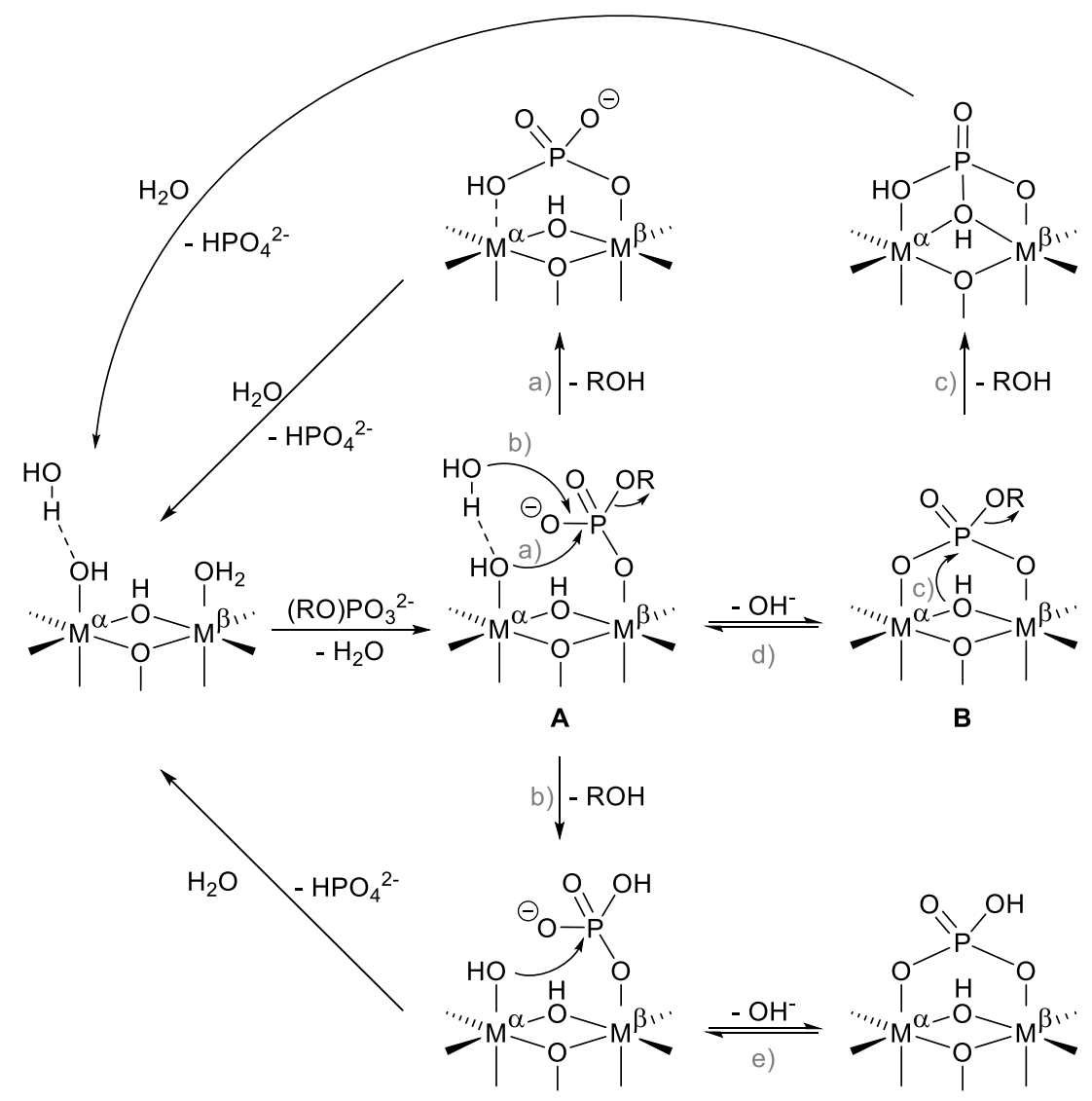

Scheme 16: Possible pathways for phosphoesterase catalyzed hydrolysis $\left(\mathrm{M}^{\alpha}=\mathrm{Fe}^{\prime \prime \prime}, \mathrm{M}^{\beta}=\mathrm{Fe} \mathrm{e}^{\prime \prime}, \mathrm{Zn}^{\prime \prime}, \mathrm{Mn}\right.$ " for PAP; $M^{\alpha}=M^{\beta}=Z n^{\prime \prime}$ for AP). ${ }^{269}$

However, stopped-flow kinetic studies with ufPAP in its native Fe ${ }^{\prime \prime \prime} \mathrm{Fe} \mathrm{e}^{\prime \prime}$ form and the artificial

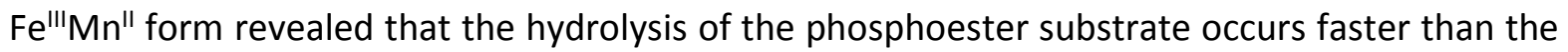
perturbation of the $\mathrm{Fe}^{\mathrm{III}}-\mathrm{O}_{\mathrm{Tyr}}$ charge transfer transition. ${ }^{82}$ This supports a monodentate substrate binding but also indicates the hydroxide nucleophile not being directly bound to the Fe ${ }^{\text {III }}$ center. In addition, EPR spectroscopic results showed that the Fe ${ }^{\text {III }}$ in bsPAP is only slowly affected during the catalytic cycle of phosphoester hydrolysis, therefore, a 'general-base' mechanism has been proposed. ${ }^{267}$ Here, the $\mathrm{M}^{\alpha}$-bound hydroxide functions as base and activates a non-coordinated water molecule, which subsequently attacks the terminal phosphoester in binding site $\beta$ (Scheme 16, pathway b); the phosphate product remains monodentately coordinated after the hydrolysis. ${ }^{269} \mathrm{~A}$ third pathway has been suggested, derived from the crystallographic data, where the phosphoester coordinates in a bridging fashion before being attacked by the bridging 
hydroxido ligand (Scheme 16, pathway c). However, the low nucleophilicity of a $\mu$-hydroxido makes pathway a and pathway b more plausible. ${ }^{25,270}$ These three different mechanisms were similarly discussed for AP consisting of two $\mathrm{Zn}^{11}$ centers in the active site. ${ }^{65,271}$ Similarly, different inhibition processes are discussed that all include formation of catalyst-substrate or catalystinhibitor (hydrolysis product) adducts, containing the phosphates in a bridging coordination mode (Scheme 16, pathway $\mathrm{d}$ and e). ${ }^{83,228}$ The presence of these equilibria was supported by labeling studies with ${ }^{18} \mathrm{O}$-phosphate, which demonstrated successive oxygen exchange between enzyme bound phosphate and water. ${ }^{272}$

It is likely that, depending on the metal composition in the active site and the substrate used, different mechanisms operate. ${ }^{66}$ However, there is an ongoing debate about (i) the substrate coordination mode (monodentate (Scheme 16, species A) vs. bridging (Scheme 16, species B)), (ii) the type of the attacking nucleophile, and (iii) the reaction type (concerted vs. stepwise). Moreover, the impact of the second coordination sphere with respect to the catalytic mechanism is still not fully understood, although it is apparent that the histidine residues proximal to the dimetallic core play an important role in the phosphoester hydrolysis activity of PAPs. ${ }^{25,82,83}$ Klabunde et al. proposed a reaction mechanism (Scheme 17) where these histidine residues, essential for catalysis, were involved in the active site of kbPAP. ${ }^{83}$

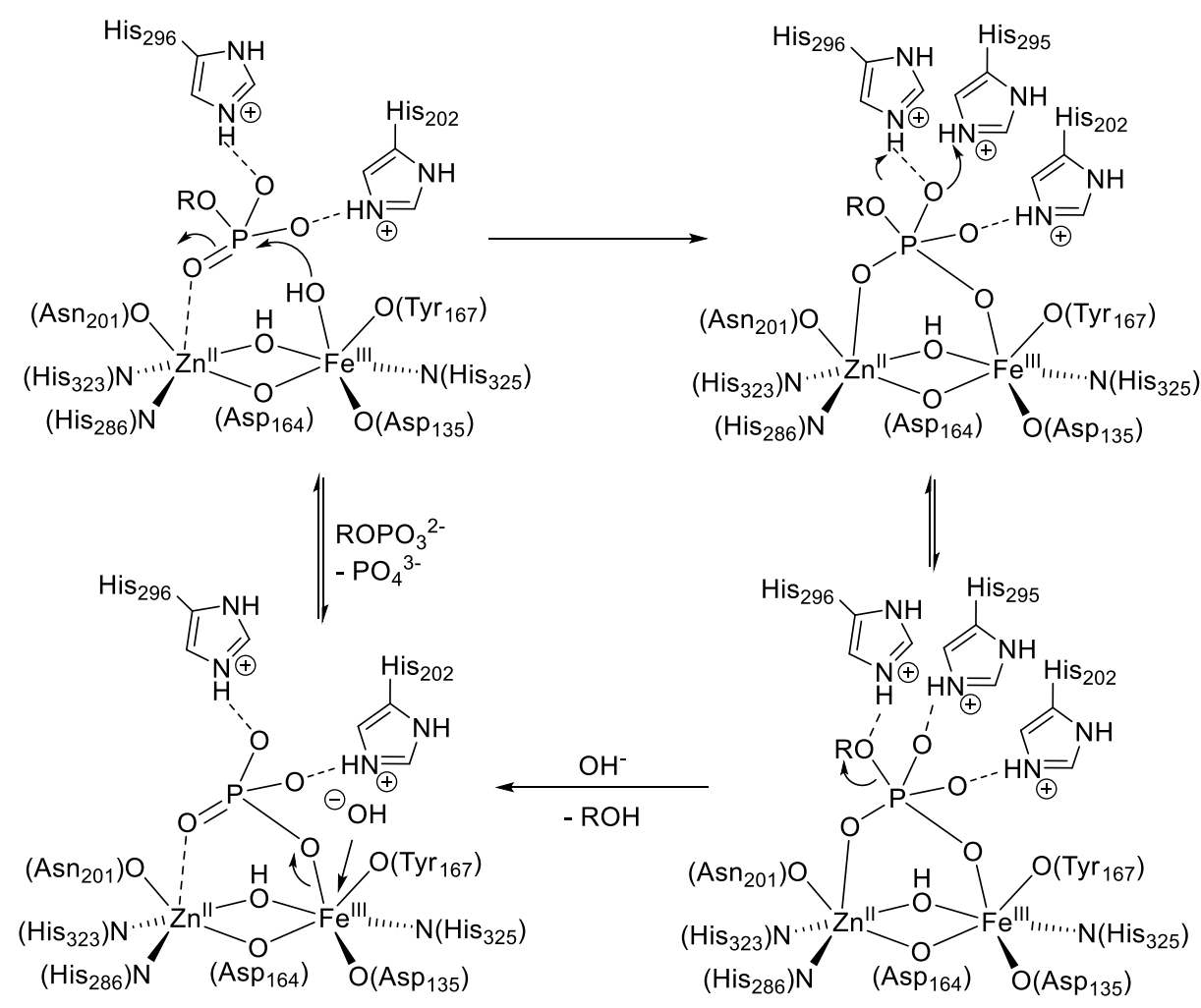

Scheme 17: Proposed reaction mechanism for PAP-catalyzed phosphoester hydrolysis, considering the second coordination sphere of the enzyme. ${ }^{83}$ 
Interaction with the protonated histidine residues promote (i) binding and activation of the substrate, (ii) transition state stabilization, and (iii) release of the leaving group, thus explaining the enhanced activity at low pH. This mechanism was supported by DFT studies, undertaken to understand the importance of hydrogen bonds within the reaction mechanism and to identify the reasons that enable the model system $\left[\mathrm{Fe}^{\prime \prime \prime}{ }_{2}\left(L^{6}\right)(\mu-O) C_{4}\right](C l)_{2}$ to hydrolyze not only the phosphodiester model substrate BDNPP but also the phosphomonoester model substrate DNPP (Scheme 18). ${ }^{108,}{ }^{109}$ Relative energies of this model complex, binding the substrates either in a monodentate or in a bridging mode, were calculated and compared. While the phosphodiester BDNPP is more likely bound in a monodentate fashion, stabilized by the formation of hydrogen bonds to the protonated cyclam ring, the phosphomonoester DNPP is proposed to interchange between the monodentate and the bridging mode due to a relatively small activation barrier. Once the phosphomonoester substrate is coordinated to one of the Fe ${ }^{I I I}$ ions two reaction pathways are possible: either the phosphoester-bridged complex is formed, leading to the deactivation of the catalyst (Scheme 18, pathway b), or the nucleophilic attack via the hydroxido ligand bound to the second Fe $\mathrm{F}^{\mathrm{III}}$ ion takes place (Scheme 18, pathway a), resulting in the hydrolysis of the substrate. These competitive reactions were compared by relaxed potential energy surface scans conducted by stepwise decrease of the distance between the noncoordinated Fe ${ }^{\text {III }}$ ion and the oxygen atom of DNPP in the case of inhibition (Scheme 18, pathway b) or the hydroxido ligand oxygen atom and the phosphorus atom of DNPP in the case of hydrolysis (Scheme 18, pathway a). ${ }^{108,109}$

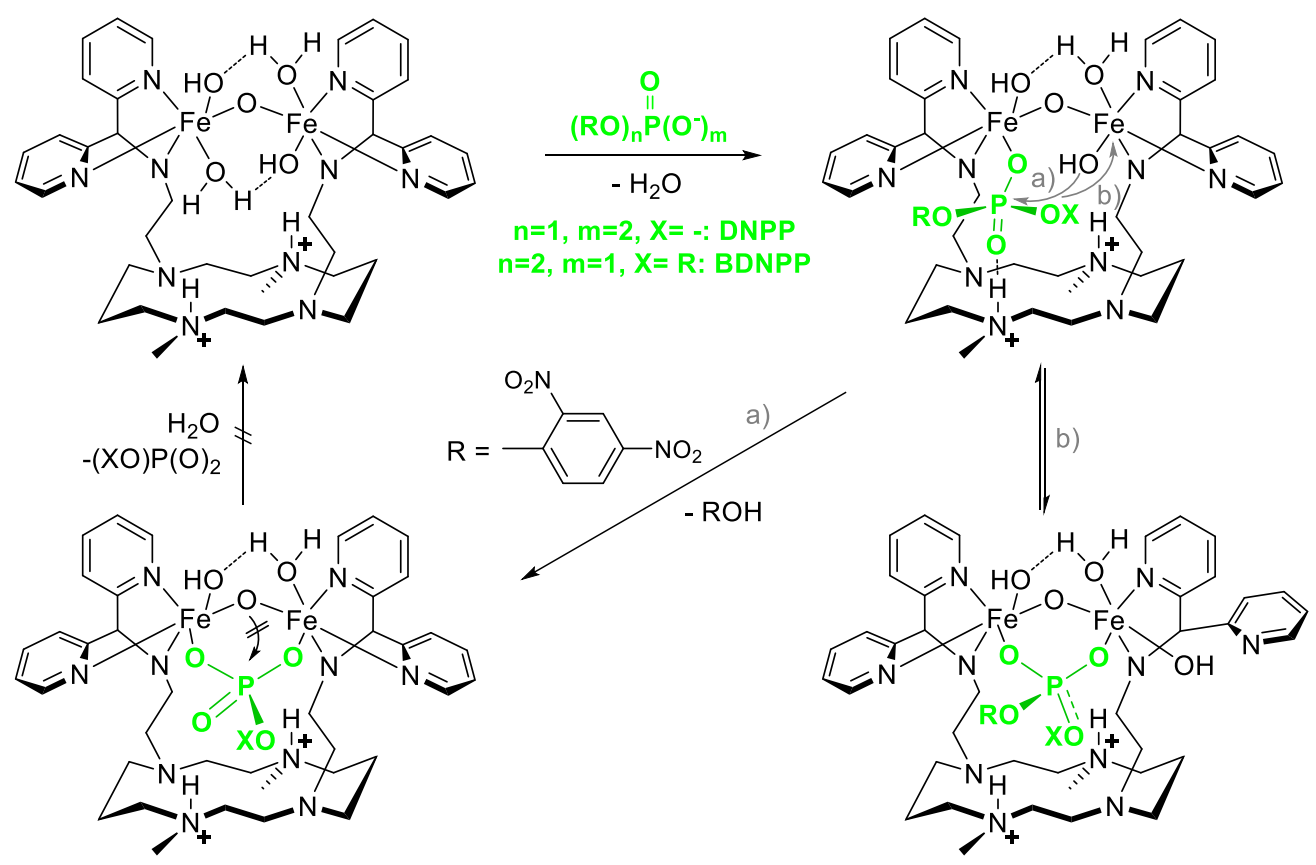

Scheme 18: Proposed catalytic cycle for the hydrolysis of BDNPP and DNPP with the use of the $\mathrm{Fe}^{\mathrm{II \prime}} 2$ complex of $\mathrm{H}_{2} \mathrm{~L}^{6} \cdot 108,109$ 
With these DFT studies it was elucidated that (i) the activation barrier of the deactivation is higher than of the cleavage of the phosphoester bond and (ii) the lack of hydrogen bond formation capacity (using a deprotonated cyclam backbone) leads to a smaller energy difference between the two possible reaction pathways. ${ }^{108,} 109$ Thus, the capacity of this model complex $\left[\mathrm{Fe}^{\prime \prime \prime}{ }_{2}\left(\mathrm{~L}^{6}\right)(\mu-\mathrm{O}) \mathrm{Cl}_{4}\right](\mathrm{Cl})_{2}$ for cleaving a phosphomonoester was ascribed to the involvement of a hydrogen bond network in the reaction mechanism.

Although the two catalytic mechanisms of PAP and AP differ in the interaction that places the phoshoester substrate in the ideal position for the nucleophilic attack and, more importantly, in the nature of the attacking nucleophile, there are some common features, i.e. (i) the activation of the substrate by Lewis acid metal ion coordination and hydrogen bonding interactions with side-chains of adjacent residues, (ii) promotion of the transition state formation by the metal ions and hydrogen bonding residues, and (iii) the assistance of the release of the alkoxide leaving groups via stabilizing interaction with the extensive hydrogen bonding network in the active site. ${ }^{11}$

However, it should not be underestimated that in addition to specific chemical features, the remarkable catalytic activity of phosphatase enzymes may also derive from the polarity and the steric demand of the environment of the active site. ${ }^{273}$ It has been suggested that the catalysis of enzymes is promoted by binding of the substrate in hydrophobic pockets with an apparent dielectric constant much lower than the surrounding water. In this way, both the equilibrium for proton transfer reactions is shifted and the strengths of hydrogen bonds, involving the substrate binding and the transition state stabilization, are changed. ${ }^{274}$ Bond cleavage in enzymes is also known to be promoted by straining of the substrate in order to support the formation of the transition state. ${ }^{11}$

In the following chapters kinetic studies with the complexes developed in this work (Chapter 3, 4 , and 5) will be discussed. The emphasis was on a correlation of the position of the hydrogen bonding residues in the active site of the model complexes with the catalytic efficiencies. However, the influence of sterically demanding residues as well as the impact of a more electronrich primary coordination site was also studied.

\subsection{Phosphoester Substrates Employed in Cleavage STUDIES}

In order to access the cleavage activity of phosphatase model complexes a number of nonnatural substrate models have been used as mimics of natural phosphate linkages. The model 
substrates employed to study the phosphoester binding with the model complexes developed in this work are 4-nitrophenylphosphate (PNPP) (as its disodium salt), bis(4-nitrophenyl)phosphate (BPNPP), 2,4-dinitrophenylphosphate (DNPP) (as its 2,6-lutidinium salt) and bis(2,4-dinitrophenyl)phosphate (BDNPP) (as its pyridinium salt); these are shown in Chart 19. PNPP and BPNPP are commercially available, BDNPP and DNPP were prepared following reported procedures as described in detail in Chapter 11.14.<smiles>O=[N+]([O-])c1ccc(OP(=O)([O-])[O-])cc1</smiles>

PNPP<smiles>O=[N+]([O-])c1ccc(OP(=O)([O-])c2ccc([N+](=O)[O-])cc2)cc1</smiles>

BPNPP<smiles>O=[N+]([O-])c1ccc(OP(=O)([O-])O)c([N+](=O)[O-])c1</smiles>

DNPP<smiles>O=[N+]([O-])c1ccc(OP(=O)([O-])Oc2ccc([N+](=O)[O-])cc2[N+](=O)[O-])c([N+](=O)[O-])c1</smiles>

BDNPP

Chart 19: Phosphoester model substrates employed in this work.

The phosphoester hydrolysis reactivity of the catalysts developed in this work was probed with a spectrophotometric assay using BDNPP (phosphodiester) and DNPP (phosphomonoester) as model substrates. These phosphoester compounds were chosen because of their welldemonstrated and -documented use with various catalysts. ${ }^{11,} 81$ They also serve as an appropriate comparison of the results obtained with those from past works since these substrates are commonly used. In Scheme 19 the hydrolysis of the phosphodiester BDNPP under basic conditions is shown. Cleavage of BDNPP forms DNPP by release of 2,4-dinitrophenolate. The phosphomonoester product DNPP can be further hydrolyzed in a second step.

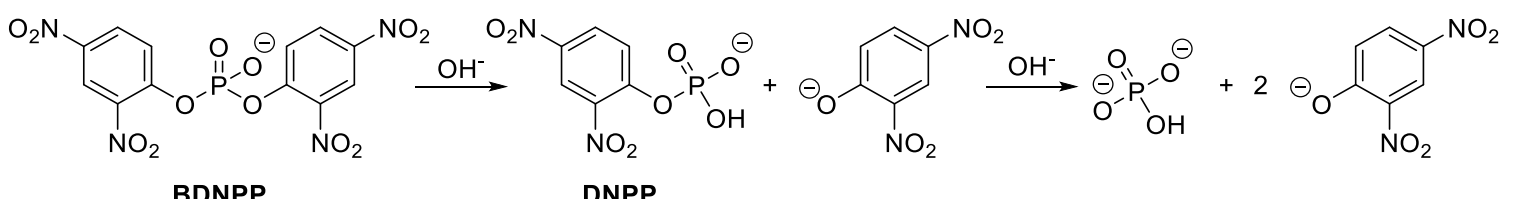

Scheme 19: Hydrolysis of BDNPP and DNPP under basic conditions releasing 2,4-dinitrophenolate.

Cleavage of the phosphorous-oxygen bond of BDNPP or DNPP was followed, for approximately three minutes, at $25^{\circ} \mathrm{C}$ by monitoring the generated product 2,4-dinitrophenolate by its strong absorption at $400 \mathrm{~nm}\left(\varepsilon=12,100 \mathrm{M}^{-1} \mathrm{~cm}^{-1}\right)$. The initial hydrolysis rate $v_{0}$ was calculated from the initial rate of the increase of the absorbance at $400 \mathrm{~nm}$.

It is important to note, that the model substrates BDNPP and DNPP are activated towards cleavage as the resulting product is strongly stabilized via resonance. Moreover, spontaneous hydrolysis of the model substrate takes place in basic solution. Therefore, all experiments were conducted in triplicate and the average data were subsequently corrected from the autohydrolysis by subtraction of the $v_{0}$ value obtained with measurements in the absence of a catalyst. 


\subsection{General Procedures}

Before the results of the kinetic investigations are discussed in the following chapters the general procedure applied will be introduced.

\subsubsection{PH DEPENDENCE ASSAYS}

By varying the $\mathrm{pH}$ of the multicomponent buffer, the $\mathrm{pH}$ dependence of the activity was studied in the $\mathrm{pH}$ range from 5 to 11 . The $\mathrm{pH}$ values refer to the aqueous component, and it should be noted that the $\mathrm{pH}$ of an aqueous solution of the buffer is the same within error as in a 1:1 mixture of buffer and acetonitrile. ${ }^{275,276}$ The resulting data were fitted either to Equation 20 describing a monoprotic system or to Equation 21 which is based on a model for a diprotic system with two active species $E^{n}$ and $E^{n-1}$ (Scheme 20)..$^{277}$

$$
\begin{array}{ll}
\text { Equation } 20 & \mathrm{v}_{0}=\frac{\mathrm{v}_{\max }}{1+\frac{\left[\mathrm{H}^{+}\right]}{K_{a}}} \\
\text { Equation 21 } & \mathrm{v}_{0}=\frac{\mathrm{v}_{\max }\left(1+\frac{\gamma \mathrm{K}_{2}}{\left[\mathrm{H}^{+}\right]}\right)}{\left(1+\frac{\left[\mathrm{H}^{+}\right]}{\left.\mathrm{K}_{\mathrm{a}_{1}}+\frac{\mathrm{K}_{2}}{\left[\mathrm{H}^{+}\right]}\right)}\right.}
\end{array}
$$

Here, $v_{0}$ is the initial reaction rate and $v_{\max }$ is the maximum reaction rate that is reached under given conditions. The factor $\gamma$ is related to the relative activity of the two active species in equilibrium ( $E^{n} S$ and $E^{n-1} S$ ); a value of $\gamma$ less than unity corresponds to a more active $E^{n} S$ adduct and a value higher than 1 considers the deprotonated adduct $E^{n-1} S$ as more active. ${ }^{277,}{ }^{278}$ The $K_{a}$ values obtained are the protonation equilibrium constants between the two relevant active species.

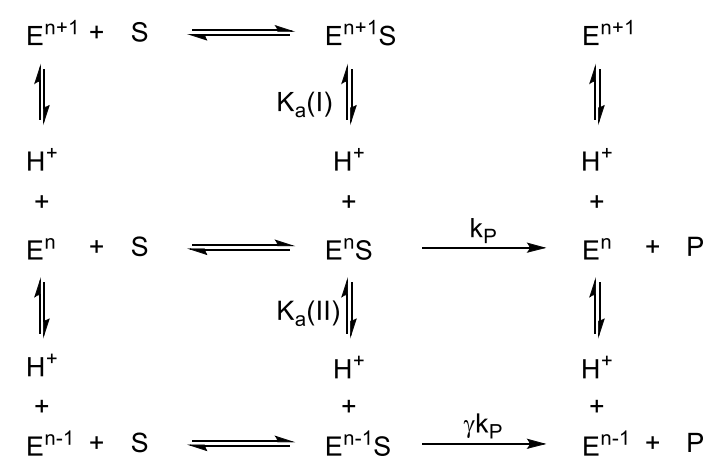

Scheme 20: Model of a diprotic system with two active species on which Equation 21 is based on. ${ }^{277}$ 


\subsubsection{SubStRate CONCENTRATION DePENDENCE AsSAYS}

The measurements of the dependence of the BDNPP hydrolysis rate on the substrate concentration were determined at a range of $\mathrm{pH}$ values to assess the catalytic behavior of different protonation states. Due to the poor solubility of BDNPP in acetonitrile, assays with a starting concentration of the substrate higher than $11.5 \mathrm{mM}$ could not be investigated. If the dependence of the rate of hydrolysis on the BDNPP concentration showed MichaelisMenten saturation behavior, the experimental data were fitted to Equation 22 in order to determine the kinetic parameter values for the Michaelis-Menten constant $K_{M}$ and the maximum reaction rate $\mathrm{V}_{\text {max. }}{ }^{279,}{ }^{280} \mathrm{~K}_{\mathrm{M}}$ describes the substrate concentration required for the enzyme/catalyst to reach one-half of its maximum cleavage reaction rate.

$$
\text { Equation } 22 \quad \mathrm{v}_{0}=\frac{\mathrm{v}_{\max }[\mathrm{BDNPP}]_{0}}{\mathrm{~K}_{\mathrm{M}}+[\mathrm{BDNPP}]_{0}}
$$

Applying Equation 23 the catalytic rate $k_{\text {cat }}$, which describes the number of substrate molecules cleaved by one active site per second, was evaluated. The efficiency of the catalyst is expressed as $\mathrm{k}_{\text {cat }} / \mathrm{K}_{\mathrm{M}}$.

$$
\text { Equation } 23 \quad \mathrm{k}_{\mathrm{cat}}=\frac{\mathrm{v}_{\max }}{[\text { complex }]_{0}}
$$

\subsubsection{TURN OVER NUMBERS}

Long-term studies regarding the catalytic activity of the catalysts developed in this thesis were conducted preparing assays with an excess of phosphoester substrate and leaving them to react at room temperature for up to two weeks. During the experiment, samples were taken at various intervals, diluted with solvent (mixture acetonitrile:MilliQ-water (1:1)), and their UV-vis spectra were recorded to determine the amount of phosphoester hydrolysis. The increase in the absorbance at $400 \mathrm{~nm}$, assigned to the hydrolysis product, 2,4dinitrophenolate, was monitored over time and turn over numbers calculated using the BeerLambert Law. 


\section{Chapter 7}

\section{Phosphatase Activity of}

Dizinc Complexes ${ }^{f}$

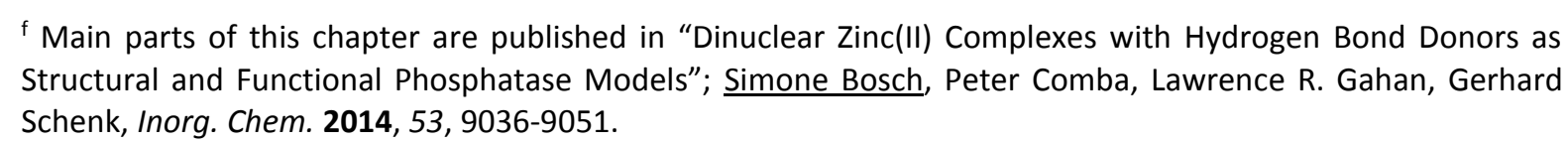




\subsection{INTRODUCTION}

The facile catalyzed cleavage of phosphoesters is a crucial reaction for living organisms, for example in processing RNA replication and bone metabolism, and it is of ecological importance in the detoxification of pesticides. Natural phosphatases, which accomplish this hydrolysis, commonly contain $\mathrm{Zn}$ " ions in their active sites. Therefore, dizinc(II) complexes have been widely used as artificial models to expand our understanding of the hydrolysis mechanism in the native enzymes and to promote the development into new therapeutic agents.

Attention in the design of model complexes has been mainly focused on the influence of (i) the

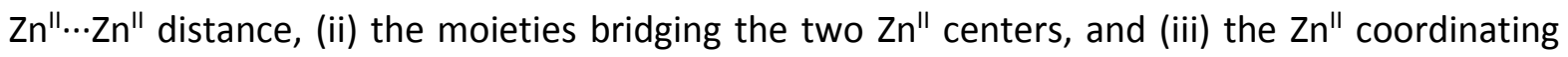
residues in the primary coordination sphere. ${ }^{28,65,271,281,282}$ Whilst many zinc(II) complexes of simple chelating ligands were reported to be effective in hydrolyzing phosphoesters, the catalytic rates generally were found to be modest compared to the native nucleases and phosphatases. Thus, in recent years emphasis has been put in the secondary coordination sphere of the $\mathrm{Zn}$ " center(s) of model complexes, because many metalloenzymes that catalyze the cleavage of phosphoesters also use amino acid side chains to preorientate the substrate molecule and to stabilize the transition state. ${ }^{80,84}$ The development of complexes incorporating functionalities, imitating the environment proximal to the dimetallic core of nucleases and phosphatases, resulted in complexes with increased acceleration capacity compared to their non-functionalized counterparts.

Before introducing examples of zinc(II) model complexes considering the secondary interactions within the active site of phosphatases, it should be noted that the kinetic studies of those complexes were often undertaken using the RNA model substrate HPNPP (2-hydroxypropyl-4nitrophenylphosphate). In the case of HPNPP the P-O bond cleavage is introduced by the nucleophilic attack of an alkoxide (in contrast to a hydroxide nucleophile for the hydrolysis of BNPP, BDNPP and DNPP (Scheme 21, bottom)), followed by the release of an alkoxide group (Scheme 21, top). Therefore, the process is termed transesterification, while the attack by a hydroxide is known as hydrolysis. ${ }^{11}$ 

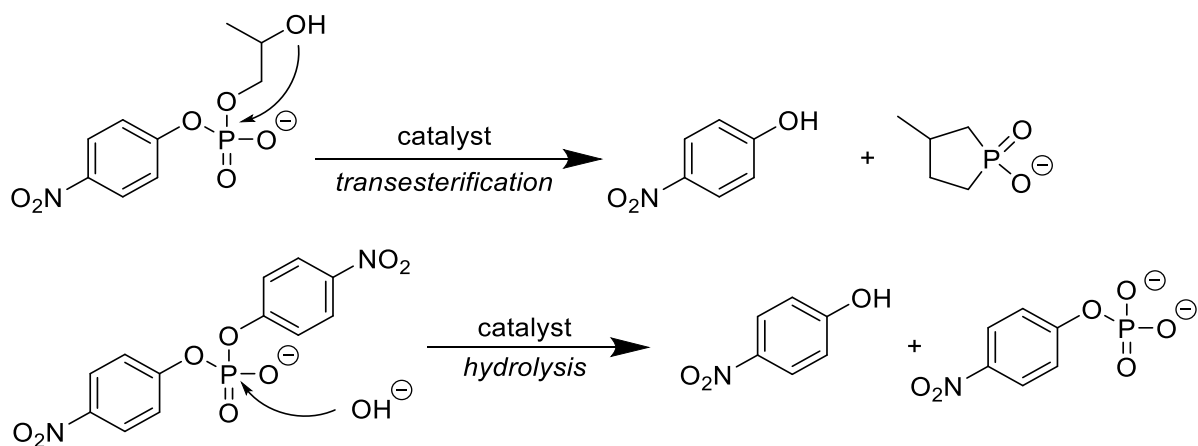

Scheme 21: Transesterification of HPNPP (top) and hydrolysis of BNPP (bottom).

Although the processes differ in the type of nucleophile initiating the cleavage reaction, they have commonalities in (i) the activation of the phosphoester by coordination to a $\mathrm{Zn}$ " center and (ii) the proposed formation of a five-coordinate trigonal bipyramidal phosphorus atom in the transition state.

Breslow et al. were the first to explore the effect of functional groups within simple metal complexes, their intention being to emulate secondary enzyme active site features that are either known or postulated to contribute to the phosphoester cleavage activity. The incorporation of basic groups such as thiophenolate or imidazole as in $\left[\mathrm{Zn}^{11}\left(\mathrm{HL}^{53}\right)\right]^{2+}$ and $\left[\mathrm{Zn}^{11}\left(\mathrm{HL}^{54}\right)\right]^{2+}$ were shown to accelerate HPNPP cleavage 9-fold and 20-fold, respectively, compared to the reference complex $\left[\mathrm{Zn}^{\prime \prime}\left(\mathrm{HL}^{52}\right)\right]^{2+}$ (Chart 20). ${ }^{283}$
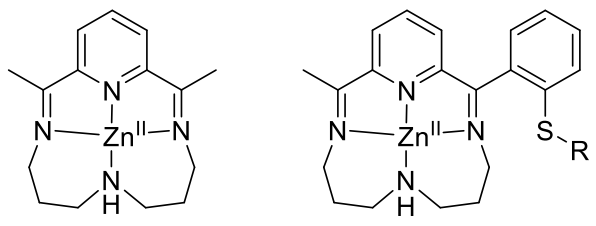

$$
\left[\mathrm{Zn} n\left(\mathrm{HL}^{52}\right)\right]^{2+}
$$

$$
\begin{aligned}
& {\left[\mathrm{Zn}^{\mathrm{II}}\left(\mathrm{HL}^{53}\right)\right]^{2+}: \mathrm{R}=\mathrm{H}} \\
& {\left[\mathrm{Zn}^{\mathrm{II}}\left(\mathrm{HL}^{54}\right)\right]^{2+}: \mathrm{R}=\text { imidazole }}
\end{aligned}
$$

Chart 20: Zinc(II) complexes employed by Breslow et al. studying the impact of basic residues proximal to the $\mathrm{Zn}$ " center. ${ }^{283}$

Subsequently Kövári and Krämer reported a significant increase in BPNPP cleavage activity by incorporation of amine groups at the end of rigid alkyne pendants of $\mathrm{Zn}$ "-bpy complexes. ${ }^{157}$ While the BPNPP hydrolysis activity for $\left[\mathrm{Zn}^{\prime \prime}\left(\mathrm{L}^{55}\right)\left(\mathrm{OH}_{2}\right)_{3}\right]^{2+}$ (Chart 21) was found to be similar to that of the parent complex $\left[\mathrm{Zn}^{\prime \prime}(\mathrm{bpy})\left(\mathrm{OH}_{2}\right)_{3}\right]^{2+}$, a significantly higher hydrolysis rate was observed with complex $\left[\mathrm{Zn}^{\prime \prime}\left(\mathrm{L}^{56}\right)\left(\mathrm{OH}_{2}\right)_{3}\right]^{2+}$ (Chart 21), ascribed to the hydrogen bonding capacity of the amino residues. ${ }^{157}$ 


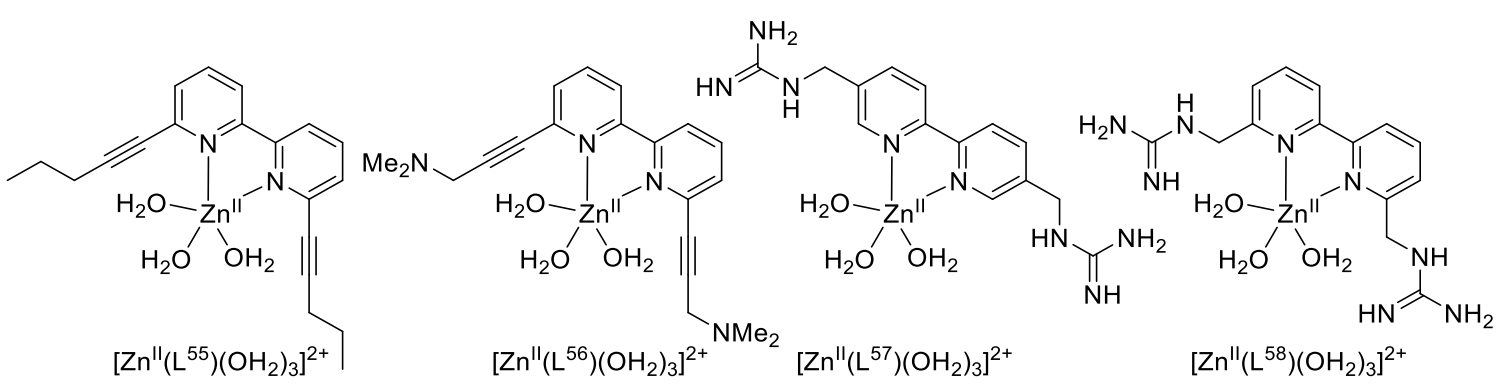

Chart 21: Zinc(II) complexes used by Kövári, Krämer and He et al. to study the effect of hydrogen bonding towards the phosphoester cleavage. ${ }^{157,284}$

With a similar model system the effect of guanidinium residues, mimicking the secondary coordination sphere of phosphatases at different positions with respect to the $\mathrm{Zn}$ " center, was investigated by He et al. ${ }^{284}$ The kinetic data revealed that $\left[\mathrm{Zn}^{\prime \prime}\left(\mathrm{L}^{57}\right)\left(\mathrm{OH}_{2}\right)_{3}\right]^{2+}$ and $\left[\mathrm{Zn}^{11}\left(\mathrm{~L}^{58}\right)\left(\mathrm{OH}_{2}\right)_{3}\right]^{2+}$ (Chart 21) accelerated BPNPP hydrolysis nearly 300-fold and 600-fold, compared to their analoguous $\left[\mathrm{Zn}^{\prime \prime}(\mathrm{bpy})\left(\mathrm{OH}_{2}\right)_{3}\right]^{2+}$ complex, missing guanidinium groups (Table 18). ${ }^{284}$

Table 18: Kinetic data $\left(\mathrm{k}_{\mathrm{cat}}\right.$ in $\left[10^{-3} \mathrm{~s}^{-1}\right], \mathrm{K}_{\mathrm{M}}$ in $[\mathrm{mM}]$ and $\mathrm{k}_{\mathrm{cat}} / \mathrm{K}_{\mathrm{M}}$ in $\left.\left[\mathrm{s}^{-1} \mathrm{M}^{-1}\right]\right)$ of BPNPP hydrolysis reported for $\left[\mathrm{Zn}^{\prime \prime}(\mathrm{bpy})\left(\mathrm{OH}_{2}\right)_{3}\right]^{2+},\left[\mathrm{Zn}^{\prime \prime}\left(\mathrm{L}^{58}\right)\left(\mathrm{OH}_{2}\right)_{3}\right]^{2+}$, and $\left[\mathrm{Zn}^{\prime \prime}\left(\mathrm{L}^{57}\right)\left(\mathrm{OH}_{2}\right)_{3}\right]^{2+}$ (all kinetic studies were undertaken in aqueous solution). ${ }^{284}$

\begin{tabular}{lllll} 
complex & $\mathrm{k}_{\text {cat }}$ & $\mathrm{K}_{\mathrm{M}}$ & $\mathrm{k}_{\text {cat }} / \mathrm{K}_{\mathrm{M}}$ & $\mathrm{pH}_{\mathrm{MM}}$ \\
\hline$\left[\mathrm{Zn} \text { "(bpy) }\left(\mathrm{OH}_{2}\right)_{3}\right]^{2+}$ & $0.57 \cdot 10^{-3}$ & 6.82 & $8.35 \cdot 10^{-5}$ & 7.2 \\
{$\left[\mathrm{Zn} "\left(\mathrm{~L}^{57}\right)\left(\mathrm{OH}_{2}\right)_{3}\right]^{2+}$} & 0.14 & 2.08 & 0.07 & 7.2 \\
{$\left[\mathrm{Zn}\left(\mathrm{L}^{58}\right)\left(\mathrm{OH}_{2}\right)_{3}\right]^{2+}$} & 0.37 & 0.70 & 0.53 & 7.2 \\
\hline
\end{tabular}

Due to the fact that the $\mathrm{K}_{\mathrm{M}}$ values for $\left[\mathrm{Zn}^{\prime \prime}\left(\mathrm{L}^{57}\right)\left(\mathrm{OH}_{2}\right)_{3}\right]^{2+}$ and $\left[\mathrm{Zn}^{\prime \prime}\left(\mathrm{L}^{58}\right)\left(\mathrm{OH}_{2}\right)_{3}\right]^{2+}$, both bearing guanidinium groups, were lower than for the reference complex $\left[\mathrm{Zn}^{\prime \prime}(\mathrm{bpy})\left(\mathrm{OH}_{2}\right)_{3}\right]^{2+}$, the hydrogen bond donors in the ligands were proposed to facilitate phosphodiester cleavage by interaction with the substrate. Furthermore, the guanidinium arms were postulated (i) to assist the binding of the phosphate substrate to the $\mathrm{Zn}^{\|}$ion, (ii) to act as additional Lewis acid, which polarizes the phosphate substrate, and (iii) potentially to protonate the leaving group. ${ }^{284}$

Similarly, the positive contribution of non-coordinating amines in $\mathrm{Zn}^{\prime \prime}$ complexes of tris(pyridylmethyl)amine towards the HPNPP cleavage efficiency was exhibited with the complexes $\left[\mathrm{Zn}^{\prime \prime}\left(\mathrm{L}^{21}\right)\left(\mathrm{OH}_{2}\right)\right]^{2+}\left(\mathrm{K}_{\mathrm{cat}} / \mathrm{K}_{\mathrm{M}}=1.0 \cdot 10^{-3} \mathrm{M}^{-1} \mathrm{~s}^{-1}\right)$ and $\left[\mathrm{Zn}^{\prime \prime}\left(\mathrm{L}^{25}\right)\left(\mathrm{OH}_{2}\right)\right]^{2+}\left(\mathrm{k}_{\text {cat }} / \mathrm{K}_{\mathrm{M}}=7.9 \cdot 10^{-2} \mathrm{M}^{-1} \mathrm{~s}^{-1}\right)$ (Chart 22), ascribed to the capacity of the amino groups to activate the bound substrate towards intramolecular nucleophilic attack via hydrogen bonding. ${ }^{11,110}$ Such interactions were confirmed by a crystal structure of $\left[\mathrm{Zn}^{\prime \prime}\left(\mathrm{L}^{24}\right)\left(\mathrm{NO}_{3}\right)\right]^{+}$derived from a similar ligand with two amino residues, forming hydrogen bonds to the coordinating oxygen atom of a $\mathrm{NO}_{3}{ }^{-}$anion. ${ }^{285}$ On the basis of DFT calculations a general base catalysis mechanism for the phosphodiester cleavage by complex $\left[\mathrm{Zn}^{\prime \prime}\left(\mathrm{L}^{25}\right)\left(\mathrm{OH}_{2}\right)\right]^{2+}$ was proposed, in which hydrogen-bonding interactions serve to stabilize the key 
five-coordinate phosphorus intermediate as well as lower the energy barrier of proton transfer reactions by enhancing solvation. ${ }^{11,286}$

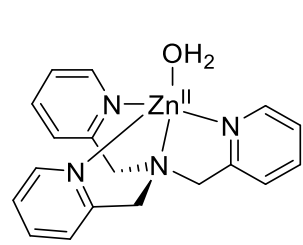

$\left[\mathrm{Zn}^{\prime \prime}\left(\mathrm{L}^{21}\right)\left(\mathrm{H}_{2} \mathrm{O}\right)\right]^{2+}$

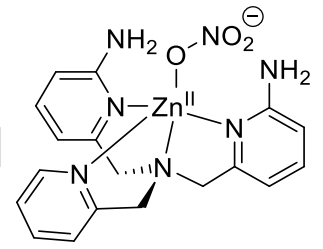

$\left[\mathrm{Zn}^{\prime \prime}\left(\mathrm{L}^{24}\right)\left(\mathrm{NO}_{3}\right)\right]^{+}$

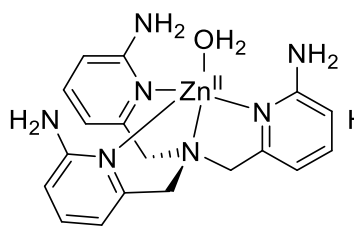

$\left[\mathrm{Zn}^{\prime \prime}\left(\mathrm{L}^{25}\right)\left(\mathrm{H}_{2} \mathrm{O}\right)\right]^{2+}$

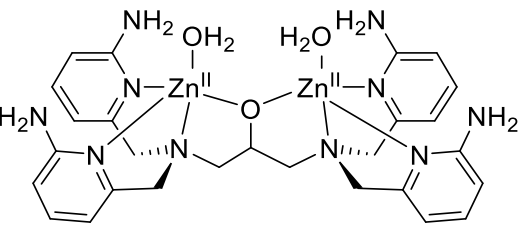

$\left[\mathrm{Zn}_{2}{ }_{2}\left(\mathrm{~L}^{7}\right)\left(\mathrm{H}_{2} \mathrm{O}\right)_{2}\right]^{4+}$

Chart 22: Zinc(II) complexes employed to study the impact of amino residues towards the cleavage of phosphodiesters. ${ }^{110,112,285}$

Recently the approach to incorporate hydrogen bond donors into zinc(II) model complexes has been extended to the synthesis of dinuclear metal-ion complexes with hydrogen bond donors in proximity to the metal centers. In this way, the dizinc(II) system $\left[\mathrm{Zn}_{2}\left(\mathrm{~L}^{7}\right)\left(\mathrm{OH}_{2}\right)_{2}\right]^{4+}$ was developed to be capable of forming four hydrogen bonds with a phosphate ester substrate as demonstrated by a X-ray structure of $\left[\mathrm{Zn}_{2}{ }_{2}\left(\mathrm{~L}^{7}\right)(\mathrm{PNPP})\right]^{3+} \cdot{ }^{112}\left[\mathrm{Zn}_{2}{ }_{2}\left(\mathrm{~L}^{7}\right)\left(\mathrm{OH}_{2}\right)_{2}\right]^{4+}$ is currently one of the most active synthetic cleavage agents, accelerating the cleavage of HPNPP by a factor of almost a million $\left(\mathrm{k}_{\mathrm{cat}}=0.017 \mathrm{~s}^{-1}, \mathrm{~K}_{\mathrm{M}}=0.32 \mathrm{mM}\right.$ at $\left.\mathrm{pH}=7.4\right)$, ascribed to the cooperation of double Lewis activation by two $\mathrm{Zn}$ " ions within the complex and the hydrogen bonding environment provided by the ligand backbone. ${ }^{112}$

Studying the phosphoester cleavage activity of $m$-xylyl based dizinc(II) complexes substituted with alkyl, amino or acetamido residues (Chart 23) an increased activity towards HPNPP transesterification while all functionalized dizinc(II) complexes $\left[\mathrm{Zn}^{\prime \prime}\left(\mathrm{L}^{60}\right)\left(\mathrm{OH}_{2}\right)_{2}\right]^{2+}$, $\left[\mathrm{Zn}^{\prime \prime}\left(\mathrm{L}^{61}\right)\left(\mathrm{OH}_{2}\right)_{2}\right]^{2+}$ and $\left[\mathrm{Zn}^{\prime \prime}\left(\mathrm{L}^{62}\right)\left(\mathrm{OH}_{2}\right)_{2}\right]^{2+}$ exhibited greater activity than the parent complex $\left[\mathrm{Zn}^{\prime \prime}\left(\mathrm{L}^{59}\right)\left(\mathrm{OH}_{2}\right)_{2}\right]^{2+} .287$

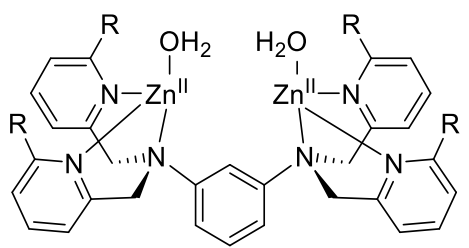

$$
\begin{aligned}
& {\left[\mathrm{Zn}_{2}{ }_{2}\left(\mathrm{~L}^{59}\right)\left(\mathrm{OH}_{2}\right)_{2}\right]^{4+}: \mathrm{R}=\mathrm{H}} \\
& {\left[\mathrm{Zn}_{2}{ }_{2}\left(\mathrm{~L}^{60}\right)\left(\mathrm{OH}_{2}\right)_{2}\right]^{4+}: \mathrm{R}=\mathrm{CH}_{3}} \\
& {\left[\mathrm{Zn}_{2}{ }_{2}\left(\mathrm{~L}^{61}\right)\left(\mathrm{OH}_{2}\right)_{2}\right]^{4+}: \mathrm{R}=\mathrm{NH}_{2}} \\
& {\left[\mathrm{Zn}_{2}{ }_{2}\left(\mathrm{~L}^{62}\right)\left(\mathrm{OH}_{2}\right)_{2}\right]^{4+}: \mathrm{R}=\mathrm{NH}(\mathrm{C}=\mathrm{O}) \mathrm{CH}_{3}}
\end{aligned}
$$

Chart 23: Dizinc(II) complexes used to study the impact of methyl, amino and acetamido functionalities proximal to the $\mathrm{Zn}$ " centers. $^{287}$

Closer inspection of the kinetic data revealed that the differences in the $\mathrm{H}$-bond donating abilities of the amino and acetamido residues are only subtly manifested in the strength of substrate binding by $\left[\mathrm{Zn}^{\prime \prime}\left(\mathrm{L}^{61}\right)\left(\mathrm{OH}_{2}\right)_{2}\right]^{2+}$ and $\left[\mathrm{Zn}^{\prime \prime}\left(\mathrm{L}^{62}\right)\left(\mathrm{OH}_{2}\right)_{2}\right]^{2+}\left(\mathrm{K}_{\mathrm{M}}\right.$ differs by $\left.\sim 3.5\right)$ and the catalytic rate constant (factor of 2). ${ }^{287}$ 
Table 19: Kinetic data $\left(\mathrm{k}_{\mathrm{cat}}\right.$ in $\left[\mathrm{s}^{-1}\right], \mathrm{K}_{\mathrm{M}}$ in $[\mathrm{mM}]$ and $\mathrm{k}_{\mathrm{cat}} / \mathrm{K}_{\mathrm{M}}$ in $\left.\left[10^{3} \mathrm{~s}^{-1} \mathrm{M}^{-1}\right]\right)$ of HPNPP transesterification reported for $\left[\mathrm{Zn}_{2}\left(\mathrm{~L}^{59}\right)\left(\mathrm{OH}_{2}\right)_{2}\right]^{4+},\left[\mathrm{Zn}_{2}\left(\mathrm{~L}^{60}\right)\left(\mathrm{OH}_{2}\right)_{2}\right]^{4+},\left[\mathrm{Zn}_{2}{ }_{2}\left(\mathrm{~L}^{61}\right)\left(\mathrm{OH}_{2}\right)_{2}\right]^{4+}$, and $\left[\mathrm{Zn}_{2}{ }_{2}\left(\mathrm{~L}^{62}\right)\left(\mathrm{OH}_{2}\right)_{2}\right]^{4+}$ (all kinetic studies were undertaken in methanol under optimal $\mathrm{pH}$ conditions). ${ }^{287}$

\begin{tabular}{llll} 
complex & $\mathrm{k}_{\text {cat }}$ & $\mathrm{K}_{\mathrm{M}}$ & $\mathrm{k}_{\text {cat }} / \mathrm{K}_{\mathrm{M}}$ \\
\hline$\left[\mathrm{Zn}_{2}{ }_{2}\left(\mathrm{~L}^{59}\right)\left(\mathrm{OH}_{2}\right)_{2}\right]^{4+}$ & 0.13 & 0.08 & 1.6 \\
{$\left[\mathrm{Zn}_{2}^{\prime \prime}\left(\mathrm{L}^{60}\right)\left(\mathrm{OH}_{2}\right)_{2}\right]^{4+}$} & 6.2 & 0.21 & 29.8 \\
{$\left[\mathrm{Zn}_{2}{ }_{2}\left(\mathrm{~L}^{61}\right)\left(\mathrm{OH}_{2}\right)_{2}\right]^{4+}$} & 3.89 & 0.24 & 16 \\
{$\left[\mathrm{Zn}_{2}{ }_{2}\left(\mathrm{~L}^{62}\right)\left(\mathrm{OH}_{2}\right)_{2}\right]^{4+}$} & 7.2 & 0.07 & 109 \\
\hline
\end{tabular}

However, comparison of the $\mathrm{K}_{\mathrm{M}}$ values reveals that (i) the acetamide derivative $\left[\mathrm{Zn}_{2}\left(\mathrm{~L}^{62}\right)\left(\mathrm{OH}_{2}\right)_{2}\right]^{4+}$ and the unsubstituted complex $\left[\mathrm{Zn}_{2}{ }_{2}\left(\mathrm{~L}^{59}\right)\left(\mathrm{OH}_{2}\right)_{2}\right]^{4+}$ have comparable substrate affinity, and (ii) both the methyl derivative $\left[\mathrm{Zn}_{2}\left(\mathrm{~L}^{60}\right)\left(\mathrm{OH}_{2}\right)_{2}\right]^{4+}$ and the amino derivative $\left[\mathrm{Zn}_{2}\left(\mathrm{~L}^{61}\right)\left(\mathrm{OH}_{2}\right)_{2}\right]^{4+}$ bind the phosphoester substrate slightly more weakly than does $\left[\mathrm{Zn}_{2}{ }_{2}\left(\mathrm{~L}^{59}\right)\left(\mathrm{OH}_{2}\right)_{2}\right]^{4+}$, missing those functionalities. Moreover, the hydrolysis rates are in the same range for all substituted complexes suggesting that the different functionalities (alkyl, amino, acetamido) are all equally capable of stabilizing the transition state. In conclusion, the effect of the functionalization of the dizinc(II) catalyst with hydrophobic and sterically demanding $\mathrm{CH}_{3}$ groups seems comparable afforded by amino and acetamido substituents. ${ }^{287}$ Therefore, other effects such as steric and local polarity seem to be as important in such systems as hydrogen-bond formation and should be considered in order to generate more active model complexes.

The function of the $\mathrm{Zn}$ " ions during transesterification is the activation of the substrate, and a positive effect of adjacent hydrogen bond donors on the substrate activation was discovered studying mono- and dinuclear model complexes, providing hydrogen bond donors proximal to the $\mathrm{Zn}^{\text {" }}$ center(s). In the case of the phosphoester hydrolysis the task of the metal ions in the model complexes is not only the phosphoester activation but also the generation of the hydroxide nucleophile. Although a positive effect of the presence of two $\mathrm{Zn}$ " ions in close distance has been reported to have an accelerating effect on the hydrolysis of phosphoester, ${ }^{86-89,} 288$ the impact of hydrogen bond donors has only been studied with a few monozinc(II) complexes providing hydrogen bonding capacity. In this work, the two strategies of (i) a dinuclear core and (ii) the provision of hydrogen bonding ability was combined to develop more accurate dizinc(II) model complexes for phosphatases. More precisely, dizinc(II) complexes have been prepared with amino and pivaloyl-amide residues in the 2-position of two of the four coordinating pyridine moieties (Chart 24). The synthesis and characterization of these dizinc(II) complexes has been discussed in Chapter 4. 


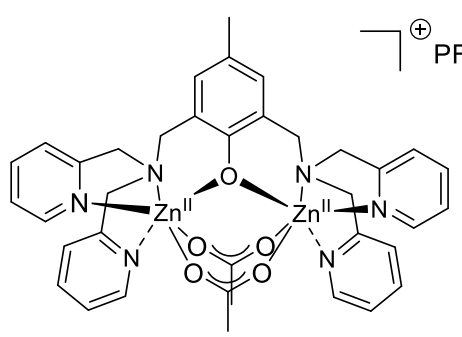

$\left[\mathrm{Zn}_{2}\left(\mathrm{~L}^{1}\right)(\mu-\mathrm{OAc})_{2}\right] \mathrm{PF} \mathrm{F}_{6}$

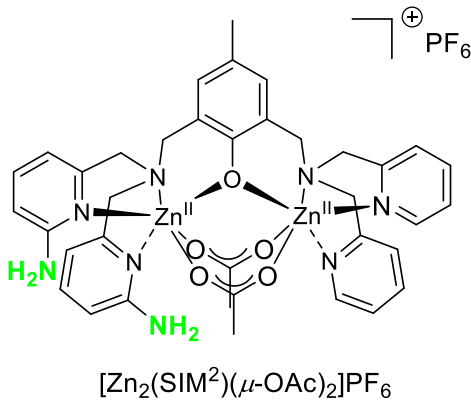

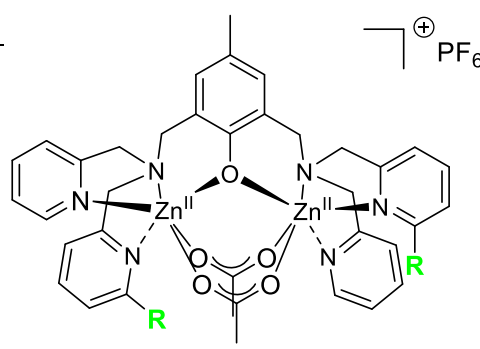

$\left[\mathrm{Zn}_{2}\left(\mathrm{H}_{2} \mathrm{~L}^{2}\right)(\mu-\mathrm{OAc})_{2}\right] \mathrm{PF} \mathrm{F}_{6}$

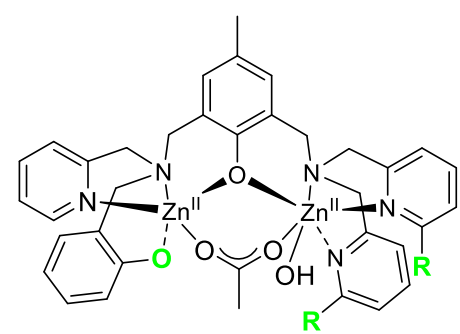

$\left[\mathrm{Zn}_{2}\left(\mathrm{H}_{2} \mathrm{SIM}^{3}\right)(\mu-\mathrm{OAc})(\mathrm{OH})\right]$

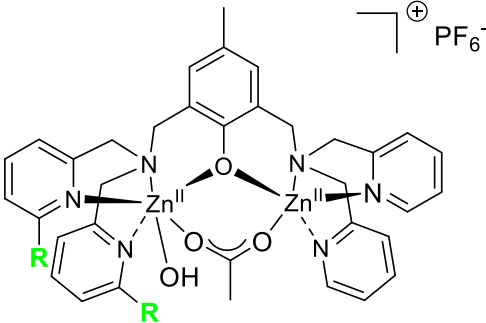

$\left[\mathrm{Zn}_{2}\left(\mathrm{H}_{2} \mathrm{SIM}^{1}\right)(\mu-\mathrm{OAc})(\mathrm{OH})\right] \mathrm{PF}_{6}$<smiles>CC(C)(C)C(=O)NC=[Fe]</smiles>

Chart 24: Dizinc(II) complexes employed in this chapter.

Comparison of the functionalized complexes $\left[\mathrm{Zn}_{2}{ }_{2}\left(\mathrm{H}_{2} \mathrm{~L}^{2}\right)(\mu-\mathrm{OAc})_{2}\right]^{+}$, $\left[\mathrm{Zn}_{2}{ }_{2}\left(\mathrm{H}_{2} \mathrm{SIM}^{1}\right)(\mu-\mathrm{OAc})(\mathrm{OH})\right]^{+}$, and $\left[\mathrm{Zn}_{2}{ }_{2}\left(\mathrm{SIM}^{2}\right)(\mu-\mathrm{OAc})_{2}\right]^{+}$to the unsubstituted complex $\left[\mathrm{Zn}_{2}{ }_{2}\left(\mathrm{~L}^{1}\right)(\mu-\mathrm{OAC})_{2}\right]^{+}$enables the study of the impact of hydrogen bond donors proximal the dizinc(II) center towards the hydrolysis of phosphoesters. Moreover, an insight in the effect of the position of hydrogen bond donors could be achieved via comparison of the two complexes $\left[\mathrm{Zn}_{2}{ }_{2}\left(\mathrm{H}_{2} \mathrm{SIM}^{1}\right)(\mu-\mathrm{OAc})(\mathrm{OH})\right]^{+}$and $\left[\mathrm{Zn}_{2}{ }_{2}\left(\mathrm{H}_{2} \mathrm{~L}^{2}\right)(\mu-\mathrm{OAc})_{2}\right]^{+}$, both bearing pivaloylamide residues, but at different positions with regard to the dizinc(II) core. Similarly, comparison of the asymmetric complexes $\left[\mathrm{Zn}_{2}\left(\mathrm{SIM}^{2}\right)(\mu-\mathrm{OAc})_{2}\right]^{+}$, bearing amino residues and $\left[\mathrm{Zn}_{2}{ }_{2}\left(\mathrm{H}_{2} \mathrm{SIM}^{1}\right)(\mu-\mathrm{OAc})(\mathrm{OH})\right]^{+}$, bearing sterically demanding pivaloyl-amide residues revealed information on the effect of a hindered $\mathrm{Zn}$ " center in a dizinc(II) complex. Furthermore, the two complexes $\left[\mathrm{Zn}_{2}{ }_{2}\left(\mathrm{H}_{2} \mathrm{SIM}^{1}\right)(\mu-\mathrm{OAc})(\mathrm{OH})\right]^{+}$and $\left[\mathrm{Zn}_{2}{ }_{2}\left(\mathrm{H}_{2} \mathrm{SIM}^{3}\right)(\mu-\mathrm{OAc})(\mathrm{OH})\right]$, which are identical with regard to the secondary coordination sphere, but differ in one of the coordinating ligands in the primary coordination sphere, enables the investigation of combining an asymmetric primary coordination sphere with an asymmetric secondary coordination sphere within a dizinc(II) complex. 


\subsection{PhOSPHOESTER HYdROlysis StUdies OF DiZINC(II) COMPLEXES}

The phosphoester hydrolysis reactivity of $\left[\mathrm{Zn}_{2}{ }_{2}\left(\mathrm{~L}^{1}\right)(\mu-\mathrm{OAC})_{2}\right]^{+}, \quad\left[\mathrm{Zn}_{2}{ }_{2}\left(\mathrm{H}_{2} \mathrm{~L}^{2}\right)(\mu-\mathrm{OAc})_{2}\right]^{+}$, $\left[\mathrm{Zn}_{2}{ }_{2}\left(\mathrm{H}_{2} \mathrm{SIM}^{1}\right)(\mu-\mathrm{OAc})(\mathrm{OH})\right]^{+}, \quad\left[\mathrm{Zn}_{2}{ }_{2}\left(\mathrm{SIM}^{2}\right)(\mu-\mathrm{OAc})_{2}\right]^{+}$, and $\left[\mathrm{Zn}_{2}\left(\mathrm{H}_{2} \mathrm{SIM}^{3}\right)(\mu-\mathrm{OAc})(\mathrm{OH})\right]$ was probed with a spectrophotometric assay using BDNPP as model substrate. The general experimental procedure explained in Chapter 6.3 was applied.

\section{pH and Concentration Dependence}

The rate vs. $\mathrm{pH}$ profiles for three of the dizinc(II) complexes show a nearly sigmoidal shape, whereas the corresponding profile for $\left[\mathrm{Zn}_{2}{ }_{2}\left(\mathrm{H}_{2} \mathrm{SIM}^{1}\right)(\mu-\mathrm{OAc})(\mathrm{OH})\right]^{+}$appears to be bell-shaped. This bell-shaped profile indicates that the catalyst-substrate complex ( $\left.E^{n} S\right)$ is most active in its mixed protonation state, where both protonation and deprotonation lead to a decrease of activity. Based on a fit adopting the equation for a monoprotic system, the assumed two active species in the case of $\left[\mathrm{Zn}_{2}{ }_{2}\left(\mathrm{~L}^{1}\right)(\mu-\mathrm{OAC})_{2}\right]^{+}$and $\left[\mathrm{Zn}_{2}\left(\mathrm{SIM}^{2}\right)(\mu-\mathrm{OAc})_{2}\right]^{+}$have the same activity. In contrast, the deprotonated active species of $\left[\mathrm{Zn}_{2}\left(\mathrm{H}_{2} \mathrm{~L}^{2}\right)(\mu-\mathrm{OAc})_{2}\right]^{+}$appears to play an important role in the catalytic cycle as the rate $v s$. $\mathrm{pH}$ profile rises steadily as the $\mathrm{pH}$ increases, leading to a $\gamma$ value of 4.19. Comparison of the profiles leads to the conclusion that the presence of two amine groups near one $\mathrm{Zn}^{\|}$center in $\left[\mathrm{Zn}_{2}\left(\mathrm{SIM}^{2}\right)(\mu-\mathrm{OAc})_{2}\right]^{+}$lowers the kinetically relevant $\mathrm{pK}_{\mathrm{a}}$ value by $1.3 \mathrm{pK}_{\mathrm{a}}$ units, in agreement with previously published results (introduced in Chapter 4). ${ }^{184}$ The influence of two amide groups at one $\mathrm{Zn}^{\text {"l }}$ center in $\left[\mathrm{Zn}_{2}{ }_{2}\left(\mathrm{H}_{2} \mathrm{SIM}^{1}\right)(\mu-\mathrm{OAc})(\mathrm{OH})\right]^{+}$is comparatively small; in contrast the symmetric incorporation of two amide moieties in $\left[\mathrm{Zn}_{2}{ }_{2}\left(\mathrm{H}_{2} \mathrm{~L}^{2}\right)(\mu-\mathrm{OAc})_{2}\right]^{+}$causes a significant effect, both in the profile shape (highest reactivity at high $\mathrm{pH}$; Figure $55 \mathrm{a}$ ) and the $\mathrm{pK}_{\mathrm{a}}$ values. 
a)

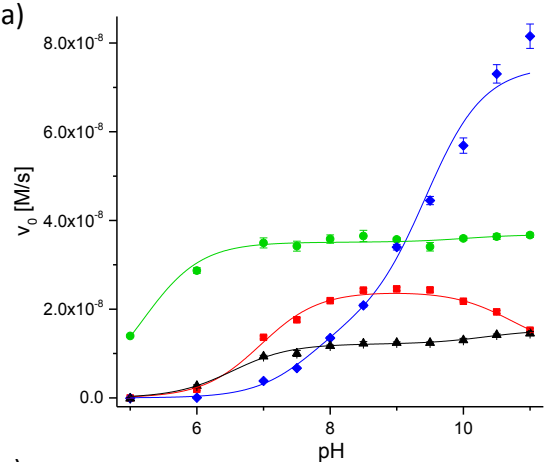

c)

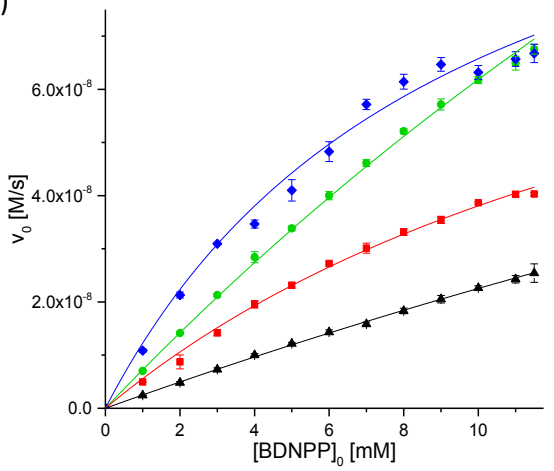

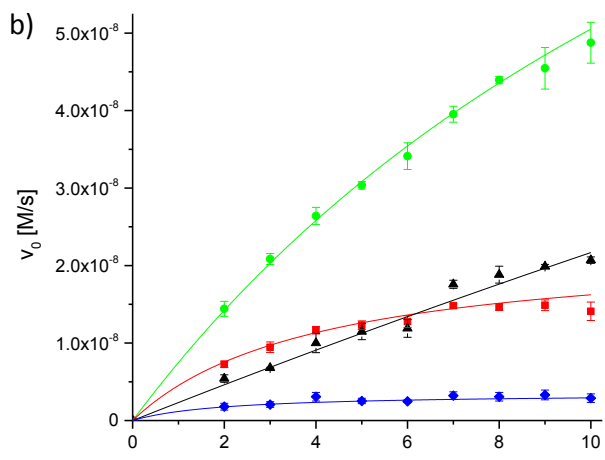

d)

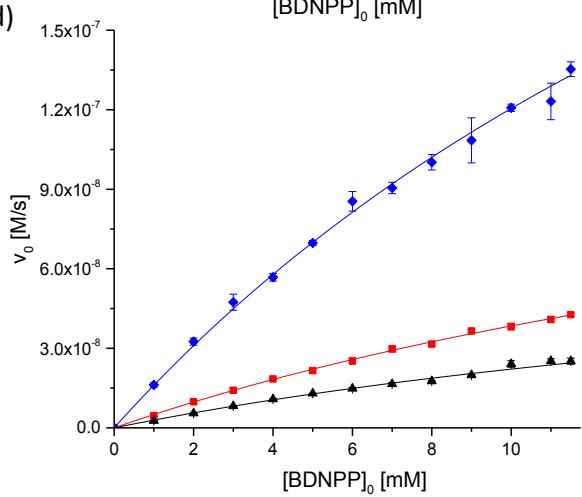

Figure 55: a) $\mathrm{pH}$ dependence of BDNPP hydrolysis activity for $\left[\mathrm{Zn}_{2}{ }_{2}\left(\mathrm{~L}^{1}\right)(\mu-\mathrm{OAc})_{2}\right]^{+}$(black triangles), $\left[\mathrm{Zn}_{2}{ }_{2}\left(\mathrm{H}_{2} \mathrm{~L}^{2}\right)(\mu-\mathrm{OAc})_{2}\right]^{+}$(blue diamonds), $\left[\mathrm{Zn}_{2}{ }_{2}\left(\mathrm{H}_{2} \mathrm{SIM}^{1}\right)(\mu-\mathrm{OAc})(\mathrm{OH})\right]^{+}$(red squares), and $\left[\mathrm{Zn}_{2}{ }_{2}\left(\mathrm{SIM}^{2}\right)(\mu-\mathrm{OAc})_{2}\right]^{+}$ (green circles) and substrate concentration dependence b) at $\mathrm{pH}=7, \mathrm{c}$ ) at pH $=9.5$ and d) at $\mathrm{pH}=11$.

The measurements of the dependence of the BDNPP hydrolysis rate on the complex concentration were determined at $\mathrm{pH}$ 9.5. The results show that for each complex the hydrolysis rates are linearly dependent on the complex concentration.

The dependence of the rate of hydrolysis on the BDNPP concentration were examined at three $\mathrm{pH}$ values (i.e. $\mathrm{pH}=7.0,9.5$ and 11 ) to assess the catalytic behavior of different protonation states (Figure $55 \mathrm{~b}, \mathrm{c}, \mathrm{d}) \cdot\left[\mathrm{Zn}_{2}{ }_{2}\left(\mathrm{SIM}^{2}\right)(\mu-\mathrm{OAc})_{2}\right]^{+}$was not tested at $\mathrm{pH}=11$ as no difference in the hydrolysis rate, compared to $\mathrm{pH}=9.5$, was detected during the previous $\mathrm{pH}$ dependence experiments. Due to the poor solubility of BDNPP in acetonitrile, assays with a starting concentration of the substrate higher than $11.5 \mathrm{mM}$ could not be investigated. The experimental data showed Michaelis-Menten saturation behavior for all complexes and were fitted to Equation 22. The derived parameter values $\mathrm{k}_{\mathrm{cat}}, \mathrm{K}_{\mathrm{M}}$ and $\mathrm{k}_{\mathrm{cat}} / \mathrm{K}_{\mathrm{M}}$ are listed in Table 20. 
Table 20. Kinetic data ( $k_{c a t}$ in $\left[10^{-3} s^{-1}\right], K_{M}$ in $[m M]$ and $k_{c a t} / K_{M}$ in $\left[s^{-1} M^{-1}\right]$ ) of BDNPP hydrolysis for $\left[\mathrm{Zn}_{2}{ }_{2}\left(\mathrm{~L}^{1}\right)(\mu-\mathrm{OAc})_{2}\right]^{+}, \quad\left[\mathrm{Zn}_{2}{ }_{2}\left(\mathrm{H}_{2} \mathrm{~L}^{2}\right)(\mu-\mathrm{OAc})_{2}\right]^{+}, \quad\left[\mathrm{Zn}_{2}{ }_{2}\left(\mathrm{H}_{2} \mathrm{SIM}^{1}\right)(\mu-\mathrm{OAc})(\mathrm{OH})\right]^{+}, \quad\left[\mathrm{Zn}_{2}\left(\mathrm{SIM}^{2}\right)(\mu-\mathrm{OAc})_{2}\right]^{+}, \quad$ and $\left[\mathrm{Zn}_{2}\left(\mathrm{H}_{2} \mathrm{SIM}^{3}\right)(\mu-\mathrm{OAC})(\mathrm{OH})\right]$.

\begin{tabular}{|c|c|c|c|c|c|c|}
\hline & $\mathrm{pH}$ & 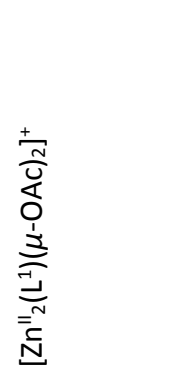 & 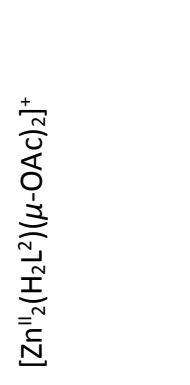 & 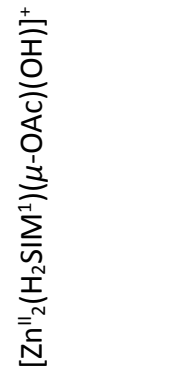 & 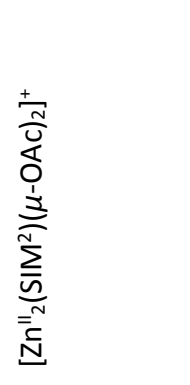 & 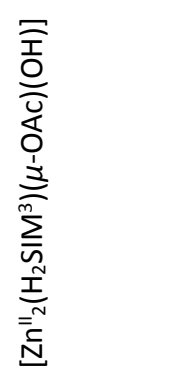 \\
\hline $\mathrm{pK}_{\mathrm{a}}(\mathrm{I})$ & & $6.53 \pm 0.05$ & $7.67 \pm 0.16$ & $6.96 \pm 0.06$ & $5.19 \pm 0.02$ & $7.33 \pm 0.16$ \\
\hline $\mathrm{pK}_{\mathrm{a}}(\mathrm{II})$ & & $10.33 \pm 0.83$ & $9.45 \pm 0.19$ & $10.72 \pm 0.28$ & $10.02 \pm 0.78$ & $10.01 \pm 0.40$ \\
\hline 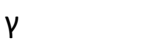 & & $1.26 \pm 0.08$ & $4.19 \pm 0.67$ & $0.45 \pm 0.14$ & $1.05 \pm 0.03$ & $2.01 \pm 0.32$ \\
\hline $\mathrm{k}_{\text {cat }}$ & 7 & $7.18 \pm 4.39$ & $0.09 \pm 0.01$ & $0.57 \pm 0.04$ & $3.49 \pm 0.25$ & \\
\hline $\mathrm{K}_{\mathrm{M}}$ & 7 & $123 \pm 79$ & $2 \pm 1$ & $4 \pm 1$ & $18 \pm 2$ & \\
\hline $\mathrm{k}_{\text {cat }} / \mathrm{K}_{\mathrm{M}}$ & 7 & 0.06 & 0.04 & 0.14 & 0.20 & \\
\hline $\mathrm{k}_{\text {cat }}$ & 9.5 & $5.08 \pm 0.85$ & $3.20 \pm 0.21$ & $2.70 \pm 0.21$ & $9.58 \pm 0.70$ & $8.67 \pm 0.89$ \\
\hline $\mathrm{K}_{\mathrm{M}}$ & 9.5 & $80 \pm 14$ & $9 \pm 1$ & $18 \pm 2$ & $52 \pm 4$ & $37 \pm 5$ \\
\hline $\mathrm{k}_{\mathrm{cat}} / \mathrm{K}_{\mathrm{M}}$ & 9.5 & 0.06 & 0.34 & 0.15 & 0.18 & 0.23 \\
\hline $\mathrm{k}_{\text {cat }}$ & 11 & $2.07 \pm 0.29$ & $10.83 \pm 0.62$ & $3.68 \pm 0.25$ & - & \\
\hline $\mathrm{K}_{\mathrm{M}}$ & 11 & $27 \pm 5$ & $26 \pm 2$ & $28 \pm 2$ & - & \\
\hline $\mathrm{k}_{\text {cat }} / \mathrm{K}_{\mathrm{M}}$ & 11 & 0.08 & 0.42 & 0.13 & - & \\
\hline $\begin{array}{l}\text { TON } \\
\text { (after } 8 d \text { ) }\end{array}$ & 9.5 & $109 \pm 5$ & $158 \pm 8$ & $101 \pm 4$ & $121 \pm 6$ & \\
\hline
\end{tabular}

While at $\mathrm{pH}=7\left[\mathrm{Zn}_{2}{ }_{2}\left(\mathrm{~L}^{1}\right)(\mu-\mathrm{OAC})_{2}\right]^{+}$, without hydrogen bond donors, exhibits the highest rate of hydrolysis, $\left[\mathrm{Zn}_{2}\left(\mathrm{SIM}^{2}\right)(\mu-\mathrm{OAc})_{2}\right]^{+}$is the most efficient catalyst due to its smaller $\mathrm{K}_{\mathrm{M}}$ value. Moreover, the Michaelis-Menten constants $\mathrm{K}_{M}$ are lower for all complexes providing a hydrogen bond network, compared to $\left[\mathrm{Zn}_{2}\left(\mathrm{~L}^{1}\right)(\mu-\mathrm{OAC})_{2}\right]^{+}$, both at $\mathrm{pH}=7$ and $\mathrm{pH}=9.5$. The net interpretation of this observation is that complexes that facilitate hydrogen bonding exhibit higher affinities towards the phosphodiester substrate and therefore reveal higher catalytic efficiencies than $\left[\mathrm{Zn}_{2}{ }_{2}\left(\mathrm{~L}^{1}\right)(\mu-\mathrm{OAc})_{2}\right]^{+}$(except for $\left[\mathrm{Zn}_{2}{ }_{2}\left(\mathrm{H}_{2} \mathrm{~L}^{2}\right)(\mu-\mathrm{OAc})_{2}\right]^{+}$at $\mathrm{pH}=7$ ).

Furthermore, the measurements at $\mathrm{pH}=11$ resulted in significantly higher $\mathrm{k}_{\text {cat }}$ values for the complexes that support hydrogen bond formation, compared to the reference complex $\left[\mathrm{Zn}_{2}{ }_{2}\left(\mathrm{~L}^{1}\right)(\mu-\mathrm{OAC})_{2}\right]^{+}$. The Michaelis-Menten constants obtained under these more basic conditions are comparable for all three complexes. Therefore, the complexes $\left[\mathrm{Zn}_{2}{ }_{2}\left(\mathrm{H}_{2} \mathrm{SIM}^{1}\right)(\mu-\mathrm{OAc})(\mathrm{OH})\right]^{+}$and $\left[\mathrm{Zn}_{2}{ }_{2}\left(\mathrm{H}_{2} \mathrm{~L}^{2}\right)(\mu-\mathrm{OAc})_{2}\right]^{+}$have twice and five times higher catalytic efficiencies than $\left[\mathrm{Zn}_{2}{ }_{2}\left(\mathrm{~L}^{1}\right)(\mu-\mathrm{OAC})_{2}\right]^{+}$.

Due to the insolubility of the neutral complex $\left[\mathrm{Zn}_{2}{ }_{2}\left(\mathrm{H}_{2} \mathrm{SIM}^{3}\right)(\mu-\mathrm{OAc})(\mathrm{OH})\right], 0.2$ vol\% perchloric acid needed to be added in order to dissolve the complex in acetonitrile. The exact appearance of the complex in slight acidic solution is not fully clarified, as discussed in Chapter 4.3. However, the 
$\mathrm{pH}$ profile of $\left[\mathrm{Zn}_{2}{ }_{2}\left(\mathrm{H}_{2} \mathrm{SIM}^{3}\right)(\mu-\mathrm{OAc})(\mathrm{OH})\right]$, depicted in Figure 56a, exhibited two deprotonation steps in the region between 5 and 11 similar to $\left[\mathrm{Zn}_{2}{ }_{2}\left(\mathrm{H}_{2} \mathrm{SIM}^{1}\right)(\mu-\mathrm{OAc})(\mathrm{OH})\right]^{+}$. Compared to complex $\left[\mathrm{Zn}_{2}{ }_{2}\left(\mathrm{H}_{2} \mathrm{SIM}^{1}\right)(\mu-\mathrm{OAc})(\mathrm{OH})\right]^{+}$, bearing only pyridine coordinating ligands besides the bridging phenolate residue, the $\mathrm{pK}_{2}(\mathrm{I})$ value of $\left[\mathrm{Zn}_{2}{ }_{2}\left(\mathrm{H}_{2} \mathrm{SIM}^{3}\right)(\mu-\mathrm{OAc})(\mathrm{OH})\right]$ is shifted to a higher value as expected due to the electron-rich terminal phenolate ligand. In contrast, the second $\mathrm{pK}_{\mathrm{a}}$ value of $\left[\mathrm{Zn}_{2}{ }_{2}\left(\mathrm{H}_{2} \mathrm{SIM}^{3}\right)(\mu-\mathrm{OAc})(\mathrm{OH})\right], \mathrm{pK}_{\mathrm{a}}(\mathrm{II})$, is shifted to lower $\mathrm{pH}$ values compared to $\left[\mathrm{Zn}_{2}{ }_{2}\left(\mathrm{H}_{2} \mathrm{SIM}^{1}\right)(\mu-\mathrm{OAc})(\mathrm{OH})\right]^{+}$. Whilst in the case of $\left[\mathrm{Zn}_{2}{ }_{2}\left(\mathrm{H}_{2} \mathrm{SIM}^{1}\right)(\mu-\mathrm{OAc})(\mathrm{OH})\right]^{+}$the singly deprotonated form was found to be the most active species, $\left[\mathrm{Zn}_{2}{ }_{2}\left(\mathrm{H}_{2} \mathrm{SIM}^{3}\right)(\mu-\mathrm{OAc})(\mathrm{OH})\right]$ exhibited the most active form after two deprotonation steps with a $\gamma$-value of 2.
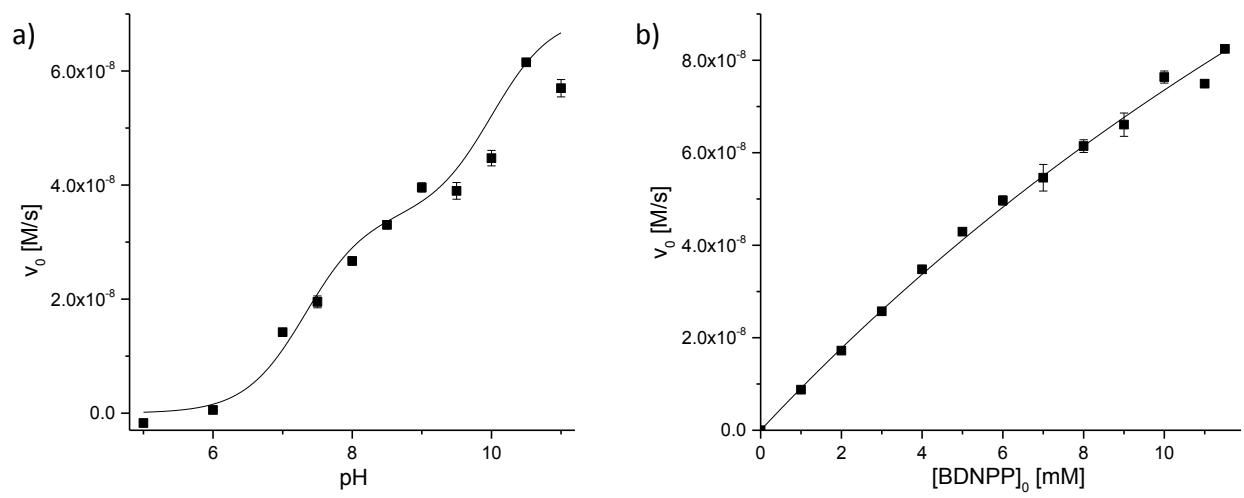

Figure 56: a) $\mathrm{pH}$ dependence of BDNPP hydrolysis activity and b) substrate concentration dependence at $\mathrm{pH}=9.5$ for $\left[\mathrm{Zn}_{2}{ }_{2}\left(\mathrm{H}_{2} \mathrm{SIM}^{3}\right)(\mu-\mathrm{OAc})(\mathrm{OH})\right]$.

The dependence of the BDNPP hydrolysis rate on the substrate concentration for $\left[\mathrm{Zn}_{2}{ }_{2}\left(\mathrm{H}_{2} \mathrm{SIM}^{3}\right)(\mu-\mathrm{OAC})(\mathrm{OH})\right]$ was investigated at $\mathrm{pH} 9.5$ and the results are depicted in Figure $56 \mathrm{~b}$. The experimental data were fitted to Equation 22 and the parameter values $\mathrm{k}_{\mathrm{cat}}, \mathrm{K}_{\mathrm{M}}$, and $\mathrm{k}_{\mathrm{cat}} / \mathrm{K}_{\mathrm{M}}$ are listed in Table 20. Comparison of the kinetic parameters of $\left[\mathrm{Zn}_{2}{ }_{2}\left(\mathrm{H}_{2} \mathrm{SIM}^{1}\right)(\mu-\mathrm{OAc})(\mathrm{OH})\right]^{+}$and $\left[\mathrm{Zn}_{2}{ }_{2}\left(\mathrm{H}_{2} \mathrm{SIM}^{3}\right)(\mu-\mathrm{OAc})(\mathrm{OH})\right]$, both with two pivaloyl-amide residues in an asymmetric manner with respect to the dizinc(II) core, but differing in the primary coordination sphere, allows statements regarding the impact of an electron-rich coordinating ligand. The respective $\mathrm{K}_{\mathrm{M}}$ values show that $\left[\mathrm{Zn}_{2}{ }_{2}\left(\mathrm{H}_{2} \mathrm{SIM}^{3}\right)(\mu-\mathrm{OAc})(\mathrm{OH})\right]$ exhibits lower substrate affinity than $\left[\mathrm{Zn}_{2}{ }_{2}\left(\mathrm{H}_{2} \mathrm{SIM}^{1}\right)(\mu-\mathrm{OAc})(\mathrm{OH})\right]^{+}$, ascribed to the more electron-rich primary coordination sphere. However, $\left[\mathrm{Zn}_{2}{ }_{2}\left(\mathrm{H}_{2} \mathrm{SIM}^{3}\right)(\mu-\mathrm{OAc})(\mathrm{OH})\right]$ revealed a three-fold increased hydrolysis rate compared to $\left[\mathrm{Zn}_{2}{ }_{2}\left(\mathrm{H}_{2} \mathrm{SIM}^{1}\right)(\mu-\mathrm{OAc})(\mathrm{OH})\right]^{+}$and following a higher catalytic efficiency.

Comparing the kinetic parameters of $\left[\mathrm{Zn}_{2}\left(\mathrm{H}_{2} \mathrm{SIM}^{3}\right)(\mu-\mathrm{OAc})(\mathrm{OH})\right]$ with all of the dizinc(II) complexes studied in this work reveals that the hydrolysis rate of $\left[\mathrm{Zn}_{2}{ }_{2}\left(\mathrm{H}_{2} \mathrm{SIM}^{3}\right)(\mu-\mathrm{OAc})(\mathrm{OH})\right]$ lies in the range of the hydrolysis rate determined for $\left[\mathrm{Zn}_{2}\left(\mathrm{SIM}^{2}\right)(\mu-\mathrm{OAc})_{2}\right]^{+}$, bearing two amino residues, and is significantly higher than the one of the reference complex $\left[\mathrm{Zn}_{2}{ }_{2}\left(\mathrm{~L}^{1}\right)(\mu-\mathrm{OAC})_{2}\right]^{+}$, 
missing hydrogen bonding capacity. Interestingly, the latter was not the case for $\left[\mathrm{Zn}_{2}{ }_{2}\left(\mathrm{H}_{2} \mathrm{SIM}^{1}\right)(\mu-\mathrm{OAc})(\mathrm{OH})\right]^{+}$, which deviates only in the primary coordination sphere from $\left[\mathrm{Zn}_{2}{ }_{2}\left(\mathrm{H}_{2} \mathrm{SIM}^{3}\right)(\mu-\mathrm{OAc})(\mathrm{OH})\right]$. This suggests that the acceleration of the BDNPP hydrolysis by a more electron-rich primary coordination sphere is more pronounced than the deceleration by two pivaloyl-amide residues adjacent one $\mathrm{Zn}^{\prime \prime}$ center.

\section{Turn Over Numbers}

Studies of the turn over number (TON) were conducted at $\mathrm{pH}=9.5$ and $25^{\circ} \mathrm{C}$ with $[\text { complex }]_{0}=15 \mathrm{nM}$ and $[\mathrm{BDNPP}]_{0}=5.25 \mu \mathrm{M}$. Samples were taken at various intervals during the experiment, diluted with solvent, and their UV-vis spectra were recorded to determine the amount of phosphoester hydrolysis. The increase in the absorbance at $400 \mathrm{~nm}$, assigned to the hydrolysis product 2,4-dinitrophenolate, was monitored over time and TON values after eight days were calculated using the Beer-Lambert Law. The resulting data are given in Figure 57 and Table 20.
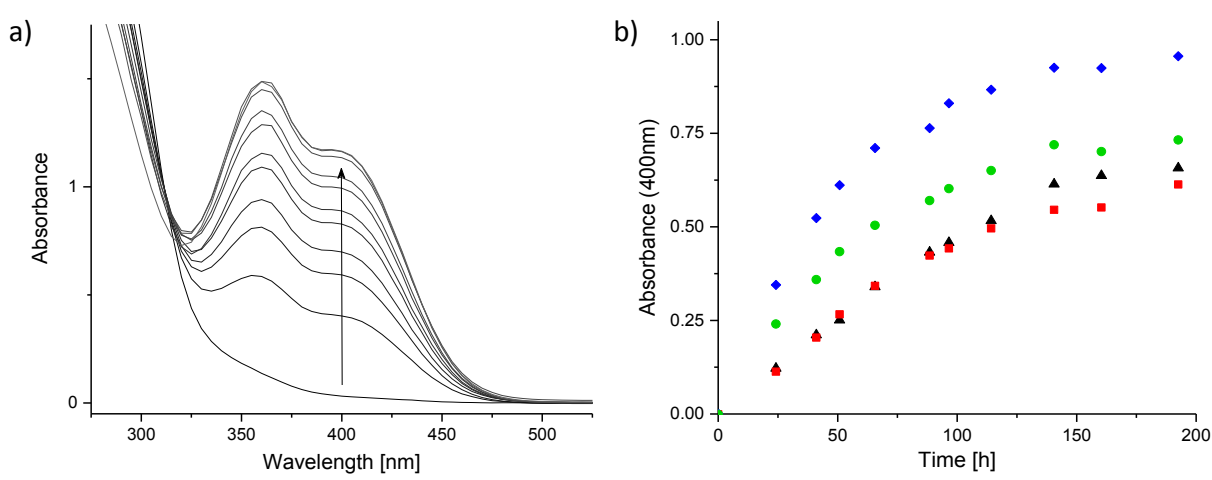

Figure 57: a) UV-vis spectra of the BDNPP hydrolysis assay with $\left[\mathrm{Zn}_{2}{ }_{2}\left(\mathrm{H}_{2} \mathrm{~L}^{2}\right)(\mu-\mathrm{OAc})_{2}\right]^{+}$, taken at different time points after substrate addition and $b$ ) time dependence of the absorbance band of 2,4-nitrophenolate (400nm) with $\left[\mathrm{Zn}_{2}{ }_{2}\left(\mathrm{~L}^{1}\right)(\mu-\mathrm{OAc})_{2}\right]^{+}$(black triangles), $\left[\mathrm{Zn}_{2}{ }_{2}\left(\mathrm{H}_{2} \mathrm{~L}^{2}\right)(\mu-\mathrm{OAc})_{2}\right]^{+}$(blue diamonds), $\left[\mathrm{Zn}_{2}{ }_{2}\left(\mathrm{H}_{2} \mathrm{SIM}^{1}\right)(\mu-\mathrm{OAc})(\mathrm{OH})\right]^{+}(\mathrm{red}$ squares) and $\left[\mathrm{Zn}_{2}{ }_{2}\left(\mathrm{SIM}^{2}\right)(\mu-\mathrm{OAC})_{2}\right]^{+}$(green circles).

Complexes generated from ligands which engender asymmetry exhibit a lower TON than the symmetric complex $\left[\mathrm{Zn}_{2}{ }_{2}\left(\mathrm{H}_{2} \mathrm{~L}^{2}\right)(\mu-\mathrm{OAc})_{2}\right]^{+}$. This implies that the presence of an amide moiety on each coordination site has a beneficial impact on the hydrolytic activity. The comparison of the time-dependent traces for the two asymmetric catalysts, $\left[\mathrm{Zn}_{2}{ }_{2}\left(\mathrm{H}_{2} \mathrm{SIM}^{1}\right)(\mu-\mathrm{OAc})(\mathrm{OH})\right]^{+}$and $\left[\mathrm{Zn}_{2}{ }_{2}\left(\mathrm{SIM}^{2}\right)(\mu-\mathrm{OAC})_{2}\right]^{+}$, indicate that there is also a decrease of the TON as a result of the bulky nature of the pivaloyl group in the former complex. Therefore, the hydrolysis rate of $\left[\mathrm{Zn}_{2}{ }_{2}\left(\mathrm{SIM}^{2}\right)(\mu-\mathrm{OAC})_{2}\right]^{+}$is in the first 70 hours significantly higher. Furthermore, the data obtained with $\left[\mathrm{Zn}_{2}{ }_{2}\left(\mathrm{H}_{2} \mathrm{SIM}^{1}\right)(\mu-\mathrm{OAc})(\mathrm{OH})\right]^{+}$are similar to those of the reference system $\left[\mathrm{Zn}_{2}{ }_{2}\left(\mathrm{~L}^{1}\right)(\mu-\mathrm{OAc})_{2}\right]^{+}$ during the first 150 hours after BDNPP addition. However, the difference in the traces after 150 hours reveal earlier inhibition in presence of two amide moieties at adjacent zinc(II) centers. It is 
proposed that an increased affinity for the hydrolysis product DNPP is the reason for this, resulting in the inhibition of the complex at an earlier point in time. The experiments demonstrate that the phosphoester hydrolysis rate of dizinc(II) complexes is influenced by (i) the availability of the catalytic center, and (ii) the possibility to interact with amine or amido hydrogen bond donors, whereas the opportunity for the formation of hydrogen bonds on both metal centers appears to have a beneficial effect for the hydrolytic activity.

Studies with a variety of complexes of bis[bis(2-substituted-pyridinyl-6-methyl)]amine type ligands have illustrated the impact of (i) enhanced $\mathrm{H}$-bonding effects in the second coordination sphere, (ii) the steric hindrance in the active site and beyond, and (iii) the polarity of the substituents, on the intramolecular hydrolysis of the substrate, for example the RNA model HPNPP. ${ }^{287}$ Moreover, an impaired BDNPP affinity was found for the dizinc(II) complex of a bulky phenolate-based ligand $\mathrm{HL}^{63}\left(\mathrm{k}_{\mathrm{cat}}=(0.97 \pm 0.21) \cdot 10^{-3} \mathrm{~s}^{-1} ; \mathrm{K}_{\mathrm{M}}=(7.01 \pm 2.57) \mathrm{mM}\right.$; $\left.\mathrm{k}_{\text {cat }} / \mathrm{K}_{\mathrm{M}}=0.14 \mathrm{~s}^{-1} \mathrm{M}^{-1}\right)$ compared to its unconcealed counterpart $\mathrm{H}_{2} \mathrm{~L}^{64}\left(\mathrm{k}_{\mathrm{cat}}=(2.45 \pm 0.27) \cdot 10^{-3} \mathrm{~s}^{-1}\right.$; $\left.\mathrm{K}_{\mathrm{M}}=(9.48 \pm 1.74) \mathrm{mM} ; \mathrm{k}_{\mathrm{cat}} / \mathrm{K}_{\mathrm{M}}=0.26 \mathrm{~s}^{-1} \mathrm{M}^{-1}\right)$, which was proposed to be a steric effect attributed to the bulky vinylbenzyl group. ${ }^{276}$

Therefore, the exact origin of the acceleration of the BDNPP hydrolysis with the dizinc(II) complexes studied in this work may be attributed in part to the enhanced $\mathrm{H}$-bonding effects, but the effects of other parameters in combination with $\mathrm{H}$-bonding, needs further investigation.

\section{Product Inhibition at the Active Site}

Studies of the hydrolysis of phosphomonoesters using DNPP as model substrate in the substrate concentration dependence assays as well as in the TON assays revealed no acceleration of phosphoester hydrolysis compared to the autohydrolysis assay for the dizinc(II) complexes $\left[\mathrm{Zn}_{2}{ }_{2}\left(\mathrm{~L}^{1}\right)(\mu-\mathrm{OAc})_{2}\right]^{+}, \quad\left[\mathrm{Zn}_{2}{ }_{2}\left(\mathrm{H}_{2} \mathrm{~L}^{2}\right)(\mu-\mathrm{OAc})_{2}\right]^{+}, \quad\left[\mathrm{Zn}_{2}{ }_{2}\left(\mathrm{H}_{2} \mathrm{SIM}^{1}\right)(\mu-\mathrm{OAc})(\mathrm{OH})\right]^{+}, \quad$ and $\left[\mathrm{Zn}_{2}\left(\mathrm{SIM}^{2}\right)(\mu-\mathrm{OAC})_{2}\right]^{+}$.

Similar to the phosphate inhibition of phosphatases, ${ }^{289,} 290$ the formation of an adduct with a bridging phosphomonoester between the two $\mathrm{Zn}^{\text {" }}$ sites is known to lead to inhibition of phosphoester hydrolysis in model complexes. ${ }^{291}$ Therefore, the influence of addition of DNPP to the reaction mixtures was explored. The presence of DNPP $(1 \mathrm{mM})$ in the kinetic assays was shown to decrease the hydrolytic rate, independently of whether the phosphomonoester was added before or simultaneously with the substrate BDNPP (Figure 58). 


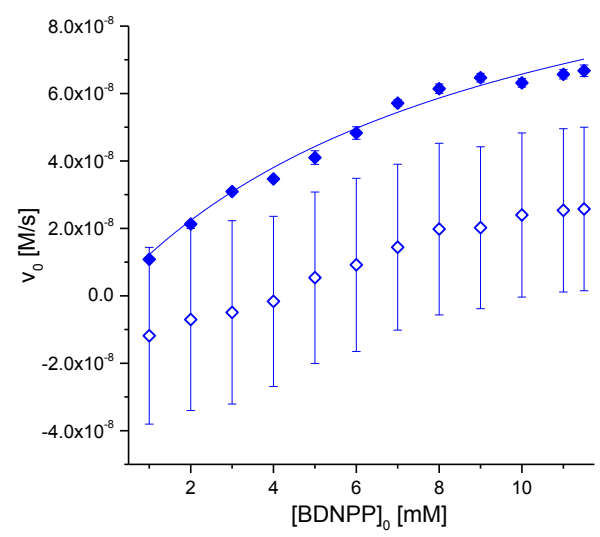

Figure 58: Initial rate vs. substrate concentration for the hydrolysis of BDNPP of $\left[\mathrm{Zn}_{2}{ }_{2}\left(\mathrm{H}_{2} \mathrm{~L}^{2}\right)(\mu-\mathrm{OAc})_{2}\right] P F_{6}$ with (unfilled symbols) and without (filled symbols) addition of DNPP.

The inhibited hydrolysis rates obtained in the presence of catalyst are, within the relatively large errors of these measurements, indistinguishable from the autohydrolysis rates under the same conditions (autohydrolysis experiments contain only phosphomono- and phosphodiester). These results demonstrate not only product inhibition but also that the hydrolysis product DNPP is more strongly bound to the complex than the phosphodiester substrate BDNPP.

\section{Crystal Structure of an Asymmetric Dizinc(II) Complex with the Phospho- monoester PNPP}

In order to explore the nature of product inhibition, the asymmetric complex $\left[\mathrm{Zn}_{2}{ }_{2}\left(\mathrm{H}_{2} \mathrm{SIM}^{1}\right)(\mu-\mathrm{OAc})(\mathrm{OH})\right]^{+}$was treated with an excess of the phosphomonoester PNPP in acetonitrile. PNPP was chosen as phosphomonoester substrate mimic due to its higher stability towards autohydrolysis compared to DNPP. After the solvent was removed and the remaining oil dissolved in methanol, colorless crystals suitable for X-ray crystallography were obtained by slow evaporation. The structure reveals that the inhibited complex contains one $\mu$-PNPP molecule, which forms a bridge between the two $\mathrm{Zn}$ " centers of the same dizinc(II) complex (Figure 59; selected structural parameters are listed in Table 21), in contrast to a previously published complex $\left[\mathrm{Zn}_{4}\left(\mathrm{~L}^{65}\right)_{2}\left(\mu-\mathrm{O}_{3} \mathrm{POC}_{6} \mathrm{H}_{4} \mathrm{NO}_{2}\right)\left(\mathrm{H}_{2} \mathrm{O}\right)_{2}\right]\left(\mathrm{PF}_{6}\right)_{2}$, in which the PNPP molecule forms not only an intramolecular bridge between the two $\mathrm{Zn}$ " ions but also a intermolecular bridge between two $\mathrm{Zn}$ " centers of adjacent dizinc(II) complexes. ${ }^{195}$ In $\left[\mathrm{Zn}_{2}{ }_{2}\left(\mathrm{H}_{2} \mathrm{SIM}^{1}\right)\left(\mu-\mathrm{O}_{3} \mathrm{POC}_{6} \mathrm{H}_{4} \mathrm{NO}_{2}\right)\right] \mathrm{PF}$, both $\mathrm{Zn}$ " ions are bound to the bridging phenoxido residue, to the nitrogen atoms of two pyridine ligands, to a tertiary amine and additionally to an oxygen atom of the bridging PNPP molecule. 


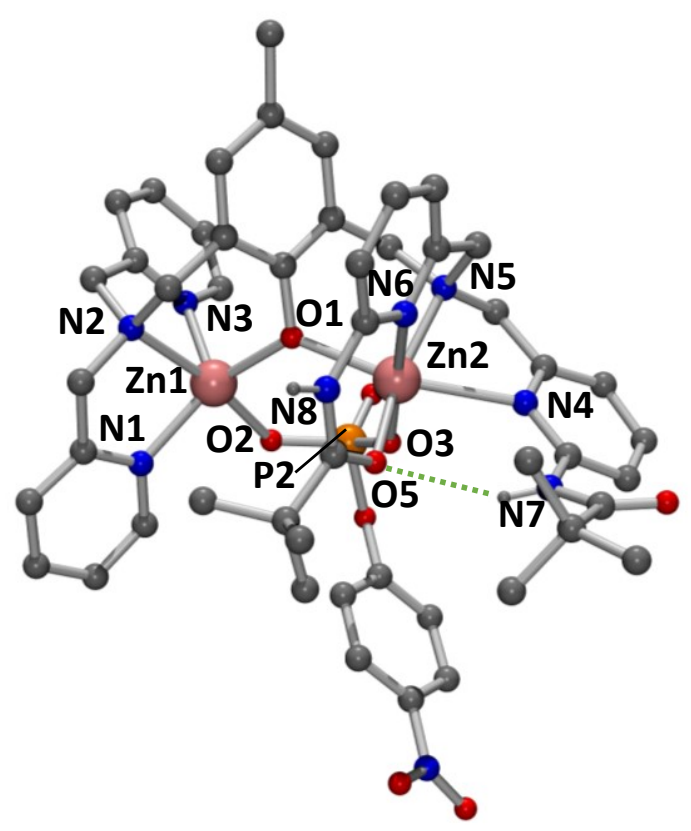

Figure 59: Structure of $\left[\mathrm{Zn}_{2}{ }_{2}\left(\mathrm{H}_{2} \mathrm{SIM}^{1}\right)\left(\mu-\mathrm{O}_{3} \mathrm{POC}_{6} \mathrm{H}_{4} \mathrm{NO}_{2}\right)\right] \mathrm{PF}_{6}$ showing hydrogen bond formation (green dotted lines; counter ions, non-coordinated solvent molecules, and hydrogen atoms, not involved in hydrogen bonding, have been omitted for clarity; crystallographic data and ORTEP plots with 50\% probability level of thermal ellipsoids appear in the Appendix, Table 34 and Figure 74).

Table 21: Selected bond lengths $(\AA \AA)$ and angles $\left({ }^{\circ}\right)$ for $\left[\mathrm{Zn}_{2}\left(\mathrm{H}_{2} \mathrm{SIM}^{1}\right)\left(\mu-\mathrm{O}_{3} \mathrm{POC}_{6} \mathrm{H}_{4} \mathrm{NO}_{2}\right)\right] \mathrm{PF} 6$ compared to $\left[\mathrm{Zn}_{2}{ }_{2}\left(\mathrm{H}_{2} \mathrm{SIM}^{1}\right)(\mu-\mathrm{OAC})(\mathrm{OH})\right] \mathrm{PF}_{6}$.

\begin{tabular}{l|ll} 
& {$\left[\mathrm{Zn}_{2}\left(\mathrm{H}_{2} \mathrm{SIM}^{1}\right)\left(\mu-\mathrm{O}_{3} \mathrm{POC}_{6} \mathrm{H}_{4} \mathrm{NO}_{2}\right)\right]^{+}$} & {$\left[\mathrm{Zn}_{2}{ }_{2}\left(\mathrm{H}_{2} \mathrm{SIM}^{1}\right)(\mu-\mathrm{OAc})(\mathrm{OH})\right]^{+}$} \\
\hline $\mathrm{Zn}(1) \cdots \mathrm{Zn}(2)$ & $3.6971(8)$ & $3.5422(7)$ \\
$\mathrm{Zn}(1)-\mathrm{O}(1)$ & $1.987(3)$ & $1.954(3)$ \\
$\mathrm{Zn}(1)-\mathrm{O}(2)$ & $2.011(3)$ & $2.003(3)$ \\
$\mathrm{Zn}(1)-\mathrm{N}(1)$ & $2.074(4)$ & $2.057(3)$ \\
$\mathrm{Zn}(1)-\mathrm{N}(2)$ & $2.269(4)$ & $2.233(2)$ \\
$\mathrm{Zn}(1)-\mathrm{N}(3)$ & $2.062(4)$ & $2.102(3)$ \\
$\mathrm{Zn}(2)-\mathrm{O}(1)$ & $2.140(3)$ & $2.120(2)$ \\
$\mathrm{Zn}(2)-\mathrm{O}(3)$ & $1.941(3)$ & $2.133(3)$ \\
$\mathrm{Zn}(2)-\mathrm{N}(4)$ & $2.443(4)$ & $2.322(3)$ \\
$\mathrm{Zn}(2)-\mathrm{N}(5)$ & $2.133(4)$ & $2.136(3)$ \\
$\mathrm{Zn}(2)-\mathrm{N}(6)$ & $2.107(4)$ & $2.336(3)$ \\
$\mathrm{Zn}(2)-\mathrm{O}(6)$ & - & $1.945(2)$ \\
$\mathrm{Zn}(2)-\mathrm{O}(5)$ & $2.122(3)$ & - \\
$\mathrm{Zn}(1)-\mathrm{O}(1)-\mathrm{Zn}(2)$ & $127.16(17)$ & $120.75(12)$ \\
$\mathrm{O}(1)-\mathrm{Zn}(1)-\mathrm{N}(1)$ & $136.52(16)$ & $118.71(12)$ \\
$\mathrm{O}(1)-\mathrm{Zn}(1)-\mathrm{N}(2)$ & $89.85(14)$ & $91.48(11)$ \\
$\mathrm{O}(1)-\mathrm{Zn}(1)-\mathrm{N}(3)$ & $100.53(16)$ & $124.82(11)$ \\
$\mathrm{O}(1)-\mathrm{Zn}(1)-\mathrm{O}(2)$ & $98.94(13)$ & $99.48(12)$ \\
$\mathrm{O}(1)-\mathrm{Zn}(2)-\mathrm{N}(4)$ & $162.62(14)$ & $88.66(10)$ \\
$\mathrm{O}(1)-\mathrm{Zn}(2)-\mathrm{N}(5)$ & $91.55(14)$ & $90.65(11)$ \\
$\mathrm{O}(1)-\mathrm{Zn}(2)-\mathrm{N}(6)$ & $87.68(14)$ & $161.27(10)$ \\
$\mathrm{O}(1)-\mathrm{Zn}(2)-\mathrm{O}(3)$ & $93.42(13)$ & $87.91(11)$ \\
$\mathrm{O}(1)-\mathrm{Zn}(2)-\mathrm{O}(6)$ & - & $102.84(10)$ \\
$\mathrm{O}(5)-\mathrm{Zn}(2)-\mathrm{O}(1)$ & $100.35(13)$ & - \\
\hline
\end{tabular}


Comparison with the structure of the corresponding acetate-bridged complex $\left[\mathrm{Zn}_{2}{ }_{2}\left(\mathrm{H}_{2} \mathrm{SIM}^{1}\right)(\mu-\mathrm{OAc})(\mathrm{OH})\right] \mathrm{PF}_{6}$ (discussed in detail in Chapter 4.3), used as the starting material for the synthesis of $\left[\mathrm{Zn}_{2}{ }_{2}\left(\mathrm{H}_{2} \mathrm{SIM}^{1}\right)\left(\mu-\mathrm{O}_{3} \mathrm{POC}_{6} \mathrm{H}_{4} \mathrm{NO}_{2}\right)\right] \mathrm{PF}_{6}$, illustrates important similarities: the $\mathrm{Zn}^{\prime \prime}$ ions in both structures are on different sides of the phenoxido plane and exhibit different coordination geometries. In the catalyst-inhibitor adduct $\left[\mathrm{Zn}_{2}{ }_{2}\left(\mathrm{H}_{2} \mathrm{SIM}^{1}\right)\left(\mu-\mathrm{O}_{3} \mathrm{POC}_{6} \mathrm{H}_{4} \mathrm{NO}_{2}\right)\right] \mathrm{PF}_{6}$, $\mathrm{Zn}(1)$ is also coordinated in a trigonal bipyramidal geometry ( $\tau$-value of 0.88 ), while $\mathrm{Zn}(2)$ is at the center of a six-coordinate site. In contrast to the catalyst complex, the remaining coordination site of $\mathrm{Zn}(2)$ is no longer completed by a terminal hydroxido group, but by the neighboring amide oxygen atom of the supporting dinucleating ligand. The coordination of the bulky PNPP inhibitor in $\left[\mathrm{Zn}_{2}{ }_{2}\left(\mathrm{H}_{2} \mathrm{SIM}^{1}\right)\left(\mu-\mathrm{O}_{3} \mathrm{POC}_{6} \mathrm{H}_{4} \mathrm{NO}_{2}\right)\right] \mathrm{PF}_{6}$, compared to the acetate co-ligand in $\left[\mathrm{Zn}_{2}{ }_{2}\left(\mathrm{H}_{2} \mathrm{SIM}^{1}\right)(\mu-\mathrm{OAc})(\mathrm{OH})\right] \mathrm{PF}_{6}$ leads to an increase of the $\mathrm{Zn}^{\prime \prime} \cdots \mathrm{Zn}^{\prime \prime}$ distance by $0.16 \AA$, accompanied by the widening of the $\mathrm{Zn}(1)-\mathrm{O}(1)-\mathrm{Zn}(2)$ angle by $6.4^{\circ}$. The structural differences in the dizinc(II) complexes of $\mathrm{H}_{3} \mathrm{SIM}^{1}$, $\left[\mathrm{Zn}_{2}{ }_{2}\left(\mathrm{H}_{2} \mathrm{SIM}^{1}\right)\left(\mu-\mathrm{O}_{3} \mathrm{POC}_{6} \mathrm{H}_{4} \mathrm{NO}_{2}\right)\right] \mathrm{PF}_{6}$ and $\left[\mathrm{Zn}_{2}{ }_{2}\left(\mathrm{H}_{2} \mathrm{SIM}^{1}\right)(\mu-\mathrm{OAc})(\mathrm{OH})\right] \mathrm{PF}_{6}$, are mainly in the angles within the triangular plane of the trigonal bipyramidal site. Due to the bulkier 4-nitrophenolate residue of PNPP in $\left[\mathrm{Zn}_{2}{ }_{2}\left(\mathrm{H}_{2} \mathrm{SIM}^{1}\right)\left(\mu-\mathrm{O}_{3} \mathrm{POC}_{6} \mathrm{H}_{4} \mathrm{NO}_{2}\right)\right] \mathrm{PF}_{6}$, compared to the acetate co-ligand in $\left[\mathrm{Zn}_{2}{ }_{2}\left(\mathrm{H}_{2} \mathrm{SIM}^{1}\right)(\mu-\mathrm{OAC})(\mathrm{OH})\right] \mathrm{PF}_{6}$, the $\mathrm{O}(1)-\mathrm{Zn}(1)-\mathrm{N}(1)$ angle is widened $\left(136.53(16)^{\mathrm{o}}\right.$ vs. $\left.118.77(16)^{\circ}\right)$, and the $\mathrm{O}(1)-\mathrm{Zn}(1)-\mathrm{N}(3)$ angle is contracted $\left(100.50(16)^{\circ}\right.$ vs. $\left.124.76(15)^{\circ}\right)$. However, the primary coordination sphere of the two complexes is similar.

Further investigation of the second coordination sphere in the amide-based complexes $\left[\mathrm{Zn}_{2}{ }_{2}\left(\mathrm{H}_{2} \mathrm{~L}^{2}\right)(\mu-\mathrm{OAc})_{2}\right] \mathrm{PF}_{6}, \quad\left[\mathrm{Zn}_{2}{ }_{2}\left(\mathrm{H}_{2} \mathrm{SIM}^{1}\right)(\mu-\mathrm{OAc})(\mathrm{OH})\right] \mathrm{PF}_{6}, \quad\left[\mathrm{Zn}_{2}{ }_{2}\left(\mathrm{H}_{2} \mathrm{SIM}^{3}\right)(\mu-\mathrm{OAc})(\mathrm{OH})\right]$ (discussed in detail in Chapter 4.3), and $\left[\mathrm{Zn}_{2}{ }_{2}\left(\mathrm{H}_{2} \mathrm{SIM}^{1}\right)\left(\mu-\mathrm{O}_{3} \mathrm{POC}_{6} \mathrm{H}_{4} \mathrm{NO}_{2}\right)\right] \mathrm{PF}_{6}$ indicates that, while the symmetric ligand $\mathrm{H}_{3} \mathrm{~L}^{2}$ forms two hydrogen bonds to the two bridging acetate coligands - one hydrogen bond to each acetate - the symmetric complexes, $\left[\mathrm{Zn}_{2}{ }_{2}\left(\mathrm{H}_{2} \mathrm{SIM}^{1}\right)(\mu-\mathrm{OAc})(\mathrm{OH})\right] P F_{6}$ and $\left[\mathrm{Zn}_{2}{ }_{2}\left(\mathrm{H}_{2} \mathrm{SIM}^{3}\right)(\mu-\mathrm{OAc})(\mathrm{OH})\right]$, form two hydrogen bonds to the hydroxido ligand, stabilizing this unusual structure. However, the introduction of the monophosphate PNPP, replacing the bridging acetate, leads to the coordination of one of the amide groups, stabilized by hydrogen bonding to the second amide function. The distance between these nitrogen and oxygen atoms of 3.210(6) $\AA$ is long compared to the corresponding distances in $\left[\mathrm{Zn}_{2}{ }_{2}\left(\mathrm{H}_{2} \mathrm{~L}^{2}\right)(\mu-\mathrm{OAc})_{2}\right] \mathrm{PF}_{6} \quad(2.982(4) \AA$ and $2.977(5) \AA \AA)$, $\left[\mathrm{Zn}_{2}{ }_{2}\left(\mathrm{H}_{2} \mathrm{SIM}^{1}\right)(\mu-\mathrm{OAc})(\mathrm{OH})\right] \mathrm{PF}_{6}(2.810(4) \AA$ and $2.875(4) \AA)$, and $\left[\mathrm{Zn}_{2}{ }_{2}\left(\mathrm{H}_{2} \mathrm{SIM}^{3}\right)(\mu-\mathrm{OAc})(\mathrm{OH})\right]$ (2.764(3) $\AA$ and 2.870(3) $\AA$ ). It is assumed that steric hindrance by the tert-butyl groups causes this elongation. Interestingly, no hydrogen bonding to the bridging phosphate, as previously observed with the amino group-containing complex $\left[\mathrm{Zn}_{2}{ }_{2}\left(\mathrm{~L}^{7}\right)(\mu-\mathrm{PNPP})\right]^{+}$, occurs. ${ }^{112}$ 


\section{${ }^{31}$ P NMR Studies of Phosphoester Binding}

\section{Phosphomonoester Binding}

In order to gain further insight into the catalytic mechanism, substrate binding was studied by ${ }^{31} \mathrm{P}$ NMR spectroscopy. ${ }^{31} \mathrm{P}$ NMR spectra of the hexafluorophosphate salts of the dizinc(II) complexes were measured with $85 \% \mathrm{H}_{3} \mathrm{PO}_{4}$ as an external standard, and the ${ }^{31} \mathrm{P}$ resonance of $\mathrm{PF}_{6}{ }^{-}$anion was then used as internal standard for the phosphoester binding experiments. First the spectra of the phosphoester substrates were measured to study the influence of the ester groups on the ${ }^{31} \mathrm{P}$ chemical shifts (Figure $60 \mathrm{a} ; \mathrm{NaPF}_{6}$ referenced to $-144.30 \mathrm{ppm}$ ). Comparison of the ${ }^{31} \mathrm{P}$ shifts in phosphoric acid, PNPP, BPNPP, DNPP, BDNPP, diphenyl phosphate and triphenyl phosphate show a linear correlation with the number of protons substituted by phenyl groups in $\mathrm{H}_{3} \mathrm{PO}_{4}$. Importantly, a comparison of the resonances of the phosphodiesters, BDNPP and BPNPP, as well as of the phosphomonoesters, DNPP and PNPP, indicates that the impact of an additional nitro substituent at the phenyl groups is rather small.

The ${ }^{31} \mathrm{P}$ NMR spectrum of the crystallographically investigated complex $\left[\mathrm{Zn}_{2}{ }_{2}\left(\mathrm{H}_{2} \mathrm{SIM}^{1}\right)\left(\mu-\mathrm{O}_{3} \mathrm{POC}_{6} \mathrm{H}_{4} \mathrm{NO}_{2}\right)\right]^{+}$in acetonitrile has a single resonance at $0.62 \mathrm{ppm}$ compared to $-5.05 \mathrm{ppm}$ for the uncoordinated phosphoester. This effect was also studied using the phosphomonoester DNPP for the four dizinc(II) complexes, in order to gain deeper understanding on the product inhibited structure during the BDNPP hydrolysis; the detected resonances are summarized in Table 22.
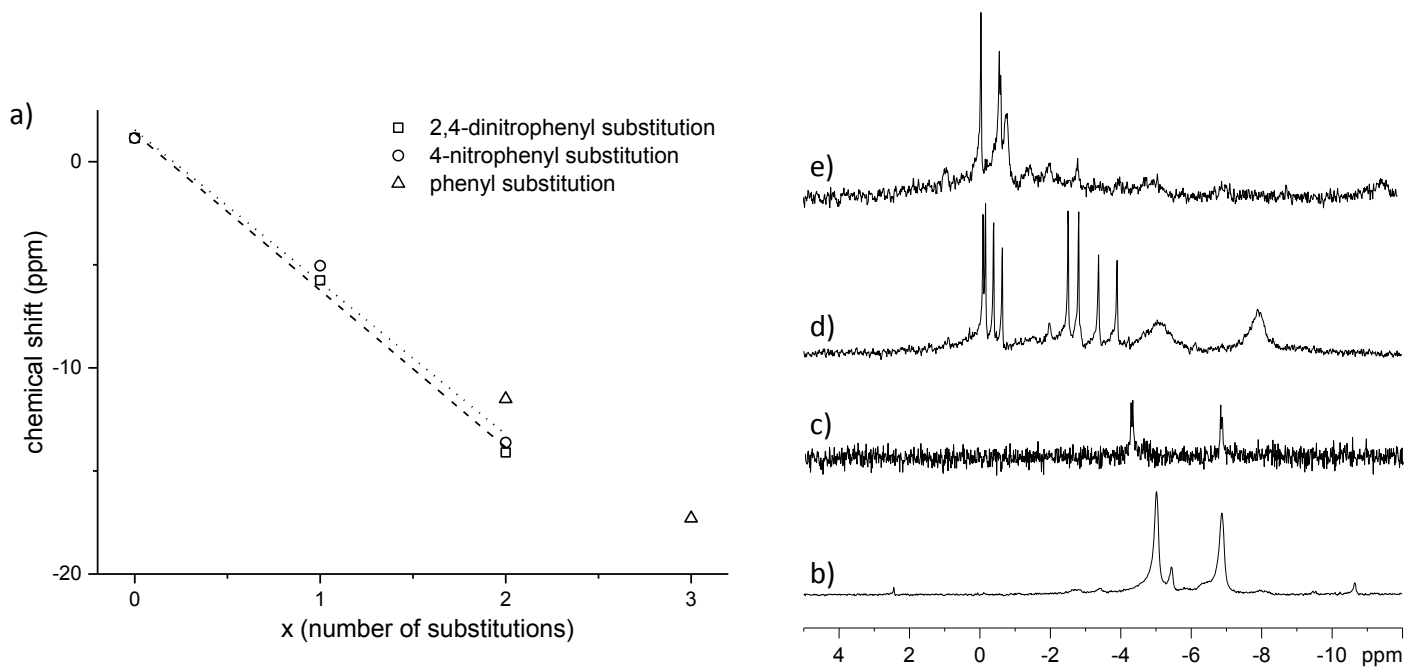

Figure 60: a) Chemical shifts of ${ }^{31} \mathrm{P} N M R$ resonances for phosphoester substrates $\left(\mathrm{OP}(\mathrm{OH})_{3-\mathrm{x}}(\mathrm{OR})_{\mathrm{x}}\right)$ in water containing $\mathrm{CD}_{3} \mathrm{CN}$ vs. extent of substitution $(\mathrm{x}$ ) of phosphoric acid with 2,4-dinitrophenyl, 4-nitrophenyl and phenyl residues; Comparison of ${ }^{31} \mathrm{P}$ NMR spectra in $\mathrm{CD}_{3} \mathrm{CN}$ after addition of DNPP to b) $\left[\mathrm{Zn}_{2}{ }_{2}\left(\mathrm{H}_{2} \mathrm{SIM}^{1}\right)(\mathrm{OAc})(\mathrm{OH})\right]^{+}$, c) $\left[\mathrm{Zn}_{2}{ }_{2}\left(\mathrm{H}_{2} \mathrm{~L}^{2}\right)(\mathrm{OAc})_{2}\right]^{+}$, d) $\left[\mathrm{Zn}_{2}{ }_{2}\left(\mathrm{SIM}^{2}\right)(\mathrm{OAc})_{2}\right]^{+}$directly after addition, and e) $\left[\mathrm{Zn}_{2}{ }_{2}\left(\mathrm{SIM}^{2}\right)(\mathrm{OAc})_{2}\right]^{+}$after 48 hours. 
Table 22: Phosphoester ${ }^{31} \mathrm{P}$ NMR resonances obtained before and after addition of dizinc(II) complexes $\left[\mathrm{Zn}_{2}{ }_{2}\left(\mathrm{H}_{2} \mathrm{~L}^{2}\right)(\mu-\mathrm{OAC})_{2}\right]^{+},\left[\mathrm{Zn}_{2}{ }_{2}\left(\mathrm{H}_{2} \mathrm{SIM}^{1}\right)(\mu-\mathrm{OAC})(\mathrm{OH})\right]^{+}$, and $\left[\mathrm{Zn}_{2}{ }_{2}\left(\mathrm{SIM}^{2}\right)(\mu-\mathrm{OAc})_{2}\right]^{+}$.

\begin{tabular}{|c|c|c|c|c|c|c|c|c|c|c|}
\hline \multirow{2}{*}{$\begin{array}{c}\text { ligand } \\
\text { backbone in } \\
\mathrm{Zn}_{2}{ }_{2} \text { complex }\end{array}$} & \multirow{2}{*}{ 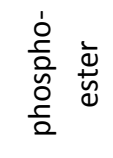 } & \multicolumn{9}{|c|}{ resonances arising from phosphoesters [ppm] } \\
\hline & & $\begin{array}{l}\text { metal- } \\
\text { free }\end{array}$ & \multicolumn{8}{|c|}{ treated with the $\mathrm{Zn}_{2}{ }_{2}$ complexes } \\
\hline $\mathrm{H}_{3} \mathrm{SIM}^{1}$ & PNPP & -5.05 & 0.62 & & & & & & & \\
\hline $\mathrm{H}_{3} \mathrm{SIM}^{1}$ & DNPP & -5.76 & -6.85 & -5.01 & -2.89 & & & & & \\
\hline after $98 \mathrm{~d}$ & DNPP & & -6.85 & -5.01 & & & & & & \\
\hline $\mathrm{H}_{3} \mathrm{~L}^{2}$ & DNPP & -5.76 & -6.80 & -4.25 & & & & & & \\
\hline after $98 \mathrm{~d}$ & DNPP & & -6.80 & -4.25 & -2.93 & & & & & \\
\hline $\mathrm{HSIM}^{2}$ & DNPP & -5.76 & -3.90 & -3.37 & -2.81 & -2.50 & -0.64 & -0.40 & -0.16 & -0.09 \\
\hline after $48 \mathrm{~h}$ & DNPP & & & & & & -0.63 & & & -0.08 \\
\hline $\mathrm{H}_{3} \mathrm{~L}^{2}$ & BDNPP & -14.10 & & & & & & & & \\
\hline & $0.25 \mathrm{eq}$ & & & -10.25 & & & & & & \\
\hline & $0.5 \mathrm{eq}$ & & & -10.20 & & & & & & \\
\hline & $0.75 \mathrm{eq}$ & & $(-11.46)$ & -10.22 & $(-6.88)$ & $(-4.58)$ & & & & \\
\hline after $24 \mathrm{~h}$ & $0.75 \mathrm{eq}$ & & & -10.21 & -6.95 & -4.54 & -1.40 & & & \\
\hline & $1.0 \mathrm{eq}$ & & -11.40 & -10.20 & -7.01 & -4.48 & -1.41 & & & \\
\hline after $21 \mathrm{~d}$ & $1.0 \mathrm{eq}$ & & & & -7.26 & -4.45 & -1.42 & & & \\
\hline
\end{tabular}

The spectra recorded after the addition of DNPP to the amine-based complex $\left[\mathrm{Zn}_{2}{ }_{2}\left(\mathrm{SIM}^{2}\right)(\mu-\mathrm{OAC})_{2}\right]^{+}$in deuterated acetonitrile ( 20 vol\% water content) show eight resonances shortly after addition $(-3.90 \mathrm{ppm},-3.37 \mathrm{ppm},-2.81 \mathrm{ppm},-2.50 \mathrm{ppm},-0.64 \mathrm{ppm}$, $-0.40 \mathrm{ppm},-0.16 \mathrm{ppm}$ and $-0.09 \mathrm{ppm}$ ) (Figure $60 \mathrm{~d}$ ). Over 48 hours further reaction results in the observation of only two resonances at $-0.61 \mathrm{ppm}$ and $-0.08 \mathrm{ppm}$ (Figure 60e). More detailed analysis of the spectrum obtained immediately after addition revealed a pattern of two sets of four resonances, in which always two resonances of the four are separated by about $0.3 \mathrm{ppm}$ (50 Hz) or $0.5 \mathrm{ppm}(78 \mathrm{~Hz}$ and $85 \mathrm{~Hz}) .{ }^{31} \mathrm{P}$ NMR studies performed with a series of cyclic phosphates disclosed that besides the electronegativity effect, the chemical shifts of ${ }^{31} \mathrm{P} N M R$ resonances are also significantly influenced by (i) the O-P-O angle, and (ii) the torsional angle $\mathrm{R}-\mathrm{O}-\mathrm{P}-\mathrm{O}(\mathrm{R})$ of the phosphate. ${ }^{292}$ Therefore, the eight resonances were attributed to four different species (-3.64 ppm, -2.66 ppm, -0.41 ppm and -0.25 ppm), each of them present in two conformers with varied positions of the 2,4-dinitrophenyl residue of the phosphoester. After 48 hours only the 'doublet' at $-0.25 \mathrm{ppm}$ remains. The chemical shift difference of $5.51 \mathrm{ppm}$ between the resonances of DNPP in its free form and bound to complex $\left[\mathrm{Zn}_{2}{ }_{2}\left(\mathrm{SIM}^{2}\right)(\text { solv })_{x}\right]^{n+}$ is in the same range as found by comparison of free PNPP and $\left[\mathrm{Zn}_{2}{ }_{2}\left(\mathrm{H}_{2} \mathrm{SIM}^{1}\right)\left(\mu-\mathrm{O}_{3} \mathrm{POC}_{6} \mathrm{H}_{4} \mathrm{NO}_{2}\right)\right]^{+}$. Therefore, a similar bridging coordination mode of DNPP to complex $\left[\mathrm{Zn}_{2}\left(\mathrm{SIM}^{2}\right)(\text { solv })_{x}\right]^{\mathrm{n}+}$ as found in $\left[\mathrm{Zn}_{2}{ }_{2}\left(\mathrm{H}_{2} \mathrm{SIM}^{1}\right)\left(\mu-\mathrm{O}_{3} \mathrm{POC}_{6} \mathrm{H}_{4} \mathrm{NO}_{2}\right)\right]^{+}$is proposed. Once more it should be emphasized that until this most stable phosphoester-bridged dizinc(II) complex was formed three different intermediate stages were detectable. 
In contrast, when the amido-based complexes $\left[\mathrm{Zn}_{2}{ }_{2}\left(\mathrm{H}_{2} \mathrm{SIM}^{1}\right)(\mu-\mathrm{OAc})(\mathrm{OH})\right]^{+}$and $\left[\mathrm{Zn}_{2}{ }_{2}\left(\mathrm{H}_{2} \mathrm{~L}^{2}\right)(\mu-\mathrm{OAC})_{2}\right]^{+}$and solved in deuterated acetonitrile ( 3.0 vol\% water content for $\left[\mathrm{Zn}_{2}{ }_{2}\left(\mathrm{H}_{2} \mathrm{~L}^{2}\right)(\mu-\mathrm{OAc})_{2}\right]^{+}$and 1.2 vol\% water content for $\left.\left[\mathrm{Zn}_{2}{ }_{2}\left(\mathrm{H}_{2} \mathrm{SIM}^{1}\right)(\mu-\mathrm{OAc})(\mathrm{OH})\right]^{+}\right)$were treated with one equivalent of DNPP, two major resonances with similar chemical shifts appeared in the ${ }^{31} \mathrm{P}$ NMR spectra (-6.85 ppm, $-5.01 \mathrm{ppm}$ and $\left.-6.80 \mathrm{ppm},-4.25 \mathrm{ppm}\right)$ (Figure 60b, c). Therefore, similar coordination modes of DNPP to $\left[\mathrm{Zn}_{2}{ }_{2}\left(\mathrm{H}_{2} \mathrm{~L}^{2}\right)(\text { solv })_{\mathrm{x}}\right]^{\mathrm{n}+}$ and $\left[\mathrm{Zn}_{2}{ }_{2}\left(\mathrm{H}_{2} \mathrm{SIM}^{1}\right)(\text { solv })_{\mathrm{x}}\right]^{\mathrm{n+}}$ are assumed. Moreover, the resonances detected were downfield and upfield shifted, respectively, compared to the free phosphomonoester. The extent of the shifts ( $0.75 \mathrm{ppm}$ to $1.51 \mathrm{ppm})$ are small compared to the cases discussed above. This suggests a rather different coordination mode of DNPP to $\left[\mathrm{Zn}_{2}{ }_{2}\left(\mathrm{H}_{2} \mathrm{SIM}^{1}\right)(\text { solv })_{x}\right]^{\mathrm{n}+}$ and $\left[\mathrm{Zn}_{2}{ }_{2}\left(\mathrm{H}_{2} \mathrm{~L}^{2}\right)(\text { solv })_{x}\right]^{\mathrm{n}+}$ than observed in the X-ray structure of $\left[\mathrm{Zn}_{2}{ }_{2}\left(\mathrm{H}_{2} \mathrm{SIM}^{1}\right)\left(\mu-\mathrm{O}_{3} \mathrm{POC}_{6} \mathrm{H}_{4} \mathrm{NO}_{2}\right)\right]^{+}$. Studies with cyclic phosphoesters have shown that the O-P-O angle has a significant impact on the ${ }^{31} \mathrm{P}$ NMR chemical shift. ${ }^{292}$ For example, an increase in the bond angle of phosphotriesters results in the better shielding of the phosphorus atom and therefore in an upfield shift of the ${ }^{31}$ P NMR resonance. ${ }^{292}$ Assuming the same binding mode for DNPP and PNPP, an upfield shift due to a larger O-P-O angle in the case of DNPP competes with a downfield shift due to coordination to two $\mathrm{Zn}$ "ions, and this could explain the smaller extent of the downfield shifts in the cases of DNPP with $\left[\mathrm{Zn}_{2}{ }_{2}\left(\mathrm{H}_{2} \mathrm{~L}^{2}\right)(\mu-\mathrm{OAc})_{2}\right]^{+}$and $\left[\mathrm{Zn}_{2}{ }_{2}\left(\mathrm{H}_{2} \mathrm{SIM}^{1}\right)(\mu-\mathrm{OAc})(\mathrm{OH})\right]^{+}$. The major resonances at around $-6.8 \mathrm{ppm}$ and -4 to $-5 \mathrm{ppm}$ can be attributed either to a complex structure with two DNPP ligands in two different electronic environments or to two different complex structures, each of them containing only one bound DNPP. Although the detection of the two major resonances upon binding DNPP by the amide-based complexes, $\left[\mathrm{Zn}_{2}{ }_{2}\left(\mathrm{H}_{2} \mathrm{~L}^{2}\right)(\text { solv })_{\mathrm{x}}\right]^{\mathrm{n+}}$ and $\left[\mathrm{Zn}_{2}{ }_{2}\left(\mathrm{H}_{2} \mathrm{SIM}^{1}\right)(\text { solv })_{\mathrm{x}}\right]^{\mathrm{n}+}$, are similar, their ${ }^{31} \mathrm{P} \mathrm{NMR}$ spectra are different: while the third resonance at $-2.89 \mathrm{ppm}$ was detected immediately after addition of DNPP to $\left[\mathrm{Zn}_{2}{ }_{2}\left(\mathrm{H}_{2} \mathrm{SIM}^{1}\right)(\mu-\mathrm{OAc})(\mathrm{OH})\right]^{+}$and disappears after 14 weeks, a species with a similar signal was not observed with $\left[\mathrm{Zn}_{2}{ }_{2}\left(\mathrm{H}_{2} \mathrm{~L}^{2}\right)(\mu-\mathrm{OAc})_{2}\right]^{+}$, but, when a spectrum was measured after 14 weeks (-2.93 ppm). This implies that both complexes form different species with DNPP in different coordination modes, however, the equilibrium constants are dependent on the symmetry of the complex backbone. This could explain the different catalytic activity of the complexes $\left[\mathrm{Zn}_{2}{ }_{2}\left(\mathrm{H}_{2} \mathrm{~L}^{2}\right)(\mu-\mathrm{OAc})_{2}\right]^{+}$and $\left[\mathrm{Zn}_{2}{ }_{2}\left(\mathrm{H}_{2} \mathrm{SIM}^{1}\right)(\mu-\mathrm{OAc})(\mathrm{OH})\right]^{+}$. 


\section{Phosphodiester Binding}

The binding of BDNPP was investigated for the most active complex $\left[\mathrm{Zn}_{2}{ }_{2}\left(\mathrm{H}_{2} \mathrm{~L}^{2}\right)(\mu-\mathrm{OAC})_{2}\right]^{+}$by stepwise addition of 0.25 equivalents of BDNPP and recording the ${ }^{31} \mathrm{P}$ NMR spectra at each step ( 1.0 vol\% water content) (Figure 61 ). Addition up to 0.5 equivalents BDNPP to a solution of the complex in $\mathrm{CD}_{3} \mathrm{CN}$ resulted in the formation of only one phosphoruscontaining species with a ${ }^{31} \mathrm{P}$ NMR resonance at $-10.22 \mathrm{ppm}$ (Figure 61b,c), in addition to free BDNPP with a resonance at $-14.43 \mathrm{ppm}$ (Figure 61a). After further addition of BDNPP two additional signals at $-6.86 \mathrm{ppm}$ and $-4.58 \mathrm{ppm}$ occurred in the ${ }^{31} \mathrm{P}$ NMR spectrum, and the signal integration increases upon increase of BDNPP concentration. Further reaction led to the disappearance of the initially detected species and formation of a new species with a resonance at $-1.41 \mathrm{ppm}$.

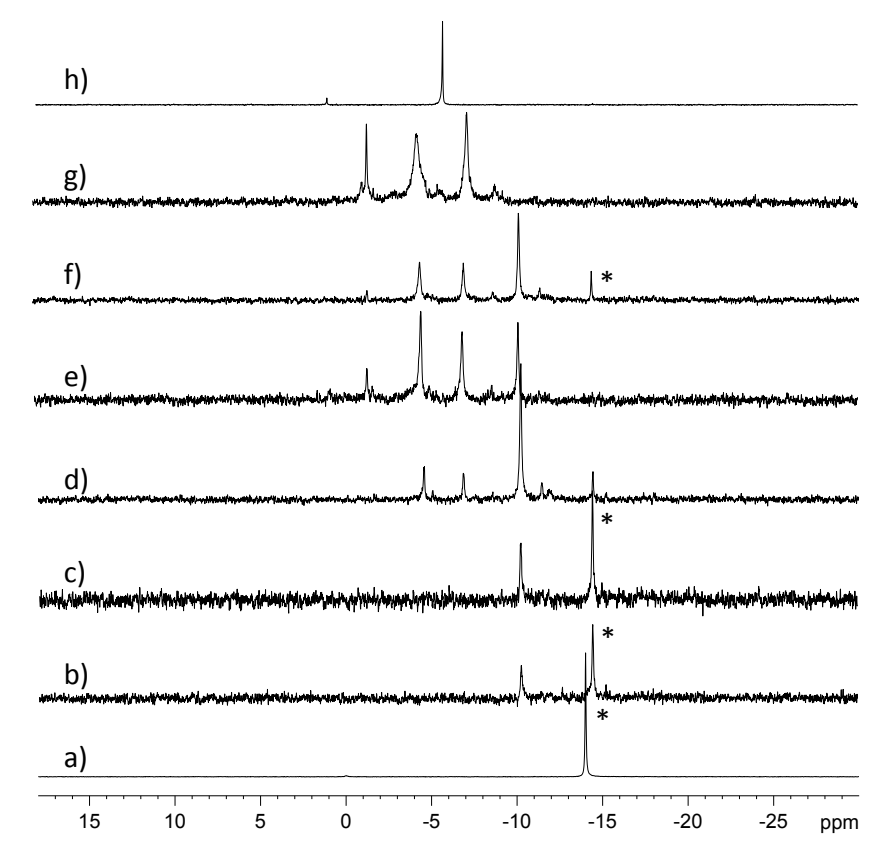

Figure 61: ${ }^{31} \mathrm{P}$ NMR measurements upon addition of BDNPP to $\left[\mathrm{Zn}_{2}{ }_{2}\left(\mathrm{H}_{2} \mathrm{~L}^{2}\right)(\mathrm{OAc})_{2}\right]^{+}$in $\mathrm{CD}_{3} \mathrm{CN}$ a) $\mathrm{BDNPP}$ reference, b) 0.25 eq BDNPP, c) 0.5 eq BDNPP, d) 0.75 eq BDNPP, e) 0.75 eq BDNPP after 24h, f) 1.0 eq BDNPP, g) 1.0 eq BDNPP after 16 days, and $h$ ) DNPP reference (* unreacted BDNPP).

Comparison to the spectra monitored after addition of the phosphomonoester DNPP to $\left[\mathrm{Zn}_{2}{ }_{2}\left(\mathrm{H}_{2} \mathrm{~L}^{2}\right)(\mu-\mathrm{OAc})_{2}\right]^{+}$(Figure 61c), assigns the resonances at $-6.86 \mathrm{ppm}$ and $-4.58 \mathrm{ppm}$ to species containing DNPP. The increase of the integrals of these two species is accompanied by the decrease of the initially detected resonance at $-10.22 \mathrm{ppm}$. Therefore, the resonance at $-10.22 \mathrm{ppm}$ is suggested to originate from a BDNPP-complex adduct. It follows that the binding of the phosphodiester to the $\mathrm{Zn}$ " ion leads to a downfield shift by $4.2 \mathrm{ppm}$ compared to free BDNPP, accompanied by the activation of the phosphodiester BDNPP. The following hydrolysis of BDNPP provides the phosphomonoester DNPP, which binds in the same way as by direct addition of DNPP to the complex. The decrease of the signal assigned to the BDNPP-complex 
adduct is accompanied with an increase of a signal at $-1.41 \mathrm{ppm}$. Although the structure of the corresponding species remains unclear, we speculate that this complex adduct is able to release the DNPP product and therefore continues to catalyze the BDNPP hydrolysis.

\section{Mass Spectrometric Investigation of Phosphoester Binding}

\section{Phosphomonoester Binding}

$\mathrm{ESI}^{+}$mass spectrometric investigations were carried out with mixtures of the different dizinc(II) complexes with either the phosphomonoester DNPP or the phosphodiester BDNPP. Treatment of $\left[\mathrm{Zn}_{2}{ }_{2}\left(\mathrm{H}_{2} \mathrm{SIM}^{1}\right)(\mu-\mathrm{OAc})(\mathrm{OH})\right]^{+}$in acetonitrile with the phosphomonoester DNPP resulted in a mass spectrum with the main peak at $\mathrm{m} / \mathrm{z} 1201.2$ corresponding to the species $\left[\mathrm{Zn}_{2}{ }_{2}\left(\mathrm{HSIM}^{1}\right)(\mathrm{DNPP})(\mathrm{HOAc})(\mathrm{HF})\right]^{+}$. This peak at $\mathrm{m} / \mathrm{z} 1201.2$ was also apparent in the spectrum recorded with the mixture of complex $\left[\mathrm{Zn}_{2}{ }_{2}\left(\mathrm{H}_{2} \mathrm{~L}^{2}\right)(\mu-\mathrm{OAc})_{2}\right]^{+}$with DNPP ascribed to the species $\left[\mathrm{Zn}_{2}{ }_{2}\left(\mathrm{HL}^{2}\right)(\mathrm{DNPP})(\mathrm{HOAC})(\mathrm{HF})\right]^{+}$, respectively. An additional signal was found in this spectrum at $\mathrm{m} / \mathrm{z} 1121.2$ matching the mass of $\left[\mathrm{Zn}_{2}\left(\mathrm{HL}^{2}\right)(\mathrm{DNPP})\right]^{+}$. Interestingly, treatment of complex $\left[\mathrm{Zn}_{2}{ }_{2}\left(\mathrm{SIM}^{2}\right)(\mu-\mathrm{OAC})_{2}\right]^{+}$with the phosphomonoester DNPP resulted in a variety of signals in the region around $\mathrm{m} / \mathrm{z} 1200$, but those could not been ascribed to specific species due to overlapping of the signals. This finding is in agreement with the above discussed ${ }^{31} \mathrm{P}$ NMR spectroscopic investigations supporting the various coordination modes of the phosphomonoester to the complex $\left[\mathrm{Zn}_{2}\left(\mathrm{SIM}^{2}\right)(\mu-\mathrm{OAC})_{2}\right]^{+}$.

\section{Phosphodiester Binding}

Samples taken during the ${ }^{31} \mathrm{P}$ NMR spectroscopic titration of BDNPP to the complex $\left[\mathrm{Zn}_{2}{ }_{2}\left(\mathrm{H}_{2} \mathrm{~L}^{2}\right)(\mu-\mathrm{OAc})_{2}\right]^{+}$resulted in $\mathrm{ESI}^{+}$mass spectra showing peaks at $\mathrm{m} / \mathrm{z} 1121.2$ and $\mathrm{m} / \mathrm{z}$ 1201.2. These two species have also been detected in the mixture of the same dizinc(II) complex with the phosphomonoester DNPP and were ascribed to the species $\left[\mathrm{Zn}_{2}{ }_{2}\left(\mathrm{HL}^{2}\right)(\mathrm{DNPP})\right]^{+}$and $\left[\mathrm{Zn}_{2}{ }_{2}\left(\mathrm{HL}^{2}\right)(\mathrm{DNPP})(\mathrm{HOAC})(\mathrm{HF})\right]^{+}$. Therefore, the coordination of the hydrolysis product has also been seen in the mass spectrometric investigations and supports the assignment during the ${ }^{31} \mathrm{P}$ NMR spectroscopic investigations. 


\subsection{SUMMARY}

Studying the phosphoester hydrolysis activity of a variety of model complexes with hydrogen bonding functionalities adjacent to the dizinc(II) core, but differing in (i) the type of the hydrogen bond donor, (ii) the position of the hydrogen bond donor, and (iii) the coordinating ligands in the primary coordination sphere, resulted in deeper insights in the impact of the second coordination sphere of phosphatases.

The impact of the hydrogen bond donors of the catalysts on their hydrolytic activity was clearly found to be $\mathrm{pH}$ dependent. Amino substitution of the two pyridine residues coordinated to one of the two $\mathrm{Zn}^{\prime \prime}$ centers as in $\left[\mathrm{Zn}_{2}{ }_{2}\left(\mathrm{SIM}^{2}\right)(\mu-\mathrm{OAc})_{2}\right]^{+}$leads to a lower $\mathrm{pH}$ maximum of the phosphoester hydrolysis activity, whilst substitution with pivaloyl-amide residues at the same positions as in $\left[\mathrm{Zn}_{2}{ }_{2}\left(\mathrm{H}_{2} \mathrm{SIM}^{1}\right)(\mu-\mathrm{OAc})(\mathrm{OH})\right]^{+}$has not a significant effect. However, positioning of one pivaloyl-amide residue adjacent to each $\mathrm{Zn}^{\text {"l }}$ center as in $\left[\mathrm{Zn}_{2}{ }_{2}\left(\mathrm{H}_{2} \mathrm{~L}^{2}\right)(\mu-\mathrm{OAC})_{2}\right]^{+}$enables activity only under strong basic conditions.

The main impact of the secondary interactions was shown to be the increased substrate affinity, and this obviously depends on the hydrogen bonding sites and the type of the hydrogen bonding groups. At pH 7 the diamino derivative $\left[\mathrm{Zn}_{2}{ }_{2}\left(\mathrm{SIM}^{2}\right)(\mu-\mathrm{OAc})_{2}\right]^{+}$is the most effective catalyst, possibly due to the flexibility in the binding mode of the phosphomonoester product, as seen in the ${ }^{31} \mathrm{P}$ NMR spectroscopic investigations. While at $\mathrm{pH} 7$ the positioning of both pivaloyl-amide residues proximal to one $\mathrm{Zn}^{\text {"l }}$ center in $\left[\mathrm{Zn}_{2}{ }_{2}\left(\mathrm{H}_{2} \mathrm{SIM}^{1}\right)(\mu-\mathrm{OAc})(\mathrm{OH})\right]^{+}$has a more positive effect towards the BDNPP hydrolysis than positioning one pivaloyl-amide adjacent to each $\mathrm{Zn}$ " center in $\left[\mathrm{Zn}_{2}{ }_{2}\left(\mathrm{H}_{2} \mathrm{~L}^{2}\right)(\mu-\mathrm{OAc})_{2}\right]^{+}$; the finding at more basic condition is inversed. Moreover, the impact of a more electron-rich primary coordination sphere in $\left[\mathrm{Zn}_{2}{ }_{2}\left(\mathrm{H}_{2} \mathrm{SIM}^{3}\right)(\mu-\mathrm{OAc})(\mathrm{OH})\right]$ is found to surpass the effect of the hydrogen bonding effect of the secondary coordination sphere. However, at strong basic conditions the hydrolysis rate of the hydrogen bonding capable complexes $\left[\mathrm{Zn}_{2}{ }_{2}\left(\mathrm{H}_{2} \mathrm{SIM}^{1}\right)(\mu-\mathrm{OAc})(\mathrm{OH})\right]^{+},\left[\mathrm{Zn}_{2}{ }_{2}\left(\mathrm{H}_{2} \mathrm{~L}^{2}\right)(\mu-\mathrm{OAc})_{2}\right]^{+}$and $\left[\mathrm{Zn}_{2}{ }_{2}\left(\mathrm{SIM}^{2}\right)(\mu-\mathrm{OAc})_{2}\right]^{+}$are significantly higher than for the parent complex $\left[\mathrm{Zn}_{2}\left(\mathrm{~L}^{1}\right)(\mu-\mathrm{OAc})_{2}\right]^{+}$missing hydrogen bond donors. Long-term catalytic studies revealed that at $\mathrm{pH} 9.5$ the symmetrical, sterically hindered, amide-based system $\left[\mathrm{Zn}_{2}\left(\mathrm{H}_{2} \mathrm{~L}^{2}\right)(\mu-\mathrm{OAc})_{2}\right]^{+}$is the most efficient dizinc(II) complex employed in this work, and this is primarily due to less stable catalyst-inhibitor complexes. In contrast, incorporation of two pivaloyl-amide residues in an asymmetrical fashion as in $\left[\mathrm{Zn}_{2}{ }_{2}\left(\mathrm{H}_{2} \mathrm{SIM}^{1}\right)(\mu-\mathrm{OAc})(\mathrm{OH})\right]^{+}$leads to the generation of a stable catalyst-inhibitor (hydrolysis 
product) adduct. The reason for this behavior was found in the crystal structure of the corresponding complex with a bridging phosphomonoester co-ligand, which reveals one of the two pivaloyl-amide moieties to function as co-ligand for the remaining vacant coordination site of the proximal $\mathrm{Zn}$ " center, stabilized by hydrogen bond formation to the second pivaloyl-amide residue. 


\section{Chapter 8}

\section{Phosphatase Activity}

\section{of Diiron Complexes}

g Main parts of this chapter will be published in "An Approach to More Accurate Model Systems for Purple Acid Phosphatases (PAPs)"; Paul V. Bernhardt, Simone Bosch, Peter Comba, Lawrence R. Gahan, Graeme R. Hanson, Valeriu Mereacre, Christopher J. Noble, Gerhard Schenk, Hubert Wadepohl; manuscript in preparation. 


\subsection{INTRODUCTION}

The hydrolysis of phosphoesters in mammals is catalyzed by phosphatases with two iron centers in the active site, i.e. PAP. The oxidation state of the two iron centers in the active site of PAP was shown to be significant, with the homovalent Fe ${ }_{2}{ }_{2}$ form of ufPAP exhibiting phosphoester hydrolysis activity of less than $1 \%$ of that of the heterovalent $\mathrm{Fe}^{111} \mathrm{Fe}{ }^{l l}$ form. ${ }^{221}$ Although phosphodiester hydrolysis was observed with a wide range of model complexes, the biological relevant hydrolysis of phosphomonoesters with model systems has rarely been reported. Recently two dinuclear PAP model compounds were reported for which the hydrolysis of the phosphomonoester model substrate DNPP was achieved. ${ }^{108,} 114$ The two complexes, $\left[\mathrm{Fe}^{\prime \prime \prime}{ }_{2}\left(\mathrm{~L}^{8}\right)(\mu-\mathrm{OH})\left(\mathrm{OH}_{2}\right)_{2}\right]\left(\mathrm{ClO}_{4}\right)_{4}$ and $\left[\mathrm{Fe}^{\prime \prime \prime \prime}{ }_{2}\left(\mathrm{~L}^{6}\right)(\mu-\mathrm{O}) \mathrm{Cl}_{4}\right](\mathrm{Cl})_{2}$, depicted in Chart 25, differ from previously studied model systems in their ability to form hydrogen bonds to the putative substrate molecule bound to the metal centers; this was accomplished by incorporation of basic residues adjacent the dimetal core. At the same time a third complex, $\left[\mathrm{Fe}_{2}{ }_{2}{ }_{2}\left(\mathrm{~L}^{2}\right)(\mu-\mathrm{OH})\right]\left(\mathrm{ClO}_{4}\right)_{4}$, was reported, and this complex included pivaloyl-amide moieties as mimics for the non-bonding histidine residues in the active site of PAPs. ${ }^{114}$ Investigations of the BDNPP hydrolysis with the $\mathrm{Fe}^{\mathrm{III}}{ }_{2}$ complex of $\mathrm{HL}^{1}$, missing hydrogen bond donors, as well as its derivatives $\left[\mathrm{Fe}^{\mathrm{III}}{ }_{2}\left(\mathrm{~L}^{8}\right)(\mu-\mathrm{OH})\left(\mathrm{OH}_{2}\right)_{2}\right]\left(\mathrm{ClO}_{4}\right)_{4}$ and $\left[\mathrm{Fe}^{\prime \prime \prime \prime}{ }_{2}\left(\mathrm{~L}^{2}\right)(\mu-\mathrm{OH})\right]\left(\mathrm{ClO}_{4}\right)_{4}$ showed that the two complexes providing hydrogen bond donors exhibited faster hydrolysis rates than the $\mathrm{Fe}^{\mathrm{III}}{ }_{2}$ complex formed with $\mathrm{HL}^{1}$. However, it should be mentioned that the complexes $\left[\mathrm{Fe}^{\prime \prime \prime \prime}{ }_{2}\left(\mathrm{~L}^{8}\right)(\mu-\mathrm{OH})\left(\mathrm{OH}_{2}\right)_{2}\right]\left(\mathrm{ClO}_{4}\right)_{4}$ and $\left[\mathrm{Fe}^{\prime \prime \prime}{ }_{2}\left(\mathrm{H}_{2} \mathrm{~L}^{2}\right)(\mu-\mathrm{OH})\right]\left(\mathrm{ClO}_{4}\right)_{4}$ exhibited similar catalytic efficiencies $\left(\mathrm{K}_{\mathrm{cat}} / \mathrm{K}_{\mathrm{M}}\right)$ to the reference complex lacking a hydrogen bond donor; the differences were due to the higher substrate affinity of the complexes $\left[\mathrm{Fe}^{\prime \prime \prime}{ }_{2}\left(\mathrm{~L}^{8}\right)(\mu-\mathrm{OH})\left(\mathrm{OH}_{2}\right)_{2}\right]\left(\mathrm{ClO}_{4}\right)_{4}$ and $\left[\mathrm{Fe}^{\prime \prime \prime \prime}{ }_{2}\left(\mathrm{H}_{2} \mathrm{~L}^{2}\right)(\mu-\mathrm{OH})\right]\left(\mathrm{ClO}_{4}\right)_{4}$ and subsequent larger substrate binding constants $\mathrm{K}_{\mathrm{M}}$, ascribed to hydrogen bond formation. ${ }^{114}$ In contrast, the complex $\left[\mathrm{Fe}^{\prime \prime \prime}{ }_{2}\left(\mathrm{~L}^{6}\right)(\mu-\mathrm{O}) \mathrm{Cl}_{4}\right](\mathrm{Cl})_{2}$ shows the highest catalytic efficiency $\mathrm{k}_{\mathrm{cat}} / \mathrm{K}_{\mathrm{M}}$ reported so far, with a similar substrate affinity compared to $\left[\mathrm{Fe}^{\prime \prime \prime}{ }_{2}\left(\mathrm{~L}^{1}\right)(\text { solv })_{x}\right]^{5+}$, but a ten-fold higher hydrolysis rate. ${ }^{108}$ Table 23 (page 170) lists kinetic data reported for different diiron complexes. 


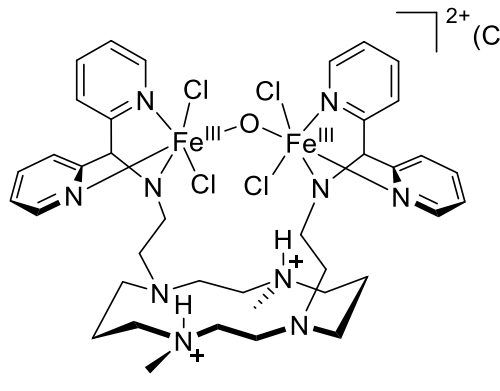

$\left[\mathrm{Fe}^{\mathrm{III}}{ }_{2}\left(\mathrm{~L}^{6}\right)(\mu-\mathrm{O}) \mathrm{Cl}_{4}\right](\mathrm{Cl})_{2}$

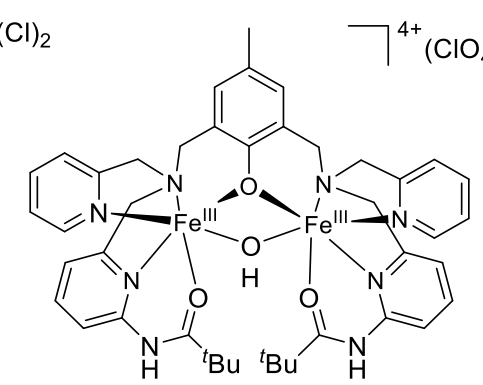

$\left[\mathrm{Fe}^{\prime \prime \prime}{ }_{2}\left(\mathrm{~L}^{2}\right)(\mu-\mathrm{OH})\right]\left(\mathrm{ClO}_{4}\right)_{4}$

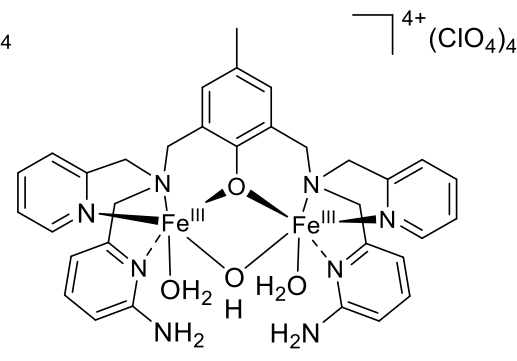

$\left[\mathrm{Fe}^{\mathrm{III}}{ }_{2}\left(\mathrm{~L}^{8}\right)(\mu-\mathrm{OH})\left(\mathrm{OH}_{2}\right)_{2}\right]\left(\mathrm{ClO}_{4}\right)_{4}$

Chart 25: Previously reported diiron(III) complexes containing hydrogen bond donors. ${ }^{108,114}$

In order to understand the impact of the hydrogen bond donors the phosphoester binding of $\left[\mathrm{Fe}_{2}{ }_{2}\left(\mathrm{~L}^{8}\right)(\mu-\mathrm{OH})\left(\mathrm{OH}_{2}\right)_{2}\right]\left(\mathrm{ClO}_{4}\right)_{4},\left[\mathrm{Fe}^{\prime \prime \prime}{ }_{2}\left(\mathrm{H}_{2} \mathrm{~L}^{2}\right)(\mu-\mathrm{OH})\right]\left(\mathrm{ClO}_{4}\right)_{4}$, and $\left[\mathrm{Fe}^{\prime \prime \prime}{ }_{2}\left(\mathrm{~L}^{6}\right)(\mu-\mathrm{O}) \mathrm{Cl}_{4}\right](\mathrm{Cl})_{2}$ was studied. These investigations revealed that (i) $\left[\mathrm{Fe}^{\prime \prime \prime \prime}{ }_{2}\left(\mathrm{~L}^{6}\right)(\mu-\mathrm{O}) \mathrm{Cl}_{4}\right](\mathrm{Cl})_{2}$ and $\left[\mathrm{Fe}^{\prime \prime \prime \prime}{ }_{2}\left(\mathrm{~L}^{8}\right)(\mu-\mathrm{OH})\left(\mathrm{OH}_{2}\right)_{2}\right]\left(\mathrm{ClO}_{4}\right)_{4}$ bind phosphomonoester DNPP in a bridging coordination mode, (ii) while the phosphodiester BDNPP was bound in a terminal fashion, and (iii) $\left[\mathrm{Fe}^{\prime \prime \prime \prime}{ }_{2}\left(\mathrm{H}_{2} \mathrm{~L}^{2}\right)(\mu-\mathrm{OH})\right]\left(\mathrm{ClO}_{4}\right)_{4}$ did not indicate terminal coordination of phosphodiesters. ${ }^{109}$ The difference in the coordination of phosphoesters of the phenolate-based complexes is reflected in their hydrolysis activity by a significantly larger activity of $\left[\mathrm{Fe}^{\prime \prime \prime}{ }_{2}\left(\mathrm{~L}^{8}\right)(\mu-\mathrm{OH})\left(\mathrm{OH}_{2}\right)_{2}\right]\left(\mathrm{ClO}_{4}\right)_{4}$ compared to $\left[\mathrm{Fe}^{\prime \prime \prime}{ }_{2}\left(\mathrm{H}_{2} \mathrm{~L}^{2}\right)(\mu-\mathrm{OH})\right]\left(\mathrm{ClO}_{4}\right)_{4}{ }^{109,}{ }^{114}$ The terminal coordination is rather unusual as many crystal structures show phenolate-based dinuclear complexes binding phosphodiesters in a bridging mode. ${ }^{293,} 294$ This unusual coordination mode of phosphoesters was attributed to the hydrogen bonding network provided by the ligand backbones 108, 109, 114 supported by computational studies with $\left[\mathrm{Fe}_{2}{ }_{2}{ }_{2}\left(\mathrm{~L}^{6}\right)(\mu-\mathrm{O}) \mathrm{Cl}_{4}\right](\mathrm{Cl})_{2}$. Commencing with a phosphomonoester molecule monodentately coordinated to complex $\left[\mathrm{Fe}^{\prime \prime \prime}{ }_{2}\left(\mathrm{~L}^{6}\right)(\mu-\mathrm{O})(\text { solv })_{3}\right]^{4+}$, the activation barrier for bridging coordination was calculated to be considerably larger than for the hydrolysis reaction, especially in case the cyclam nitrogen atoms are protonated, thus illustrating the important role of the second coordination sphere on PAP like reactivity. ${ }^{108,109}$

With the complex $\left[\mathrm{Fe}^{\prime \prime \prime}{ }_{2}\left(\mathrm{~L}^{8}\right)(\text { solv })_{x}\right]^{5+}$ the generation of its reduced $\mathrm{Fe}^{\mathrm{III}} \mathrm{Fe}^{\mathrm{II}}$ form was achieved by bulk electrolysis. Kinetic studies revealed that the heterovalent diiron complex of ligand $\mathrm{HL}^{8}$ exhibited a five-fold increased catalytic efficiency with respect to its corresponding homovalent $\mathrm{Fe}^{\prime \prime \prime}{ }_{2}$ counterpart. ${ }^{109,114}\left[\mathrm{Fe}^{\mathrm{III}} \mathrm{Fe}^{\prime \prime}\left(\mathrm{L}^{8}\right)(\text { solv })_{\mathrm{X}}\right]^{4+}$ revealed a lower substrate binding constant $\mathrm{K}_{\mathrm{M}}$ and a faster hydrolysis rate than $\left[\mathrm{Fe}^{1 I_{2}}\left(\mathrm{~L}^{8}\right)(\mathrm{solv})_{\mathrm{X}}\right]^{5+}{ }^{114}$ As discussed above, the trend of a higher activity of the heterovalent diiron form compared to the homovalent Fe ${ }^{\prime \prime}{ }_{2}$ form was also detected for the native PAP enzyme and ascribed to an increased ligand exchange rate of Fe" compared to $\mathrm{Fe}^{\prime \prime \prime} .{ }^{278}$ The release of the reaction product was proposed to be most likely the rate- 
limiting step in the catalytic mechanism, ${ }^{21,66,266,295}$ and this proposal is supported by the water exchange rates of Fe"II and Fe" $\left(10^{2} \mathrm{~s}^{-1}\right.$ and $10^{6} \mathrm{~s}^{-1}$, respectively). ${ }^{278,296-298}$

Although the complex $\left[\mathrm{Fe}_{2}{ }_{2}\left(\mathrm{H}_{2} \mathrm{~L}^{2}\right)(\mu-\mathrm{OH})\right]\left(\mathrm{ClO}_{4}\right)_{4}$ provides two hydrogen bond donors similar to $\left[\mathrm{Fe}_{2}{ }_{2}{ }_{2}\left(\mathrm{~L}^{8}\right)(\mu-\mathrm{OH})\left(\mathrm{OH}_{2}\right)_{2}\right]\left(\mathrm{ClO}_{4}\right)_{4}$ and $\left[\mathrm{Fe}^{\prime \prime \prime}{ }_{2}\left(\mathrm{~L}^{6}\right)(\mu-\mathrm{O}) \mathrm{Cl}_{4}\right](\mathrm{Cl})_{2}$, this complex exhibited no phosphomonoester hydrolysis capacity and revealed no monodetate coordination of phosphodiesters. ${ }^{109}$ The latter was rather unexpected due to the sterically demanding pivaloyl-amide residues. In order to favor terminal coordination of phosphoesters an asymmetric ligand $\mathrm{H}_{3} \mathrm{SIM}^{1}$ was designed in this work bearing two pivaloyl-amide residues similar to $\mathrm{H}_{3} \mathrm{~L}^{2}$, but both proximal to one of the two binding sites. In Chapter 2.4 the syntheses and characterization of the respective $\mathrm{Fe}_{2}{ }_{2}$ complexes, $\left[\mathrm{Fe}_{2}{ }_{2}\left(\mathrm{H}_{2} \mathrm{SIM}^{1}\right)(\mu-\mathrm{OAc})_{2}\right] \mathrm{PF}_{6}$ and $\left[\mathrm{Fe}_{2}{ }_{2}\left(\mathrm{H}_{2} \mathrm{~L}^{2}\right)(\mu-\mathrm{OAc})_{2}\right] \mathrm{PF}_{6}$, depicted in Chart 26, has been described as well as the generation of the $\mathrm{Fe}^{\prime \prime \prime} \mathrm{Fe}^{\text {Il }}$ complex of ligand $\mathrm{H}_{3} \mathrm{SIM}^{1}$.

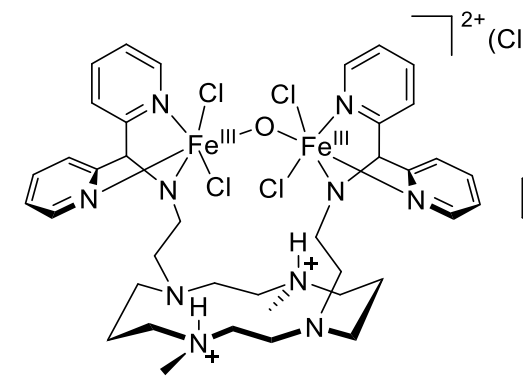

$\left[\mathrm{Fe}^{\prime \prime \prime}{ }_{2}\left(\mathrm{~L}^{6}\right)(\mu-\mathrm{O}) \mathrm{Cl}_{4}\right](\mathrm{Cl})_{2}$

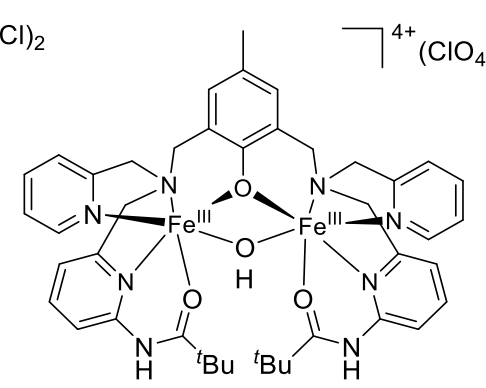

$\left[\mathrm{Fe}^{\prime \prime \prime \prime}{ }_{2}\left(\mathrm{~L}^{2}\right)(\mu-\mathrm{OH})\right]\left(\mathrm{ClO}_{4}\right)_{4}$

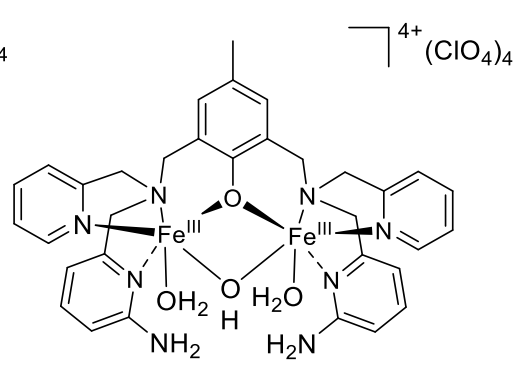

$\left[\mathrm{Fe}^{\prime \prime \prime \prime}{ }_{2}\left(\mathrm{~L}^{8}\right)(\mu-\mathrm{OH})\left(\mathrm{OH}_{2}\right)_{2}\right]\left(\mathrm{ClO}_{4}\right)_{4}$

Chart 26: Diiron(II) complexes discussed in this chapter.

Comparison of those two $\mathrm{Fe}_{2}{ }_{2}$ complexes facilitates the study of the impact of the hydrogen bond donor position. Moreover, comparison of the $\mathrm{Fe}_{2}{ }_{2}$ complex and the reported Fe ${ }^{\prime \prime \prime}{ }_{2}$ complex of the ligand $\mathrm{H}_{3} \mathrm{~L}^{2}$ will give an insight in the effect of the oxidation states of complexes providing hydrogen bonding capacity. Furthermore, the homovalent complexes $\left[\mathrm{Fe}_{2}{ }_{2}\left(\mathrm{H}_{2} \mathrm{SIM}^{1}\right)(\mu-\mathrm{OAc})_{2}\right] \mathrm{PF}_{6}$ and $\left[\mathrm{Fe}_{2}{ }_{2}\left(\mathrm{H}_{2} \mathrm{~L}^{2}\right)(\mu-\mathrm{OAC})_{2}\right] \mathrm{PF}_{6}$ and their corresponding heterovalent complexes generated by oxidation with ferrocenium hexafluorophosphate will enable to probe the effect of (i) the mixed oxidation state of the diiron core, and (ii) one or two hydrogen bond donors proximal to the Fe" center. Therefore, kinetic studies with the substrate models BDNPP and DNPP have been conducted and the obtained results are presented in the following chapter.

\subsection{PhOSPHOESTER HYDROLYSIS STUDIES OF DIIRON COMPLEXES}

The sensitivity of the $\mathrm{Fe}_{2}{ }_{2}$ complexes to oxidation made it necessary to undertake the studies of the catalytic activity of the complexes $\left[\mathrm{Fe}_{2}{ }_{2}\left(\mathrm{H}_{2} \mathrm{SIM}^{1}\right)(\mu-\mathrm{OAc})_{2}\right]^{+}$and $\left[\mathrm{Fe}_{2}{ }_{2}\left(\mathrm{H}_{2} \mathrm{~L}^{2}\right)(\mu-\mathrm{OAc})_{2}\right]^{+}$under 
inert atmosphere and therefore the experiments were carried out in a glovebox. It should be noted that the number of data points is smaller as well as the variance within the data points is significantly larger, compared to the investigations undertaken with the $\mathrm{Zn}_{2}{ }_{2}$ complexes owing to the more difficult procedures necessitated by manipulating the samples in the glovebox. The plots obtained for the dependence of the initial hydrolysis rate on the $\mathrm{pH}$ and on the initial substrate concentration are depicted in Figure 62. Unfortunately, the $v_{0} v s . p H$ curves could not been fitted following the procedures employed to model the analogous $\mathrm{Zn}_{2}$ complexes and therefore only qualitative comparison is possible.

\section{pH Dependence}

The initial rate vs. pH profiles for $\left[\mathrm{Fe}_{2}{ }_{2}\left(\mathrm{H}_{2} \mathrm{SIM}^{1}\right)(\mu-\mathrm{OAc})_{2}\right]^{+}$and $\left[\mathrm{Fe}_{2}{ }_{2}\left(\mathrm{H}_{2} \mathrm{~L}^{2}\right)(\mu-\mathrm{OAc})_{2}\right]^{+}$exhibited a similar shape. This finding is in contrast to the corresponding $\mathrm{Zn}_{2}{ }_{2}$ complexes of the ligands $\mathrm{H}_{3} \mathrm{SIM}^{1}$ and $\mathrm{H}_{3} \mathrm{~L}^{2}$ (discussed in Chapter 7.2), which showed significantly different curve shapes. The $\left[\mathrm{Fe}_{2}{ }_{2}\left(\mathrm{H}_{2} \mathrm{~L}^{2}\right)(\mu-\mathrm{OAc})_{2}\right]^{+}$complex exhibited lower hydrolysis rates compared to $\left[\mathrm{Fe}_{2}{ }_{2}\left(\mathrm{H}_{2} \mathrm{SIM}^{1}\right)(\mu-\mathrm{OAC})_{2}\right]^{+}$under the same condition. The curve shapes indicate that three active species are present in the $\mathrm{pH}$ range between $\mathrm{pH} 7$ and $\mathrm{pH}$ 10. The first deprotonation step around $\mathrm{pH} 7$ generates an active species; deprotonation of this species around pH 8.6 lowers its activity, while a third deprotonation at $\mathrm{pH}>9.5$ leads again to an increase of activity. This behavior is significantly different to the $\mathrm{Zn}_{2}{ }_{2}$ complexes $\left[\mathrm{Zn}_{2}{ }_{2}\left(\mathrm{H}_{2} \mathrm{SIM}^{1}\right)(\mu-\mathrm{OAc})(\mathrm{OH})\right]^{+}$and $\left[\mathrm{Zn}_{2}{ }_{2}\left(\mathrm{H}_{2} \mathrm{~L}^{2}\right)(\mu-\mathrm{OAC})_{2}\right]^{+}$, which showed a bell-shaped and a sigmoidal profile, respectively (Chapter 7.2).
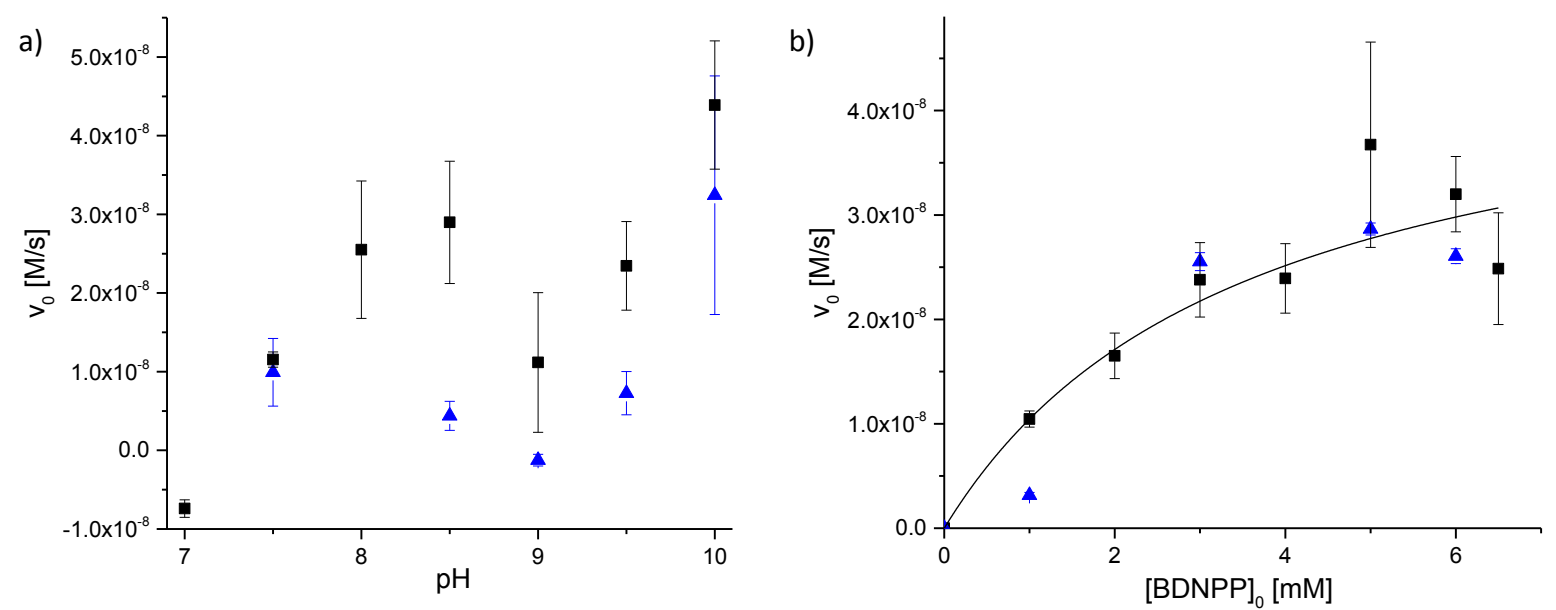

Figure 62: a) $\mathrm{pH}$ dependence and b) substrate concentration dependence at $\mathrm{pH}=9.5$ of BDNPP hydrolysis activity for $\left[\mathrm{Fe}_{2}{ }_{2}\left(\mathrm{H}_{2} \mathrm{SIM}^{1}\right)(\mathrm{OAc})_{2}\right]^{+}$(black squares) and $\left[\mathrm{Fe}_{2}{ }_{2}\left(\mathrm{H}_{2} \mathrm{~L}^{2}\right)(\mathrm{OAc})_{2}\right]^{+}$(blue triangles).

In contrast to $\left[\mathrm{Fe}_{2}{ }_{2}\left(\mathrm{H}_{2} \mathrm{~L}^{2}\right)(\mu-\mathrm{OAC})_{2}\right]^{+}$, the Fe ${ }_{2}{ }_{2}$ complex of ligand $\mathrm{H}_{3} \mathrm{~L}^{2}$ exhibited an initial rate vs. $\mathrm{pH}$ profile with a relatively sharp activity maximum at $\mathrm{pH} 6.5 .{ }^{109}$ The activity maximum in the region between $\mathrm{pH} 7$ and $\mathrm{pH} 9$ for $\left[\mathrm{Fe}_{2}{ }_{2}\left(\mathrm{H}_{2} \mathrm{~L}^{2}\right)(\mu-\mathrm{OAc})_{2}\right]^{+}$is at higher $\mathrm{pH}$ compared to 
$\left[\mathrm{Fe}^{\prime \prime \prime}{ }_{2}\left(\mathrm{H}_{2} \mathrm{~L}^{2}\right)(\text { solv })_{x}\right]^{5+}$. This activity shift to higher $\mathrm{pH}$ values is in agreement with the $\mathrm{pK}_{\mathrm{a}}$ values of iron-bound water molecules ( $\mathrm{pK}_{\mathrm{a}}=9.5$ for $\mathrm{Fe}^{\prime \prime}$ and $\mathrm{pK}_{\mathrm{a}}=2.2$ for Fe $\mathrm{e}^{\prime \prime \prime}$ ) ascribed to the lower Lewis acidity of Fe" compared to Fe"ll. ${ }^{165}$

\section{Substrate Concentration Dependence}

The dependence of the BDNPP hydrolysis rate on the substrate concentration was determined at $\mathrm{pH}=9.5$ to access a catalytic active species of the $\mathrm{Fe}_{2}{ }_{2}$ complexes and to allow comparison to the studies conducted with the respective $\mathrm{Zn}_{2}{ }_{2}$ complexes. The measurements with complex $\left[\mathrm{Fe}_{2}{ }_{2}\left(\mathrm{H}_{2} \mathrm{~L}^{2}\right)(\mu-\mathrm{OAC})_{2}\right]^{+}$, in which the two hydrogen bonding pivaloyl-amide residues are positioned in a symmetric manner, exhibited similar hydrolysis rates to $\left[\mathrm{Fe}_{2}{ }_{2}\left(\mathrm{H}_{2} \mathrm{SIM}^{1}\right)(\mu-\mathrm{OAc})_{2}\right]^{+}$, the latter bearing the hydrogen bond donors in an asymmetric fashion. Therefore, the activity of these complexes within the first minutes of catalysis appears to be independent on the position of the hydrogen bond donors. The data obtained are depicted in Figure 62b and show MichaelisMenten saturation behavior. Fitting the data of $\left[\mathrm{Fe}_{2}{ }_{2}\left(\mathrm{H}_{2} \mathrm{SIM}^{1}\right)(\mu-\mathrm{OAc})_{2}\right]^{+}$with the well-known Michaelis-Menten equation (Equation 22) provided values for the parameters $k_{c a t}, K_{M}$, and $\mathrm{k}_{\mathrm{cat}} / \mathrm{K}_{\mathrm{M}}$. Table 23 lists these values and compares them to data for other diiron complexes.

Table 23: Kinetic data ( $k_{c a t}$ in $\left[10^{-3} \mathrm{~s}^{-1}\right], K_{M}$ in $[\mathrm{mM}]$ and $k_{c a t} / K_{M}$ in $\left[s^{-1} \mathrm{M}^{-1}\right]$ ) of BDNPP hydrolysis for $\left[\mathrm{Fe}_{2}{ }_{2}\left(\mathrm{H}_{2} \mathrm{SIM}^{1}\right)(\mu-\mathrm{OAc})_{2}\right]^{+}$and reported diiron complexes (all kinetic studies were undertaken in a mixture of acetonitrile:aqueous buffer solution (1:1)).

\begin{tabular}{|c|c|c|c|c|c|c|}
\hline complex & $\mathrm{pK}_{\mathrm{a}}(\mathrm{I})$ & $\mathrm{pK}_{\mathrm{a}}(\mathrm{II})$ & $k_{\text {cat }}$ & $\mathrm{K}_{\mathrm{M}}$ & $\mathrm{k}_{\mathrm{cat}} / \mathrm{K}_{\mathrm{M}}$ & $\mathrm{pH}_{\mathrm{MM}^{\mathrm{a}}}$ \\
\hline$\left[\mathrm{Fe}^{\prime \prime}{ }_{2}\left(\mathrm{H}_{2} \mathrm{SIM}^{1}\right)(\mu-\mathrm{OAc})_{2}\right]^{+}$ & n.a. & n.a. & $2.37 \pm 0.29$ & $3.53 \pm 0.68$ & 0.67 & 9.5 \\
\hline$\left[\mathrm{Fe}^{\mathrm{III}}{ }_{2}\left(\mathrm{~L}^{1}\right)(\mathrm{solv})_{x}\right]^{5+114}$ & 5.00 & 7.15 & $0.17 \pm 0.01$ & $1.74 \pm 0.35$ & 0.10 & 6.35 \\
\hline$\left[\mathrm{Fe}^{\mathrm{el}}{ }_{4}\left(\mathrm{~L}^{66}\right)_{2}(\mu-\mathrm{OAc})_{2}(\mu-\mathrm{O})(\mu-\mathrm{OH})\left(\mathrm{OH}_{2}\right)_{2}\right]^{2} 278$ & 5.42 & 8.41 & $1.6 \pm 0.2$ & $7.4 \pm 0.6$ & 0.22 & 6.5 \\
\hline$\left[\mathrm{Fe}^{\prime \prime \prime}{ }_{2}\left(\mathrm{H}_{2} \mathrm{~L}^{2}\right)(\mathrm{solv})_{\mathrm{x}}\right]^{5+114}$ & 5.16 & 7.43 & $0.47 \pm 0.03$ & $5.92 \pm 0.62$ & 0.08 & 6.45 \\
\hline$\left[\mathrm{Fe}^{\prime \prime \prime \prime}\left(\mathrm{L}^{67}\right)(\text { solv })_{x}\right]^{5+} 114$ & 5.39 & 9.38 & $0.65 \pm 0.03$ & $7.38 \pm 0.69$ & 0.09 & 7.44 \\
\hline$\left[\mathrm{Fe}^{\prime \prime \prime \prime}\left(\mathrm{H}_{2} \mathrm{~L}^{6}\right)(\mu-\mathrm{O}) \mathrm{Cl}_{2}\right]^{2+108}$ & 5.0 & 7.3 & $1.33 \pm 0.08$ & $1.94 \pm 0.37$ & 0.68 & 6.17 \\
\hline$\left[\mathrm{Fe}^{\prime \prime \prime} \mathrm{Fe} \mathrm{el}^{\prime \prime}\left(\mathrm{L}^{4}\right)(\mu-\mathrm{OAC})_{2}\right]^{+140}$ & 5.03 & 6.93 & 1.88 & 4.63 & 0.41 & 5.6 \\
\hline$\left[\mathrm{Fe}^{11 ! \prime} \mathrm{Fe}^{\prime \prime}\left(\mathrm{L}^{67}\right)(\text { solv })_{x}\right]^{4+114}$ & 5.30 & 8.02 & $1.14 \pm 0.04$ & $2.30 \pm 0.24$ & 0.50 & 6.69 \\
\hline
\end{tabular}

${ }^{\mathrm{a}} \mathrm{pH}$ of aqueous buffer solution used for substrate dependence assays.

Compared to other examples of phenolate-bridged diiron complexes, $\left[\mathrm{Fe}_{2}{ }_{2}\left(\mathrm{H}_{2} \mathrm{SIM}^{1}\right)(\mu-\mathrm{OAc})_{2}\right]^{+}$ exhibits the highest hydrolysis rate reported to date. In addition, the catalytic efficiency is the second highest reported for diiron(III) complexes after $\left[\mathrm{Fe}^{\mathrm{III}}{ }_{2}\left(\mathrm{H}_{2} \mathrm{~L}^{6}\right)(\mu-\mathrm{O}) \mathrm{Cl}_{2}\right]^{2+}$. Although the latter complex exhibits a lower hydrolysis rate, it has a higher catalytic efficiency to $\left[\mathrm{Fe}_{2}{ }_{2}\left(\mathrm{H}_{2} \mathrm{SIM}^{1}\right)(\mu-\mathrm{OAc})_{2}\right]^{+}$due to higher substrate affinity. The Michaelis-Menten constant $\mathrm{K}_{\mathrm{M}}$ and subsequently the substrate affinity of complex $\left[\mathrm{Fe}_{2}{ }_{2}\left(\mathrm{H}_{2} \mathrm{SIM}^{1}\right)(\mu-\mathrm{OAc})_{2}\right]^{+}$is in the medium range compared to the listed diiron complexes.

Comparison of the two complexes $\left[\mathrm{Fe}^{\mathrm{III}}{ }_{2}\left(\mathrm{H}_{2} \mathrm{~L}^{2}\right)(\mathrm{solv})_{\mathrm{x}}\right]^{5+}$ and $\left[\mathrm{Fe}_{2}{ }_{2}\left(\mathrm{H}_{2} \mathrm{~L}^{2}\right)(\mu-\mathrm{OAC})_{2}\right]^{+}$with the same ligand backbone allows the identification of the impact of the oxidation state of the two iron 
centers. The reduced form exhibits a higher hydrolysis rate, a higher substrate affinity and consequently a higher catalytic efficiency, but with the cost of the maximum activity at more basic conditions. Therefore, the higher activity of the two $\mathrm{Fe}_{2}{ }_{2}$ complexes studied in this work compared to the reference complexes listed in Table 23 can be attributed to an interplay of hydrogen bonding ability and the lower Lewis acidity of the two Fe" centers (compared to the $\mathrm{Fe}^{\mathrm{III}}$ centers in the reference complexes). However, it should be noted that the $\mathrm{Fe}_{2}{ }_{2}$ complexes are less reactive at physiological $\mathrm{pH}$ than under basic conditions used for these investigations. The substrate concentration dependence measurements conducted with the $\mathrm{Zn}_{2}{ }_{2}$ complexes $\left[\mathrm{Zn}_{2}{ }_{2}\left(\mathrm{H}_{2} \mathrm{~L}^{2}\right)(\mu-\mathrm{OAc})_{2}\right]^{+}$and $\left[\mathrm{Zn}_{2}{ }_{2}\left(\mathrm{H}_{2} \mathrm{SIM}^{1}\right)(\mu-\mathrm{OAc})(\mathrm{OH})\right]^{+}$also under basic conditions (pH 9.5) resulted for both complexes in lower hydrolysis rates and higher Michaelis-Menten constants than the $\mathrm{Fe}_{2}{ }_{2}$ complex $\left[\mathrm{Fe}_{2}{ }_{2}\left(\mathrm{H}_{2} \mathrm{SIM}^{1}\right)(\mu-\mathrm{OAc})_{2}\right]^{+}$. Consequently, the catalytic efficiencies of $\left[\mathrm{Zn}_{2}{ }_{2}\left(\mathrm{H}_{2} \mathrm{~L}^{2}\right)(\mu-\mathrm{OAc})_{2}\right]^{+}$and $\left[\mathrm{Zn}_{2}{ }_{2}\left(\mathrm{H}_{2} \mathrm{SIM}^{1}\right)(\mu-\mathrm{OAc})(\mathrm{OH})\right]^{+}$are lowered compared to $\left[\mathrm{Fe}_{2}{ }_{2}\left(\mathrm{H}_{2} \mathrm{SIM}^{1}\right)(\mu-\mathrm{OAC})_{2}\right]^{+}$; two-fold in the case of $\left[\mathrm{Zn}_{2}{ }_{2}\left(\mathrm{H}_{2} \mathrm{~L}^{2}\right)(\mu-\mathrm{OAc})_{2}\right]^{+}$and four-fold in the case of $\left[\mathrm{Zn}_{2}{ }_{2}\left(\mathrm{H}_{2} \mathrm{SIM}^{1}\right)(\mu-\mathrm{OAC})(\mathrm{OH})\right]^{+}$.

\section{Turn Over Numbers}

In order to probe the long-term catalytic behavior of the diiron complexes of ligand $\mathrm{H}_{3} \mathrm{SIM}^{1}$ and $\mathrm{H}_{3} \mathrm{~L}^{2}$ studies of the TONs were conducted at $25^{\circ} \mathrm{C}$ with [complex $]_{0}=10 \mu \mathrm{M}$ and $[\mathrm{BDNPP}]_{0}=3.0 \mathrm{mM}$ at different $\mathrm{pH}$. The assays were prepared in the glovebox using $0.5 \mathrm{mM}$ solutions of $\left[\mathrm{Fe}_{2}{ }_{2}\left(\mathrm{H}_{2} \mathrm{SIM}^{1}\right)(\mu-\mathrm{OAc})_{2}\right]^{+}$and $\left[\mathrm{Fe}_{2}{ }_{2}\left(\mathrm{H}_{2} \mathrm{~L}^{2}\right)(\mu-\mathrm{OAc})_{2}\right]^{+}$as well as $0.5 \mathrm{mM}$ mixtures of $\left[\mathrm{Fe}_{2}{ }_{2}\left(\mathrm{H}_{2} \mathrm{SIM}^{1}\right)(\mu-\mathrm{OAC})_{2}\right]^{+}$and $\left[\mathrm{Fe}_{2}{ }_{2}\left(\mathrm{H}_{2} \mathrm{~L}^{2}\right)(\mu-\mathrm{OAC})_{2}\right]^{+}$with ferrocenium hexafluorophosphate (1:1), prepared 24 hours prior to the TON studies. Samples were taken from these assays at various intervals during the experiment, diluted with solvent, and their UV-vis spectra were recorded to determine the amount of phosphoester hydrolysis. The increase in the absorbance at $400 \mathrm{~nm}$, assigned to the hydrolysis product 2,4-dinitrophenolate, was monitored over time and TON values were calculated after nine days using the Beer-Lambert Law. The resulting data obtained at pH 9.5 and pH 11 are given in Figure 63 and Table 24. $\left[\mathrm{Zn}_{2}{ }_{2}\left(\mathrm{~L}^{1}\right)(\mu-\mathrm{OAC})_{2}\right] \mathrm{PF}_{6}$ was also included in the process to ensure that there is no effect given by undertaking the experiments under inert atmosphere. In fact, this $\mathrm{Zn}_{2}{ }_{2}$ complex exhibit comparable TONs to those obtained under aerobic conditions (Chapter 7.2). Furthermore, the $\mathrm{Zn}_{2}{ }_{2}$ complex lacking hydrogen bond donors exhibited the highest TONs throughout the study. 

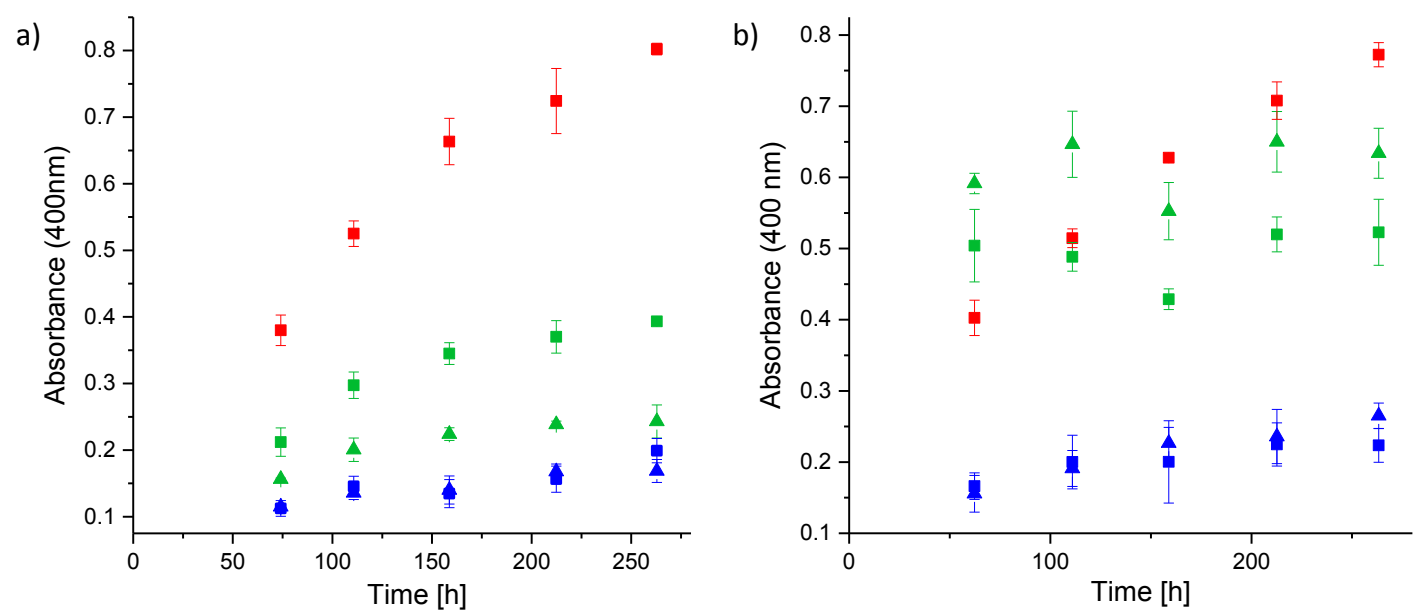

Figure 63: Time dependence of the absorbance band of 2,4-nitrophenolate (400 nm) a) at pH 9.5 and b) at pH 11 with $\left[\mathrm{Zn}_{2}{ }_{2}\left(\mathrm{~L}^{1}\right)(\mu-\mathrm{OAc})_{2}\right]^{+}(\mathrm{red}),\left[\mathrm{Fe}_{2}{ }_{2}\left(\mathrm{H}_{2} \mathrm{SIM}^{1}\right)(\mu-\mathrm{OAc})_{2}\right]^{+}$(green), and $\left[\mathrm{Fe}_{2}{ }_{2}\left(\mathrm{H}_{2} \mathrm{~L}^{2}\right)(\mu-\mathrm{OAc})_{2}\right]^{+}$(blue) with addition of 1 eq $\mathrm{FeCp}_{2} \mathrm{PF}_{6}$ (triangles) and without (squares).

The TONs for the diiron complexes increase with increasing $\mathrm{pH}$, but it should be mentioned that all four iron containing assays show similar TONs when the experiments are conducted at $\mathrm{pH}<9$. However, under more basic condition the structural variations in the diiron complexes were reflected in the differences of their determined TONs. At basic conditions $(\mathrm{pH}>9)$ the asymmetric diiron complex $\left[\mathrm{Fe}_{2}{ }_{2}\left(\mathrm{H}_{2} \mathrm{SIM}^{1}\right)(\mu-\mathrm{OAC})_{2}\right]^{+}$(depicted in green in Figure 63) showed higher TONs than the symmetric counterpart $\left[\mathrm{Fe}_{2}{ }_{2}\left(\mathrm{H}_{2} \mathrm{~L}^{2}\right)(\mu-\mathrm{OAc})_{2}\right]^{+}$(depicted in blue in Figure 63 ). This development seems independent on the oxidation state of the iron centers as the mixtures of the respective complexes with one equivalent of ferrocenium hexafluorophosphate followed the same trend. These findings lead to the conclusion that two hydrogen bond donors proximal to one $\mathrm{Fe}^{\|}$center in the $\mathrm{Fe}_{2}{ }_{2}$ model complex help to prevent more efficiently the complex being inhibited than one hydrogen bond donor proximal to each Fe"l center. Interestingly, the impact of the hydrogen bond donors was found to be reversed for the $\mathrm{Zn}_{2}{ }_{2}$ complexes: one hydrogen bond donor adjacent to each $\mathrm{Zn}$ " center is more efficient in preventing complex inhibition than two hydrogen bond donors proximal to one of the two $\mathrm{Zn}$ " centers.

Table 24: Turn over numbers (TONs) of BDNPP hydrolysis for $\left[\mathrm{Fe}_{2}{ }_{2}\left(\mathrm{H}_{2} \mathrm{SIM}^{1}\right)(\mu-\mathrm{OAc})_{2}\right]^{+}$and $\left[\mathrm{Fe}_{2}\left(\mathrm{H}_{2} \mathrm{~L}^{2}\right)(\mu-\mathrm{OAc})_{2}\right]^{+}$ as well as mixtures of those complexes with 1 eq FeCp $\mathrm{PF}_{6}$.

\begin{tabular}{|c|c|c|c|}
\hline \multirow[t]{2}{*}{ complex } & \multicolumn{3}{|c|}{ TON after $9 \mathrm{~h}$} \\
\hline & $\mathrm{pH} 9$ & $\mathrm{pH} 9.5$ & $\mathrm{pH} 11$ \\
\hline$\left[\mathrm{Fe}_{2}\left(\mathrm{H}_{2} \mathrm{SIM}^{1}\right)(\mu-\mathrm{OAC})_{2}\right]^{+}$ & $11 \pm 2$ & $61 \pm 4$ & $86 \pm 4$ \\
\hline$\left[\mathrm{Fe}_{2}\left(\mathrm{H}_{2} \mathrm{SIM}^{1}\right)(\mu-\mathrm{OAc})_{2}\right]^{+}+1$ eq $\mathrm{FeCp}_{2}{ }^{+}$ & $6 \pm 6$ & $39 \pm 1$ & $107 \pm 7$ \\
\hline$\left[\mathrm{Fe}_{2}{ }_{2}\left(\mathrm{H}_{2} \mathrm{~L}^{2}\right)(\mu-\mathrm{OAc})_{2}\right]^{+}$ & $2 \pm 2$ & $26 \pm 3$ & $37 \pm 5$ \\
\hline$\left[\mathrm{Fe}_{2}{ }_{2}\left(\mathrm{H}_{2} \mathrm{~L}^{2}\right)(\mu-\mathrm{OAc})_{2}\right]^{+}+1$ eq $\mathrm{FeCp}_{2}{ }^{+}$ & $2 \pm 2$ & $28 \pm 2$ & $39 \pm 6$ \\
\hline
\end{tabular}

Comparison of the TONs obtained with assays containing the same $\mathrm{Fe}_{2}{ }_{2}$ complex but differing in the presence of ferrocenium hexafluorophosphate enables to study the impact of the oxidation 
states of the bound iron ions. The TON of the symmetric diiron complex bearing the $\mathrm{H}_{3} \mathrm{~L}^{2}$ ligand backbone was found to be independent on the presence of ferrocenium hexafluorophosphate. This was different when the asymmetric complex $\left[\mathrm{Fe}_{2}{ }_{2}\left(\mathrm{H}_{2} \mathrm{SIM}^{1}\right)(\mu-\mathrm{OAC})_{2}\right]^{+}$was used as catalyst. The long-term measurements at $\mathrm{pH} 9$ and $\mathrm{pH} 9.5$ resulted in higher TON values in the absence of ferrocenium hexafluorophosphate indicating that the heterovalent complex [Fe"ll $\left.\mathrm{Fe}^{\prime \prime}\left(\mathrm{H}_{2} \mathrm{SIM}^{1}\right)(\text { solv })_{\mathrm{x}}\right]^{4+}$ is favored for bridging phosphate inhibition for the corresponding homovalent $\mathrm{Fe}_{2} \|_{2}$ complex. This finding is in agreement with the higher water exchange rate of $\mathrm{Fe}^{\text {Il }}$ compared to Fe $\mathrm{Fe}^{\mathrm{III}}\left(10^{6} \mathrm{~s}^{-1}\right.$ and $\left.10^{2} \mathrm{~s}^{-1}\right) \cdot{ }^{278,296-298}$ However, at strongly basic conditions ( $\left.\mathrm{pH} 11\right)$ the trend was reversed, i.e. the Fe ${ }^{I I \prime} \mathrm{Fe}^{\prime \prime}$ complex revealed a higher TON than the $\mathrm{Fe}_{2}{ }_{2}$ complex of $\mathrm{H}_{3} \mathrm{SIM}^{1}$.

Studies of the hydrolysis of phosphomonoesters using DNPP as model substrate in the TON assays revealed no acceleration of phosphoester hydrolysis compared to the autohydrolysis assay independent on (i) the symmetry, and (ii) the oxidation state of the diiron complexes.

\subsection{SUMMARY}

The investigation of the phosphoester hydrolysis undertaken with the $\mathrm{Fe}_{2}{ }_{2}$ complexes $\left[\mathrm{Fe}_{2}{ }_{2}\left(\mathrm{H}_{2} \mathrm{SIM}^{1}\right)(\mu-\mathrm{OAC})_{2}\right]^{+}$and $\left[\mathrm{Fe}_{2}{ }_{2}\left(\mathrm{H}_{2} \mathrm{~L}^{2}\right)(\mu-\mathrm{OAC})_{2}\right]^{+}$and comparison with the corresponding $\mathrm{Zn}_{2}{ }_{2}$ and Fe"ll' $\mathrm{Fe}^{\text {II }}$ complexes gave insights on the impact of (i) the secondary coordination sphere, and (ii) the Lewis acidity of the metal ion bound to the ligand backbone. The hydrolysis rate was found to be similar for both complexes $\left[\mathrm{Fe}_{2}{ }_{2}\left(\mathrm{H}_{2} \mathrm{SIM}^{1}\right)(\mu-\mathrm{OAc})_{2}\right]^{+}$and $\left[\mathrm{Fe}_{2}{ }_{2}\left(\mathrm{H}_{2} \mathrm{~L}^{2}\right)(\mu-\mathrm{OAc})_{2}\right]^{+}$; thus, the activity in the first minutes of catalysis seems to be independent on the hydrogen bond donor position. However, significant differences in long-term activity studies, i.e. the TONs, of $\left[\mathrm{Fe}_{2}{ }_{2}\left(\mathrm{H}_{2} \mathrm{SIM}^{1}\right)(\mu-\mathrm{OAC})_{2}\right]^{+}$and $\left[\mathrm{Fe}_{2}{ }_{2}\left(\mathrm{H}_{2} \mathrm{~L}^{2}\right)(\mu-\mathrm{OAC})_{2}\right]^{+}$indicate that the steric hindrance of two pivaloyl-amide residues adjacent to one Fe" center prevents inhibition by a bridging phosphomonoester molecule more efficiently than positioning one sterically demanding hydrogen bond donor proximal to each Fe"l center. Interestingly, the effect of the position of the pivaloyl-amide residues is found to be the opposite for the respective $\mathrm{Zn}_{2}$ complexes (discussed in Chapter 7.2). Of the two influences of the pivaloyl-amide residues towards the bridging phosphoester coordination, (i) the hindered access to the metal ion, and (ii) the stabilization by hydrogen bond formation, the first appears more important for the $\mathrm{Fe}_{2}{ }_{2}$ complexes while the latter seems more important for the $\mathrm{Zn}_{2}$ complexes. But it should be mentioned that both $\mathrm{Fe}_{2}{ }_{2}$ complexes exhibit lower TONs than the corresponding $\mathrm{Zn}_{2}{ }_{2}$ complexes, as expected due to the lower Lewis acidity and the faster water exchange rate of $\mathrm{Zn}^{\|}$compared to $\mathrm{Fe}^{\|}$. However, the Fe" ${ }_{2}$ complexes 
revealed higher hydrolysis rates and catalytic efficiencies than the $\mathrm{Zn}_{2}{ }_{2}$ complexes. Therefore, the higher Lewis acidity of Fe" compared to $\mathrm{Zn}^{\prime \prime}$ appears to enhance not only the hydrolysis acceleration but also the binding of inhibiting phosphomonoesters. Similarly, the oxidation of one of the two $\mathrm{Fe}^{\prime l}$ centers in $\left[\mathrm{Fe}_{2}{ }_{2}\left(\mathrm{H}_{2} \mathrm{SIM}^{1}\right)(\mu-\mathrm{OAC})_{2}\right]^{+}$forming a more accurate Fe ${ }^{\text {III }} \mathrm{Fe}{ }^{\text {Il }}$ model system for PAP, lead to a lower TON. This is in agreement with the decreased water exchange rate and the higher Lewis acidity of Fe"ll compared to Fe"l. ${ }^{164,166}$ However, the Fe" ${ }_{2}$ complex of $\mathrm{H}_{2} \mathrm{~L}^{2}$ reveales a more basic $\mathrm{pH}$ maximum of the hydrolysis activity than the respective $\mathrm{Fe}^{\mathrm{III}}{ }_{2}$ complex supporting the presumption that the role of the Fe ${ }^{\text {III }}$ center in the active site of PAP is the provision of a nucleophilic hydroxide at low pH. 


\title{
Chapter 9
}

\section{Phosphatase Activity of}

\author{
Heterovalent
}

Heterodinuclear Complexes ${ }^{\mathrm{h}}$

${ }^{\mathrm{h}}$ Main parts of this chapter will be published in "Asymmetric Mono- and Dinuclear Ga"l' and Zn" complexes as Models for Purple Acid Phosphatases (PAPs)"; Simone Bosch, Peter Comba, Lawrence R. Gahan and Gerhard Schenk; manuscript in preparation. 


\subsection{INTRODUCTION}

As a prototype of dinuclear metallohydrolases, PAPs catalyze the hydrolysis of phosphomonoesters containing two metal ions in close proximity in the active site of PAPs. More precisely, PAPs are the only dinuclear metallohydrolases which require a mixed-valent dinuclear site for their activity. ${ }^{21}$ While mammalian PAPs consist of a Fe"l'Fe" center, ${ }^{49}$ the metal ion composition in PAPs isolated from plants was found to be either Fe" ${ }^{\prime \prime \prime} n^{\prime \prime}$ or Fe ${ }^{\prime \prime \prime} \mathrm{Mn}^{\prime \prime}$. 23, 24, 67, 68 It has been shown, however, that replacement of the Fe"ll center in pig PAP and bovine spleen PAP by a Ga"' ion generates Ga"' $\mathrm{Fe}^{\text {"l }}$ and Ga"' $\mathrm{Zn}$ " derivatives that exhibit similar activities to the native enzymes, ${ }^{66,142,167,299,300}$ and showing that the diamagnetic Gall' ion was able to act as a functional analog of paramagnetic Fe ${ }^{\text {III }}$ in PAPs. ${ }^{146}$

Small heterodinuclear mixed-valent compounds modeling the structural and functional properties of metallohydrolases have been shown to be useful tools to study the roles of structural features within the active site of the corresponding enzymes. For example, the complex $\left[\mathrm{Fe}^{\prime \prime \prime} \mathrm{Zn} \mathrm{n}^{\prime \prime}\left(\mathrm{L}^{4}\right)(\mu-\mathrm{OAc})_{2}\right]^{+}$has shown that (i) the model substrate BDNPP only interacts with the $\mathrm{Zn}$ " ion, and (ii) the bridging hydroxide is a much weaker nucleophile than the terminal

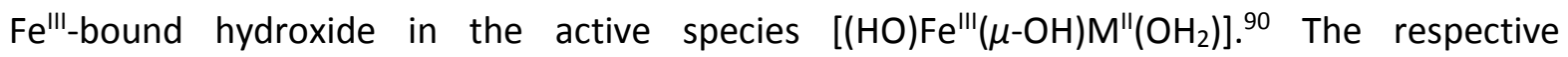
isostructural complex [Ga"l'Zn" $\left.\left(\mathrm{L}^{4}\right)(\mu-\mathrm{OAc})_{2}\right]^{+}$was also studied and exhibited a higher TON than the analogous Fe"' $\mathrm{Zn}$ " complex. ${ }^{66}$ A similar result was observed from kinetic studies of the complexes [Fe"l'Co" $\left.\left(\mathrm{L}^{4}\right)(\mu-\mathrm{OAC})_{2}\right]^{+}$and $\left[\mathrm{Ga}^{\prime \prime \prime} \mathrm{Co}^{\prime \prime}\left(\mathrm{L}^{4}\right)(\mu-\mathrm{OAC})_{2}\right]^{+}$, and Table 25 summarizes the reported kinetic data. ${ }^{146}$ As in the case of the biomimetics, the Gall' derivative of ufPAP revealed modest increased reactivity consistent with the increased nucleophilic character of the Ga'll-bound hydroxide compared to the Fe"lI-bound hydroxide. ${ }^{146}$ Moreover potentiometric titrations with [Fe"'II $\left.\mathrm{Co}^{\prime \prime}\left(\mathrm{L}^{4}\right)(\mu-\mathrm{OAC})_{2}\right]^{+}$and $\left[\mathrm{Ga}^{\prime \prime \prime} \mathrm{Co} \mathrm{C}^{\prime \prime}\left(\mathrm{L}^{4}\right)(\mu-\mathrm{OAc})_{2}\right]^{+}$supports the terminal hydroxide being the reaction initiating nucleophile, as assumed previously for [Ga"' $\left.Z n^{\prime \prime}\left(L^{4}\right)(\mu-O A c)_{2}\right]^{+}$and $\left[\mathrm{Fe}^{\prime \prime \prime} \mathrm{Zn} n^{\prime \prime}\left(\mathrm{L}^{4}\right)(\mu-\mathrm{OAc})_{2}\right]^{+}{ }^{146,66}$ Hence, the mechanism that emerges for biomimetics involves the initial monodentate coordination of the substrate to the divalent metal ion, followed by a

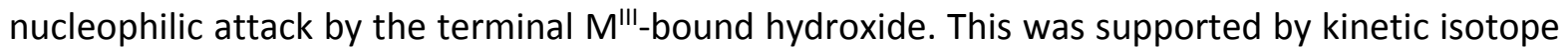
effect and UV-vis spectroscopic substrate binding studies with $\left[\mathrm{Fe}^{\prime \prime \prime} \mathrm{CO}^{\prime \prime}\left(\mathrm{L}^{4}\right)(\mu-\mathrm{OAc})_{2}\right]^{+}$and $\left[\mathrm{Ga}^{\prime \prime \prime} \mathrm{Co}^{\prime \prime}\left(\mathrm{L}^{4}\right)(\mu-\mathrm{OAC})_{2}\right]^{+} .{ }^{146}$

Kinetic studies with a Fe"l'Zn" complex anchored to 3-aminopropyl-functionalized silica (Si3AP) resulted in increased substrate affinities compared to the free catalyst. ${ }^{153}$ This effect was proposed to be caused by stabilization via hydrogen bonds with the residual amino-groups of the 
surface, supporting the role of the non-coordinating histidines in the active site of PAPs to bring the substrate in position suitable for hydrolysis.

Table 25: Kinetic data ( $\mathrm{k}_{\mathrm{cat}}$ in $\left[10^{-3} \mathrm{~s}^{-1}\right], \mathrm{K}_{\mathrm{M}}$ in $[\mathrm{mM}]$ and $\mathrm{k}_{\mathrm{cat}} / \mathrm{K}_{\mathrm{M}}$ in $\left[\mathrm{s}^{-1} \mathrm{M}^{-1}\right]$ ) of BDNPP hydrolysis (all kinetic studies were undertaken in a mixture of acetonitrile:aqueous buffer solution (1:1)).

\begin{tabular}{|c|c|c|c|c|c|c|}
\hline complex & $\mathrm{pK}_{\mathrm{a}}(\mathrm{I})$ & $\mathrm{pK}_{\mathrm{a}}(\mathrm{II})$ & $\mathrm{k}_{\text {cat }}$ & $\mathrm{K}_{\mathrm{M}}$ & $\mathrm{k}_{\mathrm{cat}} / \mathrm{K}_{\mathrm{M}}$ & $\mathrm{pH}_{\mathrm{MM}^{\mathrm{a}}}$ \\
\hline$\left[\mathrm{Fe}^{1 \mid \prime \prime} \mathrm{Zn}^{\prime \prime}\left(\mathrm{L}^{4}\right)(\mu-\mathrm{OAC})_{2}\right]^{+95}$ & 4.8 & 7.5 & 0.73 & 8.10 & 0.09 & 6.1 \\
\hline$\left[\mathrm{Fe}^{\prime \prime \prime \prime} \mathrm{Zn}\left(\mathrm{L}^{4}\right)(\mu-\mathrm{OH})_{2}\right]^{+90}$ & 5.3 & 8.1 & 0.91 & 4.20 & 0.22 & 6.5 \\
\hline$\left[\mathrm{Ga} \mathrm{III}^{\prime \prime} \mathrm{Zn} "\left(\mathrm{~L}^{4}\right)(\mu-\mathrm{OAC})_{2}\right]^{+66}$ & 5.4 & 8.6 & 1.41 & 7.15 & 0.20 & 6.8 \\
\hline$\left[\mathrm{Fe}^{\mathrm{el}} \mathrm{Co} \mathrm{O}^{\prime \prime}\left(\mathrm{L}^{4}\right)(\mu-\mathrm{OAc})_{2}\right]^{146}$ & 5.2 & 8.8 & 1.42 & 92.7 & 0.02 & 7.0 \\
\hline$\left[\mathrm{Ga} \mathrm{III}^{\prime \prime} \mathrm{Co}^{\prime \prime}\left(\mathrm{L}^{4}\right)(\mu-\mathrm{OAC})_{2}\right]^{+146}$ & 5.7 & 8.9 & 1.87 & 88.1 & 0.02 & 7.5 \\
\hline$\left[\mathrm{Fe}^{\mathrm{e} " \mathrm{Zn}}\left(\mathrm{HL}^{\mathrm{l} 8}\right)(\mu-\mathrm{OH})\left(\mathrm{H}_{2} \mathrm{O}\right)\right]^{2+153}$ & - & - & 0.90 & 3.55 & 0.25 & 7.0 \\
\hline$\left[\mathrm{Fe}^{\mathrm{III}} \mathrm{Zn}\left(\mathrm{Si} 3 \mathrm{SP}^{\prime}-\mathrm{L}^{68}\right)(\mu-\mathrm{OH})\left(\mathrm{H}_{2} \mathrm{O}\right)\right]^{2+153}$ & - & - & 0.14 & 1.54 & 0.09 & 7.0 \\
\hline
\end{tabular}

a $\mathrm{pH}$ of aqueous buffer solution used for substrate dependence assays.

Using monogallium(III) complexes of tripodal ligands, $\mathrm{H}_{2} \mathrm{~L}^{69}, \mathrm{H}_{3} \mathrm{~L}^{70}, \mathrm{H}_{3} \mathrm{~L}^{71}$, and $\mathrm{H}_{3} \mathrm{~L}^{72}$ among others (Scheme 22), the influence of the nature of the ligand donor sites on the reactivity of GallI (mimicking the $\mathrm{Fe}^{\mathrm{III}}$ ion in PAPs) has been evaluated. ${ }^{70}$ Thereby, the attack of the coordinated substrate by a Gal'-bound hydroxide (Scheme 22, path a) was shown to be the catalytic mechanism rather than the alternative, where the metal-bound hydroxide acts as a general base deprotonating incoming water, which subsequently attacks the phosphoester substrate (Scheme 22, path b). ${ }^{70}$

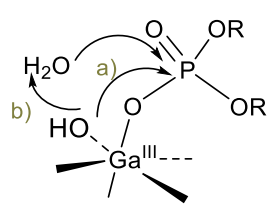<smiles>O=C(O)CN(CC(=O)O)Cc1ccccn1</smiles>

$\mathrm{H}_{2} \mathrm{~L}^{69}$<smiles>O=C(O)CN(CC(=O)O)Cc1cc([N+](=O)[O-])ccc1O</smiles>

$\mathrm{H}_{3} \mathrm{~L}^{70}$<smiles>COc1ccc(O)c(CN(CC(=O)O)CC(=O)O)c1</smiles>

Scheme 22: Substrate activation and the two possibilities for the nucleophilic attack on the phosphorus atom during phosphoester hydrolysis by monogallium(III) model compounds and ligands $\mathrm{H}_{2} \mathrm{~L}^{69}, \mathrm{H}_{3} \mathrm{~L}^{70}, \mathrm{H}_{3} \mathrm{~L}^{71}$, and $\mathrm{H}_{3} \mathrm{~L}^{72}$ employed studying the influence of the nature of the ligand donor sites on the phosphoester hydrolysis activity of Ga'"l complexes. ${ }^{70}$

During the studies, it emerged that the reactivity of the monogallium(III) complexes was enhanced by replacement of neutral nitrogen donor atoms and carboxylate groups by phenolate groups. Specifically it was proposed that the presence of strongly electron-donating phenolate groups $\left(\mathrm{H}_{3} \mathrm{~L}^{72}>\mathrm{H}_{3} \mathrm{~L}^{71}>\mathrm{H}_{3} \mathrm{~L}^{70}>\mathrm{H}_{2} \mathrm{~L}^{69}\right)$ in the coordination sphere of Gall weakens the metalhydroxide bond, thus giving rise to an enhanced nucleophilicity and higher activity of the corresponding Gal'I complexes. ${ }^{70}$ Table 26 lists the reported kinetic data. ${ }^{70}$ 
Table 26: Kinetic data $\left(\mathrm{k}_{\mathrm{cat}}\right.$ in $\left[10^{-3} \mathrm{~s}^{-1}\right], \mathrm{K}_{\mathrm{M}}$ in $[\mathrm{mM}]$ and $\mathrm{k}_{\mathrm{cat}} / \mathrm{K}_{\mathrm{M}}$ in $\left[\mathrm{s}^{-1} \mathrm{M}^{-1}\right]$ ) of BDNPP hydrolysis (all kinetic studies were undertaken in a mixture of methanol-aqueous buffer solution (1:5) at $\left.37^{\circ} \mathrm{C}\right) .{ }^{70}$

\begin{tabular}{l|llllll} 
complex & $\mathrm{pK}_{\mathrm{a}}(\mathrm{I})$ & $\mathrm{pK}_{\mathrm{a}}(\mathrm{II})$ & $\mathrm{k}_{\mathrm{cat}}$ & $\mathrm{K}_{\mathrm{M}}$ & $\mathrm{k}_{\mathrm{cat}} / \mathrm{K}_{\mathrm{M}}$ & $\mathrm{pH}_{\mathrm{MM}^{\mathrm{a}}}$ \\
\hline$\left[\mathrm{Ga} \mathrm{III}^{\prime \prime \prime}\left(\mathrm{L}^{69}\right)(\mu-\mathrm{OH})\right]_{2}$ & 5.69 & 7.12 & 3.61 & 1.34 & 2.69 & 6.4 \\
{$\left[\mathrm{Ga} a^{\prime \prime \prime}\left(\mathrm{L}^{70}\right)\left(\mathrm{H}_{2} \mathrm{O}\right)_{2}\right]$} & 5.43 & 8.59 & 21.2 & 541 & 0.04 & 7.0 \\
{$\left[\mathrm{Ga}^{\prime \prime \prime}\left(\mathrm{L}^{71}\right)\left(\mathrm{H}_{2} \mathrm{O}\right)_{2}\right]$} & 5.90 & 8.99 & 30.5 & 10.3 & 2.96 & 7.5 \\
{$\left[\mathrm{Ga}^{\prime \prime \prime}\left(\mathrm{L}^{72}\right)\left(\mathrm{H}_{2} \mathrm{O}\right)_{2}\right]$} & 7.26 & 9.72 & 46.4 & 7850 & 0.01 & 8.5 \\
\hline
\end{tabular}

${ }^{\mathrm{a}} \mathrm{pH}$ of aqueous buffer solution used for substrate dependence assays.

Comparison of the different monogallium(III) complexes emphasizes that the two most reactive complexes [Ga"' $\left.\left(\mathrm{L}^{71}\right)\left(\mathrm{H}_{2} \mathrm{O}\right)_{2}\right]$ and $\left[\mathrm{Ga}^{\prime \prime \prime}\left(\mathrm{L}^{72}\right)\left(\mathrm{H}_{2} \mathrm{O}\right)_{2}\right]$ have (i) the lowest Lewis acidities, thus providing the lowest substrate activation, and (ii) the strongest hydroxide nucleophile. ${ }^{70}$ Apparently, this reveals that of the two effects of the metal ion in the hydrolysis of phosphoesters, (i) Lewis acid activation of the substrate and (ii) nucleophile activation, the latter is more important in determining the intrinsic reactivity. ${ }^{70}$

In order to stabilize the heterovalent dinuclear core, the peptide backbone of the active site in PAPs forms two chemically distinct environments for the metal centers. In Chapter 3 the selective formation of the heterodinuclear heterovalent complexes [ $\left.\mathrm{Ga}^{\prime \prime \prime} \mathrm{Zn}^{\prime \prime}\left(\mathrm{H}_{2} \mathrm{SIM}^{1}\right)(\mathrm{solv})_{\mathrm{x}}\right]^{4+}$ and [Ga'"' $\left.\mathrm{Zn}^{\prime \prime}\left(\mathrm{H}_{2} \mathrm{SIM}^{4}\right)(\text { solv })_{x}\right]^{3+}$, depicted in Chart 27, has been illustrated. These complexes combine the three essential structural features within the active site of PAPs: (i) the distinct coordination environment in the two binding sites, (ii) the mixed-valent dinuclear metal core, and (iii) the hydrogen bonding network provided by the second coordination sphere. With hydrogen bond donors proximal to the metal centers, mimicking the non-coordinating histidine residues in the active site of PAPs, the impact of hydrogen bonding capacity on the reactivity has been examined by comparison to each other and the reported complex $\left[\mathrm{Ga}^{\prime \prime \prime} \mathrm{Zn} \mathrm{n}^{\prime \prime}\left(\mathrm{L}^{4}\right)(\mu-\mathrm{OAc})_{2}\right]^{+}$; the results are presented in the following chapter.
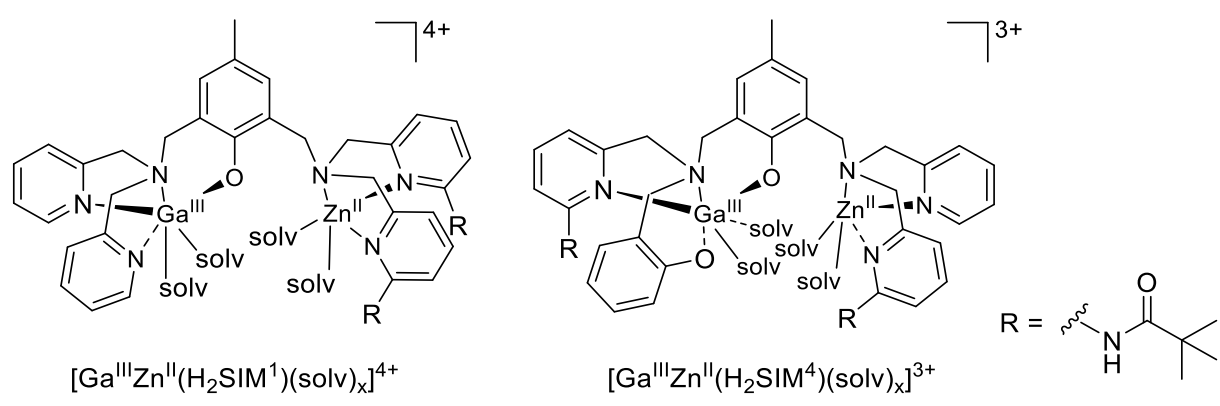

Chart 27: Ga"'Zn" complexes employed in this chapter.

Moreover, the ligands $\mathrm{H}_{3} \mathrm{SIM}^{1}$ and $\mathrm{H}_{4} \mathrm{SIM}^{4}$ have been shown to selectively form mononuclear Ga ${ }^{\text {III }}$ and $\mathrm{Zn}$ " complexes (see Chapter 3 ). Therefore, the role of the specific structural aspect were investigated not only in their Ga"'Zn" complexes but also in their mononuclear Ga"' and $\mathrm{Zn}^{\prime \prime}$ complexes as well as in their dizinc(II) complexes, depicted in Chart 28. 


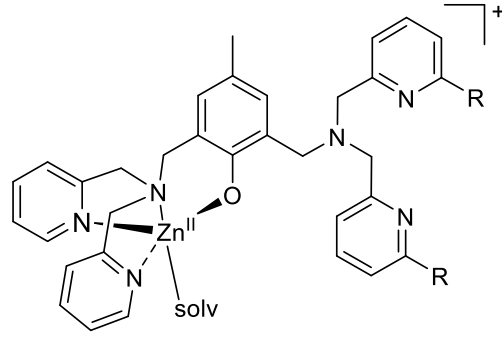

$\left[\mathrm{Zn}^{\prime \prime}\left(\mathrm{H}_{2} \mathrm{SIM}^{1}\right)(\text { solv })_{\mathrm{X}}\right]^{+}$

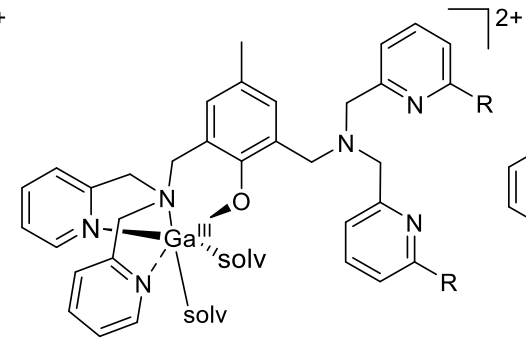

$\left[\mathrm{Ga}^{\prime \prime \prime}\left(\mathrm{H}_{2} \mathrm{SIM}^{1}\right)(\text { solv })_{\mathrm{X}}\right]^{2+}$

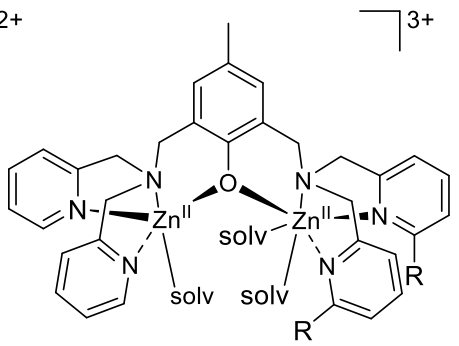

$\left[\mathrm{Zn}_{2}{ }_{2}\left(\mathrm{H}_{2} \mathrm{SIM}^{1}\right)(\text { solv })_{\mathrm{x}}\right]^{3+}$

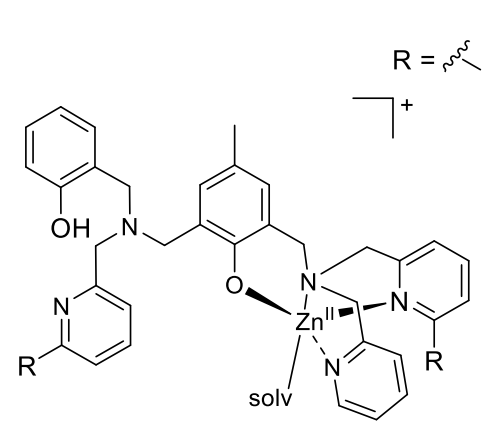

$\left[\mathrm{Zn}^{\prime \prime}\left(\mathrm{H}_{3} \mathrm{SIM}^{4}\right)(\text { solv })_{\mathrm{X}}\right]^{+}$<smiles>CC(C)(C)C(=O)O</smiles>

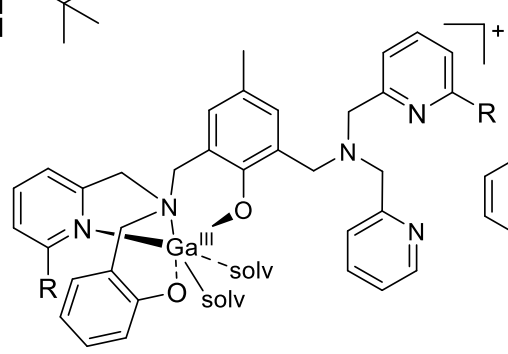

$\left[\mathrm{Ga}^{\prime \prime \prime}\left(\mathrm{H}_{2} \mathrm{SIM}^{4}\right)(\text { solv })_{\mathrm{x}}\right]^{+}$

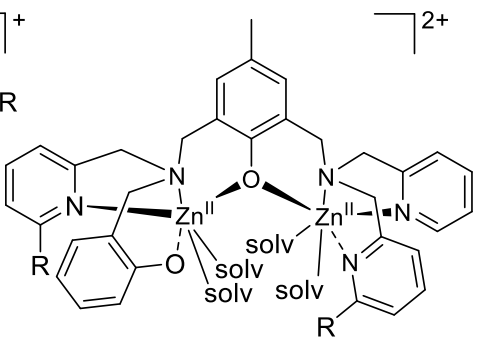

$\left[\mathrm{Zn}_{2}{ }_{2}\left(\mathrm{H}_{2} \mathrm{SIM}^{4}\right)(\text { solv })_{\mathrm{x}}\right]^{2+}$

Chart 28: $\mathrm{Zn}^{\prime \prime}, \mathrm{Ga} \mathrm{all}^{\mathrm{II}}$ and $\mathrm{Zn}_{2}{ }_{2}$ complexes referred to in this chapter.

Prior to the kinetic investigations the catalysts were prepared in solution. Separate $5 \mathrm{mM}$ acetonitrile solutions of $\mathrm{H}_{3} \mathrm{SIM}^{1}, \mathrm{H}_{4} \mathrm{SIM}^{4}$, zinc(II) perchlorate, and gallium(III) perchlorate were prepared. One equivalent of ligand solution was treated with either (i) one equivalent of zinc(II) perchlorate solution, (ii) two equivalents of zinc(II) perchlorate solution, or (iii) one equivalent of gallium(III) perchlorate solution. These mixtures were then heated at $50^{\circ} \mathrm{C}$ for one hour in the case of the zinc(II) containing mixtures and for six hours in the case of the gallium(III) containing mixtures in order to completely form the complexes $\left[\mathrm{Zn}^{\prime \prime}\left(\mathrm{H}_{2} \mathrm{SIM}^{1}\right)(\text { solv })_{\mathrm{x}}\right]^{+},\left[\mathrm{Zn}^{\prime \prime}\left(\mathrm{H}_{3} \mathrm{SIM}^{4}\right)(\mathrm{solv})_{\mathrm{x}}\right]^{+}$, $\left[\mathrm{Zn}_{2}\left(\mathrm{H}_{2} \mathrm{SIM}^{1}\right)(\text { solv })_{x}\right]^{3+},\left[\mathrm{Zn}_{2}{ }_{2}\left(\mathrm{H}_{2} \mathrm{SIM}^{4}\right)(\text { solv })_{x}\right]^{2+},\left[\mathrm{Ga}^{\text {III }}\left(\mathrm{H}_{2} \mathrm{SIM}^{1}\right)(\text { solv })_{x}\right]^{2+}$, and $\left[\mathrm{Ga}^{\text {III }}\left(\mathrm{H}_{2} \mathrm{SIM}^{4}\right)(\text { solv })_{x}\right]^{+}$. The in this way previously prepared gallium(III) complexes of $\mathrm{H}_{3} \mathrm{SIM}^{1}$ and $\mathrm{H}_{4} \mathrm{SIM}^{4}$, $\left[\mathrm{Ga}^{\prime \prime \prime}\left(\mathrm{H}_{2} \mathrm{SIM}^{1}\right)(\text { solv })_{\mathrm{x}}\right]^{2+}$ and $\left[\mathrm{Ga}^{\prime \prime \prime}\left(\mathrm{H}_{2} \mathrm{SIM}^{4}\right)(\text { solv })_{\mathrm{x}}\right]^{+}$, were treated with one equivalent of zinc(II) perchlorate and heated at $50^{\circ} \mathrm{C}$ for a further one hour to generate the Ga"' $\mathrm{Zn}$ " complexes $\left[\mathrm{Ga}^{\prime \prime \prime} \mathrm{Zn} n^{\prime \prime}\left(\mathrm{H}_{2} \mathrm{SIM}^{1}\right)(\text { solv })_{\mathrm{x}}\right]^{4+}$ and $\left[\mathrm{Ga}^{\prime \prime \prime} \mathrm{Zn}{ }^{\prime \prime}\left(\mathrm{H}_{2} \mathrm{SIM}^{4}\right)(\text { solv })_{\mathrm{x}}\right]^{3+}$. All solutions were diluted with acetonitrile in the way that the final complex concentration was $1 \mathrm{mM}$. 


\subsection{PH BEHAVIOR OF GA"III" COMPLEXES}

For determination of the $\mathrm{pK}_{\mathrm{a}}$ values of metal-bound water deprotonation in the different complexes UV-vis spectroscopic titrations were undertaken. The investigations were carried out in acetonitrile:aqueous buffer mixtures $(1: 1)$ and the reported $\mathrm{pH}$ values refer to the $\mathrm{pH}$ of the buffer solution. Figure 64a depicts as an example the resulted UV-vis spectra for the complex $\left[\mathrm{Ga}^{\prime \prime \prime} \mathrm{Zn}^{\prime \prime}\left(\mathrm{H}_{2} \mathrm{SIM}^{1}\right)(\text { solv })_{\mathrm{x}}\right]^{4+}$.

When the spectrum recorded at pH 5.5 is excluded, two isosbestic points are visible at $293 \mathrm{~nm}$ and $312 \mathrm{~nm}$, indicating an equilibrium of two species. The absorbance vs. $\mathrm{pH}$ plots at different wavelengths are given in Figure 64b. These plots were fitted according to the procedure for the dizinc(II) complexes explained in Chapter 4.3. The $\mathrm{pK}_{\mathrm{a}}$ values obtained for all complexes studied in this Chapter are given in Table 27.
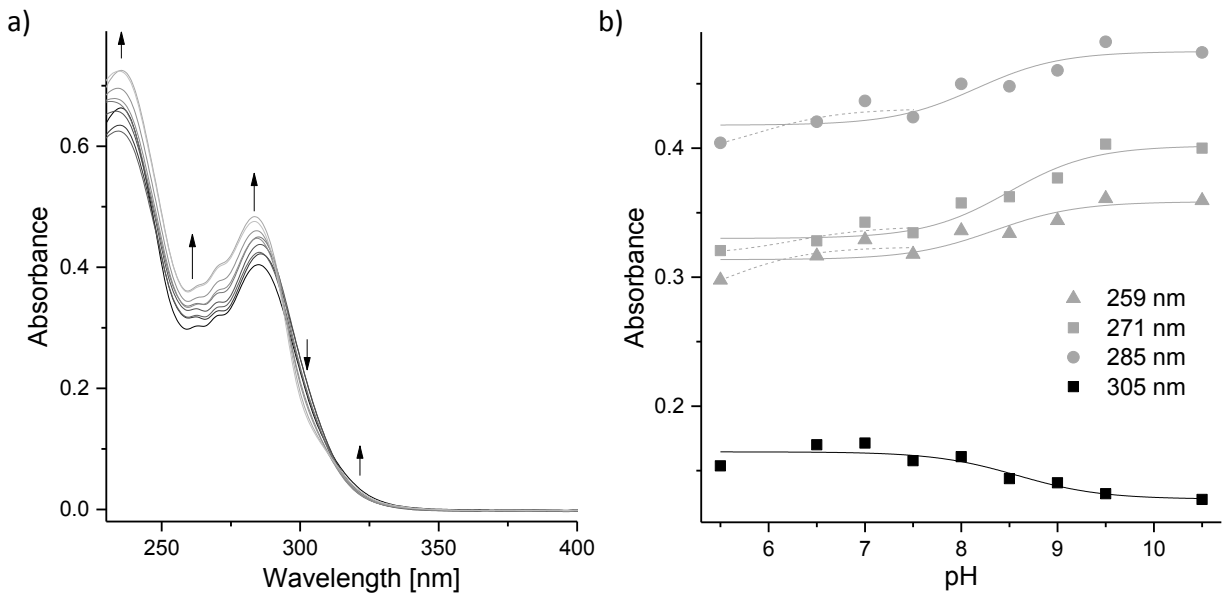

Figure 64: a) UV-vis spectroscopic titration spectra ( $25 \mu \mathrm{M}$ in acetonitrile:aqueous buffer between $\mathrm{pH} 5.5$ and 10.5) and b) absorbance vs. pH plot for $\left[\mathrm{Ga}^{\prime \prime \prime} \mathrm{Zn}^{\prime \prime}\left(\mathrm{H}_{2} \mathrm{SIM}^{1}\right)(\text { solv })_{\mathrm{x}}\right]^{4+}$.

Table 27: $\mathrm{pK}_{\mathrm{a}}$ values as determined by UV-vis-spectroscopic titration.

\begin{tabular}{|c|c|c|c|}
\hline complex & $\mathrm{pK}_{\mathrm{a}}$ & complex & $\mathrm{pK}_{\mathrm{a}}$ \\
\hline$\left[\mathrm{Zn}^{\prime \prime}\left(\mathrm{H}_{2} \mathrm{SIM}^{1}\right)(\text { solv })_{\mathrm{x}}\right]^{+}$ & $7.3 \pm 0.2$ & {$\left[\mathrm{Zn}^{\prime \prime}\left(\mathrm{H}_{2} \mathrm{SIM}^{4}\right)(\mathrm{solv})_{\mathrm{x}}\right]$} & $8.4 \pm 0.5$ \\
\hline$\left[\mathrm{Zn}_{2}{ }_{2}\left(\mathrm{H}_{2} \mathrm{SIM}^{1}\right)(\text { solv })_{x}\right]^{3+}$ & $6.9 \pm 0.6$ & {$\left[\mathrm{Zn}_{2}\left(\mathrm{H}_{2} \mathrm{SIM}^{4}\right)(\text { solv })_{\mathrm{x}}\right]^{2+}$} & $9.8 \pm 0.1$ \\
\hline \multirow[t]{2}{*}[\mathrm{Ga}^{\prime\prime\prime}(\mathrm{H}_{2}\mathrm{SIM}^{1})(\text{solv})_{\mathrm{x}}]{$^{2+}$} & $6.7 \pm 0.9$ & {$\left[\mathrm{Ga}^{\prime \prime \prime}\left(\mathrm{H}_{2} \mathrm{SIM}^{4}\right)(\text { solv })_{\mathrm{x}}\right]^{+}$} & $7.1 \pm 0.7$ \\
\hline & $9.1 \pm 0.4$ & & \\
\hline \multirow[t]{2}{*}[\mathrm{Ga}^{\prime\prime\prime}\mathrm{Zn}^{\prime\prime}(\mathrm{H}_{2}\mathrm{SIM}^{1})(\text{solv})_{\mathrm{x}}]{$^{4+}$} & $(5.9 \pm 0.4)^{a}$ & {$\left[\mathrm{Ga}^{\prime \prime \prime \prime} \mathrm{Zn} \text { " }\left(\mathrm{H}_{2} \mathrm{SIM}^{4}\right)(\text { solv })_{x}\right]^{3+}$} & $6.7 \pm 0.4$ \\
\hline & $8.4 \pm 0.2$ & & $(10.6 \pm 0.1)^{a}$ \\
\hline
\end{tabular}

${ }^{a}$ determined $\mathrm{pK}_{\mathrm{a}}$ values are close to limits of the examined $\mathrm{pH}$ range.

Fitting the data between $\mathrm{pH} 5.5$ and $\mathrm{pH} 7.5$ with a similar procedure resulted in a second $\mathrm{pKa}_{\mathrm{a}}$ value for $\left[\mathrm{Ga}^{\text {III }} \mathrm{Zn} \text { "I }\left(\mathrm{H}_{2} \mathrm{SIM}^{1}\right)(\mathrm{solv})_{\mathrm{x}}\right]^{4+}$. Similarly, a second $\mathrm{pK}_{\mathrm{a}}$ was found for $\left[\mathrm{Ga}^{\prime \prime \prime} \mathrm{Zn} \text { " }\left(\mathrm{H}_{2} \mathrm{SIM}^{4}\right)(\text { solv })_{x}\right]^{3+}$; these $\mathrm{pK}_{\mathrm{a}}$ values should, however, be interpreted with care as they are close to the limits of the $\mathrm{pH}$ range studied. The respective $\mathrm{pK}_{\mathrm{a}}$ values reported for 
$\left[\mathrm{Ga}^{\mathrm{II}} \mathrm{Zn} \mathrm{n}^{\prime \prime}\left(\mathrm{L}^{4}\right)(\mu-\mathrm{OAc})_{2}\right]^{+}, \mathrm{pK}_{\mathrm{a}}(\mathrm{I})=6.2$ and $\mathrm{pK}_{\mathrm{a}}(\mathrm{II})=8.0$, were assigned to the equilibria depicted in Scheme $2 .{ }^{66}$

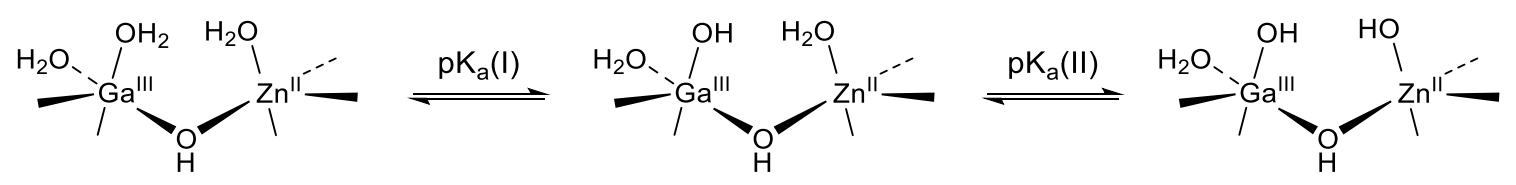

Scheme 23: Illustration of the protonation equilibria for Ga"' $\mathrm{Zn}$ " complexes present in basic solution. ${ }^{66}$

Comparison of the $\mathrm{pK}_{a}$ values obtained for $\left[\mathrm{Ga}^{\prime \prime \prime} \mathrm{Zn}^{\prime \prime}\left(\mathrm{H}_{2} \mathrm{SIM}^{4}\right)(\text { solv })_{\mathrm{x}}\right]^{3+}$ and $\left[\mathrm{Ga}^{\text {III }} \mathrm{Zn} \mathrm{n}^{\prime \prime}\left(\mathrm{L}^{4}\right)(\mathrm{OAc})_{2}\right]^{+}$ indicate a shift of $0.5 \mathrm{pH}$ units on account of a pivaloyl-amide residue proximal to the Ga"l' center. This trend was also found, but was even more pronounced, for the monozinc(II) complexes $\left[\mathrm{Zn}^{\prime \prime}\left(\mathrm{H}_{2} \mathrm{SIM}^{1}\right)(\text { solv })_{\mathrm{x}}\right]^{+}$and $\left[\mathrm{Zn}^{\prime \prime}\left(\mathrm{H}_{3} \mathrm{SIM}^{4}\right)(\text { solv })_{\mathrm{x}}\right]^{+}$.

\subsection{PhOsphoester Hydrolysis StUdies WITH GA "'ZN" COMPLEXES}

The investigation of phosphatase-like activity of the two heterovalent heterodinuclear Ga'" $\mathrm{Zn}$ "I complexes of the ligands $\mathrm{H}_{3} \mathrm{SIM}^{1}$ and $\mathrm{H}_{4} \mathrm{SIM}^{4}$ as well as their monogallium(III), monozinc(II), and dizinc(II) complexes were performed following the general procedure described in Chapter 6.3.

\section{Kinetic Investigations with Complexes Bearing the $\mathrm{H}_{3} \mathrm{SIM}^{1}$ Backbone pH Dependence}

The $\mathrm{pH}$ dependence of the hydrolysis activity towards BDNPP of the complexes described above was investigated. Figure $65 \mathrm{a}$ shows the resulting $\mathrm{v}_{0}$ vs. $\mathrm{pH}$ plot and Table 28 summarizes the $\mathrm{pK}_{\mathrm{a}}$ and $\gamma$ values obtained by fitting the data, assuming a monoprotic or diprotic system as explained in Chapter 6.3. 

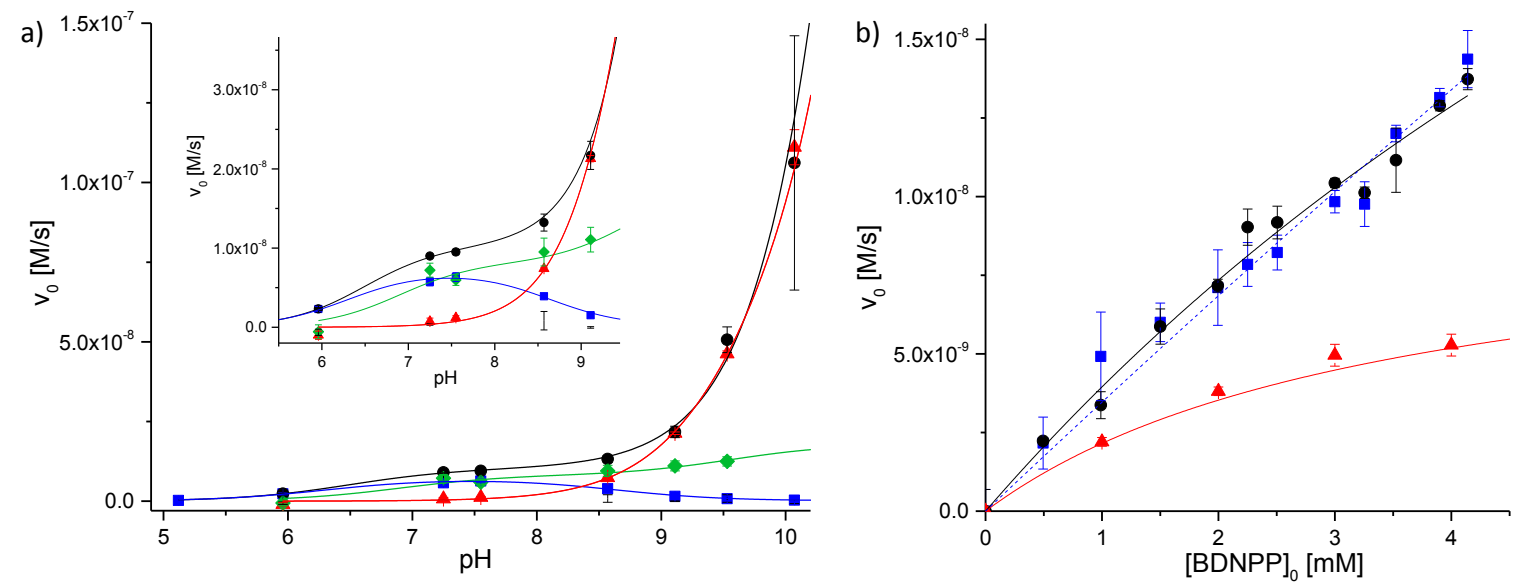

Figure 65: a) $\mathrm{pH}$ dependence and b) substrate concentration dependence at $\mathrm{pH}=7$ of BDNPP hydrolysis activity for mixtures of ligand $\mathrm{H}_{3} \mathrm{SIM}^{1}$ and with 1 eq of zinc(II) perchlorate (red triangles), 2 eq of zinc(II) perchlorate (green diamonds), 1 eq of gallium(III) perchlorate (blue squares), 1 eq zinc(II) perchlorate and 1 eq of gallium(III) perchlorate (black circles).

The examination of the BDNPP hydrolysis by the mono- and dinuclear complexes of $\mathrm{H}_{3} \mathrm{SIM}^{1}$ revealed differing $\mathrm{pH}$ dependencies. The monozinc(II) complex of $\mathrm{H}_{3} \mathrm{SIM}^{1},\left[\mathrm{Zn}^{\prime \prime}\left(\mathrm{H}_{2} \mathrm{SIM}^{1}\right)(\text { solv })_{\mathrm{x}}\right]^{+}$, with $\mathrm{Zn}$ " in the amide-free binding site $\mathrm{B}$, was only active under basic conditions and showed very little activity below $\mathrm{pH}$ 7.5. Because the $\mathrm{pK}_{\mathrm{a}}$ value for the deprotonation step of $\left[\mathrm{Zn}^{\prime \prime}\left(\mathrm{H}_{2} \mathrm{SIM}^{1}\right)(\text { solv })_{x}\right]^{+}$is close or outside of the limits of the $\mathrm{pH}$ range examined in this study, the $\mathrm{pK}_{\mathrm{a}}$ and $\gamma$ values obtained from the fit to Equation 21 need to be interpreted with care. The dizinc(II) complex of $\mathrm{HL}^{1}$ with two $\mathrm{Zn}^{\|}$centers in similar coordination environments to $\left[\mathrm{Zn}^{\prime \prime}\left(\mathrm{H}_{2} \mathrm{SIM}^{1}\right)(\text { solv })_{\mathrm{x}}\right]^{+}$and lacking hydrogen bond donors, revealed $\mathrm{pK}_{\mathrm{a}}$ values of 6.5 and 10.3 and exhibited hydrolysis activity in the $\mathrm{pH}$ range between these two $\mathrm{pK}_{\mathrm{a}}$ values. Therefore, the lack of activity at $\mathrm{pH}<7.5$ in the case of $\left[\mathrm{Zn}^{\prime \prime}\left(\mathrm{H}_{2} \mathrm{SIM}^{1}\right)(\mathrm{solv})_{x}\right]^{+}$seems to be due to the inability of cooperative interaction between two $\mathrm{Zn}$ " centers. The $\mathrm{pH}$ dependence found for the dizinc(II) complex $\left[\mathrm{Zn}_{2}{ }_{2}\left(\mathrm{H}_{2} \mathrm{SIM}^{1}\right)(\text { solv })_{x}\right]^{3+}$, with both coordination sites of $\mathrm{H}_{3} \mathrm{SIM}^{1}$ occupied by $\mathrm{Zn}^{\prime \prime}$ supports this proposal, as it exhibits BDNPP hydrolysis activity below $\mathrm{pH} 7.5$, in contrast to the monozinc(II) complex $\left[\mathrm{Zn}^{\prime \prime}\left(\mathrm{H}_{2} \mathrm{SIM}^{1}\right)(\text { solv })_{x}\right]^{+}$.

In contrast to the mono- and dizinc(II) complexes of $\mathrm{H}_{3} \mathrm{SIM}^{1}$, the monogallium(III) complex $\left[\mathrm{Ga}^{\prime \prime \prime}\left(\mathrm{H}_{2} \mathrm{SIM}^{1}\right)(\text { solv })_{x}\right]^{+}$shows a bell-shaped profile in the $\mathrm{v}_{0}$ vs. $\mathrm{pH}$ plot. This complex is only catalytically active in the $\mathrm{pH}$ range between the two $\mathrm{pK}_{\mathrm{a}}$ values of 6.3 and 8.6 , indicating that the active species is [ $\left.\mathrm{Ga}^{\prime \prime \prime}\left(\mathrm{H}_{2} \mathrm{SIM}^{1}\right)(\mathrm{OH})\left(\mathrm{OH}_{2}\right)\right]$. Both protonation and deprotonation of this active species lead to a decrease of activity.

Interestingly, when the second coordination site of the monogallium(III) complex is occupied by $\mathrm{Zn}$ in $\left[\mathrm{Ga}^{\prime \prime \prime} \mathrm{Zn} \mathrm{n}^{\prime \prime}\left(\mathrm{H}_{2} \mathrm{SIM}^{1}\right)(\text { solv })_{\mathrm{x}}\right]^{4+}$, a superposition of the profiles found for $\left[\mathrm{Zn}^{\prime \prime}\left(\mathrm{H}_{2} \mathrm{SIM}^{1}\right)(\mathrm{solv})_{\mathrm{x}}\right]^{+}$ and $\left[\mathrm{Ga}^{\prime \prime \prime}\left(\mathrm{H}_{2} \mathrm{SIM}^{1}\right)(\text { solv })_{x}\right]^{2+}$ is observed but the initial rate at $\mathrm{pH} 7$ was found to be higher than the 
sum of the corresponding initial rates of $\left[\mathrm{Zn}^{\prime \prime}\left(\mathrm{H}_{2} \mathrm{SIM}^{1}\right)(\text { solv })_{\mathrm{x}}\right]^{+}$and $\left[\mathrm{Ga}^{\prime \prime \prime}\left(\mathrm{H}_{2} \mathrm{SIM}^{1}\right)(\mathrm{solv})_{\mathrm{x}}\right]^{2+}$, indicating a cooperative effect of the two metal centers.

Comparison of the hydrolysis activity of the two mononuclear complexes of $\mathrm{H}_{3} \mathrm{SIM}^{1}$, $\left[\mathrm{Zn}^{\prime \prime}\left(\mathrm{H}_{2} \mathrm{SIM}^{1}\right)(\text { solv })_{\mathrm{x}}\right]^{+}$and $\left[\mathrm{Ga}^{\prime \prime \prime}\left(\mathrm{H}_{2} \mathrm{SIM}^{1}\right)(\text { solv })_{x}\right.$ demonstrates the role of the metal. While $\left[\mathrm{Zn}^{\prime \prime}\left(\mathrm{H}_{2} \mathrm{SIM}^{1}\right)(\text { solv })_{\mathrm{x}}\right]^{+}$shows only activity at high $\mathrm{pH},\left[\mathrm{Ga}^{\prime \prime \prime}\left(\mathrm{H}_{2} \mathrm{SIM}^{1}\right)(\text { solv })_{\mathrm{x}}\right]^{+}$is active at low $\mathrm{pH}$, indicating that in the case of a $\mathrm{Zn}$ " in the active site, a high enough concentration of hydroxide is essential to ensure phosphoester hydrolysis. The fact that the metal-free ligand as well as the ligand-free zinc(II) perchlorate did not show any activity in the entire $\mathrm{pH}$ range illustrates the requirement for the $\mathrm{Zn} \|$ ion to be coordinated to the ligand for activation and subsequent hydrolysis of the phosphoester substrate. In the case of $\left[\mathrm{Ga}^{\prime \prime \prime}\left(\mathrm{H}_{2} \mathrm{SIM}^{1}\right)(\mathrm{solv})_{\mathrm{x}}\right]^{+}$the substrate hydrolysis detected at low $\mathrm{pH}$ can be explained by the ability of the Gall center to activate coordinated water and thereby to generate the nucleophilic hydroxide. Here, the nucleophile needed for phosphoester hydrolysis is provided even at near neutral pH. Moreover, ligand-free gallium(III) perchlorate was also tested for the hydrolysis of BDNPP but did not show any activity. The fact that at basic conditions only autohydrolysis is detectable with [Ga"' $\left.\left(\mathrm{H}_{2} \mathrm{SIM}^{1}\right)(\mathrm{solv})_{\mathrm{x}}\right]^{+}$ indicates that the Ga'l' ion in the doubly deprotonated species, most likely $\left[\mathrm{Ga}^{\prime \prime \prime}\left(\mathrm{H}_{2} \mathrm{SIM}^{1}\right)(\mathrm{OH})_{2}\right]$ is not able to activate the phosphoester substrate.

\section{Substrate Concentration Dependence}

The substrate concentration dependence was probed for $\left[\mathrm{Zn}^{\prime \prime}\left(\mathrm{H}_{2} \mathrm{SIM}^{1}\right)(\mathrm{solv})_{\mathrm{x}}\right]^{+}$, $\left[\mathrm{Ga}^{\prime \prime \prime}\left(\mathrm{H}_{2} \mathrm{SIM}^{1}\right)(\text { solv })_{\mathrm{x}}\right]^{+}$, and $\left[\mathrm{Ga}^{\prime \prime \prime} \mathrm{Zn} \mathrm{n}^{\prime \prime}\left(\mathrm{H}_{2} \mathrm{SIM}^{1}\right)(\text { solv })_{\mathrm{x}}\right]^{4+}$ at $\mathrm{pH} 7\left(0.5 \mathrm{mM}<[\mathrm{BDNPP}]_{0}<4.14 \mathrm{mM}\right)$. Saturation behavior was achieved for $\left[\mathrm{Zn}^{\prime \prime}\left(\mathrm{H}_{2} \mathrm{SIM}^{1}\right)(\text { solv })_{\mathrm{x}}\right]^{+}$and $\left[\mathrm{Ga}^{\prime \prime \prime} \mathrm{Zn}^{\prime \prime}\left(\mathrm{H}_{2} \mathrm{SIM}^{1}\right)(\text { solv })_{\mathrm{x}}\right]^{4+}$ that was not the case for [Ga"' $\left.\left(\mathrm{H}_{2} \mathrm{SIM}^{1}\right)(\text { solv })_{\mathrm{X}}\right]^{+}\left(\mathrm{k}_{\mathrm{cat}}, \mathrm{K}_{\mathrm{M}}\right.$ and $\mathrm{k}_{\mathrm{cat}} / \mathrm{K}_{\mathrm{M}}$ are listed in Table 28$)$.

Table 28: Kinetic data ( $\mathrm{K}_{\mathrm{cat}}$ in $\left[10^{-3} \mathrm{~s}^{-1}\right], \mathrm{K}_{\mathrm{M}}$ in $[\mathrm{mM}]$ and $\mathrm{k}_{\mathrm{cat}} / \mathrm{K}_{\mathrm{M}}$ in $\left.\left[\mathrm{s}^{-1} \mathrm{M}^{-1}\right]\right)$ of BDNPP hydrolysis with mixtures of the ligands $\mathrm{H}_{3} \mathrm{SIM}^{1}$ or $\mathrm{H}_{4} \mathrm{SIM}^{4}$ with 1 eq of zinc(II) perchlorate, 2 eq of zinc(II) perchlorate, 1 eq of gallium(III) perchlorate, 1 eq zinc(II) perchlorate and 1 eq of gallium(III) perchlorate (all kinetic studies were undertaken in a mixture of acetonitrile-aqueous buffer solution (1:1)).

\begin{tabular}{|c|c|c|c|c|c|c|}
\hline complex & $\mathrm{pK}_{\mathrm{a}}(\mathrm{I})$ & $\mathrm{pK}_{\mathrm{a}}(\mathrm{II})$ & $\gamma$ & $\mathrm{k}_{\text {cat }}{ }^{\mathrm{a}}$ & $\mathrm{K}_{\mathrm{M}}^{\mathrm{a}}$ & $\mathrm{k}_{\mathrm{cat}} / \mathrm{K}_{\mathrm{M}}^{\mathrm{a}}$ \\
\hline$\left[\mathrm{Zn}^{\prime \prime}\left(\mathrm{H}_{2} \mathrm{SIM}^{1}\right)(\text { solv })_{\mathrm{x}}\right]^{+}$ & $(9.2 \pm 0.5)^{c}$ & $(10.9 \pm 0.5)^{c}$ & $(14.94 \pm 7.00)^{c}$ & $0.24 \pm 0.02$ & $4 \pm 1$ & 0.07 \\
\hline$\left[\mathrm{Zn}_{2}{ }_{2}\left(\mathrm{H}_{2} \mathrm{SIM}^{1}\right)(\text { solv })_{x}\right]^{3+}$ & $6.9 \pm 0.4$ & $9.6 \pm 0.3$ & $2.21 \pm 0.64$ & n.a. & n.a. & n.a. \\
\hline$\left[\mathrm{Ga}^{\prime \prime \prime}\left(\mathrm{H}_{2} \mathrm{SIM}^{1}\right)(\text { solv })_{\mathrm{x}}\right]^{2+}$ & $6.3 \pm 0.2$ & $8.6 \pm 0.2$ & $0.01 \pm 0.07$ & $-b$ & $-b$ & $-b$ \\
\hline$\left[\mathrm{Ga}^{\prime \prime \prime} \mathrm{Zn}^{\prime \prime}\left(\mathrm{H}_{2} \mathrm{SIM}^{1}\right)(\text { solv })_{\mathrm{x}}\right]^{4+}$ & $6.5 \pm 0.1$ & $10.9 \pm 0.1$ & $90.34 \pm 13.87$ & $13.03 \pm 3.51$ & $12 \pm 4$ & 1.07 \\
\hline$\left[\mathrm{Zn}^{\prime \prime}\left(\mathrm{H}_{2} \mathrm{SIM}^{4}\right)(\text { solv })_{x}\right]$ & $(12.4)^{c}$ & - & - & $0.12 \pm 0.04$ & $1 \pm 1$ & 0.10 \\
\hline$\left[\mathrm{Zn}_{2}{ }_{2}\left(\mathrm{H}_{2} \mathrm{SIM}^{4}\right)(\text { solv })_{\mathrm{x}}\right]^{2+}$ & - & - & - & n.a. & n.a. & n.a. \\
\hline$\left[\mathrm{Ga}^{\prime \prime \prime}\left(\mathrm{H}_{2} \mathrm{SIM}^{4}\right)(\text { solv })_{\mathrm{x}}\right]^{+}$ & $6.7 \pm 0.1$ & $8.7 \pm 0.2$ & $0.01 \pm 0.05$ & $0.67 \pm 0.14$ & $7 \pm 2$ & 0.10 \\
\hline$\left[\mathrm{Ga}^{\prime \prime \prime} \mathrm{Zn}^{\prime \prime}\left(\mathrm{H}_{2} \mathrm{SIM}^{4}\right)(\text { solv })_{x}\right]^{3+}$ & $6.5 \pm 0.2$ & $10.5 \pm 0.3$ & $12.84 \pm 3.62$ & $1.83 \pm 0.21$ & $7 \pm 1$ & 0.25 \\
\hline
\end{tabular}

a the assays were performed with aqueous buffer solution $\mathrm{pH}=7$, TONs were determined after 9 days.

${ }^{b}$ the kinetic data for $\left[\mathrm{Ga}^{\text {III }}\left(\mathrm{H}_{2} \mathrm{SIM}^{1}\right)(\text { solv })_{\mathrm{x}}\right]^{+}$could not be determined as Michaelis-Menten behavior was not observed.

${ }^{c}$ determined $\mathrm{pK}_{\mathrm{a}}$ values are close to limits if the examined $\mathrm{pH}$ range. 
Mareque-Rivas et al. reported that a vacant second coordination site besides the coordination site occupied by $\mathrm{Zn}^{\|}$accelerates phosphoester hydrolysis in a similar way as providing metal...metal interaction This effect was not observed for the phenolate-based complexes discussed in this chapter since the $\mathrm{Zn}_{2}{ }_{2}$ complexes of both, $\mathrm{HL}^{1}$ and $\mathrm{H}_{3} \mathrm{SIM}^{1}$, exhibit higher hydrolysis rates than $\left[\mathrm{Zn}^{\prime \prime}\left(\mathrm{H}_{2} \mathrm{SIM}^{1}\right)(\text { solv })_{x}\right]^{+} .{ }^{111}, 193$ The $\mathrm{K}_{M}$ values determined for $\left[\mathrm{Zn}^{\prime \prime}\left(\mathrm{H}_{2} \mathrm{SIM}^{1}\right)(\text { solv })_{x}\right]^{+}\left(\mathrm{K}_{\mathrm{M}}=4 \mathrm{mM}\right)$ and $\left[\mathrm{Zn}_{2}{ }_{2}\left(\mathrm{H}_{2} \mathrm{SIM}^{1}\right)(\mu-\mathrm{OAc})(\mathrm{OH})\right]^{+}\left(\mathrm{K}_{\mathrm{M}}=4 \mathrm{mM}\right.$; discussed in Chapter 7.2) are, however, in both cases much lower than for $\left[\mathrm{Zn}_{2}{ }_{2}\left(\mathrm{~L}^{1}\right)(\mu-\mathrm{OAc})_{2}\right]^{+}\left(\mathrm{K}_{\mathrm{M}}=123 \mathrm{mM}\right.$; discussed in Chapter 7.2), indicating an enhancement on the substrate affinity by the hydrogen bonding network.

Furthermore, the results obtained with the substrate concentration dependence measurements indicate that the exchange of the $\mathrm{Zn}$ in ion present in binding site $\mathrm{B}$ in $\left[\mathrm{Zn}^{\prime \prime}\left(\mathrm{H}_{2} \mathrm{SIM}^{1}\right)(\text { solv })_{x}\right]^{+}$by a Gall' ion and the subsequent coordination of $\mathrm{Zn}^{\prime \prime}$ in binding site $\mathrm{C}$ leads to a 50 -fold increase in phosphoester hydrolysis rate, but simultaneously the value for $\mathrm{K}_{M}$ rises, i.e. the substrate affinity decreases. However, the catalytic efficiency of $\left[\mathrm{Ga}^{\prime \prime \prime} \mathrm{Zn}^{\prime \prime}\left(\mathrm{H}_{2} \mathrm{SIM}^{1}\right)(\text { solv })_{\mathrm{x}}\right]^{4+}$ is still 15 -fold higher than the one detected for $\left[\mathrm{Zn}^{\prime \prime}\left(\mathrm{H}_{2} \mathrm{SIM}^{1}\right)(\text { solv })_{\mathrm{x}}\right]^{+}$.

Comparison of the kinetic parameters for the $\mathrm{Ga}^{\prime \prime \prime} \mathrm{Zn}$ " complex of $\mathrm{H}_{3} \mathrm{SIM}^{1}$ with those obtained for $\left[\mathrm{Zn}_{2}{ }_{2}\left(\mathrm{H}_{2} \mathrm{SIM}^{1}\right)(\mu-\mathrm{OAc})(\mathrm{OH})\right]^{+}\left(\mathrm{k}_{\mathrm{cat}}=(0.57 \pm 0.04) \cdot 10^{-3} \mathrm{~s}^{-1} ; \mathrm{K}_{\mathrm{M}}=(4 \pm 1) \mathrm{mM} ; \mathrm{k}_{\text {cat }} / \mathrm{K}_{\mathrm{M}}=0.14 \mathrm{~s}^{-1} \mathrm{M}^{-1} ; 301\right.$ discussed in Chapter 7.2) reveals that the heterodinuclear Ga"' $\mathrm{Zn}^{\prime \prime}$ complex hydrolyzes BDNPP twenty times faster at neutral $\mathrm{pH}$. Although the substrate affinity of the Ga"' $\mathrm{Zn}$ " complex is significantly lower, the catalytic efficiency of $\left[\mathrm{Ga}^{\prime \prime \prime} \mathrm{Zn}^{\prime \prime}\left(\mathrm{H}_{2} \mathrm{SIM}^{1}\right)(\text { solv })_{\mathrm{x}}\right]^{4+}$ is increased six-fold compared to its $\mathrm{Zn}_{2}$ counterpart illustrating the importance of the trivalent metal ion in the model complex.

\section{Kinetic Investigations with Complexes Bearing the $\mathrm{H}_{4} \mathrm{SIM}^{4}$ Backbone}

\section{pH Dependence}

The $\mathrm{pH}$ dependence experiments of the BDNPP hydrolysis for $\left[\mathrm{Zn}^{\prime \prime}\left(\mathrm{H}_{3} \mathrm{SIM}^{4}\right)(\mathrm{solv})_{\mathrm{x}}\right]^{+}$, [Ga"' $\left.\left(\mathrm{H}_{2} \mathrm{SIM}^{4}\right)(\text { solv })_{\mathrm{x}}\right]^{+}$and $\left[\mathrm{Ga}^{\prime \prime \prime} \mathrm{Zn}^{\prime \prime}\left(\mathrm{H}_{2} \mathrm{SIM}^{4}\right)(\text { solv })_{\mathrm{x}}\right]^{3+}$ resulted in similar findings as for the corresponding complexes bearing the $\mathrm{H}_{3} \mathrm{SIM}^{1}$ ligand backbone, i.e. that (i) $\left[\mathrm{Zn}^{\prime \prime}\left(\mathrm{H}_{3} \mathrm{SIM}^{4}\right)(\mathrm{solv})_{\mathrm{x}}\right]^{+}$is only active under strong basic conditions, (ii) $\left[\mathrm{Ga}^{\prime \prime \prime}\left(\mathrm{H}_{2} \mathrm{SIM}^{4}\right)(\mathrm{solv})_{\mathrm{x}}\right]^{+}$is active in a defined $\mathrm{pH}$ region between the two pKa values, 6.74 and 8.68, and (iii) $\left[\mathrm{Ga}^{\prime \prime \prime} \mathrm{Zn}^{\prime \prime}\left(\mathrm{H}_{2} \mathrm{SIM}^{4}\right)(\text { solv) }]^{3+}\right.$ exhibits a superposition of the behavior found for $\left[\mathrm{Zn}^{\prime \prime}\left(\mathrm{H}_{3} \mathrm{SIM}^{4}\right)(\text { solv })_{\mathrm{x}}\right]^{+}$and $\left[\mathrm{Ga}^{\prime \prime \prime}\left(\mathrm{H}_{2} \mathrm{SIM}^{4}\right)(\text { solv })_{\mathrm{x}}\right]^{+}$(Figure 66a). 

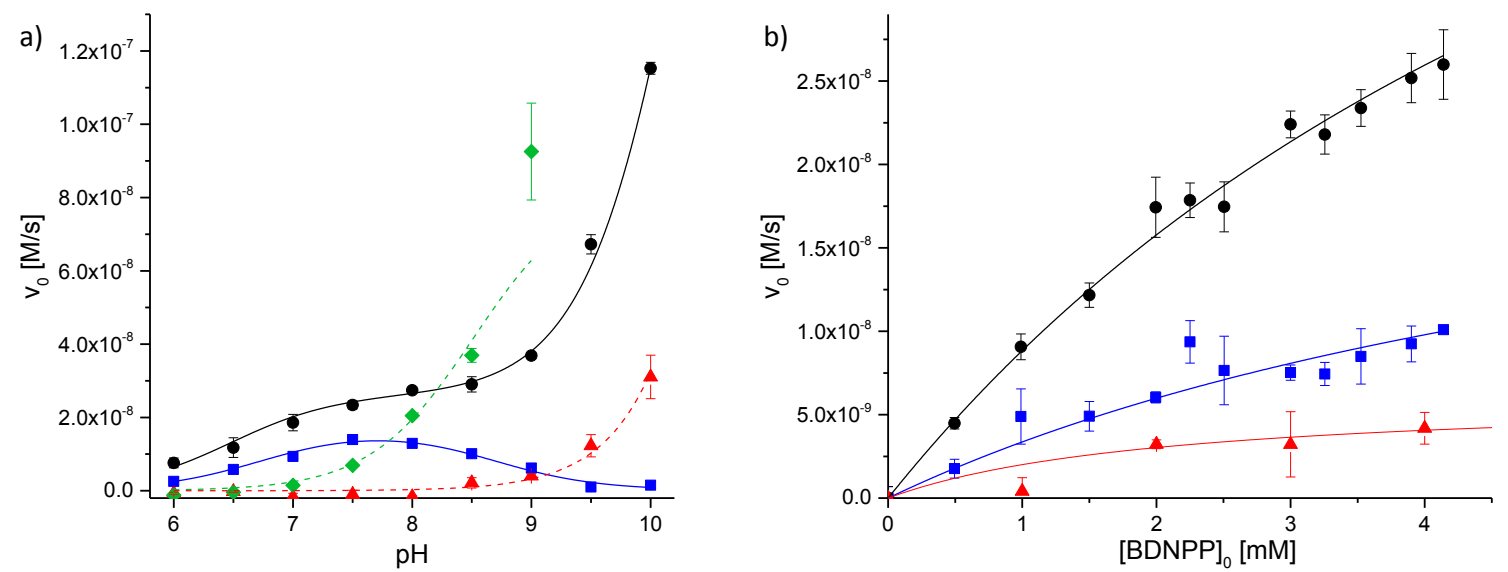

Figure 66: a) $\mathrm{pH}$ dependence and b) substrate concentration dependence at $\mathrm{pH}=7$ of BDNPP hydrolysis activity for mixtures of ligand $\mathrm{H}_{4} \mathrm{SIM}^{4}$ and with 1 eq of zinc(II) perchlorate (red triangles), 2 eq of zinc(II) perchlorate (green diamonds), 1 eq of gallium(III) perchlorate (blue squares), 1 eq zinc(II) perchlorate and 1 eq of gallium(III) perchlorate (black circles).

\section{Substrate Concentration Dependence}

The substrate concentration dependence measurements of $\left[\mathrm{Zn}^{\prime \prime}\left(\mathrm{H}_{3} \mathrm{SIM}^{4}\right)(\mathrm{solv})_{\mathrm{x}}\right]^{+}$, $\left[\mathrm{Ga}^{\prime \prime \prime}\left(\mathrm{H}_{2} \mathrm{SIM}^{4}\right)(\text { solv })_{\mathrm{x}}\right]^{+}$, and $\left[\mathrm{Ga}^{\prime \prime \prime} \mathrm{Zn}^{\prime \prime}\left(\mathrm{H}_{2} \mathrm{SIM}^{4}\right)(\text { solv })_{\mathrm{x}}\right]^{3+}$ revealed that the hydrolysis rate of the Ga"In" complex is increased three-fold compared to the monogallium(III) complex and increased six-fold compared to the monozinc(II) complex. However, the substrate affinity of the Ga"' $\mathrm{Zn}$ "I complex is similar to that of the Gall' complex and significantly lower to that of the $\mathrm{Zn}^{\text {"l }}$ complex. Due to the much faster hydrolysis rate, the catalytic efficiency of $\left[\mathrm{Ga}^{\prime \prime \prime} \mathrm{Zn}^{\prime \prime}\left(\mathrm{H}_{2} \mathrm{SIM}^{4}\right)(\mathrm{solv})_{\mathrm{x}}\right]^{3+}$ is 2.5-times higher than those of $\left[\mathrm{Zn}^{\prime \prime}\left(\mathrm{H}_{3} \mathrm{SIM}^{4}\right)(\text { solv })_{x}\right]^{+}$and $\left[\mathrm{Ga}^{\prime \prime \prime}\left(\mathrm{H}_{2} \mathrm{SIM}^{4}\right)(\text { solv })_{\mathrm{x}}\right]^{+}$.

In contrast to the corresponding complexes of $\mathrm{H}_{3} \mathrm{SIM}^{1},\left[\mathrm{Zn}^{\prime \prime}\left(\mathrm{H}_{3} \mathrm{SIM}^{4}\right)(\text { solv })\right]^{+}$bears the $\mathrm{Zn}$ "in the same binding site as [Ga'"' $\left.\mathrm{Zn}^{\prime \prime}\left(\mathrm{H}_{2} \mathrm{SIM}^{4}\right)(\text { solv })_{\mathrm{x}}\right]^{3+}$, just like $\left[\mathrm{Ga}^{\prime \prime \prime}\left(\mathrm{H}_{2} \mathrm{SIM}^{4}\right)(\text { solv })_{\mathrm{x}}\right]^{+}$contains the Ga"l in the same binding site as $\left[\mathrm{Ga}^{\prime \prime \prime} \mathrm{Zn}^{\prime \prime}\left(\mathrm{H}_{2} \mathrm{SIM}^{4}\right)(\text { solv })_{\mathrm{x}}\right]^{3+}$. Therefore, the fact that the Ga"'In" complex of $\mathrm{H}_{4} \mathrm{SIM}^{4}$ complex exhibit a higher catalytic activity than the sum of the hydrolysis rates of the monozinc(II) and monogallium(III) complexes can merely be attributed to a cooperative effect.

\section{Comparison of the $\mathrm{Zn}^{\prime \prime}, \mathrm{Zn}_{2}{ }_{2}$, Ga"', and Ga"' $\mathrm{Zn}^{\prime \prime}$ Complexes with the Ligand Backbones $\mathrm{H}_{3} \mathrm{SIM}^{1}$ and $\mathrm{H}_{4} \mathrm{SIM}^{4}$}

Independent on the ligand backbone of $\mathrm{H}_{3} \mathrm{SIM}^{1}$ and $\mathrm{H}_{4} \mathrm{SIM}^{4}$, the two complexes with the same metal ions coordinated show similar $\mathrm{pH}$ dependencies of the initial hydrolysis rate. However, the initial hydrolysis rate of $\left[\mathrm{Zn}^{\prime \prime}\left(\mathrm{H}_{2} \mathrm{SIM}^{1}\right)(\text { solv })_{x}\right]^{+}$is higher at $\mathrm{pH} 10$ than that for $\left[\mathrm{Zn}^{\prime \prime}\left(\mathrm{H}_{3} \mathrm{SIM}^{4}\right)(\mathrm{solv})_{\mathrm{x}}\right]^{+}$ indicating a shift of the $\mathrm{pK}_{\mathrm{a}}$ to higher $\mathrm{pH}$ by the pivaloyl-amide residue adjacent to the $\mathrm{Zn}$ " center in $\left[\mathrm{Zn}^{\prime \prime}\left(\mathrm{H}_{2} \mathrm{SIM}^{4}\right)(\text { solv })_{x}\right]$. The same is found for the $\mathrm{Zn}_{2}$ complexes $\left[\mathrm{Zn}_{2}{ }_{2}\left(\mathrm{H}_{2} \mathrm{SIM}^{1}\right)(\text { solv })_{x}\right]^{3+}$ and $\left[\mathrm{Zn}_{2}{ }_{2}\left(\mathrm{H}_{2} \mathrm{SIM}^{4}\right)(\text { solv })_{x}\right]^{2+}$, i.e. $\left[\mathrm{Zn}_{2}{ }_{2}\left(\mathrm{H}_{2} \mathrm{SIM}^{4}\right)(\text { solv })_{x}\right]^{2+}$ becomes active at higher $\mathrm{pH}$ than 
$\left[\mathrm{Zn}_{2}{ }_{2}\left(\mathrm{H}_{2} \mathrm{SIM}^{1}\right)(\text { solv })_{x}\right]^{3+}$, but the increase of the initial hydrolysis rate with increasing $\mathrm{pH}$ from 9 to 10 for the latter complex is more pronounced. The differences in the catalytic behavior of $\left[\mathrm{Zn}_{2}{ }_{2}\left(\mathrm{H}_{2} \mathrm{SIM}^{1}\right)(\text { solv })_{\mathrm{x}}\right]^{3+}$ and $\left[\mathrm{Zn}_{2}{ }_{2}\left(\mathrm{H}_{2} \mathrm{SIM}^{4}\right)(\text { solv })_{\mathrm{x}}\right]^{2+}$ could be a result of two structural features, (i) the unequal primary coordination sphere, and (ii) the different positioning of the hydrogen bond donors in the second coordination sphere.

Comparison of the $\mathrm{Zn}_{2}{ }_{2}$ complexes of $\mathrm{H}_{3} \mathrm{~L}^{2}$ and $\mathrm{H}_{4} \mathrm{SIM}^{4}$ provides the opportunity to study the effect of changes to the first coordination sphere, with the secondary coordination sphere the same in both complexes. While $\mathrm{H}_{3} \mathrm{~L}^{2}$ has two $\mathrm{N}_{3} \mathrm{O}$ binding sites, $\mathrm{H}_{4} \mathrm{SIM}^{4}$ offers one $\mathrm{N}_{3} \mathrm{O}$ - and one $\mathrm{N}_{2} \mathrm{O}_{2}$-coordination site. $\left[\mathrm{Zn}_{2}{ }_{2}\left(\mathrm{H}_{2} \mathrm{~L}^{2}\right)(\mu-\mathrm{OAC})_{2}\right]^{+}$(see Chapter 7.2) and $\left[\mathrm{Zn}_{2}{ }_{2}\left(\mathrm{H}_{2} \mathrm{SIM}^{4}\right)(\text { solv })_{x}\right]^{2+}$ exhibit similar behavior of their BDNPP hydrolysis activity; with increasing $\mathrm{pH}$ the initial hydrolysis rate for both increases significantly. However, the value of the initial hydrolysis rate measured at $\mathrm{pH}$ 9 is significantly higher for $\left[\mathrm{Zn}_{2}{ }_{2}\left(\mathrm{H}_{2} \mathrm{SIM}^{4}\right)(\text { solv })_{x}\right]^{2+}$ than for $\left[\mathrm{Zn}_{2}{ }_{2}\left(\mathrm{H}_{2} \mathrm{~L}^{2}\right)(\mu-\mathrm{OAc})_{2}\right]^{+}$and this is attributed to the exchange of one pyridine ligand by a more basic terminal phenolate ligand. Unfortunately, the absorbance at $400 \mathrm{~nm}$ increased to values near device limit when the experiments with $\left[\mathrm{Zn}_{2}\left(\mathrm{H}_{2} \mathrm{SIM}^{4}\right)(\text { solv })_{x}\right]^{2+}$ were undertaken at $\mathrm{pH}>9$. Based on these findings the higher initial hydrolysis rate of $\left[\mathrm{Zn}_{2}{ }_{2}\left(\mathrm{H}_{2} \mathrm{SIM}^{4}\right)(\text { solv })_{x}\right]^{2+}$ compared to $\left[\mathrm{Zn}_{2}{ }_{2}\left(\mathrm{H}_{2} \mathrm{SIM}^{1}\right)(\text { solv })_{x}\right]^{3+}$ is ascribed to the more basic phenolate donor in the former complex.

However, the most pronounced differences with respect to the two ligand backbones was found for the Gall' complexes. In the $\mathrm{pH}$ profiles of Figure 67 it is clearly demonstrated that the complexes [Ga'"' $\left(\mathrm{H}_{2} \mathrm{SIM}^{4}\right)(\text { solv) }]^{+}$and $\left[\mathrm{Ga}^{\prime \prime \prime} \mathrm{Zn}^{\prime \prime}\left(\mathrm{H}_{2} \mathrm{SIM}^{4}\right)(\text { solv })\right]^{3+}$ show higher initial hydrolysis velocities than the corresponding $\mathrm{H}_{4} \mathrm{SIM}^{4}$ complexes $\left[\mathrm{Ga}^{\prime \prime \prime}\left(\mathrm{H}_{2} \mathrm{SIM}^{1}\right)(\text { solv })_{\mathrm{x}}\right]^{2+}$ and $\left[\mathrm{Ga}^{\prime \prime \prime} \mathrm{Zn} \mathrm{n}^{\prime \prime}\left(\mathrm{H}_{2} \mathrm{SIM}^{1}\right)(\text { solv })\right]^{4+}$ in the entire $\mathrm{pH}$ range studied (Figure 67a).
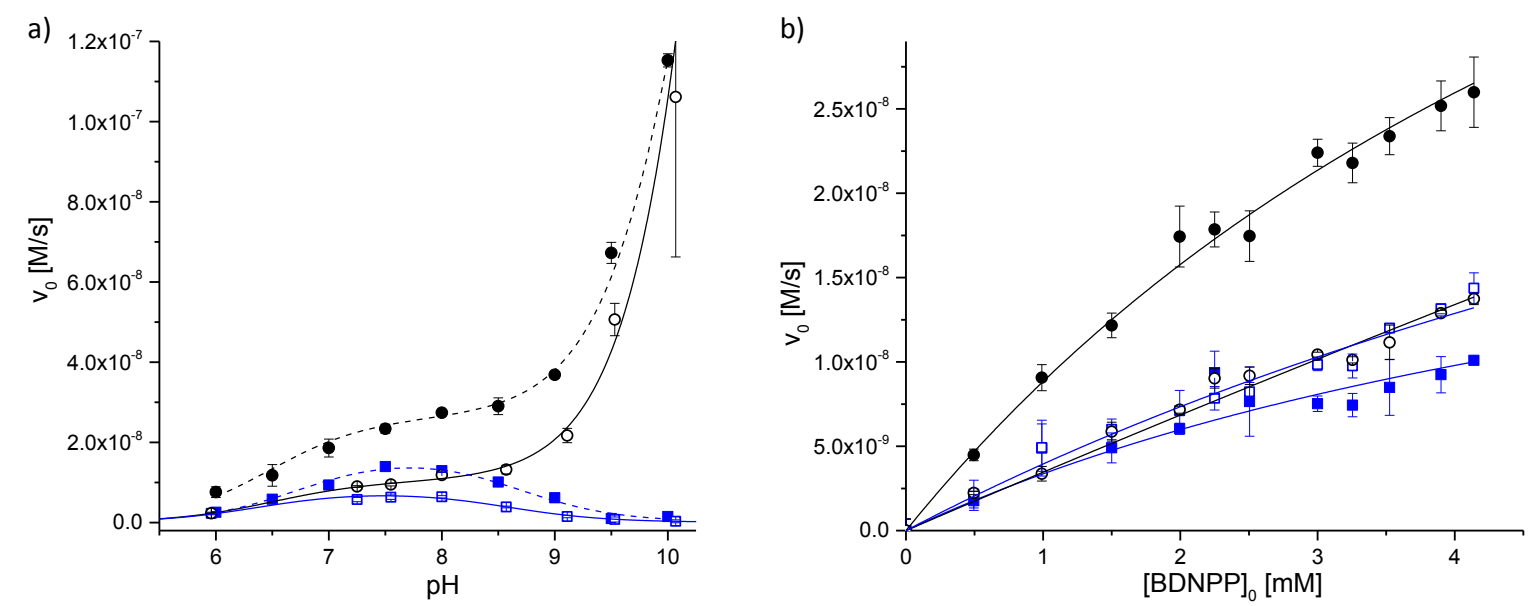

Figure 67: Comparison of a) $\mathrm{pH}$ dependence and b) substrate concentration dependence at $\mathrm{pH}=7$ of BDNPP hydrolysis activity for mixtures of ligand $\mathrm{H}_{3} \mathrm{SIM}^{1}$ (unfilled symbols) and $\mathrm{H}_{4} \mathrm{SIM}^{4}$ (filled symbols) with 1 eq of gallium(III) perchlorate (blue squares), 1 eq zinc(II) perchlorate and 1 eq of gallium(III) perchlorate (black circles). 
The Ga'"'Zn" complexes [Ga'"'Zn" $\left(\mathrm{H}_{2} \mathrm{SIM}^{4}\right)($ solv $\left.)\right]^{3+}$ and $\left[\mathrm{Ga}^{\prime \prime \prime} \mathrm{Zn}^{\prime \prime}\left(\mathrm{H}_{2} \mathrm{SIM}^{1}\right)(\text { solv })\right]^{4+}$ differ in two structural features, (i) the primary coordination environment of the Gall' center, and (ii) the position of the hydrogen bond donors mimicking the histidine residues in the second coordination sphere of the PAP active site. The previously reported complex [Ga'"'Zn" $\left.\left(L^{4}\right)(\mu-\mathrm{OAC})_{2}\right]^{+}$helps to extract the specific impact of the structural variations. ${ }^{66}$ The Ga'II environments in [Ga"' $\mathrm{Zn}^{\prime \prime}\left(\mathrm{H}_{2} \mathrm{SIM}^{4}\right)($ solv $\left.)\right]^{3+}$ and $\left[\mathrm{Ga}^{\prime \prime \prime} \mathrm{Zn}{ }^{\prime \prime}\left(\mathrm{L}^{4}\right)(\mu-\mathrm{OAC})_{2}\right]^{+}$are similar with respect to the primary coordination sphere, but differ in the secondary coordination sphere as [Ga"'Zn" $\left(\mathrm{H}_{2} \mathrm{SIM}^{4}\right)($ solv $\left.)\right]^{3+}$ bears a pivaloyl-amide residue adjacent to the Gall center while [Ga'"'Zn" $\left.\left(L^{4}\right)(\mu-\mathrm{OAC})_{2}\right]^{+}$misses a proximal hydrogen bond donor. In contrast, the Ga'l' center in both [Ga"'In" $\mathrm{n}^{\prime \prime}\left(\mathrm{H}_{2} \mathrm{SIM}^{1}\right)($ solv $\left.)\right]^{4+}$ and $\left[\mathrm{Ga}^{\prime \prime \prime} \mathrm{Zn} \mathrm{n}^{\prime \prime}\left(\mathrm{L}^{4}\right)(\mu-\mathrm{OAC})_{2}\right]^{+}$, do not have a hydrogen bond donor in close proximity and therefore do not vary in the secondary coordination sphere, but the primary coordination sphere of the two complexes is different. Therefore, comparison of [Ga"'In" $\left(\mathrm{H}_{2} \mathrm{SIM}^{4}\right)($ solv $\left.)\right]^{3+}$ with [Ga"' $\left.\mathrm{Zn}^{\prime \prime}\left(\mathrm{L}^{4}\right)(\mu-\mathrm{OAc})_{2}\right]^{+}$enables the elucidation of the effect of a hydrogen bonding donor adjacent to the Gall' center. Similarly, comparison of [Ga'"'Zn" $\left(\mathrm{H}_{2} \mathrm{SIM}^{1}\right)($ solv $\left.)\right]^{4+}$ and $\left[\mathrm{Ga}^{\prime \prime \prime} \mathrm{Zn} \mathrm{n}^{\prime \prime}\left(\mathrm{L}^{4}\right)(\mu-\mathrm{OAc})_{2}\right]^{+}$gives an insight in the impact of the exchange of a pyridine by a phenolate residue in the primary coordination environment of the Gall' center. In contrast to $\left[\mathrm{Ga}^{\prime \prime \prime} \mathrm{Zn} \mathrm{Zn}^{\prime \prime}\left(\mathrm{H}_{2} \mathrm{SIM}^{1}\right)(\text { solv })_{\mathrm{x}}\right]^{4+}$ and $\left[\mathrm{Ga}^{\prime \prime \prime} \mathrm{Zn}^{\prime \prime}\left(\mathrm{H}_{2} \mathrm{SIM}^{4}\right)(\text { solv })_{\mathrm{x}}\right]^{3+},\left[\mathrm{Ga}^{\prime \prime \prime} \mathrm{Zn}{ }^{\prime \prime}\left(\mathrm{L}^{4}\right)(\mu-\mathrm{OAc})_{2}\right]^{+}$ exhibited a sigmoidal $\mathrm{v}_{0}$ vs. $\mathrm{pH}$ curve with a relatively sharp activity maximum at $\mathrm{pH} \sim 6.8{ }^{66}$ The differences in the $\mathrm{pH}$ profile curve shapes derived for $\left[\mathrm{Ga}^{\prime \prime \prime} \mathrm{Zn}^{\prime \prime}\left(\mathrm{H}_{2} \mathrm{SIM}^{1}\right)(\mathrm{solv})_{\mathrm{x}}\right]^{4+}$ and [Ga'"'Zn" $\left.\left(\mathrm{H}_{2} \mathrm{SIM}^{4}\right)(\text { solv })_{x}\right]^{3+}$ compared to $\left[\mathrm{Ga}^{\prime \prime \prime} \mathrm{Zn} \mathrm{n}^{\prime \prime}\left(\mathrm{L}^{4}\right)(\mu-\mathrm{OAc})_{2}\right]^{+}$, more precisely the fact that the initial hydrolysis rate rises at $\mathrm{pH}$ values higher than nine for $\left[\mathrm{Ga}^{\prime \prime \prime} \mathrm{Zn}^{\prime \prime}\left(\mathrm{H}_{2} \mathrm{SIM}^{1}\right)(\text { solv })_{\mathrm{x}}\right]^{4+}$ and $\left[G^{\prime \prime \prime} \mathrm{Zn}^{\prime \prime}\left(\mathrm{H}_{2} \mathrm{SIM}^{4}\right)(\text { solv })_{x}\right]^{3+}$, in contrast to that of $\left[\mathrm{Ga}^{\prime \prime \prime} \mathrm{Zn} n^{\prime \prime}\left(\mathrm{L}^{4}\right)(\mu-\mathrm{OAc})_{2}\right]^{+}$, is attributed to the hydrogen bond donors adjacent to the $\mathrm{Zn}^{\text {" }}$ site in the former complexes.

The Ga"l' coordination environments in both [Ga"' $\left.\mathrm{Zn}^{\prime \prime}\left(\mathrm{H}_{2} \mathrm{SIM}^{1}\right)(\text { solv })_{\mathrm{x}}\right]^{4+}$ and $\left[\mathrm{Ga}^{\text {III }} \mathrm{Zn}{ }^{\prime \prime}\left(\mathrm{L}^{4}\right)(\mu-\mathrm{OAC})_{2}\right]^{+}$ do not provide hydrogen bonding nearby, but they differ in the primary coordination sphere. The Ga'll center in $\left[\mathrm{Ga}^{\prime \prime \prime} \mathrm{Zn}^{\prime \prime}\left(\mathrm{H}_{2} \mathrm{SIM}^{1}\right)(\text { solv })_{x}\right]^{4+}$ is bound in a $\mathrm{N}_{3} \mathrm{O}$-site, while the Gall' ion in [Ga"'In $\left.\mathrm{Zn}^{\prime \prime}\left(\mathrm{L}^{4}\right)(\mu-\mathrm{OAC})_{2}\right]^{+}$is coordinated to a $\mathrm{N}_{2} \mathrm{O}_{2}$-site. Therefore, the shift of the $\mathrm{pH}$ maximum by $0.8 \mathrm{pH}$ units with respect to the phosphoester hydrolysis activity in the region between $\mathrm{pH} 7$ and $\mathrm{pH} 9$ to more acidic conditions seems to be a result of the electron-rich phenolate coordinating ligand. However, it should be noted that this is in contrast to the results obtained with the monogallium(III) complexes of the tripodal ligands examined by Erxleben et al. (Scheme 22 and Table 26). ${ }^{70}$

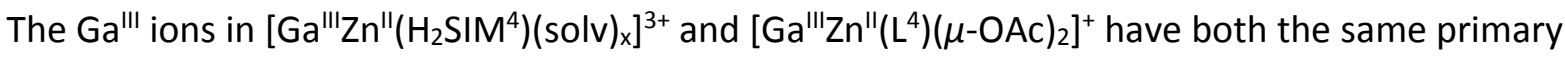
coordination sphere but in $\left[\mathrm{Ga}^{\prime \prime \prime} \mathrm{Zn}^{\prime \prime}\left(\mathrm{H}_{2} \mathrm{SIM}^{4}\right)(\text { solv })_{\mathrm{x}}\right]^{3+}$ a pivaloyl-amide residue is positioned adjacent to the $\mathrm{Ga} \mathrm{a}^{\mathrm{III}}$ ion that is missing in $\left[\mathrm{Ga}^{\prime \prime \prime} \mathrm{Zn} \mathrm{n}^{\prime \prime}\left(\mathrm{L}^{4}\right)(\mu-\mathrm{OAc})_{2}\right]^{+}$. Interestingly, the $\mathrm{pH}$ maximum 
for the hydrolysis activity in the region between $\mathrm{pH} 7$ and $\mathrm{pH} 9$ is shifted by $0.7 \mathrm{pH}$ units to more basic conditions by the incorporation of a hydrogen bond donor proximal to the Ga'll center. Thus, the capacity of hydrogen bonding has a similar effect as a less electron-rich ligand in the primary coordination sphere, resulting in a more basic activity $\mathrm{pH}$ maximum.

Although, the $\mathrm{pH}$ maxima of the hydrolysis activity in the region between $\mathrm{pH} 6$ and $\mathrm{pH} 9$ is shifted to more basic conditions for the two complexes with hydrogen bond donors proximal to the dinuclear core, [Ga"'"Zn" $\left(\mathrm{H}_{2} \mathrm{SIM}^{1}\right)(\text { solv) }]^{4+}$ and $\left[\mathrm{Ga}^{\prime \prime \prime} \mathrm{Zn}^{\prime \prime}\left(\mathrm{H}_{2} \mathrm{SIM}^{4}\right)\left(\right.\right.$ solv) ${ }^{3+}$ (see Table 28 ) exhibit at $\mathrm{pH} 7$ (i) higher hydrolysis rates $\mathrm{k}_{\mathrm{cat}}$, (ii) higher $\mathrm{K}_{\mathrm{M}}$ values and subsequently lower substrate affinity, and (iii) higher catalytic efficiencies than $\left[\mathrm{Ga}^{\prime \prime \prime} \mathrm{Zn}{ }^{\prime \prime}\left(\mathrm{L}^{4}\right)(\mu-\mathrm{OAc})_{2}\right]^{+}$(see Table 25$)$, lacking hydrogen bonding capacity. ${ }^{66}$ This is most likely due to hydrogen bond formation. Moreover, the positioning of two hydrogen bond donors adjacent to the $\mathrm{Zn}$ " binding site has the most pronounced effect.

\section{Turn Over Numbers}

In order to determine the TONs of the $\mathrm{Zn}^{\prime \prime}, \mathrm{Zn}_{2}{ }_{2}, \mathrm{Ga}^{\prime \prime \prime}$, and Ga"' $\mathrm{Zn}$ " complexes of $\mathrm{H}_{3} \mathrm{SIM}^{1}$ and $\mathrm{H}_{4} \mathrm{SIM}^{4}$ a long-term study was conducted following the general procedure explained in Chapter 6.3. The assays were prepared with the aqueous-buffer solution of $\mathrm{pH}$ 7. TON values after nine days were calculated using the Beer-Lambert Law. The resulting data are given in Figure 68 and Table 29.

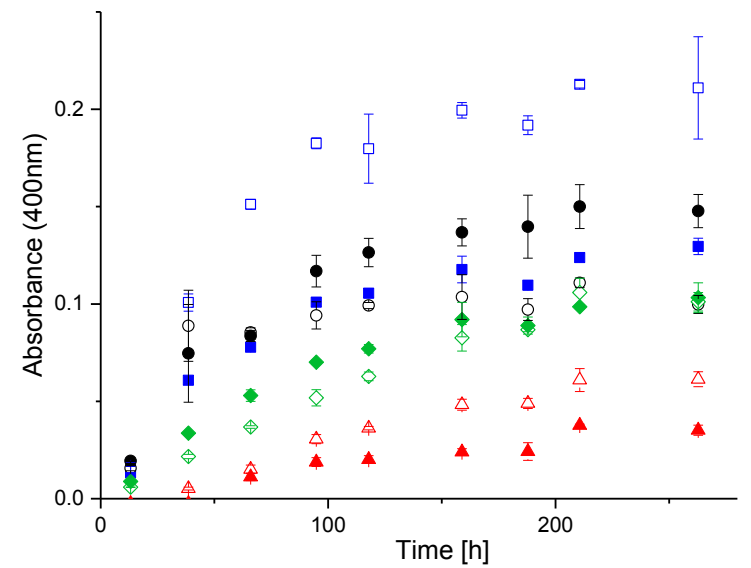

Figure 68: Time dependence of the absorbance band of 2,4-nitrophenolate $(400 \mathrm{~nm})$ at $\mathrm{pH}=7$ of BDNPP hydrolysis activity assays with mixtures of ligand $\mathrm{H}_{3} \mathrm{SIM}^{1}$ (unfilled symbols) and $\mathrm{H}_{4} \mathrm{SIM}^{4}$ (filled symbols) with 1 eq zinc(II) perchlorate (red triangles), 2 eq zinc(II) perchlorate (green diamonds), 1 eq of gallium(III) perchlorate (blue squares), 1 eq zinc(II) perchlorate and 1 eq of gallium(III) perchlorate (black circles).

Comparing the complexes with the same metal content enables the formulation of conclusions about the impact of the structural variations given by the two ligand backbones. In the case of the monozinc(II) complexes $\left[\mathrm{Zn}^{\prime \prime}\left(\mathrm{H}_{2} \mathrm{SIM}^{1}\right)(\text { solv })_{\mathrm{x}}\right]^{+}$and $\left[\mathrm{Zn}^{\prime \prime}\left(\mathrm{H}_{3} \mathrm{SIM}^{4}\right)(\text { solv })_{\mathrm{x}}\right]^{+}$the hydrolysis acceleration appears to be similar in the first 60 hours. But $\left[\mathrm{Zn}^{\prime \prime}\left(\mathrm{H}_{3} \mathrm{SIM}^{4}\right)(\text { solv })_{x}\right]^{+}$, which has a $\mathrm{Zn}$ " adjacent to a hydrogen bond donor, is, inhibited at lower product concentration than 
$\left[\mathrm{Zn}^{\prime \prime}\left(\mathrm{H}_{2} \mathrm{SIM}^{1}\right)(\text { solv })_{\mathrm{x}}\right]^{+}$lacking hydrogen bond donors proximal to the $\mathrm{Zn}^{\prime \prime}$ center, an effect possibly due to hydrogen bond formation.

Table 29: TONs of BDNPP hydrolysis with mixtures of the ligands $\mathrm{H}_{3} \mathrm{SIM}^{1}$ or $\mathrm{H}_{4} \mathrm{SIM}^{4}$ with 1 eq of zinc(II) perchlorate, 2 eq of zinc(II) perchlorate, 1 eq of gallium(III) perchlorate, 1 eq zinc(II) perchlorate and 1 eq of gallium(III) perchlorate (all kinetic studies were undertaken in a mixture of acetonitrile:aqueous buffer solution $(1: 1))$.

\begin{tabular}{l|l} 
complex & TON $^{\mathrm{a}}$ \\
\hline$\left[\mathrm{Zn}^{\prime \prime}\left(\mathrm{H}_{2} \mathrm{SIM}^{1}\right)(\text { solv })_{x}\right]^{+}$ & $10 \pm 1$ \\
{$\left[\mathrm{Zn}_{2}{ }_{2}\left(\mathrm{H}_{2} \mathrm{SIM}^{1}\right)(\text { solv })_{x}\right]^{3+}$} & $18 \pm 1$ \\
{$\left[\mathrm{Ga}^{\prime \prime \prime}\left(\mathrm{H}_{2} \mathrm{SIM}^{1}\right)(\text { solv })_{\mathrm{x}}\right]^{2+}$} & $35 \pm 1$ \\
{$\left[\mathrm{Ga}^{\prime \prime \prime} \mathrm{Zn}^{\prime \prime}\left(\mathrm{H}_{2} \mathrm{SIM}{ }^{1}\right)(\text { solv })_{\mathbf{x}}\right]^{4+}$} & $23 \pm 5$ \\
\hline$\left[\mathrm{Zn}^{\prime \prime}\left(\mathrm{H}_{2} \mathrm{SIM}^{4}\right)(\text { solv })_{\mathrm{x}}\right]$ & $6 \pm 1$ \\
{$\left[\mathrm{Zn}_{2}{ }_{2}\left(\mathrm{H}_{2} \mathrm{SIM}^{4}\right)(\text { solv })_{\mathrm{x}}\right]^{2+}$} & $16 \pm 1$ \\
{$\left[\mathrm{Ga}^{\prime \prime \prime}\left(\mathrm{H}_{2} \mathrm{SIM}^{4}\right)(\text { solv })_{\mathrm{x}}\right]^{+}$} & $21 \pm 1$ \\
{$\left[\mathrm{Ga}^{\prime \prime \prime} \mathrm{Zn}^{\prime \prime}\left(\mathrm{H}_{2} \mathrm{SIM}^{4}\right)(\text { solv })_{\mathrm{x}}\right]^{3+}$} & $25 \pm 2$ \\
\hline
\end{tabular}

a the assays were performed with aqueous buffer solution $\mathrm{pH}=7$, TONs were determined after 9 days.

For the Gall' complexes a similar result was observed since $\left[\mathrm{Ga}^{\prime \prime \prime}\left(\mathrm{H}_{2} \mathrm{SIM}^{4}\right)(\text { solv })_{\mathrm{x}}\right]^{+}$with a pivaloylamide residue adjacent to the Gall' center showed higher TONs than $\left[\mathrm{Ga}^{\prime \prime \prime}\left(\mathrm{H}_{2} \mathrm{SIM}^{1}\right)(\text { solv })_{x}\right]^{2+}$, lacking a proximal hydrogen bond donor. However, compared to the respective monozinc(II) complexes both monogallium(III) complexes exhibited higher TONs as expected from the above discussed kinetic studies. Thus, the inhibiting phosphomonoester is bound in mononuclear complexes more strongly to a $\mathrm{Zn}^{\|}$ion than to a Gall' ion and is stabilized by proximal hydrogen bond donors.

During the investigation of the TONs at pH 9.5 of various dizinc(II) complexes, discussed in Chapter 7.2, it was revealed that positioning one pivaloyl-amide residue proximal to each binding site prevents inhibition more reliably than with both at one binding site. Such an effect was not found comparing the TONs of $\left[\mathrm{Zn}_{2}{ }_{2}\left(\mathrm{H}_{2} \mathrm{SIM}^{4}\right)(\text { solv })_{x}\right]^{2+}$ and $\left[\mathrm{Zn}_{2}{ }_{2}\left(\mathrm{H}_{2} \mathrm{SIM}^{1}\right)(\text { solv })_{x}\right]^{3+}$; both exhibited similar TONs. Compared to $\left[\mathrm{Zn}_{2}{ }_{2}\left(\mathrm{H}_{2} \mathrm{~L}^{2}\right)(\mu-\mathrm{OAc})_{2}\right]^{+}$, discussed in Chapter 7.2, $\left[\mathrm{Zn}_{2}\left(\mathrm{H}_{2} \mathrm{SIM}^{4}\right)(\text { solv })_{x}\right]^{2+}$ has a terminal phenolate residue instead of a pyridine in the primary coordination sphere, while the secondary coordination sphere is in both complexes arranged in a symmetrical fashion. Therefore, the lower TON of $\left[\mathrm{Zn}_{2}{ }_{2}\left(\mathrm{H}_{2} \mathrm{SIM}^{4}\right)(\text { solv })_{x}\right]^{2+}$ compared to $\left[\mathrm{Zn}_{2}{ }_{2}\left(\mathrm{H}_{2} \mathrm{~L}^{2}\right)(\mu-\mathrm{OAc})_{2}\right]^{+}$is ascribed to the difference in the primary coordination sphere. Thus, the inhibition of dizinc(II) complexes by a phosphomonoester molecule seems to be favored by two hydrogen bond donors proximal to one binding site and an electron-rich phenolate residue in the primary coordination sphere. Comparing the two Ga"' $\mathrm{Zn}$ " complexes, [Ga"'Zn" $\left.\left(\mathrm{H}_{2} \mathrm{SIM}^{1}\right)(\text { solv })_{\mathrm{x}}\right]^{4+}$ with two privaloyl-amide residues proximal to the $\mathrm{Zn}$ " center exhibited a lower TON. This suggests that sterical hindrance does not prevent from inhibition. The higher TON of $\left[\mathrm{Ga}^{\prime \prime \prime} \mathrm{Zn}^{\prime \prime}\left(\mathrm{H}_{2} \mathrm{SIM}^{4}\right)(\text { solv })_{x}\right]^{3+}$ compared to [Ga'"' $\left.\mathrm{Zn}^{\prime \prime}\left(\mathrm{H}_{2} \mathrm{SIM}^{1}\right)(\text { solv })_{\mathrm{x}}\right]^{4+}$ is assumed to be due to the lower product affinity of the Gall center coordinated to the electron-rich terminal phenolate residue. 
The heterodinuclear complex $\left[\mathrm{Ga}^{\prime \prime \prime} \mathrm{Zn}^{\prime \prime}\left(\mathrm{H}_{2} \mathrm{SIM}^{4}\right)(\text { solv })_{x}\right]^{3+}$ revealed also a higher TON than the homodinuclear complex $\left[\mathrm{Zn}_{2}{ }_{2}\left(\mathrm{H}_{2} \mathrm{SIM}^{4}\right)(\text { solv })_{\mathrm{x}}\right]^{2+}$. It appears that a phosphoester bridge between two $\mathrm{Zn}$ " centers coordinated in $\mathrm{H}_{4} \mathrm{SIM}^{4}$ is more easily formed than between a Ga"l and a $\mathrm{Zn}^{\prime \prime}$ center, i.e. at lower product concentration. Interestingly, the TON of $\left[\mathrm{Ga}^{\prime \prime \prime} \mathrm{Zn}^{\prime \prime}\left(\mathrm{H}_{2} \mathrm{SIM}^{4}\right)(\mathrm{solv})_{\mathrm{x}}\right]^{3+}$ is also higher than for the two mononuclear complexes $\left[\mathrm{Zn}^{\prime \prime}\left(\mathrm{H}_{3} \mathrm{SIM}^{4}\right)(\mathrm{solv})_{\mathrm{x}}\right]^{+}$and $\left[\mathrm{Ga}^{\text {III }}\left(\mathrm{H}_{2} \mathrm{SIM}^{4}\right)(\mathrm{solv})_{\mathrm{x}}\right]^{+}$, indicating a positive interaction of the two metal ions in the heterodinuclear complex.

Comparing the different complexes of $\mathrm{H}_{3} \mathrm{SIM}^{1}$ without a terminal phenolate, the results suggests: the heterodinuclear complex $\left[\mathrm{Ga}^{\prime \prime \prime} \mathrm{Zn}^{\prime \prime}\left(\mathrm{H}_{2} \mathrm{SIM}^{1}\right)(\text { solv })_{\mathrm{x}}\right]^{4+}$ exhibits a similar TON as the dizinc(II) complex $\left[\mathrm{Zn}_{2}{ }_{2}\left(\mathrm{H}_{2} \mathrm{SIM}^{1}\right)(\mathrm{solv})_{x}\right]^{3+}$ indicating that the inhibition is independent of the two metal ions bound in the two binding sites of $\mathrm{H}_{3} \mathrm{SIM}^{1}$. However, [Ga"' $\left.\mathrm{Zn}^{\prime \prime}\left(\mathrm{H}_{2} \mathrm{SIM}^{1}\right)(\text { solv })_{x}\right]^{4+}$ shows a steeper increase of the absorbance at $400 \mathrm{~nm}$ with time, especially in the first fourty hours, compared to $\left[\mathrm{Zn}_{2}{ }_{2}\left(\mathrm{H}_{2} \mathrm{SIM}^{1}\right)(\text { solv })_{x}\right]^{3+}$. Thus, the hydrolysis rate depends strongly on the presence of the trivalent metal ion.

What emerged is that the Ga"l' $\mathrm{Zn}$ " complex with $\mathrm{H}_{4} \mathrm{SIM}^{4}$ is the most active catalyst, with $\mathrm{H}_{3} \mathrm{SIM}^{1}$ the monogallium(III) complex is the most active one. Surprisingly, [ $\left.\mathrm{Ga}^{\text {III }}\left(\mathrm{H}_{2} \mathrm{SIM}^{1}\right)(\text { solv })_{\mathrm{x}}\right]^{2+}$ exhibits the highest TON of all complexes studied, with a TON of 35 after nine days. The high TON of $\left[\mathrm{Ga}^{\prime \prime \prime}\left(\mathrm{H}_{2} \mathrm{SIM}^{1}\right)(\text { solv })_{x}\right]^{2+}$ is ascribed to (i) the lower product affinity of Ga"l' compared to $\mathrm{Zn}$ ", (ii) the lack of a proximal hydrogen bond donor, and (iii) the absence of a second metal ion and the resulting incapacity to bind efficiently the phosphomonoester product.

\section{Phosphomonoester Hydrolysis Studies}

Studies towards the phosphomonoester hydrolysis activity of the zinc(II), dizinc(II), gallium(III) and Ga"' $\mathrm{Zn}$ " complexes of $\mathrm{H}_{3} \mathrm{SIM}^{1}$ and $\mathrm{H}_{4} \mathrm{SIM}^{4}$ were conducted with the activated model substrate DNPP at different pHs (7, 8, 9 and 10). A significant phosphomonoester hydrolysis capacity was only observed for $\left[\mathrm{Ga}^{\prime \prime \prime} \mathrm{Zn}^{\prime \prime}\left(\mathrm{H}_{2} \mathrm{SIM}^{1}\right)(\text { solv })_{\mathrm{x}}\right]^{4+}$, while a slight increase in the 2,4-phenolate concentration in the assays of $\left[\mathrm{Ga}^{\prime \prime \prime}\left(\mathrm{H}_{2} \mathrm{SIM}^{1}\right)(\text { solv })_{x}\right]^{4+}$ at pH 7 and $\left[\mathrm{Zn}_{2}{ }_{2}\left(\mathrm{H}_{2} \mathrm{SIM}^{4}\right)(\text { solv })_{x}\right]^{2+}$ at pH 9.5 compared to autohydrolysis was detected. However, due to the instability of DNPP in the buffer solution, the deviations of the initial hydrolysis velocities obtained for $\left[\mathrm{Ga}^{\prime \prime \prime}\left(\mathrm{H}_{2} \mathrm{SIM}^{1}\right)(\mathrm{solv})_{\mathrm{x}}\right]^{4+}$ and $\left[\mathrm{Zn}_{2}{ }_{2}\left(\mathrm{H}_{2} \mathrm{SIM}^{4}\right)(\mathrm{solv})_{\mathrm{x}}\right]^{2+}$ are too high to make an explicit statement about their hydrolysis capacity. The fact that only the Ga"l'Zn" and Ga'l! complex of $\mathrm{H}_{3} \mathrm{SIM}^{1}$ showed the ability to hydrolyze the phosphomonoester DNPP in contrast to the corresponding complexes of $\mathrm{H}_{4} \mathrm{SIM}^{4}$, indicates that neither the strong electron-rich terminal phenolate nor the hydrogen bond donor proximal to the trivalent metal center cause the capacity of phosphomonoester hydrolysis in PAPs. Moreover, the dizinc(II) complex of ligand $\mathrm{H}_{3} \mathrm{SIM}^{1}$ did not reveal phosphomonoester hydrolysis. 
This indicates that the trivalent metal ion is essential for the DNPP hydrolysis. Moreover, the only model complexes reported to cleave phosphomonoester bonds contained at least one $\mathrm{Fe}^{\text {III }}$ ion. ${ }^{108,109,114}$

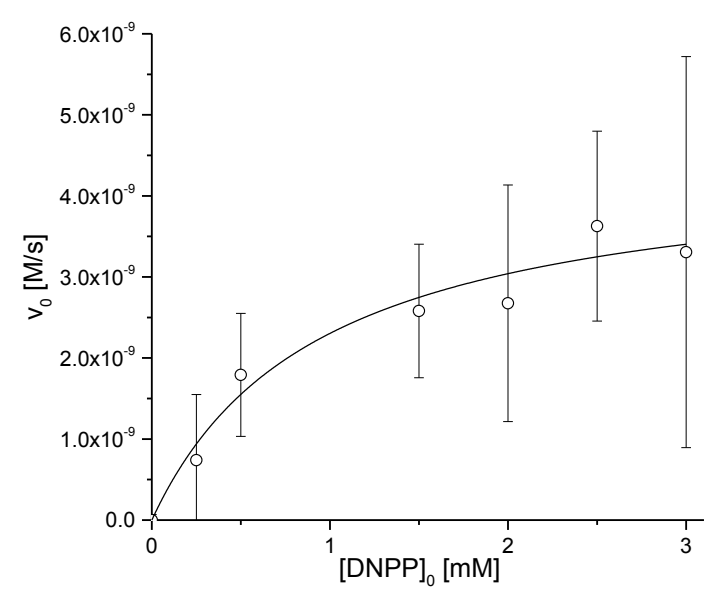

Figure 69: Substrate concentration dependence at $\mathrm{pH}=7$ of DNPP hydrolysis activity for the mixture of ligand $\mathrm{H}_{3} \mathrm{SIM}^{1}$ with 1 eq zinc(II) perchlorate and 1 eq of gallium(III) perchlorate.

The substrate concentration dependence measurements were conducted at $\mathrm{pH} 7$ in order to mimic near physiological conditions. [Ga"' $\left.\mathrm{Zn}^{\prime \prime}\left(\mathrm{H}_{2} \mathrm{SIM}^{1}\right)(\text { solv })_{x}\right]^{4+}$ showed Michaelis-Menten type behavior, and fitting the data determined the catalytic parameters $k_{\text {cat }}=(0.12 \pm 0.02) \cdot 10^{-3} \mathrm{~s}^{-1}$, $\mathrm{K}_{\mathrm{M}}=(0.94 \pm 0.32) \mathrm{mM}$ and $\mathrm{k}_{\text {cat }} / \mathrm{K}_{\mathrm{M}}=0.12 \mathrm{~s}^{-1} \mathrm{M}^{-1}$. Although the activity of [Ga"'In $\left.\mathrm{Zn}^{\prime \prime}\left(\mathrm{H}_{2} \mathrm{SIM}^{1}\right)(\mathrm{solv})_{\mathrm{x}}\right]^{4+}$ is lower than those reported for the diiron complexes, ${ }^{108,109,114}\left[\mathrm{Ga}^{\prime \prime \prime} \mathrm{Zn}^{\prime \prime}\left(\mathrm{H}_{2} \mathrm{SIM}^{1}\right)(\text { solv })_{\mathrm{x}}\right]^{4+}$ is to date the first heterodinuclear model complex able to hydrolyze phosphomonoesters.

\subsection{SUMMARY}

The investigations undertaken with the mono- and dinuclear complexes of the two asymmetric ligands $\mathrm{H}_{3} \mathrm{SIM}^{1}$ and $\mathrm{H}_{4} \mathrm{SIM}^{4}$ with differing primary and secondary coordination spheres lead to a deeper insight in the reaction mechanism of phosphoester hydrolysis with heterodinuclear biomimetics. In the proposed mechanism the phosphoester is activated by the $\mathrm{Zn}$ " center and the $\mathrm{Ga}^{\mathrm{III}}$ is responsible for providing the hydroxide nucleophile at near physiological conditions (Scheme 24). 


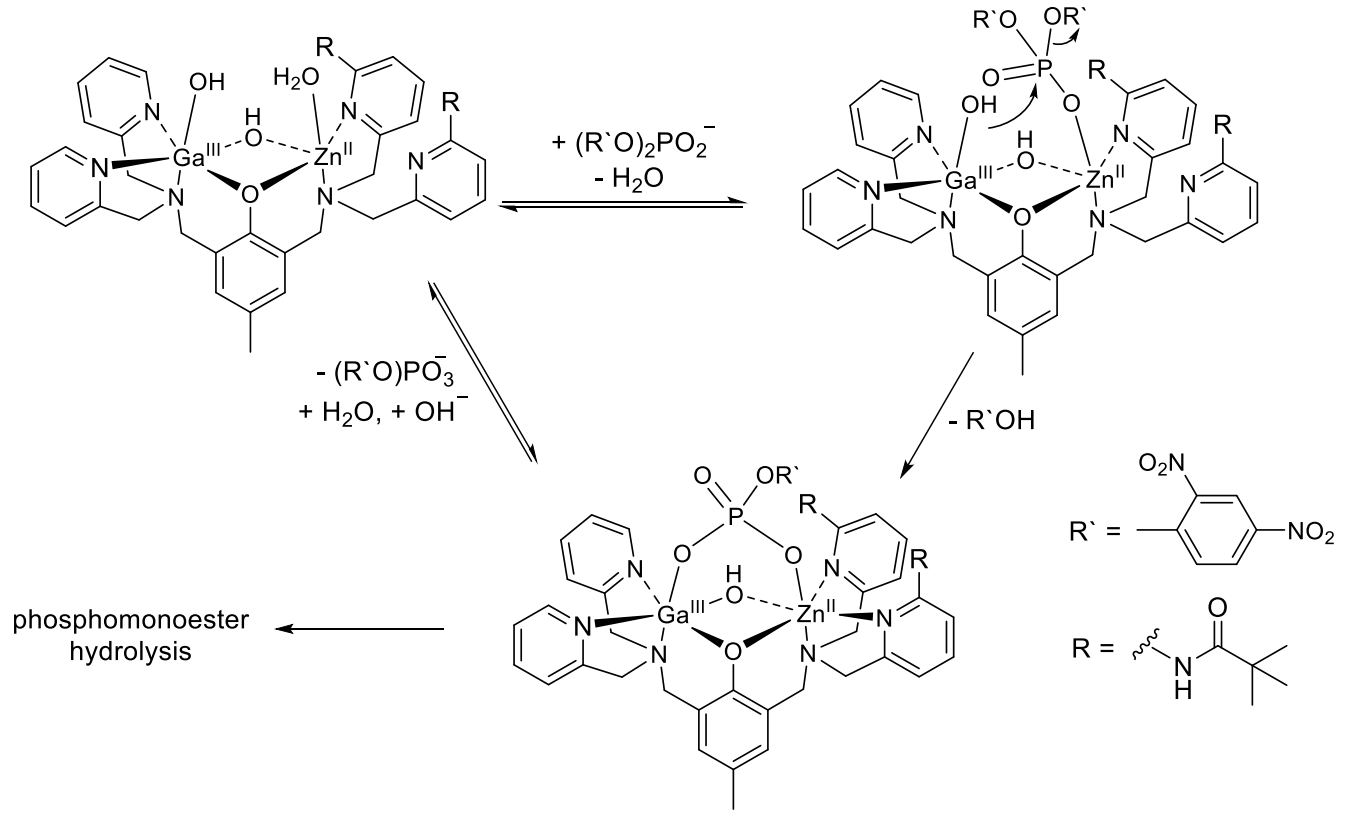

Scheme 24: Proposed mechanism for the BDNPP hydrolysis catalyzed by [Ga"' $\left.\mathrm{Zn}^{\prime \prime}\left(\mathrm{H}_{2} \mathrm{SIM}^{1}\right)(\mathrm{solv})_{\mathrm{x}}\right]^{4+}$.

It was shown that the monozinc(II) complexes are not able to hydrolyze phosphoesters at near physiological conditions, but coordination of a second $\mathrm{Zn}$ " ion in the same ligand enables the hydrolysis. A similar cooperative effect was detected for the Ga"' $\mathrm{Zn}$ " complexes. Although the substrate affinity of the complex $\left[\mathrm{Ga}^{\prime \prime \prime} \mathrm{Zn}^{\prime \prime}\left(\mathrm{H}_{2} \mathrm{SIM}^{1}\right)(\mathrm{solv})_{\mathrm{x}}\right]^{4+}$ was lower than of the corresponding $\mathrm{Zn}_{2}{ }_{2}$ complex, the heterodinuclear complex exhibits a 50-fold faster hydrolysis rate and a six-fold increased efficiency. Comparison of the activity of the two different Ga"'Zn" complexes revealed a shift of the optimum $\mathrm{pH}$ to higher values with (i) an electron-poor coordinating ligand in the primary coordination sphere, or (ii) provision of hydrogen bonding by the second coordination sphere. Furthermore, the capacity of a hydrogen bonding network proximal to the dinuclear core results in (i) higher hydrolysis rates, (ii) lower substrate affinities, and (iii) higher efficiencies, especially when the two hydrogen bond donors were placed proximal to the $\mathrm{Zn}$ " center. However, the long-time studies showed that such a sterically demanding complex is not prevented from inhibition. During the long-term studies an acceleration effect by the trivalent metal ion was detected. Furthermore, a Gall' coordinating phenolate-residue results in a higher TON than pyridine for the Ga"' $\mathrm{Zn}$ " complexes. However, the highest TON was found for the monogallium(III) complex without hydrogen bond donors in close distance to the metal center but with a vacant second amidated coordination site.

Investigation of the DNPP hydrolysis of the different complexes showed that only the complex [Ga'"' $\left.\mathrm{Zn}^{\prime \prime}\left(\mathrm{H}_{2} \mathrm{SIM}^{1}\right)(\text { solv })_{x}\right]^{4+}$ is able to cleave phosphomonoesters efficiently. Therefore, this complex does not only combine the essential structural features of the active site of PAP, but is also the first heterodinuclear model complex mimicking the essential function of PAPs, the hydrolysis of phosphomonoesters. 


\section{Chapter 10}

Conclusions and Outlook 
In course of this work the ligands $\mathrm{HL}^{1}$ and $\mathrm{H}_{2} \mathrm{~L}^{4}$, which have previously been accepted as a suitable basis for model complexes of phosphatases, were modified by the introduction of substituents capable of hydrogen bonding. In this way, the second coordination sphere of phosphatase metalloenzymes was mimicked in order to further improve the accuracy of phosphatase biomimetics as enzyme models. For the synthesis of the four asymmetric ligands, a selective procedure was evaluated, generating two different coordination sites. These ligands mimic the asymmetric peptide backbone that surrounds the dimetallic core in the active site of phosphatases.

The series of ligands studied, with no substituents, two asymmetrically disposed and two symmetrically disposed amide or two asymmetrically disposed amine anchor groups for hydrogen bonding, led to strikingly different structures of the corresponding dizinc(II) complexes. It became apparent that the steric hindrance caused by two pivaloyl-amide substitutents adjacent to one coordination site, and the subsequent inability of the corresponding dizinc(II) complexes to bind two acetate co-ligands, resulted in a dizinc(II) structure similar to the active sites of phosphatases with two different $\mathrm{Zn}$ " sites - one 6- and the other 5-coordinate. Moreover, these complexes represent rare examples of zinc(II) complexes with a hydroxido co-ligand - the potential nucleophile during the phosphoester hydrolysis reaction - stabilized by hydrogen bond formation. The desired hydrogen bonding network was also observed for the dizinc(II) and diiron(II) complexes studied, which revealed the common structure of phenoxido-bridged phosphatase models bearing two bridging acetate co-ligands.

Studying the phosphoester hydrolysis activity of the series of dizinc(II) and diiron(II) model complexes with hydrogen bonding functionalities proximal to the dimetal core enabled the analysis of the implications of (i) the type of the hydrogen bond donor, (ii) the position of the hydrogen bond donor, and (iii) the coordinating ligands in the primary coordination sphere. The main impact of the secondary interactions in the dizinc(II) complexes was found to be the increased substrate affinity, and this obviously depends on the hydrogen bonding sites and the type of the hydrogen bonding groups. In fact, at $\mathrm{pH} 7$ the diamino derivative is the most effective catalyst, and positioning of both pivaloyl-amide residues proximal to one $\mathrm{Zn}^{\prime \prime}$ center exhibited a more positive effect towards the BDNPP hydrolysis than positioning one pivaloyl-amide adjacent to each $\mathrm{Zn}^{\|}$center. Long-term catalytic studies showed that at $\mathrm{pH}$ 9.5 the symmetrical, sterically hindered, amide-based system is the most efficient dizinc(II) complex studied here, and this is primarily due to less stable catalyst-inhibitor complexes. Interestingly, the effect of the position of the pivaloyl-amide residues was shown to be the 
opposite for the corresponding diiron(II) complexes. In fact, incorporation of two pivaloylamide residues in an asymmetric fashion into a dizinc(II) complex lead to the generation of a stable catalyst-inhibitor (hydrolysis product) adduct. Herein, one of the two pivaloyl-amide moieties fills the remaining vacant coordination site and is stabilized by hydrogen bond formation to the second pivaloyl-amide residue.

Although this work showed that the incorporation of sterically demanding amide moieties into dinuclear model complexes have a beneficial effect on the phosphoester cleavage capacity, it is still not completely understood which structural feature is accounted therefore: (i) the hydrogen bonding capacity, (ii) the sterical strain, or (iii) the electronic effect caused by potential coordination of the amide-oxygen donor. In order to circumvent the latter and to preserve the steric strain of the hydrogen bonding substituents, which might promote the hydrolysis, the efforts of future work could be put in the reduction of the pivaloyl-amide functionalities of the ligands developed in this thesis. For example, reduction of ligand $\mathrm{H}_{3} \mathrm{SIM}^{1}$ with lithium aluminium hydride should generate ligand $\mathrm{H}_{4} \mathrm{~L}^{73}$ with a sterically demanding amine substituent (Chart 29). ${ }^{199}$ Using $\mathrm{H}_{4} \mathrm{~L}^{73}$ for the synthesis of a dizinc(II) complex supplies a biomimetic which maintains (i) the capacity to form hydrogen bonds as well as (ii) the steric strain comparable to $\mathrm{H}_{3} \mathrm{SIM}^{1}$, but lacks the ability to stabilize a catalyst-inhibitor complex by coordination of an adjacent donor.
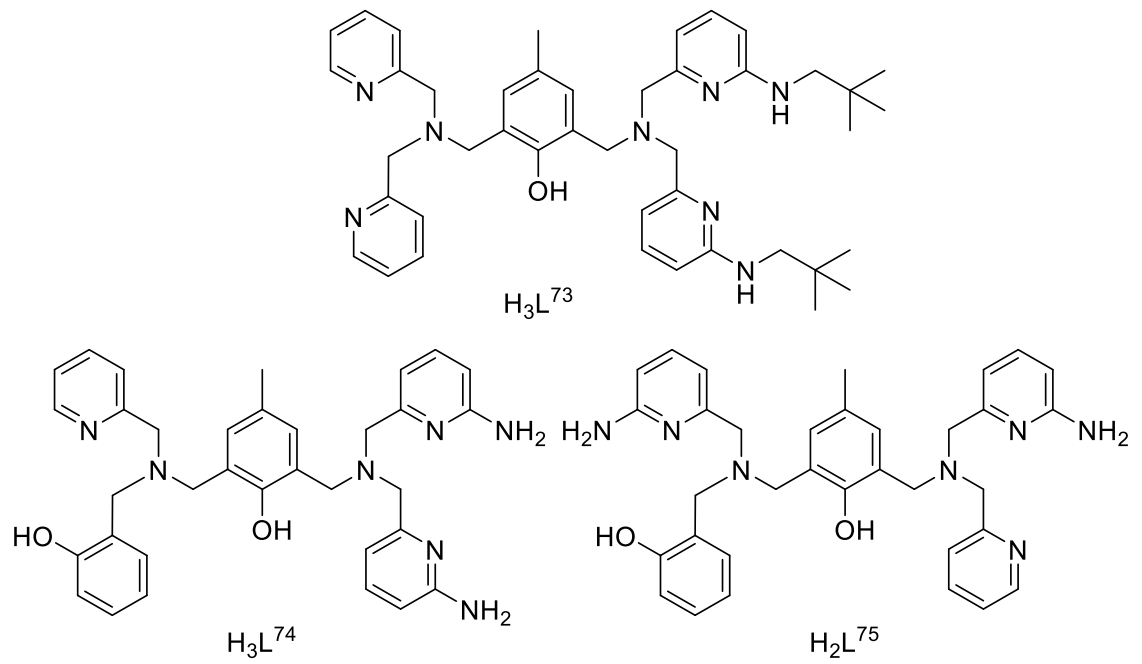

Chart 29: Depiction of the desired ligands for the generation of phosphatase biomimetics in future studies.

The more electron-rich primary coordination sphere and the amino hydrogen bond donors have been shown to support the phosphoester hydrolysis. Therefore, biomimetics which carry those structural features in tandem would be of considerable interest. The development of $\mathrm{H}_{3} \mathrm{~L}^{74}$ and $\mathrm{H}_{3} \mathrm{~L}^{75}$ (Chart 29), and complexes thereof, should have the potential to mimic the functionality of the native enzymes more accurately. 
An interesting result during the characterization of the diiron(II) complexes was the reduction capacity of Fe ${ }^{\text {III }}$ bound to the ligand $\mathrm{H}_{3} \mathrm{SIM}^{1}$ under acidic conditions. A concerted protonelectron transfer mechanism was proposed for this reaction, but a direct proof remains to be provided. Therefore, a suitable experiment could be the use of EPR spectroscopy or radical scavengers which could support the proposed mechanism via the generation of a radical species.

In order to gain a more accurate model system for the active form of mammalian PAPs the formation of a heterovalent diiron core in an asymmetric coordination environment with the capacity to form a hydrogen bond network similar to the native enzymes was probed. In fact, the successful formation of an Fe"lIFe" species via chemical oxidation of the respective diiron(II) complex with ferrocenium hexafluorophosphate under anaerobic conditions was achieved. Comparison of the catalytic activity of the parent diiron(II) and the heterovalent diiron complex supported the presumption that the role of the Fe $\mathrm{e}^{\text {III }}$ center in the active site of PAP is the provision of a nucleophilic hydroxide at low pH. Another milestone for the development of sophisticated model systems for the active form of mammalian PAPs could be the generation of diiron(II) complexes of $\mathrm{H}_{4} \mathrm{SIM}^{3}$ and $\mathrm{H}_{4} \mathrm{SIM}^{4}$ and the following study of the oxidation, analogous to the approach outlined in this thesis. Furthermore, reliable protocols for the isolation of the complexes obtained, such as extraction of the generated byproduct ferrocene need to be developed. These investigations are promising due to the provision of two chemically distinct coordination sites within these ligands.

The second strategy to generate heterovalent dinuclear model complexes which provide in parallel hydrogen bonding donors adjacent to the dimetal core was the selective generation of a Ga"' $\mathrm{Zn}$ " complex. Therefore, the coordination behavior of the three pivaloyl-amide containing ligands towards $\mathrm{Zn}$ ", $\mathrm{Cu}^{\prime \prime}, \mathrm{Ga}$ "II and Fe"ll was examined with various techniques. In this way, the selective formation of specific complexes was detected assigned to the asymmetry of the ligands. The trivalent Gall' ion was shown to coordinate in the less hindered or the more electron-rich binding site, while the remaining binding site is selectively filled by the divalent $\mathrm{Zn}$ " ion. Moreover, the catalytic activity of the monogallium(III), the monozinc(II), and the heterodinuclear Ga"' $\mathrm{Zn}$ " complexes was investigated. In this way, the previously proposed

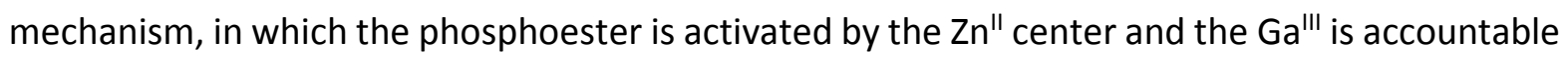
for providing the hydroxide nucleophile even at near physiological conditions, was supported in this study and a cooperative effect was detected for the Ga"'Zn" complexes. Although the substrate affinity of the Ga"' $\mathrm{Zn}$ " complex was lower than for the respective $\mathrm{Zn}_{2}$ complex, a 50-fold faster hydrolysis rate and a six-fold increased efficiency was detected in one case. 


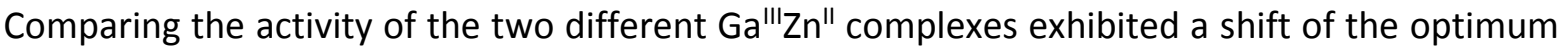
$\mathrm{pH}$ to higher values with (i) an electron-poor coordinating ligand in the primary coordination sphere, or (ii) provision of hydrogen bonding by the second coordination sphere. However, the capacity of a hydrogen bonding network proximal to the Ga"' $\mathrm{Zn}$ " core resulted in (i) higher hydrolysis rates, (ii) lower substrate affinities, and (iii) higher efficiencies, especially when the two hydrogen bond donors were placed proximal to the $\mathrm{Zn}$ " center.

With the use of long-term studies an acceleration effect by the trivalent metal ion was detected and a high TON was supported by the coordination of the Ga'l' by a terminal phenolate-residue. However, the highest TON was found for the monogallium(III) complex bearing no hydrogen bond donors at close distance to the metal center but with a vacant second amidated coordination site. The lower TON of the respective Ga"l' $\mathrm{Zn}$ " was ascribed to the inhibitory effect of a bridging product molecule, stabilized via coordination of the adjacent amide residue, as found in a similar dizinc(II) complex. Therefore, the preparation of ligand $\mathrm{H}_{3} \mathrm{~L}^{73}$ and its derivatives, in combination with the generation of a Ga"' $\mathrm{Zn}^{\prime \prime}$ complex with the two different metal ions in distinct binding sites, may shed light into the coordination capacity of the amide oxygen atom. The generation of the Ga"' $\mathrm{Zn}$ " complex of ligand $\mathrm{H}_{3} \mathrm{~L}^{73}$ is of particular interest due to the fact that the amidated counterpart $\left[\mathrm{Ga}^{\prime \prime \prime} \mathrm{Zn}^{\prime \prime}\left(\mathrm{H}_{2} \mathrm{SIM}^{1}\right)(\text { solv })_{\mathrm{x}}\right]^{4+}$ revealed the ability to cleave phosphomonoesters. Therefore, this complex does not only combine the essential structural features of the active site of PAP, but it is also the first heterodinuclear model complex found to mimick the essential function of PAPs, i.e. the hydrolysis of phosphomonoesters. However, the results of the phosphoester hydrolysis activity indicate fast inhibition, which makes the Ga"' $\mathrm{Zn}$ " complex of ligand $\mathrm{H}_{3} \mathrm{~L}^{73}$ a promising candidate for a more accurate structural and functional model of the heterovalent heterodinuclear active site of PAP, thus, leading us a step further to the well-directed development of more efficient osteoporosis therapeutic agents. 


\section{Chapter 11}

Experimental Section 


\subsection{General Remarks}

The main part of this thesis is and will be published in several scientific journals. For the NMR spectra $\left({ }^{1} \mathrm{H}\right.$ and $\left.{ }^{31} \mathrm{C}\right)$ and mass spectra for the synthesized ligands as well as titration experiments it can be refered to the Supporting Information of each of the corresponding publications. The experimental structures reported in this thesis are deposited at the "Cambridge Crystallographic Data Centre", the experimental data are not included in the Appendix, but can be obtained free of charge via www.ccdc.cam.ac.uk/data_request/cif. The corresponding CCDC-reference code of each complex is given in the Appendix.

\subsection{Material and Methods}

\subsubsection{CHEMICALS}

Chemicals and solvents used for synthesis and spectroscopy were purchased from Sigma-Aldrich and Acros. 2,4-Dinitrophenol was recrystallized and phosphoryl chloride was distilled before usage. All other chemicals were used without prior purification. Solvents for organic synthesis were of standard quality. Dry solvents were purchased from Sigma-Aldrich and Acros, stored over molecular sieves, and used without further purification. For complex syntheses and spectroscopy solvents of p.a. grade were utilized without further purification. In the kinetic assays MilliQ water was used.

\subsubsection{Physical MeAsUREMENTS}

\section{NMR Spectroscopy}

Nuclear Magnetic Resonance (NMR) spectra were recorded with Bruker AV200, AV400, AV500 and AV600 spectrometers, for ${ }^{13} \mathrm{C}$ and ${ }^{31} \mathrm{P}$ NMR spectra with ${ }^{1} \mathrm{H}$ decoupling. Two-dimensional correlation spectroscopy (COSY), nuclear Overhauser-effect spectroscopy (NOESY), heterodinuclear single quantum correlation (HSQC) and hetereodinuclear multiple bond connectivity ( $\mathrm{HMBC}$ ) experiments were used to assign each signal in the spectra. For ${ }^{1} \mathrm{H}$ and ${ }^{13} \mathrm{C}$ NMR spectra chemical shifts are reported in $\delta$-units, relative to known solvent peak references: $\delta_{\mathrm{H}}=7.27 \mathrm{ppm}, \delta_{\mathrm{C}}=77.00 \mathrm{ppm}$ for $\mathrm{CDCl}_{3} ; \delta_{\mathrm{H}}=1.94 \mathrm{ppm}, \delta_{\mathrm{C}}=1.39 \mathrm{ppm}$ for $\mathrm{CD}_{3} \mathrm{CN} ; \delta_{\mathrm{H}}=3.30 \mathrm{ppm}$, $\delta_{\mathrm{C}}=49.00 \mathrm{ppm}$ for $\mathrm{CD}_{3} \mathrm{OD}$ and $\delta_{\mathrm{H}}=2.05 \mathrm{ppm}, \delta_{\mathrm{C}}=29.92 \mathrm{ppm}$ for $\mathrm{CD}_{3} \mathrm{COCD}_{3} .85 \%$ phosphoric acid $(0 \mathrm{ppm})$ was used as external reference for ${ }^{31} \mathrm{P}$ NMR spectra. The following abbreviations 
were used: s (singlet), bs (broad singlet), d (doublet), t (triplet), dt (doublet of triplets), dd (doublet of doublets), ddd (doublet of doublets of doublets), td (triplet of doublets), sep (septet) and $\mathrm{m}$ (multiplet). The reported coupling constants ${ }^{n J}$ are for ${ }^{1} \mathrm{H}-{ }^{1} \mathrm{H}$ couplings. The software package TopSpin $^{\text {TM }}$ from BRUKER was used for data processing. ${ }^{302}$

\section{Magnetic Susceptibility Measurement by the Evans Method}

Magnetic moments in solution were measured for a solution of known concentration of complex in deuterated solvent in a capillary which was in a NMR tube carrying the same solvent. The experiments were conducted with Bruker AV200 and AV500 instruments. Magnetic moments were evaluated using Equation 24 and Equation 25, whereby appropriate corrections were considered.

$$
\begin{aligned}
& \text { Equation 24 } \chi_{\text {para,subst }}=\frac{3}{4 \pi c}\left(\frac{\Delta v}{v}\right)+\chi_{\text {dia,solv }}+\chi_{\text {dia,subst }} \\
& \text { Equation 25 } \mu=2.828 \sqrt{\chi_{\text {para,subst }} \mathrm{T}}
\end{aligned}
$$

\section{Solid State Magnetic Susceptibility Measurement with SQUID}

Magnetic susceptibility in solid state were examined with a MPMS-XL 5T superconducting quantum interference device (SQUID) from Quantum Design. The powdered samples were pressed into a PTFE band to avoid field-induced orientation and incorporated into two plastic straws as sample holder. Diamagnetic corrections for the PTFE band and the sample holder were applied.

\section{X-Ray Structure Analyses}

X-ray crystallographic data for the zinc(II) and gallium(III) complexes were collected with an Oxford Diffraction Gemini Ultra dual source (Mo or $\mathrm{Cu}$ ) CCD diffractometer. The structures were solved by direct methods (SIR-92) using the SHELXS computer program and refined by the fullmatrix least-squares method based on $\mathrm{F}^{2}$, with the SHELXL 97 computer program. ${ }^{303}$ These programs were accessed by the WINGX 1.70 .01 crystallographic collective package. ${ }^{304}$ Crystallographic data for the iron complexes were collected with a Bruker AXS Smart 1000 CCD and an Agilent Technologies SuperNova-E dual source (Mo or $\mathrm{Cu}$ ) CCD diffractometer. Using the software Olex $2,{ }^{305}$ the structures were solved with conventional direct methods and refined by full-matrix least squares based on $\mathrm{F}^{2}$. All non-hydrogen atoms were given anisotropic displacement parameters, unless they were disordered, and were placed at calculated positions. Hydrogen atoms were fixed geometrically and were not refined. The counter ion hexafluorophosphate was in some cases resolved into two disordered octahedral anions, which share the 
same phosphorus central atom. For $\left[\mathrm{Zn}_{2}{ }_{2}\left(\mathrm{H}_{2} \mathrm{~L}^{2}\right)(\mu-\mathrm{OAc})_{2}\right] \mathrm{PF}_{6}$, the structure was refined with acetone and water solvate, both with an occupancy of $50 \%$. In case of $\left[\mathrm{Fe}_{2}{ }_{2}\left(\mathrm{H}_{2} \mathrm{~L}^{2}\right)(\mu-\mathrm{OAc})_{2}\right] \mathrm{PF}_{6}$ the structure was refined with a diethylether solvate in the elemental cell. For $\left[\mathrm{Fe}_{2}{ }_{2}\left(\mathrm{H}_{2} \mathrm{SIM}^{1}\right)(\mu-\mathrm{OAc})_{2}\right] \mathrm{PF}_{6}$ and $\left\{\mathrm{Na}\left[\mathrm{Fe}_{2}{ }_{2}\left(\mathrm{H}_{2} \mathrm{SIM}^{3}\right)(\mu-\mathrm{OAc})_{2}\right]_{2}\right\} \mathrm{PF}_{6}$ electron density attributed to disordered solvent molecules was removed from the structure with the BYPASS procedure as implemented in PLATON (SQUEEZE). ${ }^{306,307}$ Partial structure factors from the solvent masks were included in the refinement as separate contributions to $F_{\text {obs. }}$.The $X$-ray structural data of the published structures were deposited at the Cambridge Crystallographic Data Centre.

\section{IR Spectroscopy}

FT-Infrared spectroscopy was carried out with a Perkin Elmer FT-IR Spectrometer SPECTRUM 2000 equipped with a Smiths DuraSamplIR II ATR diamond window.

\section{Mass Spectrometry}

Electron ionization (EI) mass spectra were recorded with a JEOL JMS-700 instrument, Matrixassisted laser desorption/ionization (MALDI) mass spectra were recorded with a Bruker ApexQe hybrid 9.4 FT-ICR instrument and Electrospray ionization (ESI) mass spectra were measured on a Finnigan LCQ spectrometer at the Institute of Organic Chemistry of the University of Heidelberg. High resolution mass spectra were collected with a Bruker microTOFQ ESI-MS spectrometer in the School of Chemistry and Molecular Biosciences of the University of Queensland and a Bruker Apex-Qe hybrid 9.4 T FT-ICR instrument at the Institute of Organic Chemistry at the University of Heidelberg. The data were processed with Bruker Compass Data Analysis software.

\section{Elemental Analyses}

Elemental microanalyses were performed in the analytic laboratories of the Institute of Organic Chemistry of the University of Heidelberg with an Elementar Vario Mikro cube machine and the Carlo Erba Elemental Analyzer model NA1500 in the School of Chemistry and Molecular Biosciences at the University of Queensland.

\section{Cyclic Voltammetry}

Cyclic voltammetric measurements were performed on a $\mathrm{CH}$ Instruments $\mathrm{CHI660D}$ electrochemical workstation equipped with a Faraday cage using a three-electrode setup consisting of a glassy carbon working electrode, a platinum wire as a counter electrode and a $\mathrm{Ag} / \mathrm{Ag}^{+}(0.1 \mathrm{mM}$ in $\mathrm{NaCl})$ reference electrode. The complex solutions were prepared with 
degassed solvents with $0.1 \mathrm{M}\left(\mathrm{NBu}_{4}\right) \mathrm{ClO}_{4}$ as electrolyte. The redox potentials of the obtained signals were determined by comparison to a ferrocen sample measured at the same conditions and referenced vs. SCE $\left(\mathrm{E}\left(\mathrm{Fc} / \mathrm{Fc}^{+}\right)=0.380 \mathrm{~V}\right.$ vs. SCE $) .^{252}$

\section{EPR Spectroscopy}

X-band EPR spectra were measured with a Bruker Biospin ELEXSYS E500 spectrometer. The microwave frequency and magnetic field were calibrated with a Bruker frequency counter and an ER036 TM or an ER035M Tesla meter. Spin-Hamiltonian parameters were determined by computer simulations of the experimental spectra using the programs XSophe ${ }^{308}$ and MoSophe. ${ }^{309}$ The simulated and experimental spectra were visualized with the software suite XeprView.

\section{UV-vis-NIR Spectroscopy}

UV-vis-NIR spectra were recorded at $25^{\circ} \mathrm{C}$ with a TIDAS II J\&M spectrophotometer and a Varian Cary50 Bio UV/visible spectrophotometer in $10 \mathrm{~mm}$ quartz cuvettes. Time-dependent UV-vis-NIR measurements were performed using a TIDAS II J\&M spectrophotometer. Time-course measurements at fixed wavelengths were monitored by a JASCO V-570 spectrophotometer and a Varian Cary50 Bio UV/visible spectrophotometer with a Peltier temperature controller.

\section{Mössbauer Spectroscopy}

${ }^{57} \mathrm{Fe}$ Mössbauer spectra of frozen solutions were acquired with a conventional spectrometer incorporating an Oxford Instruments Mössbauer-Spectromag 4000 Cryostat, equipped with a ${ }^{57} \mathrm{Co}$ source $(3.7 \mathrm{GBq})$ in a rhodium matrix in the constant-acceleration mode at $170 \mathrm{~K}$. Isomer shifts are given relative to $\alpha-F e$ at $300 \mathrm{~K}$. Spectra were fitted using the NORMOS Mössbauer Fitting Program.

\subsubsection{PHOSPHOESTER HYDROLYSIS EXPERIMENTS}

\section{General Procedure}

Phosphoester hydrolysis activity of the complexes was probed with a spectrophotometric assay using BDNPP as phosphodiester model substrate and DNPP as phosphomonoester model substrate. Cleavage of the phosphorous-oxygen bond was followed at $25^{\circ} \mathrm{C}$ by monitoring the generated product, 2,4-dinitrophenolate, by its strong absorption at $400 \mathrm{~nm}\left(\varepsilon=12,100 \mathrm{M}^{-1} \mathrm{~cm}^{-1}\right)$. All measurements were carried out in 1:1 acetonitrile-buffer mixtures and performed in 
triplicates. The complex was allowed to equilibrate in the acetonitrile-buffer mixture for one minute prior addition of substrate. When the substrate was added to the reaction mixture the starting hydrolysis activity was monitored in the time between 15 and 195 seconds and analyzed by linear regression. For each experiment autohydrolysis assays were conducted by measuring the hydrolysis rate under the same conditions, but without complex, and were substracted from the derived data.

\section{Preparation of the Multicomponent Buffer Solutions}

The aqueous buffer consisted of 2-( $N$-morpholino)ethanesulfonic acid (MES) (50 mM; pH range: 5.5 - 6.7), 4-(2-hydroxyethyl)piperazine-1-ethanesulfonic acid (HEPES) (50mM; pH range: 6.8 - 8.2), 2-(cyclohexylamino)ethanesulfonic acid (CHES) (50 mM; pH range: 8.6 - 10.0), 3-(cyclohexylamino)-1-propanesulfonic acid (CAPS) (50 mM; pH range: 9.7 - 11.1) and lithium perchlorate $(250 \mathrm{mM})$ for ionic strength control. The desired $\mathrm{pH}$ of the buffers was adjusted by addition of aqueous sodium hydroxide solution. Following treatment with Chelex $^{\circledR}$ (Chelex 100 sodium form) overnight and filtration with $45 \mu \mathrm{m}$ syringe filters ensured the absence of metal ions in the buffer solutions. The $\mathrm{pH}$ values reported refer to the aqueous component; however, it should be noted that the $\mathrm{pH}$ of a 1:1 mixture of buffer and acetonitrile was the same within error as of the corresponding buffer solution itself. ${ }^{275,} 276$

\section{Phosphodiester Hydrolysis Assays}

The activated model substrate BDNPP was initially prepared as $15 \mathrm{mM}$ solution in acetonitrile and the complex solutions were $1 \mathrm{mM}$ in acetonitrile. The $\mathrm{pH}$ dependence of the activity was studied by varying the $\mathrm{pH}$ value of the multicomponent buffer in the $\mathrm{pH}$ range of 5 to 11 . In this process the assays contained the complex $0.04 \mathrm{mM}$ and BDNPP $5 \mathrm{mM}$ in the cuvette. The resulting $\mathrm{pH}$ profiles were fitted in the Origin (OriginLab) program using Equation 20 and Equation 21 (Chapter 6.3). ${ }^{277}$

Complex concentration dependency assays were $5 \mu \mathrm{M}$ in BDNPP and 0.01-0.09 mM in complex, respectively. The data derived were fitted with a linear function using the Origin software. Substrate concentration dependence of the catalytic rate was examined with a fixed complex concentration of $0.04 \mathrm{mM}$ and the BDNPP concentration varied. The experimental data obtained were fitted in the Origin (OriginLab) program to the Michaelis-Menten equation (Equation 22). Studies of the turn over number (TON) were conducted $15 \mathrm{nM}$ in complex and $5.25 \mu \mathrm{M}$ in BDNPP. Samples were taken at various intervals during the experiment, diluted with solvent, and their UV-vis spectra were recorded to determine the progress of phosphoester hydrolysis. The 
increase in the absorbance at $400 \mathrm{~nm}$, assigned to the hydrolysis product 2,4-dinitrophenolate, was monitored over time and TON values were calculated using the Beer-Lambert Law.

\section{Phosphomonoester Hydrolysis Assays}

Phosphomonoester hydrolysis activity of the activated substrate DNPP was examined in a similar manner to the BDNPP assays, but DNPP was dissolved initially in aqueous buffer solution of the required $\mathrm{pH}$. During the $\mathrm{pH}$ dependence measurements the final concentrations in the cuvette were $0.04 \mathrm{mM}$ of complex and 0.5 mM in DNPP. Complex concentration dependent assays were $1 \mathrm{mM}$ in DNPP and the complex concentration ranged from 0.03 to $0.09 \mathrm{mM}$. In order to study the substrate concentration dependency the concentration of complex was fixed at $0.04 \mathrm{mM}$ and the DNPP concentration was varied between 0.1 and $1.5 \mathrm{mM}$. Turn-over experiment samples contained $0.01 \mathrm{mM}$ complex and $3 \mathrm{mM}$ DNPP.

\subsection{SYNTHESES OF PRECURSORS}

\subsubsection{2,6-BIS(CHLOROMETHYL)-4-METHYLPHENOL (22)}<smiles>Cc1cc(CO)c(O)c(CO)c1</smiles>

4

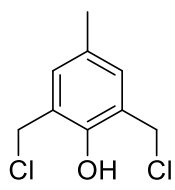

22

2,6-Bis(chloromethyl)-4-methylphenol (22) was prepared according to a known literature procedure. $^{310}$

2,6-bis(hydroxymethyl)-4-methylphenol (4) (2.5 g, $14.9 \mathrm{mmol})$ was treated with concentrated hydrochloric acid $(30 \mathrm{ml})$ and stirred at room temperature overnight. The product was extracted into dichloromethane $(3 \times 65 \mathrm{ml})$ and dried over sodium sulfate. Removal of the solvent gave 2,6-bis(chloromethyl)-4-methylphenol (22) in quantitative yield.

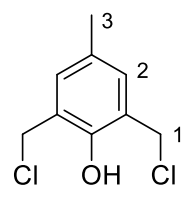

${ }^{1} \mathrm{H}$ NMR $\left(199.92 \mathrm{MHz}, \mathrm{CDCl}_{3}\right): \delta=2.29(\mathrm{~s}, 3 \mathrm{H}, \mathrm{H3}), 4.67(\mathrm{~s}, 4 \mathrm{H}, \mathrm{H} 1), 7.10(\mathrm{~s}, 2 \mathrm{H}, \mathrm{H} 2) \mathrm{ppm}$. 


\subsubsection{3-(CHLOROMETHYL)-2-HYDROXY-5-METHYLBENZALDEHYDE (5)}

\section{2-Hydroxy-3-(hydroxymethyl)-5-methylbenzaldehyde (1)}

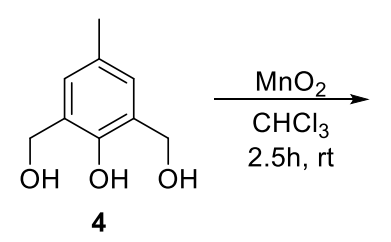<smiles>Cc1cc(C=O)c(O)c(CO)c1</smiles><smiles>Cc1cc(C=O)c(O)c(C=O)c1</smiles>

2-Hydroxy-3-(hydroxymethyl)-5-methylbenzaldehyde (1) was synthesized by modification of previously described procedures. ${ }^{120,121}$

To a solution of 2,6-bis(hydroxymethyl)-4-methylphenol (4) $(50.0 \mathrm{~g}, 0.30 \mathrm{~mol}, 1.0 \mathrm{eq}$ ) in chloroform (1.6 I) was added activated manganese oxide (191 g, $2.2 \mathrm{~mol}, 7.3 \mathrm{eq})$ in portions and the resulting suspension was stirred for 2.5 hours at room temperature (progress was monitored by $\left.\mathrm{TLC}\left(\mathrm{SiO}_{2} ; \mathrm{CH}_{2} \mathrm{Cl}_{2}: \mathrm{MeOH}=98: 2\right)\right)$. The manganese dioxide was removed via filtration through a pad of Celite $(\varnothing=3 \mathrm{~cm}, \mathrm{~L}=10 \mathrm{~cm})$. The filtrate was concentrated to a brownish solid, which contained compound $\mathbf{1}$ and compound 23. The mixture was separated by column chromatography $\left(\mathrm{SiO}_{2} ; \varnothing=5 \mathrm{~cm} ; \mathrm{L}=15 \mathrm{~cm} ; \mathrm{CH}_{2} \mathrm{Cl}_{2}: \mathrm{MeOH}=98: 2\right)$ yielding pure 2-hydroxy-3(hydroxymethyl)-5-methylbenzaldehyde (1) (23.1 g, 46\%) and 2-hydroxy-5-methylisophthalaldehyde (23) (12.8 g, $25 \%$ ), both as yellowish solids.

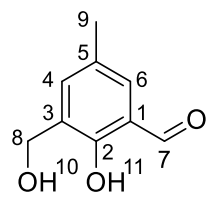

MS $\left(\mathrm{EI}^{+}\right.$, direct $): m / z=166.06\left(\left[\mathrm{C}_{9} \mathrm{H}_{10} \mathrm{O}_{3}\right]\right.$, calcd. 166.06).

${ }^{1} \mathrm{H}$ NMR $\left(600.13 \mathrm{MHz}, \mathrm{CDCl}_{3}\right): \delta=2.33(\mathrm{~s}, 3 \mathrm{H}, H 9), 2.44$ (bs, $\left.1 \mathrm{H}, H 10\right), 4.72(\mathrm{~s}, 2 \mathrm{H}, H 8), 7.28$ (s, $1 \mathrm{H}, H 6), 7.40(\mathrm{~s}, 1 \mathrm{H}, H 4), 9.85(\mathrm{~s}, 1 \mathrm{H}, H 7), 11.17(\mathrm{~s}, 1 \mathrm{H}, H 11) \mathrm{ppm}$.

${ }^{13}$ C NMR (150.90 MHz, $C D C l$ ): $\delta=20.2$ (C9), 60.5 (C8), 120.0 (C1), 129.0 (C3/C5), 129.1 (C3/C5), 132.6 (C4), 136.9 (C6), 157.3 (C2), 196.7 (C7) ppm.

Elemental analysis: Calcd. for $\mathrm{C}_{9} \mathrm{H}_{10} \mathrm{O}_{3}$ : C 65.05\%; $\mathrm{H} 6.07 \%$ Found: C $64.88 \%$; H $6.29 \%$.

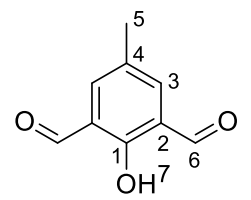

MS $\left(\mathrm{El}^{+}\right.$, direct $): m / z=164.04\left(\left[\mathrm{C}_{9} \mathrm{H}_{8} \mathrm{O}_{3}\right]\right.$, calcd. 164.05).

${ }^{1} \mathrm{H}$ NMR (200.13 MHz, CDCl 3 ): $\delta=2.40(\mathrm{~s}, 3 \mathrm{H}, H 5), 7.78(\mathrm{~s}, 2 \mathrm{H}, \mathrm{H3}), 10.23(\mathrm{~s}, 2 \mathrm{H}, H 6), 11.47(\mathrm{~s}$, $1 \mathrm{H}, H 7) \mathrm{ppm}$. 


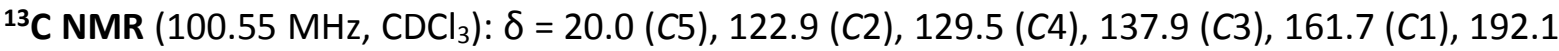
(C6) ppm.

\section{3-(Chloromethyl)-2-hydroxy-5-methylbenzaldehyde (5)}

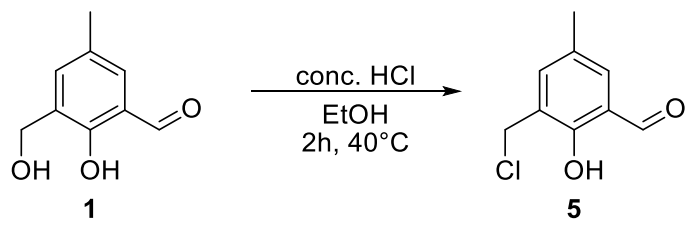

3-(Chloromethyl)-2-hydroxy-5-methylbenzaldehyde (5) was synthesized according to a known literature procedure. ${ }^{121}$

A solution of compound $1(2.50 \mathrm{~g}, 15 \mathrm{mmol})$ in ethanol $(12.5 \mathrm{ml})$ was added dropwise to warm concentrated hydrochloric acid $(37.5 \mathrm{ml})$ and the mixture was then heated at $40^{\circ} \mathrm{C}$ for two hours. The resulting precipitate was filtered and washed with water until a pH value of 8 was reached. The yellowish solid, 3-(chloromethyl)-2-hydroxy-5-methylbenzaldehyde (5), was dried under vacuum until dryness (1.85 g, 67\%).

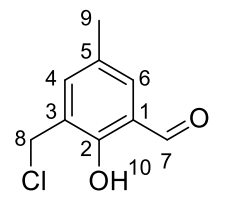

MS $\left(\mathrm{EI}^{+}\right.$, direct): $\mathrm{m} / \mathrm{z}=184.10\left(\left[\mathrm{C}_{9} \mathrm{H}_{9} \mathrm{O}_{2} \mathrm{Cl}\right]\right.$, calcd. 184.03).

${ }^{1} \mathrm{H} \operatorname{NMR}\left(200.13 \mathrm{MHz}, \mathrm{CDCl}_{3}\right): \delta=2.36(\mathrm{~s}, 3 \mathrm{H}, H 9), 4.68(\mathrm{~s}, 2 \mathrm{H}, H 8), 7.36(\mathrm{~s}, 1 \mathrm{H}, H 4), 7.47(\mathrm{~s}, 1 \mathrm{H}$, $H 6), 9.87(\mathrm{~s}, 1 \mathrm{H}, H 7), 11.26(\mathrm{~s}, 1 \mathrm{H}, H 10) \mathrm{ppm}$.

${ }^{13} \mathrm{C}$ NMR (50.32 MHz, $\left.\mathrm{CDCl}_{3}\right): \delta=20.2$ (C9), $39.8(C 8), 120.4(C 3), 125.8$ (C1/C6), 129.2 (C1/C6), 134.0 (C5), 139.0 (C4), 157.3 (C2), 196.4 (C7) ppm.

Elemental analysis: Calcd. for $\mathrm{C}_{9} \mathrm{H}_{9} \mathrm{O}_{2} \mathrm{Cl}$ : C $58.55 \% ; \mathrm{H} 4.91 \%$

Found: $\quad$ C $58.34 \%$; $5.18 \%$.

11.3.3 BIS(PYRIDIN-2-YLMETHYL)AMINE (2)

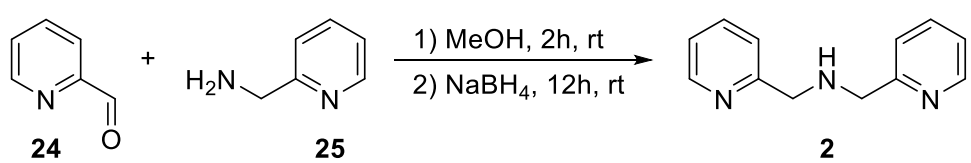

Bis(pyridine-2-ylmethyl)amine (2) was prepared according to known literature procedures. ${ }^{115}$ A solution of picolylaldehyde $(24)(9.50 \mathrm{ml}, 10.7 \mathrm{~g}, 0.10 \mathrm{~mol}, 1.0 \mathrm{eq})$ in methanol $(225 \mathrm{ml})$ was cooled with a water-ice bath and 2-(aminomethyl)pyridine (25) (10.3 ml, $10.8 \mathrm{~g}, 0.10 \mathrm{~mol}, 1.0 \mathrm{eq})$ was added dropwise. Subsequently, the reaction mixture was allowed to warm up to room 
temperature. After stirring the solution for two hours the mixture was again cooled with a waterice bath before sodium borohydride $(7.57 \mathrm{~g}, 0.20 \mathrm{~mol}, 2.0 \mathrm{eq})$ was added in portions. The reaction mixture was stirred overnight at room temperature. After the solution was concentrated under reduced pressure, the residue was treated with water $(250 \mathrm{ml})$ and neutralized with $2 \mathrm{M}$ hydrochloric acid. The product was extracted into dichloromethane $(3 \times 75 \mathrm{ml})$, washed with brine and dried over sodium sulfate. The solvent was removed under reduced pressure to give bis(pyridine-2-ylmethyl)amine (2) as a brown oil (12.5 g, 63\%).

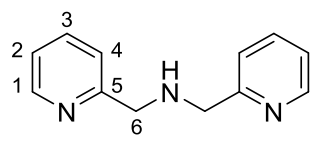

Molecular Formula: $\mathrm{C}_{12} \mathrm{H}_{13} \mathrm{~N}_{3}$

${ }^{1} \mathrm{H}$ NMR $\left(200.13 \mathrm{MHz}, \mathrm{CDCl}_{3}\right): \delta=3.26(\mathrm{~s}, 1 \mathrm{H}, \mathrm{NH}), 4.03(\mathrm{~s}, 4 \mathrm{H}, H 6), 7.17$ (dd, ${ }^{3} \mathrm{~J}=7.45 \mathrm{~Hz}$, $\left.{ }^{3} \mathrm{~J}=4.93 \mathrm{~Hz}, 2 \mathrm{H}, \mathrm{H2}\right), 7.39\left(\mathrm{~d},{ }^{3} \mathrm{~J}=7.83 \mathrm{~Hz}, 2 \mathrm{H}, H 4\right), 7.66\left(\mathrm{td},{ }^{3} \mathrm{~J}=7.46 \mathrm{~Hz},{ }^{4} \mathrm{~J}=1.79 \mathrm{~Hz}, 2 \mathrm{H}, H 3\right), 8.59$ (d, ${ }^{3} \mathrm{~J}=4.93 \mathrm{~Hz}, 2 \mathrm{H}, \mathrm{H1}$ ) ppm.

${ }^{13} \mathrm{C}$ NMR (50.32 MHz, $\left.\mathrm{CDCl}_{3}\right): \delta=54.0(C 6), 121.6$ (C2/C4), 121.9 (C2/C4), 135.9 (C3), $148.8(C 1)$, 158.7 (C5) ppm.

\subsubsection{2-(((PYRIDIN-2-YLMETHYL)AMINO)METHYL)PHENOL (16)}<smiles>O=Cc1ccccc1O</smiles>

26<smiles>NCc1ccccn1</smiles>

25<smiles>Oc1ccccc1CNCc1ccccn1</smiles>

16

2-(((Pyridine-2-ylmethyl)amino)methyl)phenol (16) was prepared according to known literature procedures. ${ }^{124,125}$

Salicylaldehyde (26) (12.2 g, $0.10 \mathrm{~mol}, 1.0 \mathrm{eq})$ was dissolved in methanol $(225.0 \mathrm{ml})$ and the solution was cooled in a water-ice bath. 2-(Aminomethyl)pyridine (25) (10.4 ml, 10.8 g, $0.10 \mathrm{~mol}$, 1.0 eq) was added dropwise and the mixture was stirred for two hours at room temperature. Subsequently, sodium borohydride $(7.57 \mathrm{~g}, 0.20 \mathrm{~mol}, 2.0 \mathrm{eq})$ was added in small portions while the solution was cooled in a water-ice bath. After completion of the addition, the reaction mixture was stirred overnight at room temperature. The solution was concentrated under reduced pressure and the residue was treated with water $(250 \mathrm{ml})$ and neutralized with $2 \mathrm{M}$ hydrochloric acid. The product was extracted into dichloromethane $(3 \times 75 \mathrm{ml})$, washed with brine and dried over sodium sulfate. Removal of the solvent yielded 2-(((pyridine-2-ylmethyl)amino)methyl)phenol (16) as a brown oil (21.2 g, 99\%). 


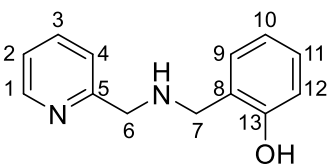

HRMS (ESI $\left.{ }^{+}, \mathrm{CH}_{3} \mathrm{OH}\right): m / z=215.1179\left(\left[\mathrm{C}_{13} \mathrm{H}_{15} \mathrm{~N}_{2} \mathrm{O}\right]^{+}\right.$, calcd. 215.1179).

${ }^{1} \mathrm{H}$ NMR (399.89 MHz, CDCl 3$): \delta=3.92(\mathrm{~s}, 2 \mathrm{H}, H 7), 4.00(\mathrm{~s}, 2 \mathrm{H}, H 6), 6.78\left(\mathrm{t},{ }^{3} \mathrm{~J}=7.40 \mathrm{~Hz}, 1 \mathrm{H}, H 10\right)$, $6.87\left(\mathrm{~d},{ }^{3} \mathrm{~J}=8.03 \mathrm{~Hz}, 1 \mathrm{H}, H 12\right), 6.98\left(\mathrm{~d},{ }^{3} \mathrm{~J}=7.28 \mathrm{~Hz}, 1 \mathrm{H}, H 9\right), 7.15-7.24(\mathrm{~m}, 3 \mathrm{H}, H 2, H 4, H 11), 7.66$ $\left(\mathrm{td},{ }^{3} \mathrm{~J}=7.62 \mathrm{~Hz},{ }^{4} \mathrm{~J}=1.19 \mathrm{~Hz}, 1 \mathrm{H}, H 3\right), 8.58\left(\mathrm{~d},{ }^{3} \mathrm{~J}=3.76 \mathrm{~Hz}, 1 \mathrm{H}, H 1\right) \mathrm{ppm}$.

${ }^{13} \mathrm{C}$ NMR (100.55 MHz, $\left.\mathrm{CDCl}_{3}\right): \delta=51.7$ (C7), 52.9 (C6), 116.3 (C12), $119.0(C 10), 122.3(C 8), 122.4$ (C2), 122.6 (C4), 128.6 (C11), 128.7 (C9), 136.6 (C3), 149.3 (C1), 157.6 (C13), 158.1 (C5) ppm.

\subsubsection{2-(AMINOMETHYL)PHENOL (20)}

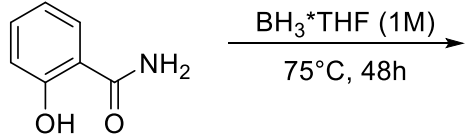

27<smiles>NCc1ccccc1O</smiles>

20

2-(Aminomethyl)phenol (20) was prepared according to published procedures with minor modifications. $^{126}$

Salicylamide (27) $(2.68 \mathrm{~g}, 19.5 \mathrm{mmol}, 1.0 \mathrm{eq})$ was treated with $1 \mathrm{M}$ borane tetrahydrofuran complex solution $(100 \mathrm{ml}, 100 \mathrm{mmol}, 5.1 \mathrm{eq})$ under argon atmosphere and heated to $75^{\circ} \mathrm{C}$ for two days. After the mixture cooled to room temperature the reaction was quenched via dropwise addition of methanol. The solvent was removed under reduced pressure. The remaining tetrahydrofuran was stripped by addition of methanol $(2 \times 25 \mathrm{ml})$ followed by removal under vacuum to obtain 2-(aminomethyl)phenol (20) as a white solid in quantitative yield.

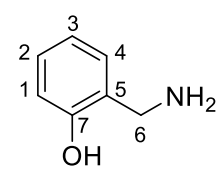

HRMS (El ${ }^{+}$, direct): $m / z=123.0697\left(\left[\mathrm{C}_{7} \mathrm{H}_{9} \mathrm{NO}\right]\right.$, calcd. 123.0684).

${ }^{1} \mathrm{H}$ NMR $\left(600.13 \mathrm{MHz}, \mathrm{CDCl}_{3}\right): \delta=4.13(\mathrm{~s}, 2 \mathrm{H}, H 6), 6.79\left(\mathrm{td},{ }^{3} \mathrm{~J}=7.42 \mathrm{~Hz},{ }^{4} \mathrm{~J}=1.11 \mathrm{~Hz}, 1 \mathrm{H}, H 3\right), 6.86$ $\left(\mathrm{dd},{ }^{3} \mathrm{~J}=8.07 \mathrm{~Hz},{ }^{4} \mathrm{~J}=0.91 \mathrm{~Hz}, 1 \mathrm{H}, H 1\right), 6.98\left(\mathrm{dt},{ }^{3} \mathrm{~J}=7.44 \mathrm{~Hz},{ }^{4} \mathrm{~J}=0.62 \mathrm{~Hz}, 1 \mathrm{H}, H 4\right), 7.18(\mathrm{td}$, $\left.{ }^{3} \mathrm{~J}=7.72 \mathrm{~Hz},{ }^{4} \mathrm{~J}=1.61 \mathrm{~Hz}, 1 \mathrm{H}, H 2\right) \mathrm{ppm}$.

${ }^{13} \mathrm{C}$ NMR (150.90 MHz, $\left.\mathrm{CDCl}_{3}\right): \delta=45.4$ (C6), 116.7 (C1), 119.0 (C3), 123.9 (C5), 127.9 (C4), 128.7 (C2), 158.3 (C7) ppm. 


\subsubsection{N-(6-PIVALOYLAMIDO-2-PYRIDYLMETHYL)-N-(2-PYRIDYL- METHYL)AMIN (18)}

\section{$N$-(6-methylpyridin-2-yl)pivalamide (9)}

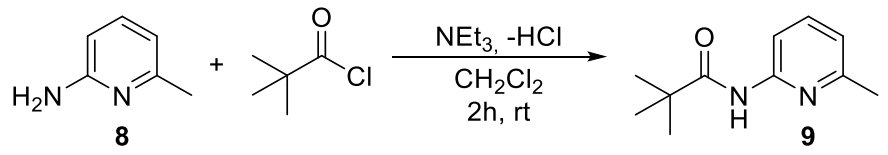

$\mathrm{N}$-(6-methylpyridin-2-yl)pivalamide (9) was prepared by slight modification of a previously described procedure. ${ }^{116}$

A solution of 2-amino-6-methylpyridine (8) $(54.1 \mathrm{~g}, 0.50 \mathrm{~mol}, 1.0 \mathrm{eq})$ in dry dichloromethane $(500 \mathrm{ml})$ was treated with triethylamine $(90.1 \mathrm{ml}, 65.8 \mathrm{~g}, 0.65 \mathrm{~mol}, 1.3 \mathrm{eq})$ and cooled to $0^{\circ} \mathrm{C}$ with an ice-water bath. Pivaloylchloride $(67.2 \mathrm{ml}, 66.3 \mathrm{~g}, 0.55 \mathrm{~mol}, 1.1 \mathrm{eq})$ was then added dropwise to the solution. The resulting mixture was warmed to room temperature and stirred for two hours. Afterwards, the reaction was quenched by addition of water $(100 \mathrm{ml})$. The phases were separated and the aqueous phase was extracted with dichloromethane $(2 \times 50 \mathrm{ml})$. The combined organic phases were dried over sodium sulfate and concentrated under reduced pressure. The yellowish solid was recrystallized three times from diethylether to give $N$-(6-methylpyridin-2-yl)pivalamide (9) as white crystals (95.6 g, 99\%).

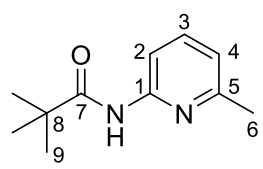

Molecular Formula: $\mathrm{C}_{11} \mathrm{H}_{16} \mathrm{~N}_{2} \mathrm{O}$

MS (El+, direct): $m / z=192.12\left(\left[\mathrm{C}_{11} \mathrm{H}_{16} \mathrm{~N}_{2} \mathrm{O}\right]\right.$, calcd. 192.13).

${ }^{1} \mathrm{H}$ NMR (199.92 MHz, CDCl 3 ): $\delta=1.29(\mathrm{~s}, 9 \mathrm{H}, H 9), 2.42(\mathrm{~s}, 3 \mathrm{H}, H 6), 6.84\left(\mathrm{~d},{ }^{3} \mathrm{~J}=7.45 \mathrm{~Hz}, 1 \mathrm{H}, H 2\right)$, $7.54\left(\mathrm{t},{ }^{3} \mathrm{~J}=7.89 \mathrm{~Hz}, 1 \mathrm{H}, H 3\right), 7.90(\mathrm{bs}, 1 \mathrm{H}, \mathrm{NH}), 8.02\left(\mathrm{~d},{ }^{3} \mathrm{~J}=8.21 \mathrm{~Hz}, 1 \mathrm{H}, H 2\right) \mathrm{ppm}$.

${ }^{13} \mathrm{C}$ NMR (100.55 MHz, $\left.\mathrm{CDCl}_{3}\right): \delta=24.0$ (C6), 27.5 (C9), 39.8 (C8), 110.7 (C2), 119.1 (C3), 138.6 (C4), 150.9 (C1), 156.7 (C5), 177.0 (C7) ppm.

Elemental analysis: Calcd. for $\mathrm{C}_{11} \mathrm{H}_{16} \mathrm{~N}_{2} \mathrm{O}$ : C 68.72\%; $\mathrm{H}$ 8.39\%; $\mathrm{N} 14.57 \%$

Found:

C $68.75 \%$; H $8.69 \%$; N $14.64 \%$. 


\section{$N$-(6-(bromomethyl)pyridin-2-yl)pivalamide (10) and $N$-(6-(dibromomethyl)- pyridin-2-yl)pivalamide (13)}<smiles>Cc1cccc(NC(=O)C(C)(C)C)n1</smiles>

9

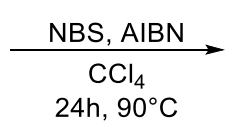

$24 \mathrm{~h}, 90^{\circ} \mathrm{C}$

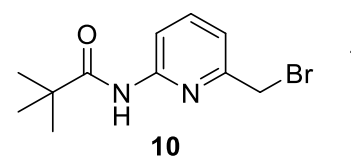

10
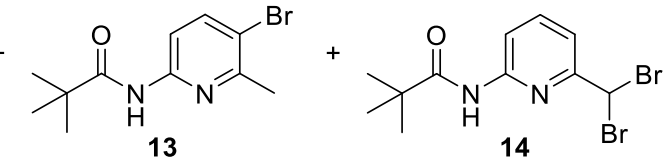

$\mathrm{N}$-(6-bromomethyl)pyridine-2-yl)pivalamide (10) was synthesized by modification of previously published procedures. ${ }^{117-119}$

$N$-(6-methylpyridin-2-yl)pivalamide (9) (50.0 g, $0.26 \mathrm{~mol}, 1.0 \mathrm{eq})$ was dissolved under argon atmosphere in anhydrous carbon tetrachloride $(500 \mathrm{ml})$ and was warmed to $70^{\circ} \mathrm{C}$. $\mathrm{N}$-bromosuccinimid (15.8 g) and a catalytic amount of AIBN was added and the mixture was heated for two hours with stirring while irradiated with a 150W UV lamp. Another portion of $\mathrm{N}$-bromosuccinimide $(15.8 \mathrm{~g})$ and catalytic amount of AIBN were added. The additions were repeated every hour; in total seven additions of NBS (111 g, $0.62 \mathrm{mmol}, 2.4 \mathrm{eq})$ were made. The mixture was then refluxed overnight. After cooling to room temperature the succinimide obtained was filtered and the filtrate concentrated under reduced pressure. The remaining brown oil was taken up in chloroform $(400 \mathrm{ml})$, washed with water $(3 \times 300 \mathrm{ml})$, brine $(300 \mathrm{ml})$ and dried over sodium sulfate. Removal of the solvent gave an orange oil containing a mixture of compound 9, the desired products, 10 and 14, and a ring-brominated species 13 . Column chromatography $\left(\mathrm{SiO}_{2} ;\right.$ Hexan:EtOAc $\left.=4: 1 ; \mathrm{R}_{\mathrm{f}}=0.38\right)$ yielded a fraction of pure compound 10 next to a mixed fraction of compound 10 , compound 13 and compound $14\left(R_{f}=0.46\right)$. The latter was separated by column chromatography $\left(\mathrm{SiO}_{2} ;\right.$ Hexane:EtOAc $\left.=10: 1 ; \mathrm{R}_{\mathrm{f}}=0.38\right)$. Ultra sonication of the orange oil obtained, treated with a small amount of hexane, yielded $\mathrm{N}$-(6-bromomethyl)pyridine-2-yl)pivalamide (10) as a white solid (30.8 g, 44\%). N-(6-(dibromomethyl)pyridin-2-yl)pivalamide (14) could not be isolated and was used in form of a mixture with the ring-brominated derivate 13 in the following reaction, after which the products were separated.

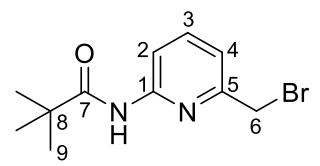

HRMS $\left(\mathrm{ESI}^{+}, \mathrm{CH}_{3} \mathrm{OH}\right): \mathrm{m} / \mathrm{z}=273.0421\left(\left[\mathrm{C}_{11} \mathrm{H}_{16} \mathrm{~N}_{2} \mathrm{OBr}\right]^{+}\right.$, calcd. 273.0419).

${ }^{1}$ H NMR $\left(600.13 \mathrm{MHz}, \mathrm{CDCl}_{3}\right): \delta=1.34(\mathrm{~s}, 9 \mathrm{H}, \mathrm{H9}), 4.43(\mathrm{~s}, 2 \mathrm{H}, \mathrm{H6}), 7.15\left(\mathrm{~d},{ }^{3} \mathrm{~J}=7.47 \mathrm{~Hz}, 1 \mathrm{H}, H 4\right)$, $7.70\left(\mathrm{t},{ }^{3} \mathrm{~J}=7.92 \mathrm{~Hz}, 1 \mathrm{H}, H 3\right), 8.02(\mathrm{bs}, 1 \mathrm{H}, \mathrm{NH}), 8.19\left(\mathrm{~d},{ }^{3} \mathrm{~J}=8.38 \mathrm{~Hz}, 1 \mathrm{H}, \mathrm{H} 2\right) \mathrm{ppm}$.

${ }^{13} \mathrm{C}$ NMR (150.90 MHz, $\mathrm{CDCl}_{3}$ ): $\delta=27.5$ (C9), $33.4(C 6), 39.8(C 8), 113.3$ (C2), $119.2(C 4), 139.4$ (C3), 151.3 (C1), 154.8 (C5), 177.2 (C7) ppm. 


\section{$N$-(6-pivaloylamido-2-pyridylmethyl)-N-(2-pyridylmethyl)amin (18)}

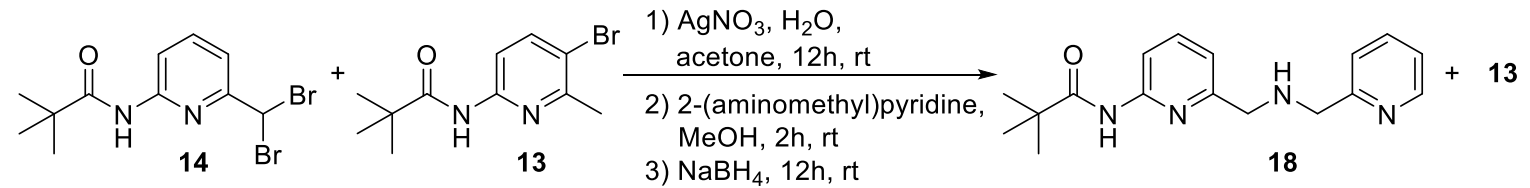

$N$-(6-formylpyridin-2-yl)pivalamide (26) and $N$-(6-pivaloylamido-2-pyridylmethyl)- $N$-(2-pyridylmethyl)amin (18) were synthesized following literature known procedures. ${ }^{114,} 234$

Compound 14 (7.68 g, $21.9 \mathrm{mmol}, 1.0 \mathrm{eq})$, in the form of a $32 \mathrm{w} \%$ mixture with compound 13, was dissolved in acetone $(210 \mathrm{ml})$ and the flask was covered with aluminium foil. Silver nitrate (33.6 g, $200 \mathrm{mmol}, 9.0 \mathrm{eq})$ in water $(18 \mathrm{ml})$ was added and the mixture stirred at room temperature overnight in the dark. The obtained precipitate was filtered and washed with dichloromethane. The yellow filtrate was concentrated to a brown oil, which was subsequently taken up in dichloromethane $(300 \mathrm{ml})$, washed with water $(100 \mathrm{ml}), 0.5 \mathrm{~N}$ sodium hydrogencarbonate solution $(100 \mathrm{ml})$, brine $(100 \mathrm{ml})$ and dried over sodium sulfate. The successful conversion of $\mathrm{N}$-(6-(dibromomethyl)pyridin-2-yl)pivalamide (14) to $\mathrm{N}$-(6-formylpyridin-2-yl)pivalamide (26) was controlled via ${ }^{1} \mathrm{H}$ NMR spectroscopy.

In the next step the $33 \mathrm{w} \%$ mixture of $N$-(6-formylpyridin-2-yl)pivalamide (26) (3.26 g, $15.8 \mathrm{mmol}$, 1.0eq) with $N$-(5-bromo-6-methylpyridin-2-yl)pivalamide (13) were dissolved in methanol (64 ml). Then, 2-(aminomethyl)pyridine $(1.78 \mathrm{ml}, 1.87 \mathrm{~g}, 17.3 \mathrm{mmol}, 1.1 \mathrm{eq})$ was added dropwise and the reaction mixture was stirred for 2.5 hours at room temperature. While cooling in an icewater bath sodium borohydride $(1.10 \mathrm{~g}, 29.0 \mathrm{mmol}, 1.8 \mathrm{eq})$ was added in small portions and the mixture was stirred at room temperature overnight. The reaction was quenched by addition of water $(70 \mathrm{ml})$ and the solution concentrated under reduced pressure. Water $(20 \mathrm{ml})$ was added to the remaining oil and extracted with ethyl acetate. Removal of the solvent gave a brown oil, which was loaded onto a silica column. Elution with ethyl acetate removed the side products before the desired product $N$-(6-pivaloylamido-2-pyridylmethyl)- $N$-(2-pyridylmethyl)amin (18) was isolated by elution with methanol ( $4.48 \mathrm{~g}, 95 \%)$.

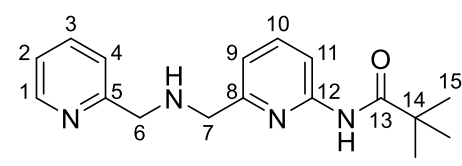

HRMS $\left(\mathrm{ESI}^{+}, \mathrm{CH}_{3} \mathrm{OH}\right): \mathrm{m} / \mathrm{z}=299.1863\left(\left[\mathrm{C}_{17} \mathrm{H}_{23} \mathrm{~N}_{4} \mathrm{O}\right]^{+}\right.$, calcd. 299.1866).

${ }^{1} \mathrm{H}$ NMR $\left(399.89 \mathrm{MHz}, \mathrm{CDCl}_{3}\right): \delta=1.31(\mathrm{~s}, 9 \mathrm{H}, H 15), 2.73\left(\mathrm{bs}, 1 \mathrm{H}, \mathrm{N}_{\mathrm{amin}} H\right), 3.85(\mathrm{~s}, 2 \mathrm{H}, H 7), 3.95$ (s, 2H, H6), $7.03\left(\mathrm{~d},{ }^{3} \mathrm{~J}=7.53 \mathrm{~Hz}, 1 \mathrm{H}, H 9\right), 7.15\left(\mathrm{dd},{ }^{3} \mathrm{~J}=7.40 \mathrm{~Hz},{ }^{3} \mathrm{~J}=4.64 \mathrm{~Hz}, 1 \mathrm{H}, H 2\right), 7.32$ (d, 
$\left.{ }^{3} \mathrm{~J}=7.78 \mathrm{~Hz}, 1 \mathrm{H}, H 4\right), 7.63\left(\mathrm{td},{ }^{3} \mathrm{~J}=7.75 \mathrm{~Hz},{ }^{4} \mathrm{~J}=1.97 \mathrm{~Hz}, 2 \mathrm{H}, H 3, H 10\right), 8.03\left(\mathrm{bs}, 1 \mathrm{H}, \mathrm{N}_{\mathrm{amid}} H\right), 8.10$ (d, $\left.{ }^{3} \mathrm{~J}=8.28 \mathrm{~Hz}, 1 \mathrm{H}, H 11\right), 8.55\left(\mathrm{~d},{ }^{3} \mathrm{~J}=4.64 \mathrm{~Hz}, 1 \mathrm{H}, H 1\right) \mathrm{ppm}$.

${ }^{13}$ C NMR (100.55 MHz, CDCl $): \delta=27.4$ (C15), 39.7 (C14), 54.2 (C7), 54.6 (C6), 112.0 (C11), 118.0 (C9), 122.0 (C2), 122.3 (C4), 136.4 (C3), 138.7 (C10), 149.2 (C1), 151.2 (C12), 157.7 (C8), 159.4 (C5), $177.0(C 13)$ ppm.

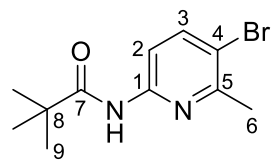

HRMS (EI ${ }^{+}$, direct): $\mathrm{m} / \mathrm{z}=270.0374\left(\left[\mathrm{C}_{11} \mathrm{H}_{15} \mathrm{~N}_{2} \mathrm{OBr}\right]\right.$, calcd. 270.0368)

${ }^{1} \mathrm{H}$ NMR (600.13 MHz, CDCl$): ~ \delta=1.31(\mathrm{~s}, 9 \mathrm{H}, \mathrm{H9}), 2.54(\mathrm{~s}, 3 \mathrm{H}, \mathrm{H6}), 7.75\left(\mathrm{~d},{ }^{3} \mathrm{~J}=8.68 \mathrm{~Hz}, 1 \mathrm{H}, \mathrm{H3}\right)$, $7.97\left(\mathrm{~d},{ }^{3} \mathrm{~J}=8.73,2 \mathrm{H}, \mathrm{NH}, \mathrm{H} 2\right) \mathrm{ppm}$.

${ }^{13} \mathrm{C}$ NMR (100.55 MHz, CDCl $)$ ): $\delta=24.4$ (C6), 27.4 (C9), 39.8 (C8), 112.5 (C2), 114.9 (C4), 141.8 (C3), 149.7 (C1), 155.2 (C5), 177.0 (C7) ppm.

\subsubsection{N,N-BIS((2-PIVALOYLAMIDOPYRIDIN-6-YL)METHYL)AMIN (3)}

\section{$N, N$-bis((2-pivaloylamidopyridin-6-yl)methyl)(2-nitrobenzene)sulfonamid}

(11)

2

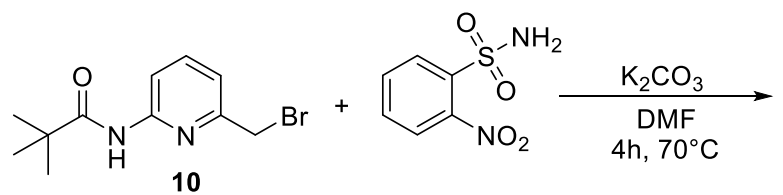

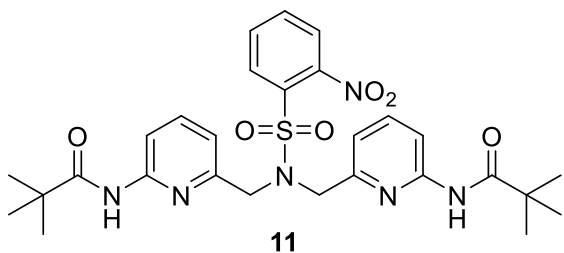

11

$\mathrm{N}, \mathrm{N}$-bis((2-pivaloylamidopyridin-6-yl)methyl)(2-nitrobenzene)sulfonamide (11) was prepared according to a previously described procedure. ${ }^{119}$

Compound 10 (4.90 g, $18.1 \mathrm{mmol}, 2.1 \mathrm{eq})$ was dissolved in dimethylformamide $(86 \mathrm{ml})$ and then treated with potassium carbonate $(2.97 \mathrm{~g}, 21.5 \mathrm{mmol}, 2.5 \mathrm{eq})$ and 2-nitrobenzoyl-sulfonamide $(1.74 \mathrm{~g}, 8.61 \mathrm{mmol}, 1.0 \mathrm{eq})$. The resulting solution was heated at $70^{\circ} \mathrm{C}$ for 4 hours. After cooling to room temperature the reaction mixture was poured onto a saturated sodium bicarbonate solution $(90 \mathrm{ml})$. Afterwards the phases were separated and the aqueous phase was extracted with ethyl acetate $(5 \times 50 \mathrm{ml})$. Then the combined organic phases were washed with half saturated sodium bicarbonate solution $(4 \times 25 \mathrm{ml})$ before being dried over sodium sulfate. Removal of the solvent under reduced pressure gave a brown oil which was purified by column chromatography $\left(\mathrm{SiO}_{2} ; \varnothing=6 \mathrm{~cm} ; \mathrm{L}=23 \mathrm{~cm}\right.$; hexane:EtOAc = 5:4; $\left.\mathrm{R}_{\mathrm{f}}=0.17\right) . \mathrm{N}, \mathrm{N}$-bis $((2$-pivaloylamidopyridin-6-yl)methyl)(2-nitrobenzene)sulfonamide (11) was obtained as a yellowish solid (4.91 g, 98\%). 


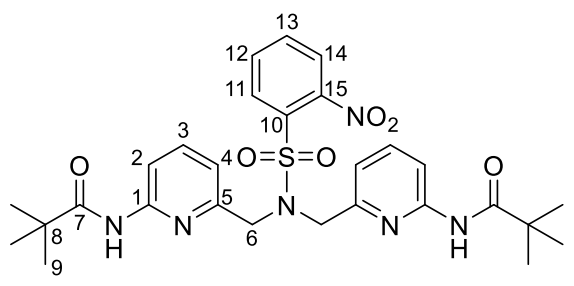

HRMS $\left(\mathrm{ESI}^{+}, \mathrm{CH}_{3} \mathrm{OH}\right): \mathrm{m} / \mathrm{z}=583.2331\left(\left[\mathrm{C}_{28} \mathrm{H}_{35} \mathrm{~N}_{6} \mathrm{O}_{6} \mathrm{~S}\right]^{+}\right.$, calcd. 583.2333).

${ }^{1}{ }^{H}$ NMR $\left(600.13 \mathrm{MHz}, \mathrm{CDCl}_{3}\right): \delta=1.32(\mathrm{~s}, 18 \mathrm{H}, H 9), 4.61(\mathrm{~s}, 4 \mathrm{H}, H 6), 6.96\left(\mathrm{~d},{ }^{3} \mathrm{~J}=7.47 \mathrm{~Hz}, 2 \mathrm{H}\right.$, $H 4), 7.53\left(\mathrm{td},{ }^{3} \mathrm{~J}=7.67 \mathrm{~Hz},{ }^{4} \mathrm{~J}=1.31 \mathrm{~Hz}, 2 \mathrm{H}, H 13\right), 7.59\left(\mathrm{t},{ }^{3} \mathrm{~J}=7.87 \mathrm{~Hz}, 2 \mathrm{H}, H 3\right), 7.64\left(\mathrm{td},{ }^{3} \mathrm{~J}=7.69 \mathrm{~Hz}\right.$, $\left.{ }^{4} \mathrm{~J}=1.26 \mathrm{~Hz}, 1 \mathrm{H}, H 12\right), 7.69\left(\mathrm{dd},{ }^{3} \mathrm{~J}=7.98 \mathrm{~Hz},{ }^{4} \mathrm{~J}=1.22,1 \mathrm{H}, H 14\right), 7.89$ (bs, $\left.2 \mathrm{H}, \mathrm{NH}\right), 7.98$ (dd, $\left.{ }^{3} \mathrm{~J}=7.97 \mathrm{~Hz},{ }^{4} \mathrm{~J}=1.11 \mathrm{~Hz}, 1 \mathrm{H}, H 11\right), 8.07\left(\mathrm{~d},{ }^{3} \mathrm{~J}=8.28 \mathrm{~Hz}, 2 \mathrm{H}, \mathrm{H} 2\right) \mathrm{ppm}$.

${ }^{13} \mathrm{C}$ NMR (150.90 MHz, $\left.\mathrm{CDCl}_{3}\right): \delta=27.1$ (C9), 39.8 (C8), 52.6 (C6), 112.6 (C2), 117.8 (C4), 124.1 (C14), 131.4 (C11), 131.7 (C13), 133.4 (C12), 134.2 (C10), 139.1 (C3), 147.6 (C15), 151.2 (C1), 153.7 (C5), 177.1 (C7) ppm.

Elemental analysis: $\quad$ Calcd. for $\mathrm{C}_{28} \mathrm{H}_{34} \mathrm{~N}_{6} \mathrm{O}_{6} \mathrm{~S} .0 .3 \mathrm{C}_{4} \mathrm{H}_{8} \mathrm{O}_{2}$ : $\quad$ C 57.58\%; $\mathrm{H}$ 6.02\%; N $13.80 \%$ Found:

\section{N,N-bis((2-pivaloylamidopyridin-6-yl)methyl)amin (3)}

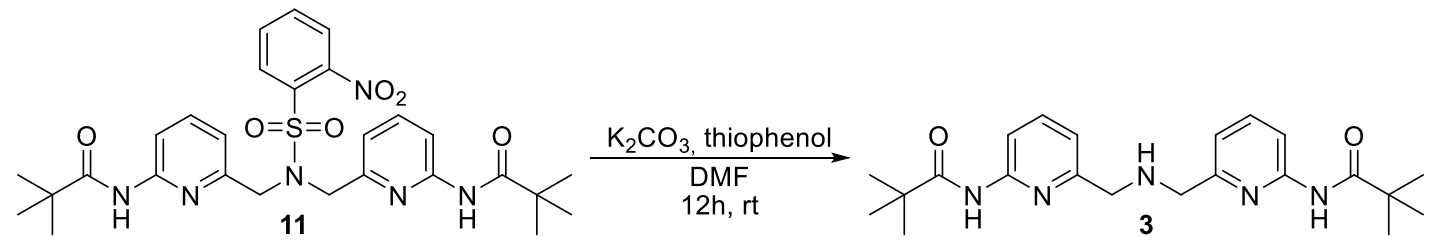

$\mathrm{N}, \mathrm{N}$-bis((2-pivaloylamidopyridin-6-yl)methyl)amin (3) was synthesized following a previously published procedure. ${ }^{119}$

A solution of compound $11(16.0 \mathrm{~g}, 27.4 \mathrm{mmol}, 1.0 \mathrm{eq})$ in anhydrous dimethylformamide (274 ml) was treated with potassium carbonate $(5.70 \mathrm{~g}, 41.1 \mathrm{mmol}, 1.5 \mathrm{eq})$ and thiophenol $(3.10 \mathrm{ml}$, $30.1 \mathrm{mmol}, 1.1 \mathrm{eq}$ ) before the reaction mixture was left stirring at room temperature overnight. Then, the reaction was quenched via decantation into a saturated sodium bicarbonate solution. Subsequently, the product was extracted into ethyl acetate, washed with half saturated sodium bicarbonate solution and dried over sodium sulfate. Removal of the solvent gave a crude product, which was purified by column chromatography $\left(\mathrm{SiO}_{2} ; \mathrm{CH}_{2} \mathrm{Cl}_{2}: \mathrm{MeOH}: \mathrm{NEt}_{3}=980: 14: 8 ; \mathrm{R}_{\mathrm{f}}=0.12\right)$ yielding $N, N$-bis((2-pivaloylamidopyridin-6-yl)methyl)amin (3) as a yellowish solid (8.78 g, $81 \%)$.

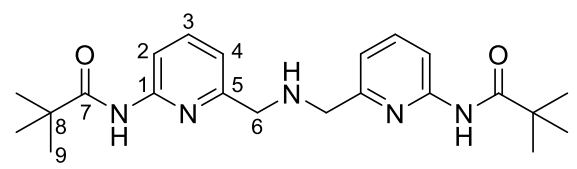

HRMS $\left(\mathrm{ESI}^{+}, \mathrm{CH}_{3} \mathrm{OH}\right): \mathrm{m} / \mathrm{z}=398.2546\left(\left[\mathrm{C}_{22} \mathrm{H}_{32} \mathrm{~N}_{5} \mathrm{O}_{2}\right]^{+}\right.$, calcd. 398.2551). 
${ }^{1} \mathrm{H}$ NMR $\left(600.13 \mathrm{MHz}, \mathrm{CDCl}_{3}\right): \delta=1.30(\mathrm{~s}, 18 \mathrm{H}, \mathrm{H9}), 3.85(\mathrm{~s}, 4 \mathrm{H}, \mathrm{H6}), 7.01\left(\mathrm{~d},{ }^{3} \mathrm{~J}=7.53 \mathrm{~Hz}, 2 \mathrm{H}\right.$, H4), $7.63\left(\mathrm{t},{ }^{3} \mathrm{~J}=7.84 \mathrm{~Hz}, 2 \mathrm{H}, H 3\right), 8.05$ (bs, $\left.2 \mathrm{H}, \mathrm{NH}\right), 8.10$ (d, ${ }^{3} \mathrm{~J}=8.28 \mathrm{~Hz}, 2 \mathrm{H}, \mathrm{H2}$ ) ppm.

${ }^{13} \mathrm{C}$ NMR (100.55 MHz, CDCl $)$ ): $\delta=27.4$ (C9), 39.7 (C8), 54.0 (C6), 112.0 (C2), 118.0 (C4), 138.7 (C3), $151.2(C 1), 157.4$ (C5), 177.0 (C7) ppm.

\subsection{SYNTHESIS OF LIGAND $\mathrm{H}_{3} \mathrm{~L}^{2}$}

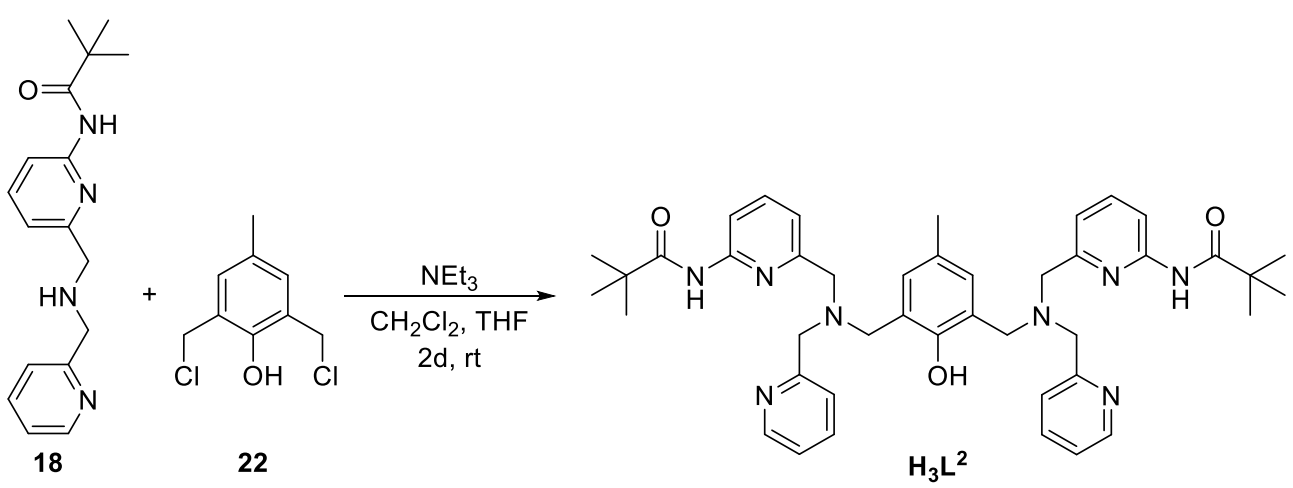

Ligand $\mathrm{H}_{3} \mathrm{~L}^{2}$ was synthesized following a literature known procedure. ${ }^{114}$

A mixture of compound $18(2.57 \mathrm{~g}, 8.61 \mathrm{mmol}, 2.0 \mathrm{eq})$ and triethylamine $(1.20 \mathrm{ml}, 0.86 \mathrm{~g}$, $8.61 \mathrm{mmol}, 2.0 \mathrm{eq})$ in anhydrous tetrahydrofuran $(7.75 \mathrm{ml}$ ) was added dropwise to a cold suspension of 2,6-bis(chloromethyl)-4-methylphenol (22) (0.89 g, $4.34 \mathrm{mmol}, 1.0 \mathrm{eq})$ in anhydrous dichloromethane $(6.90 \mathrm{ml})$ at $0^{\circ} \mathrm{C}$. The reaction mixture was allowed to stir for two days at room temperature and filtered. The filtrate was concentrated under reduced pressure and the remaining residue was taken up in dichloromethane $(100 \mathrm{ml})$, washed with brine $(50 \mathrm{ml})$ and dried over sodium sulfate. Removal of solvent gave a crude product, which was purified via column chromatography $\left(\mathrm{SiO}_{2} ; \mathrm{DEE}: \mathrm{NH}_{3}(7 \mathrm{~N}\right.$ in $\left.\mathrm{MeOH})=98: 2 ; \mathrm{R}_{\mathrm{f}}=0.22\right)$ yielding the desired ligand $\mathrm{H}_{3} \mathrm{~L}^{2}$ as a white solid (1.14 g, 36\%).

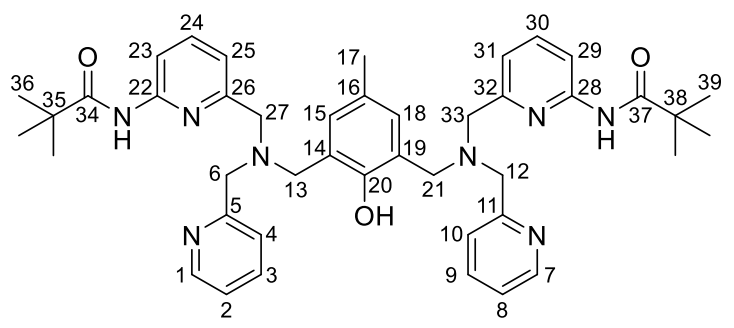

HRMS $\left(\mathrm{ESI}^{+}, \mathrm{CH}_{3} \mathrm{CN}\right): m / z=729.4235\left(\left[\mathrm{C}_{43} \mathrm{H}_{53} \mathrm{~N}_{8} \mathrm{O}_{3}\right]^{+}\right.$, calcd. 729.4235).

${ }^{1} \mathrm{H}$ NMR $\left(500.13 \mathrm{MHz}, \mathrm{CD}_{3} \mathrm{CN}\right): \delta=1.24(\mathrm{~s}, 18 \mathrm{H}, H 36, H 39), 2.21(\mathrm{~s}, 3 \mathrm{H}, H 17), 3.72(\mathrm{~s}, 4 \mathrm{H}, H 13$, $H 21$ ), 3.75 (s, 4H, H27, H33), 3.78 (s, 4H, H6, H12), 7.04 (s, 2H, H15, H18), 7.15 (m, 4H, H2, H8, $H 25, H 31), 7.46\left(\mathrm{~d},{ }^{3} \mathrm{~J}=8.07 \mathrm{~Hz}, 2 \mathrm{H}, H 4, H 10\right), 7.62\left(\mathrm{td},{ }^{3} \mathrm{~J}=7.70 \mathrm{~Hz},{ }^{4} \mathrm{~J}=1.83 \mathrm{~Hz}, 2 \mathrm{H}, H 3, H 9\right), 7.64$ $\left(\mathrm{t},{ }^{3} \mathrm{~J}=7.89 \mathrm{~Hz}, 2 \mathrm{H}, H 24, H 30\right), 7.97\left(\mathrm{~d},{ }^{3} \mathrm{~J}=8.44 \mathrm{~Hz}, 2 \mathrm{H}, H 23, H 29\right), 8.28$ (bs, 2H, NH), 8.44 (ddd, $\left.{ }^{3} \mathrm{~J}=4.91 \mathrm{~Hz},{ }^{4} \mathrm{~J}=1.78 \mathrm{~Hz}, 5 \mathrm{~J}=0.93 \mathrm{~Hz}, 2 \mathrm{H}, H 1, H 7\right) \mathrm{ppm}$. 
${ }^{13} \mathrm{C}$ NMR (100.55 MHz, CDCl $): \delta=20.6$ (C17), 27.5 (C36, C39), 39.8 (C35, C38), 54.7 (C13, C21), $59.0(C 27, C 33), 59.9$ (C6, C12), 111.7 (C23, C29), 118.0 (C25, C31), 121.9 (C2, C8), 122.7 (C4, C10), $123.8(C 14, C 19), 127.6(C 16), 129.7$ (C15, C18), 136.5 (C3, C9), 138.9 (C24, C30), $148.7(C 1, C 7)$, 151.0 (C22, C28), 153.1 (C20), 157.3 (C26, C32), 159.4 (C5, C11), 177.1 (C34, C37) ppm.

FT-IR spectroscopy: $v=3438(v\{\mathrm{~N}-\mathrm{H}\}), 3389(\mathrm{v}\{\mathrm{O}-\mathrm{H}\}), 2964(\mathrm{v}\{\mathrm{C}-\mathrm{H}\}), 2869(\mathrm{v}\{\mathrm{C}-\mathrm{H}\}), 2817(\mathrm{v}\{\mathrm{C}-\mathrm{H}\})$, $1686(v\{\mathrm{C}=\mathrm{O}\}), 1523(\mathrm{v}\{\mathrm{C}-\mathrm{N}\}), \delta\{\mathrm{CNH}\}), 1405\left(\mathrm{v}\left\{\mathrm{C}-\left(\mathrm{CH}_{3}\right)_{3}\right\}\right), 797(\delta\{\mathrm{py}-\mathrm{H}\}) \mathrm{cm}^{-1}$.

Elemental analysis: Calcd. for $\mathrm{C}_{43} \mathrm{H}_{52} \mathrm{~N}_{8} \mathrm{O}_{3} \cdot \mathrm{CH}_{3} \mathrm{OH}: \mathrm{C}$ 69.45\%; $\mathrm{H} 7.42 \%$; $\mathrm{N} 14.73 \%$ Found:

C $69.47 \%$; $7.10 \%$; N $14.98 \%$.

\subsection{SYNTHESIS OF LIGAND $\mathrm{H}_{3} \mathrm{SIM}^{1}$}

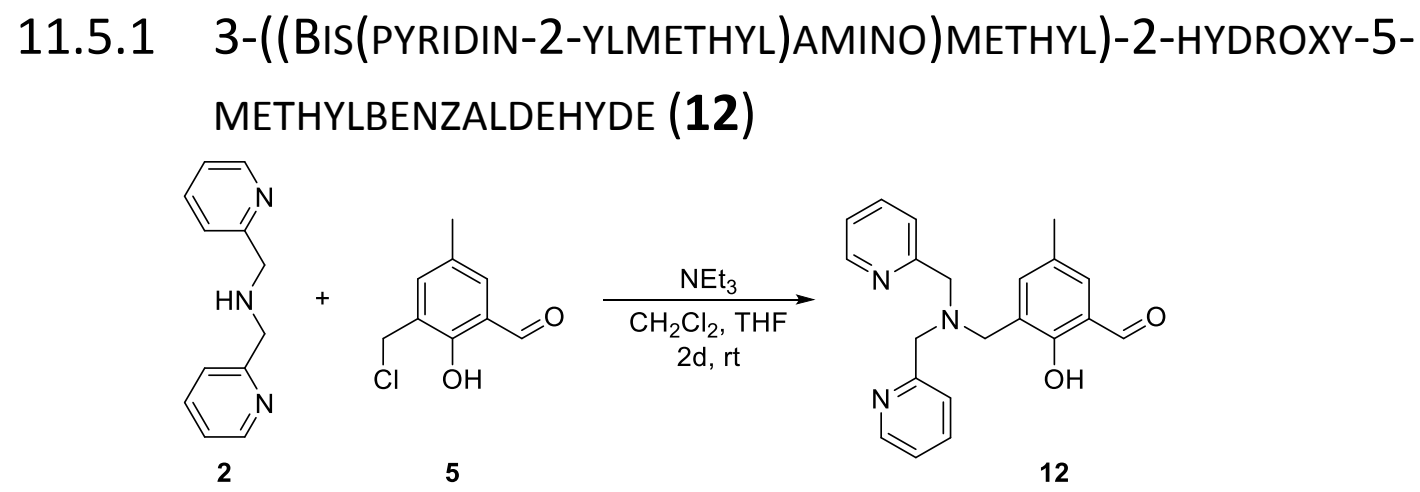

3-((Bis(pyridine-2-ylmethyl)amino)methyl)2-hydroxy-5-methylbenzaldehyde

(12) was synthesized by modification of the previously reported method. ${ }^{106}$

3-(Chloromethyl)-2-hydroxy-5-methylbenzaldehyde (5) (0.54 g, $2.7 \mathrm{mmol}, 1.0 \mathrm{eq}$ ) was dissolved in dichloromethane $(4.0 \mathrm{ml})$ and cooled to $0^{\circ} \mathrm{C}$ in an water ice bath. A solution of bis(pyridin-2ylmethyl)amine (2) $(0.50 \mathrm{~g}, 2.7 \mathrm{mmol}, 1.0 \mathrm{eq})$ in tetrahydrofuran $(4.5 \mathrm{ml})$ which was treated with triethylamine $(0.70 \mathrm{ml}, 0.51 \mathrm{~g}, 5.0 \mathrm{mmol}, 1.9 \mathrm{eq})$, was added dropwise. Afterwards, the reaction mixture was brought to room temperature and stirred for two days. The precipitate obtained was filtered and washed with a small amount of dichloromethane. The filtrate was concentrated under reduced pressure, taken up in dichloromethane $(10 \mathrm{ml})$, washed with saturated sodium bicarbonate solution $(10 \mathrm{ml})$ and dried over sodium sulfate. The yellow oil obtained after removal of the solvent solidified while keeping at $-8^{\circ} \mathrm{C}$. Recrystallisation from dichloromethane/hexane (1:1) yield 3-((bis(pyridine-2-ylmethyl)amino)methyl)2-hydroxy-5-methylbenzaldehyde (12) as a yellowish solid $(0.57 \mathrm{~g}, 61 \%)$. 


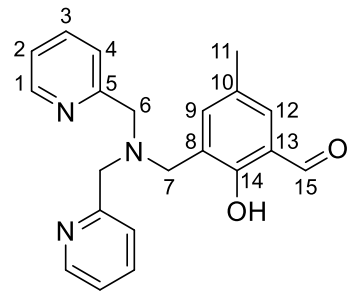

HRMS (ESI $\left.{ }^{+}, \mathrm{CH}_{3} \mathrm{OH}\right): m / z=348.1707\left(\left[\mathrm{C}_{21} \mathrm{H}_{22} \mathrm{~N}_{3} \mathrm{O}_{2}\right]^{+}\right.$, calcd. 348.1707).

${ }^{1} \mathrm{H}$ NMR $\left(600.13 \mathrm{MHz}, \mathrm{CD}_{3} \mathrm{CN}\right): \delta=2.25(\mathrm{~s}, 3 \mathrm{H}, H 11), 3.78(\mathrm{~s}, 2 \mathrm{H}, H 7), 3.84(\mathrm{~s}, 4 \mathrm{H}, H 6), 7.22$ (ddd, $\left.{ }^{3} \mathrm{~J}=7.42 \mathrm{~Hz},{ }^{3} \mathrm{~J}=4.89 \mathrm{~Hz},{ }^{4} \mathrm{~J}=1.01 \mathrm{~Hz}, 2 \mathrm{H}, H 2\right), 7.27\left(\mathrm{~d},{ }^{4} \mathrm{~J}=2.32,1 \mathrm{H}, H 9\right), 7.36\left(\mathrm{~d},{ }^{3} \mathrm{~J}=7.87 \mathrm{~Hz}, 2 \mathrm{H}\right.$, $H 4), 7.40\left(\mathrm{~d},{ }^{4} \mathrm{~J}=1.61 \mathrm{~Hz}, 1 \mathrm{H}, H 12\right), 7.69\left(\mathrm{td},{ }^{3} \mathrm{~J}=7.69 \mathrm{~Hz},{ }^{4} \mathrm{~J}=1.77 \mathrm{~Hz}, 2 \mathrm{H}, H 3\right), 8.46$ (ddd, $\left.{ }^{3} \mathrm{~J}=4.95 \mathrm{~Hz},{ }^{4} \mathrm{~J}=1.64 \mathrm{~Hz},{ }^{5} \mathrm{~J}=0.92 \mathrm{~Hz}, 2 \mathrm{H}, H 1\right), 10.41(\mathrm{~s}, 1 \mathrm{H}, H 15) \mathrm{ppm}$.

${ }^{13} \mathrm{C}$ NMR (150.90 MHz, CD 3 CN): $\delta=20.3$ (C11), 56.1 (C7), 59.7 (C6), 123.4 (C2), 123.9 (C13), 124.2 (C4), 126.6 (C8), 128.3 (C12), 129.0 (C10), 137.9 (C3), 138.8 (C9), 149.8 (C1), 159.4 (C5), 160.3 (C14), 192.1 (C15) ppm.

\subsubsection{LIGAND $\mathrm{H}_{3} \mathrm{SIM}^{1}$}
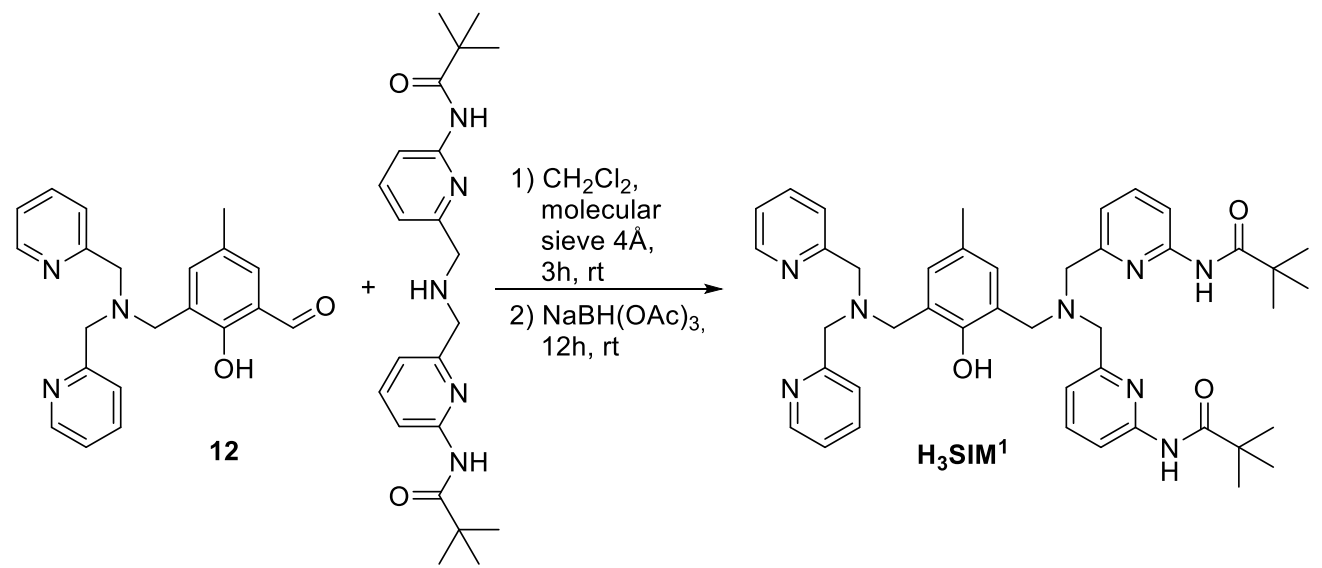

3

Molecular sieves (4 A) were added to a solution of compound 12 (3.13 g, $9.0 \mathrm{mmol}, 1.0 \mathrm{eq})$ in anhydrous dichloromethane $(23 \mathrm{ml})$, followed by the dropwise addition of a solution of compound 3 ( $3.58 \mathrm{~g}, 9.0 \mathrm{mmol}, 1.0 \mathrm{eq})$ in anhydrous dichloromethane $(10 \mathrm{ml})$. The mixture was stirred for three hours at room temperature. The solution was then treated with sodium triacetoxyborohydride $(4.20 \mathrm{~g}, 20 \mathrm{mmol}, 2.2 \mathrm{eq})$ by addition in portions and the mixture stirred at room temperature overnight. The reaction was quenched by addition of saturated sodium bicarbonate solution ( $30 \mathrm{ml}$ ) and stirring for 45 minutes. The $\mathrm{pH}$ value was subsequently adjusted to 8 by addition of solid sodium carbonate. The phases were separated and the aqueous phase was extracted with dichloromethane $(3 \times 30 \mathrm{ml})$. The combined organic phases were washed with saturated sodium carbonate solution $(20 \mathrm{ml})$ and brine $(20 \mathrm{ml})$ and dried over sodium sulfate. Removal of the solvent under reduced pressure gave a brownish foam. Purification by column 
chromatography $\left(\mathrm{SiO}_{2} ; \mathrm{DEE}: \mathrm{NH}_{3}(7 \mathrm{~N}\right.$ in $\left.\mathrm{MeOH})=98: 2 ; \mathrm{R}_{\mathrm{f}}=0.48\right)$ yielded $\mathrm{H}_{3} \mathrm{SIM}^{1}$ as a white solid (1.94 g, 30\%).

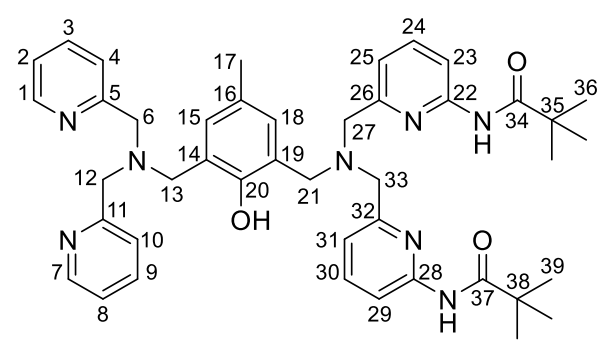

HRMS $\left(\mathrm{ESI}^{+}, \mathrm{CH}_{3} \mathrm{CN}\right): \mathrm{m} / z=729.4232\left(\left[\mathrm{C}_{43} \mathrm{H}_{53} \mathrm{~N}_{8} \mathrm{O}_{3}\right]^{+}\right.$, calcd. 729.4235).

${ }^{1} \mathrm{H}$ NMR (CD $\left.{ }_{3} \mathrm{CN}, 500.13 \mathrm{MHz}\right): \delta=1.25(\mathrm{~s}, 18 \mathrm{H}, H 36, H 39), 2.21(\mathrm{~s}, 3 \mathrm{H}, H 17), 3.69(\mathrm{~s}, 2 \mathrm{H}, H 21)$, $3.73(\mathrm{~s}, 4 \mathrm{H}, H 27, H 33), 3.74$ (s, 2H, H13), 3.79 (s, 4H, H6, H12), 6.88 (d, 4 J = $1.74 \mathrm{~Hz}, 1 \mathrm{H}, H 18$ ), 7.10 $\left(\mathrm{d},{ }^{3} \mathrm{~J}=8.06 \mathrm{~Hz}, 2 \mathrm{H}, H 25, H 31\right), 7.15\left(\mathrm{~d},{ }^{4} \mathrm{~J}=0.92 \mathrm{~Hz}, 1 \mathrm{H}, H 15\right), 7.15(\mathrm{~m}, 2 \mathrm{H}, H 2, H 8), 7.50$ (d, $\left.{ }^{3} \mathrm{~J}=8.07 \mathrm{~Hz}, 2 \mathrm{H}, H 4, H 10\right), 7.62\left(\mathrm{t},{ }^{3} \mathrm{~J}=8.44 \mathrm{~Hz}, 2 \mathrm{H}, H 24, H 30\right), 7.64\left(\mathrm{dt},{ }^{3} \mathrm{~J}=7.70 \mathrm{~Hz},{ }^{4} \mathrm{~J}=1.83 \mathrm{~Hz}\right.$, $2 \mathrm{H}, H 3, H 9$ ), $7.96\left(\mathrm{~d},{ }^{3} \mathrm{~J}=8.44 \mathrm{~Hz}, 2 \mathrm{H}, H 23, H 29\right.$ ), 8.28 (bs, $2 \mathrm{H}, \mathrm{NH}$ ), 8.46 (ddd, ${ }^{3} \mathrm{~J}=4.83 \mathrm{~Hz}$, $\left.{ }^{4} \mathrm{~J}=1.78 \mathrm{~Hz}, 5 \mathrm{~J}=1.05 \mathrm{~Hz}, 2 \mathrm{H}, H 1, H 7\right) \mathrm{ppm}$.

${ }^{13} \mathrm{C}$ NMR ( $\left.\mathrm{CD}_{3} \mathrm{CN}, 100.61 \mathrm{MHz}\right): \delta=20.8$ (C17), 27.7 (C36, C39), 40.5 (C35, C38), 54.4 (C13), 56.3 (C21), 59.6 (C27, C33), 60.8 (C6, C12), 112.5 (C23, C29), 119.2 (C25, C31), 123.1 (C2, C8), 123.8 (C4, C10), 124.7 (C19), 125.6 (C14), 128.5 (C16), 130.7 (C15), 131.0 (C18), 137.4 (C3, C9), 139.9 (C24, C30), 149.9 (C1, C7), 152.3 (C22, C28), 154.4 (C20), 158.7 (C26, C32), 160.6 (C5, C11), 177.9 (C34, C37) ppm.

FT-IR spectroscopy: $v=3438(v\{\mathrm{~N}-\mathrm{H}\}), 3389(\mathrm{v}\{\mathrm{O}-\mathrm{H}\}), 2964(\mathrm{v}\{\mathrm{C}-\mathrm{H}\}), 2869(\mathrm{v}\{\mathrm{C}-\mathrm{H}\}), 2817(\mathrm{v}\{\mathrm{C}-\mathrm{H}\})$, $1687(v\{\mathrm{C}=\mathrm{O}\}), 1517(\mathrm{v}\{\mathrm{C}-\mathrm{N}\}, \delta\{\mathrm{CNH}\}), 1403\left(\mathrm{v}\left\{\mathrm{C}-\left(\mathrm{CH}_{3}\right)_{3}\right\}\right), 798\left(\delta\{\right.$ py-H\}$) \mathrm{cm}^{-1}$.

Elemental analysis: Calcd. for $\mathrm{C}_{43} \mathrm{H}_{52} \mathrm{~N}_{8} \mathrm{O}_{3}: \quad$ C 70.85\%; $\mathrm{H} 7.19 \%$; $\mathrm{N} 15.37 \%$ Found: C $70.63 \%$; H 7.06\%; N $15.29 \%$.

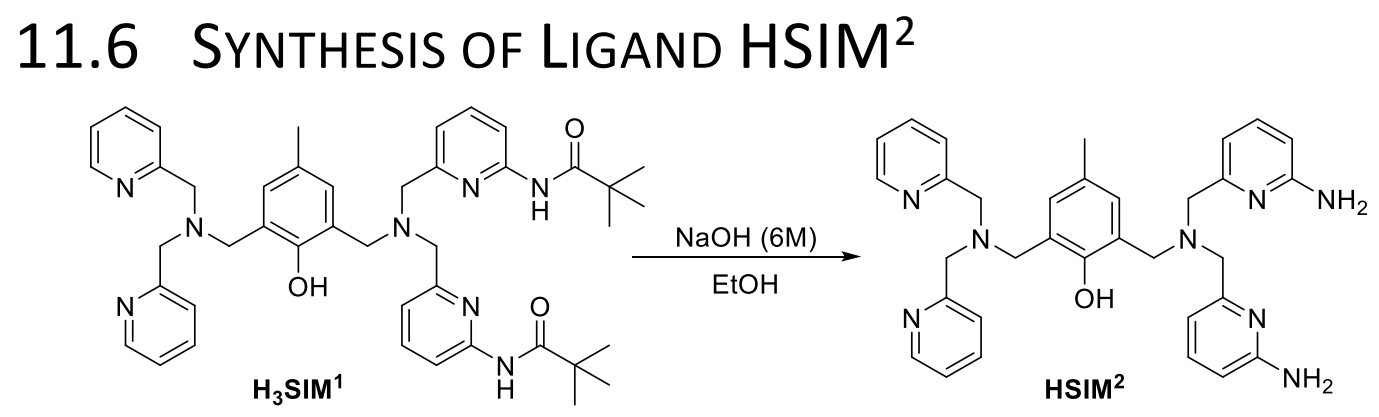

A solution of $\mathrm{H}_{3} \mathrm{SIM}^{1}$ (300 $\left.\mathrm{mg}, 0.41 \mathrm{mmol}\right)$ in ethanol $(10 \mathrm{ml})$ was treated with aqueous sodium hydroxide solution $(6 \mathrm{M}, 2.0 \mathrm{ml})$, after which the mixture was heated at $70^{\circ} \mathrm{C}$ for three days while stirring. Subsequently the solvent was removed under reduced pressure and the remaining solid was taken up in water $(10 \mathrm{ml})$. Concentrated hydrochloric acid was added until the $\mathrm{pH}$ value 
reached 1 and the $\mathrm{pH}$ value was subsequently adjusted to 7 using saturated sodium bicarbonate solution. After the product was extracted into dichloromethane $(3 \times 10 \mathrm{ml})$ the combined organic phases were washed with saturated sodium bicarbonate solution $(10 \mathrm{ml})$ and dried over sodium sulfate. Removal of the solvent yielded a crude product which was purified by column chromatography (Alox neutral; $\mathrm{MeOH}$ ) yielding the desired product $\mathrm{HSIM}^{2}$ as a white solid (109 mg, $47 \%)$.

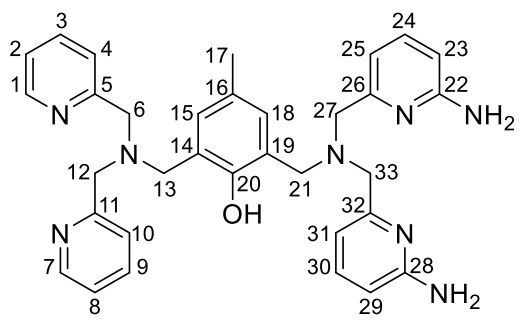

HRMS $\left(\mathrm{ESI}^{+}, \mathrm{CH}_{3} \mathrm{CN}\right): \mathrm{m} / \mathrm{z}=561.3080\left(\left[\mathrm{C}_{33} \mathrm{H}_{37} \mathrm{~N}_{8} \mathrm{O}\right]^{+}\right.$, calcd. 561.3085).

${ }^{1} \mathrm{H}$ NMR $\left(\mathrm{CD}_{3} \mathrm{CN}, 500.13 \mathrm{MHz}\right): \delta=2.18(\mathrm{~s}, 3 \mathrm{H}, H 17), 3.51(\mathrm{~s}, 4 \mathrm{H}, H 27, H 33), 3.57(\mathrm{~s}, 2 \mathrm{H}, H 21)$, $3.70(\mathrm{~s}, 2 \mathrm{H}, H 13), 3.77(\mathrm{~s}, 4 \mathrm{H}, H 6, H 12), 6.35$ (d, $\left.{ }^{3} \mathrm{~J}=8.07 \mathrm{~Hz}, 2 \mathrm{H}, H 23, H 29\right), 6.61\left(\mathrm{~d},{ }^{3} \mathrm{~J}=6.97 \mathrm{~Hz}\right.$, $2 \mathrm{H}, H 25, H 31), 6.88(\mathrm{~s}, 1 \mathrm{H}, H 18), 6.95(\mathrm{~s}, 1 \mathrm{H}, H 15), 7.17\left(\mathrm{ddd},{ }^{3} \mathrm{~J}=6.24 \mathrm{~Hz},{ }^{3} \mathrm{~J}=5.14 \mathrm{~Hz},{ }^{4} \mathrm{~J}=1.10 \mathrm{~Hz}\right.$, $2 \mathrm{H}, H 2, H 8), 7.33\left(\mathrm{t},{ }^{3} \mathrm{~J}=7.70 \mathrm{~Hz}, 2 \mathrm{H}, H 24, H 30\right), 7.46\left(\mathrm{~d},{ }^{3} \mathrm{~J}=7.70 \mathrm{~Hz}, 2 \mathrm{H}, H 4, H 10\right), 7.66$ (td, $\left.{ }^{3} \mathrm{~J}=7.70 \mathrm{~Hz},{ }^{4} \mathrm{~J}=1.83 \mathrm{~Hz}, 2 \mathrm{H}, H 3, H 9\right), 8.48\left(\mathrm{~d},{ }^{3} \mathrm{~J}=5.14 \mathrm{~Hz}, 2 \mathrm{H}, H 1, H 7\right) \mathrm{ppm}$.

${ }^{13} \mathrm{C}$ NMR (CD $\left.{ }_{3} \mathrm{CN}, 100.61 \mathrm{MHz}\right): \delta=20.5$ (C17), 52.5 (C13), 55.0 (C21), 60.2 (C27, C33), 60.5 (C6, C12), 107.8 (C23, C29), 113.1 (C25, C31), 123.2 (C2, C8), 124.2 (C4, C10), 124.7 (C14), 125.0 (C19), 131.2 (C15), 131.4 (C18), 137.7 (C3, C9), 139.2 (C24, C30), 149.9 (C1, C7), 157.9 (C26, C32), 160.0 (C5/C11/C22/C28), 160.2 (C5/C11/C22/C28) ppm.

FT-IR spectroscopy: $v=3322(\mathrm{v}\{\mathrm{N}-\mathrm{H}\}), 3199(\mathrm{v}\{\mathrm{N}-\mathrm{H}\}), 3059(\mathrm{v}\{\mathrm{Ar}-\mathrm{H}\}), 3011(\mathrm{v}\{\mathrm{Ar}-\mathrm{H}\}), 2917$ $(v\{C-H\}), 2816(v\{C-H\}), 1594(v\{C=C\}), 1574(v\{C=C\}), 1466(v\{C=C\}), 1435(v\{C=C\}), 1299(v\{C-N\})$, $1221(v\{C-N\}), 758(\delta\{p y-H\}) \mathrm{cm}^{-1}$. 


\subsection{SYNTHESIS OF LIGAND $\mathrm{H}_{4} \mathrm{SIM}^{3}$}

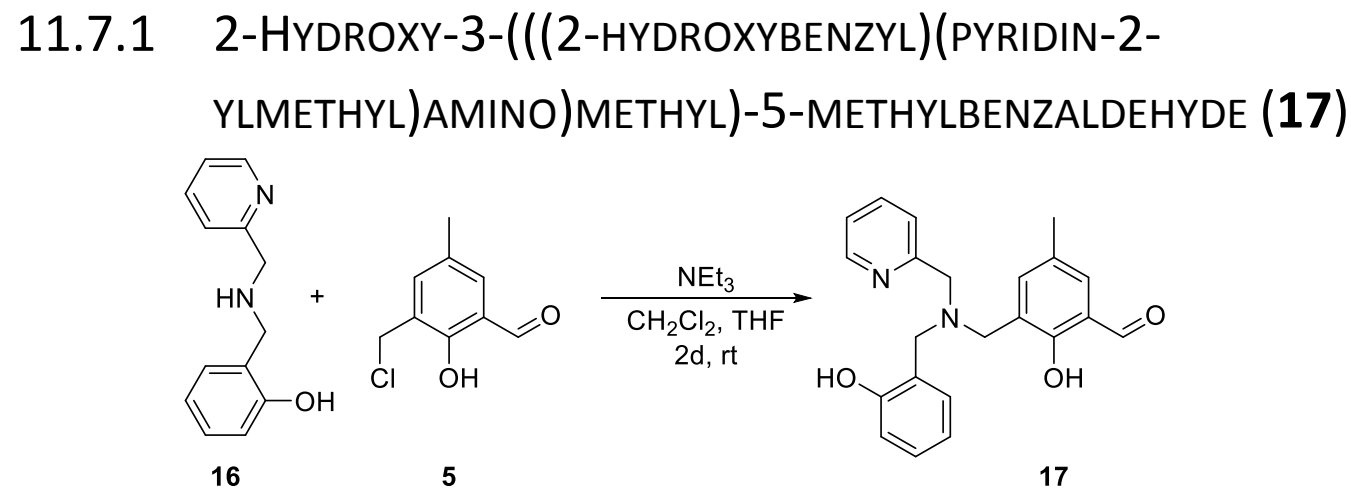

2-Hydroxy-3-(((2-hydroxybenzyl)(pyridine-2-ylmethyl)amino)methyl)-5-methylbenzaldehyde (17) was prepared according to a literature known procedures. ${ }^{311}$

3-(Chloromethyl)-2-hydroxy-5-methylbenzaldehyde (5) $(4.62 \mathrm{~g}, 25.0 \mathrm{mmol}, 1.0 \mathrm{eq})$ was dissolved in dichloromethane $(40.0 \mathrm{ml})$ and the solution was cooled in a water-ice-bath. Then, a mixture of 2-(((pyridin-2-ylmethyl)amino)methyl)phenol (16) (5.36 g, $25.0 \mathrm{mmol}, 1.0 \mathrm{eq})$ in tetrahydrofuran $(45.0 \mathrm{ml})$, treated with triethylamine $(12.8 \mathrm{ml}, 9.29 \mathrm{~g}, 50.0 \mathrm{mmol}, 2.0 \mathrm{eq})$, was added dropwise. After stirring the reaction mixture at room temperature for two days the precipitate obtained was filtered and washed with a small amount of dichloromethane. The filtrate was concentrated under reduced pressure and the residue taken up in dichloromethane $(50 \mathrm{ml})$ before being washed with brine $(30 \mathrm{ml})$ and dried over sodium sulfate. Removal of the solvent and recrystallization from dichloromethane/hexane (1:1) yielded 2-hydroxy-3-(((2-hydroxybenzyl)(pyridine-2-ylmethyl)amino)methyl)-5-methylbenzaldehyde (17) as a pale yellow solid (4.99 $\mathrm{g}$, $55 \%)$.

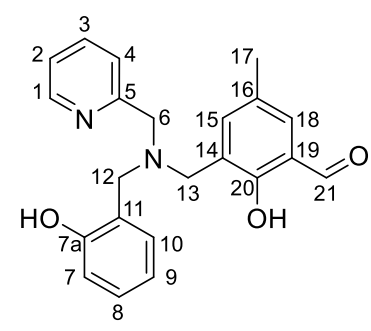

HRMS $\left(\mathrm{ESI}^{+}, \mathrm{CH}_{3} \mathrm{OH}\right): \mathrm{m} / z=363.1706\left(\left[\mathrm{C}_{22} \mathrm{H}_{23} \mathrm{~N}_{2} \mathrm{O}_{3}\right]^{+}\right.$, calcd. 363.1703).

${ }^{1} \mathrm{H}$ NMR (600.13 MHz, CD $3 \mathrm{CN}$ ): $\delta=2.23$ (s, 3H, H17), $3.74(\mathrm{~s}, 2 \mathrm{H}, H 13), 3.77(\mathrm{~s}, 2 \mathrm{H}, H 12), 3.85$ (s, $2 \mathrm{H}, H 6), 6.75-6.78(\mathrm{~m}, 2 \mathrm{H}, H 7, H 9), 7.10\left(\mathrm{dd},{ }^{3} \mathrm{~J}=7.27 \mathrm{~Hz},{ }^{4} \mathrm{~J}=1.01 \mathrm{~Hz}, 1 \mathrm{H}, H 10\right), 7.13$ (td, $\left.{ }^{3} \mathrm{~J}=7.70 \mathrm{~Hz},{ }^{4} \mathrm{~J}=1.67 \mathrm{~Hz}, 1 \mathrm{H}, H 8\right), 7.27-7.30(\mathrm{~m}, 2 \mathrm{H}, H 2, H 4), 7.31\left(\mathrm{~d},{ }^{4} \mathrm{~J}=1.82 \mathrm{~Hz}, H 15\right), 7.36$ (d, $\left.{ }^{4} \mathrm{~J}=1.61 \mathrm{~Hz}, H 18\right), 7.74\left(\mathrm{td},{ }^{3} \mathrm{~J}=7.67 \mathrm{~Hz},{ }^{4} \mathrm{~J}=1.82 \mathrm{~Hz}, 1 \mathrm{H}, H 3\right), 8.57\left(\mathrm{dd},{ }^{3} \mathrm{~J}=4.95 \mathrm{~Hz},{ }^{4} \mathrm{~J}=0.71 \mathrm{~Hz}\right.$, H1), 10.07 (s, 1H, H21), 11.09 (bs, 2H, OH) ppm. 
${ }^{13} \mathrm{C}$ NMR (150.90 MHz, CD ${ }_{3} \mathrm{CN}$ ): $\delta$ = 20.3 (C17), 54.0 (C13), 57.3 (C12), 58.5 (C6), 117.1 (C7), 120.0 (C9), 122.7 (C19), 123.6 (C11), 123.7 (C2), 124.6 (C4), 126.0 (C14), 129.7 (C16), 130.1 (C8), 131.3 (C10/C18), 131.3 (C10/C18), 138.4 (C3), 139.5 (C15), 149.3 (C1), 158.2 (C5), 158.5 (C7a), 159.3 (C20), 195.1 (C21) ppm.

Elemental analysis: Calcd. for $\mathrm{C}_{22} \mathrm{H}_{22} \mathrm{~N}_{2} \mathrm{O}_{3} \cdot 0.05 \mathrm{CH}_{2} \mathrm{Cl}_{2}$ : $\quad \mathrm{C} 72.23 \% ; \mathrm{H} 6.08 \%$; $\mathrm{N}$ 7.64\% Found:

C 71.76\%; H 6.31\%; N 7.43\%.

\subsubsection{LIGAND $\mathrm{H}_{4} \mathrm{SIM}^{3}$}

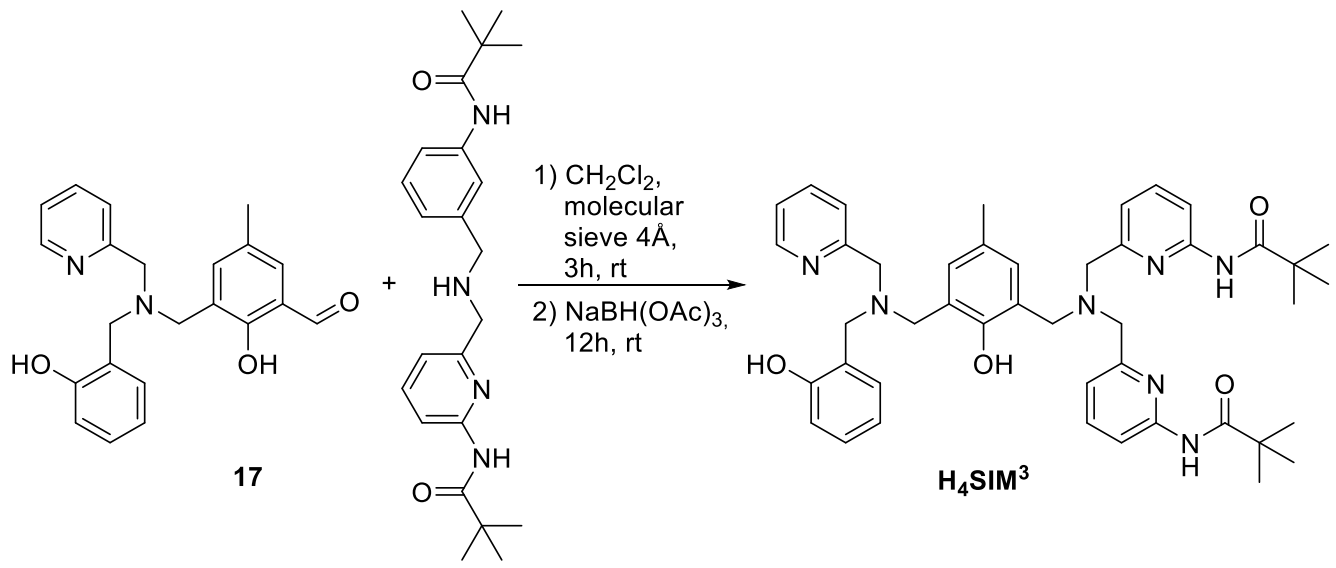

3

A solution of compound 17 ( $3.56 \mathrm{~g}, 9.80 \mathrm{mmol}, 1.0 \mathrm{eq})$ in anhydrous dichloromethane $(25 \mathrm{ml})$ was treated with molecular sieves (4 Å) before a solution of compound 3 (4.10 g, $10.3 \mathrm{mmol}$, $1.1 \mathrm{eq})$ in anhydrous dichloromethane $(11 \mathrm{ml})$ was added dropwise. After the mixture was stirred for three hours at room temperature sodium triacetoxyborohydride (4.59 g, $21.7 \mathrm{mmol}, 2.2 \mathrm{eq}$ ) was added in small portions and the reaction mixture was left to react at room temperature while stirring. The reaction was quenched by addition of saturated sodium bicarbonate solution $(30 \mathrm{ml})$ and the mixture was stirred for 45 minutes before the $\mathrm{pH}$ value was adjusted to 8 by addition of solid sodium carbonate. The phases were separated and the aqueous phase was extracted with dichloromethane $(3 \times 30 \mathrm{ml})$. The combined organic phases were washed with saturated sodium carbonate solution $(20 \mathrm{ml})$, brine $(20 \mathrm{ml})$ and dried over sodium sulfate. Removal of the solvent under reduced pressure gave a orange foam. Purification by column chromatography $\left(\mathrm{SiO}_{2}\right.$; DEE: $n$-hexane: $\mathrm{NH}_{3}(7 \mathrm{~N}$ in $\left.\mathrm{MeOH})=90: 8: 2 ; \mathrm{R}_{\mathrm{f}}=0.57\right)$ yielded $\mathrm{H}_{4} \mathrm{SIM}^{3}$ as a white solid $(4.50 \mathrm{~g}, 62 \%)$. 


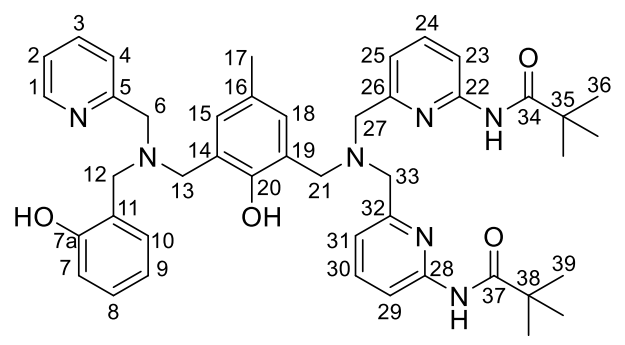

Molecular Formula: $\mathrm{C}_{44} \mathrm{H}_{53} \mathrm{~N}_{7} \mathrm{O}_{4}$

HRMS $\left(\mathrm{ESI}^{+}, \mathrm{CH}_{3} \mathrm{CN}\right): \mathrm{m} / \mathrm{z}=744.4231\left(\left[\mathrm{C}_{44} \mathrm{H}_{54} \mathrm{~N}_{7} \mathrm{O}_{4}\right]^{+}\right.$, calcd. 744.4235).

${ }^{1} \mathrm{H}$ NMR (500.13 MHz, CD ${ }_{3} \mathrm{CN}$ ): $\delta=1.26(\mathrm{~s}, 18 \mathrm{H}, H 36, H 39), 2.16(\mathrm{~s}, 3 \mathrm{H}, H 17), 3.69(\mathrm{~s}, 2 \mathrm{H}, H 21)$, $3.74(\mathrm{~s}, 4 \mathrm{H}, H 27, H 33), 3.75$ (s, 2H, H13), $3.76(\mathrm{~s}, 2 \mathrm{H}, H 6), 3.78$ (s, 2H, H12), $6.74(\mathrm{~m}, 2 \mathrm{H}, H 7, H 9)$, $6.85\left(\mathrm{~d},{ }^{4} \mathrm{~J}=1.83 \mathrm{~Hz}, 1 \mathrm{H}, H 18\right), 6.99\left(\mathrm{~d},{ }^{4} \mathrm{~J}=1.47 \mathrm{~Hz}, 1 \mathrm{H}, H 15\right), 7.05$ (d, 3 J = $7.34 \mathrm{~Hz}, 3 \mathrm{H}, H 10, H 25$, $H 31$ ), 7.11 (td, $\left.{ }^{3} \mathrm{~J}=7.70 \mathrm{~Hz},{ }^{4} \mathrm{~J}=1.83 \mathrm{~Hz}, 1 \mathrm{H}, H 8\right), 7.14$ (d, ${ }^{3} \mathrm{~J}=7.70 \mathrm{~Hz}, 1 \mathrm{H}, H 4$ ), 7.19 (ddd, $\left.{ }^{3} \mathrm{~J}=7.70 \mathrm{~Hz},{ }^{3} \mathrm{~J}=6.60 \mathrm{~Hz},{ }^{4} \mathrm{~J}=1.10 \mathrm{~Hz}, 1 \mathrm{H}, H 2\right), 7.58\left(\mathrm{td},{ }^{3} \mathrm{~J}=7.70 \mathrm{~Hz},{ }^{4} \mathrm{~J}=1.83 \mathrm{~Hz}, 1 \mathrm{H}, H 3\right), 7.61(\mathrm{t}$, ${ }^{3} \mathrm{~J}=7.70 \mathrm{~Hz}, 2 \mathrm{H}, H 24, H 30$ ), 7.97 (d, ${ }^{3} \mathrm{~J}=7.70 \mathrm{~Hz}, 2 \mathrm{H}, H 23, H 29$ ), 8.33 (bs, 2H, NH), 8.52 (ddd, $\left.{ }^{3} \mathrm{~J}=4.95 \mathrm{~Hz},{ }^{4} \mathrm{~J}=1.76 \mathrm{~Hz}, 5 \mathrm{~J}=0.91 \mathrm{~Hz}, 1 \mathrm{H}, H 1\right) \mathrm{ppm}$.

${ }^{13} \mathrm{C}$ NMR (100.61 MHz, CD $\left.{ }_{3} \mathrm{CN}\right): \delta=20.6$ (C17), 27.7 (C36, C39), 40.5 (C35, C38), 53.2 (C13), 57.2 (C21), 57.9 (C12), 59.3 (C27, C33), 59.4 (C6), 112.6 (C23, C29), 116.9 (C9), 119.1 (C25, C31), 119.9 (C7), 123.3 (C2), 124.1 (C11), 124.4 (C4), 124.5 (C14/C19), 124.9 (C14/C19), 128.7 (C16), 129.7 (C8), 130.8 (C10), 131.3 (C18), 131.6 (C15), 137.7 (C3), 140.0 (C24, C30), 149.9 (C1), 152.4 (C22, C28), 154.5 (C20), 158.2 (C26, C32), 158.8 (C5/C11), 158.9 (C5/C11), 178.0 (C34, C37) ppm.

FT-IR spectroscopy: $v=3392(v\{\mathrm{~N}-\mathrm{H}\}), 2968(\mathrm{v}\{\mathrm{C}-\mathrm{H}\}), 2870(\mathrm{v}\{\mathrm{C}-\mathrm{H}\}), 2824(\mathrm{v}\{\mathrm{C}-\mathrm{H}\}), 1689(\mathrm{v}\{\mathrm{C}=\mathrm{O}\})$, $1522(v\{\mathrm{C}-\mathrm{N}\}, \delta\{\mathrm{CNH}\}), 1450\left(\mathrm{v}\left\{\mathrm{C}-\left(\mathrm{CH}_{3}\right)_{3}\right\}\right), 751(\delta\{\mathrm{py}-\mathrm{H}\}) \mathrm{cm}^{-1}$.

Elemental analysis: Calcd. for $\mathrm{C}_{43} \mathrm{H}_{52} \mathrm{~N}_{8} \mathrm{O}_{3}$ : C 71.04\%; $\mathrm{H} 7.18 \%$; 13.18

Found: $\quad$ C $71.11 \% ; H$ H $7.26 \%$; N 12.93. 


\subsection{SYNTHESIS OF LIGAND $\mathrm{H}_{4} \mathrm{SIM}^{4}$}

\subsubsection{N-(6-(((3-FORMYL-2-HYDROXY-5-METHYLBENZYL)(PYRIDIN-2- YLMETHYL)AMINO)METHYL)PYRIDIN-2-YL)PIVALAMIDE (19)}

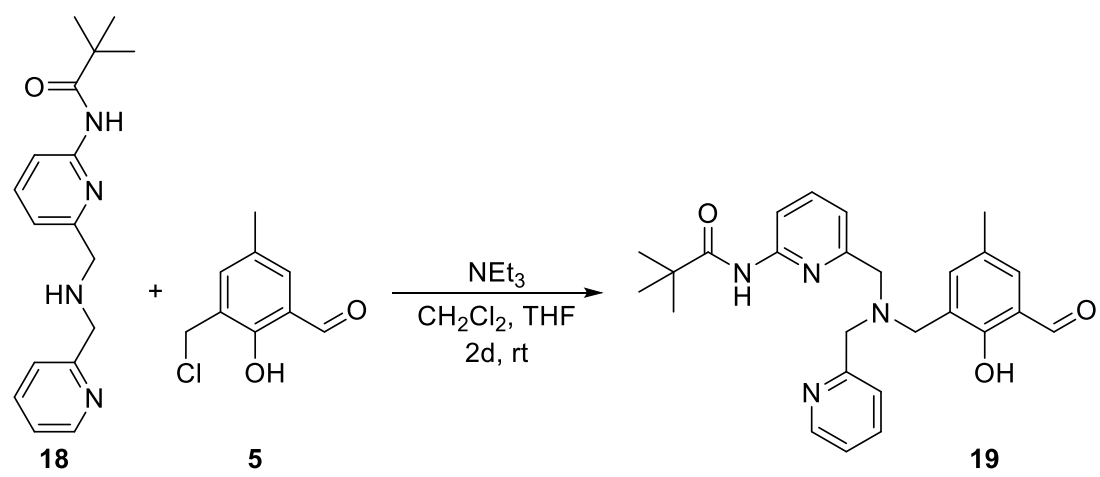

$N$-(6-pivaloylamido-2-pyridylmethyl)- $N$-(2-pyridylmethyl)amine (18) (4.92 g, $16.5 \mathrm{mmol}, 1.0$ eq) was dissolved in anhydrous dichloromethane $(26.5 \mathrm{ml})$ and treated with triethylamine $(4.30 \mathrm{ml}$, $3.09 \mathrm{~g}, 16.5 \mathrm{mmol}, 1.0 \mathrm{eq})$. This solution was then added dropwise to a cold mixture of 3-(chloromethyl)-2-hydroxy-5-methylbenzaldehyde (5) (3.05 g, $16.5 \mathrm{mmol}, 1.0 \mathrm{eq})$ in tetrahydrofuran $(30.0 \mathrm{ml})$ at $0^{\circ} \mathrm{C}$. Afterwards the mixture was stirred for two days at room temperature and the white precipitate obtained was filtered, washed with a small amount of dichloromethane and the filtrate was concentrated under reduced pressure. The remaining yellow oil was taken up in dichloromethane $(50 \mathrm{ml})$, washed with half concentrated brine $(20 \mathrm{ml})$ and dried over sodium sulfate. The solvent was evaporated under vacuum to yield $\mathrm{N}$-(6-(/(3formyl-2-hydroxy-5-methylbenzyl)(pyridin-2-ylmethyl)amino)methyl)pyridin-2-yl)pivalamide (19) as a yellow solid (7.07 g, 96\%).

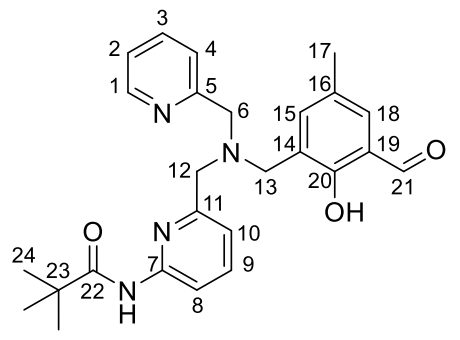

HRMS $\left(\mathrm{ESI}^{+}, \mathrm{CH}_{3} \mathrm{OH}\right): \mathrm{m} / \mathrm{z}=447.2391\left(\left[\mathrm{C}_{26} \mathrm{H}_{31} \mathrm{~N}_{4} \mathrm{O}_{3}\right]^{+}\right.$, calcd. 447.2391).

${ }^{1} \mathbf{H}$ NMR (399.89 MHz, CDCl 3 ): $\delta=1.38(\mathrm{~s}, 9 \mathrm{H}, H 24), 2,29(\mathrm{~s}, 3 \mathrm{H}, H 17), 3.81(\mathrm{~s}, 2 \mathrm{H}, H 13), 3.89(\mathrm{~s}$, $4 \mathrm{H}, H 6, H 12), 6.98\left(\mathrm{~d},{ }^{3} \mathrm{~J}=7.53 \mathrm{~Hz}, 1 \mathrm{H}, H 10\right), 7.15\left(\mathrm{dd},{ }^{3} \mathrm{~J}=7.28 \mathrm{~Hz},{ }^{3} \mathrm{~J}=5.24 \mathrm{~Hz}, 1 \mathrm{H}, H 2\right), 7.20$ (d, $\left.{ }^{4} \mathrm{~J}=1.51 \mathrm{~Hz}, 1 \mathrm{H}, H 15\right), 7.37\left(\mathrm{~d},{ }^{3} \mathrm{~J}=7.91 \mathrm{~Hz}, 1 \mathrm{H}, H 4\right), 7.49\left(\mathrm{~d},{ }^{4} \mathrm{~J}=1.63 \mathrm{~Hz}, 1 \mathrm{H}, H 18\right), 7.60-7.67(\mathrm{~m}$, $2 \mathrm{H}, H 3, H 9), 8.14\left(\mathrm{~d},{ }^{3} \mathrm{~J}=8.28 \mathrm{~Hz}, 1 \mathrm{H}, H 8\right), 8.46$ (bs, $\left.1 \mathrm{H}, \mathrm{NH}\right), 8.52$ (d, $\left.{ }^{3} \mathrm{~J}=4.77 \mathrm{~Hz}, 1 \mathrm{H}, H 1\right), 10.52$ $(\mathrm{s}, 1 \mathrm{H}, H 21), 11.74(\mathrm{bs}, 1 \mathrm{H}, \mathrm{OH}) \mathrm{ppm}$. 
${ }^{13} \mathrm{C}$ NMR (100.55 MHz, $\left.\mathrm{CDCl}_{3}\right): \delta=20.2$ (C17), 27.4 (C24), 39.8 (C23), 55.7 (C13), 58.3 (C12), 59.4 (C6), 112.0 (C8), 118.0 (C10), 122.2 (C2), 122.9 (C4), 125.0 (C14), 128.1 (C16, C19), 128.3 (C18), 136.8 (C3), 137.8 (C15), 139.2 (C9), 148.8 (C1), 151.3 (C7), 156.1 (C11), 158.2 (C5), 158.7 (C20), 177.2 (C22), 191.1 (C21) ppm.

\subsubsection{N-(6-(((2-HYDROXY-3-(((2-HYDROXYBENZYL)AMINO)METHYL)-} 5-METHYLBENZYL)(PYRIDIN-2-YLMETHYL)AMINO)METHYL)PYRIDIN-2-YL)PIVALAMIDE (21)<smiles>Cc1cc(CNCc2ccccc2O)c(O)c(CN(Cc2ccccn2)Cc2cccc(NC(=O)C(C)(C)C)n2)c1</smiles>

A mixture of compound 19 (6.25 g, $14.0 \mathrm{mmol}, 1.0 \mathrm{eq})$ and 2-(aminomethyl)phenol (20) (2.42 g, $19.6 \mathrm{mmol}, 1.4 \mathrm{eq})$ in methanol $(300 \mathrm{ml})$ was heated at $50^{\circ} \mathrm{C}$ for three hours. The solution was cooled with an ice-water bath and sodium borohydride $(1.99 \mathrm{~g}, 52.6 \mathrm{mmol}, 3.8 \mathrm{eq})$ was added in portions. After addition, the reaction mixture was refluxed for three hours. After cooling to room temperature the solution was concentrated under vacuum. The remaining oil was taken up in dichloromethane $(100 \mathrm{ml})$ and saturated sodium bicarbonate solution $(100 \mathrm{ml})$ was added carefully. Using solid sodium carbonate the $\mathrm{pH}$ value was adjusted to 14 . The phases were separated and the aqeuous phase was extracted with dichloromethane $(4 \times 75 \mathrm{ml})$. The combined organic phases were washed with brine $(20 \mathrm{ml})$ and dried over sodium sulfate. Removal of the solvent yielded $\mathrm{N}$-(6-(((2-hydroxy-3-(((2-hydroxybenzyl)amino)methyl)-5-methylbenzyl)(pyridin2-ylmethyl)amino)methyl)pyridin-2-yl)pivalamide (21) as a yellowish solid which was used in the next step without further purification.

HRMS $\left(\mathrm{ESI}^{+}, \mathrm{CH}_{3} \mathrm{OH}\right): m / z=554.3125\left(\left[\mathrm{C}_{33} \mathrm{H}_{40} \mathrm{~N}_{5} \mathrm{O}_{3}\right]^{+}\right.$, calcd. 554.3126).

${ }^{1} \mathrm{H}$ NMR $\left(200.13 \mathrm{MHz}, \mathrm{CDCl}_{3}\right): \delta=1.27\left(\mathrm{~s}, 6 \mathrm{H},\left(\mathrm{CH}_{3}\right) \mathrm{C}\right), 1.30\left(\mathrm{~s}, 3 \mathrm{H},\left(\mathrm{CH}_{3}\right) \mathrm{C}\right), 2.17\left(\mathrm{~s}, 3 \mathrm{H}, \mathrm{CH}_{3}\right), 3.66$ $\left(\mathrm{s}, 2 \mathrm{H}, \mathrm{CH}_{2}\right), 3.75\left(\mathrm{~s}, 2 \mathrm{H}, \mathrm{CH}_{2}\right), 3.77\left(\mathrm{~s}, 2 \mathrm{H}, \mathrm{CH}_{2}\right), 3.82\left(\mathrm{~s}, 2 \mathrm{H}, \mathrm{CH}_{2}\right), 3.86\left(\mathrm{~s}, 2 \mathrm{H}, \mathrm{CH}_{2}\right), 4.69(\mathrm{~s}, 1 \mathrm{H}$, $\left.\mathrm{N}_{\text {amin }} H\right), 6.76\left(\mathrm{td},{ }^{3} \mathrm{~J}=7.32,{ }^{4} \mathrm{~J}=1.21,1 \mathrm{H}, H_{\text {aryl }}\right), 6.84-6.94\left(\mathrm{~m}, 4 \mathrm{H}, H_{\text {aryl }}\right), 7.03\left(\mathrm{~s}, 1 \mathrm{H}, H_{\text {aryl }}\right)$, $7.09-7.22\left(\mathrm{~m}, 3 \mathrm{H}, H_{\text {aryl }}\right), 7.26-7.34\left(\mathrm{~m}, 1 \mathrm{H}, H_{\text {aryl }}\right), 7.52-7.68\left(\mathrm{~m}, 2 \mathrm{H}, H_{\text {aryl }}\right), 8.13\left(\mathrm{~d},{ }^{3} \mathrm{~J}=8.34,2 \mathrm{H}\right.$, $\left.H_{\text {aryl }}\right), 8.45\left(\mathrm{~s}, 1 \mathrm{H}, \mathrm{N}_{\text {amid }} H\right), 8.51\left(\mathrm{ddd},{ }^{3} \mathrm{~J}=4.89,{ }^{4} \mathrm{~J}=1.86,5 \mathrm{~J}=0.82,1 \mathrm{H}, H_{\text {aryl }}\right) \mathrm{ppm}$. 


\subsubsection{LIGAND $\mathrm{H}_{4} \mathrm{SIM}^{4}$}

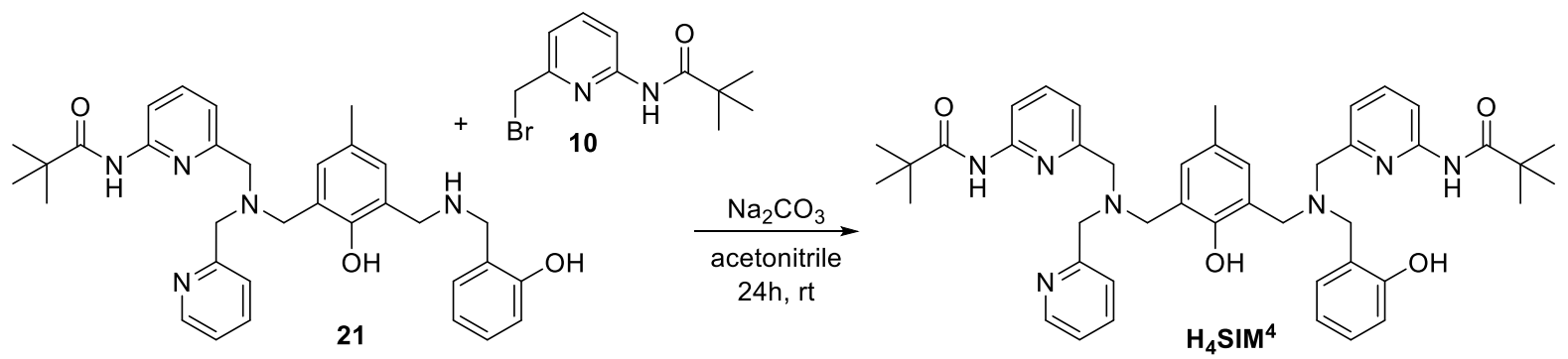

Compound 21 (7.75 g, $14.0 \mathrm{mmol}, 1.0 \mathrm{eq})$ was dissolved in acetonitrile $(400 \mathrm{ml})$ and treated with sodium carbonate $(1.48 \mathrm{~g}, 14.0 \mathrm{mmol}, 1.0 \mathrm{eq})$. Then, $N$-(6-bromomethyl)pyridine-2-yl)pivalamide (10) $(3.80 \mathrm{~g}, 14.0 \mathrm{mmol}, 1.0 \mathrm{eq})$ was added and the reaction mixture was stirred at room temperature for 24 hours. The solution was concentrated to a brown oil, which was taken up in dichloromethane $(100 \mathrm{ml})$ and saturated sodium bicarbonate solution $(100 \mathrm{ml})$. The product was extracted into dichloromethane $(4 \times 50 \mathrm{ml})$, washed with brine $(30 \mathrm{ml})$ and dried over sodium sulfate. After removal of the solvent the crude product was purified via column chromatography ( $\mathrm{SiO}_{2} ; \mathrm{DEE}: n$-hexane: $\mathrm{NH}_{3}(7 \mathrm{~N}$ in $\left.\mathrm{MeOH})=90: 8: 2 ; \mathrm{R}_{\mathrm{f}}=0.48\right)$ to yield $\mathrm{H}_{4} \mathrm{SIM}^{4}$ as yellowish solid (2.12 g, 22\%).

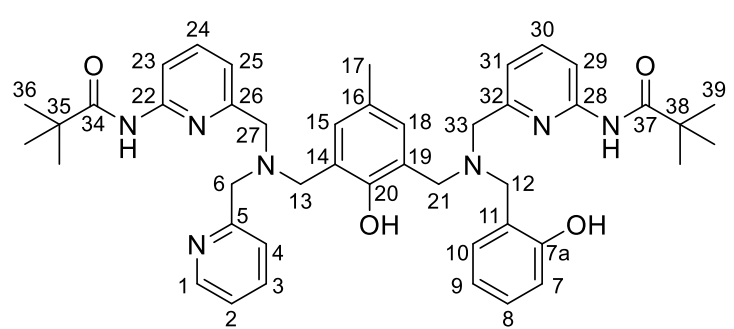

HRMS $\left(\mathrm{ESI}^{+}, \mathrm{CH}_{3} \mathrm{OH}\right): \mathrm{m} / z=744.4237\left(\left[\mathrm{C}_{44} \mathrm{H}_{54} \mathrm{~N}_{7} \mathrm{O}_{4}\right]^{+}\right.$, calcd. 744.4232).

${ }^{1}{ }_{H}$ NMR (500.13 MHz, CD ${ }_{3} \mathrm{CN}$ ): $\delta=1.28$ (s, 9H, H36/39), 1.28 (s, 9H, H36/39), 2.13 (s, 3H, H17), $3.67(\mathrm{~s}, 2 \mathrm{H}, H 13 / 21), 3.73$ (s, 2H, H13/21), 3.74 (s, 4H, H6, H27/H30), 3.76 (s, 2H, H27/H30), 3.77 (s, 2H, H12), 6.74-6.79 (m, 2H, H7/H9), 6.81 (d, $\left.{ }^{4} \mathrm{~J}=1.83 \mathrm{~Hz}, 1 \mathrm{H}, H 15 / H 18\right), 6.84$ (dd, ${ }^{3} \mathrm{~J}=7.70 \mathrm{~Hz}$, $\left.{ }^{4} \mathrm{~J}=0.73 \mathrm{~Hz}, 1 \mathrm{H}, \mathrm{H} 25 / \mathrm{H} 31\right), 6.96$ (d, $\left.{ }^{4} \mathrm{~J}=2.20 \mathrm{~Hz}, 1 \mathrm{H}, H 15 / H 18\right), 6.99$ (dd, ${ }^{3} \mathrm{~J}=7.70 \mathrm{~Hz},{ }^{4} \mathrm{~J}=0.73 \mathrm{~Hz}$, $1 \mathrm{H}, H 25 / H 31), 7.09$ (dd, $\left.{ }^{3} \mathrm{~J}=7.34 \mathrm{~Hz},{ }^{4} \mathrm{~J}=1.47 \mathrm{~Hz}, 1 \mathrm{H}, H 10\right), 7.12-7.15(\mathrm{~m}, 2 \mathrm{H}, H 2 / H 8), 7.33$ (d, $\left.{ }^{3} \mathrm{~J}=7.70 \mathrm{~Hz}, 1 \mathrm{H}, H 4\right), 7.56-7.61(\mathrm{~m}, 2 \mathrm{H}, \mathrm{H} 3, \mathrm{H} 24 / \mathrm{H} 30), 7.64\left(\mathrm{t},{ }^{3} \mathrm{~J}=7.89 \mathrm{~Hz}, 1 \mathrm{H}, \mathrm{H} 24 / \mathrm{H} 30\right), 7.97$ (d, $\left.{ }^{3} \mathrm{~J}=8.07 \mathrm{~Hz}, 1 \mathrm{H}, H 23 / H 29\right), 8.00\left(\mathrm{~d},{ }^{3} \mathrm{~J}=7.70 \mathrm{~Hz}, 1 \mathrm{H}, H 23 / H 29\right), 8.42$ (bs, $\left.1 \mathrm{H}, \mathrm{NH}\right), 8.45$ (ddd, $\left.{ }^{3} \mathrm{~J}=4.86 \mathrm{~Hz},{ }^{4} \mathrm{~J}=1.82 \mathrm{~Hz},{ }^{5} \mathrm{~J}=0.84 \mathrm{~Hz}, 1 \mathrm{H}, H 1\right), 8.48(\mathrm{bs}, 1 \mathrm{H}, \mathrm{NH}) \mathrm{ppm}$.

${ }^{13} \mathrm{C}$ NMR (125.76 MHz, CD ${ }_{3} \mathrm{CN}$ ): $\delta$ = 20.6 (C17), 27.6 (C36/C39), 27.7 (C36/C39), 40.5 (C35/C38), 40.5 (C35/C38), 52.9 (C13/C21), 57.2 (C13/C21), 58.0 (C12), 58.7 (C27/C33), 59.0 (C27/C33), 59.8 (C6), 112.3 (C23/C29), 112.4 (C23/C29), 116.8 (C7/C9), 119.1 (C25/C31), 119.3 (C25/C31), 119.9 (C7/C9), 123.1 (C2), 123.9 (C4), 124.29 (C14/C20), 124.4 (C11), 124.9 (C14/C20), 128.5 (C16), 129.7 (C8), 131.0 (C10, C15/C18), 131.1 (C10, C15/C18), 131.4 (C10, C15/C18), 137.6 (C3), 140.0 
(C24/C30), 140.0 (C24/C30), 149.8 (C1), 152.3 (C22/C28), 152.4 (C22/C28), 154.5 (C20), 157.7 (C26/C32), 157.9 (C26/C32), 158.6 (C7a), 159.6 (C5), 177.9 (C34/C37).

FT-IR spectroscopy: $v=3669(v\{\mathrm{O}-\mathrm{H}\}), 3388(\mathrm{v}\{\mathrm{N}-\mathrm{H}\}), 2971(\mathrm{v}\{\mathrm{C}-\mathrm{H}\}), 2901(\mathrm{v}\{\mathrm{C}-\mathrm{H}\}), 2819(\mathrm{v}\{\mathrm{C}-\mathrm{H}\})$, $1688(v\{\mathrm{C}=\mathrm{O}\}), 1520(v\{\mathrm{C}-\mathrm{N}\}, \delta\{\mathrm{CNH}\}), 1449\left(v\left\{\mathrm{C}-\left(\mathrm{CH}_{3}\right)_{3}\right\}\right), 755(\delta\{\mathrm{py}-\mathrm{H}\}) \mathrm{cm}^{-1}$.

Elemental analysis: Calcd. for $\mathrm{C}_{44} \mathrm{H}_{53} \mathrm{~N}_{7} \mathrm{O}_{4}$ : C 71.04\%; $\mathrm{H}$ 7.18\%; $\mathrm{N} 13.18 \%$

Found: $\quad$ C $70.66 \%$; H $7.31 \%$; N $13.35 \%$.

\subsection{SYNTHESIS OF DIZINC(II) COMPLEXES}

\subsubsection{General Procedure}

The ligand $\left(0.137 \mathrm{mmol}, 1.0 \mathrm{eq}\right.$ ) was dissolved in methanol $\left(5.0 \mathrm{ml}\right.$ ) (when $\mathrm{H}_{4} \mathrm{SIM}^{3}$ was used as ligand the solution was treated with $2 \mathrm{M}$ sodium hydroxide solution $(69 \mu \mathrm{l}, 1.0 \mathrm{eq}))$. After addition of $\mathrm{Zn}^{\text {"l }}$ acetate $\left(57.8 \mathrm{mg}, 0.274 \mathrm{mmol}, 2.0 \mathrm{eq}\right.$ ) the mixture was heated to $50^{\circ} \mathrm{C}$ for 20 minutes. Sodium hexafluorophosphate $(46.0 \mathrm{mg}, 0.274 \mathrm{mmol}, 2.0 \mathrm{eq})$ was added to the hot solution. The compounds were obtained as colourless crystals upon slow evaporation of solvent and these were collected by filtration.

\subsubsection{SYNTHESIS OF $\left[\mathrm{ZN}_{2}{ }_{2}\left(\mathrm{~L}^{1}\right)(\mu-\mathrm{OAC})_{2}\right] \mathrm{PF}_{6}$}

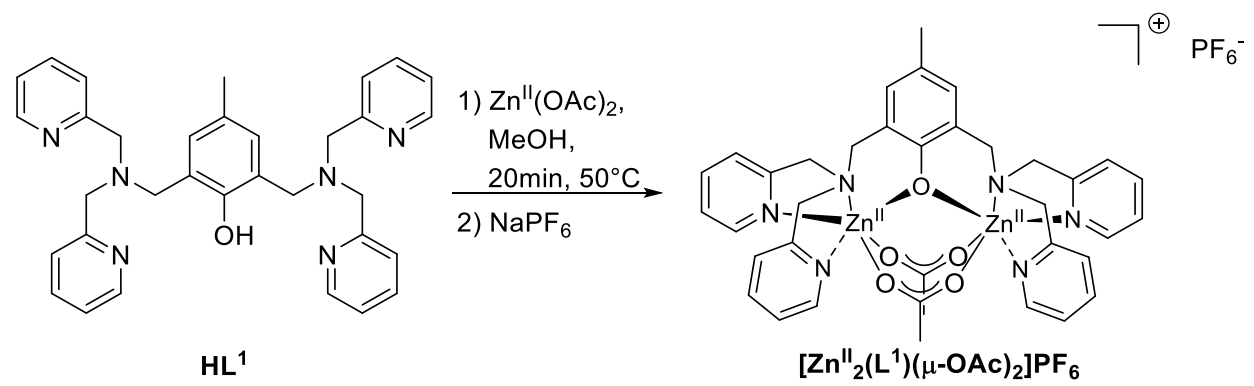

Yield: $102.5 \mathrm{mg}(81 \%)$.

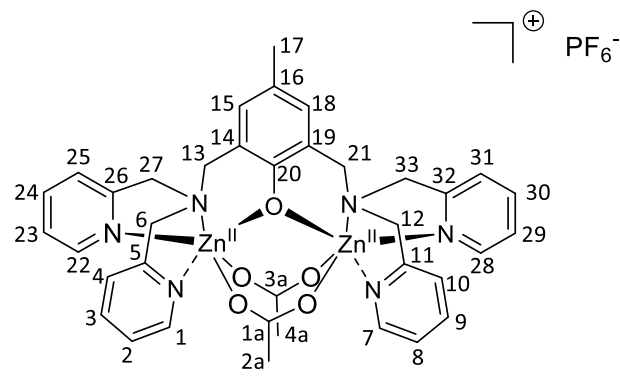

CCDC no.: 998888

HRMS (ESI $\left.{ }^{+}, \mathrm{CH}_{3} \mathrm{CN}\right): m / z=360.0704\left(\left[\mathrm{C}_{35} \mathrm{H}_{36} \mathrm{~N}_{6} \mathrm{O}_{3} \mathrm{Zn}_{2}\right]^{2+}\right.$, calcd. 360.0689); 765.1352 $\left(\left[\mathrm{C}_{36} \mathrm{H}_{37} \mathrm{~N}_{6} \mathrm{O}_{5} \mathrm{Zn}_{2}\right]^{+}\right.$, calcd. 765.1360). 
${ }^{1} \mathrm{H}$ NMR $\left(500.13 \mathrm{MHz}, \mathrm{CD}_{3} \mathrm{CN}\right): \delta=1.95$ (s, 3H, H17), $1.99(\mathrm{~s}, 6 \mathrm{H}, \mathrm{H} 2 \mathrm{a}, \mathrm{H4a}), 3.17$ (d, ${ }^{2} \mathrm{~J}=11.37 \mathrm{~Hz}$, $2 \mathrm{H}, H 13, H 21), 3.35$ (d, $\left.{ }^{2} \mathrm{~J}=16.51 \mathrm{~Hz}, 2 \mathrm{H}, H 12, H 33\right), 3.48$ (d, $\left.{ }^{2} \mathrm{~J}=16.14 \mathrm{~Hz}, 2 \mathrm{H}, H 12, H 33\right), 3.88$ (d, $\left.{ }^{2} \mathrm{~J}=11.37 \mathrm{~Hz}, 2 \mathrm{H}, H 13, H 21\right), 3.97\left(\mathrm{~d},{ }^{2} \mathrm{~J}=14.31 \mathrm{~Hz}, 2 \mathrm{H}, H 6, H 27\right), 4.50\left(\mathrm{~d},{ }^{2} \mathrm{~J}=14.31 \mathrm{~Hz}, 2 \mathrm{H}, H 6\right.$, $H 27$ ), 6.40 (s, 2H, H15, H18), 6.44 (d, $\left.{ }^{3} \mathrm{~J}=7.70 \mathrm{~Hz}, 2 \mathrm{H}, H 10, H 31\right), 7.01$ (dd, ${ }^{3} \mathrm{~J}=7.34 \mathrm{~Hz},{ }^{3} \mathrm{~J}=5.14 \mathrm{~Hz}$, $2 \mathrm{H}, H 8, H 29), 7.29\left(\mathrm{td},{ }^{3} \mathrm{~J}=7.70 \mathrm{~Hz},{ }^{4} \mathrm{~J}=1.47 \mathrm{~Hz}, 2 \mathrm{H}, H 9, H 30\right), 7.43\left(\mathrm{dd},{ }^{3} \mathrm{~J}=7.34 \mathrm{~Hz},{ }^{3} \mathrm{~J}=5.14 \mathrm{~Hz}\right.$, $2 \mathrm{H}, H 2, H 23), 7.48\left(\mathrm{~d},{ }^{3} \mathrm{~J}=8.07 \mathrm{~Hz}, 2 \mathrm{H}, H 4, H 25\right), 7.92\left(\mathrm{td},{ }^{3} \mathrm{~J}=7.79 \mathrm{~Hz},{ }^{4} \mathrm{~J}=1.65 \mathrm{~Hz}, 2 \mathrm{H}, H 3, H 24\right)$, $8.24\left(\mathrm{~d},{ }^{3} \mathrm{~J}=4.77 \mathrm{~Hz}, 2 \mathrm{H}, H 7, H 28\right), 8.76\left(\mathrm{~d},{ }^{3} \mathrm{~J}=4.40 \mathrm{~Hz}, 2 \mathrm{H}, H 1, H 22\right) \mathrm{ppm}$.

${ }^{13} \mathrm{C}$ NMR (100.61 MHz, CD $\left.{ }_{3} \mathrm{CN}\right): \delta=20.2$ (C17), 24.9 (C2a, C4a), 58.5 (C12, C33), 60.7 (C13, C21), $60.8(C 6, C 27), 121.9(C 10, C 31), 123.6$ (C8, C29), 124.3 (C14, C19), $125.0(C 2, C 23), 125.1$ (C16), 125.2 (C4, C25), 131.9 (C15, C18), 138.6 (C9, C30), 140.3 (C3, C24), 147.2 (C7, C28), 148.6 (C1, C22), 155.8 (C11, C32), 156.3 (C5, C26), 160.2 (C20), 179.3 (C1a, C3a) ppm.

FT-IR spectroscopy: $v=2996(v\{C-H\}), 2931(v\{C-H\}), 2844(v\{C-H\}), 1600\left(v_{a}\{\mathrm{OAc}\}\right), 1406$ $\left(v_{s}\{O A c\}\right), 831(v\{P-F\}), 650(\delta\{p y-H\}), 556(\delta\{F-P-F\}) c^{-1}$.

Elemental analysis: Calcd. for $\mathrm{C}_{37} \mathrm{H}_{39} \mathrm{~N}_{6} \mathrm{O}_{5} \mathrm{PF}_{6} \mathrm{Zn}_{2}$ : C 48.10\%; $\mathrm{H} \quad 4.26 \% ; \mathrm{N} 9.11 \%$ Found:

\subsubsection{SYNTHESIS OF $\left[\mathrm{ZN}_{2}{ }_{2}\left(\mathrm{H}_{2} \mathrm{~L}^{2}\right)(\mu-\mathrm{OAC})_{2}\right] \mathrm{PF}_{6}$}

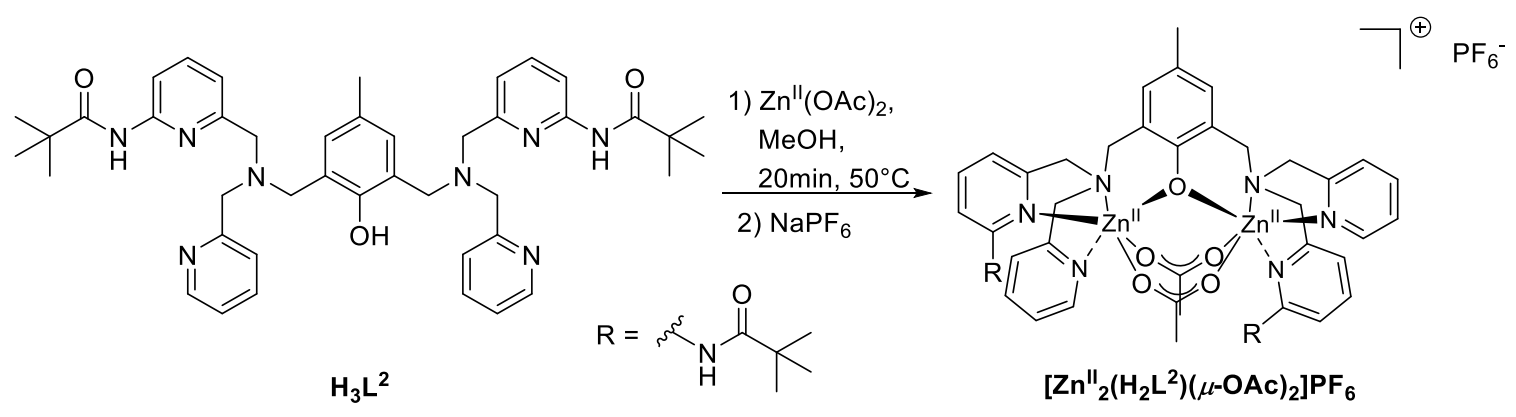

Yield: $116.6 \mathrm{mg}(76 \%)$.

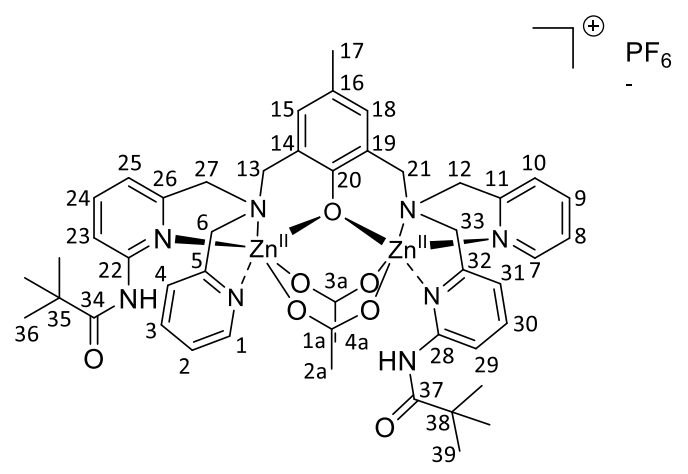

CCDC no.: 998889

HRMS $\left(\mathrm{ESI}^{+}, \mathrm{CH}_{3} \mathrm{CN}\right): \mathrm{m} / \mathrm{z}=429.1283\left(\left[\mathrm{C}_{43} \mathrm{H}_{50} \mathrm{~N}_{8} \mathrm{O}_{3} \mathrm{Zn}_{2}\right]^{2+}\right.$, calcd. 429.1268).

${ }^{1} \mathrm{H}$ NMR $\left(500.13 \mathrm{MHz}, \mathrm{CD}_{3} \mathrm{CN}\right): \delta=1.27(\mathrm{~s}, 18 \mathrm{H}, H 36, H 39), 1.91(\mathrm{~s}, 3 \mathrm{H}, H 17), 2.12(\mathrm{~s}, 6 \mathrm{H}, H 2 \mathrm{a}$, $H 4 \mathrm{a}), 3.19\left(\mathrm{~d},{ }^{2} \mathrm{~J}=11.37 \mathrm{~Hz}, 2 \mathrm{H}, H 13, H 21\right), 3.42-3.56(\mathrm{~m}, 4 \mathrm{H}, H 6, H 12), 3.82\left(\mathrm{~d},{ }^{2} \mathrm{~J}=11.74 \mathrm{~Hz}, 2 \mathrm{H}\right.$, $H 13, H 21), 3.97\left(\mathrm{~d},{ }^{2} \mathrm{~J}=15.04 \mathrm{~Hz}, 2 \mathrm{H}, H 27, H 33\right), 4.59\left(\mathrm{~d},{ }^{2} \mathrm{~J}=15.04 \mathrm{~Hz}, 2 \mathrm{H}, H 27, H 33\right), 6.40(\mathrm{~s}, 2 \mathrm{H}$, 
$H 15, H 18), 6.59\left(\mathrm{~d},{ }^{3} \mathrm{~J}=7.70 \mathrm{~Hz}, 2 \mathrm{H}, H 4, H 10\right), 7.05\left(\mathrm{dd},{ }^{3} \mathrm{~J}=6.79 \mathrm{~Hz},{ }^{3} \mathrm{~J}=6.05 \mathrm{~Hz}, 2 \mathrm{H}, H 2, H 8\right), 7.16$ (d, $\left.{ }^{3} \mathrm{~J}=7.70 \mathrm{~Hz}, 2 \mathrm{H}, H 25, H 31\right), 7.38\left(\mathrm{td},{ }^{3} \mathrm{~J}=7.70 \mathrm{~Hz},{ }^{4} \mathrm{~J}=1.47 \mathrm{~Hz}, 2 \mathrm{H}, H 3, H 9\right), 7.85\left(\mathrm{t},{ }^{3} \mathrm{~J}=7.70 \mathrm{~Hz}\right.$, $2 \mathrm{H}, H 24, H 30), 8.14\left(\mathrm{~d},{ }^{3} \mathrm{~J}=8.44 \mathrm{~Hz}, 2 \mathrm{H}, H 23, H 29\right), 8.39(\mathrm{~m}, 2 \mathrm{H}, H 1, H 7), 9.89(\mathrm{~s}, 2 \mathrm{H}, \mathrm{NH}) \mathrm{ppm}$.

${ }^{13} \mathrm{C}$ NMR $\left(100.61 \mathrm{MHz}, \mathrm{CD}_{3} \mathrm{CN}\right): \delta=19.2$ (C17), 25.3 (C2a, C4a) 26.7 (C36, C39), 39.8 (C35, C38), 57.1 (C6, C12), 60.2 (C13, C21), 61.1 (C27, C33), 115.9 (C23, C29), 119.1 (C25, C31), 121.7 (C4, C10), 122.9 (C2/C8/C14/C19), 122.9 (C2/C8/C14/C19), 124.7 (C16), 130.4 (C15, C18), 138.9 (C3, C9), 141.0 (C24, C30), 146.2 (C1, C7), 151.98 (C22, C28), 154.4 (C26, C32), 155.1 (C5, C11), 159.0 (C20), 177.8 (C34, C37), 178.9 (C1a, C3a) ppm.

${ }^{31}$ P NMR (100.61 MHz, CD 3 CN): $\delta=-144.3$ (sep, $\left.\mathrm{PF}_{6}{ }^{-}\right)$ppm.

FT-IR spectroscopy: v = $3293(v\{\mathrm{~N}-\mathrm{H}\}), 2976(\mathrm{v}\{\mathrm{C}-\mathrm{H}\}), 2924(\mathrm{v}\{\mathrm{C}-\mathrm{H}\}), 2877(\mathrm{v}\{\mathrm{C}-\mathrm{H}\}), 1700(\mathrm{v}\{\mathrm{C}=\mathrm{O}\})$, $1600\left(v_{a}\{\mathrm{OAc}\}\right), 1536(\mathrm{v}\{\mathrm{C}-\mathrm{N}\}, \delta\{\mathrm{CNH}\}), 1458\left(\mathrm{v}\left\{\mathrm{C}-\left(\mathrm{CH}_{3}\right)_{3}\right\}\right), 1406\left(\mathrm{v}_{\mathrm{s}}\{\mathrm{OAc}\}\right), 1277(\mathrm{v}\{\mathrm{C}-\mathrm{N}\}), 841(\mathrm{v}\{\mathrm{P}-$ F\}), 650 ( $\delta\{$ py-H\}), 557 ( $\delta\{F-P-F\}) \mathrm{cm}^{-1}$.

Elemental analysis: Calcd. for $\mathrm{C}_{47} \mathrm{H}_{57} \mathrm{~N}_{8} \mathrm{O}_{7} \mathrm{PF}_{6} \mathrm{Zn}_{2}$ : C 50.32\%; $\mathrm{H} \mathrm{5.12 \% ;} \mathrm{N} \mathrm{9.99 \%}$ Found: $\quad$ C $50.09 \%$; H $5.26 \%$; N $9.96 \%$.

\subsubsection{SYNTHESIS OF $\left[\mathrm{ZN}_{2}{ }_{2}\left(\mathrm{H}_{2} \mathrm{SIM}^{1}\right)(\mu-\mathrm{OAC})(\mathrm{OH})\right] \mathrm{PF}_{6}$}

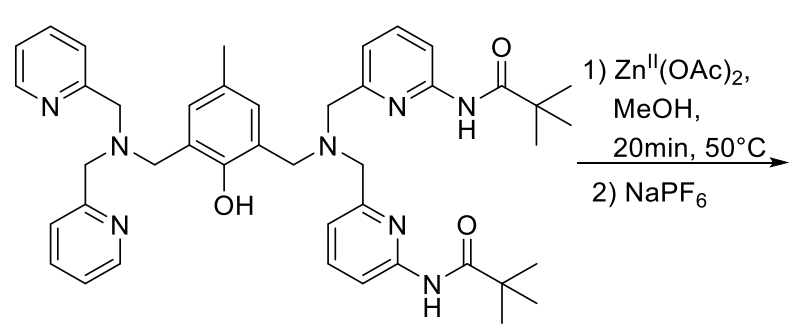

$\mathrm{H}_{3} \mathrm{SIM}^{1}$

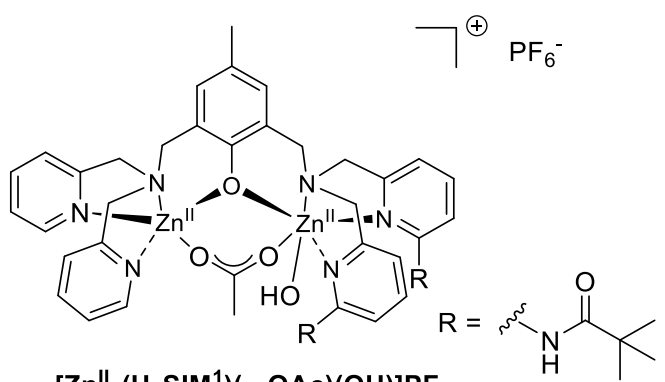

$\left[\mathrm{Zn}_{2}{ }_{2}\left(\mathrm{H}_{2} \mathrm{SIM}^{1}\right)(\mu-\mathrm{OAc})(\mathrm{OH})\right] P F_{6}$

Yield: $23.2 \mathrm{mg}(16 \%)$.

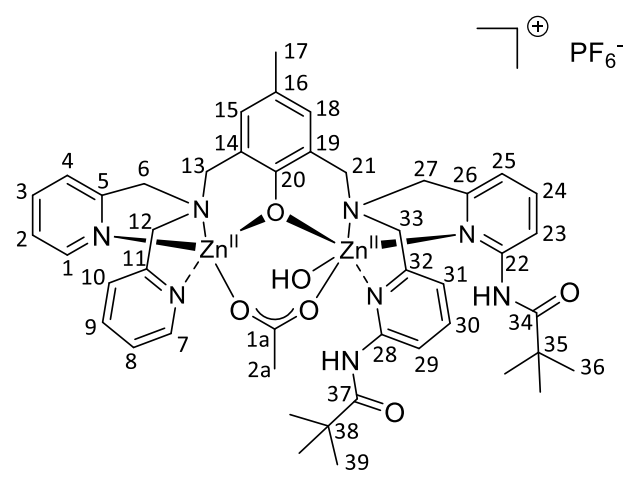

CCDC no.: 998887

HRMS $\left(\mathrm{ESI}^{+}, \mathrm{CH}_{3} \mathrm{CN}\right): \mathrm{m} / \mathrm{z}=429.1298\left(\left[\mathrm{C}_{43} \mathrm{H}_{50} \mathrm{~N}_{8} \mathrm{O}_{3} \mathrm{Zn}_{2}\right]^{2+}\right.$, calcd. 429.1268); 877.2596 $\left(\left[\mathrm{C}_{43} \mathrm{H}_{50} \mathrm{~N}_{8} \mathrm{O}_{3} \mathrm{Zn}_{2} \mathrm{~F}\right]^{+}\right.$, calcd. 877.2525).

${ }^{1} \mathrm{H}$ NMR (500.13 MHz, CD ${ }_{3} \mathrm{CN}$ ): $\delta=0.85$ (s, 9H, H36/H39), 1.35 (s, 9H, H36/H39), 1.99 (s, 3H, H17), $2.03(\mathrm{~s}, 3 \mathrm{H}, \mathrm{H} 2 \mathrm{a}), 3.36\left(\mathrm{~d},{ }^{2} \mathrm{~J}=11.74 \mathrm{~Hz}, 1 \mathrm{H}, H 13\right), 3.40\left(\mathrm{~d},{ }^{2} \mathrm{~J}=11.74 \mathrm{~Hz}, 1 \mathrm{H}, H 21\right), 3.56(\mathrm{~d}$, 
$\left.{ }^{2} \mathrm{~J}=15.77 \mathrm{~Hz}, 1 \mathrm{H}, H 27\right), 3.67\left(\mathrm{~d},{ }^{2} \mathrm{~J}=15.41 \mathrm{~Hz}, 1 \mathrm{H}, H 27\right), 3.78\left(\mathrm{~d},{ }^{2} \mathrm{~J}=16.87 \mathrm{~Hz}, 1 \mathrm{H}, H 12\right), 3.84(\mathrm{~d}$, $\left.{ }^{2} \mathrm{~J}=17.24 \mathrm{~Hz}, 1 \mathrm{H}, H 12\right), 3.92\left(\mathrm{~d},{ }^{2} \mathrm{~J}=14.31 \mathrm{~Hz}, 1 \mathrm{H}, H 33\right), 4.03\left(\mathrm{~d},{ }^{2} \mathrm{~J}=11.37 \mathrm{~Hz}, 1 \mathrm{H}, H 13\right), 4.12-4.21$ $(\mathrm{m}, 2 \mathrm{H}, H 6, H 21), 4.28\left(\mathrm{~d},{ }^{2} \mathrm{~J}=17.97 \mathrm{~Hz}, 1 \mathrm{H}, H 6\right), 4.38\left(\mathrm{~d},{ }^{2} \mathrm{~J}=14.31 \mathrm{~Hz}, 1 \mathrm{H}, H 33\right), 6.29(\mathrm{~d}$, $\left.{ }^{3} \mathrm{~J}=7.34 \mathrm{~Hz}, 1 \mathrm{H}, H 25\right), 6.61\left(\mathrm{~d},{ }^{4} \mathrm{~J}=1.83 \mathrm{~Hz}, 1 \mathrm{H}, H 15\right), 6.64\left(\mathrm{~d},{ }^{4} \mathrm{~J}=1.83 \mathrm{~Hz}, 1 \mathrm{H}, H 18\right), 7.10(\mathrm{~d}$, $\left.{ }^{3} \mathrm{~J}=7.70 \mathrm{~Hz}, 1 \mathrm{H}, \mathrm{H31}\right), 7.14\left(\mathrm{~d},{ }^{3} \mathrm{~J}=7.70 \mathrm{~Hz}, 1 \mathrm{H}, H 10\right), 7.25\left(\mathrm{dd},{ }^{3} \mathrm{~J}=8.44 \mathrm{~Hz},{ }^{3} \mathrm{~J}=7.70 \mathrm{~Hz}, 1 \mathrm{H}, H 24\right)$, $7.36\left(\mathrm{dd},{ }^{3} \mathrm{~J}=6.20 \mathrm{~Hz}, 1 \mathrm{H}, H 8\right), 7.53\left(\mathrm{~d},{ }^{3} \mathrm{~J}=8.07 \mathrm{~Hz}, 1 \mathrm{H}, H 4\right), 7.60\left(\mathrm{dd},{ }^{3} \mathrm{~J}=7.59 \mathrm{~Hz},{ }^{3} \mathrm{~J}=6.20 \mathrm{~Hz}\right.$, $1 \mathrm{H}, H 2), 7.80\left(\mathrm{td},{ }^{3} \mathrm{~J}=7.79 \mathrm{~Hz},{ }^{4} \mathrm{~J}=1.65 \mathrm{~Hz}, 1 \mathrm{H}, H 9\right), 7.81-7.87(\mathrm{~m}, 2 \mathrm{H}, H 23, H 30), 8.08$ (td, $\left.{ }^{3} \mathrm{~J}=7.79 \mathrm{~Hz},{ }^{4} \mathrm{~J}=1.65 \mathrm{~Hz}, 1 \mathrm{H}, H 3\right), 8.33\left(\mathrm{~d},{ }^{3} \mathrm{~J}=8.44 \mathrm{~Hz}, 1 \mathrm{H}, H 29\right), 8.59\left(\mathrm{~d},{ }^{3} \mathrm{~J}=4.77 \mathrm{~Hz}, 1 \mathrm{H}, H 7\right)$, $8.80\left(\mathrm{~d},{ }^{3} \mathrm{~J}=5.14 \mathrm{~Hz}, 1 \mathrm{H}, H 1\right) \mathrm{ppm}$.

${ }^{13} \mathrm{C}$ NMR (125.76 MHz, CD 3 CN): $\delta$ = 20.2 (C17), 27.8 (C16/C39), 27.9 (C16/C39), 40.5 (C35/C38), 41.2 (C35/C38), 57.7 (C12), 58.9 (C13), 59.9 (C27), 61.3 (C33), 61.4 (C6), 61.7 (C21), 114.6 (C23), 115.7 (C29), 117.7 (C25), 119.2 (C31), 124.7 (C10/C14), 124.7 (C10/C14), 125.0 (C4), 125.3 (C8/C19), 125.3 (C8/C19), 126.1 (C2), 127.4 (C16), 133.2 (C18), 133.4 (C15), 139.9 (C24), 141.4 (C30), 141.7 (C9), 142.6 (C3), 149.1 (C7), 149.8 (C1), 153.4 (C22/C28), 153.7 (C26), 154.3 (C32), 155.1 (C22/C28), 156.6 (C5), 157.1 (C11), 160.0 (C20), 179.60 (C34/C37), 179.6 (C34/C37), 180.7 (C1a) ppm.

${ }^{31} \mathrm{P}$ NMR (100.61 MHz, $\left.\mathrm{CD}_{3} \mathrm{CN}\right): \delta=-144.3\left(\mathrm{sep}, \mathrm{PF}_{6}{ }^{-}\right) \mathrm{ppm}$.

FT-IR spectroscopy: $v=2982(v\{C-H\}), 2937(v\{C-H\}), 2876(v\{C-H\}), 1687(v\{C=O\}), 1581\left(v_{a}\{\mathrm{OAc}\}\right)$,

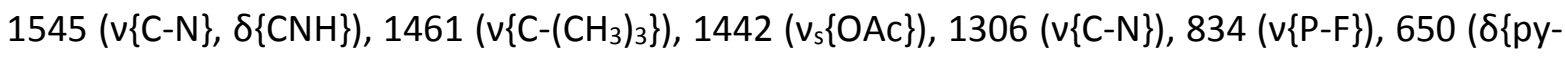
$\mathrm{H}\}), 556(\delta\{\mathrm{F}-\mathrm{P}-\mathrm{F}\}) \mathrm{cm}^{-1}$.

Elemental analysis: Calcd. for $\mathrm{C}_{45} \mathrm{H}_{55} \mathrm{~N}_{8} \mathrm{O}_{6} \mathrm{PF}_{6} \mathrm{Zn}_{2}: \quad \mathrm{C} 50.06 \%$; $\mathrm{H}$ 5.13\%; $\mathrm{N} 10.38 \%$ Found:

C $50.46 \%$; H $5.38 \%$; N $10.57 \%$.

\subsubsection{SYNTHESIS OF $\left[\mathrm{ZN}_{2}{ }_{2}\left(\mathrm{SIM}^{2}\right)(\mu-\mathrm{OAC})_{2}\right] \mathrm{PF}_{6}$}

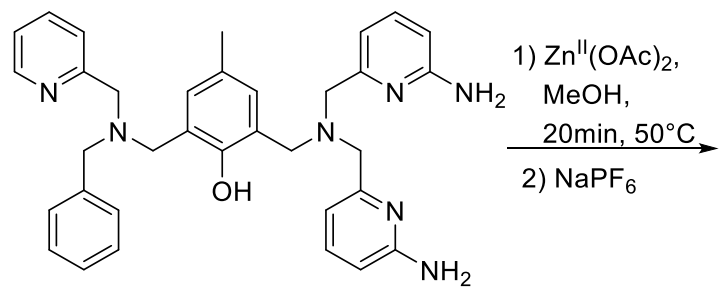

$\mathrm{HSIM}^{2}$

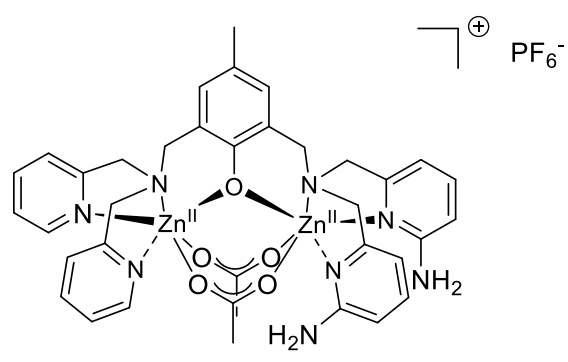

$\left[\mathrm{Zn}_{2}{ }_{2}\left(\mathrm{SIM}^{2}\right)(\mu-\mathrm{OAC})_{2}\right] \mathrm{PF}_{6}$

Yield: $47.5 \mathrm{mg}(50 \%)$. 


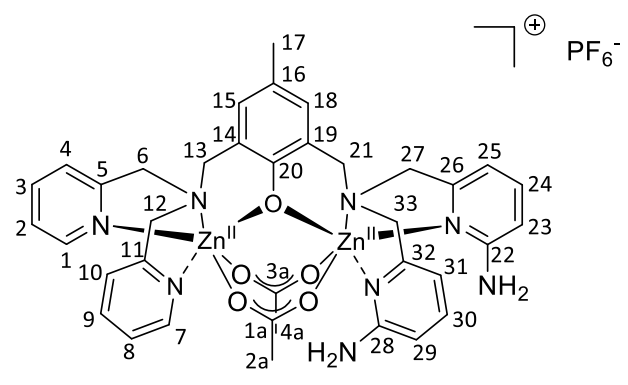

CCDC no.: 998890

HRMS $\left(\mathrm{ESI}^{+}, \mathrm{CH}_{3} \mathrm{CN}\right): \mathrm{m} / \mathrm{z}=344.0747\left(\left[\mathrm{C}_{33} \mathrm{H}_{34} \mathrm{~N}_{8} \mathrm{OZn}_{2}\right]^{2+}\right.$, calcd. 344.0698); 749.1529 $\left(\left[\mathrm{C}_{33} \mathrm{H}_{34} \mathrm{~N}_{8} \mathrm{OZn}_{2} \mathrm{CH}_{3} \mathrm{COO}\right]^{+}\right.$, calcd. 749.1524).

${ }^{1} \mathrm{H}$ NMR (500.13 MHz, CD $3 \mathrm{CN}$ ): $\delta=1.85$ (s, 3H, H2a/H4a), 1.94 (s, 3H, H17), 2.05 (s, 3H, H2a/H4a), $3.02\left(\mathrm{~d},{ }^{2} \mathrm{~J}=11.37 \mathrm{~Hz}, 1 \mathrm{H}, H 13 / H 21\right), 3.17\left(\mathrm{~d},{ }^{2} \mathrm{~J}=15.77 \mathrm{~Hz}, 1 \mathrm{H}, H 33\right), 3.24\left(\mathrm{~d},{ }^{2} \mathrm{~J}=15.77 \mathrm{~Hz}, 1 \mathrm{H}\right.$, H33), $3.28\left(\mathrm{~d},{ }^{2} \mathrm{~J}=10.64 \mathrm{~Hz}, 1 \mathrm{H}, H 13 / H 21\right), 3.35\left(\mathrm{~d},{ }^{2} \mathrm{~J}=16.51 \mathrm{~Hz}, 1 \mathrm{H}, H 12\right), 3.50\left(\mathrm{~d},{ }^{2} \mathrm{~J}=16.51 \mathrm{~Hz}\right.$, $1 \mathrm{H}, H 12), 3.57\left(\mathrm{~d},{ }^{2} \mathrm{~J}=13.94 \mathrm{~Hz}, 1 \mathrm{H}, H 27\right), 3.80\left(\mathrm{~d},{ }^{2} \mathrm{~J}=11.37 \mathrm{~Hz}, 1 \mathrm{H}, H 13 / H 21\right), 4.03$ (d, $\left.{ }^{2} \mathrm{~J}=14.67 \mathrm{~Hz}, 1 \mathrm{H}, H 6\right), 4.33\left(\mathrm{~d},{ }^{2} \mathrm{~J}=10.64 \mathrm{~Hz}, 1 \mathrm{H}, H 13 / H 21\right), 4.40\left(\mathrm{dd},{ }^{2} \mathrm{~J}=14.12 \mathrm{~Hz},{ }^{2} \mathrm{~J}=10.09 \mathrm{~Hz}\right.$, $2 \mathrm{H}, H 6, H 27), 5.58$ (d, ${ }^{3} \mathrm{~J}=7.34 \mathrm{~Hz}, 1 \mathrm{H}, H 31$ ), 5.78 (bs, $2 \mathrm{H}, \mathrm{NH}_{2}$ ), 6.05 (d, ${ }^{3} \mathrm{~J}=8.10 \mathrm{~Hz}, 1 \mathrm{H}, H 29$ ), $6.29\left(\mathrm{~d},{ }^{4} \mathrm{~J}=1.83 \mathrm{~Hz}, 1 \mathrm{H}, H 15 / H 18\right), 6.45-6.53$ (m, 3H, H10, H15/H18, H23), 6.55 (d, 3 J = $7.34 \mathrm{~Hz}$, $1 \mathrm{H}, \mathrm{H} 25), 6.67$ (bs, $2 \mathrm{H}, \mathrm{NH}_{2}$ ), $6.88\left(\mathrm{dd},{ }^{3} \mathrm{~J}=8.44 \mathrm{~Hz},{ }^{3} \mathrm{~J}=7.34 \mathrm{~Hz}, 1 \mathrm{H}, H 30\right), 6.98-7.03(\mathrm{~m}, 1 \mathrm{H}, H 8)$, $7.33\left(\mathrm{td},{ }^{3} \mathrm{~J}=7.61 \mathrm{~Hz},{ }^{4} \mathrm{~J}=1.65 \mathrm{~Hz}, 1 \mathrm{H}, \mathrm{H} 9\right), 7.41-7.50(\mathrm{~m}, 3 \mathrm{H}, \mathrm{H} 2, H 4, H 24), 7.93\left(\mathrm{td},{ }^{3} \mathrm{~J}=7.79 \mathrm{~Hz}\right.$, $4 \mathrm{~J}=1.65 \mathrm{~Hz}, 1 \mathrm{H}, H 3), 8.28(\mathrm{~m}, 1 \mathrm{H}, H 7), 8.72(\mathrm{~m}, 1 \mathrm{H}, H 1) \mathrm{ppm}$.

${ }^{13} \mathrm{C}$ NMR (100.62 MHz, CD $3 \mathrm{CN}$ ): $\delta=20.2$ (C17), 25.0 (C2a/C4a) 25.9 (C2a/C4a), 58.7 (C12), 58.7 (C33), 60.4 (C13/C21), 60.7 (C6), 60.8 (C27), 61.0 (C13/C21), 108.7 (C29), 110.5 (C31), 110.8 (C23/C25), 112.7 (C23/C25), 122.0 (C10), 123.6 (C8), 124.4 (C19/C14), 124.9 (C2/C19/C14), 125.0 (C2/C19/C14), 125.2 (C4/C16), 125.3 (C4/C16), 131.6 (C15/C18), 131.7 (C15/C18), 138.9 (C9), 139.2 (C30), 140.3 (C3), 140.6 (C24), 147.1 (C7), 148.5 (C1), 153.9 (C26/C32), 153.9 (C26/C32), 156.1 (C5/C11), 156.1 (C5/C11), 160.0 (C28), 160.4 (C22), 160.51 (C20), 179.1 (C1a/C3a), 179.3 (C1a/C3a) ppm.

${ }^{31} \mathrm{P}$ NMR (100.61 MHz, $\left.\mathrm{CD}_{3} \mathrm{CN}\right): \delta=-144.3\left(\mathrm{sep}, \mathrm{PF}_{6}{ }^{-}\right) \mathrm{ppm}$.

FT-IR spectroscopy: $v=3484(v\{\mathrm{~N}-\mathrm{H}\}), 3332(\mathrm{v}\{\mathrm{N}-\mathrm{H}\}), 3218(\mathrm{v}\{\mathrm{N}-\mathrm{H}\}), 2984(\mathrm{v}\{\mathrm{C}-\mathrm{H}\}), 2935(\mathrm{v}\{\mathrm{C}-\mathrm{H}\})$, $2906(v\{C-H\}), 1643(\delta\{C N H\}), 1606\left(v_{a}\{\mathrm{OAC}\}\right), 1476\left(v_{s}\{\mathrm{OAc}\}\right), 1275(\mathrm{v}\{\mathrm{C}-\mathrm{N}\}), 827(\mathrm{v}\{\mathrm{P}-\mathrm{F}\}), 658$ (ठ\{py-H\}), $557(\delta\{F-P-F\}) \mathrm{cm}^{-1}$.

Elemental analysis: Calcd. for $\mathrm{C}_{37} \mathrm{H}_{41} \mathrm{~N}_{8} \mathrm{O}_{5} \mathrm{PF}_{6} \mathrm{Zn}_{2}: \quad \mathrm{C} 46.61 \%$; $\mathrm{H} 4.33 \%$; $\mathrm{N} 11.75 \%$ Found: $\quad$ C $46.42 \%$; H $4.34 \%$; N $11.74 \%$. 
11.9.6 SYNTHESIS OF $\left[\mathrm{ZN}_{2}\left(\mathrm{H}_{2} \mathrm{SIM}^{3}\right)(\mu-\mathrm{OAC})(\mathrm{OH})\right]$
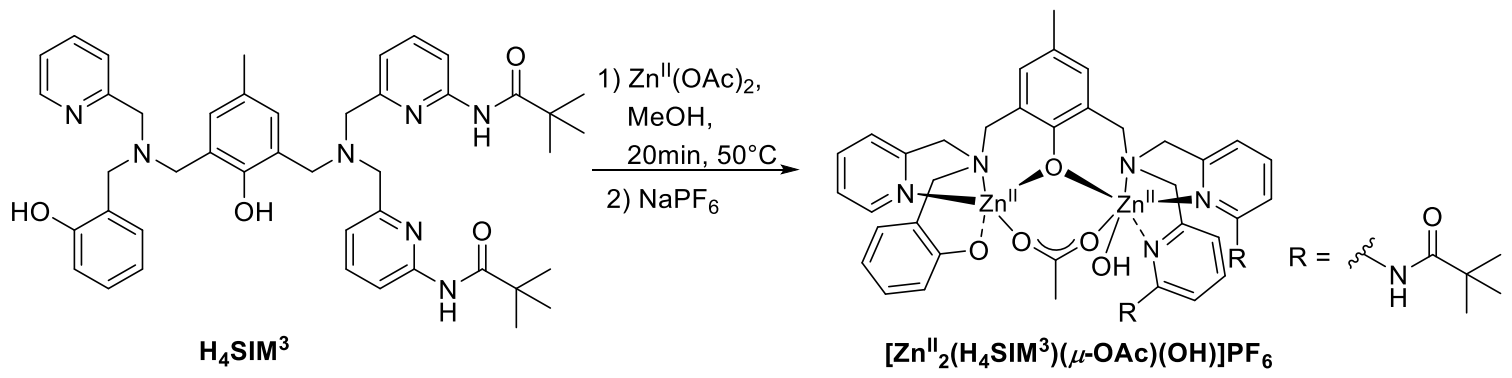

Yield: $85.7 \mathrm{mg}(67 \%)$.

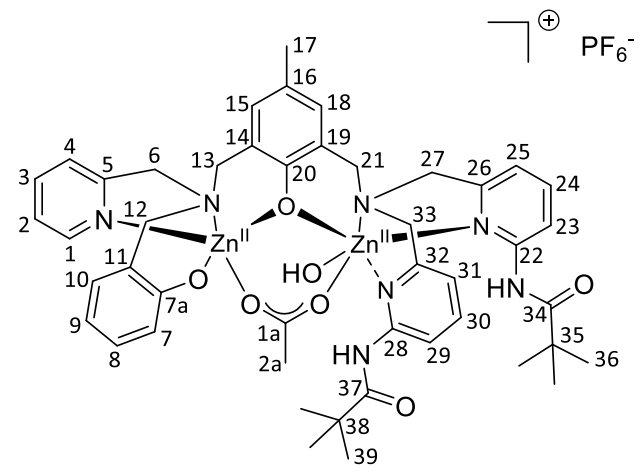

CCDC no.: 1009842

HRMS $\left(\mathrm{ESI}^{+}, \mathrm{CH}_{3} \mathrm{CN}\right): \mathrm{m} / \mathrm{z}=434.6237\left(\left[\mathrm{C}_{44} \mathrm{H}_{51} \mathrm{~N}_{7} \mathrm{O}_{4} \mathrm{Zn}_{2}\right]^{2+}\right.$, calcd. 434.6287).

${ }^{1} \mathrm{H}$ NMR (500.13 MHz, CD ${ }_{3} \mathrm{CN}\left(0.4\right.$ vol\% $\left.\left.\mathrm{HClO}_{4}\right)\right): \delta=1.35$ (s, 18H, H36, H39), 1.95 (s, 3H, H2a), $2.29(\mathrm{~s}, 3 \mathrm{H}, H 17), 3.82\left(\mathrm{~d},{ }^{2} \mathrm{~J}=16.51 \mathrm{~Hz}, 2 \mathrm{H}, H 27, H 33\right), 3.96(\mathrm{~s}, 2 \mathrm{H}, H 21), 4.11\left(\mathrm{~d},{ }^{2} \mathrm{~J}=16.14 \mathrm{~Hz}\right.$, $2 \mathrm{H}, H 27, H 33), 4.40(\mathrm{~s}, 2 \mathrm{H}, H 6, H 12), 4.41(\mathrm{~s}, 2 \mathrm{H}, H 6, H 12), 4.43(\mathrm{~s}, 2 \mathrm{H}, H 13), 6.87\left(\mathrm{~d},{ }^{3} \mathrm{~J}=8.07 \mathrm{~Hz}\right.$, $1 \mathrm{H}, H 7), 6.92\left(\mathrm{t},{ }^{3} \mathrm{~J}=7.34 \mathrm{~Hz}, 1 \mathrm{H}, H 9\right), 7.20-7.39$ (m, 9H, H4, H8, H10, H15, H18, H23, H25, H29, $H 31), 7.39\left(\mathrm{dd},{ }^{3} \mathrm{~J}=6.97 \mathrm{~Hz},{ }^{3} \mathrm{~J}=5.50 \mathrm{~Hz}, 1 \mathrm{H}, H 2\right), 7.82\left(\mathrm{t},{ }^{3} \mathrm{~J}=7.70 \mathrm{~Hz}, 1 \mathrm{H}, H 3\right), 8.02\left(\mathrm{t},{ }^{3} \mathrm{~J}=7.89 \mathrm{~Hz}\right.$, $2 \mathrm{H}, H 24, H 30), 8.48\left(\mathrm{~d},{ }^{3} \mathrm{~J}=4.40 \mathrm{~Hz}, 1 \mathrm{H}, H 1\right), 9.26(\mathrm{~s}, 2 \mathrm{H}, \mathrm{NH}) \mathrm{ppm}$.

${ }^{13} \mathrm{C} \mathrm{NMR}\left(125.76 \mathrm{MHz}, \mathrm{CD}_{3} \mathrm{CN}\left(0.4\right.\right.$ vol\% $\left.\left.\mathrm{HClO}_{4}\right)\right): \delta=20.5$ (C17), 20.7 (C2a), 27.4 (C36), 41.9 (C35), 52.0 (C21), 55.6 (C27), 56.7 (C6, C12/C13), 56.8 (C6, C12/C13), 116.4 (C23), 116.5 (C7), 117.5 (C7a), 121.8 (C10), 121.9 (C25), 125.3 (C4), 125.5 (C2), 132.2 (C16), 132.7 (C8/C9), 133.2 (C8/C9), 135.2 (C15/C18), 137.1 (C15/C18), 139.9 (C3), 143.5 (C24), 149.4 (C1), 153.4 (C26), 153.8 (C14/C19), 156.4 (C5/C11), 180.8 (C1a) ppm.

FT-IR spectroscopy: $v=2978(v(C-H)), 2932(v(C-H)), 2867(v(C-H)), 1681(v(C=O)), 1581\left(v_{a}(O A C)\right)$, $1453\left(\mathrm{v}\left(\mathrm{C}-\left(\mathrm{CH}_{3}\right)_{3}\right), 1433\left(\mathrm{v}_{\mathrm{s}}(\mathrm{OAc})\right), 1305(\mathrm{v}(\mathrm{C}-\mathrm{N})), 770(\delta(\mathrm{py}-\mathrm{H})) \mathrm{cm}^{-1}\right.$.

Elemental analysis: $\quad$ Calcd. for $\mathrm{C}_{46} \mathrm{H}_{55} \mathrm{~N}_{7} \mathrm{O}_{7} \mathrm{Zn}_{2}: \quad$ C 58.24\%; $\mathrm{H} 5.84 \%$; $\mathrm{N} 10.33 \%$

Found: $\quad$ C $58.02 \%$; H $5.57 \%$; N $10.19 \%$. 


\subsection{SYNTHESES OF DIIRON(II) COMPLEXES}

\subsubsection{General Procedure}

The ligand (68.6 $\mu \mathrm{mol}, 1.0 \mathrm{eq})$ was dissolved under inert atmosphere in anhydrous, degassed methanol $(2.5 \mathrm{ml})$. The solution was treated with iron(II) acetate $(137 \mu \mathrm{mol}, 2.0 \mathrm{eq})$ and stirred at room temperature overnight. Sodium hexafluorophosphate $(140 \mu \mathrm{mol}, 2.0 \mathrm{eq})$ was added and the mixture stirred for ten minutes before filtration through a syringe filter. Crystallization from the filtrate was achieved with diffusion of diethyl ether, whereby yellow crystals were obtained, which were collected by filtration.

\subsubsection{SYNTHESIS OF $\left[\mathrm{FE}_{2}{ }_{2}\left(\mathrm{H}_{2} \mathrm{SIM}^{1}\right)(\mu-\mathrm{OAC})_{2}\right] \mathrm{PF}_{6}$}<smiles>Cc1cc(CN(Cc2cccc(CN(Cc3ccccn3)Cc3ccccn3)n2)Cc2cccc(NC(=O)C(C)(C)C)n2)c(O)c(C(=O)NC(=O)C(C)(C)C)c1</smiles>

1) $\mathrm{Fe}^{\prime \prime}(\mathrm{OAc})_{2}$ $\mathrm{MeOH}$, $12 \mathrm{~h}, \mathrm{rt}$ 2) $\mathrm{NaPF}_{6}$

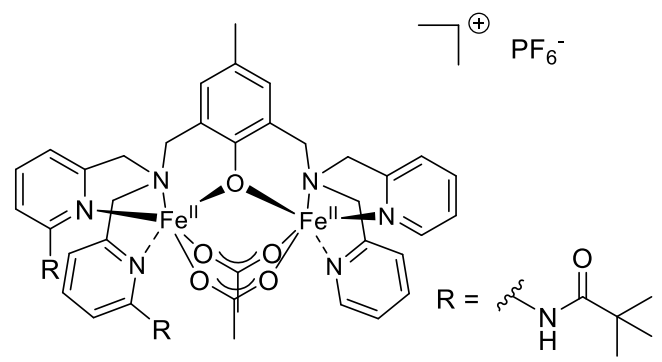

$\left[\mathrm{Fe}_{2}{ }_{2}\left(\mathrm{H}_{2} \mathrm{SIM}^{1}\right)(\mu-\mathrm{OAc})_{2}\right] \mathrm{PF}_{6}$

Yield: $37.0 \mathrm{mg}$ (49\%).

HRMS $\left(\mathrm{ESI}^{+}, \mathrm{CH}_{2} \mathrm{Cl}_{2}\right): m / z=957.3087\left(\left[\mathrm{C}_{47} \mathrm{H}_{57} \mathrm{~N}_{8} \mathrm{O}_{7} \mathrm{Fe}_{2}\right]^{+}\right.$, calcd. 957.3046).

${ }^{1} \mathrm{H}$ NMR $\left(500.13 \mathrm{MHz}, \mathrm{CD}_{3} \mathrm{CN}\right): \delta=-31.71$ (bs, $\left.1 \mathrm{H}\right),-17.11$ (bs, $\left.1 \mathrm{H}\right),-12.52$ (bs, $\left.1 \mathrm{H}\right),-5.86$ (bs, $\left.1 \mathrm{H}\right)$, -1.20 (s, 1H), $3.86(\mathrm{~s}, 9 \mathrm{H}), 4.08(\mathrm{~s}, 9 \mathrm{H}), 7.49(\mathrm{~s}, 1 \mathrm{H}), 9.48(\mathrm{~s}, 1 \mathrm{H}), 9.67(\mathrm{~s}, 1 \mathrm{H}), 12.52(\mathrm{~s}, 1 \mathrm{H}), 17.21$ (bs, 1H), $22.61(\mathrm{~s}, 1 \mathrm{H}), 27.49(\mathrm{~s}, 1 \mathrm{H}), 32.89(\mathrm{~s}, 1 \mathrm{H}), 36.04(\mathrm{~s}, 1 \mathrm{H}), 36.11(\mathrm{~s}, 4 \mathrm{H}), 41.10(\mathrm{~s}, 3 \mathrm{H}), 41.55$ (s, 1H), $46.06(s, 1 H), 50.39(s, 2 H), 52.42(s, 1 H), 57.01(s, 3 H), 67.36(s, 1 H), 69.39(s, 1 H), 73.79$ (s, 1H), 77.16 (s, 1H), 93.51 (s, 1H), 120.21 (bs, 1H), 148.20 (bs, 1H), 166.91 (s, 1H), $189.14(\mathrm{~s}, 1 \mathrm{H})$ ppm.

Elemental analysis: Calcd. for $\mathrm{C}_{47} \mathrm{H}_{57} \mathrm{~N}_{8} \mathrm{O}_{7} \mathrm{Fe}_{2} \mathrm{PF}_{6}$ : C 51.20\%; $\mathrm{H} 5.21 \%$; $\mathrm{N} 10.16 \%$ Found: C $51.17 \%$; H 5.30\%; N $10.05 \%$. 
11.10.3 SYNTHESIS OF $\left[\mathrm{FE}_{2}{ }_{2}\left(\mathrm{H}_{2} \mathrm{~L}^{2}\right)(\mu-\mathrm{OAC})_{2}\right] \mathrm{PF}_{6}$

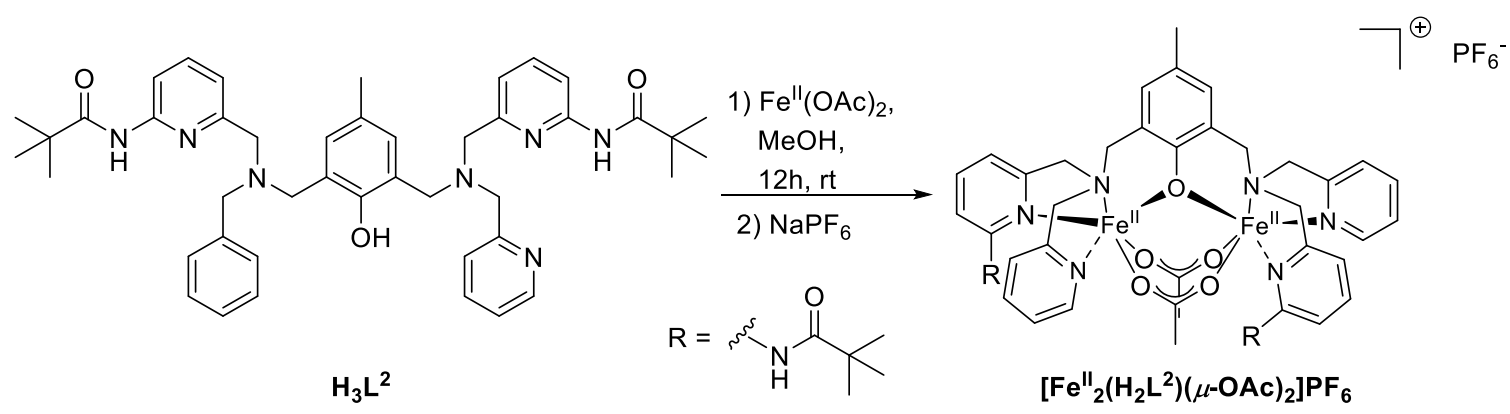

Yield: $48.2 \mathrm{mg}$ (63\%).

HRMS $\left(\mathrm{ESI}^{+}, \mathrm{CH}_{2} \mathrm{Cl}_{2}\right): \mathrm{m} / z=957.3074\left(\left[\mathrm{C}_{47} \mathrm{H}_{57} \mathrm{~N}_{8} \mathrm{O}_{7} \mathrm{Fe}_{2}\right]^{+}\right.$, calcd. 957.3046).

${ }^{1} \mathrm{H}$ NMR $\left(500.13 \mathrm{MHz}, \mathrm{CD}_{3} \mathrm{CN}\right): \delta=2.12(\mathrm{~s}, 2 \mathrm{H}), 5.40(\mathrm{~s}, 18 \mathrm{H}), 5.68(\mathrm{~s}, 2 \mathrm{H}), 5.76(\mathrm{~s}, 2 \mathrm{H}), 10.70$ (bs, 2H), 13.00 (bs, 2H), $17.06(\mathrm{~s}, 2 \mathrm{H}), 26.15(\mathrm{~s}, 2 \mathrm{H}), 29.54(\mathrm{~s}, 3 \mathrm{H}), 41.15(\mathrm{~s}, 3 \mathrm{H}), 41.37(\mathrm{~s}, 5 \mathrm{H}), 45.70$ (bs, 2H), 55.14 (s, 2H), 57.07 (s, 2H), 80.81 (bs, 2H), 107.33 (bs, 2H), 157.62 (bs, 2H), 188.50 (s, 2H) ppm.

Elemental analysis: $\quad$ Calcd. for $\mathrm{C}_{47} \mathrm{H}_{57} \mathrm{~N}_{8} \mathrm{O}_{7} \mathrm{Fe}_{2} \mathrm{PF}_{6}$ : C 51.20\%; $\mathrm{H}$ 5.21\%; N $10.16 \%$ Found: $\quad$ C $51.28 \%$; H $5.46 \%$; N $10.20 \%$. 


\subsection{SyNTHESES OF PHOSPHOESTER SUBSTRATES}

\subsubsection{SYNTHESIS OF BDNPP}<smiles>O=[N+]([O-])c1ccc(O)c([N+](=O)[O-])c1</smiles>

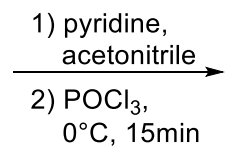

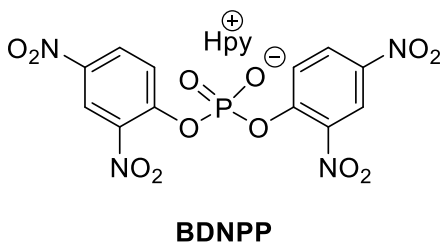

BDNPP was synthesized following published procedures with minor modifications. ${ }^{312}$

Dried 2,6-dinitrophenol (4.15 g, $22.5 \mathrm{mmol}, 2.8 \mathrm{eq}$ ) was dissolved in anhydrous acetonitrile $(45 \mathrm{ml})$ and anhydrous pyridine $(3.60 \mathrm{ml}, 3.68 \mathrm{~g}, 46.5 \mathrm{mmol}, 5.7 \mathrm{eq})$ was added. The solution was cooled to $0^{\circ} \mathrm{C}$ with an ice-water bath and freshly distilled phosphoryl chloride $(0.75 \mathrm{ml}, 1.24 \mathrm{~g}$, $8.1 \mathrm{mmol}, 1.0 \mathrm{eq}$ ) was added dropwise. After stirring for 15 minutes the mixture was poured onto an ice-water mixture $(400 \mathrm{ml})$ and the mixture was kept in the fridge overnight. The resulting precipitate was filtered and washed with a small amount of cold water. The crude product was recrystallized three times from hot acetone yielding BDNPP pyridinium salt as a white powder (2.81 g, 69\%).

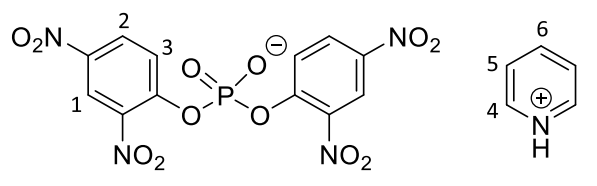

Molecular Formula: $\mathrm{C}_{17} \mathrm{H}_{12} \mathrm{~N}_{5} \mathrm{O}_{12} \mathrm{P}$

${ }^{1} \mathrm{H}$ NMR (399.89 MHz, $\left.\mathrm{CD}_{3} \mathrm{COCD}_{3}\right): \delta=8.12\left(\mathrm{~d},{ }^{3} \mathrm{~J}=9.25 \mathrm{~Hz}, 2 \mathrm{H}, H 3\right.$ ), 8.25 (bs, $\left.2 \mathrm{H}, H 5\right), 8.50$ (dd, $\left.{ }^{3} \mathrm{~J}=9.25 \mathrm{~Hz},{ }^{4} \mathrm{~J}=3.13 \mathrm{~Hz}, 2 \mathrm{H}, H 2\right), 8.71\left(\mathrm{~d},{ }^{4} \mathrm{~J}=2.78 \mathrm{~Hz}, 2 \mathrm{H}, H 1\right), 8.77\left(\mathrm{t},{ }^{3} \mathrm{~J}=7.65 \mathrm{~Hz}, 1 \mathrm{H}, H 6\right), 9.03$ (bs, $2 \mathrm{H}, \mathrm{H} 4$ ) ppm.

${ }^{31}$ P NMR $\left(161.88 \mathrm{MHz}, \mathrm{CD}_{3} \mathrm{COCD}_{3}\right): \delta=-14.2 \mathrm{ppm}$.

Elemental analysis: $\quad$ Calcd. for $\mathrm{C}_{17} \mathrm{H}_{12} \mathrm{~N}_{5} \mathrm{O}_{12} \mathrm{P}: \quad \mathrm{C} 40.09 \%$; $\mathrm{H} 2.37 \%$; $\mathrm{N} 13.75 \%$

Found:

C $40.06 \%$; $2.29 \%$; N $13.65 \%$. 


\subsubsection{SYNTHESIS OF DNPP}

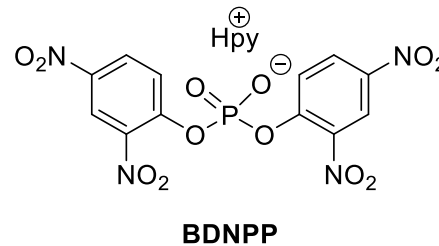
1) Dowex $50 \mathrm{WX2}$, 2) $0.3 \mathrm{M} \mathrm{NaOH}, \mathrm{rt}, 1 \mathrm{~h}$ 3) $5 \mathrm{M} \mathrm{HCl}, 0^{\circ} \mathrm{C}$
4) 2,6-lutidine, $\mathrm{Et}_{2} \mathrm{O}$<smiles>O=[N+]([O-])c1ccc(OP(=O)([O-])O)c([N+](=O)[O-])c1</smiles>

DNPP

DNPP was synthesized following a published procedure. ${ }^{313}$

BDNPP pyridinium salt $(1.28 \mathrm{~g}, 2.83 \mathrm{mmol}, 1.0 \mathrm{eq})$ dissolved in methanol $(700 \mathrm{ml})$ was passed through a Dowex 50 WX2 ion exchange column (sodium form). The solvent was removed in vacuum $\left(\mathrm{T}<40^{\circ} \mathrm{C}\right)$ and the successful exchange of the pyridinium ion for sodium ion was controlled via ${ }^{1} \mathrm{H}$ NMR spectroscopy. BDNPP sodium salt was treated with $0.3 \mathrm{M}$ sodium hydroxide solution $(50 \mathrm{ml}$ ) at room temperature for one hour. Then the solution was made strongly acidic by addition of 5M hydrochloric acid $(\sim 15 \mathrm{ml})$ and the resulting precipitate was removed by filtration. The filtrate was concentrated and the residue was taken up in diethyl ether $(50 \mathrm{ml})$. The colorless solution was filtered and 2,6-lutidine $(0.33 \mathrm{ml}, 0.30 \mathrm{~g}, 2.83 \mathrm{mmol}, 1.0 \mathrm{eq})$ was added dropwise while stirring until the solution turned slightly yellow. Filtration of the obtained precipitate and recrystallization from hot ethanol gave DNPP lutidinium salt as yellowish needles $(0.16 \mathrm{~g}, 15 \%)$.

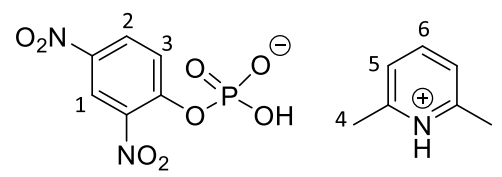

${ }^{1} \mathrm{H}$ NMR $\left(400.13 \mathrm{MHz}, \mathrm{CD}_{3} \mathrm{CN}\right): \delta=2.66(\mathrm{~s}, 6 \mathrm{H}, \mathrm{H4}), 7.47\left(\mathrm{~d},{ }^{3} \mathrm{~J}=7.92 \mathrm{~Hz}, 2 \mathrm{H}, \mathrm{H5}\right.$ ), 7.93 (dd, $\left.{ }^{3} \mathrm{~J}=9.39 \mathrm{~Hz},{ }^{5} \mathrm{~J}=0.88 \mathrm{~Hz}, 1 \mathrm{H}, H 3\right), 8.11\left(\mathrm{t},{ }^{3} \mathrm{~J}=7.92 \mathrm{~Hz}, 1 \mathrm{H}, H 6\right), 8.32\left(\mathrm{dd},{ }^{3} \mathrm{~J}=9.24 \mathrm{~Hz},{ }^{4} \mathrm{~J}=2.79 \mathrm{~Hz}\right.$, $1 \mathrm{H}, H 2), 8.57\left(\mathrm{dd},{ }^{4} \mathrm{~J}=2.93 \mathrm{~Hz},{ }^{5} \mathrm{~J}=0.88 \mathrm{~Hz}, 1 \mathrm{H}, H 1\right) \mathrm{ppm}$.

${ }^{31} \mathrm{P}$ NMR $\left(161.98 \mathrm{MHz}, \mathrm{CD}_{3} \mathrm{CN}\right): \delta=-5.0 \mathrm{ppm}$.

Elemental analysis: $\quad$ Calcd. for $\mathrm{C}_{13} \mathrm{H}_{14} \mathrm{~N}_{3} \mathrm{O}_{8} \mathrm{P}: \quad \mathrm{C} 42.06 \% ; \mathrm{H} 3.80 \% ; \mathrm{N} 11.32 \%$

Found:

C $41.92 \%$; H 3.81\%; N $11.34 \%$. 


\section{BIBLIOGRAPHY}

1. Gass, M.; Dawson-Hughes, B., Am. J. Med. 2006, 119, 3-11.

2. O'Neill, S.; MacLennan, A.; Bass, S.; Diamond, T.; Ebeling, P.; Findlay, D.; Flicker, L.; Markwell, A.; Nowson, C.; Pocock, N.; Sambrook, P.; Singh, M. F., Aust. Fam. Physician 2004, 33, 910-919.

3. Sambrook, P.; Olver, I.; Goss, A., Aust. Fam. Physician 2006, 35, 801-803.

4. Halleen, J. M.; Alatalo, S. L.; Suominen, H.; Cheng, S.; Janckila, A. J.; Väänänen, H. K., J. Bone Miner. Res. 2000, 15, 1337-1345.

5. Moss, D. W.; Raymond, F. D.; Wile, D. B., Crit. Rev. Clin. Lab. Sci. 1995, 32, 431-467.

6. Hayman, A. R.; Jones, S. J.; Boyde, A.; Foster, D.; Colledge, W. H.; Carlton, M. B.; Evans, M. J.; Cox, T. M., Development 1996, 122, 3151-3162.

7. Angel, N. Z.; Walsh, N.; Forwood, M. R.; Ostrowski, M. C.; Cassady, A. I.; Hume, D. A., J. Bone Miner. Res. 2000, 15, 103-110.

8. Vääräniemi, J.; Halleen, J. M.; Kaarlonen, K.; Ylipahkala, H.; Alatalo, S. L.; Andersson, G.; Kaija, H.; Vihko, P.; Väänänen, H. K., J. Bone Miner. Res. 2004, 19, 1432-1440.

9. Oddie, G. W.; Schenk, G.; Angel, N. Z.; Walsh, N.; Guddat, L. W.; de Jersey, J.; Cassady, A. I.; Hamilton, S. E.; Hume, D. A., Bone 2000, 27, 575-584.

10. Valizadeh, M.; Schenk, G.; Nash, K.; Oddie, G. W.; Guddat, L. W.; Hume, D. A.; de Jersey, J.; Burke, T. R.; Hamilton, S., Arch. Biochem. Biophys. 2004, 424, 154-162.

11. Desbouis, D.; Troitsky, I. P.; Belousoff, M. J.; Spiccia, L.; Graham, B., Coord. Chem. Rev. 2012, 256, 897-937.

12. Schroeder, G. K.; Lad, C.; Wyman, P.; Williams, N. H.; Wolfenden, R., Proc. Natl. Acad. Sci. USA 2006, 103, 4052-4055.

13. Cozzone, A. J., Annu. Rev. Microbiol. 1988, 42, 97-125.

14. Chang, C.; Stewart, R. C., Plant Physiol. 1998, 117, 723-731.

15. Mildvan, A. S., Proteins: Struct., Funct., Genet. 1997, 29, 401-416.

16. Shenoy, A. R.; Sreenath, N.; Podobnik, M.; Kovacevic, M.; Visweswariah, S. S., Biochemistry 2005, 44, 15695-15704.

17. Shenoy, A. R.; Capuder, M.; Draskovic, P.; Lamba, D.; Visweswariah, S. S.; Podobnik, M., J. Mol. Biol. 2007, 365, 211-225.

18. Ghanem, E.; Li, Y.; Xu, C.; Raushel, F. M., Biochemistry 2007, 46, 9032-9040.

19. Jackson, C. J.; Carr, P. D.; Kim, H. K.; Liu, J. W.; Ollis, D. L., Acta Cryst. F 2006, 62, 659661.

20. Jackson, C. J.; Carr, P. D.; Liu, J. W.; Watt, S. J.; Beck, J. L.; Ollis, D. L., J. Mol. Biol. 2007, 367, 1047-1062.

21. Mitic, N.; Smith, S. J.; Neves, A.; Guddat, L. W.; Gahan, L. R.; Schenk, G., Chem. Rev. 2006, 106, 3338-3363.

22. Schenk, G.; Mitic, N.; Gahan, L.; Smith, S.; Hadler, K., Eurobic 9: Proceedings of the 9th European Biological Inorganic Chemistry Conference 2008, 29-38.

23. Durmus, A.; Eicken, C.; Sift, B. H.; Kratel, A.; Kappl, R.; Hüttermann, J.; Krebs, B., Eur. J. Biochem. 1999, 260, 709-716.

24. Schenk, G.; Ge, Y.; Carrington, L. E.; Wynne, C. J.; Searle, I. R.; Carroll, B. J.; Hamilton, S.; de Jersey, J., Arch. Biochem. Biophys. 1999, 370, 183-189.

25. Schenk, G.; Elliott, T. W.; Leung, E.; Carrington, L. E.; Mitic, N.; Gahan, L. R.; Guddat, L. W., BMC Struct. Biol. 2008, 8:6.

26. Guddat, L. W.; McAlpine, A. S.; Hume, D.; Hamilton, S.; de Jersey, J.; Martin, J. L., Structure 1999, 7, 757-767. 
27. Sträter, N.; Jasper, B.; Scholte, M.; Krebs, B.; Duff, A. P.; Langley, D. B.; Han, R.; Averill, B. A.; Freeman, H. C.; Guss, J. M., J. Mol. Biol. 2005, 351, 233-246.

28. Daumann, L. J.; Schenk, G.; Ollis, D. L.; Gahan, L. R., Dalton Trans. 2014, 43, 910-928.

29. Schenk, G.; Mitić, N.; Hanson, G. R.; Comba, P., Coord. Chem. Rev. 2013, 257, 473-482.

30. Coleman, J. E., Annu. Rev. Biochem. 1992, 61, 897-946.

31. Millan, J. L., Purinergic Signal. 2006, 2, 335-341.

32. Millán, J. L., Mammalian alkaline phosphatases: from biology to applications in medicine and biotechnology, WILEY-VCH: Weinheim, 2006.

33. Narisawa, S.; Huang, L.; Iwasaki, A.; Hasegawa, H.; Alpers, D. H.; Millan, J. L., Mol. Cell. Biol. 2003, 23, 7525-7530.

34. Narisawa, S.; Hoylaerts, M. F.; Doctor, K. S.; Fukuda, M. N.; Alpers, D. H.; Millan, J. L., Am. J. Physiol. 2007, 293, G1068-G1077.

35. Akiba, Y.; Mizumori, M.; Guth, P. H.; Engel, E.; Kaunitz, J. D., Am. J. Physiol. 2007, 293, G1223-G1233.

36. Mizumori, M.; Ham, M.; Guth, P. H.; Engel, E.; Kaunitz, J. D.; Akiba, Y., J. Physiol. 2009, 587, 3651-3663.

37. Poelstra, K.; Bakker, W. W.; Klok, P. A.; Hardonk, M. J.; Meijier, D. K. F., Lab. Invest. 1997, 76, 319-327.

38. Poelstra, K.; Bakker, W. W.; Klok, P. A.; Kamps, J. A.; Hardonk, M. J.; Meijer, D. K., Am. J. Pathol. 1997, 151, 1163-1169.

39. Goldberg, R. F.; Austen, W. G., Jr.; Zhang, X.; Munene, G.; Mostafa, G.; Biswas, S.; McCormack, M.; Eberlin, K. R.; Nguyen, J. T.; Tatlidede, H. S.; Warren, H. S.; Narisawa, S.; Millan, J. L.; Hodin, R. A., Proc. Natl. Acad. Sci. USA 2008, 105, 3551-3556.

40. Lalles, J.-P., Nutr. Rev. 2010, 68, 323-332.

41. Minkin, C., Calcif. Tissue Int. 1982, 34, 285-290.

42. Efstratiadis, T.; Moss, D. W., Enzyme 1985, 34, 140-143.

43. Hayman, A. R.; Bune, A. J.; Bradley, J. R.; Rashbass, J.; Cox, T. M., J. Histochem. Cytochem. 2000, 48, 219-227.

44. Nuttleman, P. R.; Roberts, R. M., J. Biol. Chem. 1990, 265, 12192-12199.

45. Kaija, H.; Alatalo, S. L.; Halleen, J. M.; Lindqvist, Y.; Schneider, G.; Kalervo Väänänen, H.; Vihko, P., Biochem. Biophys. Res. Commun. 2002, 292, 128-132.

46. Ek-Rylander, B.; Flores, M.; Wendel, M.; Heinegaard, D.; Andersson, G., J. Biol. Chem. 1994, 269, 14853-14856.

47. Raeisaenen, S. R.; Alatalo, S. L.; Ylipahkala, H.; Halleen, J. M.; Cassady, A. I.; Hume, D. A.; Vaeaenaenen, H. K., Biochem. Biophys. Res. Commun. 2005, 331, 120-126.

48. Mitic, N.; Valizadeh, M.; Leung, E. W. W.; de Jersey, J.; Hamilton, S.; Hume, D. A.; Cassady, A. I.; Schenk, G., Arch. Biochem. Biophys. 2005, 439, 154-164.

49. Schenk, G.; Guddat, L. W.; Ge, Y.; Carrington, L. E.; Hume, D. A.; Hamilton, S.; de Jersey, J., Gene 2000, 250, 117-125.

50. Bozzo, G. G.; Raghothama, K. G.; Plaxton, W. C., Eur. J. Biochem. 2002, 269, 62786286.

51. Bozzo, G. G.; Raghothama, K. G.; Plaxton, W. C., Biochem. J. 2004, 377, 419-428.

52. Bozzo, G. G.; Dunn, E. L.; Plaxton, W. C., Plant Cell Environ. 2006, 29, 303-313.

53. Zimmermann, P.; Regierer, B.; Kossmann, J.; Frossard, E.; Amrhein, N.; Bucher, M., Plant Biol. 2004, 6, 519-528.

54. Del Pozo, J. C.; Allona, I.; Rubio, V.; Leyva, A.; De la Pena, A.; Aragoncillo, C.; Paz-Ares, J., Plant J. 1999, 19, 579-589.

55. Li, D.; Zhu, H.; Liu, K.; Liu, X.; Leggewie, G.; Udvardi, M.; Wang, D., J. Biol. Chem. 2002, 277, 27772-27781. 
56. Veljanovski, V.; Vanderbeld, B.; Knowles, V. L.; Snedden, W. A.; Plaxton, W. C., Plant Physiol. 2006, 142, 1282-1293.

57. Cashikar, A. G.; Kumaresan, R.; Rao, N. M., Plant Physiol. 1997, 114, 907-915.

58. Olczak, M.; Watorek, W., Physiol. Plant. 2003, 118, 491-498.

59. Duff, S. M. G.; Sarath, G.; Plaxton, W. C., Physiol. Plant. 1994, 90, 791-800.

60. Hegeman, C. E.; Grabau, E. A., Plant Physiol. 2001, 126, 1598-1608.

61. Wojtaszek, P.; Trethowan, J.; Bolwell, G. P., FEBS Lett. 1997, 405, 95-98.

62. Hayman, A. R.; Cox, T. M., J. Biol. Chem. 1994, 269, 1294-1300.

63. Sibille, J. C.; Doi, K.; Aisen, P., J. Biol. Chem. 1987, 262, 59-62.

64. Kim, E. E.; Wyckoff, H. W., J. Mol. Biol. 1991, 218, 449-464.

65. Weston, J., Chem. Rev. 2005, 105, 2151-2174.

66. Smith, S.; Casellato, A.; Hadler, K.; Mitić, N.; Riley, M.; Bortoluzzi, A.; Szpoganicz, B.; Schenk, G.; Neves, A.; Gahan, L., J. Biol. Inorg. Chem. 2007, 12, 1207-1220.

67. Schenk, G.; Boutchard, C. L.; Carrington, L. E.; Noble, C. J.; Moubaraki, B.; Murray, K. S.; de Jersey, J.; Hanson, G. R.; Hamilton, S., J. Biol. Chem. 2001, 276, 19084-19088.

68. Beck, J. L.; McConachie, L. A.; Summors, A. C.; Arnold, W. N.; De Jersey, J.; Zerner, B., Biochim. Biophys. Acta, Protein Struct. Mol. Enzymol. 1986, 869, 61-68.

69. Yang, Y.-S.; McCormick, J. M.; Solomon, E. I., J. Am. Chem. Soc. 1997, 119, 1183211842.

70. Coleman, F.; Hynes, M. J.; Erxleben, A., Inorg. Chem. 2010, 49, 6725-6733.

71. Butler-Ransohoff, J. E.; Kendall, D. A.; Kaiser, E. T., Proc. Natl. Acad. Sci. USA 1988, 85, 4276-4278.

72. Chaidaroglou, A.; Brezinski, D. J.; Middleton, S. A.; Kantrowitz, E. R., Biochemistry 1988, 27, 8338-8343.

73. O'Brien, P. J.; Herschlag, D., Biochemistry 2001, 40, 5691-5699.

74. Funhoff, E. G.; Wang, Y.; Andersson, G.; Averill, B. A., FEBS Journal 2005, 272, 29682977.

75. Zhao, M.; Wang, H.-B.; Ji, L.-N.; Mao, Z.-W., Chem. Soc. Rev. 2013, 42, 8360-8375.

76. Youngme, S.; Chaichit, N.; Kongsaeree, P.; van Albada, G. A.; Reedijk, J., Inorg. Chim. Acta 2001, 324, 232-240.

77. Daumann, L. J., PhD Thesis: Spectroscopic and Mechanistic Studies of Dinuclear Metallohydrolases and their Biomimetic Complexes, The University of Queensland, Brisbane, 2013.

78. Holm, R. H.; Kennepohl, P.; Solomon, E. I., Chem. Rev. 1996, 96, 2239-2314.

79. Twitchett, M. B.; Geoffrey Sykes, A., Eur. J. Inorg. Chem. 1999, 1999, 2105-2115.

80. Wilcox, D. E., Chem. Rev. 1996, 96, 2435-2458.

81. Gahan, L. R.; Smith, S. J.; Neves, A.; Schenk, G., Eur. J. Inorg. Chem. 2009, 2009, 27452758.

82. Mitic, N.; Hadler, K. S.; Gahan, L. R.; Hengge, A. C.; Schenk, G., J. Am. Chem. Soc. 2010, 132, 7049-7054.

83. Klabunde, T.; Sträter, N.; Fröhlich, R.; Witzel, H.; Krebs, B., J. Mol. Biol. 1996, 259, 737748.

84. Straeter, N.; Lipscomb, W. N.; Klabunde, T.; Krebs, B., Angew. Chem. Int. Ed. 1996, 35, 2025-2055.

85. Schenk, G.; Gahan, L. R.; Carrington, L. E.; Mitic, N.; Valizadeh, M.; Hamilton, S. E.; de Jersey, J.; Guddat, L. W., Proc. Natl. Acad. Sci. USA 2005, 102, 273-278.

86. Molenveld, P.; Engbersen, J. F. J.; Reinhoudt, D. N., Chem. Soc. Rev. 2000, 29, 75-86.

87. Williams, N. H.; Takasaki, B.; Wall, M.; Chin, J., Acc. Chem. Res. 1999, 32, 485-493.

88. Iranzo, O.; Kovalevsky, A. Y.; Morrow, J. R.; Richard, J. P., J. Am. Chem. Soc. 2003, 125, 1988-1993. 
89. Chin, J., Curr. Opin. Chem. Biol. 1997, 1, 514-521.

90. Neves, A.; Lanznaster, M.; Bortoluzzi, A. J.; Peralta, R. A.; Casellato, A.; Castellano, E. E.; Herrald, P.; Riley, M. J.; Schenk, G., J. Am. Chem. Soc. 2007, 129, 7486-7487.

91. Belle, C.; Pierre, J.-L., Eur. J. Inorg. Chem. 2003, 2003, 4137-4146.

92. Lanznaster, M.; Neves, A.; Bortoluzzi, A.; Aires, V.; Szpoganicz, B.; Terenzi, H.; Severino, P.; Fuller, J.; Drew, S.; Gahan, L.; Hanson, G.; Riley, M.; Schenk, G., J. Biol. Inorg. Chem. 2005, 10, 319-332.

93. Schepers, K.; Bremer, B.; Krebs, B.; Henkel, G.; Althaus, E.; Mosel, B.; MüllerWarmuth, W., Angew. Chem. Int. Ed. 1990, 29, 531-533.

94. Neves, A.; Aires de Brito, M.; Drago, V.; Griesar, K.; Haase, W., Inorg. Chim. Acta 1995, 237, 131-135.

95. Lanznaster, M.; Neves, A.; Bortoluzzi, A. J.; Szpoganicz, B.; Schwingel, E., Inorg. Chem. 2002, 41, 5641-5643.

96. Karsten, P.; Neves, A.; Bortoluzzi, A. J.; Lanznaster, M.; Drago, V., Inorg. Chem. 2002, 41, 4624-4626.

97. Than, R.; Feldmann, A. A.; Krebs, B., Coord. Chem. Rev. 1999, 182, 211-241.

98. Jarenmark, M.; Carlsson, H.; Nordlander, E., C. R. Chim. 2007, 10, 433-462.

99. Albedyhl, S.; Averbuch-Pouchot, Marie T.; Belle, C.; Krebs, B.; Pierre, Jean L.; SaintAman, E.; Torelli, S., Eur. J. Inorg. Chem. 2001, 2001, 1457-1464.

100. Suzuki, M.; Kanatomi, H.; Murase, I., Chem. Lett. 1981, 10, 1745-1748.

101. Neves, A.; de Brito, M. A.; Vencato, I.; Drago, V.; Griesar, K.; Haase, W.; Mascarenhas, Y. P., Inorg. Chim. Acta 1993, 214, 5-8.

102. Horn Jr, A.; Vencato, I.; Bortoluzzi, A. J.; Hörner, R.; Silva, R. A. N.; Spoganicz, B.; Drago, V.; Terenzi, H.; de Oliveira, M. C. B.; Werner, R.; Haase, W.; Neves, A., Inorg. Chim. Acta 2005, 358, 339-351.

103. Bortoluzzi, A. J.; Neves, A.; Rey, N. A., Acta Crystallogr. C 2007, 63, o84-086.

104. Andruh, M.; Kahn, O.; Sainton, J.; Dromzee, Y.; Jeannin, S., Inorg. Chem. 1993, 32, 1623-1628.

105. Roth, A.; Spielberg, E. T.; Plass, W., Inorg. Chem. 2007, 46, 4362-4364.

106. Lambert, E.; Chabut, B.; Chardon-Noblat, S.; Deronzier, A.; Chottard, G.; Bousseksou, A.; Tuchagues, J.-P.; Laugier, J.; Bardet, M.; Latour, J.-M., J. Am. Chem. Soc. 1997, 119, 9424-9437.

107. Jarenmark, M.; Kappen, S.; Haukka, M.; Nordlander, E., Dalton Trans. 2008, 993-996.

108. Comba, P.; Gahan, L. R.; Hanson, G. R.; Mereacre, V.; Noble, C. J.; Powell, A. K.; Prisecaru, I.; Schenk, G.; Zajaczkowski-Fischer, M., Chem. Eur. J. 2012, 18, 1700-1710.

109. Zajaczkowski-Fischer, M., Dissertation: Second Generation Model Complexes for the Enzyme Purple Acid Phosphatase, The University of Heidelberg, Heidelberg, 2010.

110. Feng, G.; Mareque-Rivas, J. C.; Torres Martín de Rosales, R.; Williams, N. H., J. Am. Chem. Soc. 2005, 127, 13470-13471.

111. Feng, G.; Mareque-Rivas, J. C.; Williams, N. H., Chem. Commun. 2006, 1845-1847.

112. Feng, G.; Natale, D.; Prabaharan, R.; Mareque-Rivas, J. C.; Williams, N. H., Angew. Chem. Int. Ed. 2006, 45, 7056-7059.

113. Linjalahti, H.; Feng, G.; Mareque-Rivas, J. C.; Mikkola, S.; Williams, N. H., J. Am. Chem. Soc. 2008, 130, 4232-4233.

114. Comba, P.; Gahan, L. R.; Mereacre, V.; Hanson, G. R.; Powell, A. K.; Schenk, G.; Zajaczkowski-Fischer, M., Inorg. Chem. 2012, 51, 12195-12209.

115. Carvalho, N. M. F.; Horn Jr, A.; Bortoluzzi, A. J.; Drago, V.; Antunes, O. A. C., Inorg. Chim. Acta 2006, 359, 90-98.

116. Ośmiałowski, B.; Kolehmainen, E.; Dobosz, R.; Gawinecki, R.; Kauppinen, R.; Valkonen, A.; Koivukorpi, J.; Rissanen, K., J. Phys. Chem. A 2010, 114, 10421-10426. 
117. Soo, H. S.; Komor, A. C.; lavarone, A. T.; Chang, C. J., Inorg. Chem. 2009, 48, 1002410035.

118. Goswami, S.; Das, N. K.; Sen, D.; Hoong-Kun, F., Supramol. Chem. 2010, 22, 532-543.

119. Drewry, J. A.; Fletcher, S.; Hassan, H.; Gunning, P. T., Org. Biomol. Chem. 2009, 7, 5074-5077.

120. Carlsson, H.; Haukka, M.; Bousseksou, A.; Latour, J.-M.; Nordlander, E., Inorg. Chem. 2004, 43, 8252-8262.

121. Sun, G.-C.; He, Z.-H.; Li, Z.-J.; Yuan, X.-D.; Yang, Z.-J.; Wang, G.-X.; Wang, L.-F.; Liu, C.R., Molecules 2001, 6, 1001-1005.

122. Abe, A. M. M.; Helaja, J.; Koskinen, A. M. P., Org. Lett. 2006, 8, 4537-4540.

123. Gisch, N.; Balzarini, J.; Meier, C., J. Med. Chem. 2008, 51, 6752-6760.

124. Johansson, A.; Abrahamsson, M.; Magnuson, A.; Huang, P.; Mårtensson, J.; Styring, S.; Hammarström, L.; Sun, L.; Åkermark, B., Inorg. Chem. 2003, 42, 7502-7511.

125. Krebs, B.; Schepers, K.; Bremer, B.; Henkel, G.; Althaus, E.; Mueller-Warmuth, W.; Griesar, K.; Haase, W., Inorg. Chem. 1994, 33, 1907-1914.

126. Woods, M.; Sherry, A. D., Inorg. Chem. 2003, 42, 4401-4408.

127. Xavier, F. R.; Bortoluzzi, A. J.; Neves, A., Chem. Biodiversity 2012, 9, 1794-1805.

128. Strater, N.; Klabunde, T.; Tucker, P.; Witzel, H.; Krebs, B., Science 1995, 268, 1489 1492.

129. Uppenberg, J.; Lindqvist, F.; Svensson, C.; Ek-Rylander, B.; Andersson, G., J. Mol. Biol. 1999, 290, 201-211.

130. Egloff, M.-P.; Cohen, P. T. W.; Reinemer, P.; Barford, D., J. Mol. Biol. 1995, 254, 942959.

131. Tainer, J. A.; Getzoff, E. D.; Richardson, J. S.; Richardson, D. C., Nature 1983, 306, 284287.

132. Tainer, J. A.; Getzoff, E. D.; Beem, K. M.; Richardson, J. S.; Richardson, D. C., J. Mol. Biol. 1982, 160, 181-217.

133. Ghosh, D.; Kundu, N.; Maity, G.; Choi, K.-Y.; Caneschi, A.; Endo, A.; Chaudhury, M., Inorg. Chem. 2004, 43, 6015-6023.

134. Carlsson, H.; Nordlander, E.; Jarenmark, M., C. R. Chim. 2007, 10, 433-462.

135. Jarenmark, M.; Csapo, E.; Singh, J.; Wockel, S.; Farkas, E.; Meyer, F.; Haukka, M.; Nordlander, E., Dalt. Trans. 2010, 39, 8183-8194.

136. Carboni, M.; Latour, J.-M., Coord. Chem. Rev. 2011, 255, 186-202.

137. Jarenmark, M.; Haukka, M.; Demeshko, S.; Tuczek, F.; Zuppiroli, L.; Meyer, F.; Nordlander, E., Inorg. Chem. 2011, 50, 3866-3887.

138. Lee, J.; Jung, D. J.; Lee, H.-J.; Lee, K.-B.; Hur, N. H.; Jang, H. G., Bull. Korean Chem. Soc. 2000, 21, 1025-1030.

139. Borovik, A. S.; Papaefthymiou, V.; Taylor, L. F.; Anderson, O. P.; Que, L., J. Am. Chem. Soc. 1989, 111, 6183-6195.

140. Smith, S. J.; Peralta, R. A.; Jovito, R.; Horn, A.; Bortoluzzi, A. J.; Noble, C. J.; Hanson, G. R.; Stranger, R.; Jayaratne, V.; Cavigliasso, G.; Gahan, L. R.; Schenk, G.; Nascimento, O. R.; Cavalett, A.; Bortolotto, T.; Razzera, G.; Terenzi, H.; Neves, A.; Riley, M. J., Inorg. Chem. 2012, 51, 2065-2078.

141. Batista, S. C.; Neves, A.; Bortoluzzi, A. J.; Vencato, I.; Peralta, R. A.; Szpoganicz, B.; Aires, V. V. E.; Terenzi, H.; Severino, P. C., Inorg. Chem. Commun. 2003, 6, 1161-1165.

142. Schenk, G.; Peralta, R. A.; Batista, S.; Bortoluzzi, A. J.; Szpoganicz, B.; Dick, A. K.; Herrald, P.; Hanson, G. R.; Szilagyi, R.; Riley, M. J.; Gahan, L. R.; Neves, A., J. Biol. Inorg. Chem. 2008, 13, 139-155.

143. Danford, J. J.; Dobrowolski, P.; Berreau, L. M., Inorg. Chem. 2009, 48, 11352-11361. 
144. Peralta, R. A.; Bortoluzzi, A. J.; de Souza, B.; Jovito, R.; Xavier, F. R.; Couto, R. A. A.; Casellato, A.; Nome, F.; Dick, A.; Gahan, L. R.; Schenk, G.; Hanson, G. R.; de Paula, F. v. C. S.; Pereira-Maia, E. C.; de P. Machado, S.; Severino, P. C.; Pich, C.; Bortolotto, T.; Terenzi, H. n.; Castellano, E. E.; Neves, A.; Riley, M. J., Inorg. Chem. 2010, 49, 1142111438.

145. Xavier, F. R.; Peralta, R. A.; Bortoluzzi, A. J.; Drago, V.; Castellano, E. E.; Haase, W.; Tomkowicz, Z.; Neves, A., J. Inorg. Biochem. 2011, 105, 1740-1752.

146. Xavier, F. R.; Neves, A.; Casellato, A.; Peralta, R. A.; Bortoluzzi, A. J.; Szpoganicz, B.; Severino, P. C.; Terenzi, H.; Tomkowicz, Z.; Ostrovsky, S.; Haase, W.; Ozarowski, A.; Krzystek, J.; Telser, J.; Schenk, G.; Gahan, L. R., Inorg. Chem. 2009, 48, 7905-7921.

147. Karsten, P.; Neves, A.; Bortoluzzi, A. J.; Strähle, J.; Maichle-Mössmer, C., Inorg. Chem. Commun. 2002, 5, 434-438.

148. Smith, S. J.; Riley, M. J.; Noble, C. J.; Hanson, G. R.; Stranger, R.; Jayaratne, V.; Cavigliasso, G.; Schenk, G.; Gahan, L. R., Inorg. Chem. 2009, 48, 10036-10048.

149. Belle, C.; Gautier-Luneau, I.; Karmazin, L.; Pierre, J.-L.; Albedyhl, S.; Krebs, B.; Bonin, M., Eur. J. Inorg. Chem. 2002, 2002, 3087-3090.

150. Belle, C.; Gautier-Luneau, I.; Gellon, G.; Pierre, J.-L.; Morgenstern-Badarau, I.; SaintAman, E., Dalton Trans. 1997, 3543-3546.

151. de Souza, B.; Kreft, G. L.; Bortolotto, T.; Terenzi, H.; Bortoluzzi, A. J.; Castellano, E. E.; Peralta, R. A.; Domingos, J. B.; Neves, A., Inorg. Chem. 2013, 52, 3594-3596.

152. Retegan, M.; Milet, A.; Jamet, H., J. Phys. Chem. A 2010, 114, 7110-7116.

153. Piovezan, C.; Jovito, R.; Bortoluzzi, A. J.; Terenzi, H. n.; Fischer, F. L.; Severino, P. C.; Pich, C. T.; Azzolini, G. G.; Peralta, R. A.; Rossi, L. M.; Neves, A., Inorg. Chem. 2010, 49, 2580-2582.

154. Vallee, B. L.; Auld, D. S., Proc. Natl. Acad. Sci. USA 1990, 87, 220-224.

155. Christianson, D. W., Adv. Protein Chem. 1991, 42, 281-355.

156. McCall, K. A.; Huang, C.-C.; Fierke, C. A., J. Nutr. 2000, 130, 1437S-1446S.

157. Kövári, E.; Krämer, R., Chem. Ber. 1994, 127, 2151-2157.

158. Kovari, E.; Heitker, J.; Kramer, R., J. Chem. Soc., Chem. Commun. 1995, 1205-1206.

159. Kövári, E.; Krämer, R., J. Am. Chem. Soc. 1996, 118, 12704-12709.

160. Rossi, L. M.; Neves, A.; Bortoluzzi, A. J.; Hoerner, R.; Szpoganicz, B.; Terenzi, H.; Mangrich, A. S.; Pereira-Maia, E.; Castellano, E. E.; Haase, W., Inorg. Chim. Acta 2005, 358, 1807-1822.

161. Osório, R. E. H. M. B.; Peralta, R. A.; Bortoluzzi, A. J.; de Almeida, V. R.; Szpoganicz, B.; Fischer, F. L.; Terenzi, H.; Mangrich, A. S.; Mantovani, K. M.; Ferreira, D. E. C.; Rocha, W. R.; Haase, W.; Tomkowicz, Z.; Anjos, A. d.; Neves, A., Inorg. Chem. 2012, 51, 15691589.

162. Wall, M.; Linkletter, B.; Williams, D.; Hynes, R. C.; Chin, J., J. Am. Chem. Soc. 1999, 121, 4710-4711.

163. Comba, P.; Gahan, L. R.; Hanson, G. R.; Westphal, M., Chem. Commun. 2012, 48, 9364-9366.

164. Lide, D. R., CRC handbook of chemistry and physics: a ready-reference book of chemical and physical data, 89. ed.; CRC Press: Boca Raton, 2008.

165. Baes, C. F.; Mesmer, R. E., The hydrolysis of cations, Wiley: New York, 1976.

166. Richens, D. T., The chemistry of aqua ions: synthesis, structure and reactivity; a tour through the periodic table of the elements, Wiley: Chichester, 1997.

167. Merkx, M.; Averill, B. A., Biochemistry 1998, 37, 8490-8497.

168. Mareque Rivas, J. C.; Torres Martin de Rosales, R.; Parsons, S., Dalton Trans. 2003, 2156-2163. 
169. Smith, S. J.; Noble, C. J.; Palmer, R. C.; Hanson, G. R.; Schenk, G.; Gahan, L. R.; Riley, M. J., J. Biol. Inorg. Chem. 2008, 13, 499-510.

170. Lötzbeyer, L., Dissertation: Synthese neuer Patellamid-Derivate und Eigenschaften ihrer Kupfer(II)-Komplexe,, Ruprecht-Karls Universität Heidelberg, Heidelberg, 2001.

171. Addison, A. W.; Rao, T. N.; Reedijk, J.; van Rijn, J.; Verschoor, G. C., Dalton Trans. 1984, 1349-1356.

172. Kozlevcar, B.; Segedin, P., Croat. Chem. Acta 2008, 81, 369-379.

173. Kahn, O., Molecular magnetism, VCH: New York, 1993.

174. Clarke, J.; Braginski, A. I., The SQUID Handbook, Vol. II: Applications of SQUIDs and SQUID Systems, Wiley-VCH: Weinheim, 2006.

175. de Almeida, V. R.; Xavier, F. R.; Osorio, R. E. H. M. B.; Bessa, L. M.; Schilling, E. L.; Costa, T. G.; Bortolotto, T.; Cavalett, A.; Castro, F. A. V.; Vilhena, F.; Alves, O. C.; Terenzi, H.; Eleutherio, E. C. A.; Pereira, M. D.; Haase, W.; Tomkowicz, Z.; Szpoganicz, B.; Bortoluzzi, A. J.; Neves, A., Dalton Trans. 2013, 42, 7059-7073.

176. Rey, N. A.; Neves, A.; Bortoluzzi, A. J.; Haase, W.; Tomkowicz, Z., Dalton Trans. 2012, 41, 7196-7200.

177. Chaudhuri, P.; Wagner, R.; Weyhermueller, T., Inorg. Chem. 2007, 46, 5134-5136.

178. Ruiz, E.; Alemany, P.; Alvarez, S.; Cano, J., Inorg. Chem. 1997, 36, 3683-3688.

179. Ruiz, E.; Alemany, P.; Alvarez, S.; Cano, J., J. Am. Chem. Soc. 1997, 119, 1297-1303.

180. Paine, T. K.; Weyhermueller, T.; Wieghardt, K.; Chaudhuri, P., Inorg. Chem. 2002, 41, 6538-6540.

181. Grillo, V. A.; Hanson, G. R.; Hambley, T. W.; Gahan, L. R.; Murray, K. S.; Moubaraki, B., J. Chem. Soc., Dalton Trans. 1997, 305-311.

182. Holman, T. R.; Wang, Z.; Hendrich, M. P.; Que, L., Inorg. Chem. 1995, 34, 134-139.

183. Yan, S.; Pan, X.; Taylor, L. F.; Zhang, J. H.; O'Connor, C. J.; Britton, D.; Anderson, O. P.; Que Jr, L., Inorg. Chim. Acta 1996, 243, 1-8.

184. Mareque-Rivas, J. C.; Prabaharan, R.; Martin de Rosales, R. T., Chem. Commun. 2004, 76-77.

185. Vallee, B. L.; Auld, D. S., Acc. Chem. Res. 1993, 26, 543-551.

186. Bertini, I.; Luchinat, C.; Rosi, M.; Sgamellotti, A.; Tarantelli, F., Inorg. Chem. 1990, 29, 1460-1463.

187. Kodama, M.; Kimura, E., Dalton Trans. 1977, 2269-2276.

188. Zompa, L. J., Inorg. Chem. 1978, 17, 2531-2536.

189. Kimura, E.; Shiota, T.; Koike, T.; Shiro, M.; Kodama, M., J. Am. Chem. Soc. 1990, 112, 5805-5811.

190. Mareque-Rivas, J. C.; Prabaharan, R.; Parsons, S., Dalton Trans. 2004, 1648-1655.

191. Chiu, Y.-H.; Canary, J. W., Inorg. Chem. 2003, 42, 5107-5116.

192. Christianson, D. W.; Fierke, C. A., Acc. Chem. Res. 1996, 29, 331-339.

193. Hernandez-Gil, J.; Ferrer, S.; Salvador, E.; Calvo, J.; Garcia-Espana, E.; Mareque-Rivas, J. C., Chem. Commun. 2013, 49, 3655-3657.

194. Huang, F.; Cheng, C.; Feng, G., J. Org. Chem. 2012, 77, 11405-11408.

195. Daumann, L. J.; Dalle, K. E.; Schenk, G.; McGeary, R. P.; Bernhardt, P. V.; Ollis, D. L.; Gahan, L. R., Dalton Trans. 2012, 41, 1695-1708.

196. Adams, H.; Bradshaw, D.; Fenton, D. E., Inorg. Chim. Acta 2002, 332, 195-200.

197. Das, B.; Haukka, M.; Nordlander, E., Acta Cryst. E 2014, 70, m120-m121.

198. Selmeczi, K.; Michel, C.; Milet, A.; Gautier-Luneau, I.; Philouze, C.; Pierre, J.-L.; Schnieders, D.; Rompel, A.; Belle, C., Chem. Eur. J. 2007, 13, 9093-9106.

199. Berreau, L. M.; Saha, A.; Arif, A. M., Dalton Trans. 2006, 183-192.

200. Matsufuji, K.; Shiraishi, H.; Miyasato, Y.; Shiga, T.; Ohba, M.; Yokoyama, T.; Okawa, H., Bull. Chem. Soc. Jpn. 2005, 78, 851-858. 
201. Hough, E.; Hansen, L. K.; Birknes, B.; Jynge, K.; Hansen, S.; Hordvik, A.; Little, C.; Dodson, E.; Derewenda, Z., Nature 1989, 338, 357-360.

202. Goldberg, J.; Huang, H.-b.; Kwon, Y.-g.; Greengard, P.; Nairn, A. C.; Kuriyan, J., Nature 1995, 376, 745-753.

203. M. Hesse; H. Meier; Zeeh, B., Spectroscopic methods in organic chemistry, G. Thieme: NY, 1997.

204. H. Günzler; H.-U. Gremlich, IR Spectroscopy An Introduction, 1. ed.; 2002.

205. Nakamoto, K., Infrared and Raman spectra of inorganic and coordination compounds, Wiley: NY, 1978.

206. Deacon, G. B.; Phillips, R. J., Coord. Chem. Rev. 1980, 33, 227-250.

207. Gross, F., Iron Metabolism: An International Symposium, Springer: Berlin, Heidelberg, 1964.

208. Lippard, S. J., Angew. Chem. Int. Ed. 1988, 27, 344-361.

209. Vincent, J. B.; Olivier-Lilley, G. L.; Averill, B. A., Chem. Rev. 1990, 90, 1447-1467.

210. Fontecave, M.; Ménage, S.; Duboc-Toia, C., Coord. Chem. Rev. 1998, 178-180, 15551572.

211. Friedle, S.; Reisner, E.; Lippard, S. J., Chem. Soc. Rev. 2010, 39, 2768-2779.

212. Merkx, M.; Kopp, D. A.; Sazinsky, M. H.; Blazyk, J. L.; Müller, J.; Lippard, S. J., Angew. Chem. Int. Ed. 2001, 40, 2782-2807.

213. Sazinsky, M. H.; Lippard, S. J., Acc. Chem. Res. 2006, 39, 558-566.

214. Stubbe, J.; Nocera, D. G.; Yee, C. S.; Chang, M. C. Y., Chem. Rev. 2003, 103, 2167-2201.

215. Nordlund, P.; Reichard, P., Annu. Rev. Biochem. 2006, 75, 681-706.

216. Kurtz, D. M., Jr., Dalton Trans. 2007, 4115-4121.

217. Antanaitis, B. C.; Aisen, P.; Lilienthal, H. R., J. Biol. Chem. 1983, 258, 3166-3172.

218. Hill, H. A. O.; Sadler, P. J.; Thomson, A. J., Metal Sites in Proteins and Models, 1. ed.; Springer: Berlin, Heidelberg, 1997; Vol. 89.

219. Averill, B. A.; Davis, J. C.; Burman, S.; Zirino, T.; Sanders-Loehr, J.; Loehr, T. M.; Sage, J. T.; Debrunner, P. G., J. Am. Chem. Soc. 1987, 109, 3760-3767.

220. Wilkins, R. G., Chem. Soc. Rev. 1992, 21, 171-178.

221. Merkx, M.; Averill, B. A., Biochemistry 1998, 37, 11223-11231.

222. Wang, D. L.; Holz, R. C.; David, S. S.; Que, L., Jr.; Stankovich, M. T., Biochemistry 1991, 30, 8187-8194.

223. Bernhardt, P. V.; Schenk, G.; Wilson, G. J., Biochemistry 2004, 43, 10387-10392.

224. Debrunner, P. G.; Hendrich, M. P.; Jersey, J. d.; Keough, D. T.; Sage, J. T.; Zerner, B., Biochim. Biophys. Acta, Protein Struct. Mol. Enzymol. 1983, 745, 103-106.

225. Rodriguez, J. H.; Ok, H. N.; Xia, Y. M.; Debrunner, P. G.; Hinrichs, B. E.; Meyer, T.; Packard, N. H., J. Phys. Chem. 1996, 100, 6849-6862.

226. Day, E. P.; David, S. S.; Peterson, J.; Dunham, W. R.; Bonvoisin, J. J.; Sands, R. H.; Que, L., J. Biol. Chem. 1988, 263, 15561-15567.

227. Crowder, M. W.; Vincent, J. B.; Averill, B. A., Biochemistry 1992, 31, 9603-9608.

228. Dietrich, M.; Münstermann, D.; Suerbaum, H.; Witzel, H., Eur. J. Biochem. 1991, 199, 105-113.

229. Eulering, B.; Ahlers, F.; Zippel, F.; Schmidt, M.; Nolting, H. F.; Krebs, B., Chem. Commun. 1995, 1305-1307.

230. Nie, H.; Aubin, S.; Mashuta, M. S.; Wu, J.; Richardson, J. F.; Hendrickson, D. N.; Buchanan, R. M., Inorg. Chem. 1995, 34, 2382-2388.

231. Suzuki, M.; Uehara, A.; Oshio, H.; Endo, K.; Yanaga, M.; Kida, S.; Saito, K., Bull. Chem. Soc. Jpn. 1987, 60, 3547-3555.

232. Yim, S. H.; Lee, H. J.; Lee, K.-B.; Kang, S. J.; Hur, N. H.; Jang, H. G., Bull. Korean Chem. Soc. 1998, 19, 654-660. 
233. Maeda, Y.; Yamamoto, M.; Hayami, S., J. Radioanal. Nucl. Chem. 2007, 272, 651-656.

234. Inomata, T.; Shinozaki, K.; Hayashi, Y.; Arii, H.; Funahashi, Y.; Ozawa, T.; Masuda, H., Chem. Commun. 2008, 392-394.

235. Borovik, A. S.; Que, L., Jr., J. Am. Chem. Soc. 1988, 110, 2345-2347.

236. O'Connor, C. J.; Lippard, S. J., Prog. Inorg. Chem. 1982, 203-283.

237. Jang, H. G.; Hendrich, M. P.; Que, L., Jr., Inorg. Chem. 1993, 32, 911-918.

238. Tolman, W. B.; Liu, S.; Bentsen, J. G.; Lippard, S. J., J. Am. Chem. Soc. 1991, 113, 152164.

239. Satcher, J. H., Jr.; Balch, A. L.; Olmstead, M. M.; Droege, M. W., Inorg. Chem. 1996, 35, 1749-1750.

240. Hayashi, Y.; Kayatani, T.; Sugimoto, H.; Suzuki, M.; Inomata, K.; Uehara, A.; Mizutani, Y.; Kitagawa, T.; Maeda, Y., J. Am. Chem. Soc. 1995, 117, 11220-11229.

241. Dong, Y.; Menage, S.; Brennan, B. A.; Elgren, T. E.; Jang, H. G.; Pearce, L. L.; Que, L., Jr., J. Am. Chem. Soc. 1993, 115, 1851-1859.

242. Evans, D. F., J. Chem. Soc. 1959, 2003-2005.

243. Bertini, I.; Luchinat, C.; Parigi, G., Solution NMR of paramagnetic molecules: applications to metallobiomolecules and models, 1. ed.; Elsevier: Amsterdam, 2001.

244. Carboni, M.; Clémancey, M.; Molton, F.; Pécaut, J.; Lebrun, C.; Dubois, L.; Blondin, G.; Latour, J. M., Inorg. Chem. 2012, 51, 10447-10460.

245. Wang, Z.; Holman, T. R.; Que, L., Magn. Reson. Chem. 1993, 31, S78-S84.

246. Gonsor, U., Mössbauer spectroscopy, Springer: Berlin, 1975.

247. Gütlich, P., Chem. unserer Zeit 1970, 4, 133-144.

248. Brant, R. A. NORMOS Mössbauer Fit. Progr. Pack., Duisburg, Germany, 1994.

249. Dong, Y.; Fujii, H.; Hendrich, M. P.; Leising, R. A.; Pan, G.; Randall, C. R.; Wilkinson, E. C.; Zang, Y.; Que, L., Jr.; et, a., J. Am. Chem. Soc. 1995, 117, 2778-2792.

250. Rhile, I. J.; Markle, T. F.; Nagao, H.; DiPasquale, A. G.; Lam, O. P.; Lockwood, M. A.; Rotter, K.; Mayer, J. M., J. Am. Chem. Soc. 2006, 128, 6075-6088.

251. Markle, T. F.; Tronic, T. A.; DiPasquale, A. G.; Kaminsky, W.; Mayer, J. M., J. Phys. Chem. A 2012, 116, 12249-12259.

252. Pavlishchuk, V. V.; Addison, A. W., Inorg. Chim. Acta 2000, 298, 97-102.

253. Manago, T.; Hayami, S.; Oshio, H.; Osaki, S.; Hasuyama, H.; Herber, R. H.; Maeda, Y., J. Chem. Soc., Dalton Trans. 1999, 1001-1012.

254. Alimi, M.; Allam, A.; Selkti, M.; Tomas, A.; Roussel, P.; Galardon, E.; Artaud, I., Inorg. Chem. 2012, 51, 9350-9356.

255. Rivas, J. C. M.; Hinchley, S. L.; Metteau, L.; Parsons, S., Dalton Trans. 2006, 2316-2322.

256. Arii, H.; Nagatomo, S.; Kitagawa, T.; Miwa, T.; Jitsukawa, K.; Einaga, H.; Masuda, H., J. Inorg. Biochem. 2000, 82, 153-162.

257. Zheng, S.; Berto, T. C.; Dahl, E. W.; Hoffman, M. B.; Speelman, A. L.; Lehnert, N., J. Am. Chem. Soc. 2013, 135, 4902-4905.

258. Yan, S.; Cheng, P.; Wang, Q.; Liao, D.; Jiang, Z.; Wang, G., Sci. China, Ser. B: Chem. 2000, 43, 405-411.

259. Ainscough, E. W.; Brodie, A. M.; Plowman, J. E.; Brown, K. L.; Addison, A. W.; Gainsford, A. R., Inorg. Chem. 1980, 19, 3655-3663.

260. Gaber, B. P.; Miskowski, V.; Spiro, T. G., J. Am. Chem. Soc. 1974, 96, 6868-6873.

261. Weil, J. A.; Bolton, J. R., Electron paramagnetic resonance: elementary theory and practical applications, 2. ed. ed.; Wiley-Interscience: Hoboken, NJ, 2007.

262. Suzuki, M.; Oshio, H.; Uehara, A.; Endo, K.; Yanaga, M.; Kida, S.; Saito, K., Bull. Chem. Soc. Jpn. 1988, 61, 3907-3913.

263. Stec, B.; Hehir, M. J.; Brennan, C.; Nolte, M.; Kantrowitz, E. R., J. Mol. Biol. 1998, 277, 647-662. 
264. Stec, B.; Holtz, K. M.; Kantrowitz, E. R., J. Mol. Biol. 2000, 299, 1303-1311.

265. Aquino, M. A. S.; Lim, J. S.; Sykes, A. G., J. Chem. Soc., Dalton Trans. 1994, 429-436.

266. Twitchett, M. B.; Schenk, G.; Aquino, M. A. S.; Yiu, D. T. Y.; Lau, T.-C.; Sykes, A. G., Inorg. Chem. 2002, 41, 5787-5794.

267. Smoukov, S. K.; Quaroni, L.; Wang, X.; Doan, P. E.; Hoffman, B. M.; Que, L., J. Am. Chem. Soc. 2002, 124, 2595-2603.

268. Wang, X.; Ho, R. Y. N.; Whiting, A. K.; Que, L., Jr., J. Am. Chem. Soc. 1999, 121, 92359236.

269. Merkx, M.; Averill, B. A., J. Am. Chem. Soc. 1999, 121, 6683-6689.

270. Wang, X.; Randall, C. R.; Que, L., Jr., Biochemistry 1996, 35, 13946-13954.

271. Kimura, E., Curr. Opin. Chem. Biol. 2000, 4, 207-213.

272. Wynne, C. J.; Hamilton, S. E.; Dionysius, D. A.; Beck, J. L.; de Jersey, J., Arch. Biochem. Biophys. 1995, 319, 133-141.

273. Neverov, A. A.; Lu, Z.-L.; Maxwell, C. I.; Mohamed, M. F.; White, C. J.; Tsang, J. S. W.; Brown, R. S., J. Am. Chem. Soc. 2006, 128, 16398-16405.

274. Richard, J. P.; Amyes, T. L., Bioorg. Chem. 2004, 32, 354-366.

275. Daumann, L. J.; Gahan, L. R.; Comba, P.; Schenk, G., Inorg. Chem. 2012, 51, 76697681.

276. Daumann, L. J.; Marty, L.; Schenk, G.; Gahan, L. R., Dalton Trans. 2013, 42, 9574-9584.

277. Segel, I. H., Enzyme Kinetics: Behavior and analysis of rapid equilibrium and steadystate enzyme systems, Wiley-Interscience: NY, 1975.

278. Kantacha, A.; Buchholz, R.; Smith, S.; Schenk, G.; Gahan, L., J. Biol. Inorg. Chem. 2011, 16, 25-32.

279. Michaelis, L.; Menten, M. L., Biochem. Z. 1913, 49, 333-369.

280. Johnson, K. A.; Goody, R. S., Biochemistry 2011, 50, 8264-8269.

281. Meyer, F., Eur. J. Inorg. Chem. 2006, 3789-3800.

282. Parkin, G., Chem. Rev. 2004, 104, 699-768.

283. Breslow, R.; Berger, D.; Huang, D. L., J. Am. Chem. Soc. 1990, 112, 3686-3687.

284. He, J.; Sun, J.; Mao, Z.-W.; Ji, L.-N.; Sun, H., J. Inorg. Biochem. 2009, 103, 851-858.

285. Mareque-Rivas, J. C.; Torres Martin de Rosales, R.; Parsons, S., Chem. Commun. 2004, 610-611.

286. Fan, Y.; Gao, Y. Q., J. Am. Chem. Soc. 2007, 129, 905-913.

287. Mohamed, M. F.; Brown, R. S., J. Org. Chem. 2010, 75, 8471-8477.

288. Aoki, S.; Kimura, E., Rev. Mol. Biotechnol. 2002, 90, 129-155.

289. Coburn, S. P.; Mahuren, J. D.; Jain, M.; Zubovic, Y.; Wortsman, J., J. Clin. Endocrinol. Metab. 1998, 83, 3951-3957.

290. H. N. Fernley, P. G. W., Biochem. J. 1967, 104, 1011-1018.

291. Kinoshita, E.; Takahashi, M.; Takeda, H.; Shiro, M.; Koike, T., Dalton Trans. 2004, 11891193.

292. Gorenstein, D. G., Phosphorus-31 NMR: principles and applications, Acad. Pr.: Orlando, 1984.

293. Norman, R. E.; Yan, S.; Que, L., Jr.; Backes, G.; Ling, J.; Sanders-Loehr, J.; Zhang, J. H.; O'Connor, C. J., J. Am. Chem. Soc. 1990, 112, 1554-1562.

294. Turowski, P. N.; Armstrong, W. H.; Liu, S.; Brown, S. N.; Lippard, S. J., Inorg. Chem. 1994, 33, 636-645.

295. Duboc-Toia, C.; Menage, S.; Vincent, J.-M.; Averbuch-Pouchot, M. T.; Fontecave, M., Inorg. Chem. 1997, 36, 6148-6149.

296. Helm, L.; Merbach, A. E., Chem. Rev. 2005, 105, 1923-1959.

297. Tanaka, M.; Yamada, S., Chem. Commun. 1976, 178-179.

298. Grant, M.; Jordan, R. B., Inorg. Chem. 1981, 20, 55-60. 
299. Davis, J. C.; Averill, B. A., Proc. Natl. Acad. Sci. USA 1982, 79, 4623-4627.

300. David, S. S.; Que, L., Jr., J. Am. Chem. Soc. 1990, 112, 6455-6463.

301. Bosch, S.; Comba, P.; Gahan, L. R.; Schenk, G., Inorg. Chem. 2014, 53, 9036-9051.

302. Bruker TopSpin 2.0 Software for NMR data processing.

303. Sheldrick, G. M. SHELXL97: Program for the refinement of crystal structures, University of Gottingen: Germany, 1997.

304. Farrugia, L. J., J. Appl. Crystallogr. 1999, 32, 837-838.

305. Dolomanov, O. V.; Bourhis, L. J.; Gildea, R. J.; Howard, J. A. K.; Puschmann, H., J. Appl. Crystallogr. 2009, 42, 339-341.

306. Van der Sluis, P.; Spek, A. L., Acta Cryst. A 1990, A46, 194-201.

307. Spek, A. L., J. Appl. Crystallogr. 2003, 36, 7-13.

308. Hanson, G. R.; Gates, K. E.; Noble, C. J.; Griffin, M.; Mitchell, A.; Benson, S., J. Inorg. Biochem. 2004, 98, 903-916.

309. Hanson, G. R. Molecular sophe simulation suite, Bruker Biospin: Germany, Switherland, France, U.S., 2007.

310. Paine, R. T.; Tan, Y.-C.; Gan, X.-M., Inorg. Chem. 2001, 40, 7009-7013.

311. Jovito, R.; Neves, A.; Bortoluzzi, A. J.; Lanznaster, M.; Drago, V.; Haase, W., Inorg. Chem. Commun. 2005, 8, 323-327.

312. Bunton, C. A.; Farber, S. J., J. Org. Chem. 1969, 34, 767-772.

313. Rawji, G.; Milburn, R. M., J. Org. Chem. 1981, 46, 1205-1206. 


\section{APPENDIX}

Table 30: Crystallographic data for $\left[\mathrm{Ga}^{\prime \prime \prime}\left(\mathrm{H}_{2} \mathrm{SIM}^{1}\right)(\mu-\mathrm{OH})\right]_{2}\left(\mathrm{ClO}_{4}\right)_{2} \cdot \mathrm{CH}_{3} \mathrm{CN} \cdot \mathrm{Et} 2 \mathrm{O}$, $\left[\mathrm{Ga}^{\prime \prime \prime} \mathrm{Zn}{ }^{\prime \prime}\left(\mathrm{H}_{2} \mathrm{SIM}^{1}\right)(\mathrm{OAc})_{2}(\mathrm{OH})\right] \mathrm{PF}_{6} \cdot \mathrm{Et}_{2} \mathrm{O}$ and $\left[\mathrm{Cu}_{2}\left(\mathrm{HSIM}^{1}\right)(\mu-\mathrm{OAc})\right] \mathrm{PF}_{6}$.

$\left[\mathrm{Ga}^{\prime \prime \prime}\left(\mathrm{H}_{2} \mathrm{SIM}^{1}\right)(\mu-\mathrm{OH})\right]_{2}\left(\mathrm{ClO}_{4}\right)_{2} \quad\left[\mathrm{Ga}^{\prime \prime \prime} \mathrm{Zn}\left(\mathrm{n}_{2}\left(\mathrm{H}_{2} \mathrm{SIM}^{1}\right)(\mathrm{OAc})_{2}(\mathrm{OH})\right] \mathrm{PF}_{6} \quad\left[\mathrm{Cu}_{2}{ }_{2}\left(\mathrm{HSIM}^{1}\right)(\mu-\mathrm{OAc})\right] \mathrm{PF} F_{6}\right.$ $\cdot \mathrm{CH}_{3} \mathrm{CN} \cdot \mathrm{Et}_{2} \mathrm{O}$ $\cdot \mathrm{Et}_{2} \mathrm{O}$

\begin{tabular}{|c|c|c|c|}
\hline $\begin{array}{l}\text { Empirical } \\
\text { formula }\end{array}$ & $\mathrm{C}_{49} \mathrm{H}_{65} \mathrm{Cl}_{2} \mathrm{GaN}_{9} \mathrm{O}_{13}$ & $\mathrm{C}_{51} \mathrm{H}_{68} \mathrm{~F}_{6} \mathrm{GaN}_{8} \mathrm{O}_{9} \mathrm{PZn}$ & $\mathrm{C}_{45} \mathrm{H}_{53} \mathrm{Cu}_{2} \mathrm{~F}_{6} \mathrm{~N}_{8} \mathrm{O}_{5} \mathrm{P}$ \\
\hline Formula weight & 1128.72 & 1217.19 & 1058.00 \\
\hline Temperature (K) & $120(1)$ & $200(2)$ & $100(1)$ \\
\hline Wavelength $(\AA ̊)$ & 1.54184 & 0.71073 & 0.71073 \\
\hline Crystal system & Triclinic & Monoclinic & Triclinic \\
\hline Space group & $P-1$ & C $12 / c 1$ & P -1 \\
\hline$a(\AA ̊)$ & $10.5436(3)$ & $18.8817(7)$ & $12.887(7)$ \\
\hline$b(\AA)$ & $13.6018(4)$ & $18.7090(10)$ & $13.394(8)$ \\
\hline$c(\AA ̊)$ & $19.3106(6)$ & $32.536(2)$ & $15.137(9)$ \\
\hline$\alpha\left({ }^{\circ}\right)$ & $78.857(3)$ & 90 & $79.960(13)$ \\
\hline$\beta\left({ }^{0}\right)$ & $82.0085(3)$ & $103.756(5)$ & $73.452(17)$ \\
\hline$\gamma\left({ }^{0}\right)$ & $77.117(3)$ & 90 & $70.673(11)$ \\
\hline $\operatorname{Vol}\left(\AA^{3}\right)$ & $2635.77(15)$ & 11163.9(10) & $2354(2)$ \\
\hline Z & 2 & 8 & 2 \\
\hline$\mu\left(\mathrm{mm}^{-1}\right)$ & 2.248 & 1.022 & 1.015 \\
\hline$F(000)$ & 1182 & 5056 & 1092 \\
\hline $\mathrm{P}\left(\mathrm{Mg} / \mathrm{m}^{3}\right)$ & 1.422 & 1.448 & 1.492 \\
\hline $\begin{array}{l}\text { Reflections } \\
\text { collected }\end{array}$ & 59276 & 23354 & 56089 \\
\hline $\begin{array}{l}\text { Independent } \\
\text { reflections }\left[R_{\text {int }}\right]\end{array}$ & 9785 [0.0736] & $9829[0.0556]$ & $14368[0.0424]$ \\
\hline $\begin{array}{l}\Theta \text { range for data } \\
\text { collection }\left({ }^{\circ}\right)\end{array}$ & $3.777-70.622$ & $2.91-29.24$ & $1.727-30.555$ \\
\hline GOOF on $F^{2}$ & 1.059 & 1.035 & 1.014 \\
\hline Final $R$ indices & $R_{1}=0.0827$ & $R_{1}=0.0772$ & $R_{1}=0.0382$ \\
\hline$[1>2 \sigma(I)]$ & $w R_{2}=0.2212$ & $w R_{2}=0.1893$ & $w R_{2}=0.0873$ \\
\hline$R$ indices & $R_{1}=0.1090$ & $R_{1}=0.1089$ & $R_{1}=0.0632$ \\
\hline (all data) & $w R_{2}=0.2400$ & $w R_{2}=0.2103$ & $w R_{2}=0.0993$ \\
\hline $\begin{array}{l}\text { Largest diff. } \\
\text { peak and hole } \\
\left.\text { (e } \AA^{-3}\right)\end{array}$ & 1.347 and -0.796 & 1.223 and -0.695 & 0.669 and -0.394 \\
\hline $\begin{array}{l}\text { Identification } \\
\text { code }\end{array}$ & co_sb15 & - & co_sb14 \\
\hline CCDC number & - & 1024620 & - \\
\hline
\end{tabular}




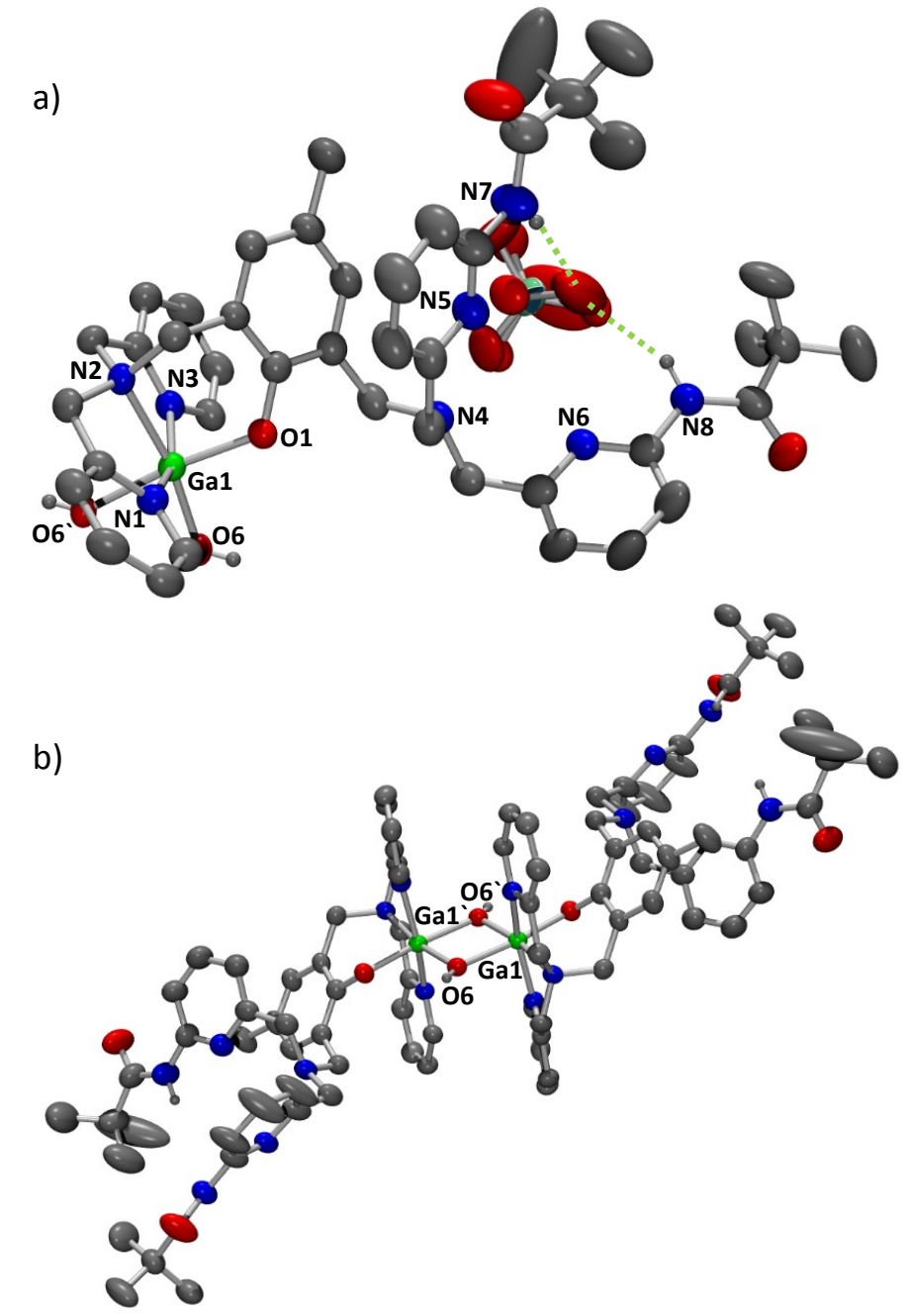

Figure 70: ORTEP plots of a) $\left[\mathrm{Ga}^{\prime \prime \prime}\left(\mathrm{H}_{2} \mathrm{SIM}^{1}\right)(\mu-\mathrm{OH})\right] \mathrm{ClO}_{4}$ and $\left.\mathrm{b}\right)$ the respective dimer $\left[\mathrm{Ga}^{\prime \prime \prime}\left(\mathrm{H}_{2} \mathrm{SIM}^{1}\right)(\mu-\mathrm{OH})\right]_{2}{ }^{2+}$ with $50 \%$ probability level of thermal ellipsoids. Counter ions, non-coordinated solvent molecules and hydrogen atoms not involved in hydrogen bonding are omitted for clarity. 

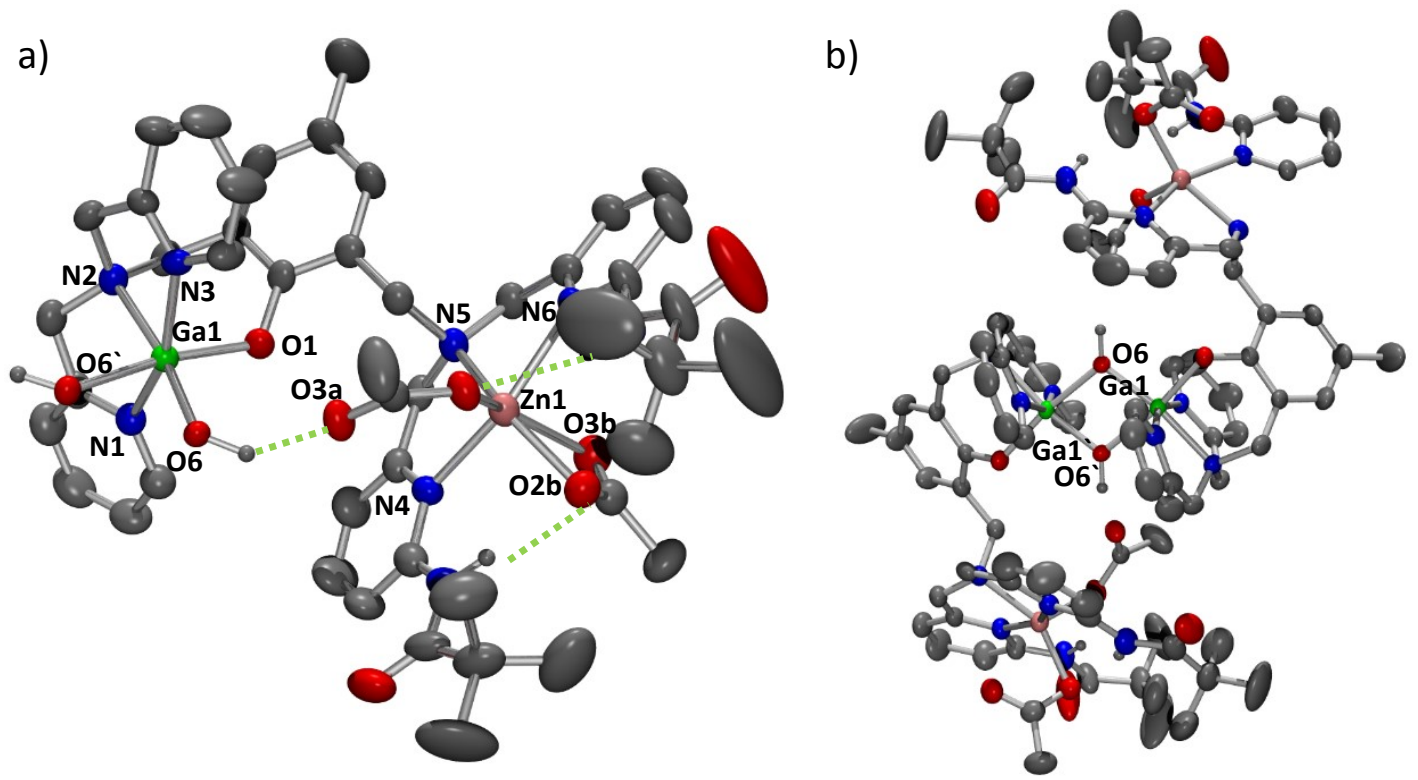

Figure 71: ORTEP plots of a) [ $\mathrm{Ga}^{\prime \prime \prime} \mathrm{Zn}^{\prime \prime}\left(\mathrm{H}_{2} \mathrm{SIM}^{1}\right)(\mathrm{OAc})_{2}(\mathrm{OH}) \mathrm{PF}_{6}$ and $\left.\mathrm{b}\right)$ the respective dimeric structure with $50 \%$ probability level of thermal ellipsoids showing hydrogen bonds as green, dotted lines. Counter ions, noncoordinated solvent molecules and hydrogen atoms not involved in hydrogen bonding are omitted for clarity.

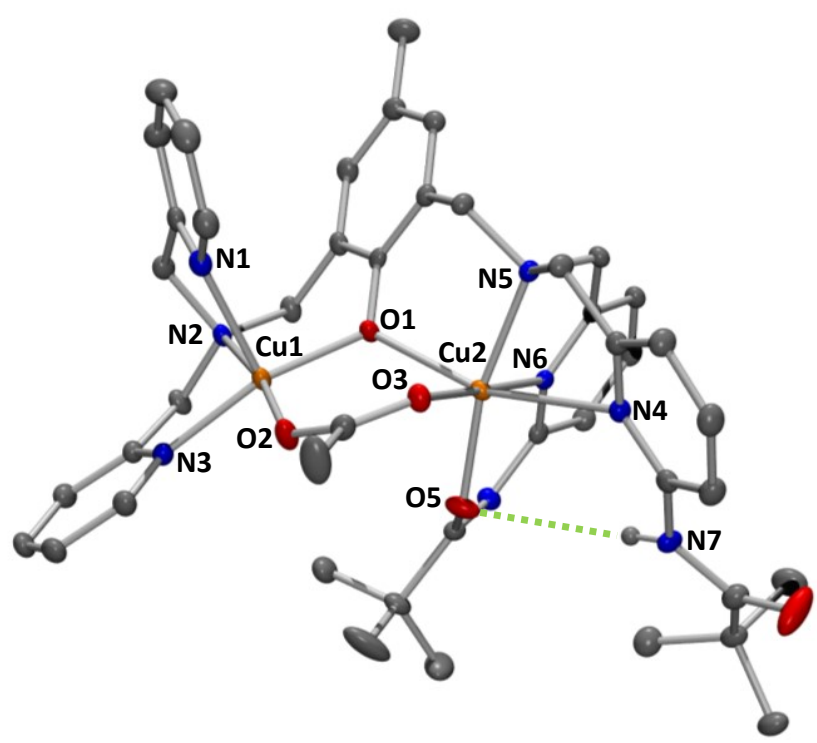

Figure 72: ORTEP plot of $\left[\mathrm{Cu}_{2}{ }_{2}\left(\mathrm{HSIM}^{1}\right)(\mu-\mathrm{OAc})\right] \mathrm{PF}_{6}$ with $50 \%$ probability level of thermal ellipsoids showing hydrogen bonds as green, dotted lines. Counter ion and hydrogen atoms not involved in hydrogen bonding are omitted for clarity. 
Table 31: Crystallographic data for $\left\{\mathrm{Na}\left[\mathrm{Fe}^{\prime \prime \prime}\left(\mathrm{H}_{2} \mathrm{SIM}^{2}\right)\right]_{2}(\mu-\mathrm{OAC})\left(\mu-\mathrm{OH}_{2}\right)\right\}\left(\mathrm{BF}_{4}\right)_{2} \cdot 3 \mathrm{H}_{2} \mathrm{O}$.

\begin{tabular}{|c|c|}
\hline & $\left\{\mathrm{Na}\left[\mathrm{Fe}\left(\mathrm{H}_{2} \mathrm{~L}^{4}\right)\right]_{2}(\mu-\mathrm{OAc})\left(\mu-\mathrm{OH}_{2}\right)\right\}\left(\mathrm{BF}_{4}\right)_{2} \cdot 3 \mathrm{H}_{2} \mathrm{O}$ \\
\hline Empirical formula & $\mathrm{C}_{45} \mathrm{H}_{53} \mathrm{~N}_{7} \mathrm{O}_{6} \mathrm{BF}_{4} \mathrm{Na}_{0.5} \mathrm{Fe}$ \\
\hline Formula weight & 942.10 \\
\hline Temperature (K) & 293(2) \\
\hline Wavelength $(\AA ̊)$ & 0.71073 \\
\hline Crystal system & Triclinic \\
\hline Space group & $P-1$ \\
\hline a $(\AA ̊)$ & $17.6526(17)$ \\
\hline$b(\AA)$ & $17.6900(17)$ \\
\hline$c(\AA)$ & $19.16(3)$ \\
\hline$\alpha\left({ }^{\circ}\right)$ & $77.164(10)$ \\
\hline$\beta\left({ }^{\circ}\right)$ & $63.144(12)$ \\
\hline$\gamma\left({ }^{\circ}\right)$ & $84.177(8)$ \\
\hline $\operatorname{Vol}\left(\AA^{3}\right)$ & $5204(8)$ \\
\hline Z & 4 \\
\hline$\mu\left(\mathrm{mm}^{-1}\right)$ & 0.357 \\
\hline$F(000)$ & 1970 \\
\hline $\mathrm{P}\left(\mathrm{Mg} / \mathrm{m}^{3}\right)$ & 1.202 \\
\hline Reflections collected & 39653 \\
\hline Independent reflections $\left[R_{\text {int }}\right]$ & $18269[0.1091]$ \\
\hline$\theta$ range for data collection $\left({ }^{\circ}\right)$ & $2.84-25.00$ \\
\hline GOOF on $\mathrm{F}^{2}$ & 0.976 \\
\hline Final $R$ indices & $R_{1}=0.1245$ \\
\hline$[1>2 \sigma(I)]$ & $w R_{2}=0.2919$ \\
\hline$R$ indices & $R_{1}=0.2396$ \\
\hline (all data) & $w R_{2}=0.3768$ \\
\hline Largest diff. peak and hole (e $\left.\AA^{-3}\right)$ & 0.667 and -0.507 \\
\hline
\end{tabular}

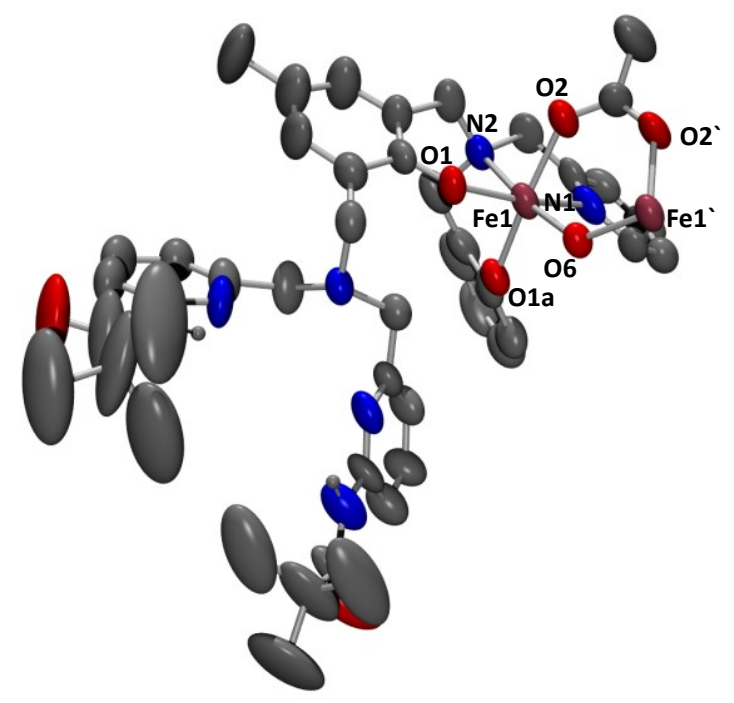

Figure 73: ORTEP plot of the monomeric structure of $\left\{\mathrm{Na}\left[\mathrm{Fe}^{\prime \prime \prime}\left(\mathrm{H}_{2} \mathrm{SIM}^{2}\right)\right]_{2}(\mu-\mathrm{OAc})\left(\mu-\mathrm{OH}_{2}\right)\right\}\left(\mathrm{BF}_{4}\right)_{2}$ including bridging acetate and aqua ligands with $50 \%$ probability level of thermal ellipsoids. Counter ion and hydrogen atoms not involved in hydrogen bonding are omitted for clarity. 
Table 32: Atomic coordinates $\left(\cdot 10^{4}\right)$ and equivalent isotropic displacement parameters $\left(\AA^{2} \cdot 10^{3}\right)$ for $\left\{\mathrm{Na}\left[\mathrm{Fe}^{\mathrm{III}}\left(\mathrm{H}_{2} \mathrm{SIM}^{2}\right)\right]_{2}(\mu-\mathrm{OAc})\left(\mu-\mathrm{OH}_{2}\right)\right\}\left(\mathrm{BF}_{4}\right)_{2} ; \mathrm{U}(\mathrm{eq})$ is defined as one third of the trace of the orthogonalized $\mathrm{U}^{\mathrm{ij}}$ tensor.

\begin{tabular}{|c|c|c|c|c|}
\hline & $x$ & $y$ & $z$ & $\mathrm{U}(\mathrm{eq})$ \\
\hline $\mathrm{Fe}(1)$ & $6817(1)$ & 1666(1) & $2786(1)$ & $53(1)$ \\
\hline $\mathrm{Fe}(2)$ & $7525(1)$ & $617(1)$ & $4202(1)$ & $53(1)$ \\
\hline C(28') & $9046(5)$ & $4446(5)$ & $3652(6)$ & $73(3)$ \\
\hline $\mathrm{O}(1)$ & $7668(3)$ & $1468(3)$ & $1778(3)$ & $56(1)$ \\
\hline$O(1 \mathrm{~A})$ & $6990(3)$ & $2797(3)$ & $2502(3)$ & $54(1)$ \\
\hline$O\left(1 A^{\prime}\right)$ & $6852(3)$ & $1026(3)$ & $5114(3)$ & $60(2)$ \\
\hline$O\left(1^{\prime}\right)$ & $8592(3)$ & $983(3)$ & $4059(3)$ & $54(1)$ \\
\hline$C(29)$ & $9814(5)$ & $4897(5)$ & $1163(6)$ & $81(3)$ \\
\hline$C\left(34^{\prime}\right)$ & 13920(6) & $1685(6)$ & $1044(8)$ & $88(3)$ \\
\hline$N(5)$ & $9373(4)$ & $2569(3)$ & $364(4)$ & $54(2)$ \\
\hline$O(6)$ & $7464(3)$ & $1472(3)$ & $3402(3)$ & $49(1)$ \\
\hline$N\left(6^{\prime}\right)$ & $9211(4)$ & $3817(4)$ & $3348(4)$ & $64(2)$ \\
\hline$C(37)$ & $11742(6)$ & $4812(7)$ & $476(8)$ & $96(4)$ \\
\hline$C(3)$ & $4193(6)$ & $2608(5)$ & $4695(8)$ & $89(4)$ \\
\hline$N(2)$ & $5930(4)$ & $1810(4)$ & $2246(4)$ & $57(2)$ \\
\hline$O(2)$ & $6367(3)$ & $570(3)$ & $3195(4)$ & $68(2)$ \\
\hline $\mathrm{O}\left(4^{\prime}\right)$ & $14449(4)$ & $1808(5)$ & $1249(5)$ & $112(3)$ \\
\hline$N\left(4^{\prime}\right)$ & $11868(4)$ & $2042(4)$ & $2538(5)$ & $64(2)$ \\
\hline$N\left(5^{\prime}\right)$ & $10029(4)$ & $2241(4)$ & $3172(4)$ & $59(2)$ \\
\hline$O\left(2^{\prime}\right)$ & $6495(4)$ & $72(3)$ & $4295(4)$ & $75(2)$ \\
\hline$C(28)$ & $10300(5)$ & $4298(5)$ & $830(6)$ & $69(3)$ \\
\hline$C\left(23^{\prime}\right)$ & 13110(7) & $2646(6)$ & $2376(8)$ & $88(3)$ \\
\hline$N(6)$ & 9993(4) & $3669(4)$ & $801(4)$ & $66(2)$ \\
\hline$N\left(2^{\prime}\right)$ & $7687(4)$ & $-457(3)$ & $4967(4)$ & $55(2)$ \\
\hline$N(1)$ & $5641(4)$ & 1934(4) & $3739(5)$ & $67(2)$ \\
\hline$C\left(14^{\prime}\right)$ & $9220(5)$ & $-171(4)$ & $4466(5)$ & $57(2)$ \\
\hline$C\left(20^{\prime}\right)$ & $9279(4)$ & $541(4)$ & $3970(5)$ & $54(2)$ \\
\hline C(33') & 10197(5) & 2955(4) & $2551(5)$ & $61(2)$ \\
\hline$N\left(7^{\prime}\right)$ & 13099(4) & $1826(4)$ & $1466(5)$ & $79(2)$ \\
\hline$N\left(8^{\prime}\right)$ & $8200(4)$ & $4634(4)$ & $4117(5)$ & $80(3)$ \\
\hline$N(4)$ & $10631(6)$ & $2804(5)$ & $-1148(5)$ & $84(3)$ \\
\hline$C(4)$ & $4231(5)$ & $2347(5)$ & $4068(7)$ & $70(3)$ \\
\hline$C(32)$ & 9139(5) & $3625(4)$ & $1085(5)$ & $54(2)$ \\
\hline$C\left(16^{\prime}\right)$ & $10724(5)$ & $-414(5)$ & $3699(6)$ & $69(3)$ \\
\hline$C\left(32^{\prime}\right)$ & $10021(5)$ & $3673(5)$ & $2885(5)$ & $60(2)$ \\
\hline$C\left(11^{\prime}\right)$ & $6640(5)$ & $-3(6)$ & $6210(5)$ & $65(2)$ \\
\hline$N\left(1^{\prime}\right)$ & $8269(4)$ & $-103(4)$ & $3369(4)$ & $62(2)$ \\
\hline
\end{tabular}




\begin{tabular}{|c|c|c|c|c|}
\hline \multicolumn{5}{|l|}{ PENDIX } \\
\hline$C\left(24^{\prime}\right)$ & $12657(9)$ & $2951(7)$ & $3013(9)$ & $108(4)$ \\
\hline$C(39 A)$ & $12926(8)$ & $4295(12)$ & $-653(12)$ & 197(10) \\
\hline$C(11)$ & $5864(5)$ & $3251(5)$ & $2168(6)$ & $63(3)$ \\
\hline $\mathrm{O}(5)$ & $11481(4)$ & $5386(4)$ & $710(6)$ & $121(4)$ \\
\hline$C(19)$ & $8466(6)$ & $1516(5)$ & $439(6)$ & $71(3)$ \\
\hline$C(26)$ & $9852(7)$ & $3004(5)$ & $-1097(6)$ & $76(3)$ \\
\hline$C\left(18^{\prime}\right)$ & $10760(5)$ & 299(5) & $3228(5)$ & $63(2)$ \\
\hline$F(4)$ & $1712(5)$ & $3813(4)$ & $5661(4)$ & $128(3)$ \\
\hline$C(5)$ & $4967(5)$ & $2021(5)$ & $3586(5)$ & $60(2)$ \\
\hline$C(7)$ & $6275(5)$ & $3945(5)$ & $2876(5)$ & $69(3)$ \\
\hline$C\left(21^{\prime}\right)$ & $10128(5)$ & $1536(5)$ & 2791(5) & $63(2)$ \\
\hline$C\left(13^{\prime}\right)$ & $8373(5)$ & $-395(4)$ & $5189(5)$ & $56(2)$ \\
\hline$C(38)$ & $12663(7)$ & $4617(8)$ & $112(12)$ & $138(7)$ \\
\hline$C\left(22^{\prime}\right)$ & $12703(5)$ & $2182(5)$ & $2160(6)$ & $69(3)$ \\
\hline$O\left(5^{\prime}\right)$ & $7490(4)$ & $3561(4)$ & $4355(5)$ & $106(3)$ \\
\hline$C(33)$ & $8847(5)$ & $2847(4)$ & $1125(5)$ & $58(2)$ \\
\hline$C\left(15^{\prime}\right)$ & $9929(5)$ & $-627(5)$ & $4335(6)$ & $67(3)$ \\
\hline$C(21)$ & $9255(5)$ & $1713(4)$ & $443(5)$ & $62(2)$ \\
\hline$C(13)$ & $6113(5)$ & $1218(6)$ & $1777(5)$ & $69(3)$ \\
\hline$N(8)$ & 11199(4) & $4277(4)$ & $532(5)$ & $87(3)$ \\
\hline$C\left(19^{\prime}\right)$ & $10066(4)$ & $784(4)$ & $3341(5)$ & $51(2)$ \\
\hline$F(1)$ & $2375(5)$ & $3124(6)$ & $4759(7)$ & $205(5)$ \\
\hline $\mathrm{N}(7)$ & $12038(6)$ & $2577(7)$ & $-1872(6)$ & $136(4)$ \\
\hline C(27’) & $10522(6)$ & $2187(5)$ & $3642(6)$ & $77(3)$ \\
\hline$C(25)$ & $9691(7)$ & $3159(5)$ & $-1722(7)$ & $80(3)$ \\
\hline$F(2)$ & $1057(6)$ & 2959(4) & $5449(6)$ & 171(4) \\
\hline C(17’) & $11472(6)$ & $-929(6)$ & $3566(7)$ & $97(4)$ \\
\hline$F(3)$ & $1561(6)$ & $4028(4)$ & $4585(6)$ & 168(4) \\
\hline$C\left(26^{\prime}\right)$ & $11444(6)$ & $2364(5)$ & $3173(7)$ & $74(3)$ \\
\hline$C(7 A)$ & $6398(5)$ & $3331(5)$ & $2511(5)$ & $62(2)$ \\
\hline$C\left(7 A^{\prime}\right)$ & $6624(5)$ & $782(6)$ & $5890(6)$ & $68(3)$ \\
\hline$C(27)$ & $9208(6)$ & $3051(6)$ & $-270(6)$ & $78(3)$ \\
\hline$C(30)$ & $8957(6)$ & $4841(5)$ & $1420(6)$ & $77(3)$ \\
\hline$C(35)$ & $13574(14)$ & $2320(20)$ & $-2341(11)$ & $230(14)$ \\
\hline$C\left(38^{\prime}\right)$ & $6677(6)$ & $4635(6)$ & $4854(8)$ & $91(4)$ \\
\hline$C(31)$ & $8610(5)$ & $4193(5)$ & $1393(6)$ & $69(3)$ \\
\hline$C\left(12^{\prime}\right)$ & $6879(5)$ & $-616(5)$ & $5728(5)$ & $69(3)$ \\
\hline$C(18)$ & $8496(7)$ & $1395(7)$ & $-277(7)$ & $100(4)$ \\
\hline$C(22)$ & $11271(7)$ & $2787(5)$ & $-1871(6)$ & $83(3)$ \\
\hline $\mathrm{O}(4)$ & $12893(7)$ & $2658(8)$ & $-3172(6)$ & 181(5) \\
\hline$C(14)$ & $6961(5)$ & $1299(5)$ & $1058(6)$ & $67(2)$ \\
\hline
\end{tabular}




\begin{tabular}{|c|c|c|c|c|}
\hline$C(12)$ & $6009(5)$ & $2608(5)$ & $1729(6)$ & $78(3)$ \\
\hline$C(1)$ & $5604(5)$ & $2190(5)$ & $4357(6)$ & $61(2)$ \\
\hline$C\left(4^{\prime}\right)$ & $8971(7)$ & $-1322(5)$ & $3234(7)$ & $98(4)$ \\
\hline$C\left(2^{\prime}\right)$ & $9307(8)$ & $-326(6)$ & $2105(6)$ & $110(4)$ \\
\hline C(37’) & $7502(6)$ & $4235(6)$ & $4413(6)$ & $81(3)$ \\
\hline$C\left(5^{\prime}\right)$ & $8386(6)$ & $-849(5)$ & $3696(5)$ & $66(2)$ \\
\hline$C\left(8^{\prime}\right)$ & $6096(7)$ & $1088(8)$ & $7184(7)$ & $111(4)$ \\
\hline$C(23)$ & $11137(8)$ & $2966(6)$ & $-2542(7)$ & $93(3)$ \\
\hline$C(2)$ & $4900(6)$ & $2519(5)$ & $4854(6)$ & $67(3)$ \\
\hline$C(6)$ & $5067(4)$ & 1693(5) & $2913(6)$ & $67(3)$ \\
\hline$c\left(31^{\prime}\right)$ & $10676(6)$ & $4150(5)$ & $2689(7)$ & $84(3)$ \\
\hline$C(8)$ & $5669(7)$ & $4473(6)$ & $2890(7)$ & $89(4)$ \\
\hline$C(24)$ & $10321(8)$ & $3155(6)$ & $-2462(7)$ & $97(3)$ \\
\hline$C(39 D)$ & $5952(6)$ & $4100(7)$ & $5122(8)$ & $129(6)$ \\
\hline$c\left(35^{\prime}\right)$ & $14183(7)$ & $1403(8)$ & $308(8)$ & $98(4)$ \\
\hline$C\left(6^{\prime}\right)$ & $7830(6)$ & $-1105(4)$ & $4548(5)$ & $66(3)$ \\
\hline$C(9)$ & $5175(7)$ & $4429(7)$ & $2546(8)$ & $111(5)$ \\
\hline$C\left(25^{\prime}\right)$ & 11818(9) & $2820(6)$ & $3422(8)$ & $97(4)$ \\
\hline$C(36 B)$ & $13596(10)$ & $2943(16)$ & $-1864(14)$ & $253(14)$ \\
\hline$C(20)$ & $7697(5)$ & $1416(4)$ & $1113(5)$ & $62(2)$ \\
\hline$C\left(7^{\prime}\right)$ & $6366(5)$ & $1326(6)$ & $6377(6)$ & $81(3)$ \\
\hline$C(10)$ & $5241(5)$ & $3797(6)$ & 2192(7) & $92(4)$ \\
\hline$C\left(10^{\prime}\right)$ & $6389(6)$ & $-209(7)$ & $7036(6)$ & $88(3)$ \\
\hline$C(16)$ & $7809(9)$ & 1219(9) & $-337(8)$ & $131(5)$ \\
\hline$C\left(9^{\prime}\right)$ & $6109(7)$ & $328(8)$ & $7516(7)$ & $103(4)$ \\
\hline$C\left(3^{\prime}\right)$ & $9478(9)$ & $-1067(6)$ & $2432(8)$ & $129(6)$ \\
\hline$C\left(1^{\prime}\right)$ & $8715(6)$ & $134(5)$ & $2599(6)$ & $75(3)$ \\
\hline$C(39 C)$ & 13161(8) & $5357(9)$ & $-122(12)$ & $200(9)$ \\
\hline$C(2 A)$ & $5507(8)$ & $-491(7)$ & $4067(8)$ & $136(5)$ \\
\hline$C(39 E)$ & $6593(7)$ & $5391(7)$ & $4311(10)$ & $142(6)$ \\
\hline$C(1 \mathrm{~A})$ & $6178(6)$ & $87(5)$ & $3828(6)$ & $69(3)$ \\
\hline$C\left(29^{\prime}\right)$ & $9655(6)$ & $4935(5)$ & $3510(8)$ & $110(5)$ \\
\hline$C(36 D)$ & $15111(7)$ & 1124(9) & $16(9)$ & $146(6)$ \\
\hline$C(34)$ & 12799(10) & $2559(11)$ & $-2512(9)$ & $166(8)$ \\
\hline$C(15)$ & $7031(7)$ & $1202(8)$ & $336(7)$ & $117(4)$ \\
\hline$C(39 B)$ & $12856(7)$ & $3990(10)$ & $662(11)$ & 198(10) \\
\hline$C(39 F)$ & $6695(7)$ & $4825(9)$ & $5562(9)$ & $149(6)$ \\
\hline$C(36 E)$ & 13644(8) & $749(9)$ & $425(9)$ & 139(5) \\
\hline$C(36 F)$ & 14073(9) & $2053(10)$ & $-308(8)$ & $168(7)$ \\
\hline$B(1)$ & $1723(8)$ & $3461(8)$ & $5115(8)$ & $79(4)$ \\
\hline$C(17)$ & $7872(9)$ & $1066(11)$ & $-1100(8)$ & 189(9) \\
\hline
\end{tabular}




\begin{tabular}{lllll} 
APPENDIX & & & \\
$\mathrm{C}\left(300^{\prime}\right)$ & $10477(7)$ & $4779(7)$ & $3034(10)$ & $132(6)$ \\
$\mathrm{C}(36 \mathrm{~A})$ & $13523(17)$ & $1564(14)$ & $-1878(14)$ & $305(19)$ \\
$\mathrm{C}(36 \mathrm{C})$ & $14383(10)$ & $2522(15)$ & $-3127(11)$ & $265(14)$ \\
$\mathrm{F}(5)$ & $603(3)$ & $1941(3)$ & $884(4)$ & $99(2)$ \\
$\mathrm{F}(6)$ & $1773(4)$ & $1479(7)$ & $942(5)$ & $182(4)$ \\
$\mathrm{F}(8)$ & $1702(5)$ & $2623(7)$ & $491(15)$ & $393(16)$ \\
$\mathrm{B}(2)$ & $1490(12)$ & $1989(11)$ & $605(12)$ & $174(13)$ \\
$\mathrm{F}(7)$ & $1807(8)$ & $1875(13)$ & $-150(6)$ & $299(9)$ \\
$\mathrm{O}(9)$ & $8545(4)$ & $2292(3)$ & $4613(4)$ & $79(2)$ \\
$\mathrm{Na}(1)$ & $8251(3)$ & $2827(3)$ & $2974(3)$ & $133(2)$ \\
$\mathrm{O}(8)$ & $9030(20)$ & $3694(18)$ & $-4100(20)$ & $259(14)$ \\
$\mathrm{O}(7)$ & $11161(13)$ & $6458(12)$ & $3191(16)$ & $165(8)$ \\
\hline
\end{tabular}

Table 33: Crystallographic data for $\left[\mathrm{Zn}_{2}{ }_{2}\left(\mathrm{~L}^{1}\right)(\mu-\mathrm{OAC})_{2}\right] \mathrm{PF} \mathrm{F}_{6},\left[\mathrm{Zn}_{2}{ }_{2}\left(\mathrm{H}_{2} \mathrm{~L}^{2}\right)(\mu-\mathrm{OAc})_{2}\right] \mathrm{PF} 6 \cdot 0.5 \mathrm{H}_{2} \mathrm{O} \cdot 0.5 \mathrm{CH}_{3} \mathrm{COCH}_{3}$ and $\left[\mathrm{Zn}_{2}{ }_{2}\left(\mathrm{SIM}^{2}\right)(\mu-\mathrm{OAc})_{2}\right] \mathrm{PF} 6$.

$\left[\mathrm{Zn}_{2}\left(\mathrm{~L}^{1}\right)(\mu-\mathrm{OAC})_{2}\right] \mathrm{PF}_{6} \quad\left[\mathrm{Zn}_{2}\left(\mathrm{H}_{2} \mathrm{~L}^{2}\right)(\mu-\mathrm{OAC})_{2}\right] \mathrm{PF}_{6} \quad\left[\mathrm{Zn}_{2}\left(\mathrm{SIM}^{2}\right)(\mu-\mathrm{OAC})_{2}\right] \mathrm{PF}_{6}$ $\begin{array}{ll}\cdot 0.5 \mathrm{H}_{2} \mathrm{O} \cdot 0.5 \mathrm{CH}_{3} \mathrm{COCH}_{3} & \\ \mathrm{C}_{48.50} \mathrm{H}_{61} \mathrm{~F}_{6} \mathrm{~N}_{8} \mathrm{O}_{8} \mathrm{PZn}_{2} & \mathrm{C}_{37} \mathrm{H}_{41} \mathrm{~F}_{6} \mathrm{~N}_{8} \mathrm{O}_{5} \mathrm{PZn}_{2}\end{array}$

\begin{tabular}{|c|c|c|c|}
\hline Empirical formula & $\mathrm{C}_{37} \mathrm{H}_{39} \mathrm{~F}_{6} \mathrm{~N}_{6} \mathrm{O}_{5} \mathrm{PZn}_{2}$ & $\mathrm{C}_{48.50} \mathrm{H}_{61} \mathrm{~F}_{6} \mathrm{~N}_{8} \mathrm{O}_{8} \mathrm{PZn}_{2}$ & $\mathrm{C}_{37} \mathrm{H}_{41} \mathrm{~F}_{6} \mathrm{~N}_{8} \mathrm{O}_{5} \mathrm{PZn}_{2}$ \\
\hline Formula weight & 923.45 & 1159.76 & 953.49 \\
\hline Temperature (K) & $293(2)$ & $293(2)$ & $200(2)$ \\
\hline Wavelength $(\AA ̊)$ & 1.54180 & 0.71073 & 0.71073 \\
\hline Crystal system & Triclinic & Monoclinic & Orthorhombic \\
\hline Space group & $P-1$ & $\mathrm{P} 2_{1} / \mathrm{n}$ & P21 2121 \\
\hline $\mathrm{a}(\AA \cap)$ & $10.2562(4)$ & $16.2410(10)$ & $11.1444(6)$ \\
\hline b (Å) & $12.5876(5)$ & $21.5930(10)$ & $17.0144(9)$ \\
\hline$c(\AA ̊)$ & $16.7528(7)$ & $16.3693(8)$ & $20.6731(8)$ \\
\hline$\alpha\left({ }^{\circ}\right)$ & $89.561(3)$ & 90 & 90 \\
\hline$\beta\left({ }^{\circ}\right)$ & 74.354(3) & $100.533(6)$ & 90 \\
\hline$\gamma\left({ }^{\circ}\right)$ & $74.696(3)$ & 90 & 90 \\
\hline $\operatorname{Vol}\left(\AA^{3}\right)$ & 2004.11(14) & $5643.9(5)$ & 3919.9(3) \\
\hline Z & 2 & 4 & 4 \\
\hline$\mu\left(\mathrm{mm}^{-1}\right)$ & 2.531 & 0.953 & 1.348 \\
\hline$F(000)$ & 944 & 2404 & 1952 \\
\hline $\mathrm{P}\left(\mathrm{Mg} / \mathrm{m}^{3}\right)$ & 1.530 & 1.365 & 1.616 \\
\hline Reflections collected & 17469 & 25999 & 10212 \\
\hline $\begin{array}{l}\text { Independent } \\
\text { reflections }\left[R_{\text {int }}\right]\end{array}$ & $6308[0.0222]$ & 9929 [0.0342] & $6482[0.0394]$ \\
\hline $\begin{array}{l}\Theta \text { range for data } \\
\text { collection }\left({ }^{\circ}\right)\end{array}$ & $3.65-62.25$ & $3.10-25.00$ & $2.94-25.00$ \\
\hline GOOF on $F^{2}$ & 1.026 & 1.058 & 1.025 \\
\hline Final $R$ indices & $R_{1}=0.0303$ & $R_{1}=0.0564$ & $R_{1}=0.0523$ \\
\hline$[1>2 \sigma(I)]$ & $w R_{2}=0.0792$ & $w R_{2}=0.1520$ & $w R_{2}=0.1185$ \\
\hline$R$ indices & $R_{1}=0.0362$ & $R_{1}=0.0775$ & $R_{1}=0.0629$ \\
\hline (all data) & $w R_{2}=0.0847$ & $w R_{2}=0.1643$ & $w R_{2}=0.1263$ \\
\hline $\begin{array}{l}\text { Largest diff. peak and } \\
\text { hole }\left(\mathrm{e} \AA^{-3}\right)\end{array}$ & 0.371 and -0.251 & 1.013 and -0.340 & 1.087 and -0.363 \\
\hline CCDC number & 998888 & 998889 & 998890 \\
\hline
\end{tabular}


Table 34: Crystallographic data for $\left[\mathrm{Zn}_{2}{ }_{2}\left(\mathrm{H}_{2} \mathrm{SIM}^{1}\right)(\mu-\mathrm{OAc})(\mathrm{OH})\right] \mathrm{PF}_{6}$, $\left[\mathrm{Zn}_{2}{ }_{2}\left(\mathrm{H}_{2} \mathrm{SIM}^{3}\right)\left(\mu-\mathrm{O}_{3} \mathrm{POC}_{6} \mathrm{H}_{4} \mathrm{NO}_{2}\right)\right] \mathrm{PF}_{6}$ $\cdot \mathrm{H}_{2} \mathrm{O} \cdot \mathrm{CH}_{3} \mathrm{OH}$ and $\left[\mathrm{Zn}_{2}{ }_{2}\left(\mathrm{H}_{2} \mathrm{SIM}^{3}\right)(\mu-\mathrm{OAC})(\mathrm{OH})\right]$.

$\left[\mathrm{Zn}_{2}\left(\mathrm{H}_{2} \mathrm{SIM}^{1}\right)(\mu-\mathrm{OAC})(\mathrm{OH})\right] \mathrm{PF}_{6} \quad\left[\mathrm{Zn}_{2}\left(\mathrm{H}_{2} \mathrm{SIM}^{1}\right)\left(\mu-\mathrm{O}_{3} \mathrm{POC}_{6} \mathrm{H}_{4} \mathrm{NO}_{2}\right)\right] \mathrm{PF}_{6} \quad\left[\mathrm{Zn}_{2}\left(\mathrm{H}_{2} \mathrm{SIM}^{3}\right)(\mu-\mathrm{OAC})(\mathrm{OH})\right]$ $\cdot \mathrm{H}_{2} \mathrm{O} \cdot \mathrm{CH}_{3} \mathrm{OH}$

\begin{tabular}{|c|c|c|c|}
\hline Empirical formula & $\mathrm{C}_{45} \mathrm{H}_{55} \mathrm{~F}_{6} \mathrm{~N}_{8} \mathrm{O}_{6} \mathrm{PZn}_{2}$ & $\mathrm{C}_{50} \mathrm{H}_{61} \mathrm{~F}_{6} \mathrm{~N}_{9} \mathrm{O}_{11} \mathrm{P}_{2} \mathrm{Zn}_{2}$ & $\mathrm{C}_{46} \mathrm{H}_{55} \mathrm{~N}_{7} \mathrm{O}_{7} \mathrm{Zn}_{2}$ \\
\hline Formula weight & 1079.68 & 1270.76 & 948.71 \\
\hline Temperature (K) & $293(2)$ & $190(2)$ & $200(2)$ \\
\hline Wavelength (Å) & 1.54180 & 0.71073 & 1.54180 \\
\hline Crystal system & Monoclinic & Monoclinic & Triclinic \\
\hline Space group & $\mathrm{P} 2_{1} / \mathrm{n}$ & $\mathrm{P} 2_{1} / \mathrm{n}$ & P-1 \\
\hline $\mathrm{a}(\AA ̊)$ & $16.8815(6)$ & $14.5695(6)$ & $13.3627(8)$ \\
\hline b (Å) & $13.5366(5)$ & $23.3831(8)$ & $14.0727(8)$ \\
\hline$c(\AA ̊)$ & $21.8837(9)$ & $17.1987(5)$ & $14.7688(10)$ \\
\hline$\alpha\left({ }^{\circ}\right)$ & 90 & 90 & $70.854(6)$ \\
\hline$\beta\left(^{\circ}\right)$ & $105.077(4)$ & $107.817(4)$ & $66.835(6)$ \\
\hline$\gamma\left({ }^{\circ}\right)$ & 90 & 90 & $62.523(6)$ \\
\hline $\operatorname{Vol}\left(\AA^{3}\right)$ & $4828.7(3)$ & $5578.2(3)$ & $2228.5(2)$ \\
\hline Z & 4 & 4 & 2 \\
\hline$\mu\left(\mathrm{mm}^{-1}\right)$ & 2.215 & 1.004 & 1.803 \\
\hline$F(000)$ & 2232 & 2624 & 992 \\
\hline $\mathrm{P}\left(\mathrm{Mg} / \mathrm{m}^{3}\right)$ & 1.485 & 1.513 & 1.414 \\
\hline $\begin{array}{l}\text { Reflections } \\
\text { collected }\end{array}$ & 53093 & 22528 & 20772 \\
\hline $\begin{array}{l}\text { Independent } \\
\text { reflections }\left[R_{\text {int }}\right]\end{array}$ & $7633[0.0738]$ & $9829[0.0664]$ & 6963 [0.0508] \\
\hline $\begin{array}{l}\Theta \text { range for data } \\
\text { collection }\left(^{\circ}\right)\end{array}$ & $2.96-62.39$ & $2.89-25.00$ & $3.31-62.41$ \\
\hline GOOF on $F^{2}$ & 1.018 & 1.030 & 1.051 \\
\hline Final $R$ indices & $R_{1}=0.0485$ & $R_{1}=0.0671$ & $R_{1}=0.049$ \\
\hline$[/>2 \sigma(I)]$ & $w R_{2}=0.1198$ & $w R_{2}=0.1291$ & $w R_{2}=0.127$ \\
\hline$R$ indices & $R_{1}=0.0669$ & $R_{1}=0.1115$ & $R_{1}=0.0563$ \\
\hline (all data) & $w R_{2}=0.1324$ & $w R_{2}=0.1482$ & $w R_{2}=0.1359$ \\
\hline $\begin{array}{l}\text { Largest diff. peak } \\
\left.\text { and hole (e } \AA^{-3}\right)\end{array}$ & 0.513 and -0.386 & 0.883 and -0.690 & $\begin{array}{l}1.038 \text { and } \\
-0.382\end{array}$ \\
\hline CCDC number & 998887 & 998891 & 1009842 \\
\hline
\end{tabular}




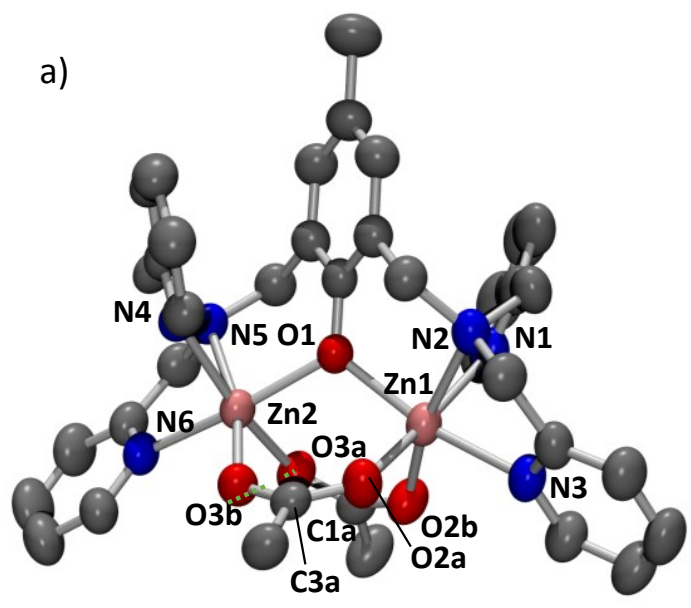

b)

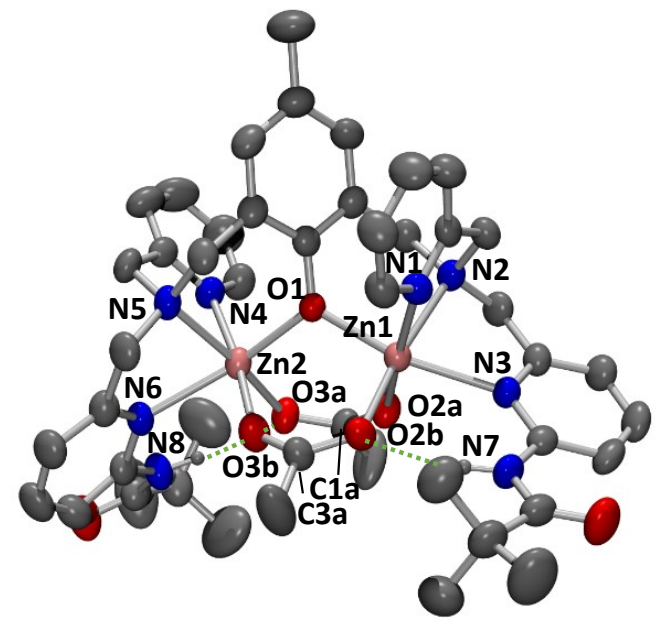

c)

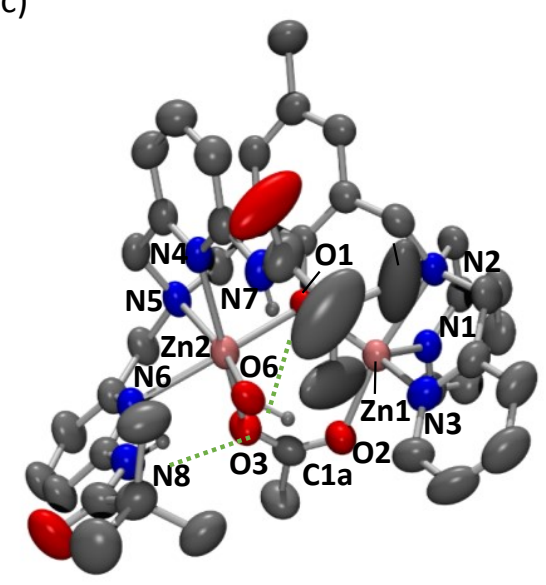

e)

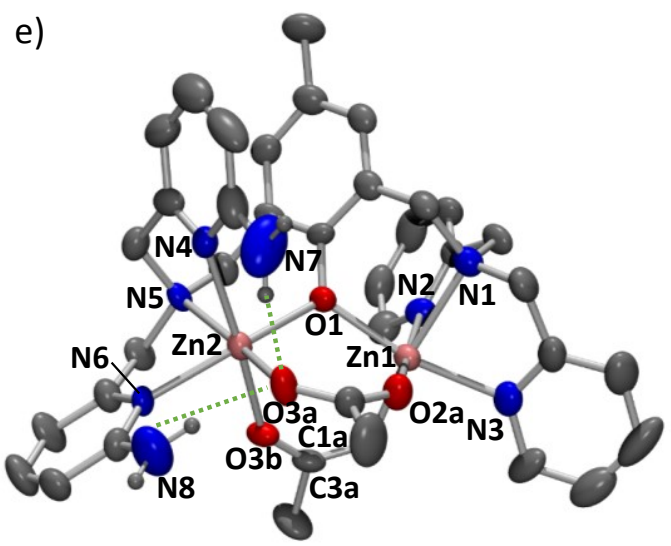

d)

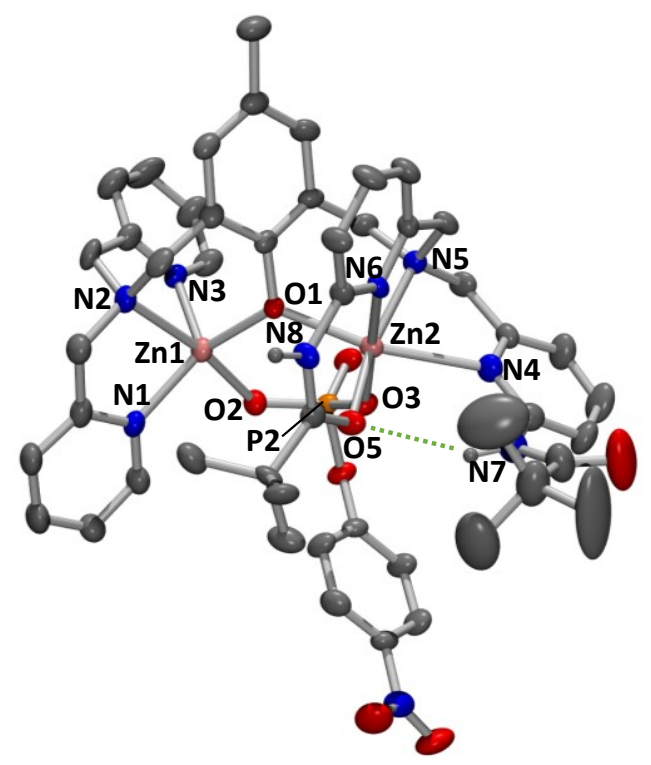

f)

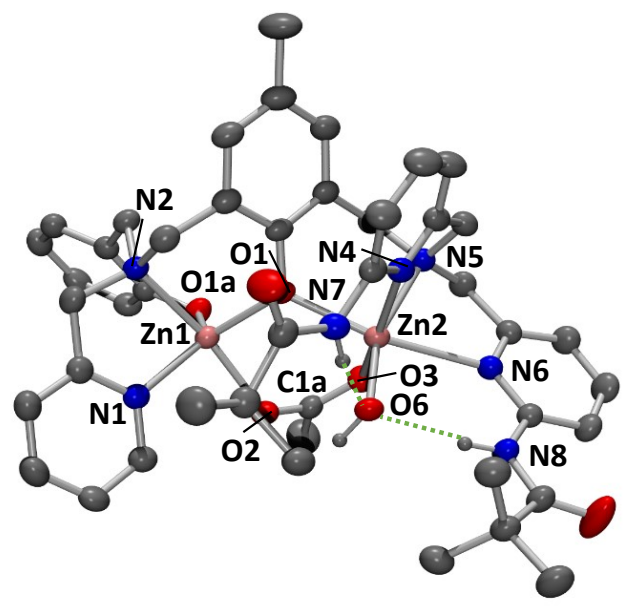

Figure 74: ORTEP plots of a) $\left.\left.\left[\mathrm{Zn}_{2}{ }_{2}\left(\mathrm{~L}^{1}\right)(\mu-\mathrm{OAc})_{2}\right] \mathrm{PF}{ }_{6}, \mathrm{~b}\right)\left[\mathrm{Zn}_{2}{ }_{2}\left(\mathrm{H}_{2} \mathrm{~L}^{2}\right)(\mu-\mathrm{OAc})_{2}\right] \mathrm{PF} 6_{6}, \mathrm{c}\right)\left[\mathrm{Zn}{ }_{2}\left(\mathrm{H}_{2} \mathrm{SIM}^{1}\right)(\mu-\mathrm{OAc})(\mathrm{OH})\right] \mathrm{PF}{ }_{6}$, d) $\left[\mathrm{Zn}_{2}{ }_{2}\left(\mathrm{H}_{2} \mathrm{SIM}^{1}\right)\left(\mu-\mathrm{O}_{3} \mathrm{POC}_{6} \mathrm{H}_{4} \mathrm{NO}_{2}\right)\right] \mathrm{PF} F_{6}$, e) $\left[\mathrm{Zn}_{2}{ }_{2}\left(\mathrm{SIM}^{2}\right)(\mu-\mathrm{OAc})_{2}\right] \mathrm{PF}_{6}$ and f) $\left[\mathrm{Zn}_{2}{ }_{2}\left(\mathrm{H}_{2} \mathrm{SIM}^{3}\right)(\mu-\mathrm{OAc})(\mathrm{OH})\right]$ with $50 \%$ probability level of thermal ellipsoids. Counter ions, non-coordinated solvent molecules and hydrogen atoms not involved in hydrogen bonding are omitted for clarity. 
Table 35: Crystallographic data for $\left[\mathrm{Fe}_{2}{ }_{2}\left(\mathrm{H}_{2} \mathrm{SIM}^{1}\right)(\mu-\mathrm{OAc})_{2}\right] \mathrm{PF} 6, \quad\left[\mathrm{Fe}_{2}{ }_{2}\left(\mathrm{H}_{2} \mathrm{~L}^{2}\right)(\mu-\mathrm{OAc})_{2}\right] \mathrm{PF}_{6} \cdot \mathrm{Et}_{2} \mathrm{O}$ and $\left\{\mathrm{Na}\left[\mathrm{Fe}_{2}{ }_{2}\left(\mathrm{H}_{2} \mathrm{SIM}^{3}\right)(\mu-\mathrm{OAC})_{2}\right]_{2}\right\} P \mathrm{~F}_{6}$.

$\left[\mathrm{Fe}_{2}{ }_{2}\left(\mathrm{H}_{2} \mathrm{SIM}^{1}\right)(\mu-\mathrm{OAC})_{2}\right] \mathrm{PF}_{6} \quad\left[\mathrm{Fe}_{2}{ }_{2}\left(\mathrm{H}_{2} \mathrm{~L}^{2}\right)(\mu-\mathrm{OAC})_{2}\right] \mathrm{PF}_{6} \quad\left\{\mathrm{Na}\left[\mathrm{Fe}_{2}{ }_{2}\left(\mathrm{H}_{2} \mathrm{SIM}^{3}\right)(\mu-\mathrm{OAC})_{2}\right]_{2}\right\} \mathrm{PF}_{6}$ $\cdot \mathrm{Et}_{2} \mathrm{O}$

\begin{tabular}{|c|c|c|c|}
\hline Empirical formula & $\mathrm{C}_{47} \mathrm{H}_{57} \mathrm{~F}_{6} \mathrm{Fe}_{2} \mathrm{~N}_{8} \mathrm{O}_{7} \mathrm{P}$ & $\mathrm{C}_{51} \mathrm{H}_{67} \mathrm{~F}_{6} \mathrm{Fe}_{2} \mathrm{~N}_{8} \mathrm{O}_{8} \mathrm{P}$ & $\mathrm{C}_{96} \mathrm{H}_{114} \mathrm{~F}_{6} \mathrm{Fe}_{4} \mathrm{~N}_{14} \mathrm{NaO}_{16} \mathrm{P}$ \\
\hline Formula weight & 1102.67 & 1176.79 & 2111.36 \\
\hline Temperature (K) & $120(1)$ & $110(1)$ & $100(1)$ \\
\hline Wavelength $(\AA)$ & 0.71073 & 0.7107 & 0.71073 \\
\hline Crystal system & Monoclinic & Monoclinic & Monoclinic \\
\hline Space group & P 21/n & P 21/n & P 21/n \\
\hline a $(\AA)$ & $14.9220(5)$ & $14.79581(6)$ & $20.237(9)$ \\
\hline$b(\AA)$ & 19.7653(5) & $22.51003(10)$ & $25.243(12)$ \\
\hline$c(\AA)$ & $18.4489(5)$ & $16.47614(8)$ & $23.062(12)$ \\
\hline$\alpha\left({ }^{\circ}\right)$ & 90 & 90 & 90 \\
\hline$\beta\left({ }^{\circ}\right)$ & $91.110(3)$ & $94.4616(4)$ & 109.169(16) \\
\hline$\gamma\left({ }^{\circ}\right)$ & 90 & 90 & 90 \\
\hline $\operatorname{Vol}\left(\AA^{3}\right)$ & $5440.3(3)$ & $5470.82(4)$ & $11128(9)$ \\
\hline Z & 4 & 4 & 4 \\
\hline$\mu\left(\mathrm{mm}^{-1}\right)$ & 0.637 & 0.639 & 0.603 \\
\hline$F(000)$ & 2288 & 2456 & 4400 \\
\hline $\mathrm{P}\left(\mathrm{Mg} / \mathrm{m}^{3}\right)$ & 1.346 & 1.429 & 1.260 \\
\hline $\begin{array}{l}\text { Reflections } \\
\text { collected }\end{array}$ & 77359 & 236690 & 195127 \\
\hline $\begin{array}{l}\text { Independent } \\
\text { reflections }\left[R_{\text {int }}\right]\end{array}$ & $9711[0.0887]$ & $13964[0.0358]$ & $21219[0.0778]$ \\
\hline $\begin{array}{l}\Theta \text { range for data } \\
\text { collection }\left({ }^{\circ}\right)\end{array}$ & $3.298-25.142$ & $3.243-28.909$ & $1.336-25.740$ \\
\hline GOOF on $F^{2}$ & 1.031 & 1.038 & 1.024 \\
\hline Final $R$ indices & $R_{1}=0.0908$ & $R_{1}=0.0437$ & $R_{1}=0.0634$ \\
\hline$[I>2 \sigma(I)]$ & $w R_{2}=0.2418$ & $w R_{2}=0.1087$ & $w R_{2}=0.1589$ \\
\hline$R$ indices & $R_{1}=0.1223$ & $R_{1}=0.0499$ & $R_{1}=0.0859$ \\
\hline (all data) & $w R_{2}=0.2675$ & $w R_{2}=0.1125$ & $w R_{2}=0.1733$ \\
\hline $\begin{array}{l}\text { Largest diff. peak } \\
\text { and hole }\left(\mathrm{e} \AA^{-3}\right)\end{array}$ & 1.714 and -0.890 & 1.044 and -0.867 & 1.655 and -1.052 \\
\hline Identification code & co_sb12 & co_sb4 & co_sb5 \\
\hline
\end{tabular}



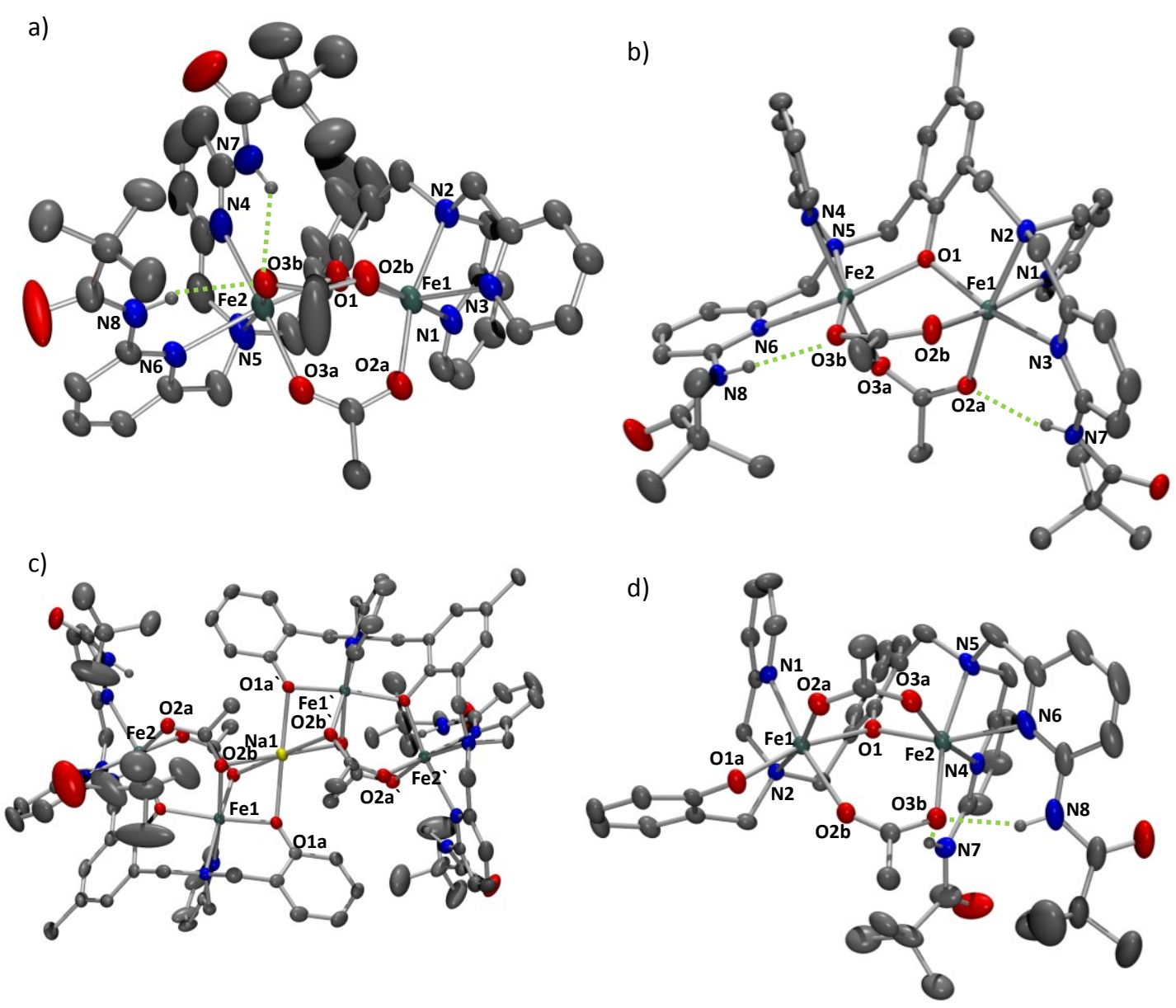

Figure 75: ORTEP plots of a) $\left[\mathrm{Fe}_{2}{ }_{2}\left(\mathrm{H}_{2} \mathrm{SIM}^{1}\right)(\mu-\mathrm{OAc})_{2}\right] \mathrm{PF}$, b) $\left[\mathrm{Fe}_{2}{ }_{2}\left(\mathrm{H}_{2} \mathrm{~L}^{2}\right)(\mu-\mathrm{OAc})_{2}\right] \mathrm{PF}_{6}, \quad$ c) $\left\{\mathrm{Na}\left[\mathrm{Fe}_{2}{ }_{2}\left(\mathrm{H}_{2} \mathrm{SIM}^{3}\right)(\mu-\mathrm{OAC})_{2}\right]_{2}\right\} \mathrm{PF}_{6}$ and $\left.\mathrm{d}\right)$ the respective monomer $\left[\mathrm{Fe}_{2}{ }_{2}\left(\mathrm{H}_{2} \mathrm{SIM}^{3}\right)(\mu-\mathrm{OAc})_{2}\right]$ with $50 \%$ probability level of thermal ellipsoids showing hydrogen bonds as green, dotted lines. Counter ions, non-coordinated solvent molecules and hydrogen atoms not involved in hydrogen bonding are omitted for clarity. 
Table 36: Crystallographic data for $\left[\mathrm{Fe}{ }^{I I I} \mathrm{Na}\left(\mathrm{H}_{2} \mathrm{SIM}^{1}\right)(\mu-\mathrm{OAc})_{2}\right] \mathrm{PF}_{6}$.

\begin{tabular}{|c|c|}
\hline & 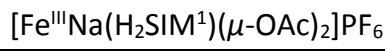 \\
\hline Empirical formula & $\mathrm{C}_{47} \mathrm{H}_{57} \mathrm{~F}_{6} \mathrm{FeN}_{8} \mathrm{NaO}_{7} \mathrm{P}$ \\
\hline Formula weight & 1069.81 \\
\hline Temperature (K) & $110(1)$ \\
\hline Wavelength ( $\AA$ ) & 0.7107 \\
\hline Crystal system & Monoclinic \\
\hline Space group & P 21/c \\
\hline a $(\AA ̊)$ & $22.1770(3)$ \\
\hline$b(\AA ̊)$ & $14.66804(16)$ \\
\hline c $(\AA ̊)$ & $15.3946(2)$ \\
\hline$\alpha\left({ }^{\circ}\right)$ & 90 \\
\hline$\beta\left({ }^{0}\right)$ & $99.1136(12)$ \\
\hline$\gamma\left({ }^{0}\right)$ & 90 \\
\hline $\operatorname{Vol}\left(\AA^{3}\right)$ & $4944.54(11)$ \\
\hline Z & 4 \\
\hline$\mu\left(\mathrm{mm}^{-1}\right)$ & 0.428 \\
\hline$F(000)$ & 2228 \\
\hline $\mathrm{P}\left(\mathrm{Mg} / \mathrm{m}^{3}\right)$ & 1.437 \\
\hline Reflections collected & 156660 \\
\hline Independent reflections $\left[R_{\text {int }}\right]$ & $15102[0.0720]$ \\
\hline$\Theta$ range for data collection $\left({ }^{\circ}\right)$ & $3.214-30.548$ \\
\hline GOOF on $F^{2}$ & 1.035 \\
\hline Final $R$ indices & $R_{1}=0.0424$ \\
\hline$[I>2 \sigma(I)]$ & $w R_{2}=0.0957$ \\
\hline$R$ indices & $R_{1}=0.0568$ \\
\hline (all data) & $w R_{2}=0.1027$ \\
\hline Largest diff. peak and hole (e $\left.\AA^{-3}\right)$ & 0.625 and -0.440 \\
\hline Identification code & co_sb8 \\
\hline
\end{tabular}

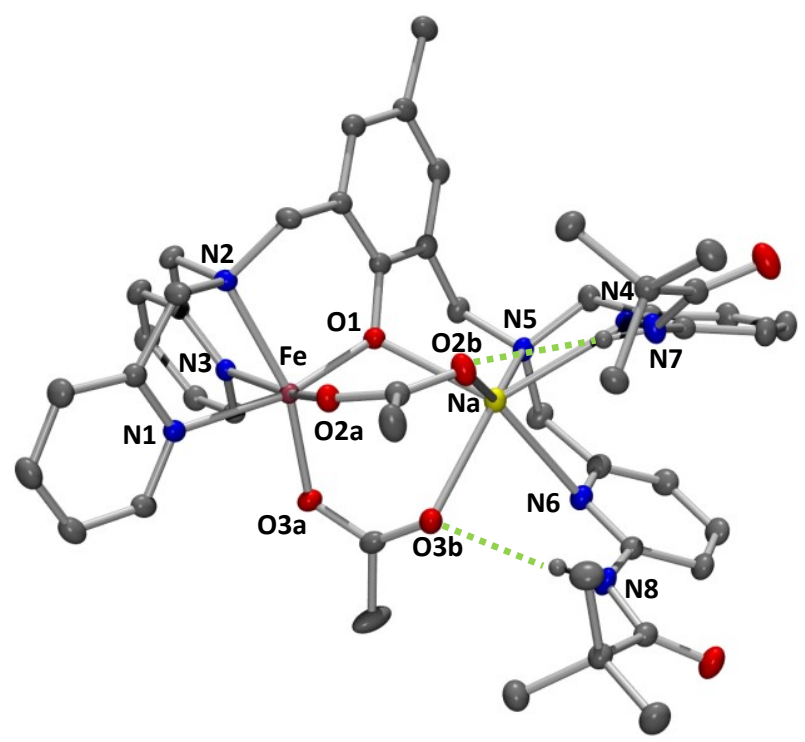

Figure 76: ORTEP plot of [ $\left.\mathrm{Fe}^{\text {III }} \mathrm{Na}\left(\mathrm{H}_{2} \mathrm{SIM}^{1}\right)(\mu-\mathrm{OAc})_{2}\right] \mathrm{PF}_{6}$ with $50 \%$ probability level of thermal ellipsoids showing hydrogen bonds as green, dotted lines. Counter ions, non-coordinated solvent molecules and hydrogen atoms not involved in hydrogen bonding are omitted for clarity. 


\section{Eidesstattliche Versicherung gemäß § 8 der Promotionsordnung der Naturwissenschaftlich-Mathematischen Gesamtfakultät der Universität Heidelberg}

1. Bei der eingereichten Dissertation zum Thema

The Impact of the Second Coordination Sphere in Phosphatase Model Complexes handelt es sich um meine eigenständig erbrachte Leistung.

2. Ich habe nur die angegebenen Quellen und Hilfsmittel benutzt und mich keiner unzulässigen Hilfe Dritter bedient. Insbesondere habe ich wörtlich oder sinngemäß aus anderen Werken übernommene Inhalte als solche kenntlich gemacht.

3. Die Arbeit oder Teile davon werde ich an einer Hochschule des In- oder Auslands als Bestandteil einer Prüfungs- oder Qualifikationsleistung im Rahmen eines binationalen Promotionsverfahren vorlegen.

Titel der Arbeit: The impact of the Second Coordination Sphere in Phosphatase Model Complexes Hochschule und Jahr: The University of Queensland, Australia in 2015

Art der Prüfungs- oder Qualifikationsleistung: Degree of Doctor of Philosophy (PhD)

4. Die Richtigkeit der vorstehenden Erklärungen bestätige ich.

5. Die Bedeutung der eidesstatllichen Versicherung und die strafrechtlichen Folgen einer unrichtigen oder unvollständigen eidesstattlichen Versicherung sind mir bekannt.

Ich versichere an Eides statt, dass ich nach bestem Wissen die reine Wahrheit erklärt und nichts verschwiegen habe.

Ort und Datum

Unterschrift 